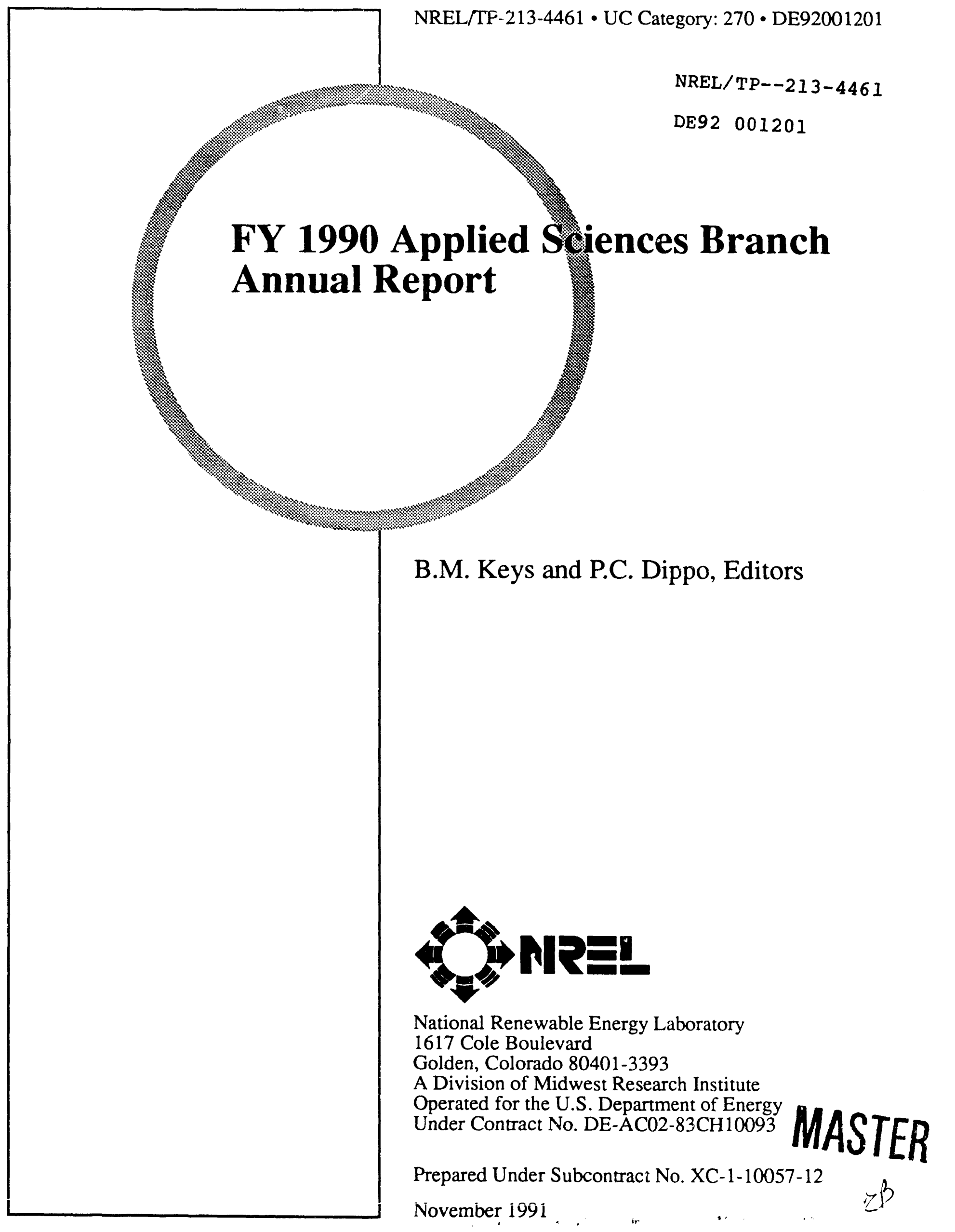


On September 16, 1991, the Solar Energy Research Institute was designated a national laboratory, and Its name was changed to the Natlonal Renewable Energy Laboratory.

\section{NOTICE}

This repon was prepared as an account of work sponsored by an agency of the United States government. Neither the United States government nor any agency thereof, nor any of their employees, makes any warranty, express or implied, or assumes any legal liability or responsibility for the accuracy, completeness, or usefulness of any information, apparatus, product, or process disclosed, or represents that its use would not infringe privately owned rights. Reference herein to any specific commercial product. process, or service by trade name, trademark, manufacturer, or otherwise does not necessarily constitute or imply its endorsement, recommendation, or favoring by the United States government or any agency thereot. The views and opinions of authors expressed herein do not necessarily state or reflert those of the United States government or any agency thereof.

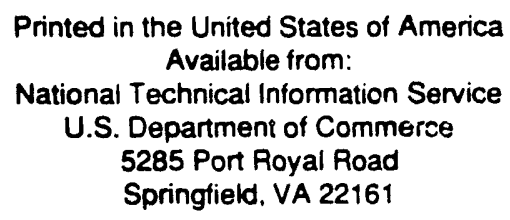

Price: Microfiche A01

Printed Copy A09

Codes are used for pricing all publications. The code is determined by the number of pages in the publication. Information pertaining to the pricing codes can be found in the current issue of the following publications which are generally available in most libraries: Energy Research Abstracts (ERA); Government Reports Announcements and Index (GRA and I); Scientific and Technical Abstract Reports (STAR); and publication NTIS-PR-360 available from NTIS at the above address. 


\section{Table of Contents}

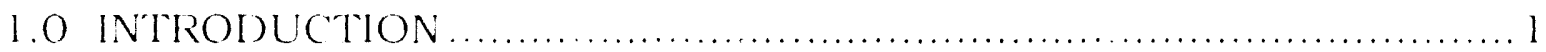

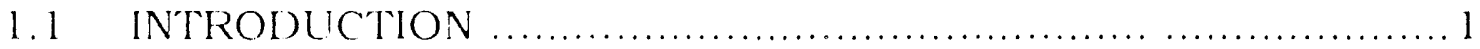

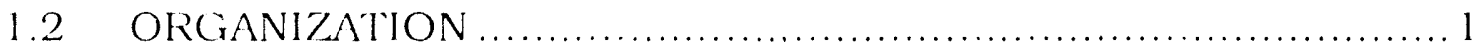

1.2.1 Surface and interface Analysis ............................. 1

1.2.2 Materials Characterization ................................. 2

1.2 .3 Deviee Development ........................................ 2

1.2.4 Electro-Optical Characterization ............................. 2

1.2.5 Advaned Module Testing and Performance ...................3

1.2.6 Cell Performance ...........................................

1.2.7 Surface Interactions. Modification. and Stability ............3

1.2.8 FrIR Spectroseopie and Research Center .................... 4

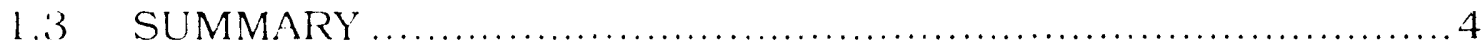

2.0 SURFACE AND INTERFACE ANALYSIS ................................ 5

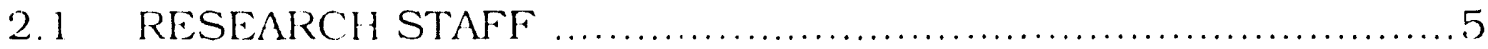

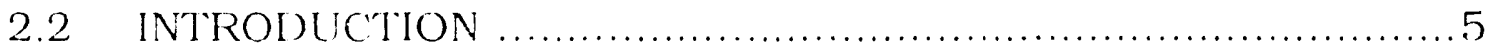

2.3 ANALYTICAL CAPABILITIES .....................................6

2.3.1 Scanning Auger Microprobes ..................................6

2.3.2 Photoemission Systems .................................... 7

2.3.3 Scamning Tumneling Microscope ............................ 7

2.3 .4 Ion Microprobes .......................................... 8

2.3.5 Molectular Beam Epitaxy .................................... \&

2.4 ANALYTICAL TECHNIQUUES ...................................... 8

2.4.1 Auger Electron Spectra ......................................... 8

2.4.2 Multiple-Point Analysis ....................................... 9

2.4.3 Auger Line Scans...............................................

2.4.4 Aiger Compositional Maps ....................................... 9

2.4.5 Auger Line-Shape Analysis ................................... 10

2.4.6 Auge Voltage Contrast ....................................... 10

2.4 .7 XIS Speetra .................................................. 10

2.4 .8 EELS Spectra ............................................... 10

2.4.9 Scanning Tiunneling Microseopy ............................. 11

2.4.10 Secondary Ion Mass Spectrometry ........................... 11

2.4.11 Molcoular Beam Epitaxy (MBE) .......................... 11

2.4.12 Migration Enhanced Epitaxy (MEE) ........................ 11

2.4.13 Reflection High Energy Electron Diffraction (RIEED) and RHEED Intensity Oscillation (RIO) Analysis .......... 12

2.5 EQUIPMENT/ANALYSIS DEVELOPMENT ..................... 12

2.5.1 Elererom Cyclotron Resonance (ECR) Plasma Source .... 12

2.5.2 Migration Enhanced Epitaxy I)evelopment ................. 14

2.5.3 RHEED) Intensity Oscillation Fre(fucency Domain Fhax Transient Analysis .......................................... 17 


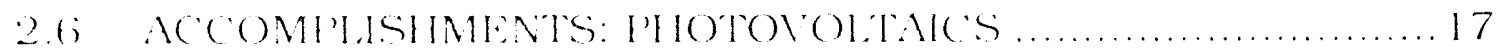

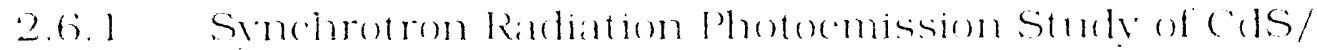
Cunsise Hetorojumetion Formaltion ....................... 17

2.6 .2 Chatraterization of the Native Oxide of Counse 2 Using Synchrotron Ratliation Photocmission ....................29

2.6 .3 Stress Analysis in Lattice Mismatehed Heterostructures ...............................................

2.6.4 Ijifusion in CoTe/CaS Thin Films Studied by SIMS ....37

2.7 AC COMPLISHMENTS: RELATEI RESEARCH AREAS ........ 42

2.7.1 AES Line-Shape Analysis at Gain Bomblaries of Oriented Grained YBa Cu, O

2.7.2 SIMS Investigations of Substrate/Film Interelifiusion in YBa. Cu, O, Films Grown on SrTiO. LaAlO.

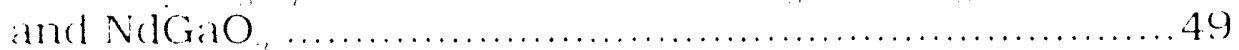

2.8 FUTURE WORK 56

3.O MATERIALS CHARACTERIZATION ...............................59)

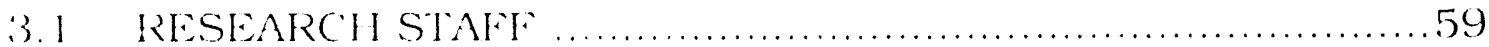

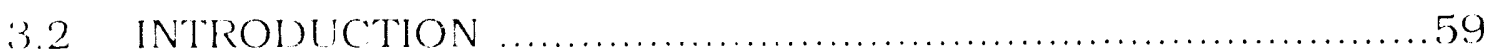

(3.3) ANALYTICAL CAPABILITIES ANI) TECHINIQUES ..............59

3.3.1 Electrom Probe X-ray Mirroanalysis (EPMA) ..............59

3.3.2 Transmission Electron Microscopy (TFM) .................60

3.3.3 Seanning Electron Microscopy (SEM) .....................60

3.4 ACCOMPLISHMENTS: PHOTOVOLTAICS .....................6l

3.4.1 SEM Investigation of Hrelrogen Passivation in

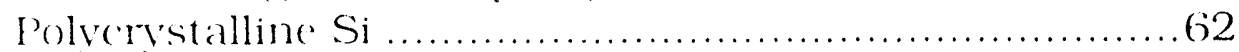

3.4.2 The Characterization of III-V Semicondenetors

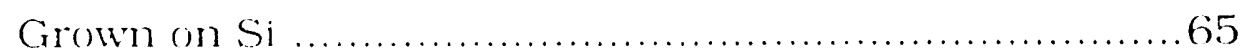

3.4.3 SEM Studies of Crvitalline CulnSe

3.4.4 SEM Studies of Thin-film Polvervatalline CulnSe, .......73

3.4.5 Phosphorms-loper Epitaxial ColTe Thin Films ............. 73

3.4.6 Microstrueture of Polyerystalline CotTe Thin Films ...... 76

3.4.7 TEM Investigation of Hvelrogen-mplanted

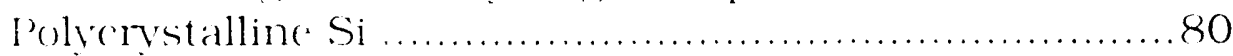

3.5 ACCOMPIISHMENTS: REIATEI) RESEARCH AREAS ..........

3.5 .1 Analysis of Hoh-Temperature Suprerondurting

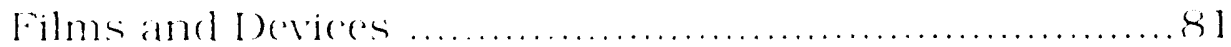

3.5 .2 The Microstruteture of Leatl Zirenate Titamate

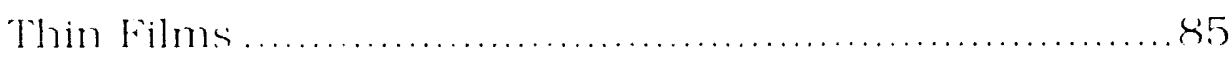

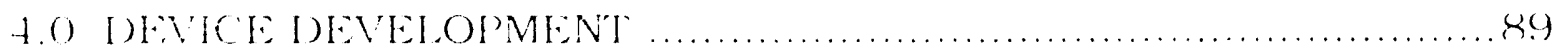

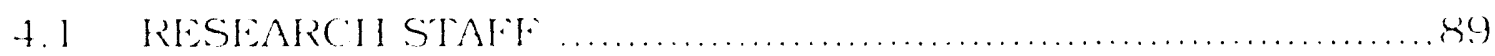

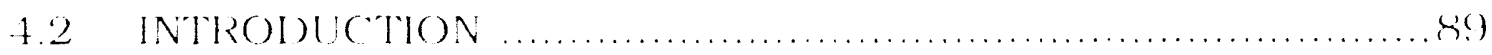

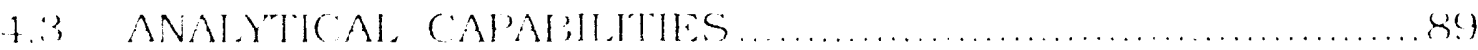

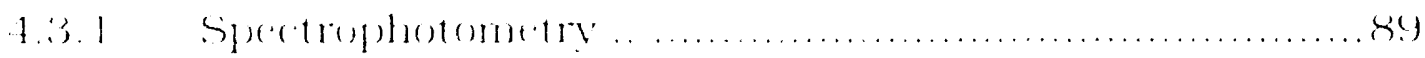


4.3.2 Ellipsometry ................................................. 90

4.3.3 Hall Probe ........................................................ 90

4.3.4 Admittance Spectroscopy .................................... 90

4.3.5 Contact Resistance ........................................ 91

4.4 DEVICE TECHNOLOGY ..................................................

4.4.1 Grid Optimization ............................................ 91

4.4.2 Antireflection Coatings (ARC) ................................92

4.4.2.1 Development of a Dedicated ARC Deposition

System ...................................................992

4.4.2.2 Use of $\mathrm{Al}_{2} \mathrm{O}_{3}$ as an Intermediate Refractive Index ARC Material ..............................................992

4.4.2.3 Combination Front- and Back-Side ARC Modeling and Fabrication ....................................... 93

4.4.2.4 ARC for Use With Entech Covers ...................... 93

4.4.2.5 Entech Covering Technology Development ...........93

4.4.2.6 Ellipsometry ........................................... 94

4.4.3 Solar Cell Fabrication ........................................... 94

4.4.3.1 Clean Room ........................................... 94

4.4.3.2 Photolithography Photomask Pattern Generation and Mask Alignment ..................................... 95

4.4.3.3 Metallization ............................................ 95

4.4.3.3.1 Photolithographic Patterning for Metallization .......................................96 96

4.4.3.3.2 Adhesion of Metallization ......................97

4.4.3.3.3 Specific Contact Resistance ( $\mathrm{rc}$ ) of Metallization ........................................99

4.4.3.3.4 Diffusion of Metallization ..................... 100

4.4.4 Solution Growth of CdS ........................................ 101

4.4.4.1 Device Fabrication Procedure ......................... 101

4.4.4.2 Initial results .......................................... 101

4.5 EQUIPMENT/ANALYSIS DEVELOPMENT ........................ 102

4.5.1 Sputter Deposition Systems ..................................... 102

4.5.1.1 ULTEK System Modification ......................... 102

4.5.2 Vacuum Evaporation Systems ............................... 103

4.5.2.1 NRC' 3115 System .................................... 103

4.5.2.2 Metallization System .................................. 103

4.5.3 Plasma Exposure Systems ................................... 103

4.5.3.1 Technics Planaretch II System ...................... 103

4.5.3.2 Technics Model 500 II ................................... 104

4.5.4 Spectrophotometry ............................................ 104

4.5.4.1 Measurement using the Cary 2300

spectrophotometer ..................................... 104

4.5.4.2 Nitrogen purging ...................................... 105

4.5.4.3 Photoemission measurements ....................... 105

4.5.4.4 Software development .............................. 105 
4.5.5 Spectroscopic Ellipsometry ............................ 106

4.5.5.1 Design Features of the Ellipsometer ................ 106

4.5.5.2 Applications of the ellipsometer .................... 108

4.6 ACCOMPLISHMENTS: PHOTOVOLTAICS ....................... 11 14

4.6.1 Low-bandgap, InP-based solar cells ....................... 114

4.6.1.1 Introduction .......................................... 114

4.6.1.2 GaxIn As- $\mathrm{As}_{\mathrm{y}} \mathrm{P}_{1-\mathrm{y}} / \mathrm{InP}$ Solar Cells .................... 117

4.6.1.3 Summary ............................................. 122

4.6.2 Development of CdTe Thin Film Cells ....................... 122

4.6.2.1 Background ......................................... 122

4.6.2.2 Objectives ........................................... 122

4.6.2.3 CdTe Film Deposition ................................ 122

4.6.2.4 Film properties ..................................... 123

4.6.3 CulnSe ${ }_{2}$ Single Crystals .................................. 123

4.6.3.1 Photoluminescence of $\mathrm{n}$ and $\mathrm{p}$-type Surfaces ..... 124

4.6.3.2 Spectroscopic atomic imaging....................... 127

4.6.3.3 Surface processing effects ......................... 127

4.6.3.4 Interface Characterization .......................... 130

4.6.3.5 Summary ............................................ 136

4.6.4 Spectrophotometery Measurements ........................ 137

4.6.4.1 Measurements on EVA samples..................... 137

4.6.4.2 Measurements on ISET CuInSe2 films .............. 137

4.6.4.3 Measurements on ARCO ZnO ........................ 138

4.6.4.4 Internal Quantum Efficiency of InP Cells ........... 138

4.7 ACCOMPLISHMENTS: RELATED RESEARCH AREAS .......... 138

4.7.1 NASA Interagency Contract No. C-3000-K ................. 138

4.7.1.1 Pure Hydrogen Plasma Treatments .................. 138

4.7.1.2 TCO Layer Improvement ............................. 139

4.7.1.3 Large Area $4 \mathrm{~cm}^{2}$ Cell Development ................. 139

4.7.1.4 Small Scale Production of $4 \mathrm{~cm}^{2}$ ITO/InP Cells .. 140

4.7.2 Spire Corporation .......................................... 141

4.7.2.1 Effect of Ag Stress on the $\mathrm{Cr} / \mathrm{Au} / \mathrm{Ag}$ Contact ..... 141

4.7.2.2 Diffusion of $\mathrm{Cr}$ and $\mathrm{Au}$ into InGaAs and InP ..... 142

4.7.2.3 Alternative Metallization Stack ....................... 142

4.7.3 NRL ....................................................... 142

4.7.4 Varian Associates ............................................. 146

4.7.5 DDF Projects .............................................. 148

4.7.5.1 Heteroepitaxial InP solar cells on GaAs

and Si Substrates....................................... 148

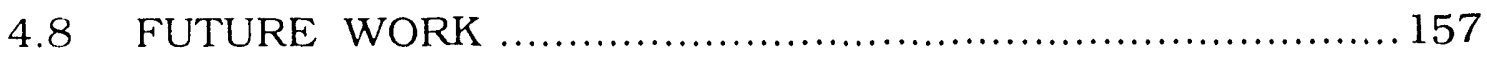

5.0 ELECTRO-OPTICAL CHARACTERIZATION ............................ 161

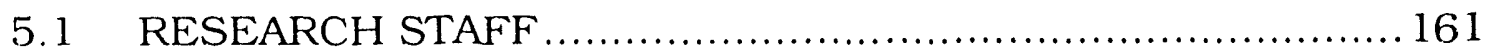

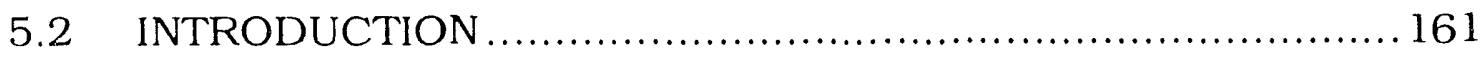

5.3 ANALYTICAL CAPABILITIES AND TECHNIGUES ............... 161 
5.3.1 The Laser Characterization Facility ........................ 161

5.3.1.1 Time-Resolved Photoluminescence .................. 161

5.3.1.2 Measurement Diagnosis ............................... 163

5.3.1.3 Photoluminescence Spectroscopy ................... 164

5.3.1.4 Diffusion Time-Of-Flight ............................ 164

5.3.1.5 Deep-Level Transient Spectroscopy (DLTS) ........ 165

5.3.2 Electrical Characterization of High-Temperature

Superconductors ............................................ 165

5.3.2.1 Resistance-Temperature Measurements ........... 165

5.3.2.2 Meissner Mutual Inductance .......................... 165

5.3.2.3 Critical Current Apparatus ............................. 166

5.4 ACCOMPLISHMENTS: PHOTOVOLTAICS ........................ 166

5.4.1 Time-Resolved Photoluminescence ......................... 166

5.4.1.1 AlGaAs/GaAs Quantum Well Structures ........... 166

5.4.1.2 Optimization of Growth Conditions for PV Devices: Spire Corporation ....................... 166

5.4.1.3 AlGaAs Mlaterials Development:

Research Triangle Institute ......................... 167

5.4.1.4 AlGaAs Materials Development:

Kopin Corporation ..................................... 168

5.4.1.5 Polycrystalline CdTe: Photon Energy ................. 169

5.4.2 Photoluminescence Spectroscopy ........................... 169

5.4.3 Time-of-Flight (TOF) ....................................... 170

5.4.4 Deep-Level Transient Spectroscopy (DLTS) ................ 171

5.5 ACCOMPLISHMENTS: RELATED RESEARCH AREAS .......... 172

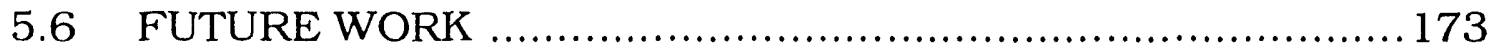

6.0 ADVANCED PHOTOVOLTAIC MODULE PERFORMANCE

TESTING AND RELIABILITY RESEARCH …............................ 175

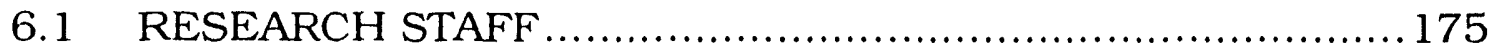

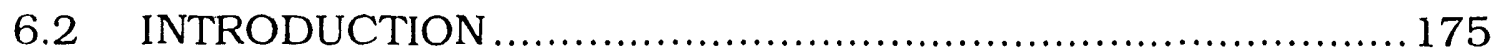

6.3 ANALYTICAL CAPABILITIES AND TECHNIQUES ................ 176

6.3.1 PV Module Outdoor Performance Characterization and Evaluation Laboratory ................................ 176

6.3.2 PV Module Outdoor Stability and Energy Performance Test and Evaluation Laboratory ........................... 178

6.3.3 Simulation Test and Evaluation Laboratory .............. 179

6.3.4 PV Module Encapsulation Research Laboratory .......... 179

6.3.5 Accelerated Environmental Test and Evaluation Laboratory .................................................. 179

6.4 ACCOMPLISHMENTS: PHOTOVOLTAICS ....................... 180

6.4.1 Module and Submodule Characterization Testing ....... 180

6.4.2 Module Stability Performance Testing and Evaluation . 181

6.4.3 Interim Qualification Test for Terrestrial Photovoltaic Thin-Film Flat-Module Modules .......................... 183 
6.4.5 Ethylene-Vinyl Acetate (EVA) Encapsulant

Degradation ................................................. 183

6.4.5.1 EVA Formulation and Encapsulation................ 185

6.4.5.2 EVA Materials Studied ................................ 186

6.4.5.3 Analytical Procedure/Methods ....................... 186

6.4.5.4 Thermal Stability of EVA ............................ 186

6.4.5.5 EVA Gel Uniformity ................................ 188

6.4.5.6 Quality of Raw EVA Material (Elvax 150) ........... 189

6.4.5.7 Thermal Processing Effects on EVA ................. 189

6.4.5.8 Characterization of Degraded EVA ................... 189

6.4.5.9 Structural Analysis ..................................... 193

6.4.5.10 Uniformity of EVA Degradation...................... 194

6.4.5.11 Effects of EVA Yellowing on Solar Cell Efficiency 194

6.4.5.12 Conclusions ............................................ 196

6.4.5.13 Accomplishments ................................... 198

6.4.6 Photovoltaic Module Reliability Workshop ................. 198

6.4.7 Standards Development Activities ........................... 199

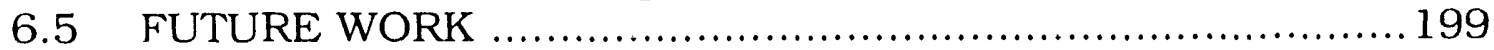

7.0 CELL PERFORMANCE CHARACTERIZATION ...........................201

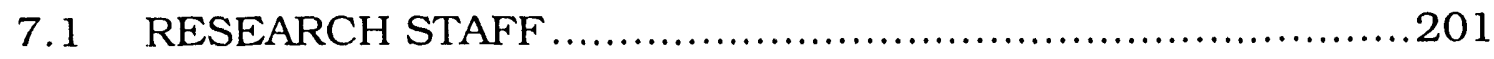

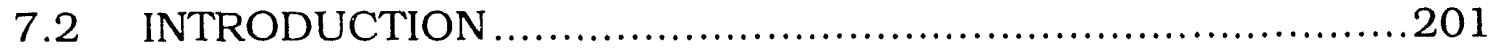

7.3. ANALYTICAL CAPABILITIES AND TECHNIQUES ...............201

7.3.1 Efficiency with Respect to Standard Reporting Conditions .....................................................201

7.3.2 Spectral Response Measurement System .................204

7.3.3 Primary Reference Cell Calibration Facility ................204

7.4 EQUIPMENT/ANALYSIS DEVELOPMENT .......................205

7.4.1 Modeling of the Direct-Beam Solar Spectrum from Spectroradiometric Measurements ..........................205

7.4.2 0.1 to 200 Suns Continuous Illumination Concentrator Measurement ................................208

7.4.3 PV Performance Modeling .................................208

8.0 SURFACE INTERACTIONS, MODIFICATION, AND STABILITY

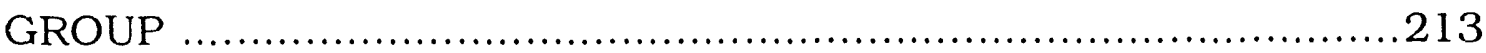

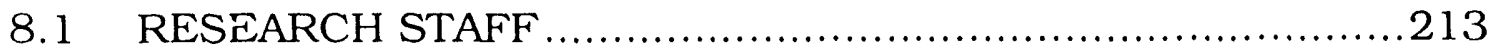

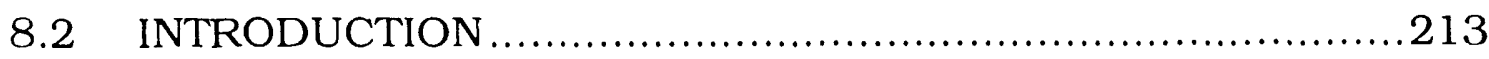

8.3 ANALYTICAL CAPABILITIES ................................... 214

8.4 ANALYTICAL TECHNIQUES ................................... 214

8.4.1 Exposing Solid Surfaces to High Flux Densities of Solar Radiation .......................................... 214

8.4.2 Techniques for Preparing and Studying Organized Molecular Assemblies with Metal Overlayers .............215

8.4.3 Water Adsorption/Desorption Using a Quartz 
Crystal Microbalance .........................................2 217

8.6 ACCOMPLISHMENTS: PHOTOVOLTAICS .........................2 218

8.7 ACCOMPLISHMENTS: RELATED RESEARCH AREAS .........219

8.7.1 Solar Induced Surface Transformations and

Modifications (SISTM) ........................................2 219

8.7.1.1 Introduction ......................................... 219

8.7.1.2 Phase Transformation Hardening ...................220

8.7.1.3 Cladding .............................................220

8.7.1.3 Radiative Joining .................................22 21

8.7.1.4 Self-Propagating, High-Temperature Synthesis

[SHS] Reactions .....................................222

8.7.1.4 Thin-Film Growth in Controlled Atmospheres ....222

8.7.2 EXPS and ISS of Organized Molecular

Assembly/Copper Interfaces ...............................2223

8.7.2.1 Accomplishments ....................................224

8.7.2.2 Benefits to Solar Energy Research .................224

8.7.3 Advanced Desiccant Materials Research ...................225

8.7.3.1 Accomplishments ....................................225

8.7.3.2 Benefits to Photovoltaic Research ...................226

8.7.4 Evaluation Criteria and Test Methods for

Electrochromic Windows .................................227

8.7.5 Surface Processing Workshop ..............................228

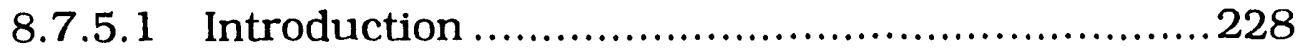

8.7.5.2 Accomplishments .....................................2230

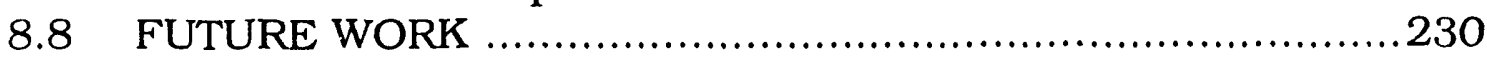

9.0 FTIR SPECTROSCOPIC RESEARCH AND SERVICE CENTER .......233

9.1 RESEARCH STAFF ....................................................233

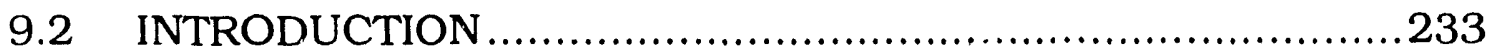

9.3 ANALYTICAL CAPABILITIES .......................................2235

9.4 ANALYTICAL TECHNIGUES ........................................ 235

9.4.1 FTIR Advantage: Mechanical Simplicity

(One Moving Part) ...............................................2236

9.4.2 FTIR Advantage: Increased Speed and Sensitivity (Felgett Advantage) ..........................................2236

9.4.3 FTIR Advantage: Greater Optical Throughput (Jayuinot Advantage) .........................................236

9.4.4 FTIR Advantage: Internal Laser Frequency Reference (Connes Advantage) .............................................2237

9.4.5 FTIR Advantage: Freedom from Stray Light and Sample Emission Artifacts ..................................2237

9.4.6 Discussion of Analytical Techniques ......................2237

9.4.7 FTIR Microscope .............................................238

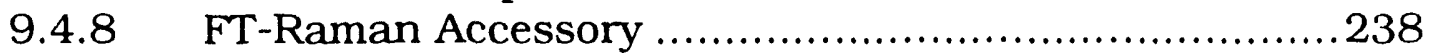

9.4.9 Far-IR FTIR Spectrophotometer ..........................239 
9.5 EQUIPMENT/ANALYSIS DEVELOPMENT .........................239

9.6 ACCOMPLISHMENTS: PHOTOVOLTAICS ........................240

9.7 ACCOMPLISHMENTS: RELATED RESEARCH AREAS ..........241

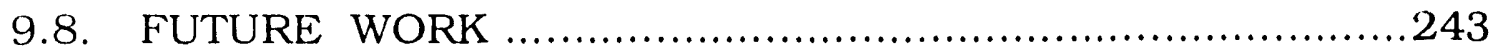

APPENDIX A: STAFF BIOGRAPHIES .....................................245 APPENDIX B: COOPERATING LABORATORIES …......................251 APPENDIX C: APPLIED SCIENCES BRANCH BIBLIOGRAPHY ….......255 APPENDIX D: MAJOR INSRTUMENTATION ANDTEST EQUIPMENT . . 290

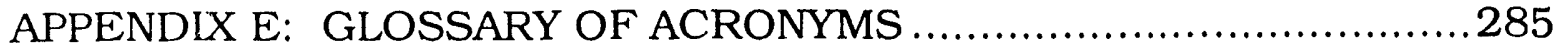




\subsection{INTRODUCTION}

\subsection{INTRODUCTION}

The Applied Sciences Branch actively supports the advancement of DOE/ SERI goals for the development and implementation of the solar photovoltaic technology. The primary focus of the laboratories is to provide state-of-theart analytical capabilities for materials and device characterization and fabrication. The branch houses a comprehensive facility which is capable of providing information on the full range of photovoltaic components. A major objective of the branch is to aggressively pursue collaborative research with other government laboratories, universities, and industrial firms for the advancement of photovoltaic technologies. Members of the branch disseminate research findings to the technical community in publications and presentations. Along with detailed accounts of the individual group's accomplishments over the past year, this report contains five appendices which detail the following information: the capabilities and technical qualifications of the staff (Appendix A); the outside laboratories with which members of the branch have collaborated in the past year (Appendix B); technical publications which have emanated from the branch in the past fiscal year (Appendix C); major equipment and capabilities of the various groups (Appendix D); and a partial list of acronyms used in this report (Appendix E).

\subsection{ORGANIZATION}

The Branch has undergone one organizational change since the previous reporting period. This last year saw the formation of of the FTIR Spectroscopic and Research Center. This center will significantly enhance the research already taking place in this branch, as well as the institute and the photovoltaics community at large.

The Applied Scinces Branch now encompasses eight coordinated research groups, providing integrated research and development which covers all aspects of photovoltaic materials/devices characterization, fabrication and modeling. A summary of the capabilities of each group is given in Appendix D. The support research and services available are explained briefly in the following paragraphs.

\subsubsection{Surface and Interface Analysis}

The work of this group entails determinations of the chemical, compositional, and microelectrical characteristics of materials, surfaces, and device interfaces with extremely high depth and spatial resolution. Techniques include secondary ion mass spectrometry (SIMS): Auger electron spectroscopy 
(AES); scanning Auger microscopy (SAM); X-ray photoelectron spectroscopy (XPS); electron energy loss spectroscopy (EELS); electron-stimulated desorption spectroscopy (ESD); electron-beam-induce d current and voltage (EBIC and EBIV); Auger voltage contrast (AVC); high-resolution elemental and ionic mapping, including volume-indexing; cooperative soft $x$-ray synchrotron-source analysis; scanning tunneling microscopy (STM): and molecular beam epitaxy (MBE).

\subsubsection{Materials Characterization}

This group employs advanced microscopy and microanalytical techniques in the determination of crystallographic, defect topographic, compositional, and microelectrical properties of photovoltaic materials and devices. These include electron probe microanalysis (EPMA) with energy dispersive and wavelength dispersive spectroscopy (EDS and WDS); scanning electron microscopy (SEM) to liquid helium temperatures; scanning transmission electron microscopy (TEM and STEM); voltage contrast; electron-beaminduced current and voltage characterization; electron diffraction; electron channeling; image analysis: X-ray diffraction; X-ray fluorescence; microcathodoluminescence; cooperative high-resolution interface analysis using high-voltage electron microscopy; and complete sample preparation facilities, including ion etching with integral SIMS analysis.

\subsubsection{Device Development}

This group provides modeling and fabrication support for the development of cell structures, including; diagnostir device preparation, optical conducting film fabrication and studies, thin-film and space cells, and computer analysis and design. Capabilities include; precision sputtering equipment for deposition of optical films, antireflection coatings, metallizations, transparent conductors, etc.; research clean room facilities; III-V cell processing capabilities; photolithography and mask design and fabrication; InP-alloy metal-organic chemical vapor deposition (MOCVD); optical and electrical charactcrization for films and coatings; plasma deposition; photoconductivity, and ellipsometry; contact resistance determinations; and computer modeling programs for optical coatings and solar cell design.

\subsubsection{Electro-Optical Characterization}

The work of this group involves determination of the electrical and optical properties of materials and solar cells; and laser spectroscopies, including photoluminescence and specialized determinations of minority-carrier properties. Capabilities and techniques include capacitance-voltage, conductance-voltage, and temperature dependences; Hall effect/van der Pauw measurements; laser scanning; deep-level transient spectroscopy 
(DLTS): photoluminescence (PL); minority-carrier lifetime (to the picosecond range) and diffusion-length measurements; determination of minoritycarrier mobility by time-of-flight (TOF) measurements; picosecond laser spectroscopies; optical spectrophotometry; and ellipsometry. In addition, new facilities have been developed for the electrical characterization of high temperature superconducting materials. This includes measurements of resistance versus temperature ( $R$ vs. T), Meissner Mutual Inductance (MMI), and critical current.

\subsubsection{Advanced Module Testing and Performance}

The work of this group includes testing and investigations of module and submodule performance and reliability/lifetime properties, both under simulated and actual sunlight conditions. Capabilities include outdoor and indoor testing for stability and reliability; module arıd submodule efficiency and energy measurements; environmental and temperature/humidity test chambers for module conditioning and exposure; accelerated lifetime testing and simulations; module infrared (IR) thermography and electrical insulation (voltage breakdown and current leakage) array system performance and load simulator, extensive outdoor, and user-oriented cooperative test facilities.

\subsubsection{Cell Performance}

The work of this group includes calibrating and maintaining primary photovoltaic reference cells; performing efficiency measurements under standard reporting conditions; calibrating secondary reference cells for the photovoltaic community; evaluating photovoltaic device parameters. Capabilities and techniques include current versus voltage measurements as functions of temperature, spectral and total irradiance; spectral response as functions of temperature, voltage bias, spectral and total irradiance; laser

photo response mapping. and detection of measurement related artifacts present in other current versus voltage measurement systems.

\subsubsection{Surface Interactions, Modification, and Stability}

The work of this group involves the fabrication, modification, and characterization of surfaces and interfaces to better understand their properties and structures. Capabilities include surface spectroscopies (ion scattering spectrometry (ISS). XPS, SIMS, fast atom bombardment (FAB), AES. SAM): Fourier Transform Infrared spectroscopy (FT-IR); contact angle measurements, ultramicrogravimetry, and quartz crystal microbalance measurements. The group is able to perform reactions in the high pressure chamber on the LHS-10 system, or in a solar furnace. In addition, the group has experience in the preparation and characterization of polymer films and organized molecular assemblies (OMA). 


\subsubsection{FTIR Spectroscopic and Research Center}

This is an Institute-wide service center providing Fourier-transform infrared (FTIR) spectroscopic analysis and experimental design for determination of the covalent or lattice bonding structure of materials, the effects of chemical reactions on materials, as well as the chemical composition and sub-ppm levels of impurities or dopants in samples. Techniques include: highresolution, mid-infrared FTIR spectroscopy in transmittance, specular reflectance, diffuse reclectance, and attenuated total reflectance (ATR) sampling modes using $5 \mathrm{~cm}^{2}, 0.5 \mathrm{~cm}^{2}$, and fiber-optic ATR probes, along with environmental control for in-situ analysis and measurement of reaction kinetics for most sampling modes; FT-Raman spectroscopy in both reflective and refractive modes; and FTIR microscopy for obtaining mid-infrared transmittance or refectance spectra of sample areas as small as $10 \times 10 \mu \mathrm{m}$. and far-infrared spectroscopy $\left(650-50 \mathrm{~cm}^{-1}\right)$ with transmittance and diffuse reflectance sampling modes.

\subsection{SUMMARY}

These laboratories have been established to support the US Photovoltaic Program. Researchers involved in the Program, as well as the Photovoltaic Industry and internationai research groups, are invited to utilize and interact with the Applied Sciences Branch for the advancement of their technologies. 


\title{
2.0 SURFACE AND INTERFACE ANALYSIS
}

\section{$2.1 \quad$ RESEARCH STAFF}

\author{
A.J. Nelson, Senior Scientist and Group Leader \\ P. Sheldon, Senior Scientist \\ S.E. Asher, Senior Scientist \\ A.B. Swartzlander-Franz, Associate Scientist \\ S. Gebhard, Postdoctoral Research Associate \\ L.L. Kazmerski, Principal Scientist
}

\subsection{INTRODUCTION}

The chemical, compositional, and microelectrical properties of photovoltaic materials and devices are analyzed in these laboratories. Staff researchers support both internal and external researchers and provide timely analysis on fundamental compositional problems that limit the performance and operating lifetime of specific material systems and photovoltaic cells/ modules.

The Surface and Interface Analysis Laboratories specialize in analyzing individual layers of a photovoltaic material, device or module. Examining materials a monolayer at a time, researchers determine the types of impurities present, their chemical or electrical makeup, and/or their relative or absolute concentration and location. The staff correlates the presence of impurities with the electrical properties and performance of the cell or module.

Using sophisticated surface microprobe and epitaxial growth systems, the staff can conduct complementary, compositional and electrical analyses with multiple techniques applied concurrently or consecutively to the same area as well as grow diagnostic device structures. Additionally, research scientists have extensive experience in photovoltaic device physics, epitaxial growth and surface analysis, providing unique capabilities in the area of device characterization.

Recent activities in this research area include continued development of Auger line-shape analysis for determining chemical bonding at surfaces and grain boundaries in photovoltaic and superconducting materials. In addition to the in-house photoemission activities, several experiments were completed at the Synchrotron Radiation Center. Specifically, (1) the band diagram for the CdS/CuInSe ${ }_{2}$ heterojunction and (2) initial surface chemistry of CuInSe were directly determined from these photoemission measurements. SIMS is continuing to be used to characterize the chemistry of the high $\mathrm{T}$. 
superconductor/substrate interfaces. Atomic-level scanning tunneling microscopy (STM) was used as well.

The molecular beam epitaxy (MBE) laboratory utilizes the controlled growth environment and in-situ diagnostics available with MBE to study problems relevant to photovoltaics. This work, performed in collaboration with both internal and external researchers, falls into two categories. The first of these. is to support the Applied Sciences Branch by designing and growing electrical and structural diagnostic devices for instrument qualification. For example: (1) to provide electrically characterized standards necessary for quantitative SIMS analysis; (2) to provide precisely grown layered structures for sputter rate determination and depth resolution qualification for AES and SIMS analysis; and (3) to provide specialized structures for instrument development (DLTS, CV, and minority carrier lifetime measurements). The second category involves internal research which over the past year has focused on MBE instrument development. We have expanded both our insitudiagnostic and growth capabilities through the development of advanced frequency domain RHEED oscillation analysis techniques (used to quantify flux transients) and the development of an automated migration enhanced epitaxy (MEE) growth system. In addition, we have expanded our research efforts in analyzing stress variations and their effect on heteroepitaxial photovoltaic materials.

Secondary ion mass spectrometry (SIMS) was used to study the diffusion of $\mathrm{S}$ and Te at the CdTe/CdS heterointerface. SIMS was also used to characterize the diffusion of substrate elements into thin films of high $T_{\text {. }}$ superconductors.

\subsection{ANALYTICAL CAPABILITIES}

Appendix D provides a listing of the major instrumentaion and test equipment, their features, and specifications.

\subsubsection{Scanning Auger Microprobes}

The Perkin-Elmer/Physical Electronics Models 590 and 600 Scanning Auger Micropı obes provide scanning Auger microscopy (SAM), Auger electron spectroscopy (AES), and secondary ion mass spectrometry (SIMS). Each system uses AES to perform compositional analysis and compositional depth profiling of selected elements by recording the Auger intensities as a function of energy or sputtering time, respectively. SAM uses AES in a raster scanning mode to provide an image of the lateral distribution of elements on the sample surface. SIMS detects secondary ions sputtered from the surface and identifies elements or molecular species by their mass-to-charge ratios. 
The two systems are distinguished by different spatial resolution capabilities and examination techniques. The Model 600 is used in cases where fine spatial resolution $(\sim 300 \AA)$ is needed. The Model 590 is operated when spatial resolution is less critical. Other distinguishing features include the ability of the Model 600 to operate at lower current levels. All systems have EBIC (electron beam induced current), EBIV (electron beam induced voltage) and AVC (Auger voltage contrast) functions for performing microelectrical análysis. Both systems also have a sample fracturing stage for examining the internal properties of samples without exposing them to atmospheric contamination. The Model 600 also has the capability to cool a sample to $100 \mathrm{~K}$ during analysis - a feature used when analyzing samples which are composed of high vapor pressure elements (e.g., $\mathrm{HgCdTe}, \mathrm{TlBaCaCuO}_{x}$ ) - and the capability to heat a sample to $600^{\circ} \mathrm{C}$ for in-situ annealing.

\subsubsection{Photoemission Systems}

The scanning AES capability of the Perkin-Elmer/Physical Electronics Model 550 XPS/Auger system has greater energy resolution than the 590 or the 600. This is particularly functional in X-ray photoelectron spectroscopy (XPS), electron energy loss spectroscopy (EELS), and ultraviolet photoelectron spectroscopy (UPS). XPS is used for performing chemical evaluations that require molecular structure and chemical bonding information. Data on valence band electron energy levels are obtained with the UPS. Band gap data can be acquired when UPS is used in conjunction with EELS. The system also has a quadrapole mass analyzer (QMA) for monitoring the partial pressure of dosing or residual gases in the system during analysis.

Research is aiso being conducted in the Aladdin ring ( $1 \mathrm{GeV})$ located at the Synchrotron Radiation Center of the University of Wisconsin-Madison. Soft $\mathrm{X}$-ray photoemission experiments ( $50 \mathrm{meV}$ energy resolution) are being used to further study surface and interface reactions of PV materials in order to understand some of the more difficult problems requiring the higher energy resolution.

\subsubsection{Scanning Tunneling Microscope}

The RHK and McAllister Technical Services' Scanning Tunneling Microscopes (STM) provide atomic resolution images of the electronic properties of metal and semiconductor surfaces in an ultra-high vacuum (UHV) environment. Computer control of the STM allows researchers to obtain atomic-scale resolution (1-2 $\AA$ ) images of surface topography, electronic structure (including work function maps) of a surface and location of absorbates or impurities on a surface. 


\subsubsection{Ion Microprobes}

The Cameca IMS-3f Ion Microprobes are high resolution SIMS instruments dedicated primarily to the analysis of elemental distribution (mapping) and high sensitivity (ppm-ppb) depth profiling. These systems are approximately two to three orders of magnitude more sensitive to elements than the QMAbased SIMS instruments. They have greater atomic mass resolution and are capable of faster sputtering rates for depth profiling. These systems can detect dopants and trace elements to $1 \times 10^{15} \mathrm{~cm}^{-3}$, and perform dopant depth profiling. Using their ion mapping capabilities, the systems can determine the location and amount of species present on a sample surface. Moreover, SIMS is the only surface analysis technique that directly detects the presence of hydrogen in materials.

\subsubsection{Molecular Beam Epitaxy}

The Perkin-Elmer/Physical Electronics MBE400 system is used to grow various III-V based structures, in a controlled environment, for photovoltaic and diagnostic applications. The system contains the UHV chambers used for sample introduction, surface analysis and epitaxial growth, respectively. In general, MBE/MEE utilizes low growth rates and low substrate temperatures to achieve abrupt interfaces with minimal interdiffusion. These attributes allow device structures to be tailored to a specific application. The MBE/MEE system has several in-situ analysis techniques that allow quantitative analysis of the substrate and epitaxial layers. both before and during growth. These in-situ techniques include: scanning Auger electron spectroscopy (AES), residuai gas analysis, reflection high energy electron diffraction (RHEED), and RHEED intensity oscillation (RIO) analysis. AES analysis allows for detection and quantification of surface contaminants that can adversely affect the growth and nucleation of the epitaxial layer. Likewise, in -situ RHEED analysis can yield information on the substrate surface quality prior to growth as well as information on the epitaxial surface layer morphology. crystallinity, and reconstruction, during growth. RHEED intensity oscillations can be used to calibrate growth rates and alloy compositions with monolayer accuracy. These analytical techniques allow the operator to optimize both substrate preparation techniques and epitaxial growth conditions.

\subsection{ANALYTICAL TECHNIGUES}

\subsubsection{Auger Electron Spectra}

Auger spectra are used to identify surface constituents or to determine the composition of specific surface features. Spectra can be generated in two ways, the point analysis mode. wherein a stationary beam probes a selected 
point, or the area-averaged mode where the beam is scanned over the surface at television (TV) rates. With either method, spectral data are collected and stored in the $\mathrm{N}(\mathrm{E})$ format (i.e., the electron energy analyzer is stepped through a selected energy range and the electron signal level at each step is measured, stored, and can be scanned repetitively to obtain the desired signal level). Mathematical routines such as differentiation, smoothing, expansion, and background subtraction can then be applied to the stored data. A multiplex format, whereby selected energy regions containing Auger peaks of interest are scanned, is also available. This format is used for automatic data acquisition and quantification (e.g.. sputter depth profiling) and uses computer routines along with elemental sensitivity factors derived from standards. A.uger analysis performed simultaneously with ion beam sputter etching provides information on elemental composition as a function of depth into the material and is termed "compositional depth profiling."

\subsubsection{Multiple-Point Analysis}

Multiple-point analysis is appropriate when several features within the imaged area are of interest. Using an image stored on the storage monitor as a guide and the scanning system controls, the coordinates for up to 20 separate points within the imaged area are selected by the operator and stored in memory. The operator then specifies acquisition parameters and the system automatically sequences through the points, performs the analyses, and stores the data for processing and printout.

\subsubsection{Auger Line Scans}

An Auger line scan shows the relative concentration of a specific element along a line across the specimen. The electron beam is stepped point-bypoint along the selected line. The Auger peak height and background level are measured and the difference is stored. The system can scan up to 5 vertical or horizontal lines and will monitor up to 10 elements per line in a single sequence. Stored data can be printed or superimposed on a photomicrograph. Line scans can also be corrected for topography.

\subsubsection{Auger Compositional Maps}

An Auger image, or elemental map, shows the surface distribution of an element. An elemental map is obtained by setting the analyzer to a specfic Auger peak energy and scanning the electron beam over the selected sample area point-by-point. The peak intensity above the adjacent background at each point is measured and stored for later output. The matrix size as specified by the operator can be up to 250 lines with up to 250 points per line. 
The stored image can be photographed with a 4- to 256-level gray-scale format or a pseudo color format.

\subsubsection{Auger Line-Shape Analysis}

Auger line-shape analysis involves the resolution of observed changes in KVV, LVV. MVV or NVV Auger transitions due to different chemical environments in a material. AES data is recorded in N(E) mode and corrected for secondary electron background as well as inelastic backscatter. Curve fitting is then accomplished by using a weighted-least-squares fit of a Gaussian function to the data values by means of stepwise Gauss-Newton iterations. A Gaussian fit to the actual data is used since the data are taken in a pulse count mode and the pulse-height distribution is a Gaussian. In most cases, theoretical Auger line shxpes are used as models for the curve fit parameters.

\subsubsection{Auger Voltage Contrast}

Auger voltage contrast (AVC) involves the measurement of energy shifts (usually on the order of $0.5 \mathrm{eV}$ ) of Auger transitions associated with an internal potential at a $\mathrm{p}-\mathrm{n}$ junction dise to differences in carrier type and concentration. This technique is used to obtain surface potential images of $\mathrm{p}-\mathrm{n}$ junctions. A narrow energy window is set on the low-energy slope of an Auger peak while the electron beam is scanned across the sample in the Auger compositional mapping mode. The small energy differences are measured and displayed as a surface potential map of the $\mathrm{p}-\mathrm{n}$ junction. This technique requires an angle lapped surface.

\subsubsection{XPS Spectra}

$\mathrm{X}$-ray photoelectron spectroscopy is used for elemental identification and quantitative analysis. In addition, more detailed chemicai bonding information is obtained with XPS from high-resolution scans of selected peaks. Chemical bonding information is deduced from changes in peak shapes and peak energy shifts. Furthermore, inhomogeneous broadening of XPS core level spectra can be used to qualitatively indentify disorder phenomena in a material.

\subsubsection{EELS Spectra}

Electron energy loss spectroscopy is used to investigate energy levels at the solid-vacuum interface using a low energy incicient electron beam $(<500 \mathrm{eV})$. EELS involves single particle excitations from filled to empty states including interband transitions between the conduction band and the valence band or 
between surface states. The spectra also contains peaks caused by plasmon excitations.

\subsubsection{Scanning Tunneling Microscopy}

The scanning tunneling microscope is based on the continuous maintainence of the tunnel current that flows between a very sharp metal tip and the surface that is being imaged. The basic STM experiment consists of rastering the tunneling tip over a conductive surface with piezo-ceramic drivers. Quantum tunneling of electrons between the tip and the surface occurs whenever the distance of separation is on the order of the electron wavelength. Several contrast mechanisms are used to image the surface: (1) constant current mode providing a topographic image; (2) constant height mode for relatively flat surfaces; (3) barrier height mode where the tip-tosample distance is modulated by a small sinusoidal displacement; and (4) I/V spectroscopic mode to probe the local density of states at the surface.

\subsubsection{Secondary Ion Mass Spectrometry}

Secondary ion mass spectrometry uses a focussed beam of primary ions $\mathrm{O}_{2}{ }^{+}$ or $\mathrm{Cs}^{+}$) to remove material from a sample surface. A fraction of the material sputtered from the surface is ionized either positively or negatively. The secondary ions are accelerated into a mass spectrometer and analyzed according to their mass to charge ratio. A depth profile is obtained by following the secondary ion signals for chosen elements as successive layers of the sample are removed. Mass spectra are obtained by scanning the magnet through the entire mass range and observing the secondary ion signal. Secondary ion signals are quantified by applying relative sensitivity factors generated from external standards.

\subsubsection{Molecular Beam Epitaxy (MBE)}

MBE is a technique that is used to produce epitaxial thin films with abrupt interfaces and reproducible composition profiles. In its simplest form, MBE growth consists of directing constituent fluxes (in this case group III and group V species) to a heated substrate. Epitaxial growth is obtained when these species have sufficient thermal energy to migrate across the semiconductor surface to the appropriate lattice site. The growth process is governed by many factors, including flux rates, sticking coefficients, and the surface mobilities of the evaporating species.

\subsubsection{Migration Enhanced Epitaxy (MEE)}

MEE is an extension of MBE and is based on the rapid surface migration effect, which is characteristic of $\mathrm{Ga}$ and $\mathrm{Al}$ atoms in an As-free atmosphere 
over a GaAs surface. MEE growth consists of alternately pulsing molecular beams, from effusion cells, by opening and closing shutters at a frequency corresponding to approximately one monolayer of material. For example, in the first cycle of GaAs growth, the number of Ga atoms supplied to the surface in an As-free environment is approximately equal to the number of available sites. The Ga surface migration is enhanced. relative to an As-rich surface, thus producing a single monolayer of Ga without island formation. On the subsequent cycle, $\mathrm{As}_{4}$ is transferred to the surface, in a Ga-free environment, and incorporated into the lattice through catalytic decomposition. This layer-by-layer growth produces material with exceptionally smooth surfaces and good electronic and crystallographic properties at growth temperatures as low as $200^{\circ} \mathrm{C}$.

\subsubsection{Reflectic i High Energy Electron Diffraction (RHEED) and RHEED Intensity Oscillation (RIO) Analysis}

RHEED analysis is accomplished using a low-energy electron beam incident, at a glancing angle, on the substrate surface. The diffracted beam is then imaged on a phosphor screen located directly opposite the electron gun. RHEED provides detailed information on the surface topography (sensitive on a monolayer scale), the surface reconstruction, and the surface crystallinity.

RHEED can also be used to precisely measure growth rates, in-situ, on a realtime basis by monitoring RHEED intensity oscillations. The RIO system, shown schematically in Fig. 2.1, consists of a photodiode/objective assembly which is focused on the specularly reflected diffraction spot imaged on the phosphor RHEED screen. The signal from the photodiode is then amplified and digitized for further analysis. The intensity oscillations in the specularly reflected beam. depicted in the RHEED output inset of Fig. 2.1, are related to the changes in surface roughness during growth. For example, prior to growth the equilibrium surface is quite smooth, corresponding to high reflectivity of the specular zeroth order beam. Upon initiating growth, islands begin to nucleate at random positions on the surface, leading to a decrease in reflectivity. When $50 \%$ coverage is achieved ( 0.5 monolayers) the reflectivity reaches a minimum. The surface will then begin to smooth, until complete coverage is achieved, at which point the process will repeat. This sequence leads to periodic oscillations where each period corresponds to exactly one monolayer of material. This information is invaluable when accurate growth rate and alloy composition data are required.

\subsection{EQUIPMENT/ANALYSIS DEVELOPMENT}

\subsubsection{Electron Cyclotron Resonance (ECR) Plasma Source}

A microwave discharge which uses electron cyclotron resonance (ECR) can be used for plasma etching, surface modification and thin film growth. The 


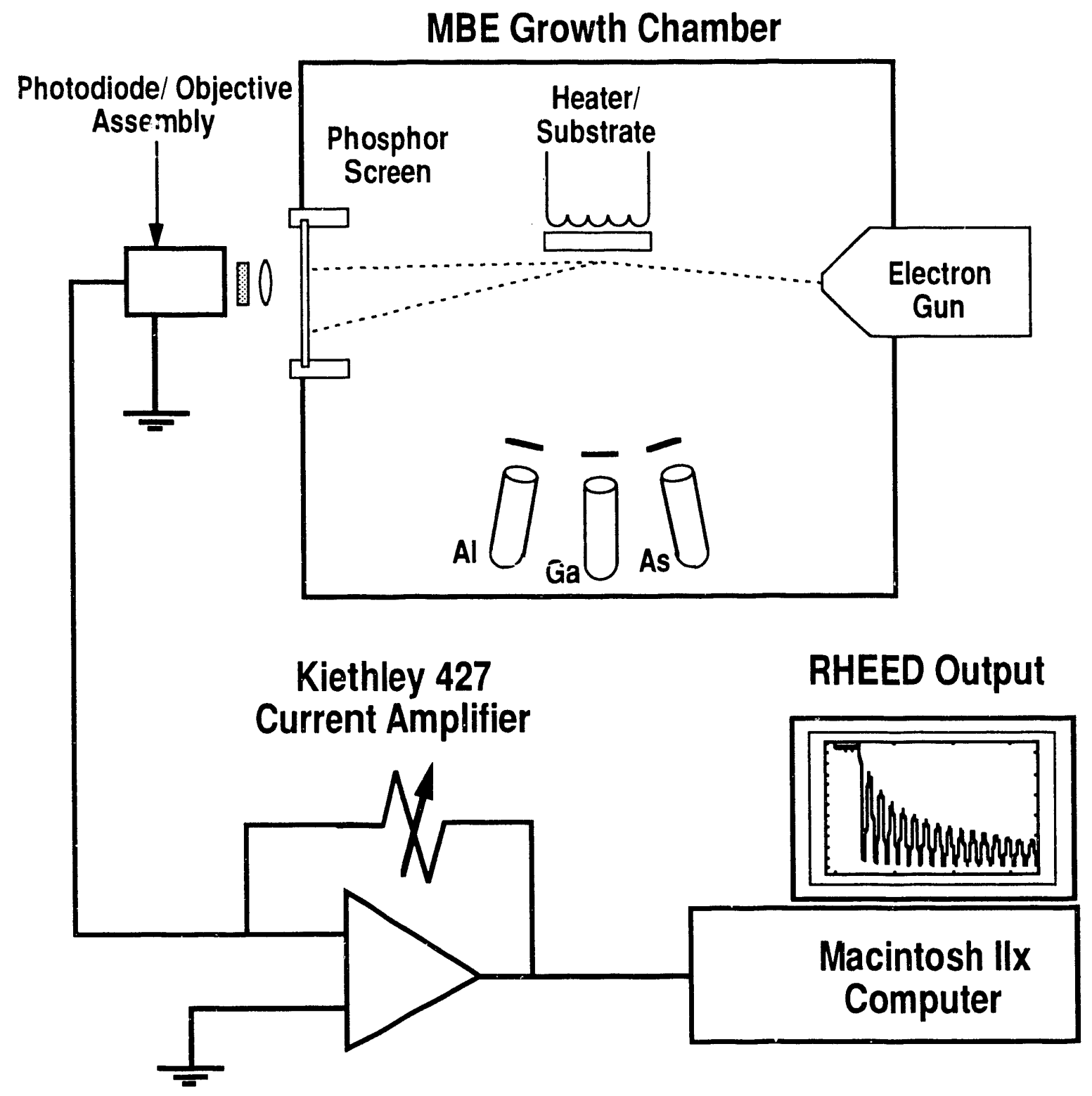

Figure 2.1: Schematic of the RHEED intensity oscillation system.

wide range of applications for an ECR plasma source make the technique an attractive and promising research and development tool. Hence, a Compact ECR Source was purchased from Applied Science and Technology, Inc.

Excitation of the Compact ECR source is by $2.45 \mathrm{GHz}$ microwave energy (10250 watts) coupled into the source through a vacuum window. There are no filaments. cathodes or grids to contaminate the system or process. A strong axial magnetic field (875 Gauss) is used to create electron cyclotron resonance, promote efficient coupling of the microwaves to the plasma, and 
minimize plasma contact with the interior stainless-steel walls of the source. Plasma densities of $10^{11}$ to $10^{12} \mathrm{~cm}^{-3}$ are obtained, giving high ion currents and good gas efficiency.

The ions produced by an ECR plasma have energies of 10 to $25 \mathrm{eV}$ at pressures in the $10^{-3}$ to $10^{-4}$ torr range which is much less than the ion energies produced by an rf plasma. Further reduction of these ion energies is possible through substrate biasing.

The first application of this ECR plasma source will be for post-growth oxidation of high $T_{\text {c }}$ superconductors (HTSC). The use of a microwave plasma for atomic oxygen generation has been shown to be effective in producing fully oxidized HTSC films. In addition to ionized species, the ECR source provides atomic species, excited neutrals, and radicals. This technique will be applied to electrodeposited HTSC thin films in hopes of improving the transport properties.

The second application for this ECR plasma source will be for fundamental studies on plasma etched surfaces of III-V and I-III-VI $\mathrm{I}_{2}$ semiconductors aimed at improving device performance.

The third application for the ECR plasma source wiii he for the low temperature $\left(\sim 370^{\circ} \mathrm{C}\right)$ growth of polycrystalline diamond films.

\subsubsection{Migration Enhanced Epitaxy Development}

Recent interest in MEE growth stems largely from its ability to produce ultraabrupt interfaces which, when properly applied, result in optimized device performance. This is best demonstrated by the example shown in Fig. 2.2, where time resolved PL data is shown for double heterostructure (DH) devices grown both with and without an MEE nucleating layer, Figs. 2(b) and (a), respectively. We found that by incorporating a simple MEE nucleating layer beneath the DH structure (see inset in Fig. 2.2), we were able to markedly improve the AlGaAs/GaAs interface quality. This is reflected in the order of magnitude improvement in the lifetime ( $t=42.3 \mathrm{~ns})$, and the subsequent reduction in the interface recombination velocity $(\mathrm{S}=1200 \mathrm{~cm} /$ s) that resulted when the MEE layer was included.

As a result of the promising data obtained using MEE, one of our goals was to upgrade the MEE portion of the MBE system so that it is both more versatile and reliable. MEE growths severely strain the shutter actuator bellows, frequently leading to premature failures (a typical MEE growth risn will use over 2000 shutter cycles). In the original design, the bellows were welded directly to the source flange; as a result a bellows failure would result in a repair that was both expensive and time consuming. Therefore. in order

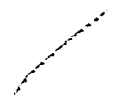




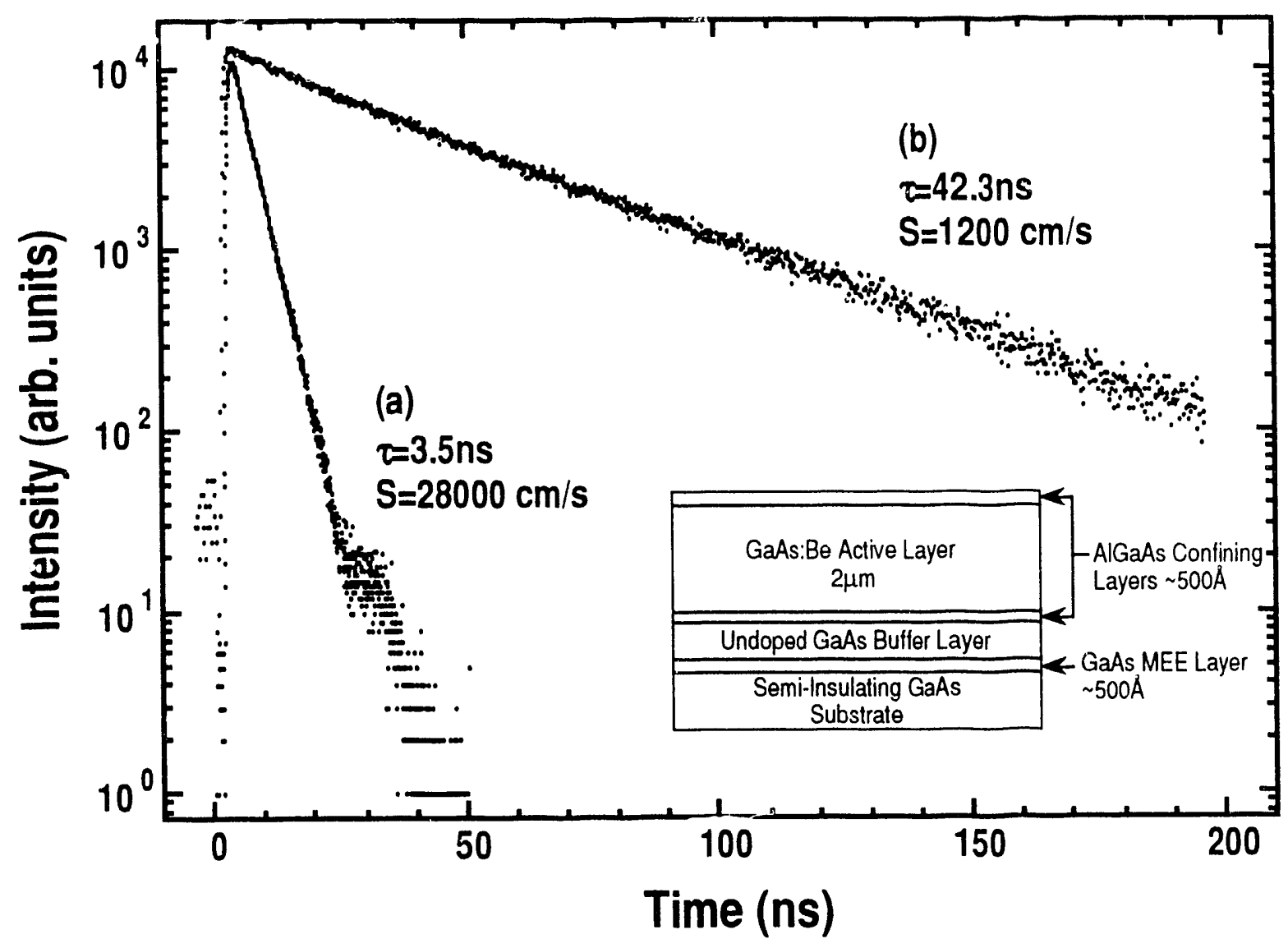

Figure 2.2: Time resolved PL data for AlGaAs/GaAs/AlGaAs DH structures (shown schematically in inset) for samples grown (a) by conventional MBE and (b) with a thin (500A) nucleating MEE layer followed by conventional MBE growth.

to rontinue research using MEE growth, it was necessary to design a new source flange specifically for this purpose. The result of this effort is shown in Fig. 2.3. The feature that distinguishes this design from the original, is the ease with which the shutter actuators can be replaced. Now when a leak develops in a shutter bellows, it can be replaced by removing the actuator assemibly at the conflat flange surface and replacing it with a new one. This procedure takes less than an hour and costs $\sim \$ 400$. In contrast, the old design required removal of the entire source flange, which was then sent to the manufacture for repair. This process took a minimum of four weeks, at a cost of $\sim \$ 7500$.

In addition to the hardware modifications described, it was necessary to design custom software that could be used to accurately control MEE shutter events as well as other important aspects of the growth. The software developed has five primary functions. (1) The software can be used to setup elaborate MEE growth sequences, precisely controlling shutters with a 


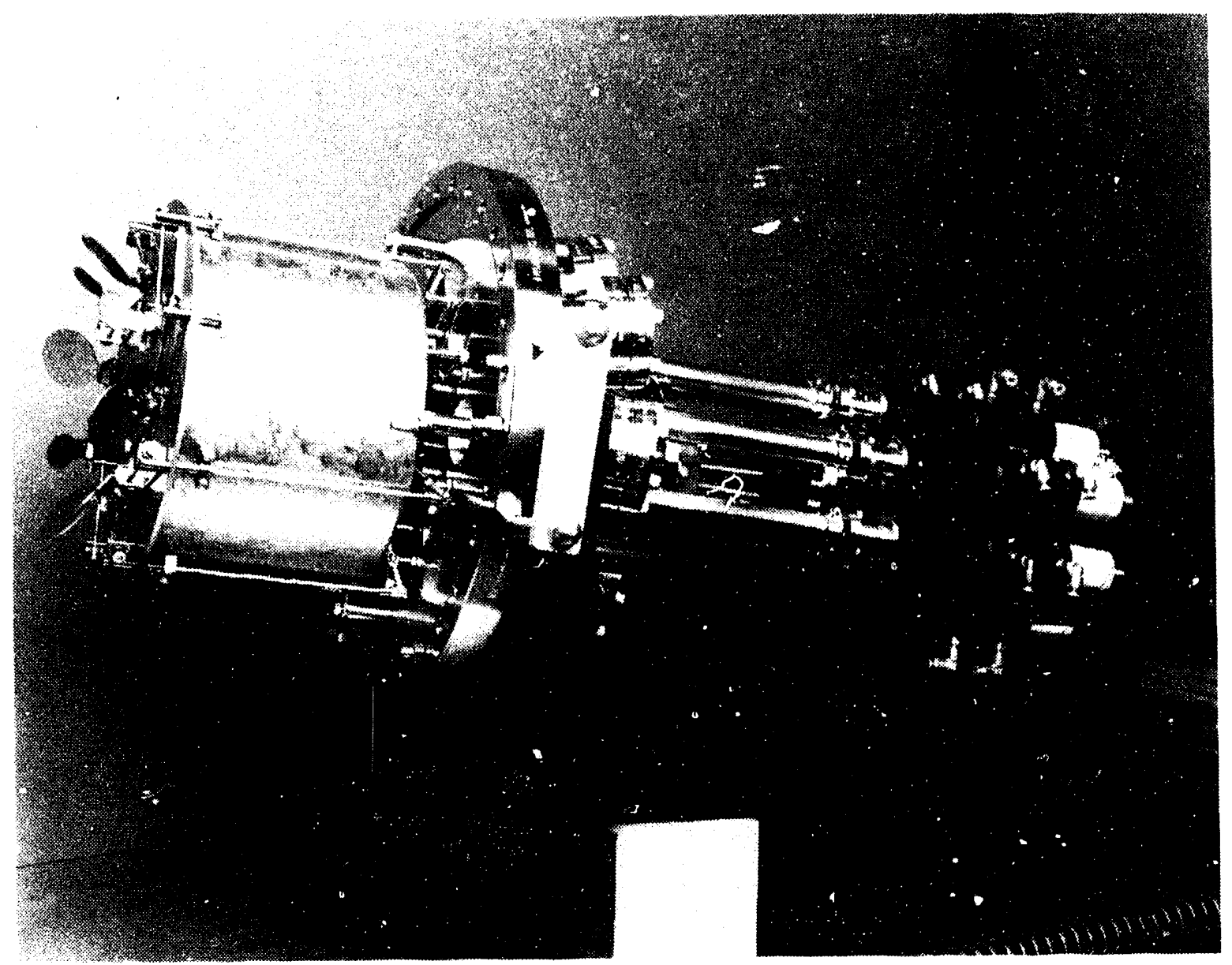

Figure 2.3: Photograph of the redesigned MBE/MEE source flanga taken prior to installation. This design allows shutter actuators to be replaced in the field. a feature lacking from the original design.

resolution of better than $16.6 \mathrm{msec}$. Growth rate information, determined using RHEED oscillation analysis, can then be used to precisely adjust the shutter frequencies to ensure the deposition of single monolayers during MEE growth. (2) The software can acquire and analyze RHEED intensity oscillation data and is capable of performing flux transient analysis using a fast fourier transform algorithm. (3) The software periodically acquires critical growth data establishing a data base for each derosition. The system logs data from over 40 sources including growth pressure, oven temperatures. oven power consumption, substrate temperature, substrate power consumption, liquid nitrogen temperature, and shutter status. This information provides a complete profile of the entire growth run. (4) The software fully automates data acquisition and control of the UTI 100C quadrupole mass spectrometer providing information on background partial pressures and constituent fluxes. (5) The software fully automates data acquisition and control of the Perkin-Elmer Physical Electronics scanning Auger spectrometer, allowing full control of Auger surface scans and greatly enhancing the operators ability to identify the peak position of Auger transitions. 


\subsubsection{RHEED Intensity Oscillation Frequency Do main Flux Transient Analysis}

Although RHEED intensity oscillation analysis is an invalıable and accurate technique for determining growth rates, results can often be misinterpreted. The reason for this is that flux transients, from effusion cells, are not always accounted for. These flux transients result when a closed shutter reflects heat, from the effusion cell, back to the melt surface. This raises the melt surface temperature, relative to the bulk, which produces a higher growth rate when the shutter is initiclly opened. After the shutter is opened, the melt surface reaches equilibrium and the growth rate stabilizes. This phenomena is observed when the data shown in Fig. 2.4 is carefully analyzed. Figure 2.4(a) is RIO data taken from a typical growth run. The fast fourier transform (FFT) of these oscillations are shown in Fig. 2.4(b). Using the arbitrarily selected threshold level shown in Fis. 2.4(b) as a baseline, it is evident that there is a significant frequency distribution in the analyzed data. This indicates that the flux rate is unstable upon initiating growth.

If accurate growth rates are required, it is important to separate the initial flux transient from the steady state growth rate. Therefore, we have developed custom software which allows us to analyze specific portions of the RIO data. The benefits of this effort can be seen by examining Figs. 2.5 and 2.6. Figure 2.5 is the same RIO data presented in Fig. 2.4(a) except that it is separated into three regions. The region marked (a) is the RIO data taken from the initial $10 \mathrm{~s}$ of growth. and the region marked (bj is the RIO data taken from the final $10 \mathrm{~s}$ of growth. Figure 2.6 shows the results of FFT analysis performed on regions (a) and (b) of Fig. 2.5. The results of the FFT analysis obtained from the initial set of RIO data, shown in Fig. 2.6(a). shows significant frequency dispersion. This indicates that as the shutter is opened the growth rate fluctuates. In contrast, the FF $\Gamma$ analysis obtained from the final set of RIO data, shown in Fig. 2.6(b), show a single frequency component indicating that a steady state flux has been achieved. This data shows that the Ga flux is more than $20 \%$ higher than ine steady state flux, immediately after opening the Ga shutter. This newly developed analysis technique will provide valuable information for MEE growths as well as accurate calibration of all vy compositions.

\subsection{ACCOMPLISHMENTS: PHOTOVOLTAICS}

\subsubsection{Synchrotron Radiation Photoemission Study of CdS/ CuInSe $_{2}$ Heterojunction Formation}

The ternary $\mathrm{A}^{\prime} \mathrm{B}^{\prime \prime \prime} \mathrm{X}_{2}{ }^{17}$ chalcopyrite semiconductor. CuInSe.. exhibits superior performance as an absorber in heterojunction solar cells. Polycrystalline thin-film and single-crystal photovoltaic devices with efficiencies exceeding 


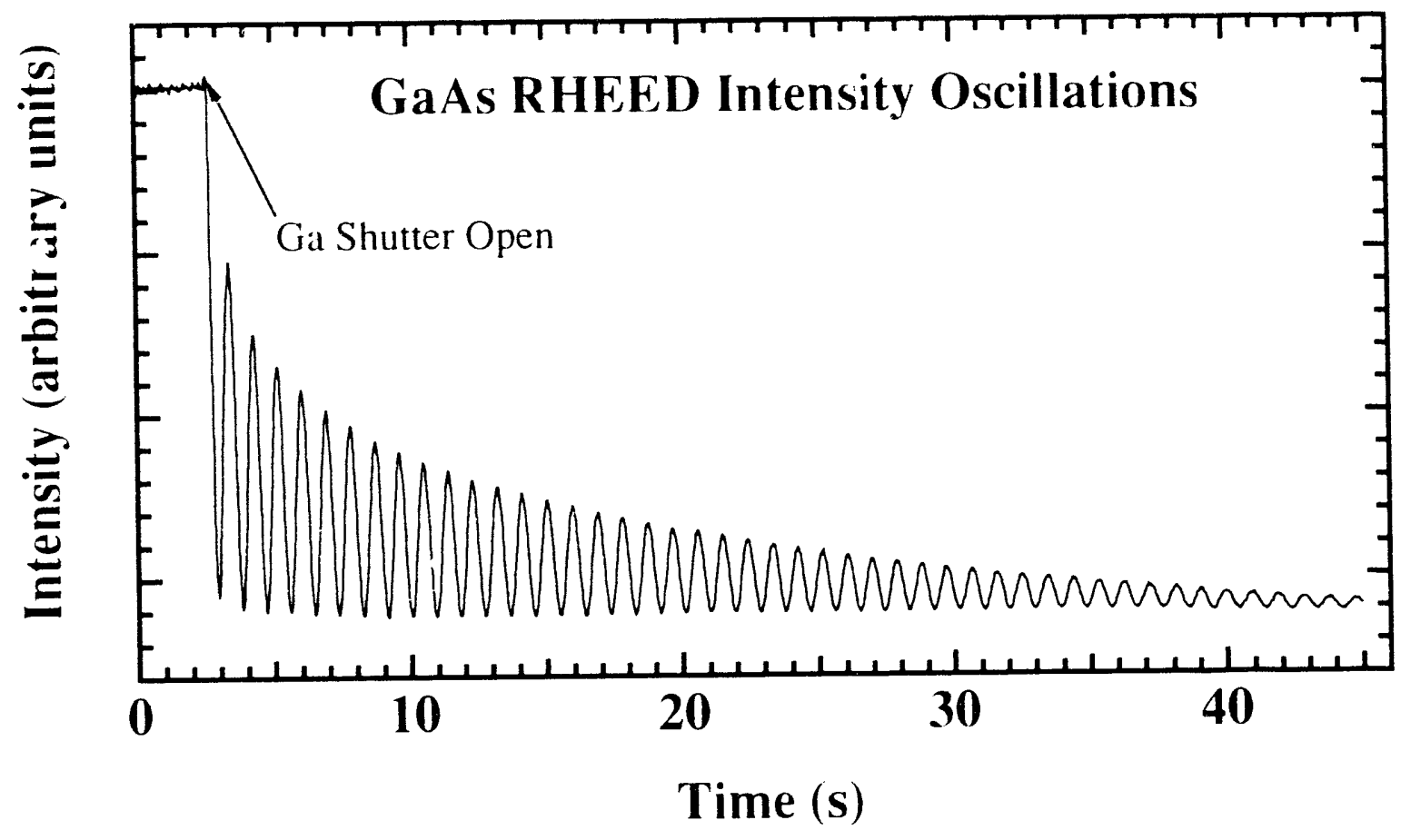

(a)

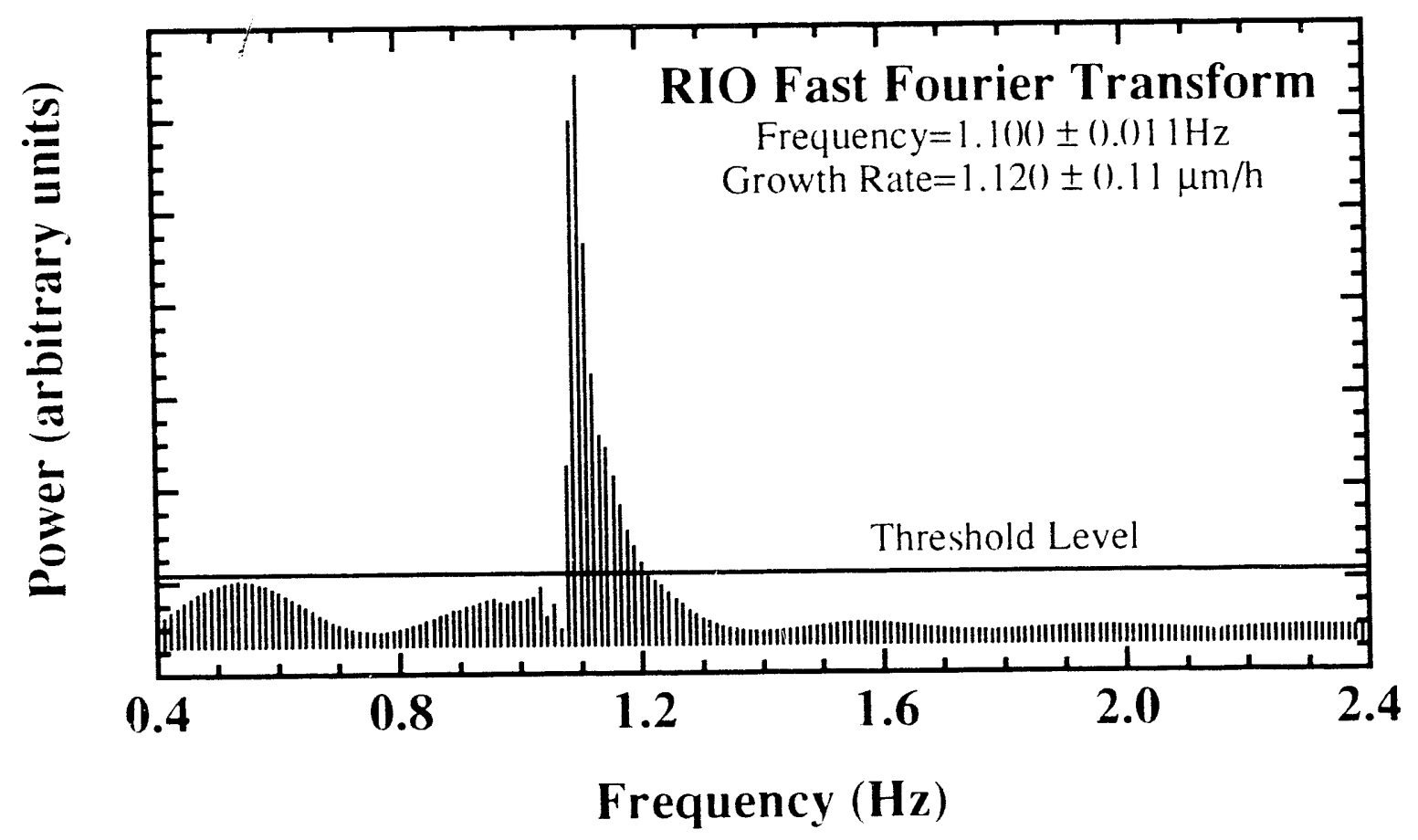

(b)

Figure 2.4: (a) GaAs RHEED intensity oscillation data taken from a (001) surface in the [110] azimuth. (b) Fast fourier power spectrum of the complete set of RIO data presented in Figure MBE-4(a). 


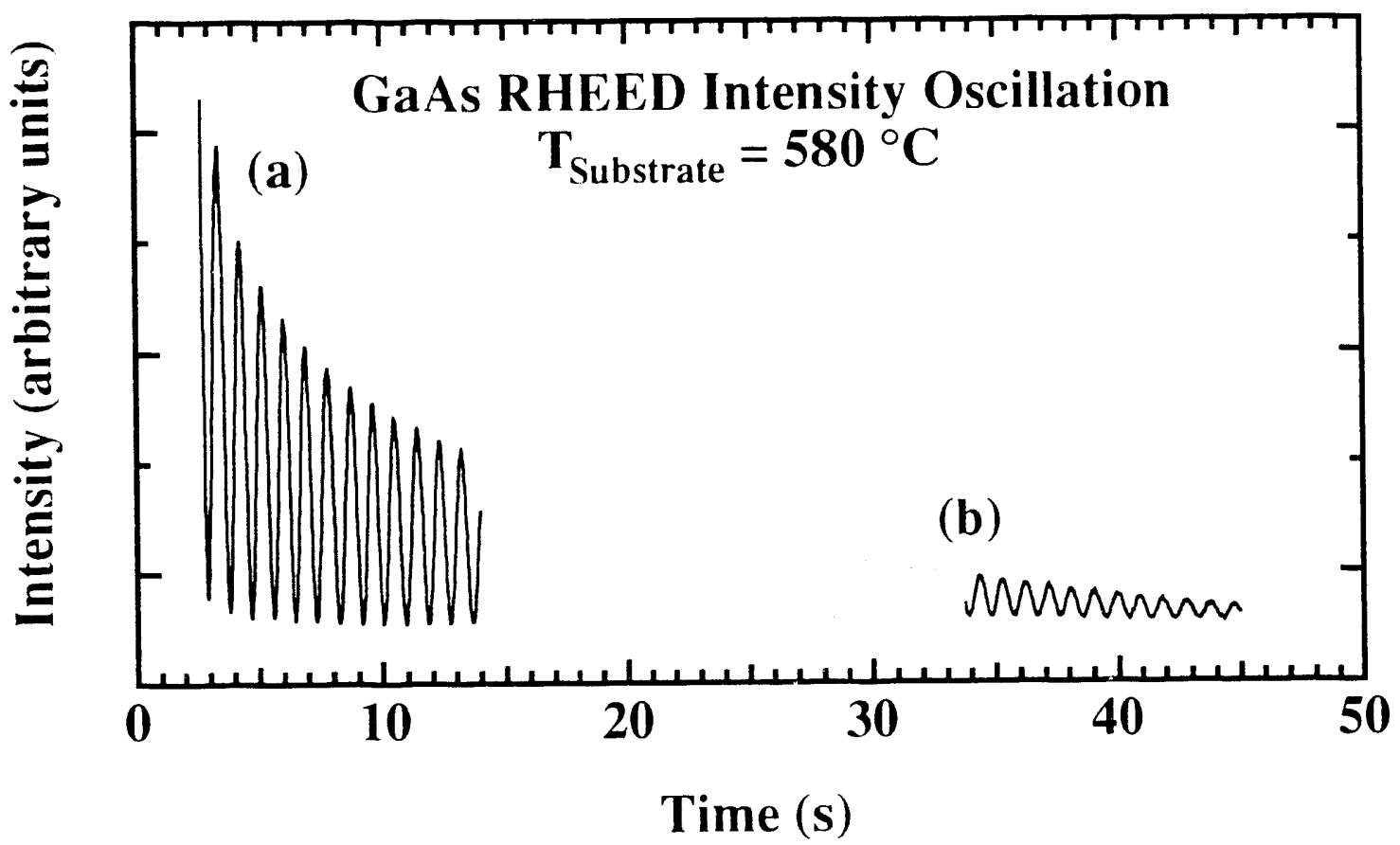

Figure 2.5: GaAs RHEED intensity oscillation data taken from a (001) surface in the [110] azimuth. (a) Initial RHEED oscillations where flux transients would dominate and (b) steady state RHEED oscillation data.

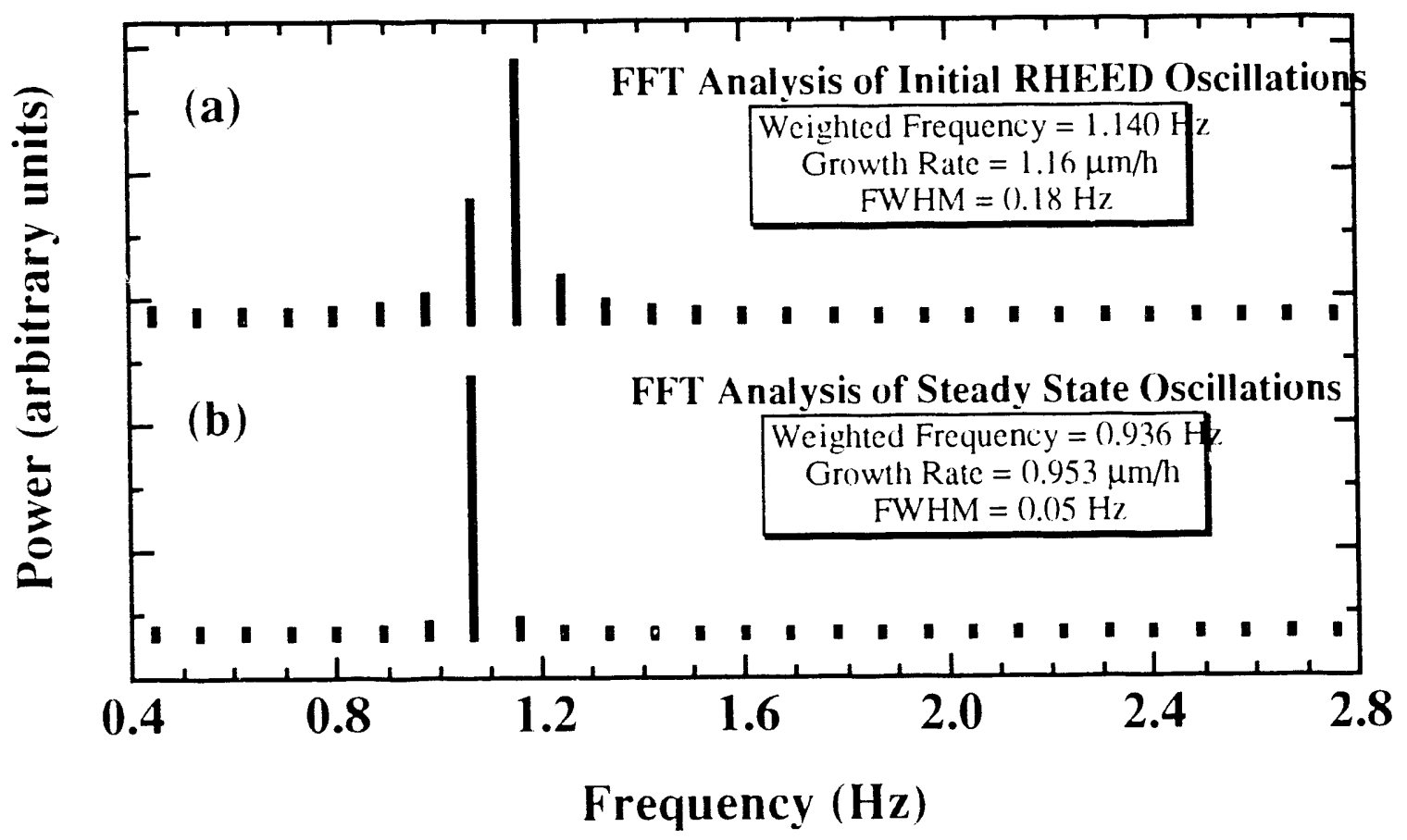

Figure 2.6: (a) Fast fourier power spectrum of the initial RHEED oscillation data presented in Figure MBE-5(a). (b) Fast fourier power spectrum of the steady state RHEED oscillation data presented in Figure MBE-5(b). 
$12 \%$ have been reported. The theoretical electronic structure of Culnse, has been determined but experimental data is still somewhat limited. In addition. theoretical and experimental data on the formation of the CdS/ CulnSe, heterojunction is limited and inconclusive. Thus, important gaps exist in our knowledge of the electronic properties of this technologically important heterojunction.

Synchrotron radiation soft X-ray photoemission spectroscopy (performed at the $1 \mathrm{GeV}$ storage ring (Aladdin) at the University of Wisconsin Synchrotron Radiation Center) was used to investigate the development of the electronic structure at the CdS/CuInSe ${ }_{2}$ heterojunction interface. CdS overlayers were deposited in steps on single-crystal p- and n-type CuInSe $\mathrm{C}_{2}$ at $250^{\circ} \mathrm{C}$. Results indicate that the CdS grows in registry with the substrate, initially in a two dimensional growth mode followed by three dimensional island growth as is corroborated by RHEED analysis. Photoemission measurements were acquired after each growth in order to observe changes in the valence band electronic structure as well as changes in the In $4 d$, Se $3 d, C d 4 d$ and $\mathrm{S} 2 \mathrm{p}$ core lines.

In order to fully characterize the CuInSe ${ }_{2}$ substrate prior to CdS growth, the valence-band (VB) spectra of the p-type and n-type CuInSe, were acquired with several photon energies with the typical $n$-type VB spectra series presented in Fig. 2.7. Comparison of the experimental VB structure to the theoretically predicted band structure as determined by a self-consistent potential-variation mixed-basis (PVMB) approach shows the following:

(1) The upper valence band $\left(\mathrm{T}_{4 v}-\mathrm{G}_{4 v}-\mathrm{N}_{1 v}\right.$. where $\mathrm{T}$ and $\mathrm{N}$ are symmetry points in a body-centered tetragonal Brillouin zone) has a calculated width of $4.8 \mathrm{eV}$ and consists of a mixture of Se $4 \mathrm{p}$ and $\mathrm{Cu} 3 \mathrm{~d}$ orbitals which, due to the relative proximity of the $\mathrm{Se} 4 \mathrm{p}$ and $\mathrm{Cu} 3 \mathrm{~d}$ orbital energies, interact. The observed upper valence band appears as a two peak structure corresponding to the two branches of the $\mathrm{Cu} d$ bands. The calculated peak density of states is near $E_{v}-3.3 \mathrm{eV}$ which is higher than the observed value of $\mathrm{E}_{\mathrm{v}}-2.4 \mathrm{eV}$ by $0.9 \mathrm{eV}\left(\mathrm{E}_{\mathrm{v}}\right.$ is the valence band maximum (VBM), and is determined from the linear extrapolation of the leading edge as outlined below). The observed upper valence band structures for both single-crystal $n$-and p-type CuInSe ${ }_{2}$ are thus in reasonably good agreement with theory. The observed emission

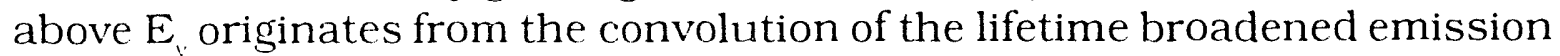
with the finite energy resolution of the electron analyzer and monochromator.

(2) The In-Se band $\left(T 5_{v}-G_{2 v}-N_{1 v}\right)$ has its peak density of states near $E_{v}$ - $6.0 \mathrm{eV}$ and a calculated maximum width of $0.7 \mathrm{eV}$. This band is only observed at the lower photon energies due to variations in the corresponding photoionization cross sections and is observed at $E_{v}-6.3 \mathrm{eV}$ for both $\mathrm{p}-$ and n-type CuInSe . $_{2}$ 


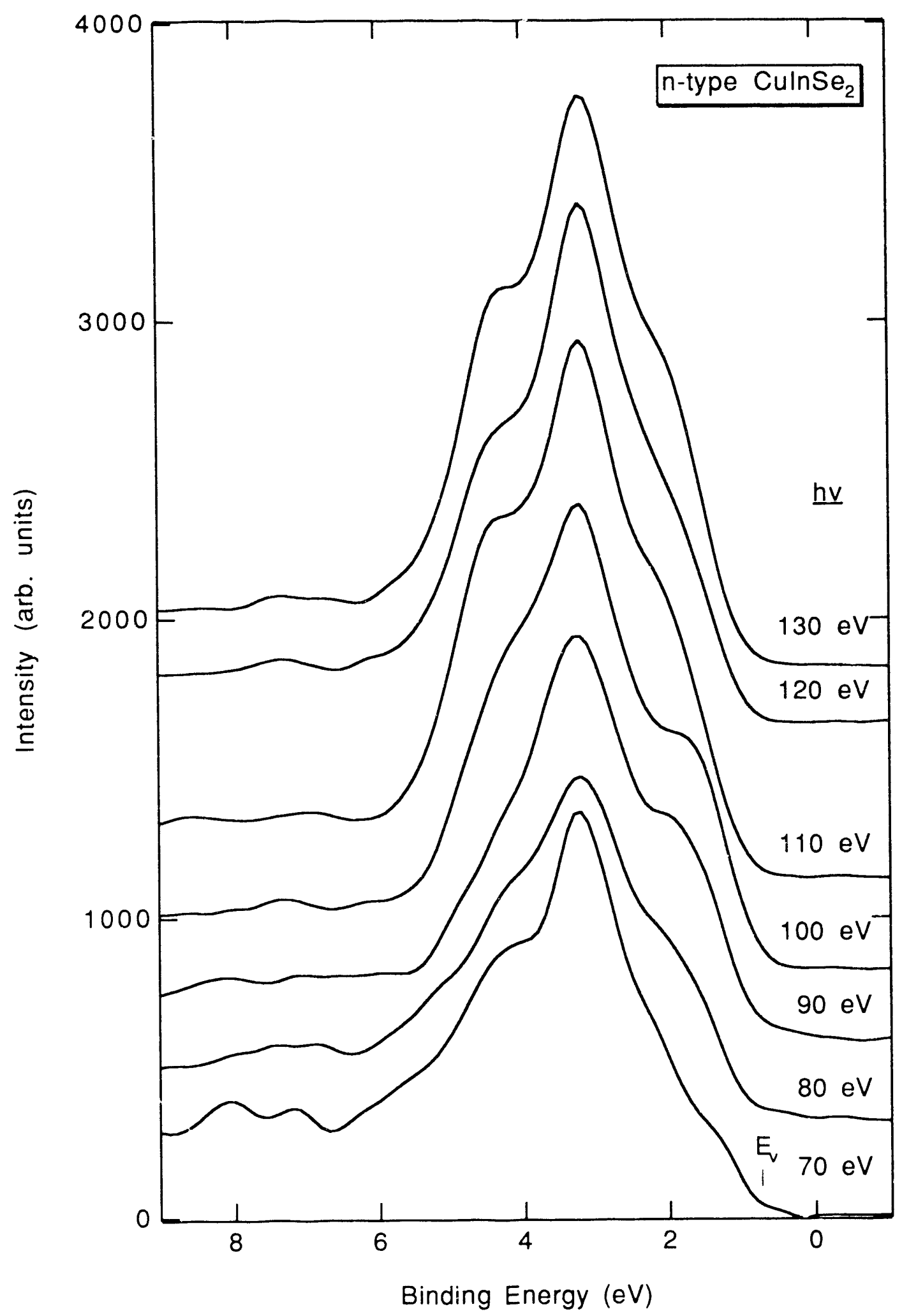

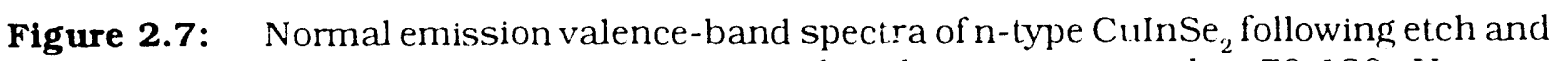
in situ sputter cleaning acquired in the energy range $h \bar{\omega}=70-130 \mathrm{eV}$. 
(3) At yet higher binding energies, theory predicts a Se $4 \mathrm{~s}$ band ( $T 5_{4}-\mathrm{G}_{1 \mathrm{v}}$ $-\mathrm{N}_{1 \mathrm{v}}$ ) between $\mathrm{E}_{\mathrm{v}}-12.8 \mathrm{eV}$ and $\mathrm{E}_{\mathrm{v}}-13.2 \mathrm{eV}$. This band was not observed due to its low photoionization cross section in the photon energy range used for this study.

(4) The calculated binding energy of the In $4 \mathrm{~d}$ core level peak relative to the valence band maximum (VBM) is at $16.9 \mathrm{eV}$. Figure 2.8 displays the In $4 \mathrm{~d}$ peak as a function of CdS coverage. At zero coverage, this peak is observed at $\mathrm{E}_{\mathrm{v}}-17.4 \mathrm{eV}$ for both $\mathrm{p}-$ and n-type CuInSe $\mathrm{e}_{2}$, which is in agreement with values found in the literature.

Figure 2.9 shows the normal emission valence band spectra of the CdS/ CulnSe2 interface as a function of the effective CdS coverage for the n-type crystal. The evolution of the valence band maximum (VBM) shown in these figures is a direct indication of the evolution of the electronic structure leading to the CdS/CuInSe, heterojunction formation. The position of the $\operatorname{VBM}\left(\mathrm{E}_{\mathrm{v}}\right)$ for each coverage is determined from the linear extrapolation of the leading edge. Linear extrapolation of the leading edge of VB emission is somewhat arbitrary and can account for errors in the determination of the VBM. However, the band-mapping analysis necessary for the precise determination of $E_{v}$ was impractical for this investigation and would not significantly affect the value of the valence band discontinuity. Therefore, in the case of the $n$-type CuInSe $e_{2}, \mathrm{E}_{v}$ shifts by $0.3 \pm 0.1 \mathrm{eV}$ when going from clean n-type CuInSe ${ }_{2}$ to the $70 \AA$ CdS overlayer spectrum. Similarly, E $E_{v}$ shifts by $0.3 \pm 0.1 \mathrm{eV}$ when going from clean p-type CuInSe ${ }_{2}$ to the $300 \AA \mathrm{CdS}$ overlayer spectrum. These shifts do not directly correspond to the valence band discontinuity, $\Delta \mathrm{E}_{\mathrm{v},}$ between $\mathrm{CuInSe}_{{ }_{2}}$ and CdS since the position of the substrate valence band edge at the interface is modified by adatom induced changes in the band bending. Therefore, in order to determine $\mathrm{DE}_{\mathrm{v}}$, one needs an independent estimate of the band bending potential for both $\mathrm{p}$ - and n-type substrates. Specifically, three quantities are necessary to determine $\mathrm{DE}_{\mathrm{v}}$ for this heterojunction. two of which are the core-level to valence-band maximum energy separation for each constituent of the heterojunction. The third quantity is the core-level binding energy difference $\Delta \mathrm{E}_{\mathrm{r}, \mathrm{f}}$ for a core-level on each side of the heterojunction referenced to the Fermi energy $\left(E_{\mathrm{F}}\right)$.

Band bending potentials can be determined by monitoring the corresponding shifts of the substrate core level photoemission peaks as long as contributions due to chemical shifts can be separated. It has been determined that for most binary semiconductors, the anion core levels are severely affected by adatom induced changes in the chemical shift and therefore cannot be used to estimate band bending variations. However. it has also been determined that the cation core levels are not affected by changes in the chemical shift 


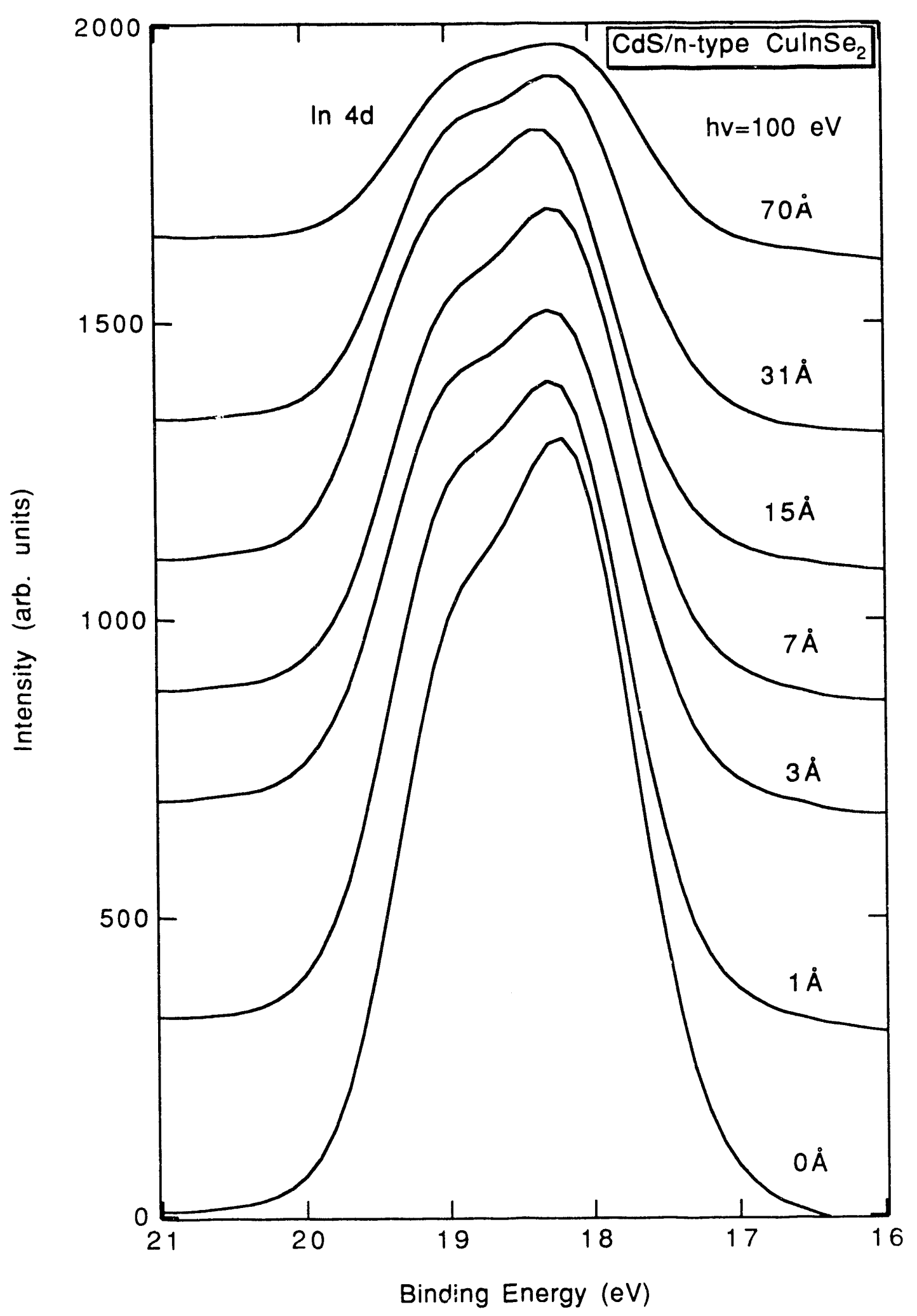

Figure 2.8: Characteristic In $4 d$ core-level emission for CuInSe $e_{2}$ as a function of the effective CdS coverage. 


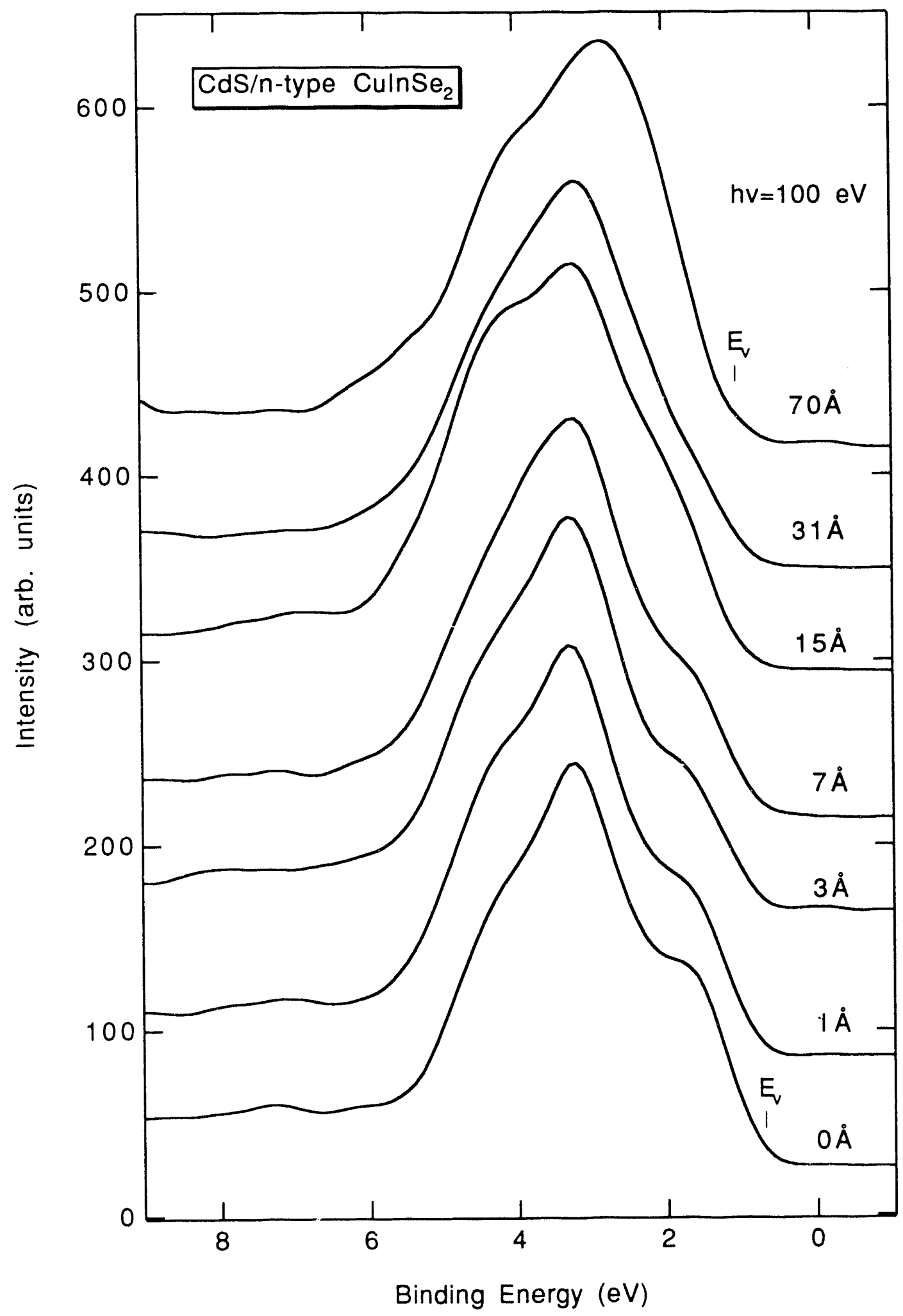

Figure 2.9: Normal emission valence-band spectra of the CdS/CuInSe ${ }_{2}$ interface as a function of the effective CdS coverage for the n-type crystals. 
and thus their adatom induced shifts can be used directly to determine band bending variations.

The In $4 \mathrm{~d}$ core level emission for $\mathrm{n}$ - and p-type CuInSe ${ }_{2}$, as a function of the effective CdS coverage, depicts the evolution of the In $4 \mathrm{~d}$ peak during the formation of the CdS/CuInSe ${ }_{2}$ interface. The peak position at zero coverage is $E_{v}-17.4 \mathrm{eV}$ and shifts to $E_{v}-17.5 \mathrm{eV}$ at the lower coverages (1, 3 and $7 \AA$ ). This shift reflects the adatom induced changes in the substrate band bending. At total coverage, the peak position is $\mathrm{E}_{\mathrm{v}}-17.1 \mathrm{eV}$. Thus, the first quantity needed to determine the valence band discontinuity, specifically $\mathrm{E}_{\mathrm{In} 4 \mathrm{~d}}-\mathrm{E}_{\mathrm{v}}$ in the CuInSe ${ }_{2}$, is $17.4 \mathrm{eV}$.

The representative $\mathrm{Cd} 4 \mathrm{~d}$ core level emission for $\mathrm{n}$ - and p-type CuInSe ${ }_{2}$, as a function of the effective CdS coverage, is presented in Fig. 2.10. For the lower CdS coverages, the peak position is $E_{v}-10.5 \mathrm{eV}$ and shifts to $E_{v}-10.1$ $\mathrm{eV}$ at the higher coverages. Thus, the last two quantities necessary for the determination of the valence band discontinuity are extracted, specifically, $\mathrm{E}_{\mathrm{Cd} 4 \mathrm{~d}}-\mathrm{E}_{\mathrm{v}}$ in CdS is $10.1 \mathrm{eV}$ and $\mathrm{E}_{\mathrm{ln} 4 \mathrm{~d}}-\mathrm{E}_{\mathrm{Cd} d 4 \mathrm{~d}}$ across the heterojunction is 7.0 $\mathrm{eV}$ (referenced to $\mathrm{E}_{\mathrm{F}}$ ).

Using the results of these three measurements it is clear that the valence band discontinuity $\Delta \mathrm{E}_{\mathrm{v}}$ is $0.3 \mathrm{eV}$ for the $\mathrm{CdS} / \mathrm{n}-\mathrm{CuInSe}_{2}$ heterojunction. Similarly, the valence band discontinuity for the CaS/p-CuInSe ${ }_{2}$ heterojunction is $0.3 \mathrm{eV}$. Combining these results with $\mathrm{E}_{\mathrm{g}}\left(\mathrm{CuInSe}_{2}\right)=1.04 \mathrm{eV}$ and $\mathrm{E}_{\mathrm{g}}(\mathrm{CdS})=2.42 \mathrm{eV}$, one may determine the conduction band discontinuity $\mathrm{DE}_{\mathrm{c}}$ to be $1.08 \mathrm{eV}$ for an abrupt CdS/n-CuInSe ${ }_{2}$ or CdS/p-CuInSe ${ }_{2}$ heterojunction. These results are summarized schematically in Fig. 2.11 for the n-type CuInSe ${ }_{2}$ heterojunction. The experimental data shows no band bending for either case due to (1) the 3-5 ^ probe depth and (2) the low carrier concentration since the width of the space charge region is inversely proportional to the square-root of the carrier concentration.

These CdS/CuInSe ${ }_{2}$ heterojunctions are most commonly used for solar cell applications. It is clear that no solar cell could operate with weakly-doped CdS, not only because of the high series resistance which would result in the CdS, but also because of the presence of a substantial barrier to conduction of either carrier type from the CuInSe ${ }_{2}$ to the CdS (Fig. 2.11). Normally, solar cells are fabricated with CdS heavily doped with In, making it strongly ntype. Furthermore, there is a possibility that In will diffuse across the heterojunction further enhancing the conductivity of the CdS. In this case, the Fermi level in the CdS would rise to near the conduction band edge and the CuInSe ${ }_{2}$ band edges would equalize to match the Fermi levels across the junction. This would lead to strong band bending near the heterojunction. If the CdS is much more heavily doped than the CuInSe ${ }_{2}$, most of the band bending will occur in the CuInSe ${ }_{2}$. The amount of band bending will exceed 


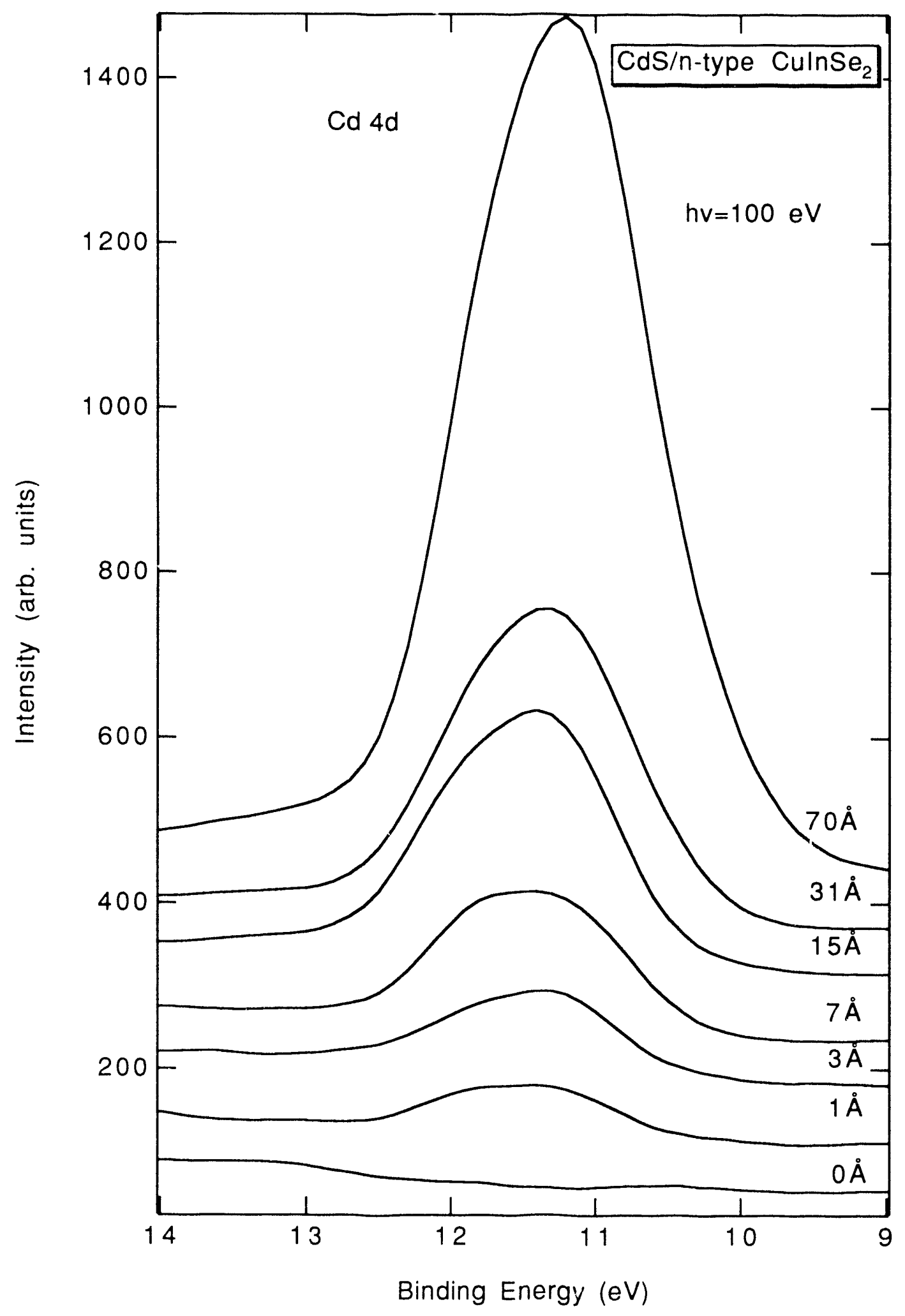

Figure 2.10: Characteristic Cd $4 d$ core-level emission for CuInSe ${ }_{2}$ as a function of the effective CdS coverage. 


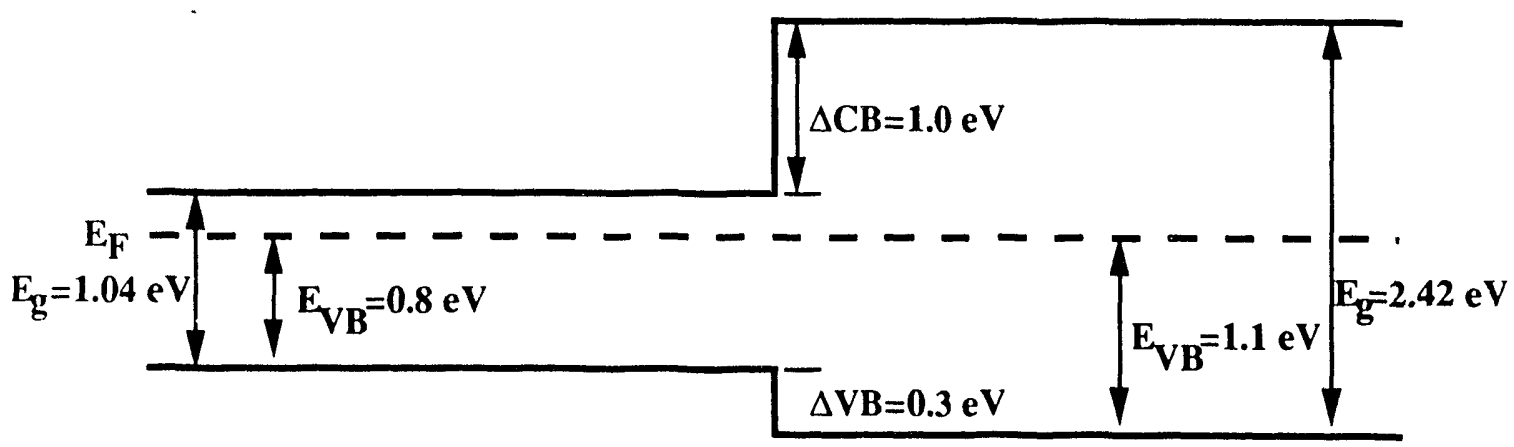

Figure 2.11: Schematic of the experimentally determined band lineup across the CdS/ $\mathrm{CuInSe}_{2}$ heterojuntion.

the energy gap of the CuInSe ${ }_{2}$ and thus a thin 2-dimensional electron gas will develop near the heterojunction due to diffusion of electrons out of the $n^{+} \mathrm{CdS}$ as shown schematically in Fig. 2.12a. This electron gas will act as a metal and will be present for any type or level of doping in the CuInSe ${ }_{2}$ but would be smallest in the heavily $\mathrm{n}$-type material.

This model only applies to single-crystal CuInSe ${ }_{2} / \mathrm{CdS}$ heterojunctions but may aid in explaining several previously unexplained observations concerning $\mathrm{CdS} / \mathrm{CuInSe}_{2}$ heterojunctions and solar cells, in general. Specifically, the apparent lack of interface-dominated recombination of minority carriers, the presence of buried homojunctions in the $\mathrm{CuInSe}_{2}$ as observed by electron-beam-induced conductivity (EBIC), and the broad range of $\mathrm{Cu}_{1} \mathrm{inSe}_{2}$ compositions around stoichiometry for which good sular cells can be fabricated. All of these observations are consistent with the presence of an induced homojunction. The presence of a 2-dimensional electron gas in the $\mathrm{CuInSe}_{2}$ near the heterojunction will produce an essentially metallic contact which will be relatively insensitive to the type of epitaxy or quality of the CdS/ $\mathrm{CuInSe}_{2}$ interface. When the CuInSe ${ }_{2}$ is sub-stoichiometric it tends to be weakly p-type. Thus, the formation of an electron gas near the heterojunction will invert the local carrier population in the CuInSe ${ }_{2}$, forming an induced homojunction. The depth of this homojunction will depend on the doping level of the CuInSe $e_{2}$ and will increase with the resistivity of the material. In fact, such an induced homojunction would appear even for $\mathrm{n}^{-} \mathrm{CuInSe}_{2}$, as observed.

In the case of thin films, as the composition of the CuInSe ${ }_{2}$ becomes In-rich, the films are observed to be weakly n-type with carrier concentrations in the 


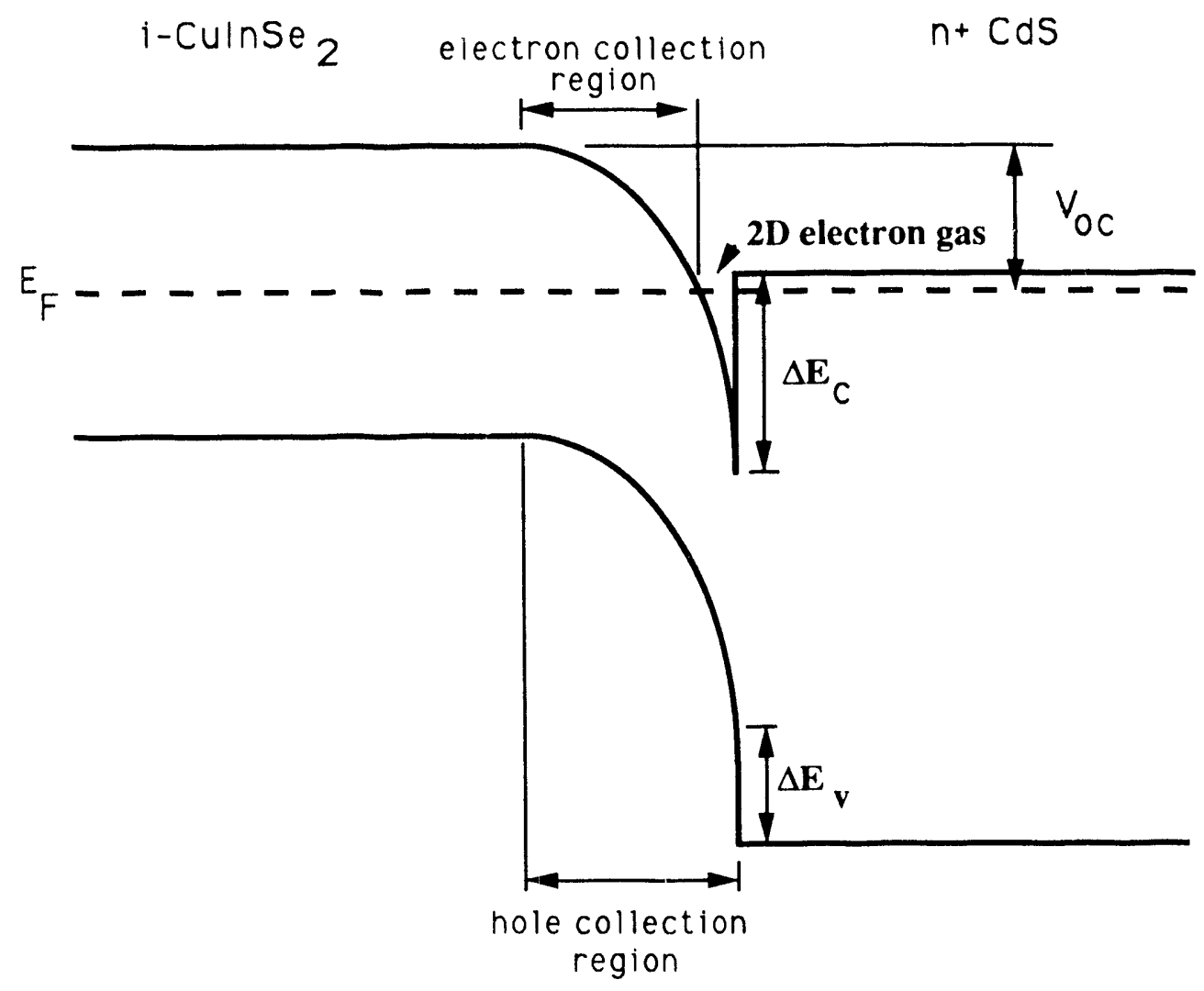

(a)
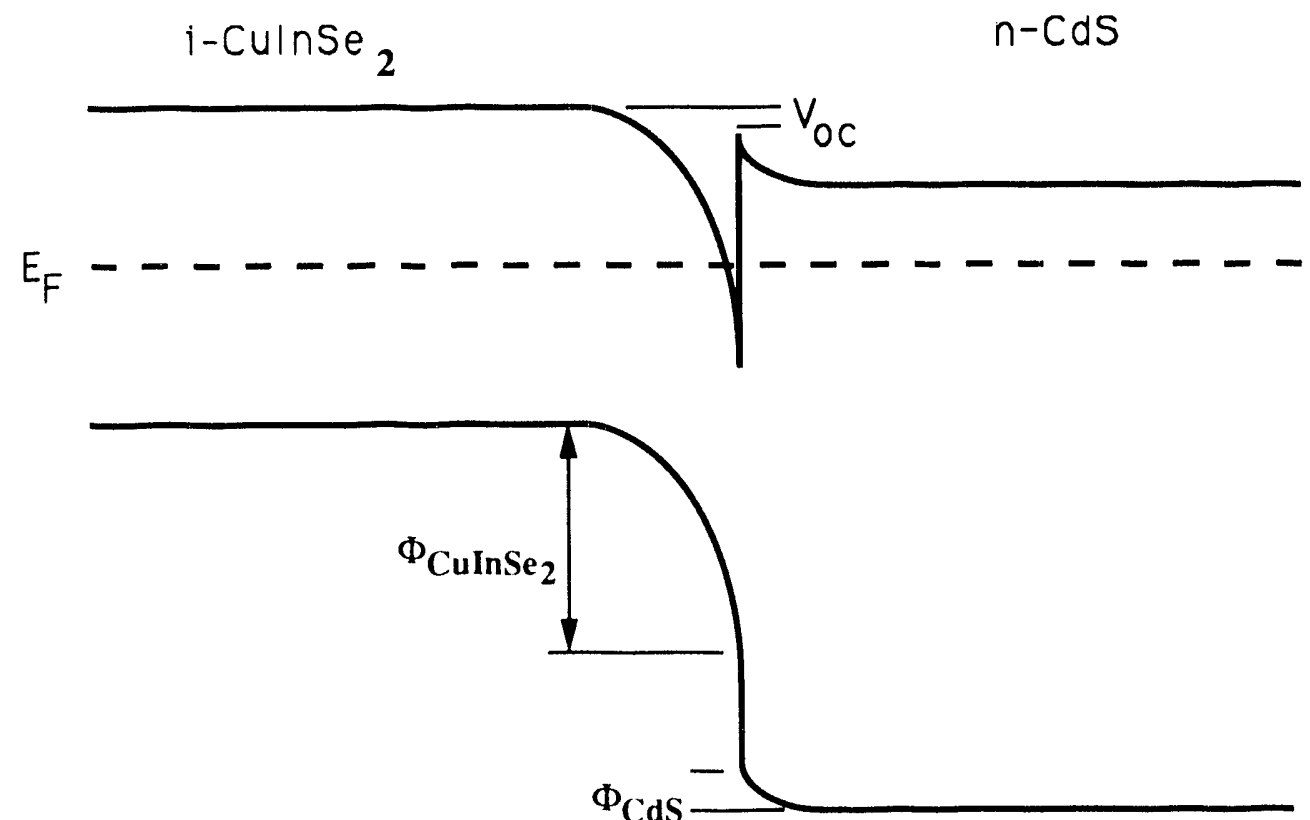

(b)

Figure 2.12: CdS/CuInSe $e_{2}$ heterojunction models for (a) band bending in the CuInSe ${ }_{2}$ and (b) with band bending in the CdS. 
$10^{15} \mathrm{~cm}^{\cdot 3}$ range for up to $\sim 2 \%$ excess In. Near stoichiometry, the carrier concentration changes to p-type and rises rapidly. It is reasonable to assume that the Fermi level lies near the center of the band gap for both Inrich and stoichiometric material, falling toward $\mathrm{E}_{\mathrm{v}}$ as the material becomes $\mathrm{Cu}$-rich. As long as band bending occurs primarily in the CuInSe ${ }_{2}$, the open circuit voltage $\left(\mathrm{V}_{\mathrm{oc}}\right)$ of the solar cell will be determined by $\left(\mathrm{E}_{\mathrm{c}}-\mathrm{E}_{\mathrm{F}}\right)$ in the $\mathrm{CuInSe}_{2}$ (Fig. 2.12a). The open circuit voltage will drop significantly if band bending occurs in the CdS as shown schematically in Fig. 2.12b.

It has been previously suggested that the band edge discontinuity should occur primarily in the valence band. This conclusion is based on applying a generalized empirical method to the temperature dependence of the solar cell and on photoemission data for $\mathrm{CdS} / \mathrm{Si}$ and $\mathrm{CuInSe}{ }_{2} / \mathrm{Si}_{\text {heterojunctions. }}$ However, this method is unreliable in heterojunctions where significant Fermi level pinning may occur in one of the materials at the interface and where the local structure and chemistry of the interface depends strongly on the heterojunction in question. On the basis of the above arguments showing that the observed band edge discontinuities are consistent with observed heterojunction behavior, we conclude that the majority of the band edge discontinuity actually occurs in the conduction band edge for singlecrystal CuInSe ${ }_{2} /$ CdS heterostructures.

\subsubsection{Characterization of the Native Oxide of CuInSe ${ }_{2}$ Using Synchrotron Radiation Photoemission}

The initial surface composition of the CuInSe $\mathrm{Cis}_{2}$ important in determining interfacial properties of the CdS/CuInSe ${ }_{2}$ solar cell and can dramatically affect the device performance. Specifically, the presence of an oxide on this surface has significant implications for device stability and operation as well as for the development of other device structures. Some previous work has reported results on the initial oxidation of CuInSe ${ }_{2}$ thin-films and crystai. showing the oxide to be composed of primarily $\operatorname{In}_{2} \mathrm{O}_{3}$ with some $\mathrm{SeO}_{2}$ and interfacial $\mathrm{Cu}_{x} \mathrm{Se}$. This study expands upon these prior results using high resolution synchrotron radiation soft $\mathrm{x}$-ray photoemission spectroscopy to investigate the native oxide of $n$-type single-crystal CuInSe ${ }_{2}$. Photoemission measurements were acquired on the oxide surface before and after removal by sputter etching.

Photoemission experiments were performed using a "Grasshopper" monochrometer on the $1 \mathrm{GeV}$ storage ring (Aladdin) at the University of Wisconsin Synchrotron Radiation Center. The photoemitted electrons were analyzed using a hemispherical sector analyzer with $1.5^{\circ}$ angular acceptance mounted on a two-axis goniometer. Photoemission spectra were taken with a photon energy of $100 \mathrm{eV}$. The combined energy resolution of the monochrometer and the analyzer was $\Delta \mathrm{E} \approx 0.1 \mathrm{eV}$. All spectra were measured 
at normal emission with a photon angle of incidence of $45^{\circ}$. Following the initial analysis, the crystal surface was cleaned, in-situ. by sputtering at normal incidence with $1 \mathrm{keV}$ Ar ions $(\sim 2 \mu \mathrm{A}$ beam current) in order to obtain a clean valence band spectra. Sputter induced damage was removed by annealing for $\sim 2$ minutes at $500^{\circ} \mathrm{C}$. These process parameters were determined from previous work which concluded that $\mathrm{CuInSe}_{2}$ crystals could be sputtered with up to $1.5 \mathrm{keV}$ Ar ions and annealed at $500^{\circ} \mathrm{C}$ without changing the surface composition.

Figure 2.13 displays the progression of the normal emission valence band (VB) structure as a function of sputter etching time. The upper valence band for CuInSe $e_{2}$ consists of a mixture of Se $4 p$ and $\mathrm{Cu} 3 \mathrm{~d}$ orbitals and appears as a two peak structure corresponding to the two branches of the $\mathrm{Cu} d$ bands. The valence band maximum $\left(\mathrm{E}_{\mathrm{v}}\right)$ occurs $0.8 \mathrm{eV}$ below the Fermi energy $\left(E_{\mathrm{F}}\right)$ and the main $\mathrm{Cu} 3 \mathrm{~d}$ feature occurs at $3.2 \mathrm{eV}\left(\mathrm{E}_{\mathrm{v}}-2.4 \mathrm{eV}\right)$. The feature at $4.3 \mathrm{eV}\left(\mathrm{E}_{\mathrm{v}}-3.5 \mathrm{eV}\right)$ in the oxidized VB spectra is a shifted $\mathrm{Cu} 3 \mathrm{~d}$ line which is assigned to $\mathrm{Cu}_{2} \mathrm{Se}$ as supported by the Se $3 \mathrm{~d}$ data discussed below. The broadening of the entire VB after 1 hour of sputtering is indicative of residual ion damage at the surface after annealing.

Figure 2.14 displays the normal emission In $4 \mathrm{~d}$ core level spectra as a function of sputter time for the oxidized CuInSe ${ }_{2}$ surface. The kinetic energy of these photoemitted electrons is $\sim 75 \mathrm{eV}$ which is at the minimum of the universal escape depth curve with an actual escape depth of 3-5 $\AA$. The In $4 \mathrm{~d}$ line, corresponding to the oxidized surface, is resolved into two components. The higher energy component at $18.9 \mathrm{eV}\left(\mathrm{E}_{\mathrm{v}}-18.1 \mathrm{eV}\right)$ is a mixture of the In $4 \mathrm{~d}_{5 / 2}$ line representative of $\operatorname{In}_{2} \mathrm{O}_{3}$ and the $\ln 4 \mathrm{~d}_{3 / 2}$ line representative of CulnSe $_{2}$. The largest feature at $18.2 \mathrm{eV}\left(\mathrm{E}_{\mathrm{v}}-17.4 \mathrm{eV}\right)$ corresponds to the In $4 \mathrm{~d}_{5 / 2}$ line representative of CuInSe ${ }_{2}$ which is in agreement with values found in the literature. Sputter etching removes this oxide leaving only the In $4 \mathrm{~d}$ core line representative of $\operatorname{In}^{3+}$ in CuInSe ${ }_{2}$.

Figure 2.15 displays the normal Se $3 \mathrm{~d}$ core level emission spectra as a function of sputter time for the oxidized CuInSe ${ }_{2}$ surface. The kinetic energy of these photoemitted electrons is $\sim 39 \mathrm{eV}$ which corresponds to an escape depth, from the universal escape depth curve, of 3- $\tau \AA$. The Se $3 d$ line corresponding to the oxidized surface clearly has several components. The major features at $54.9 \mathrm{eV}\left(\mathrm{E}_{\mathrm{v}}-54.1 \mathrm{eV}\right)$ and $55.7 \mathrm{eV}\left(\mathrm{E}_{\mathrm{v}}-54.9 \mathrm{eV}\right)$ correspond to the $\mathrm{Se} 3 \mathrm{~d}_{5 / 2}$ and Se $3 \mathrm{~d}_{3 / 2}$ lines, respectively, representative of $\mathrm{Se}^{2-}$ in $\mathrm{Cu}_{2} \mathrm{Se}$ This is in agreement with the aforementioned $\mathrm{Cu} 3 \mathrm{~d}$ result. The feature which initially occurs at $54.2 \mathrm{eV}$ and shifts to $54.4 \mathrm{eV}\left(\mathrm{E}_{\mathrm{v}}-53.6 \mathrm{eV}\right)$ after sputtering corresponds to $\mathrm{Se}^{2}$ in CuInSe $\mathrm{C}_{2}$. No features representative of $\mathrm{SeO}_{2}$ were evident. This may be due to the fact that the presence of the interfacial $\mathrm{Cu}_{2} \mathrm{Se}$ would inhibit the formation of $\mathrm{SeO}_{2}$. 


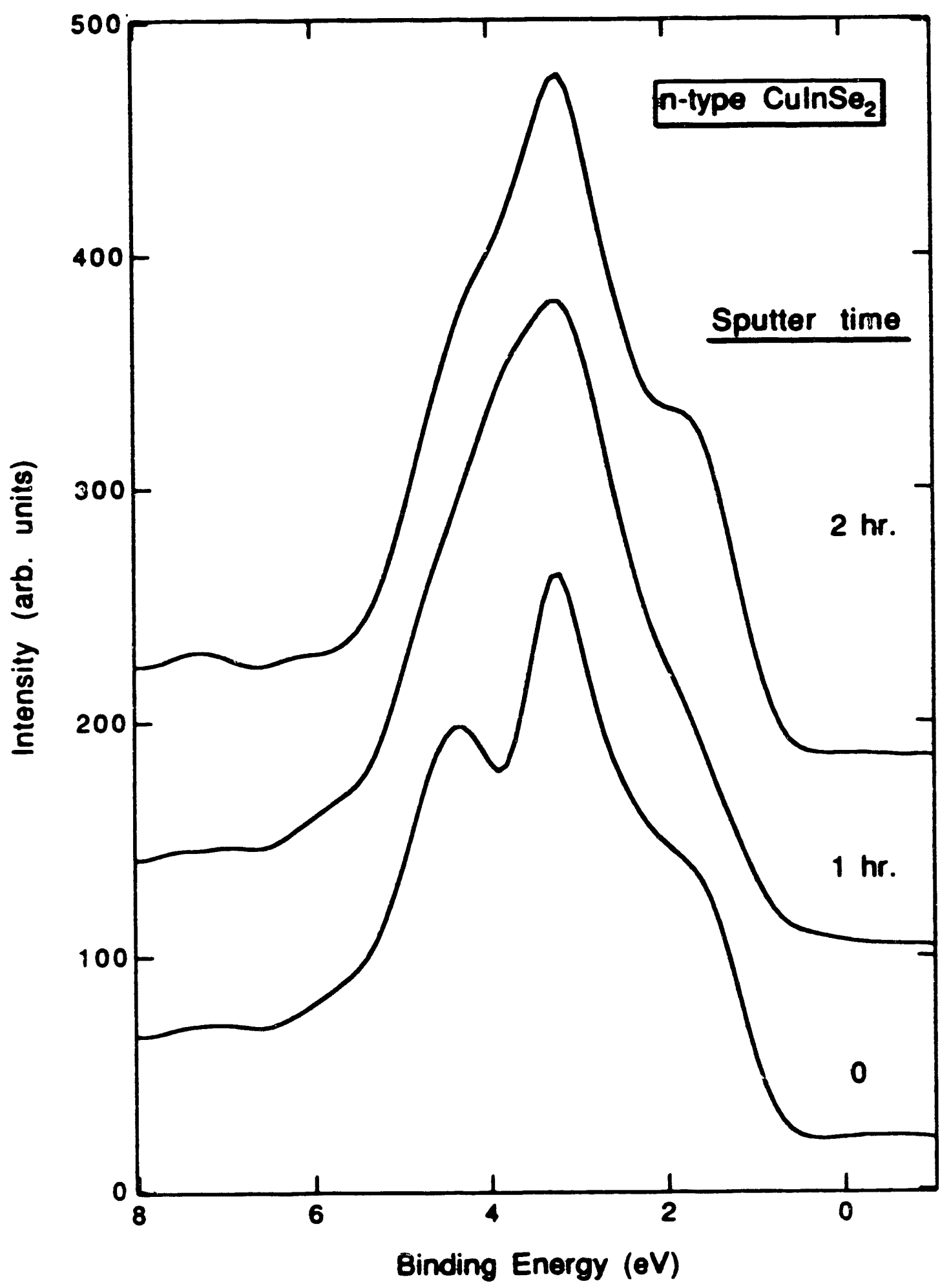

Figure 2.13: Normal emission valence-band spectra of $n$-type CulnSe as a function of sputter cleaning acquired with a photon energy of $h \omega=10 \mathrm{eV}$. 


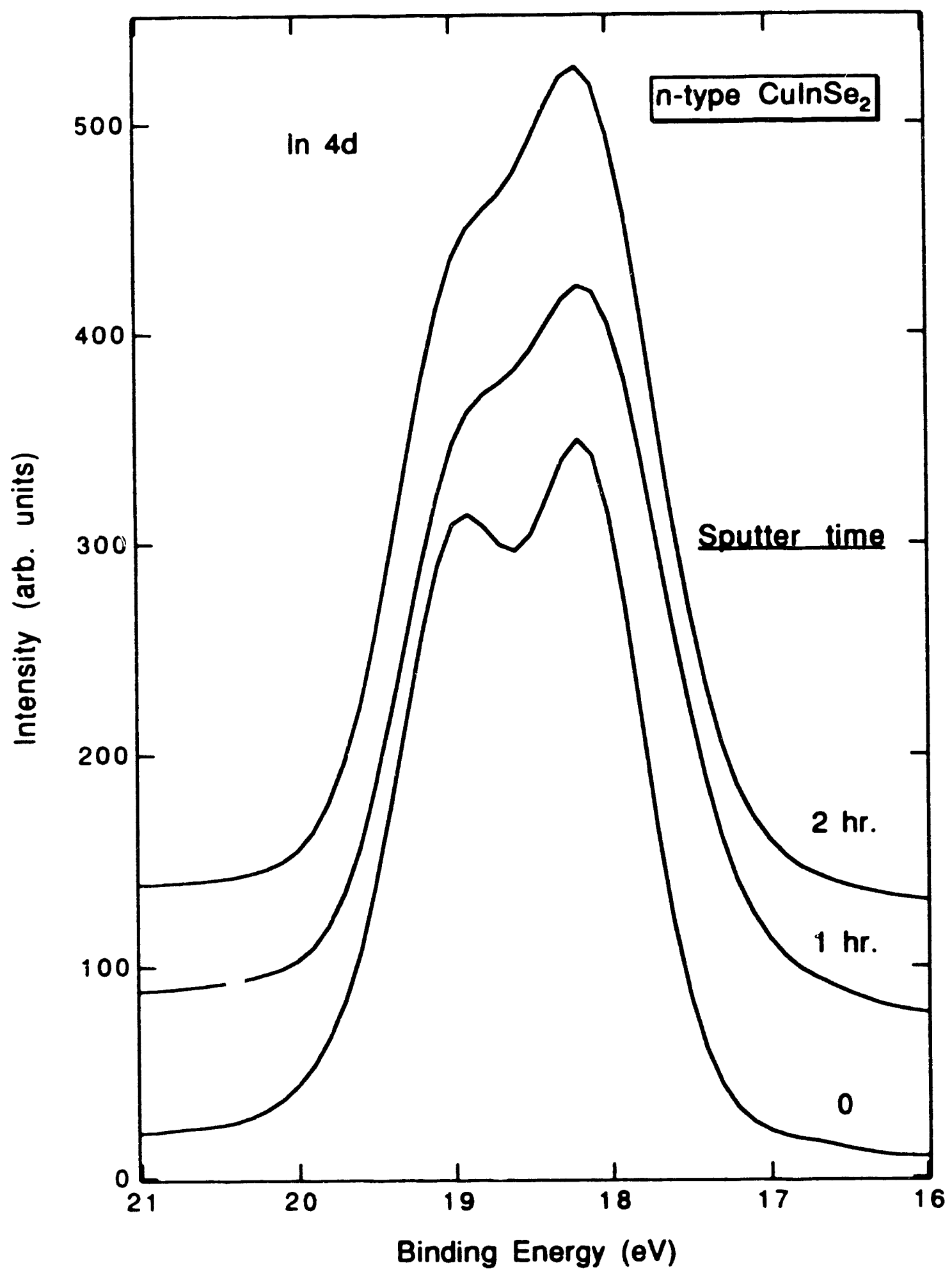

Figure 2.14: Normal emission In $4 d$ core level spectra as a function of sputter time for the oxidized n-type CuInSe $e_{2}$ surface acquired with a photon energy of $h \varpi=100$ $\mathrm{eV}$. 


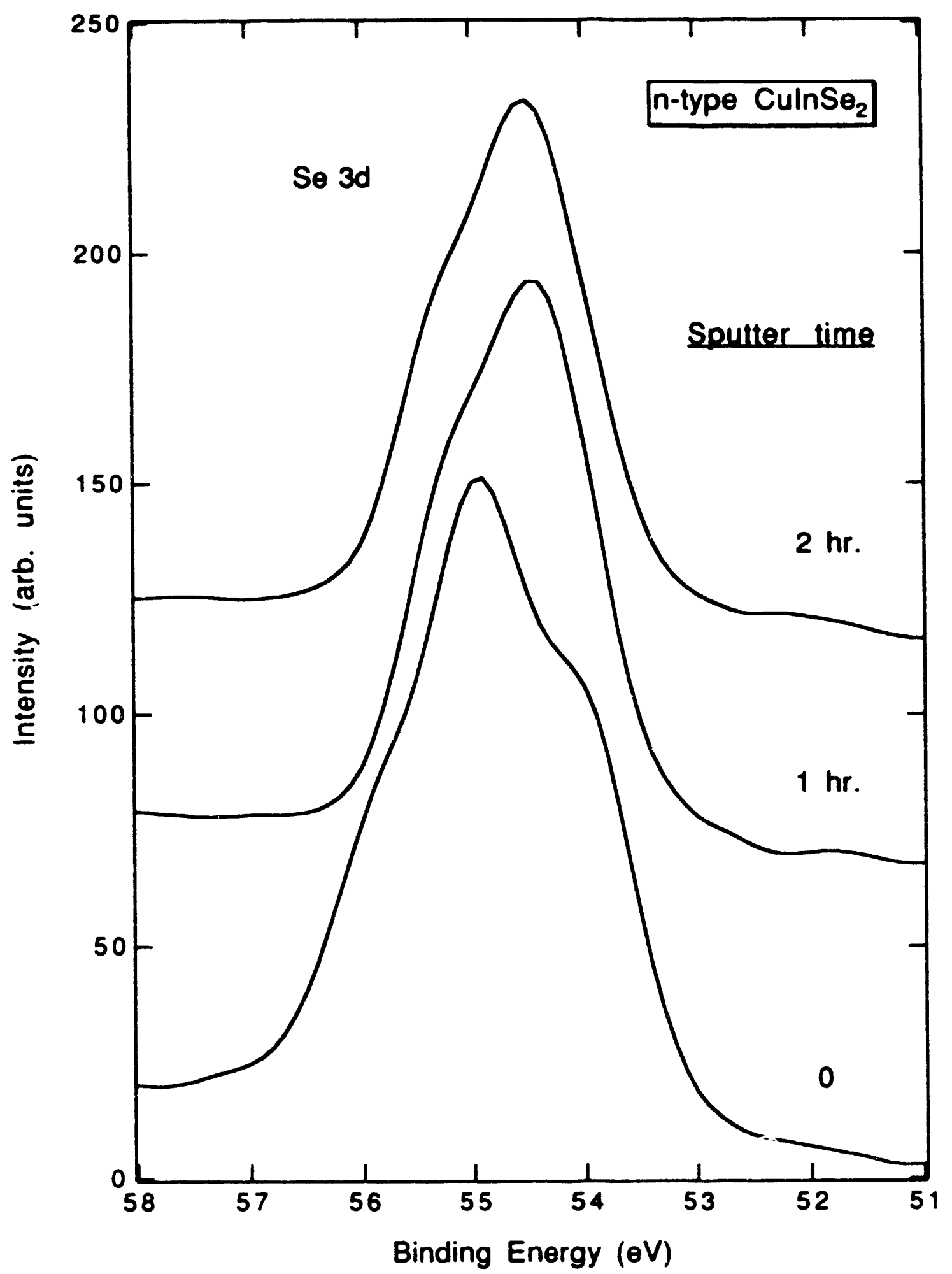

Figure 2.15: Normal emission Se $3 d$ core level spectra as a function of sputter time for the oxidized n-type CuInSe, surface acquired with a photon energy of ha $=100$ $\mathrm{eV}$. 
In summary, the native oxide of $\mathrm{CuInSe}_{2}$ has been studied using high resolution synchrotron radiation soft $\mathrm{x}$-ray photoemission spectroscopy. Results show the native oxide to be composed of an $\operatorname{In}_{2} \mathrm{O}_{33}$ outer layer (no $\mathrm{SeO}_{2}$ ) with an additional $\mathrm{Cu}_{2} \mathrm{Se}$ interface layer.

\subsubsection{Stress Analysis in Lattice Mismatched Heterostructures [100]}

In collaboration with GTE Laboratories, work analyzing stress in lattice mismatched epitaxial layers has continued. Information about stress nonuniformities in heteroepitaxial systems is important for device applications because stress leads to band structure modifications, which affect optical and electrical properties of the enilayer. Also, there is evidence that stress induced dislocation migration may lead to device degradation. For these reasons, it is important to understand how stress influences the optoelectronic properties of materials and to find a means of controlling the stress in these heterostructures.

In this work, we have 1 sed cathodoluminescence (CL) as a means of mapping out the spatial nonuniformities of stress associated with the metallization of multiple quantum well (MQW) structures ${ }^{1}$. Metallization in such structures is required for the application of electric fields, which modulate the optical properties of the layers, the formation of waveguides associated with the strain-induced variation in the refractive index produced underneath the edge of the metal stripes. In photovoltaic structures metallized areas are used for current collecting grids.

An example of the effect of gold metallization on the luminescence properties of MBE-grown GaAs/AlGaAs MQW's underneath gold stripes ( $100 \mu$ m wide and $0.4-\mu \mathrm{m}$ thick separated by $8 \mu \mathrm{m}$ openings) is shown in Fig. 2.16. In this case, CL observations were performed on cross-sections of cleaved samples. Figure 2.16a is a backscattered electron image that clearly highlights a gold contact from the MQW structure. In this image, an interface between the GaAs substrate and the MBE-grown epitaxial layers is also visible. Figure $2.16 \mathrm{~b}$ presents the $\mathrm{CL}$ monochromatic image recorded at $790 \mathrm{~nm}$ (at a temperature of about $20 \mathrm{~K}$ ), which is the peak of the emission spectrum from the quantum wells underneath the gold contact edges. Spectra recorded from the region in-between the bright regions in Fig. $2.16 \mathrm{~b}$ indicated a shift to shorter wavelengths of $\approx 1.5 \mathrm{~nm}$. This corresponds to a tensile stress of about $1 \mathrm{kbar}$ in the quantum well region underneath the gold stripe edges. This tensile stress results in a change in the refractive index $\Delta n \approx 0.01$, which is sufficient to support a single mode (at $\lambda \approx 1 \mu \mathrm{m}$ ) in a waveguide $\sim 1 \mu \mathrm{m}$ thick $^{\prime}$. A schematic diagram of another structure examined is illustrated in Fig. 2.17. which shows openings D $1(50 \mu \mathrm{m})$ and D2 $(150 \mu \mathrm{m})$ that have been chemically etched. In this structure the thickness of the metal contact layer 


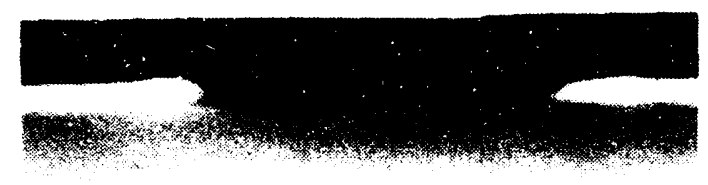

(a)

$1 \stackrel{1}{\mu m}$

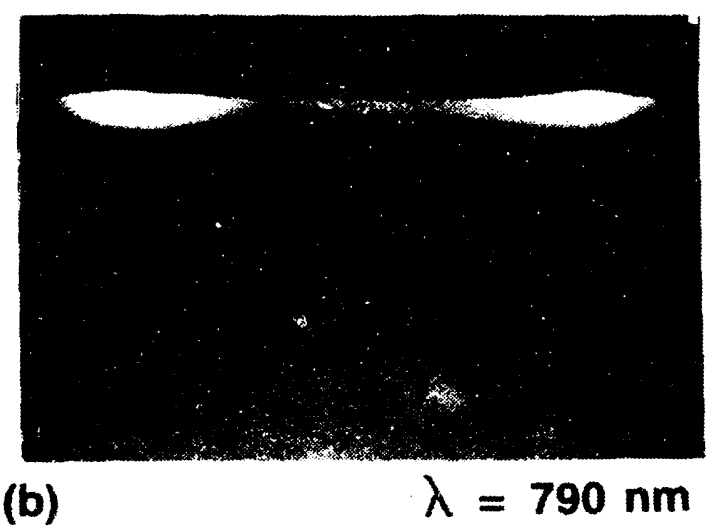

Figure 2.16: SEM micrographs of the waveguide structure: (a) backscattered electron image, and (b) monochromatic CL image recorded at $790 \mathrm{~nm}$. The thickness of the metal layer is about $0.4 \mu \mathrm{m}$.

was $\sim 0.4 \mu \mathrm{m}$ and the thickness of the etched region was $\sim 2 \mu \mathrm{m}$. In order to examine the region of the layer underneath the top gold contact, the $\mathrm{CL}$ was measured from the opening D2 of the structure. The CL monochromatic image, recorded at $780 \mathrm{~nm}$, of the MQW region is shown in Fig. 2.18, in which the dark ring corresponds to the region underneath the edge of the metal ring. Spectroscopic CL measurements indicated that the emission from the dark ring in Fig. 2.18 is shifted by $\sim 2 \mathrm{~nm}$ towards a longer wavelength relative to the region away from the ring. This indicates the presence of tensile stress on the order of $1 \mathrm{kbar}$ in the region of the dark ring ${ }^{1}$.

In summary. CL microscopy and spectroscopy, in conjunction with the known behavior of the optical transitions in the presence of stress, can be employed for both the qualitative and quantitative analyses of stress in epitaxial films. 


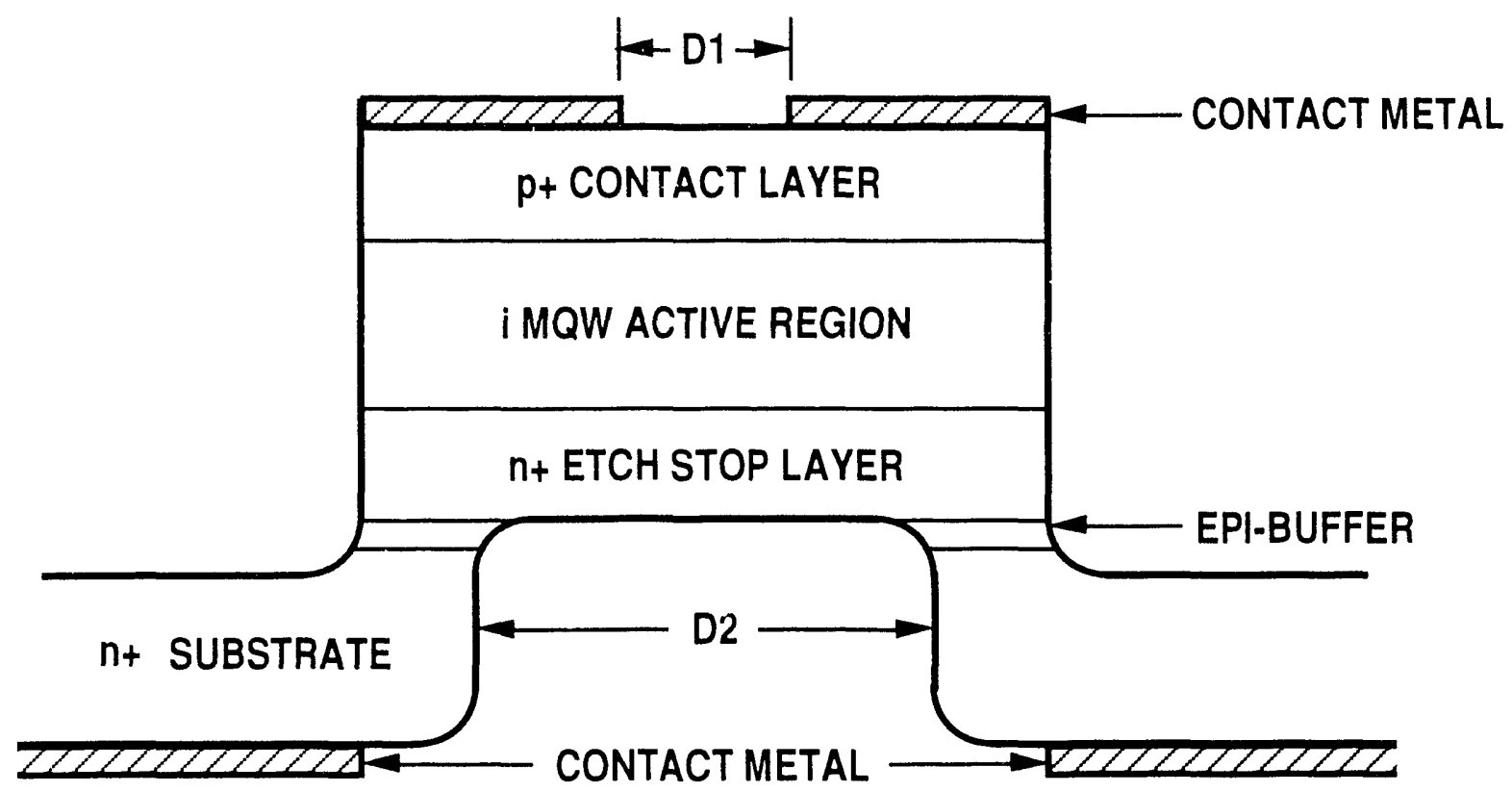

Figure 2.17: Schematic diagram of the structure used in CL measurements. MQW active region contains 25 periods of $70 \mathrm{~A}$ GaAs quantum wells separated by $80 \AA$ $\mathrm{Al}_{x} \mathrm{Ga}_{1-x} \mathrm{As}$ barriers $(\mathrm{x} \approx 0.2)$. $\mathrm{D} 1$ and $\mathrm{D} 2$ are 50 and $150 \mu \mathrm{m}$, respectively. The thickness of the contact metal is $0.4 \mu \mathrm{m}$.

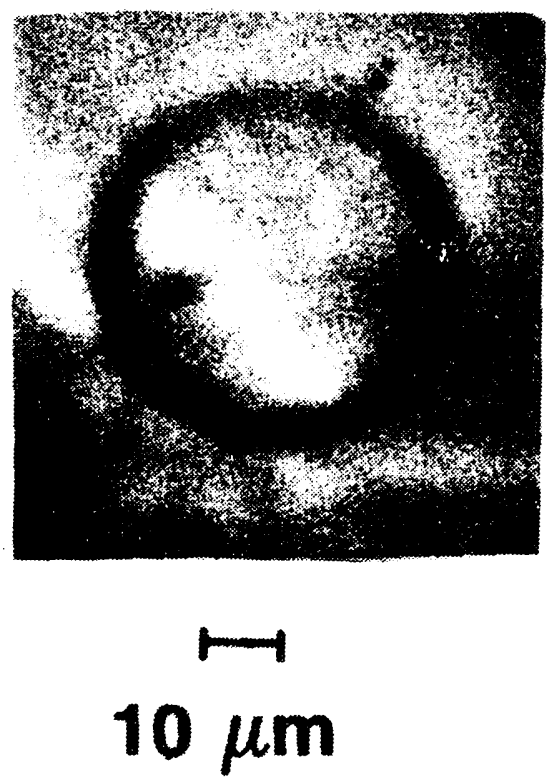

Figure 2.18: Monochromatic CL image, recorded at $780 \mathrm{~nm}$ at the sample temperature of about $20 \mathrm{~K}$, of the MQW region shown in Figure 16. 


\subsubsection{Diffusion in CdTe/CdS Thin Films Studied by SIMS}

Most CdTe used for photovoltaic applications requires a post-deposition heat treatment to optimize device performance. The intent of this study was to determine the amount of interdiffusion which occurred in the CdTe/CdS heterostructure during fabrication and post deposition annealing. Polycrystalline thin films of CdTe on CdS fabricated by two different methods were used. The first set of CdTe films were electroplated from solution onto $\mathrm{CdS} / \mathrm{SnO}_{\mathrm{x}} /$ glass to a thickness of approximately $2 \mu \mathrm{m}$. The second set was deposited by spray processing onto $\mathrm{CdS} / \mathrm{SnO}_{x} /$ glass to a thickness of approximately $6 \mu \mathrm{m}$. For both sets, the CdS was deposited by spray processing to a thickness of approximately $0.15 \mu \mathrm{m}$ and $6 \mu \mathrm{m}$, respectively.

A SIMS depth profile obtained from the as-deposited electroplated film is shown in Fig. 2.19. This profile shows that there has been no $\mathrm{S}$ or Te diffusion during deposition. To observe the effect of the post-deposition anneal, the film shown in Fig. 2.19 was divided into pieces and these were annealed at $375^{\circ}, 390^{\circ}, 410^{\circ}$ and $425^{\circ} \mathrm{C}$ for one hour each. Figure 2.20 shows a montage of the SIMS depth profile data obtained for $\mathrm{S}$ and Te from these samples. It is clear from this figure that even at the lowest temperature there has been a significant amount of S diffusion into the CdTe layer. It is also notable that the Te profile has not been significantly changed by the annealing process, indicating that while $\mathrm{S}$ is highly mobile in CdTe, Te does not move freely into the CdS layer. A depth profile obtained from one of the spray processed CdTe films is shown in Fig. 2.21. This profile appears to show that a significant amount of diffusion has occurred throughout both layers. This result is in contrast to the finding for electrodeposited films, where Te was found not to diffuse into the CdS layer.

Depth profile data can be influenced by the surface geometry, thus it is often necessary to examine the sample surface by scanning electron microscopy (SEM) to fully interpret the results. SEM images of the CdTe films used in this study are shown in Figs. 2.22a-f. The micrographs show the asdeposited electroplated films before sputtering, (a), and after sputtering. (d). Figure $2.22 \mathrm{~b}$, e show the $425^{\circ} \mathrm{C}$ annealed electroplated films before and after sputtering, respectively. In the electroplated films the CdTe layers are relatively smooth before sputtering and their surfaces are not changed by the ion beam during analysis. The surface of the spray processed film is shown in Figs. 2.22c,f. The CdTe layer of this film has significant topography before sputtering (Fig. 2.22c), and the ion beam causes the formation of large sputter cones during SIMS analysis (Fig. 2.22f). This last set of micrographs indicates that while the depth resolution is adequate for the analysis of the electroplated films, the SIMS analysis of the spray processed films will be severely limited by the original surface topography and the topography which develops as a result of ion bombardment. It is thus difficult to 


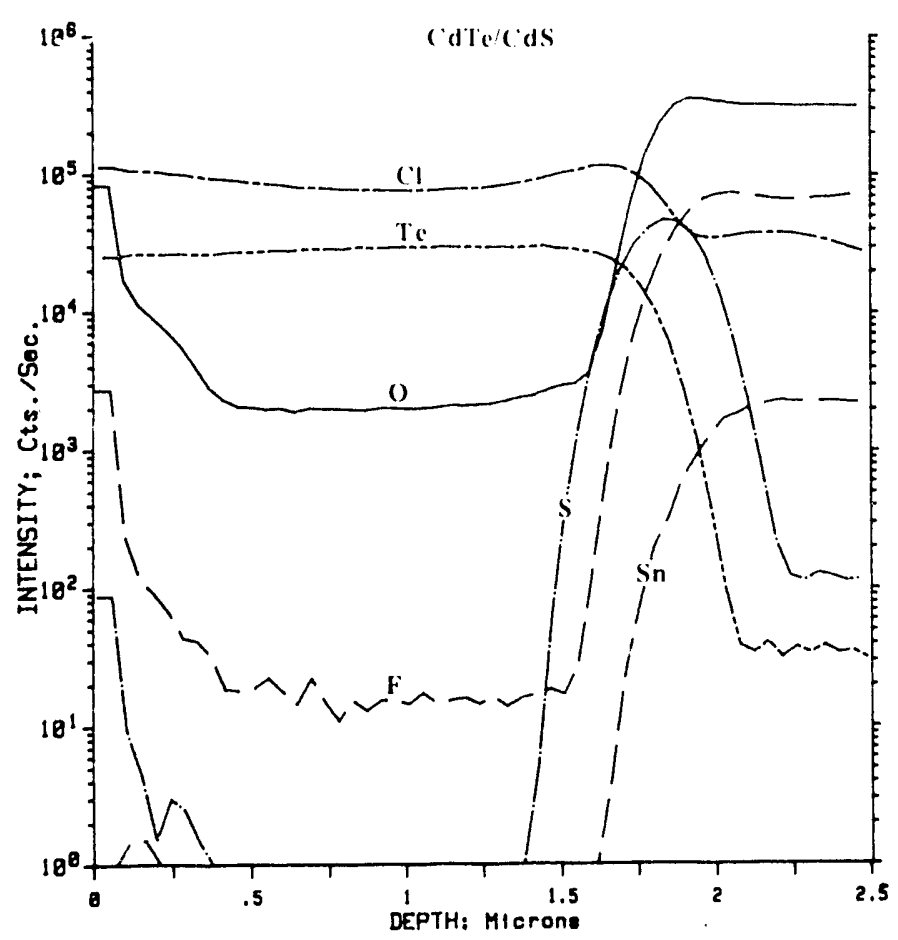

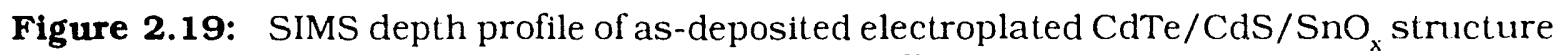
showing no apparent $\mathrm{S}$ or Te interdiffusion.

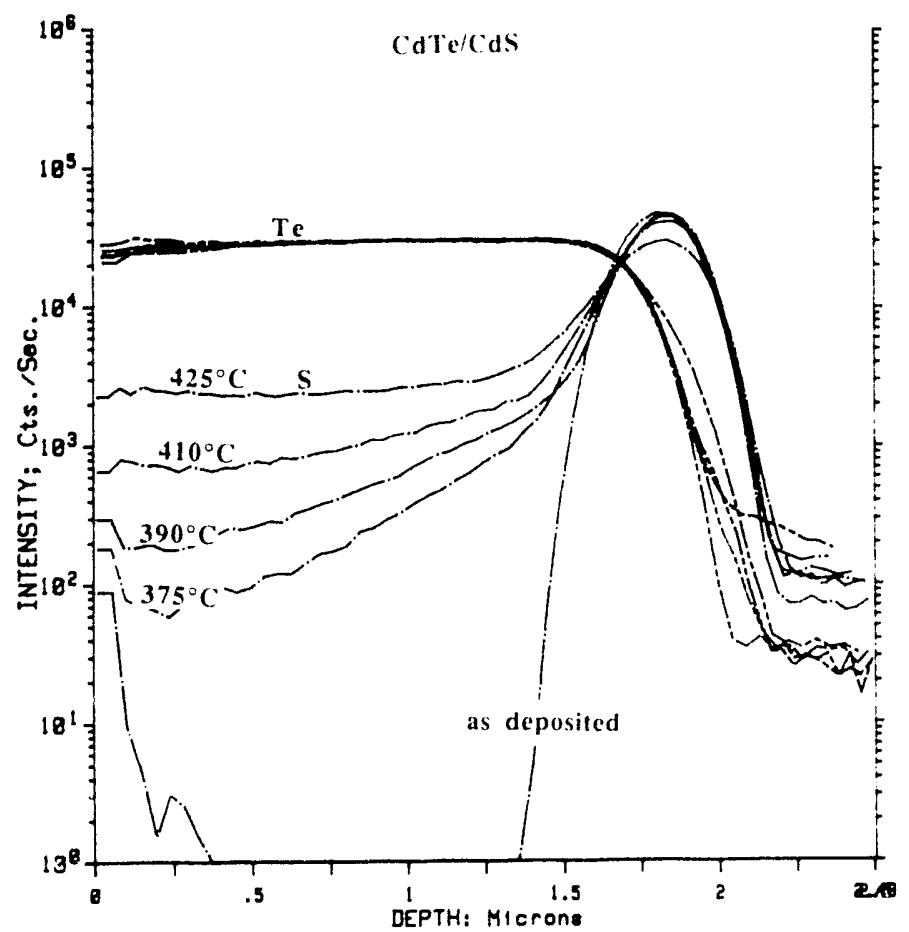

Figure 2.20: Showing the effect of post-deposition annealing on $S$ and Te interdiffusion. Montage of S and Te SIMS depth profiles generated by overlaying data from four different anneal temperatures onto the profiles from the as-deposited sample. Annealing temperatures were $375^{\circ}, 390^{\circ}, 410^{\circ}$, and $425^{\circ} \mathrm{C}$ for $1 \mathrm{~h}$ each. 


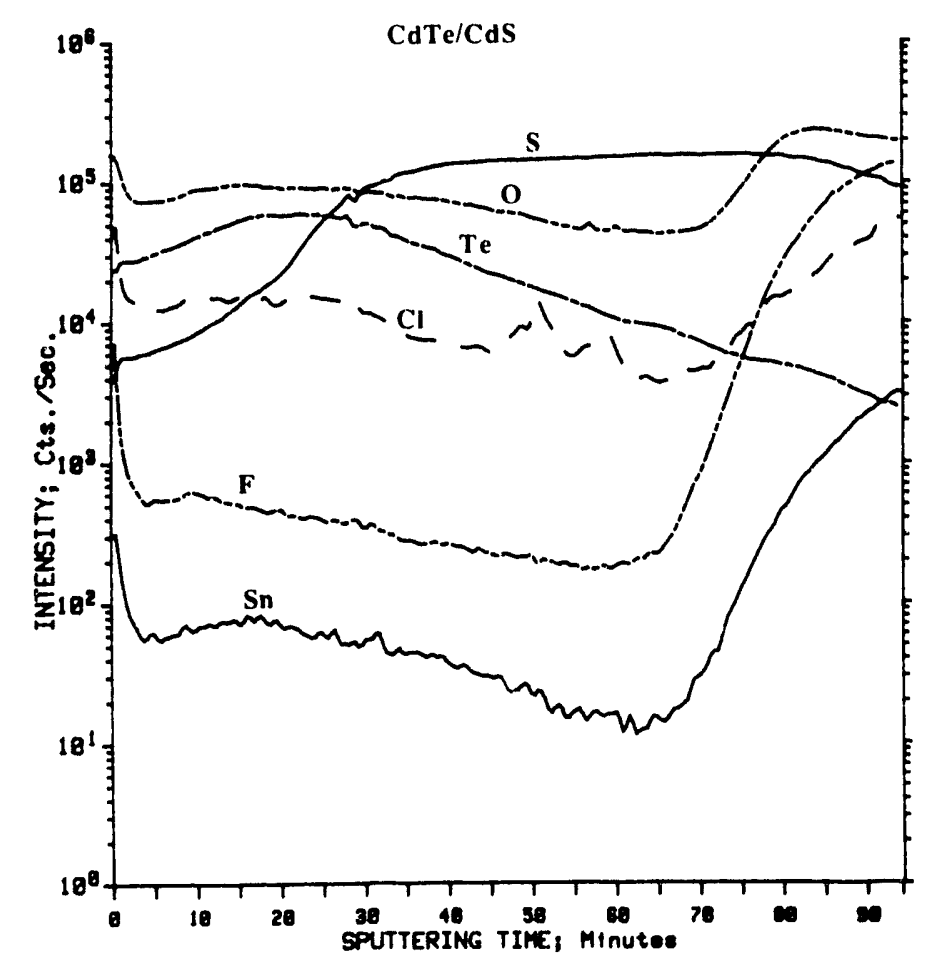

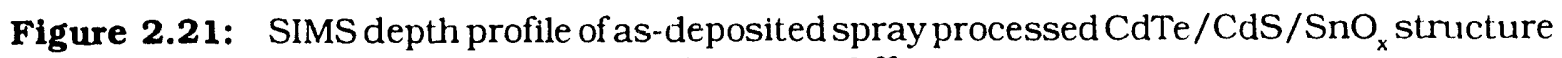
showing apparent $\mathrm{S}$ and Te interdiffusion.

determine, from SIMS analysis alone, whether or not diffusion has occurred in the spray processed film.

The formation of sputter cones presents a problem for any depth profiling technique that uses an ion beam to remove material from the surface. It is known that polycrystalline metals often exhibit surface roughening under ion bombardment due to variations in the sputter rates between the different crystal faces. SIMS is frequently used to study polycrystalline materıals. The preceeding samples were used to determine whether the initial surface topography or the sample crystallinity was more important in the development of sputter cones.

In this study, X-ray diffraction was used to determine the extent of crystallinity in the CdTe layers. The diffraction spectra for the three films shown in Figs. 2.22a-c are shown in Figs. 2.23a-c. The diffraction spectrum from the as-deposited electroplated film (Fig. 2.23a) shows that the film is cubic and highly oriented along the [111] direction. This is also evidenced in the SEM micrograph (Fig. 2.22a), which shows the surface of this film to be uniformly covered with a highly faceted, pyramid-like topography. The diffraction spectrum from the electroplated $425^{\circ} \mathrm{C}$ annealed film (Fig. 2.23b) shows that a significant change in the crystallinity has occurred. The spectrum now contains all the significant peaks from a randomly distributed 

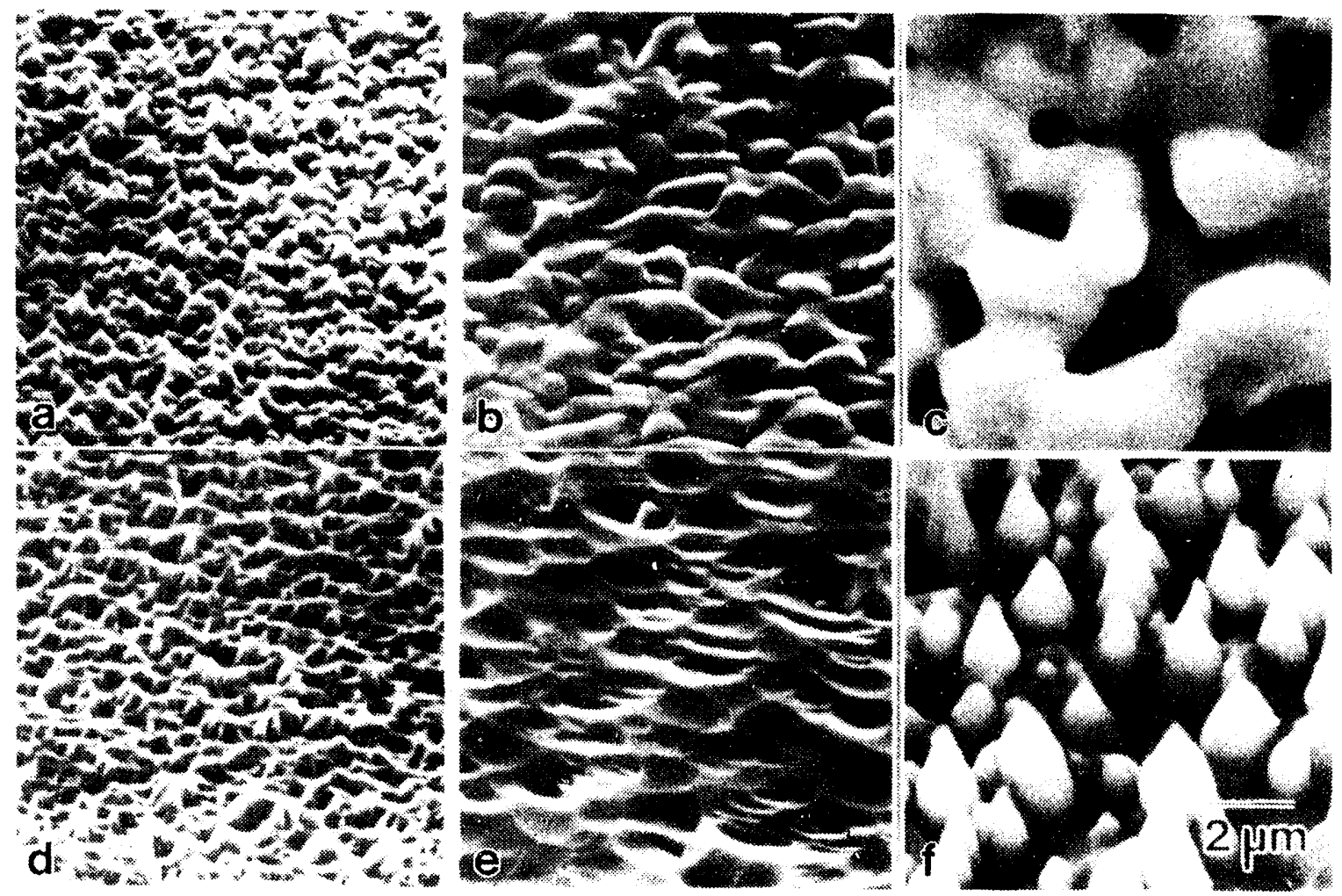

Figure 2.22: SEM micrographs of: (a) as-deposited electroplated CdTe surface before ion beam sputtering, (b) electroplated CdTe surface annealed at $425^{\circ} \mathrm{C}$ for 1 hour before ion beam sputtering, (c) spray processed CdTe surface before ion beam sputtering, (d) as-deposited electroplated CdTe surface after ion beam sputtering, (e) electroplated CdTe surface annealed at $425^{\circ} \mathrm{C}$ for 1 hour after ion beam sputtering, (f) spray processed CdTe surface after ion beam sputtering.

cubic polycrystalline film with no preferred orientation. In addition, a broad low angle peak is visible indicating some amorphous material is present. Note also that the SEM micrograph of this film (Fig. 2.22b) no longer exhibits the highly faceted structure observed in the as-deposited film (Fig. 2.22a). An unexpected result is the systematic decrease of the d-spacings in the annealed film, which is consistent with the incorporation of the smaller S atom into the Te sublattice. The diffraction spectrum in Fig. $2.23 \mathrm{c}$ is from the spray processed film. This spectrum is almost identical to the one obtained from the $425^{\circ} \mathrm{C}$ annealed film (Fig. 2.23b). It also contains the peaks expected from randomly distributed cubic material, however, no low angle amorphous peak is present. The spray processed film also exhibits the same systematic decrease in the d-spacings observed in the $425^{\circ} \mathrm{C}$ annealed film, an indication of probable S diffusion. Thus, from X-ray diffraction both the annealed electroplated film and the spray processed film are composed of randomly oriented. polycrystalline grains with a distorted crystal lattice that is smaller than pure CdTe. For sputter depth profiling these results imply that the more important parameter for these films is the original surface topography and not the crystallinity. 

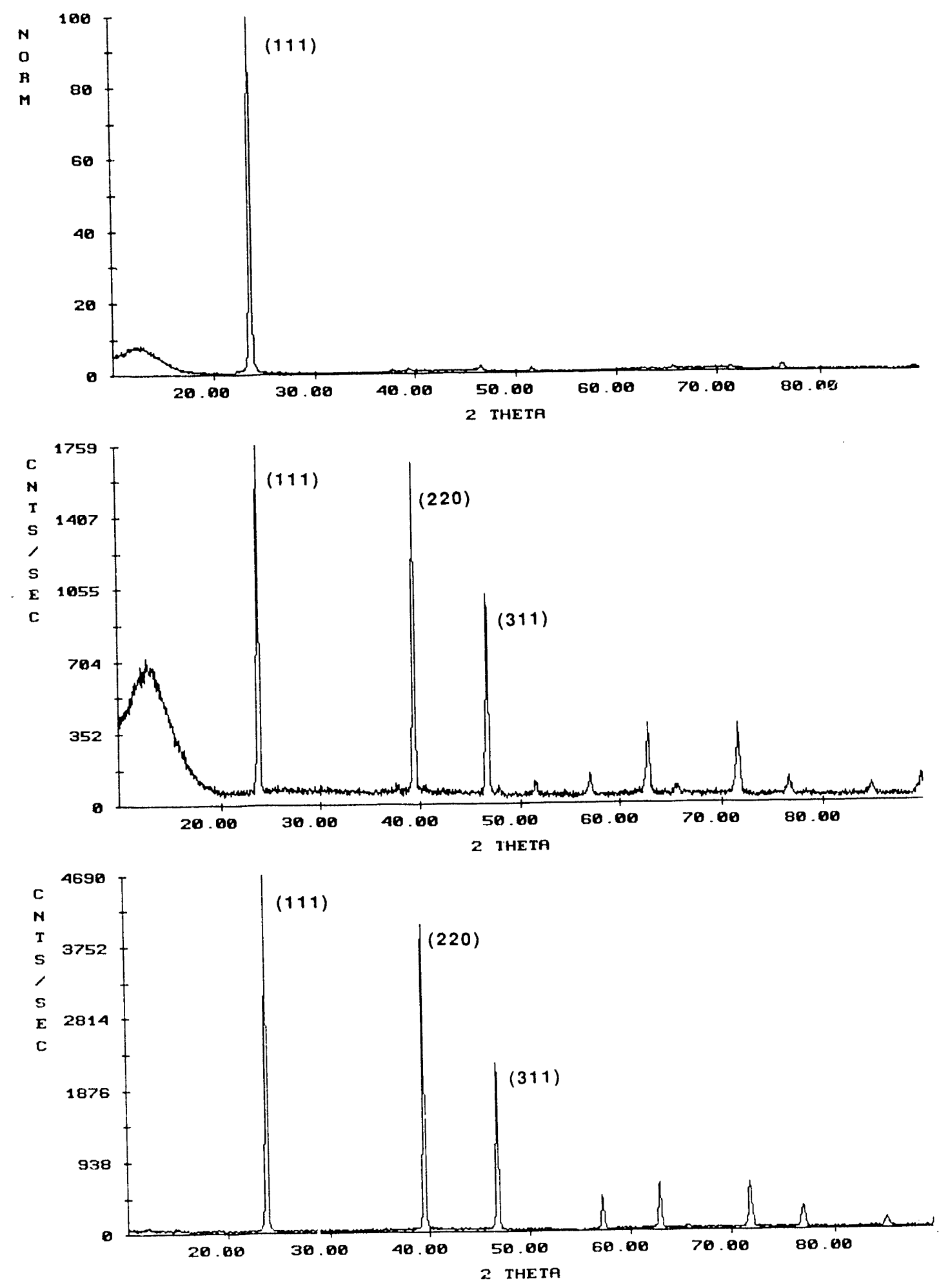

Figure 2.23: X-ray diffraction spectra from polycrystalline CdTe, (a) as-deposited electroplated film, (b) $425^{\circ} \mathrm{C}$ annealed electroplated film, (c) as-deposited spray processed film. Indices for the major cubic lattice peaks are labelled. 
The diffraction data suggested $\mathrm{S}$ diffusion in the annealed electroplated and the spray processed films. As an independent check of the S contents of these films, bulk compositional analysis was performed by electron probe microanalysis (EPMA). The S contents of the as-deposited electroplated film was found to be at or below the detection limit ( 0.1 at.\%) for EPMA. The S content of the $425^{\circ}$ annealed sample was found to be 1.9 at.\%. The spray processed film was found to contain a bulk S concentration of 3.5 at.\%. The $S$ values were consistent across each sample and for all films measured, confirming the $\mathrm{S}$ diffusion indicated by $\mathrm{X}$-ray diffraction.

In summary. SIMS was used to study the interdiffusion of $\mathrm{S}$ and Te in electroplated polycrystalline thin films of CdTe on CiS. At the temperatures studied $\left(375-425^{\circ} \mathrm{C}\right)$. S diffusion into CdTe is rapid. however, Te was not found to diffuse into CdS. After annealing at $425^{\circ} \mathrm{C}$ for one hour, the concentration of $\mathrm{S}$ in the electroplated film was found to be uniform throughout the CdTe layer (by SIMS) at a level of 1.9 at.\% (by EPMA). The as-deposited film was found to be cubic, and highly oriented along (111). The annealed film was found to be composed of randomly oriented cubic polycrystalline grains, with some amorphous material. The SIMS investigation of the spray processed film was hindered by the extreme topography of the CdTe layer. In addition, the ion beam caused the development of sputter cones which further obscured diffusion effects. Sulphur diffusion in this film was confirmed by a combination of EPMA and X-ray diffraction. The S content of the spray processed film was found to be 3.5 at.\%. Finally, the development of sputter induced topography was found to be more dependent on the initial surface roughness than on the sample crystallinity.

\subsection{ACCOMPLISHMENTS: RELATED RESEARCH AREAS}

\subsubsection{AES Line-Shape Analysis at Grain Boundaries of Oriented

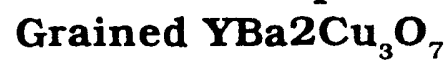

The major impediment to the technological application of the bulk $\mathrm{YBa}_{2} \mathrm{Cu}_{33} \mathrm{O}_{7}$ high temperature superconductor is its low transport critical current density $(J)$. typically on the order of $10-10^{3} \mathrm{~A} / \mathrm{cm}^{2}$ at $77 \mathrm{~K}$. This limitation in performance can be traced to the grain boundaries and can be explained by Josephson type weak-links between superconducting grains. Several studies have shown deviations in composition at grain boundaries even in the absence of impurity segregation in addition to structural disorder. This weak-link problem, caused by grain boundary inhomogeneities. can be overcome through the proper control of microstructure and stoichiometry yielding $\mathrm{J}_{1}$ 's of $\sim 17.000 \mathrm{~A} / \mathrm{cm}^{2}$ for bulk melt textured $\mathrm{YBa}_{2} \mathrm{Cu}_{3,3} \mathrm{O}_{x}$ ceramics and $~ 75.000 \mathrm{~A} / \mathrm{cm}^{2}$ for bulk texture-modified $\mathrm{YBa}_{2} \mathrm{Cu}_{3} \mathrm{O}_{x}$ material. In order to improve $\mathrm{J}_{\text {. ' }} \mathrm{s}$, it is necessary to accurately determine the exact composition and chemistry of the grain boundary and any secondary phase(s) to assist 
in developing process parameters which will effectively reduce or eliminate these compositional inhomogeneities and secondary phases. Auger electron spectroscopy (AES) is an ideal technique for investigating the local chemical environment of atomic species at grain boundaries in $\mathrm{YBa}_{2} \mathrm{Cu}_{3} \mathrm{O}_{7}$ due to its sensitivity to variations of the local atomic charge density across the valence band (VB), its high spatial resolution $(\sim 300 \AA)$ and its depth resolution $(\sim 10$ A).

This section presents and discusses the results of a high resolution Auger line-shape analysis of the $\mathrm{Y}(\mathrm{LMM}), \mathrm{Ba}(\mathrm{MNN}), \mathrm{Cu}(\mathrm{LMM})$ and $\mathrm{O}(\mathrm{KLL})$ peaks on the surface of grains and at grain boundaries of vacuum cleaved, orientedgrained bulk $\mathrm{YBa}_{2} \mathrm{Cu}_{3} \mathrm{O}_{7}$. Observed changes in the line shapes between grains and grain boundaries are related to the presence of compositional inhomogeneities and intergranular phases, with phase identification based on comparisons with spectra from the literature.

The oriented-grained bulk $\mathrm{YBa}_{2} \mathrm{Cu}_{3} \mathrm{O}_{7}$ samples were fabricated by a liquid phase processing method developed by K. Salama at the Texas Center for Superconductivity, University of Houston. A sintered bar sample, 4 to 6.5 $\mathrm{cm}$ long, is vertically introduced into a furnace which is preheated to about $1100^{\circ} \mathrm{C}$. and is maintained at this temperature for 5 to 10 minutes. During this period, the superconducting phase melts incongruently into $\mathrm{Y}_{2} \mathrm{BaCuO}_{5}$ and a liquid phase. The sample is then rapidly cooled to $1030^{\circ} \mathrm{C}$ and then cooled at a rate of 1 to $2^{\circ} \mathrm{C}$ /hour to temperatures ranging from $980-925^{\circ} \mathrm{C}$. Slow cooling through the peritectic temperature permits the reaction of the decomposed phases to form the 1-2-3 phase. In this process, no thermal gradient exists in the sample allowing the whole melt to solidify at a slow rate through the peritectic temperature. The sample is cooled further, at a rate of $1^{\circ} \mathrm{C} /$ minute to room temperature with a hold at $550^{\circ} \mathrm{C}$. The sample is then annealed in oxygen for 12 hours at $600^{\circ} \mathrm{C}$ and cooled to room temperature with long holds at $500^{\circ} \mathrm{C}$ and $400^{\circ} \mathrm{C}$. The samples prepared by this method have a microstructure with long plate-type thick grains oriented over a wide area parallel to the $a-b$ plane.

Results of the multipoint EMPA compositional analysis indicates that the bulk composition of the oriented-grained $\mathrm{YBa}_{2} \mathrm{Cu}_{3} \mathrm{O}_{7}$ sample consists of 8.9 at. $\% \mathrm{Y}, 16.6$ at. $\% \mathrm{Ba}, 21.8$ at. $\% \mathrm{Cu}$ and 52.7 at.\% $\mathrm{O}$. These results indicate that the bulk sample is stoichiometric and homogeneous.

Figure 2.24 shows a photomicrograph of the vacuum fractured orientedgrained $\mathrm{YBa}_{2} \mathrm{Cu}_{3} \mathrm{O}_{7}$ surface used for this investigation. The flat features are parallel to the $a-b$ plane and are only partially exposed by the fracture. Specifically, the surfaces which extend under other material, are judged to be c-axis grain boundaries. Thus, the points where Auger data was acquired are labeled $\mathrm{A}, \mathrm{B}$ and $\mathrm{C}$ and are representative of $\mathrm{c}$-axis surface grain 


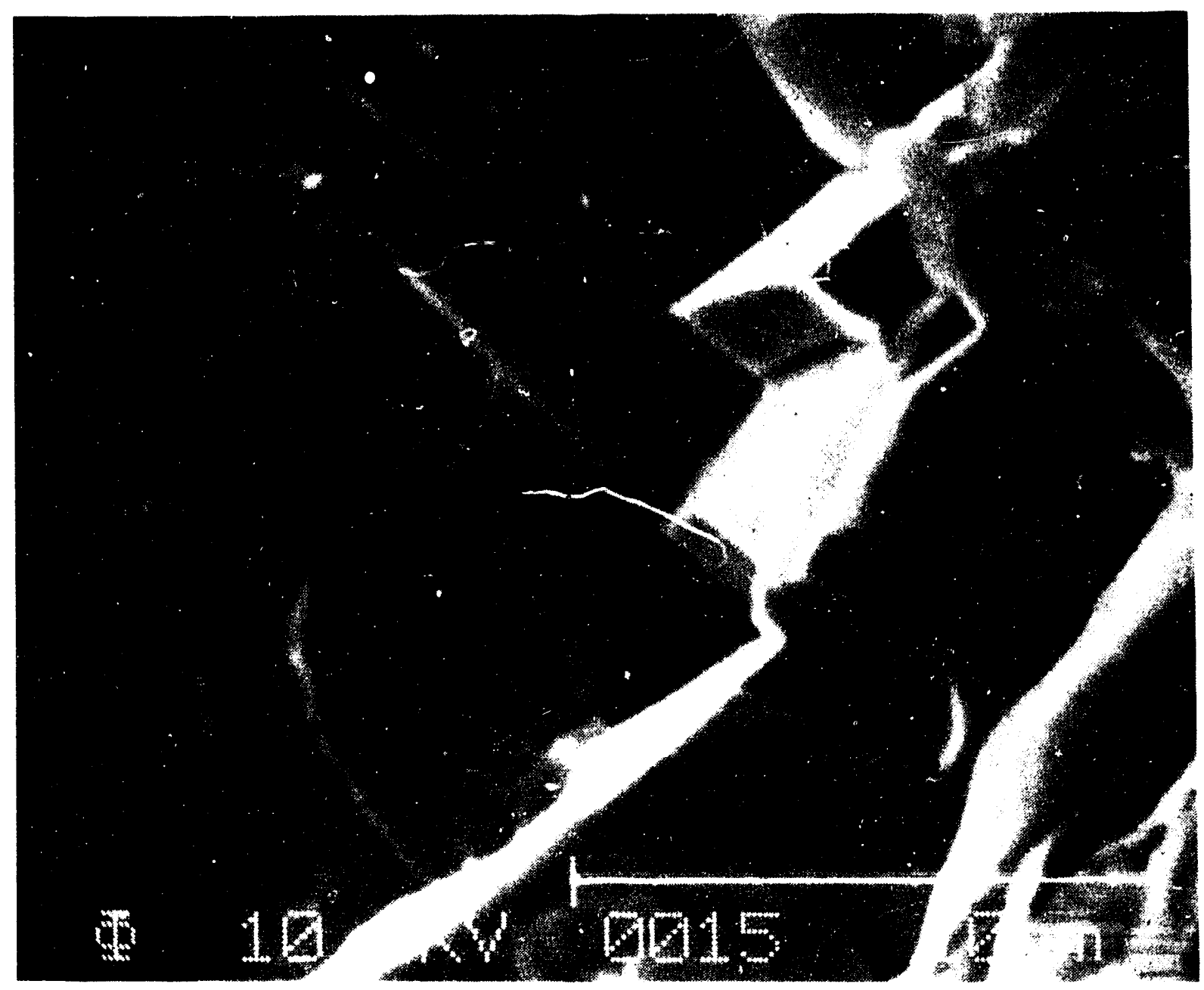

Figure 2.24: A photoniciograph of the vacmun fractured oriented-grained $\mathrm{YBa}_{22} \mathrm{Cu}_{3} \mathrm{O}_{7}$ surfare.

boundaries (A.C) and a-b grain surfaces (B) as referenced in the accompanying spectra.

Auger results for the Ba $M_{1} N_{4} N_{4}$ ine shapes obtained on a grain boundary and at a grain surface are presented in Fig. 2.25. The line shape and peak energy are strongly influenced by the (hemical environment since the Auger electron emission involves valence electrons and the core levei binding energy. Line shape analvsis of the Y(i,MM) Auger line revealed no pertinent chemical effects and thus is not induded in this presentation. The Ba MNN spectratequired at the grain houndary is relatively narrow and centered at a kinetic concrgy of 581.0 ( V Vhereas the spectra acquired at the grain surface is relatively hoad with its centroid shifted by $-1 \mathrm{eV}$ to $582.0 \mathrm{eV}$. The most stratghtforwarel explanation for the froadened line shape is that it romsistsof asmperpositionof the surfare Ba core level with the bulk Ba core- 


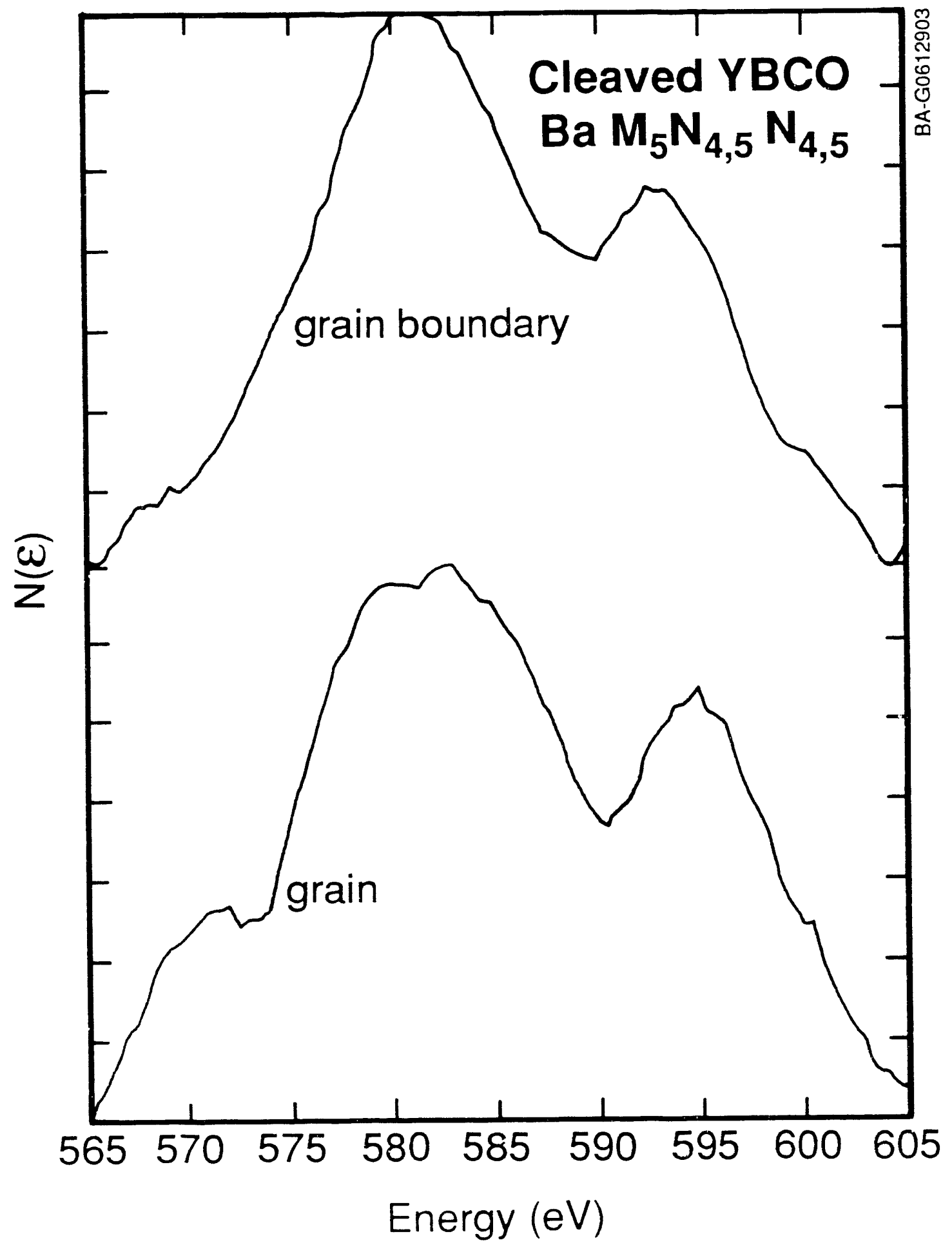

Figure 2.25: Auger results for the Ba $M N_{4} N_{4}$ - line shapes obtained on a grain boundary and at a grain surface. 
level. The surface Ba core-level shift indicates that the Ba atoms are in an altered surface environment possible due to charge transfer effects. reduced core-hole screening of the final state. or effects of the altered surface Madelung potential. Based on this interpretation, the differences between the Ba MNN line shapes acquired at a c-axis grain boundary and at the a$\mathrm{b}$ grain surface are not due to the presence of additional Ba phases.

The $\mathrm{Cu} \mathrm{L} \mathrm{L}_{3} \mathrm{M}_{4.5} \mathrm{M}_{4.5}$ line shapes obtained on a grain boundary and at a grain surface are presented in Fig. 2.26. The initial states of the $\mathrm{Cu} \mathrm{L}_{3} \mathrm{VV}$ Auger line are formed from the $3 \mathrm{~d}^{-1}$ and $3 \mathrm{~d}^{10} \underline{\mathrm{L}}$ states $[\underline{L}$ designates a ligand $(\mathrm{O} 2 \mathrm{p})$ holel of divalent copper with the final states formed by the $3 \mathrm{~d}^{7}$ and $3 \mathrm{~d}^{8} \underline{\mathrm{L}}$ multiplets with the $3 \mathrm{~d}^{\mathrm{s}} \underline{\mathrm{L}}$ states dominate. The Cu LMM spectra obtained at the grain boundary is also relatively narrow and is centered at a kinetic energy of $913.5 \mathrm{eV}$. The spectra acquired at the grain surface has broadened and shifted relative to the grain boundary spectra. Comparison of these $\mathrm{Cu}$ LVV spectra with $\mathrm{Cu}$ LVV spectra shown in the literature for $\mathrm{YBa}_{2} \mathrm{Cu}_{3} \mathrm{O}_{7}$. $\mathrm{Y}_{2} \mathrm{BaCuO}_{5}$. $\mathrm{CuO} . \mathrm{Cu}_{2} \mathrm{O}$ and $\mathrm{Cu}$ metal indicates that the grain boundary has an overlayer of $\mathrm{CuO}$, the thickness of which can be estimated from the escape depth of the secondary electrons. Based on the escape depth for $910 \mathrm{eV}$ electrons ( 10 to $15 \AA$ ) obtained from the universal escape depth curve and the fact that no substrate features are distinguishable in the Cu LVV spectra acquired at a grain boundary, the thickness of the $\mathrm{CuO}$ overlayer is estimated to be $\leq 15 \AA$ A. Additionally, the Cu LVV spectra obtained on a grain surface compares quite well with the $\mathrm{Cu} \mathrm{LVV} \mathrm{spectra} \mathrm{for} \mathrm{YBa}_{2} \mathrm{Cu}_{3:} \mathrm{O}_{7}$, further enhancing the viability of the Auger line-shape technique in determining chemical bonding.

The $O \mathrm{~K}_{1} \mathrm{~L}_{2.3 .3} \mathrm{~L}_{2.3 .3}$ i;- shape, shown in Figure 2.27 for a grain boundary and at a grain surface. tend to support this interpretation. The O KLL spectra acquired at the grain boundary is relatively broad indicating multiple $O$ states in contrast to the narrow $\mathrm{Ba}$ and $\mathrm{Cu}$ lines obtained over the same area. This broadened line shape probably consists of a superposition of the bulk $\mathrm{O}$ core-level component at $509.0 \mathrm{eV}$ and a shifted grain boundary $\mathrm{O}$ corelevel component at $506.0 \mathrm{eV}$. The shifted grain boundary component represents $\mathrm{O}$ in $\mathrm{CuO}$ where the important basis states are $\mathrm{Cu} 3 \mathrm{~d}^{\prime \prime} 4 \mathrm{~s}^{\prime \prime} \mathrm{O} 2(\mathrm{sp})^{\prime \prime}$ and $\mathrm{Cu} 3 \mathrm{~d}^{10} 4 \mathrm{~s}^{\prime \prime} \mathrm{O} 2(\mathrm{sp})^{7}$ and the chemical shift would be towards higher energy as observed.

The presence of the thin $\mathrm{CuO}$ layer at $\mathrm{c}$-axis grain boundaries (A.C) of this bulk texture-modified $\mathrm{YBa}_{2} \mathrm{Cu}_{33} \mathrm{O}_{x}$ material will certainly affect the transport properties along the c-axis depending on whether the layer is insulating. semiconducting or metallic at $\mathrm{T}_{\text {.. }}$. If the intergranular $\mathrm{CuO}$ layer is insulating or semiconducting. poor transport properties (conductivity $\left(\sigma_{2}\right)$ and $J_{\text {. }}$ ) are observed. depending on the thickness. The $a-b$ grain surfaces (B). which ronnert conducting $\mathrm{CuO}_{2}$ planes. do not show $\mathrm{CuO}$ segregates and thus 


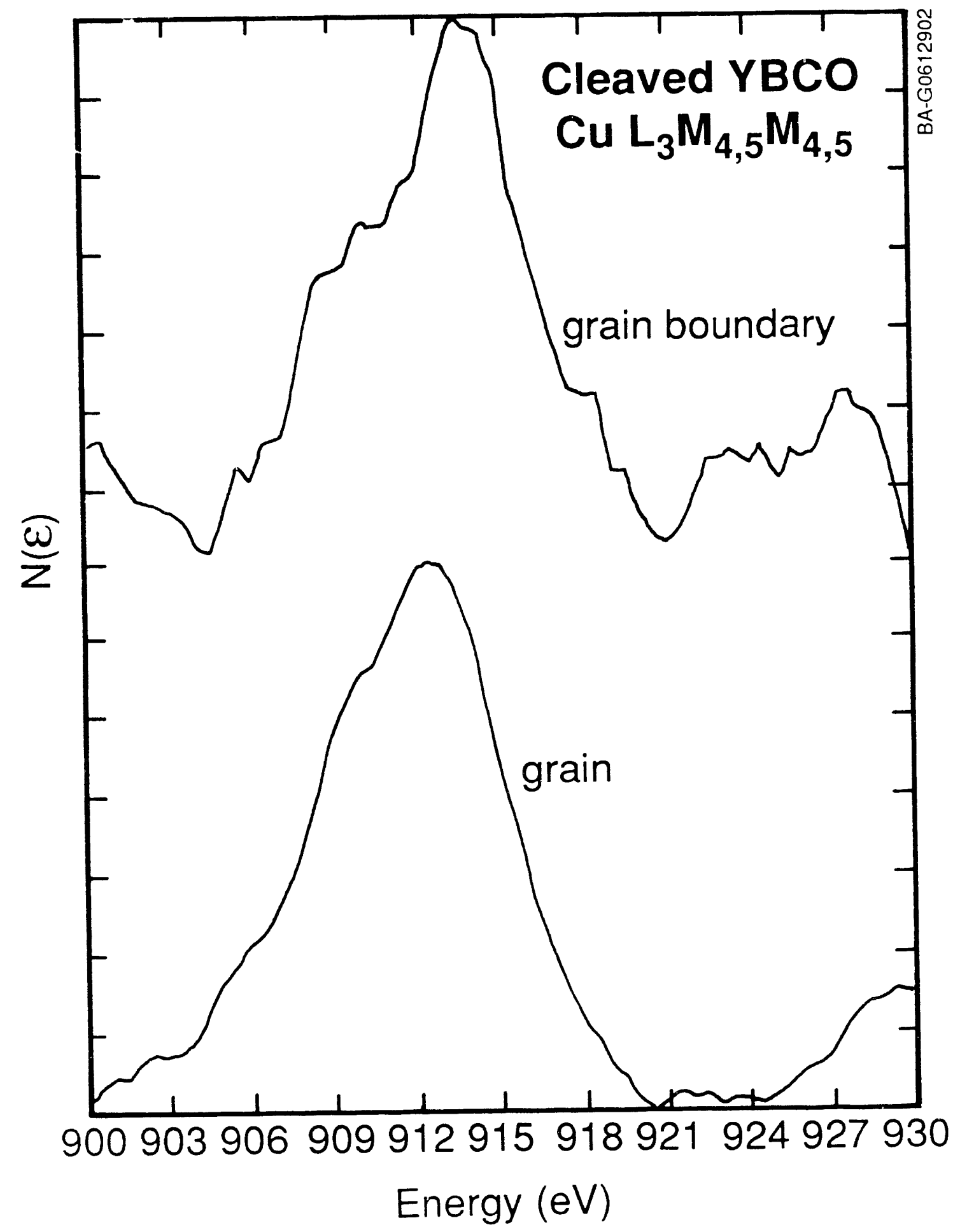

Figure 2.26: The $\mathrm{Cu} \mathrm{L}_{3} \mathrm{M}_{4,3} \mathrm{M}_{4}$. line shapes obtained on a grain boundary and at a grain surface. 


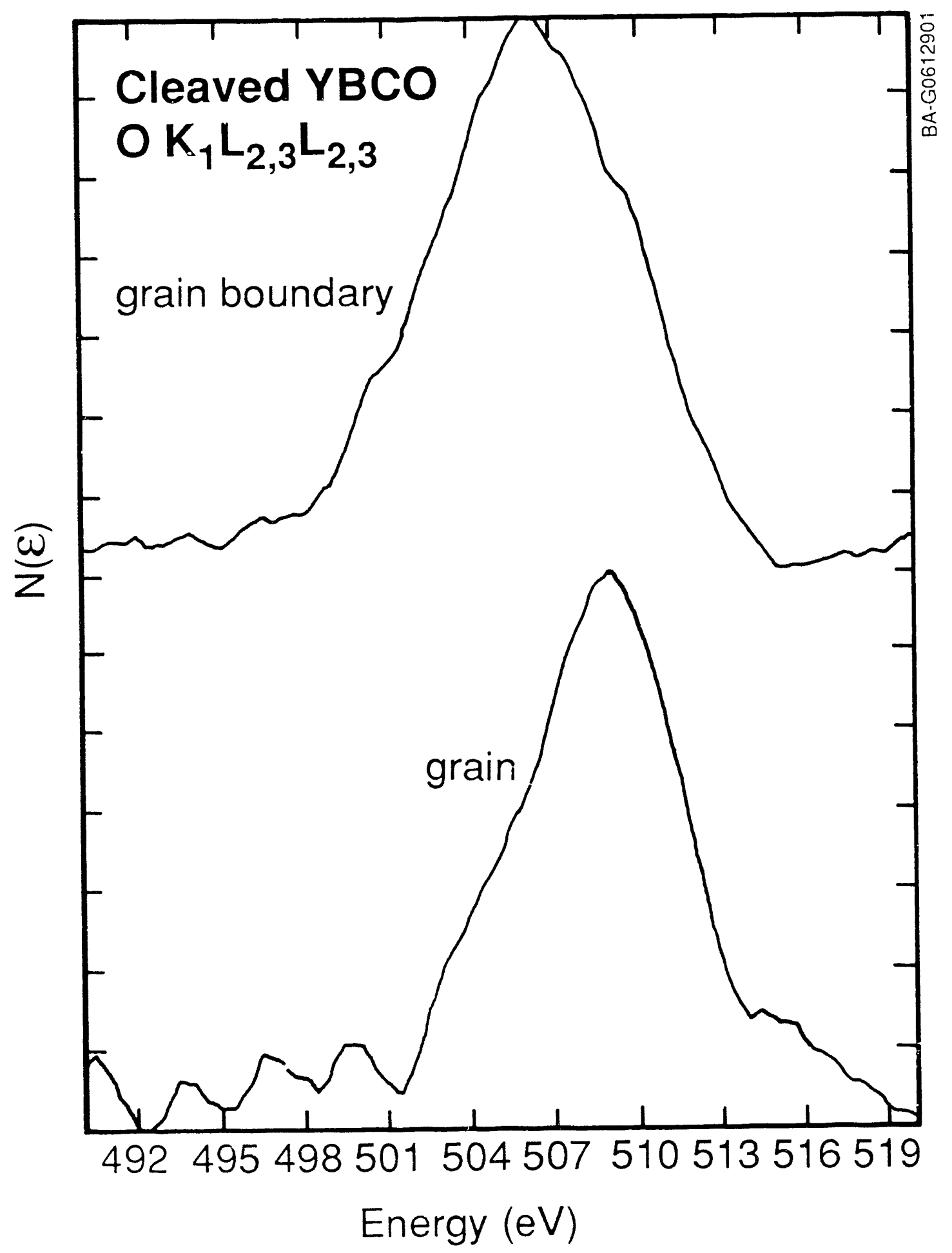

Figure 2.27: The $O K_{1} L_{2, i} L_{2, i}$ line shape for a grain boundary and at a grain surface. 
yield a higher $\sigma_{1}$ and $J_{1,11}$ (by a factor of $\sim 20$ ). If the $\mathrm{CuO}$ was metallic, the layer would act as a normal metal with a large coherence length which would become superconducting by the proximity effect. Unfortunately, no clear determination of the physical properties of the CuO layer can be made from these measurements.

In summary. Auger line shape analysis was used to characterize the surface and grain boundary chemistry of oriented-grained bulk $\mathrm{YBa}_{2} \mathrm{Cu}_{33} \mathrm{O}_{7}$ which had been cleaved in vacuum. The bulk material was fabricated using a liquid phase processing method which utilizes slow cooling through the peritectic transformation to control the microstructure yielding long, thick platelets exhibiting high critical current density. Observed changes in the $B a M_{5} N_{4.5} N_{4.5}$. $\mathrm{Cu} \mathrm{L} \mathrm{L}_{3,3} \mathrm{M}_{4,5} \mathrm{M}_{4.5}$ and $\mathrm{OK} \mathrm{L}_{1,3.3} \mathrm{~L}_{2,3}$ line shapes between grains and grain boundaries are related to the presence of $\mathrm{YBa}_{2} \mathrm{Cu}_{33} \mathrm{O}_{7}$ grains and a thin $\mathrm{CuO}$ intergranular phase. The presence of his thin intergranular phase may be responsible for Josephson type weak coupling between grains which limits current transport across grain boundaries.

\subsubsection{SIMS Investigations of Substrate/Film Interdiffusion in $\mathrm{YBa}_{2} \mathrm{Cu}_{3} \mathrm{O}_{7}$ Films Grown on $\mathrm{SrTiO}_{3}, \mathrm{LaAlO}_{3}$, and $\mathrm{NdGaO}_{3}$}

Contamination at the grain boundaries of high temperature superconducting (HTSC) thin films may limit the critical current densities $\left(J_{r}\right)$ of the films. One potential source of contamination is the diffusion of substrate elements into the $\mathrm{YBa}_{2} \mathrm{Cu}_{33} \mathrm{O}_{7}(\mathrm{YBCO})$ during high temperature annealing. Post-deposition annealing is often necessary to produce or enhance the superconducting properties of the film. An early study performed with SIMS found that significant diffusion of substrate elements had occurred at annealing temperatures of $850^{\circ} \mathrm{C}$. This result appeared to correlate with the superconducting transition temperature $\left(\mathrm{T}_{1}\right)$. A second study, however, found little correlation between $J_{1}$ and the apparent diffusion of substrate elements. The current study had two goals; to correlate the YBCO film morphology with the observed SIMS depth profile, and to use these results to perform a controlled study of substrate element interdiffusion using several different substrates.

SIMS is a useful technique for the study of elemental interdiffusion. however. when analyzing thin films it is important to correlate the observed depth profile with the film morphology. Surface topography can cause distortions in the profile by altering the depth resolution. The depth resolution is determined, in part. by the various layers of the sample which are exposed to the primary ion beam at one time. If secondary ions are simultaneously emitted from a wide range of depths. then the profile appears as a mixture of layers similar to diffusion. These artifacts are more pronounced in thin films because anomalies in the film surface often have 
similar dimensions to the film thickness. This can cause distortion throughout the entire depth profile. This effect was found to be significant for YBCO thin films and is illustrated in the following section.

The following depth profiles were obtained from two multilayer structures composed of the following layers, $\mathrm{YBCO} / \mathrm{LaAlO}_{3:} /-\mathrm{YBCO} / \mathrm{LaAlO}_{33}$ substrate $\left(\mathrm{LaAlO}_{3}=\mathrm{LAO}\right)$, with the approximate thicknesses of $200 \mathrm{~nm} / 100 \mathrm{~nm} / 200$ $\mathrm{nm} /$ substrate, respectively. Figure 2.28 shows a typical depth profile through a structure of this type. Here the layers appear to have sharp interfaces with a minimum of interdiffusion. Figure 2.29 shows an SEM micrograph of the surface of this sample. The surface is relatively featureless and does not contain pinholes or extra material. The depth profile shown in Fig. 2.30 is from a second multilayer sample. This profile appears to show that the layers of the structure have undergone significant interdiffusion at all of the interfaces. Although the structures were fabricated by different methods they were not expected to vary greatly in the amount of interdiffusion. Figure 2.31 shows an SEM image of the surface of the second structure. This sample has a highly structured surface which contains large valleys and peaks with dimensions on the same order of magnitude as the film thickness. The uneven surface of the second sample results in secondary ion emission from different depths in the film. The subsequent depth profile from this sample is distorted and appears to show interdiffusion. Although not shown here, depth profiles were also found to be distorted by the presence of extra material on the surface of the films. The results of these analyses show that in order to observe diffusion effects it is necessary to avoid rough or non-uniform areas during SIMS analysis.

The YBCO growth method most suited for the study of diffusion is laser ablation. Laser ablated films are attractive for two reasons: (1) The films are superconducting without the added step of a post-deposition anneal in flowing $\mathrm{O}_{2}$; and (2) the films are, in general, smooth and featureless, containing little if any topography that would obscure diffusion information. The former reason is important because YBCO undergoes a morphological transformation at approximately $800^{\circ} \mathrm{C}$. This transformation can cause an otherwise smooth film to become rough and textured. Some YBCO deposition techniques require post-deposition annealing above this limit to produce superconducting films. The annealing temperature for this study was chosen to be below the onset of the morphological change.

YBCO films were deposited by laser ablation from a stoichiometric target with a laser power of $1.8 \mathrm{~J} / \mathrm{cm}^{2}$. The samples were kept under flowing $\mathrm{O}_{2}$ at a pressure of 27 pascals and the deposition rate was $60-80 \mathrm{~nm} / \mathrm{min}$. The substrate temperature was held at $730^{\circ} \mathrm{C}$ during the deposition. Total film thicknesses were between $180-300 \mathrm{~nm}$. Three different substrates were studied. SrTiO, $\mathrm{LaAlO}_{3,}$, and $\mathrm{NdGaO}_{33}$. The post-deposition anneals were 


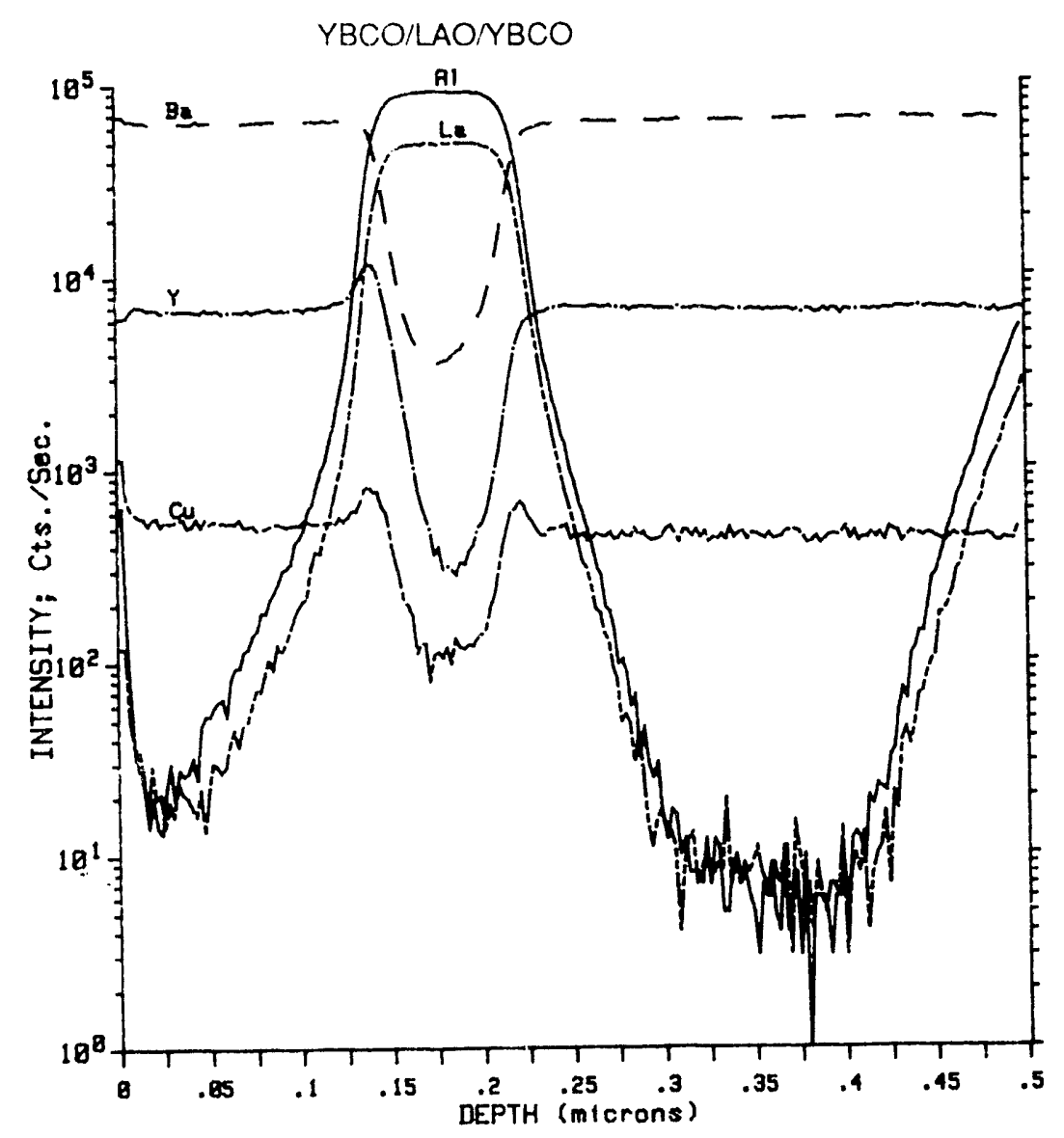

Figure 2.28: SIMS depth profile of YBCO/LAO/YBCO/LAO substrate mult ilayer structure showing sharp interfaces and minimal interdiffusion.

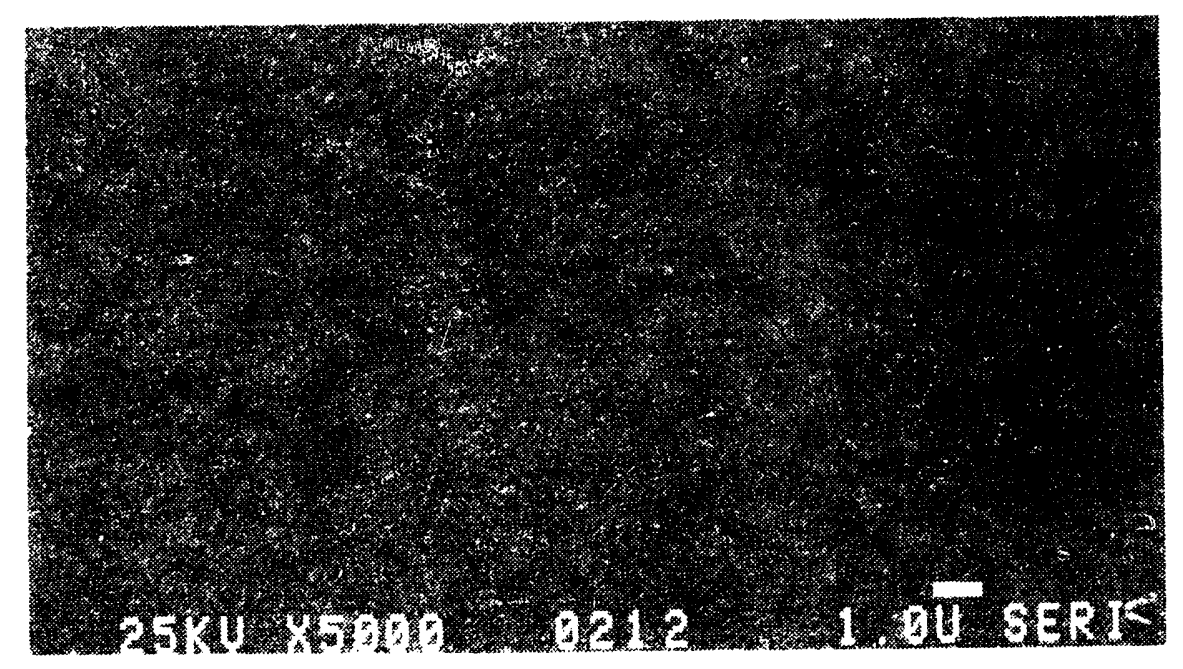

Figure 2.29: SEM nicrograph of the surface of the sample shown in Figure 23. The sample surface is featureless with a minimal amount of lopography. 


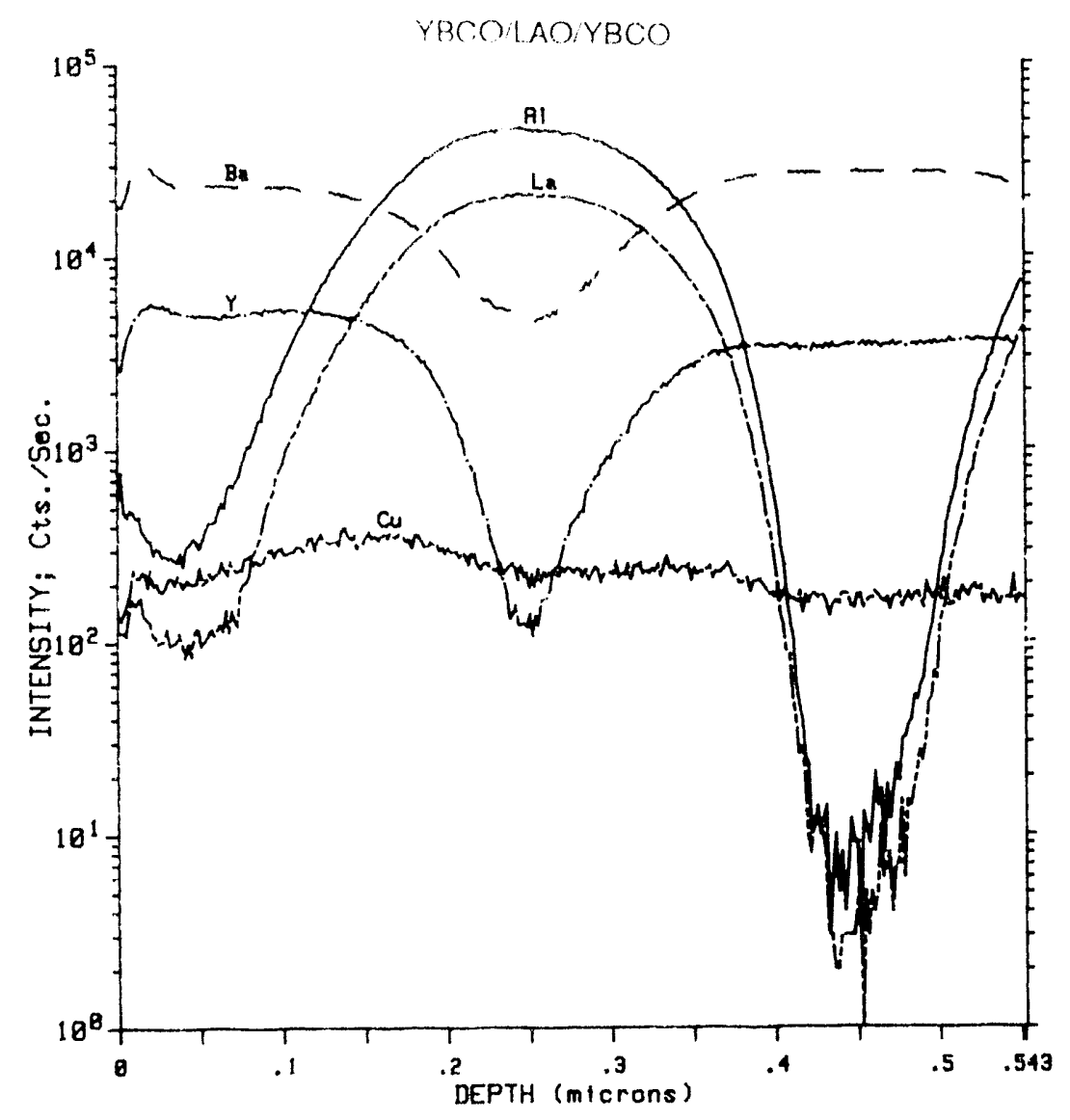

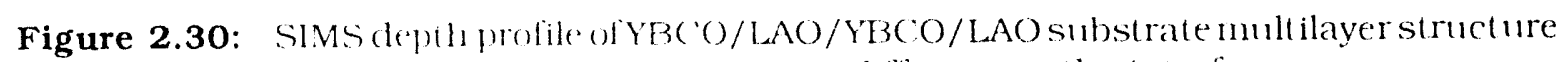
stuming alplarentextemsive interdifusion at the interfaces.

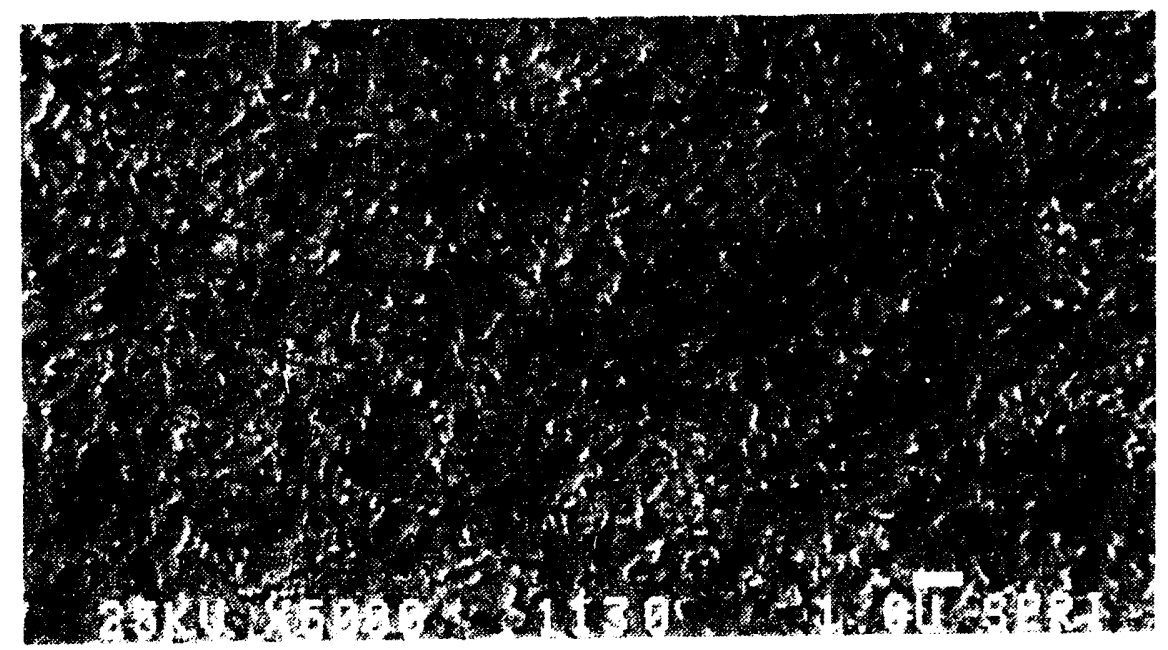

Figure 2.31: SHM nutrograph of he suface of the sample shown in Figure SIMS8. The

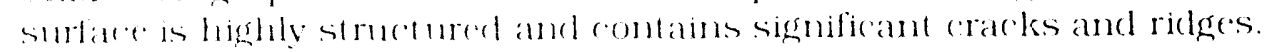


performed at $750^{\circ} \mathrm{C}$ in a diffusion furnace under flowing $\mathrm{O}_{2}$. The films were cooled in the furnace under $\mathrm{O}_{2}$. The annealing times for each set of films are given in Table 2.1.

Table 2.1: Annealing times for the films.

\begin{tabular}{|c|c|}
\hline Substrate & Anneal Times (min) \\
\hline $\mathrm{LaAlO}_{3}$ & $\begin{array}{llll}0 & 15 & 31 & 44\end{array}$ \\
\hline $\mathrm{SrTiO}_{33}$ & $\begin{array}{lll}0 & 30 & 60\end{array}$ \\
\hline $\mathrm{NdGaO}_{3}$ & $\begin{array}{lll}0 & 30 & 60\end{array}$ \\
\hline
\end{tabular}

Prior to SIMS analysis each film was examined by SEM to determine the quality of the surface. In general, the surfaces were smooth and uniform. although all the samples contained areas with extra material 'spattered' on the surface. These areas invariably yielded depth profiles showing the apparent 'diffusion' of substrate elements through the film to the surface. Areas which were free of extraneous material yielded quite different results. In the smooth areas, there was little evidence for diffusion of substrate elements for any of the three substrates studied. This indicates that for films with few grain boundaries there is no diffusion. Depth profiles from the four $\mathrm{LaAlO}_{33}$ films are shown in Figs. 2.32a-d. This series of profiles illustrates that there is little to no diffusion of substrate elements into the YBCO for the annealing temperature used in this study. For the longest anneal (Fig. 2.32d), it appears that a small amount of La has diffused into the film. This is the only sample out of the three substrates studied which showed any evidence of diffusion. SEM micrographs of the film surface showed that some small pinholes had formed after annealing. One possible explanation for the La signal is that the substrate was visible through the pinholes from the beginning of the profile. This explanation is suspect, however, because there is no Al signal. La and Al have similar sensitivities, thus if the substrate was showing through a pinhole, there should also be an Al signal present. A second explanation is that La moves up the edges of the pinhole from the substrate. This would imply that La is mobile at the edges of grains (along the boundaries) but not inside the individual grains. It also implies that La is more mobile than Al. More study is necessary to understand this result.

In summary, this work has shown that no diffusion of substrate elements is observed following high temperature annealing of YBCO thin films on various substrates. Anneals were performed at a temperature of $750^{\circ} \mathrm{C}$ for times up to $60 \mathrm{~min}$. Three different substrates were studied, $\mathrm{SrTiO}_{3}, \mathrm{LaAlO}_{3}$, and $\mathrm{NdGaO}_{3}$. The critical parameter for obtaining reproducible diffusion data by SIMS was shown to be the uniformity of the YBCO surface. Surface topography and/or extraneous material on the surface distorts the observed depth profile and can mask the true profile shape. Diffusion may occur at imperfections in the film such as pinholes, however, more study is needed to confirm this hypothesis. 


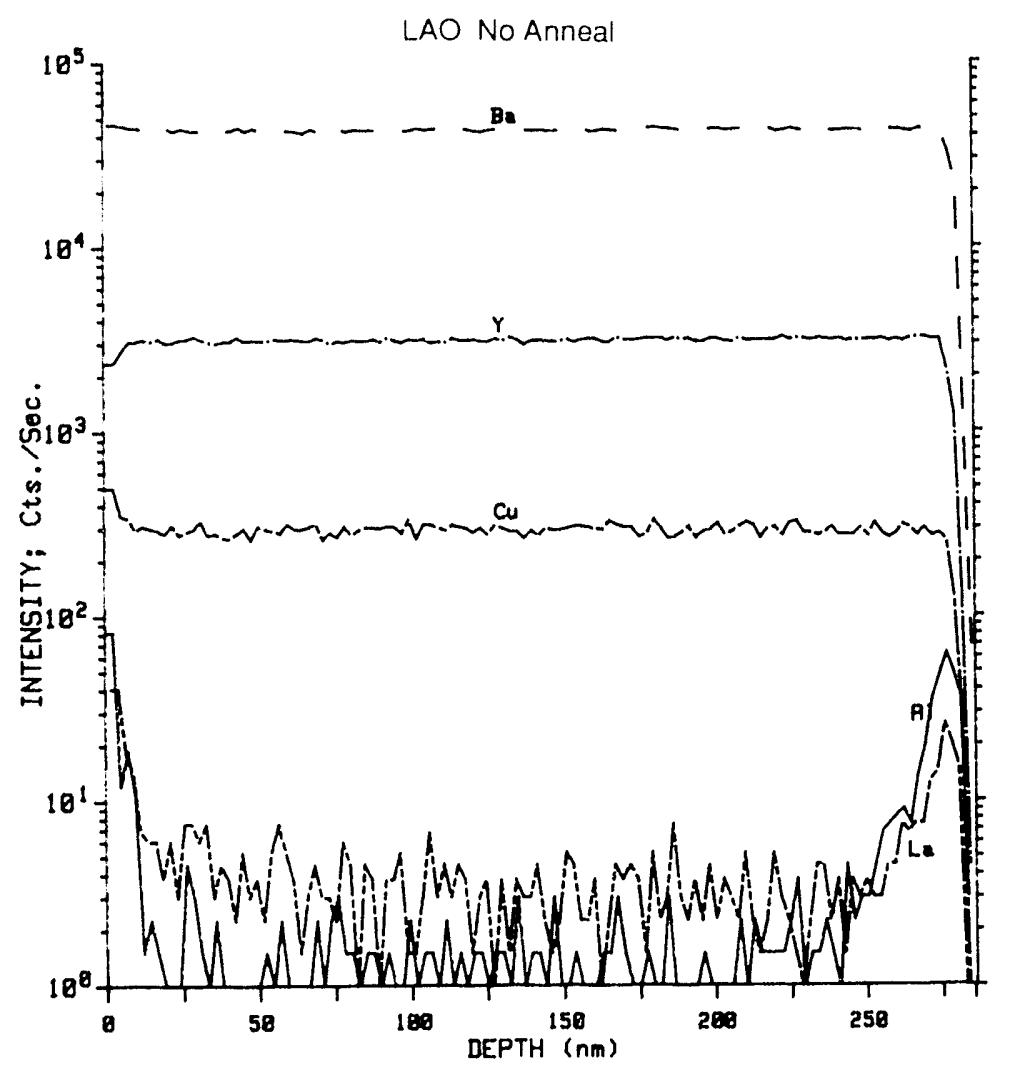

(a)

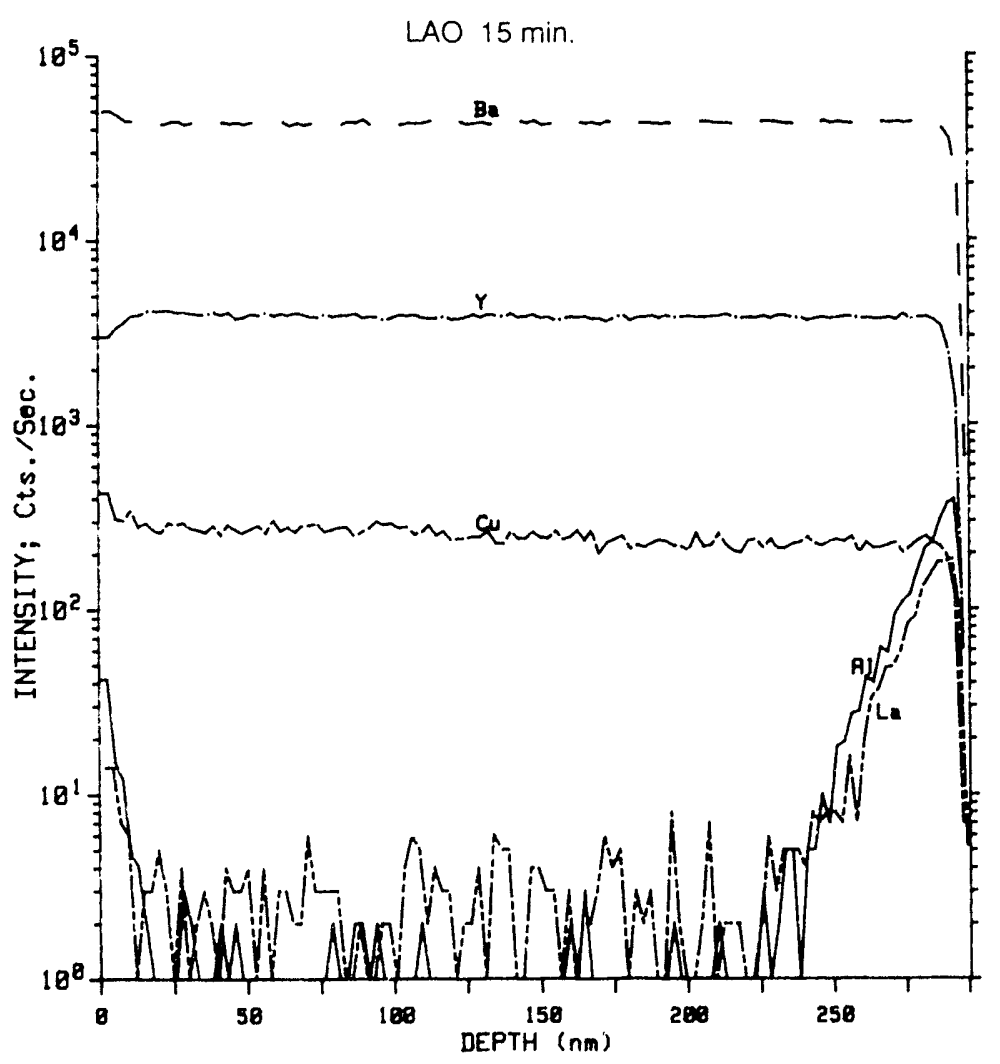

(b) 


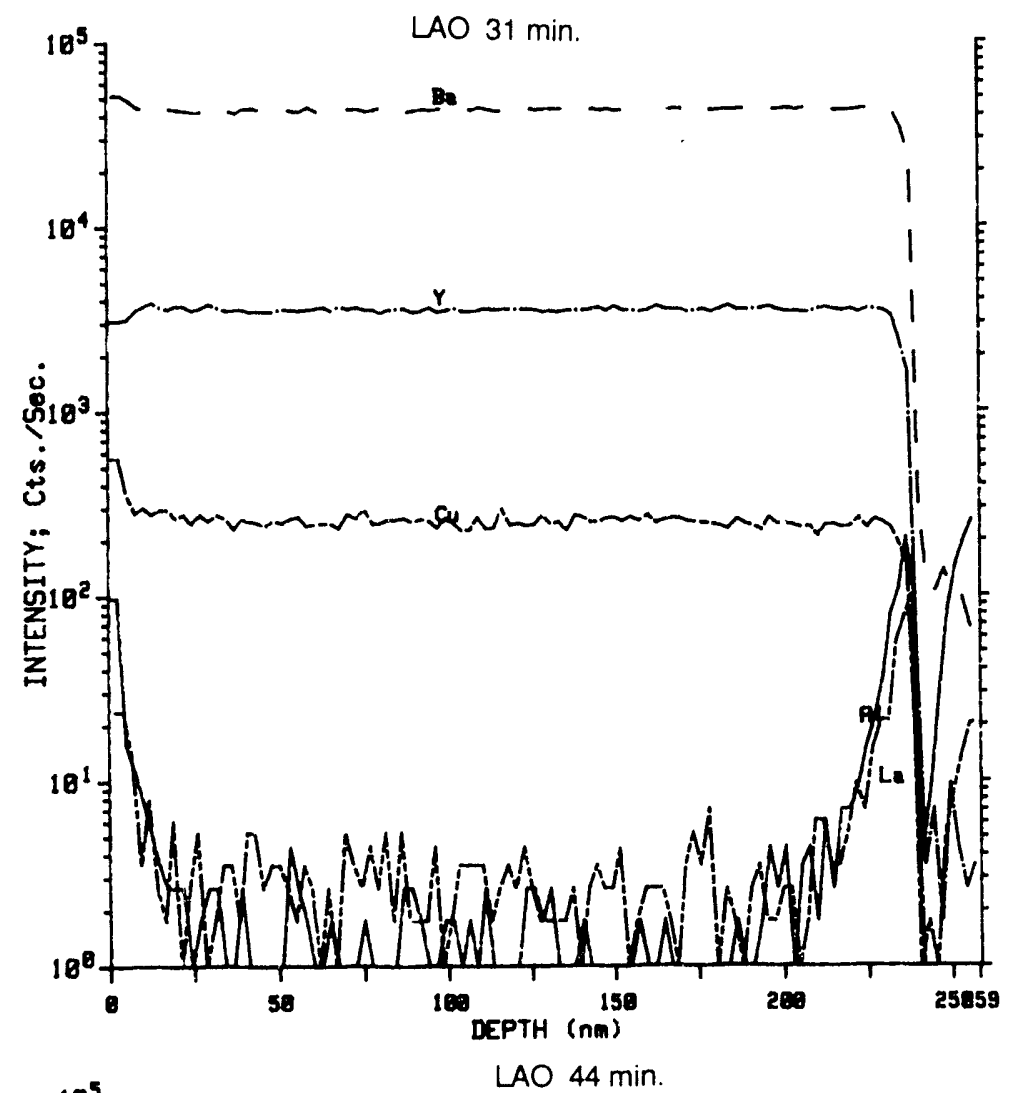

(c)

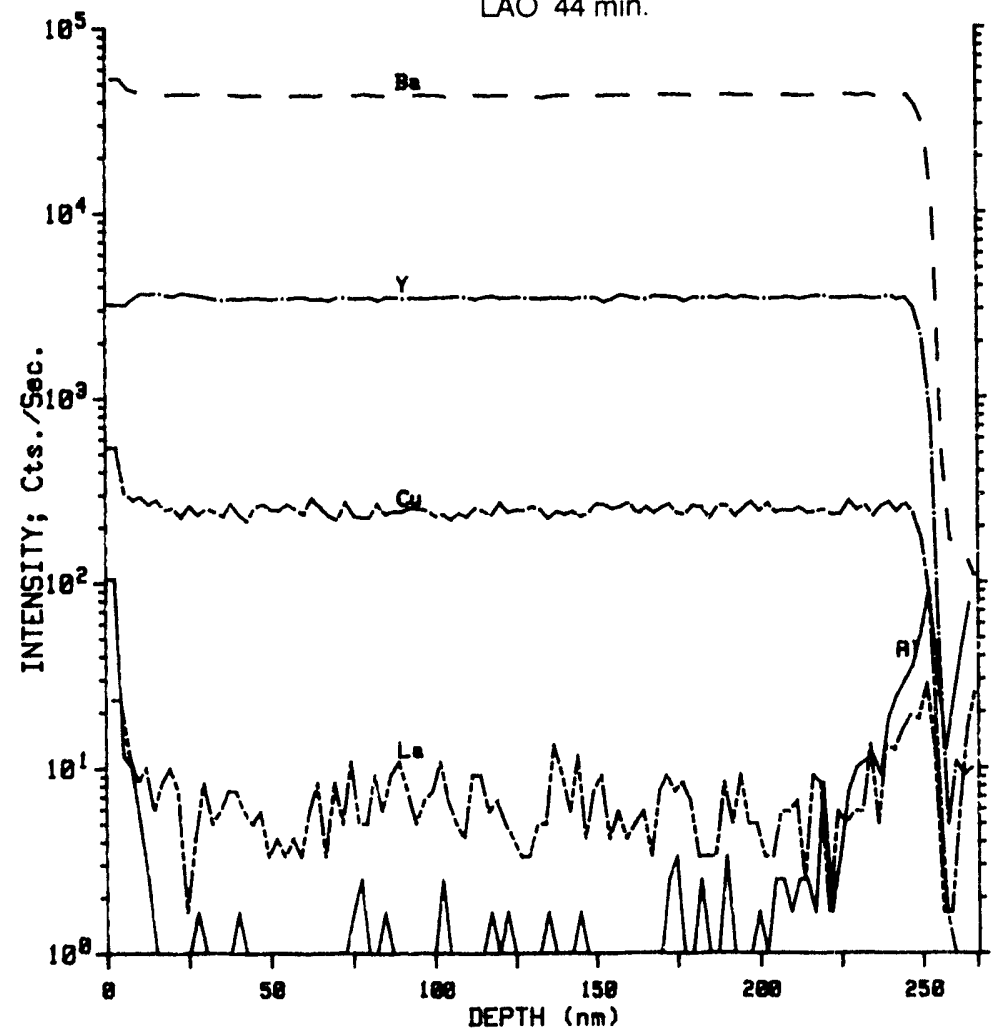

(d)

Figure 2.32: SIMS depth profiles from YBCO films on LAO substrates and annealed for various times at $750^{\circ} \mathrm{C}$; (a) as-deposited film with no anneal, (b) film annealed for $15 \mathrm{~min}$., (c) film annealed for $31 \mathrm{~min}$., and (d) film annealed for $44 \mathrm{~min}$. 


\subsection{FUTURE WORK}

Collaborative research will continue with the staff at the Synchrotron Radiation Center (SRC). University of Wisconsin-Madison, utilizing high resolution photoemission techniques to characterize the Cd(In)S/CuInSe ${ }_{2}$ and metal/CuInSe, interfaces. Results of these investigations will provide further information on heterojunction formation and on the formation mechanisms of ohmic and Schottky contacts to single crystal CuInSe ${ }_{2}$. Additionally, CuInSe, will be grown by MBE and characterized in-situ at the $\mathrm{SRC}$ in order to understand the basic physics in volved in order/disorder phenomena.

Research will be performed on characterizing the effects of plasma etching for the purpose of modifying surfaces of high-T superconductors (which has been shown to improve transport properties) and for oxidation of HTSCs during growth to produce fully oxidized films.

An effort to understand the effects of reactive ion etching of III-V and I-III$\mathrm{VI}_{2}$, materials for process development will be initiated. UV and soft X-ray photoemission will be used to characterize reactively ion etched (using $\mathrm{H}_{2} \mathrm{~S}$,

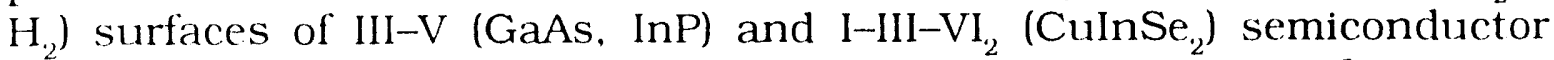
crystals. The goal is to develop a process that would pin the surface Fermi energy of these semiconductors to improve device performance as well as to understand the basic physics involved.

Research will begin on the growth and in-situ characterization of CdTe and CdS in an effort to understand the order/disorder phenomena and to develop processes necessary for optimum II-VI device performance.

Work will continue in the development of the RHEED intensity oscillation analysis system. Valuable information regarding interface and surface morphology could be obtained if RIO data could be acquired concurrently with MEE growth. Presently, this is not possible because the processor, responsible for control and data acquisition, is tied up controlling the MEE shutter events. We plan to modify the software and acquire a buffered FIFO data acquisition board. This will allow us to collect RIO data during MEE shutter event sequences without planing additional demands for processor time. Acquiring RIO data concurrent with MEE timing sequences will allow us to optimize MEE growth of critical device structures.

With the completion of the MEE growth facility, research will begin utilizing MEE to produce ultra-abrupt AlGaAs/GaAs interfaces. The purpose of this research is to determine if this growth technique can be used to reduce the interface recombination velocity in structures containing heterojunction 
interfaces. If successful, MEE growth could be used to improve the performance of photovoltaic and other devices which incorporate these problematic interfaces. Additionally. MEE will be used to explore novel doping schemes in an effort to extend the limits attainable by conventional MBE growth. This would be particularly valuable in photovoltaic devices employing a heavily doped region such as tunnel junctions.

1. Yacobi, B.G., B. Elman, C. Jagannath. A.N.M. Masum Choudhury. and M. Urban. Appl. Phys. Lett. Vol. 52, p. 1806 (1988). 


\subsection{MATERIALS CHARACTERIZATION}

\subsection{RESEARCH STAFF}

M.M. Al-Jassim. Senior Scientist and Group Leader

J.P. Goral. Staff Scientist

K.M. Jones. Staff Scientist

A.R. Mason. Master Technician

R.J. Matson. Staff Scientist

\subsection{INTRODUCTION}

Using advanced electron microscopy and microanalytical techniques, the Materials Characterization Group investigates the compositional. crvstallographic. structural, defect. luminescent. and topographic properties of photovoltaic (PV) and semiconducting materials and devices. The group collaborates extensively with internal and DOE subcontracted researchers and provides analyses crucial to understanding and developing state-of-theart PV devices. The work can be divided into: (1) routine support service provided to in-house and subcontracted researchers le.g.. mieasurement of a sample's chemical composition. measuring the density of defects in a material and determining the crystallinity): (2) long-term studies of particular problems pertaining to PV devices. This is done in close collaboration with a great variety of PV researchers. and the results are often correlated with the crystal growth conditions and/or with the electrical properties and cell performance: and (3) characterization support in areas other than PV, but in concert with SERI's mission le.g.. the study and determination of the composition and crystal structure of high-temperature superconductors and our business development efforit.

\subsection{ANALYTICAL CAPABILITIES AND TECHNIGUES}

Appendix $D$ provides a listing of the major instrumentation and test equipment. their features. and specifications.

\subsubsection{Electron Probe X-ray Microanalysis (EPMA)}

This technique is uset? primarily to determine the chemical composition of the top 0.5 to $2 \mu \mathrm{m}$ of a specimen surface. This is accomplished by both energy dispersive spec uroscopy (EDS) and wavelength dispersive spectroscopy (WDS). The instrument used is a fully automated Cameca MBX Electron Microprobe with a Tracor Northern TN-2000 data acquisition system.

EDS is nominally ised for quicitative cvaluation. while WDS is used to quantifi- elemental concentrations to \pm 0.5 atomic percent (at. \%) accuracy. 
This system can analyze all elements heavier than boron and in concentrations $>0.5$ at. $\%$. Computer automation allows compositional mapping of predetermined regions of interest in a sample.

\subsubsection{Transmission Electron Microscopy (TEM)}

This instrument and associated techniques are used primarily for structural and analytical studies involving the examination of thin (200 to $2000 \AA$ ) plan-view and cross-sectional samples. Electron diffraction is used to study the crystal structure of the r naterials examined and to reveal grain orientation. crystallographic relationship between grains. and any preferred orientation of polycrystalline films. Bright-field and dark-field imaging are used to i.vestigate the type. density, origin and three-dimensional distribution of defects such as dislocations, stacking faults, and grain boundaries. In addition. the instrument can perform X-ray EDS analysis. which is used to evaluate local chemical compositions and compositional inhomogeneities, and electron energy loss spectroscopy (EELS). which yields qualitative information on elements of low atomic number. High resolution (lattice imaging) is often utilized to study the structure and abruptness of interfaces in multilayer structures and to investigate atomic ordering in various compound semiconductors.

The current system consists of a Philips CM-30 300-keV TEM with a scanning transmission electron microscope (STEM) attachment. a Kevex Delta Class EDS analyzer, and a Gatan 807 EELS spectrometer. The pointto-point resolution of the srstem is $2.1 \mathrm{~A}$ in the TEM mode and $\sim 15 \AA$ in the STEM mode. Furthermore, the double-tilt goniometer specimen holder allows a \pm 60 tilt.

\subsubsection{Scanning Electron Microscopy (SEM)}

The group supports and applies two extensively developed scanning electron microscope-based systems. The first SEM is a JEOL JSM-35c with the following capabilities: electron channeling (EC), energy dispersive spectroscopy (EDS)/X-ray analysis augmented by a TN-2000 image automation and $\mathrm{X}$-ray analysis system. backscattered electron imaging (BEI) and cathodoluminescence (CL) imaging. in addition to the usual secondary electron imaging (SEI. resolution: $5.0 \mathrm{~nm}$ ). The system was further developed to include temperature-dependent ( $80 \mathrm{~K}$ to $700 \mathrm{~K}$ ). lightand voltage-biased. planar and cross-sectional electron-beam-induced curı ent and voltase (EBIC. EBIV) analysis capabilities. The selected area electron channeling allows the instrument to determine the crystalline type. orientation. and atomic lattice spacing of areas down to $20 \mu \mathrm{m}$ in diameter. The superiosition of SEI and X-ray maps allows compositional and topographical teatures to be correlated. EBIC or charge coilection mucroscopy 
(CCM), constitutes the primary use of this system. Junction. or crosssectional. EBIC is used routinely to determine the position of the electrical junction with respect to the device surface and the intended layers of the device structure. The electrical behavior of the device junction(s) can be monitored as a function of voltage and/or light biasing over a wide range of temperatures. Planar EBIC is used to determine both the density and electrical recombination behavior of crystalline defects such as dislocations in mismatched heterostructures. This in turn can be correlated with device performance parameters. Finally. either junction or planar EBIC can be used for determining the local minority carrier diffusion lengths in a sample material or device. The synergistic power of the system is considerable in its ability to characterize and correlate compositional, structural, electrical. luminescent. and topographical features and properties of a sample at one sitting.

The second SEM system is a JEOL Model JSM-840. which is dedicated to temperature-dependent. panchromatic. and spectral $\mathrm{CL}$. CL is used to study the recombination behavior of defects and to measure the relative impurity concentrations. the density and distribution of defects, and minority carrier diffusion lengths and lifetimes. The special temperature controlled liquid helium CL stage allows these properties to be studied at temperatures ranging from room temperaiure down to $10^{\circ} \mathrm{K}$, with the lower temperatures veing us ful for greater luminescent yield and increased spectral resolution. The panchromatic (or total light) CL imaging is generally used for mapping nonuniformities in the recombination behavior of a material without having to form a device, which would be necessary for EBIC analysis.

\subsection{ACCOMPLISHMENTS: PHOTOVOLTAICS}

The electron microprobe continued to have the largest sample analysis throughput of the branch's analytical capabilities (on average 200 to 250 samples per month). supporting almost all internal and subcontract groups with accurate and quantitative compositional analysis. Samples analyzed were in both thin-film and bulk form. A very wide variety of PV materials have been examined. including CuInSe, CuGaSe, $_{2}$. ZnCdS. CdTe. CdSe, CuGalnSe, GaAlAs. GaInAs. GaAsP. GaInP. ITO, amorphous Si. as well as high T, superconductors.

The SEMs and TEM were used extensively to study surface morphology. crystallinity, and defect properties: more than 700 samples were logged during the past year. For these studies. SEM secondary electron and EBIC imaging. selected-area electron channeling patterns, and TEM plan-view examination have been employed. 
In addition to these routine investigations, many collaborative research projects invclving internal and external research groups and PV industry researchers have focused on specific and critical problems encountered in PV materials and devices. Selected examples demonstrating the usefulness and range of the available analysis techniques follow.

\subsubsection{SEM Investigation of Hydrogen Passivation in Polycrystalline Si}

Hydrogen passivation of grain boundaries (GBs) in commercial (Solarex) polycrystalline silicon solar cells from the backside of the cell was studied by EBIC in the scanning electron microscope (SEM). Back-surface passivation is being investigated since front-side passivation causes serious surface damage with resultant surface recombination velocities as high as $7 \times 10^{7}$ $\mathrm{cm} / \mathrm{sec}^{1.2 .3 .4}$. The damage could be due to the hydrogen implantation and/ or impurity contaminations introduced during the implantation process ${ }^{5.6}$. Front-side hydrogenation also restricts solar cell fabrication processes ${ }^{\gamma}$. Since the volume near the front surface $p-n$ junction is what needs to be passivated. particular emphasis has been placed on the depth to which the passivation occurs.

The hydrogen passivation was carried out in a Kaufman ion beam system at a beam energy of $1.5 \mathrm{keV}$ and beam current of $55 \mathrm{~mA}$ for 15 minutes. The $\mathrm{EBIC}$ technique was used to monitor the recombination rate of a given $\mathrm{GB}$ (Fig. 3.1), which is indicative of the degree of passivation. The EBIC contrast. C. defined as (Imax - Imin)/Imax from a quantitative EBIC linescan. measures the short-circuit current (Isc) loss at the GB caused by minority carrier recombination at defects. Although the overall trend of passivation effects among the GBs was investigated. one location on a uniform grain boundary was characterized before and after passivation with EBIC linescans and maps to measure the diffusion length and effective surface recombination at the GB (Fig. 3.2). EBIC maps represent the variation of the local Isc due to both defects/recombination sites in the bulk and. as we see in Fig. 3.2. obstruction of the beam by the metal contact (the wider line). Figure 3.2 compares the recombination at the GB (a) before passivation . (b) after backside passivation. and (c) after a subsequent heat treatment at $340^{\circ} \mathrm{C}$ for 5 hours in vacuum. The se three figures have an $\mathrm{EBIC}$ contrast of $48 \%, \sim 18 \%$, and $45 \%$. respectively. Where the thin dark line is the GB itself. the thin varying white line is the EBIC linescan showing that the quantitative contrast and the horizontal line are both the actual location of the linescan and the zero beam current reference line. Given that the same relative EBIC contrasts were ofserved at beam voltages of $1 \mathrm{kV}$ (penetration depth of $\sim 3 \mathrm{~nm}$ ). it is reasonable to conclude that hydrogen passivation occurred all the way through the thickness of the Si wafer. Besides indicating reduced current loss, the lighter GB (Fig. 3.2b) indicates 


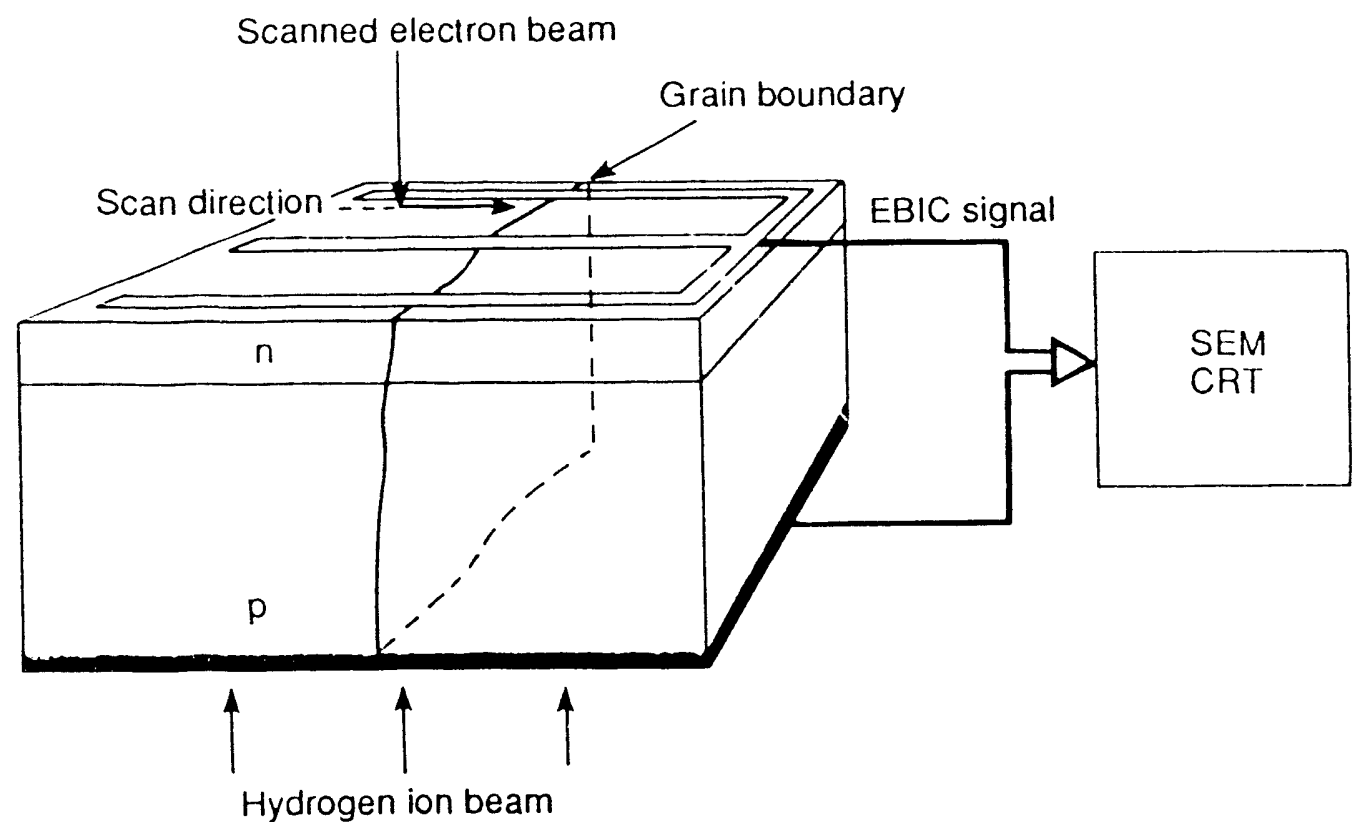

Figure 3.1: Configuration used in EBIC measurements to characterize hydrogen passivation depth from the backside.

considerable nonuniformity (varying from $10 \%$ to $20 \%$ contrast) in the passivation effect along the same GB that showed higher, yet uniform, recombination before passivation. After heat treatment the GB had essentially recovered most or all of its previous recombination and uniformity. The reasons for the nonuniformity in the passivation effect are not clear. However. this is currently believed to be due to the distribution of hydrogen in the GB as shown by our TEM studies.

Quantitative studies of the recombination velocity (S) and the minority carrier diffusion length (L) near the GB were performed based on Donolato's theory ${ }^{4}$ for the analysis of EBIC GB linescan profiles. In summary, and taking a simple average, it was determined that:

$\begin{array}{lll}\text { before passivation: } & \mathrm{S}=5.1 \times 10^{5}(\mathrm{~cm} / \mathrm{s}), & \mathrm{L}=8.6 \mu \mathrm{m} \\ \text { after passivation: } & \mathrm{S}=5.6 \times 10^{4}(\mathrm{~cm} / \mathrm{s}), & \mathrm{L}=13.0 \mu \mathrm{m} .\end{array}$

In other words, the recombination velocity dropped by about an order of magnitude and the diffusion length near the GB increased by $\sim 50 \%$ because of the backside passivation method.

The passivation mechanism is not well understood at this stage. One possible explanation is that hydrogen is saturating Si dangling bonds in the $\mathrm{GB}$ to form $\mathrm{Si}: \mathrm{H}$ bonds. However, the chemical bond dissociation energy of 


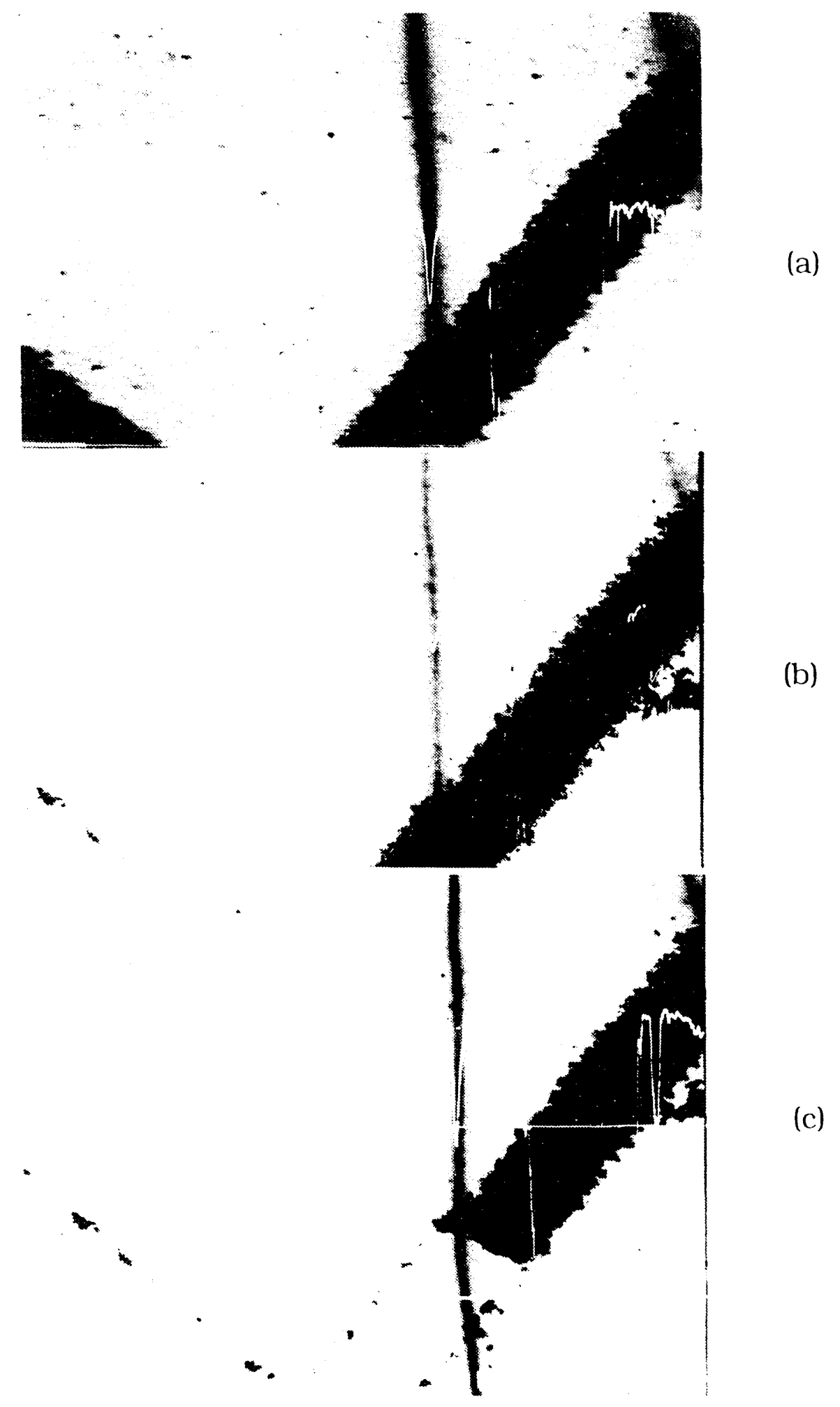

Figure 3.2: EBIC images/maps and quantitative linescans of a grain boundary before hydrogen passivation (a), after passivation (b), and after subsequent heat treatment (c). Beam conditions: $35 \mathrm{kV}, 1 \mathrm{nA}$. The linescans were taken from the same location on the grain boundary insofar as was possible. 
the Si:H bond is in the range of 1.5 to $3.0 \mathrm{eV}^{11}$. but the thermal energy provided in the annealing experiment is only $\sim 0.05 \mathrm{eV}$. Therefore, the simple saturation of the dangling bonds model does not account for the annealing result.

In summary, a hydrogen diffusion and passivation depth of $\sim 250 \mu \mathrm{m}$ was observed. Both nonuniform and reversible passivation along the GB studied suggest the presence of differing defects and their distribution and corresponding passivation mechanisms.

\subsubsection{The Characterization of III-V Semiconductors Grown on Si}

TEM and high resolution electron microscopy (HREM) were used to characterize GaAs and InP layers grown by metal organic chemical vapor deposition (MOCVD) on Si substrates. Photoluminescence examination, carried out by the Electro-optical Characterization Group, was used to measure the minority carriers lifetime. The main motivation for this work is the need for a low-cost, high thermal conductivity and light substrate material such as Si. However, the high defect densities $\left(>108 \mathrm{~cm}^{-2}\right)$ in these structures, which are largely caused by the high lattice mismatch, remains the primary obstacle to the fabrication of high-quality minority carrier devices. In this regard, we have collaborated with a number of in-house and outside researchers to study the nature, density, and origin of defects in these structures in an attempt to devise means of reducing their density and improving the minority-carrier lifetime.

TEM cross-sectional examination showed that the growth of InP on $\mathrm{Si}$ at $400^{\circ} \mathrm{C}$ is highly three-dimensional, proceeding by the nucleation and coalescence of well-defined islands (Fig. 3.3). These islands are heavily faulted and mostly faceted on low index planes. Detailed HREM examination (Fig. 3.4) revealed a variety of line and planar defects. The predominant types of defects are microtwins and stacking faults, which partially accommodate the $8 \%$ misfit between InP and Si. The generation of such planar defects has been attributed to errors in deposition on faceted nuclei. Therefore, twodimensional growth should suppress their generation and result in lower defect density. Growing InP at lower temperatures may favor a more twodimensional growth in this highly mismatched system. However, the cracking of phosphine at temperatures lower than $350^{\circ} \mathrm{C}$ becomes considerably more difficult and a high (larger than 1000) V/III ratio becomes essential ".

Observing the above-mentioned constraints on nucleation temperature, a two-step growth was followed in which nucleation took place at $350^{\circ}$ to $400^{\circ} \mathrm{C}$ and growth at $600^{\circ} \mathrm{C}$. TEM examination of such structures showed that complete Si surface coverage is achieved by the first InP layer (its 


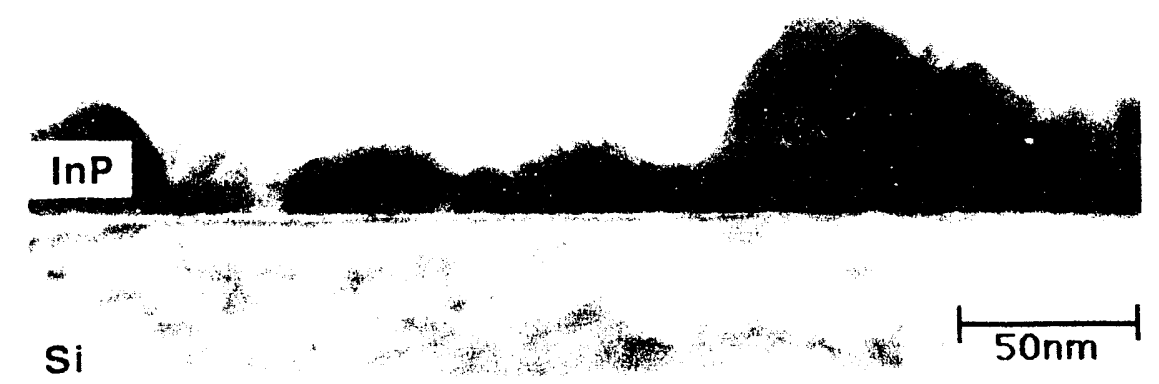

Figure 3.3: The three-dimensional nucleation of InP on Si.

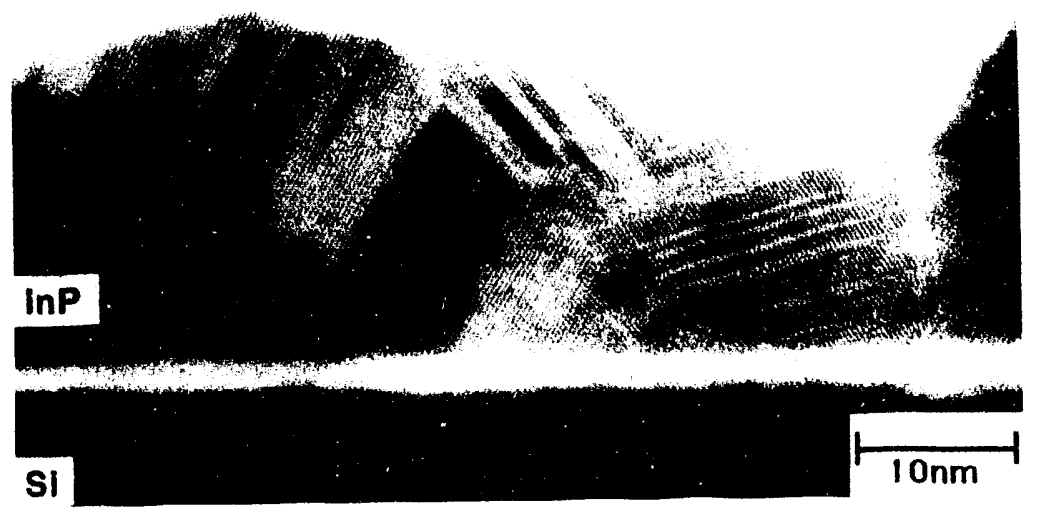

Figure 3.4: HREM image of defects in InP nuclei. 
thickness is typically $200 \AA$ ), rendering the remainder of growth as homoepitaxial. The defect density in these structures decreased ninarkedly as a function of layer thickness. The microtwins never seem to propagate very far into the layer, while stacking faults may propagate into the layer surface unless they intersect faults on other $\{1111\}$ planes. However, 3 to 4 $\mu \mathrm{m}$-thick InP layers contain stacking faults and dislocation densities in the $5-10 \times 10^{4} \mathrm{~cm}^{-2}$ range and exhibited minority carrier lifetimes less than 300 ps. These findings, and the difficulty in nucleating InP directly on $\mathrm{Si}$, led us to use a variety of bufer layers.

GaAs buffer layers were grown on Si using a two-step growth process in which 200 to $300 \AA$ were nucleated at $400^{\circ} \mathrm{C}$ followed by growth at $650^{\circ} \mathrm{C}$. The use of GaAs/Si as substrates for the subsequent growth of InP reduced the mismatch by a factor of two, and resulted in markedly better morphology and a significantly improved reproducibility. The thickness of the GaAs buffer was optimized and its effects on the structural quality of the InP epilayers were investigated. It is crucial to minimize the total epilayer thickness in these structures in order to avoid layer cracking. Using thicknesses $<0.1 \mu \mathrm{m}$ resulted in defect densities in excess of $109 \mathrm{~cm}^{-2}$. Figure 3.5 shows the dependence of the defect density in the InP layers on the buffer layer thickness. Clearly, there is no significant advantage in going beyond $1-\mu \mathrm{m}$-thick buffer layers.

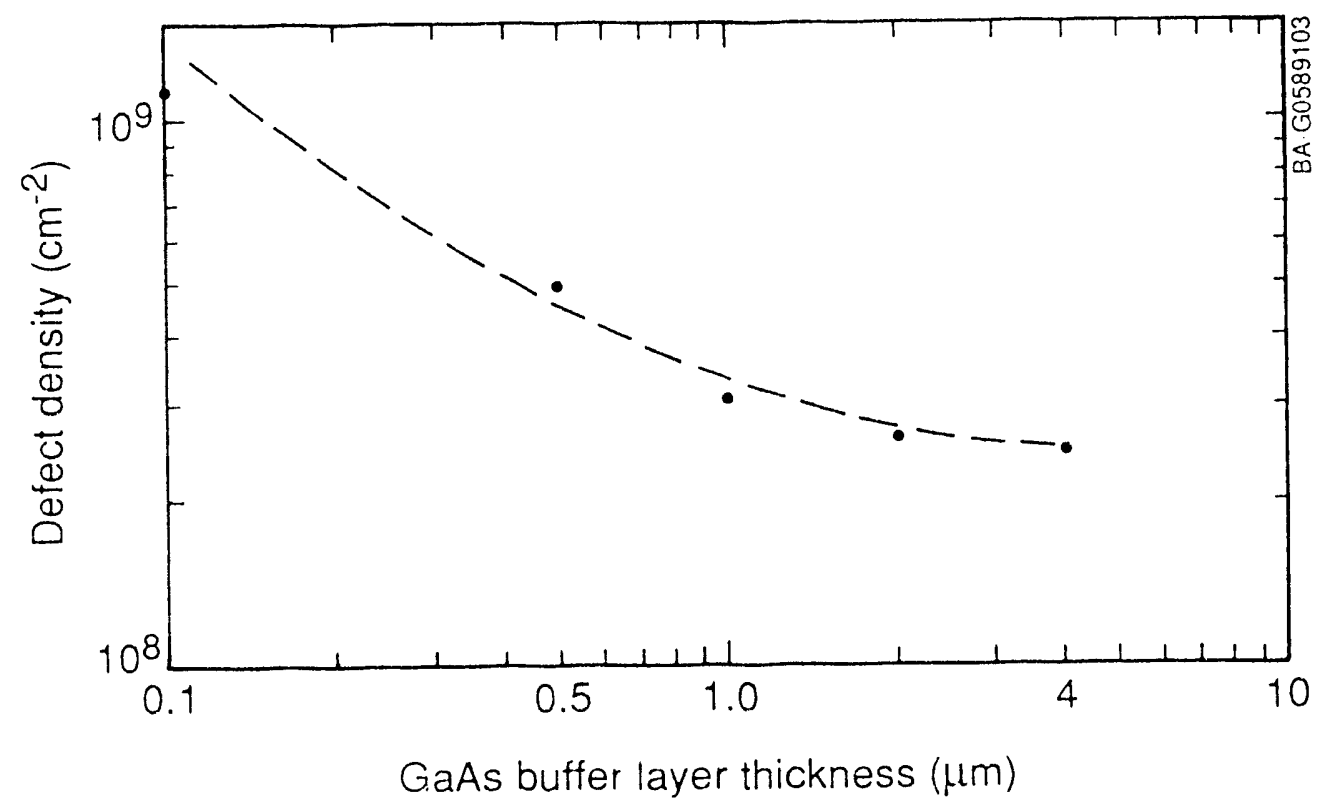

Figure 3.5: The variation of the defect densily in InP layers with the thickness of the GaAs buffer layer. 
The importance of the structural perfection of the GaAs buffer layers was studied by simultaneously growing InP' on bulk InP. bulk GaAs, TCG GaAs/ Si. and standard MOCVD-grown GaAs/Si. The GaAs layer thickness used was $3 \mu \mathrm{m}$. The results are summarized in Table $3-1$. They clearly show that the best quality material was obtained in the homoepitaxial case, which was used as a control. Despite the wide range of defect density in the underlying GaAs (from $10^{-4}$ for bulk GaAs to $4 \times 10^{4} \mathrm{~cm}^{-2}$ for regular GaAs/Si) the defect densities in the InP layer were comparable. Furthermore, the same trend was observed for the photoluminescence $(\mathrm{PL})$ and $\mathrm{X}$-ray rocking curves data. This indicates that the defect density in the InP layers and the minoritycarrier properties are dictated by the $4 \%$ lattice mismatch between $\operatorname{InP}$ and GaAs. To further improve the structural quality of the InP epilayers, TCG growth was attempted. This was performed by interrupting the growth and annealing at temperatures between $800^{\circ}$ and $850^{\circ} \mathrm{C}$. The amount of phosphorus overpressure used and the annealing temperature proved to be critical. Using annealing temperatures higher than $850^{\circ} \mathrm{C}$ and/or inadequate phosphorus overpressure resulted in a serious degradation of the layer's surface, which caused the generation of high defect densities (Fig. 3.6). Nonetheless, when the right combination of phosphorus overpressure and annealing temperature was chosen, the effect of annealing was clearly evident in TEM cross sections (Fig. 3.7). Some dislocation interaction and annihilation were observed and the formation of closed dislocation loops took place. However. only a factor of 2 reduction in defect density was obtained. Similarly, only a small improvement in the minority-carrier lifetime was noticed as it increased from 0.4 to $0.8 \mathrm{~ns}$.

The above results show that thermal cycle growth of InP is not as effective as it is with GaAs/ $\mathrm{Si}^{12}$. Additionally, they indicate that the defect density in the InP layers is primarily dictated by the $4 \%$ mismatch between GaAs and InP. When such a high mismatch is relieved in one step, a very dense dislocation network with an average spacing of $\sim 7 \mathrm{~nm}$ (assuming that the majority of dislocations are of the pure edge type) is required for a full

Table 3-1: Experimental data concernong InP grown on Si.

\begin{tabular}{|c|c|c|c|c|}
\hline $\begin{array}{c}\text { Substrate } \\
\text { Material }\end{array}$ & $\begin{array}{c}\text { PL Full Width at } \\
\text { Half Max } \\
\text { (FWHM) [eV] }\end{array}$ & $\begin{array}{c}\text { X-ray } \\
\text { FWHM [s] }\end{array}$ & $\begin{array}{c}\text { PL Lifetime } \\
\text { [ns] }\end{array}$ & $\begin{array}{c}\text { Dislocation } \\
\text { Density } \\
{\left[\text { [ } / \mathrm{cm}^{2}\right]}\end{array}$ \\
\hline Bulk InP & 0.085 & 15 & 3.7 & $<5 \times 106$ \\
\hline Bulk GaAs & 0.124 & 236 & 0.415 & $3.9 \times 108$ \\
\hline $\begin{array}{c}\text { Regular } \\
\text { GaAs/ Si }\end{array}$ & 0.122 & 340 & 0.475 & $3.5 \times 108$ \\
\hline TCG GaAs/Si & 0.117 & 286 & 0.464 & $2.5 \times 108$ \\
\hline
\end{tabular}




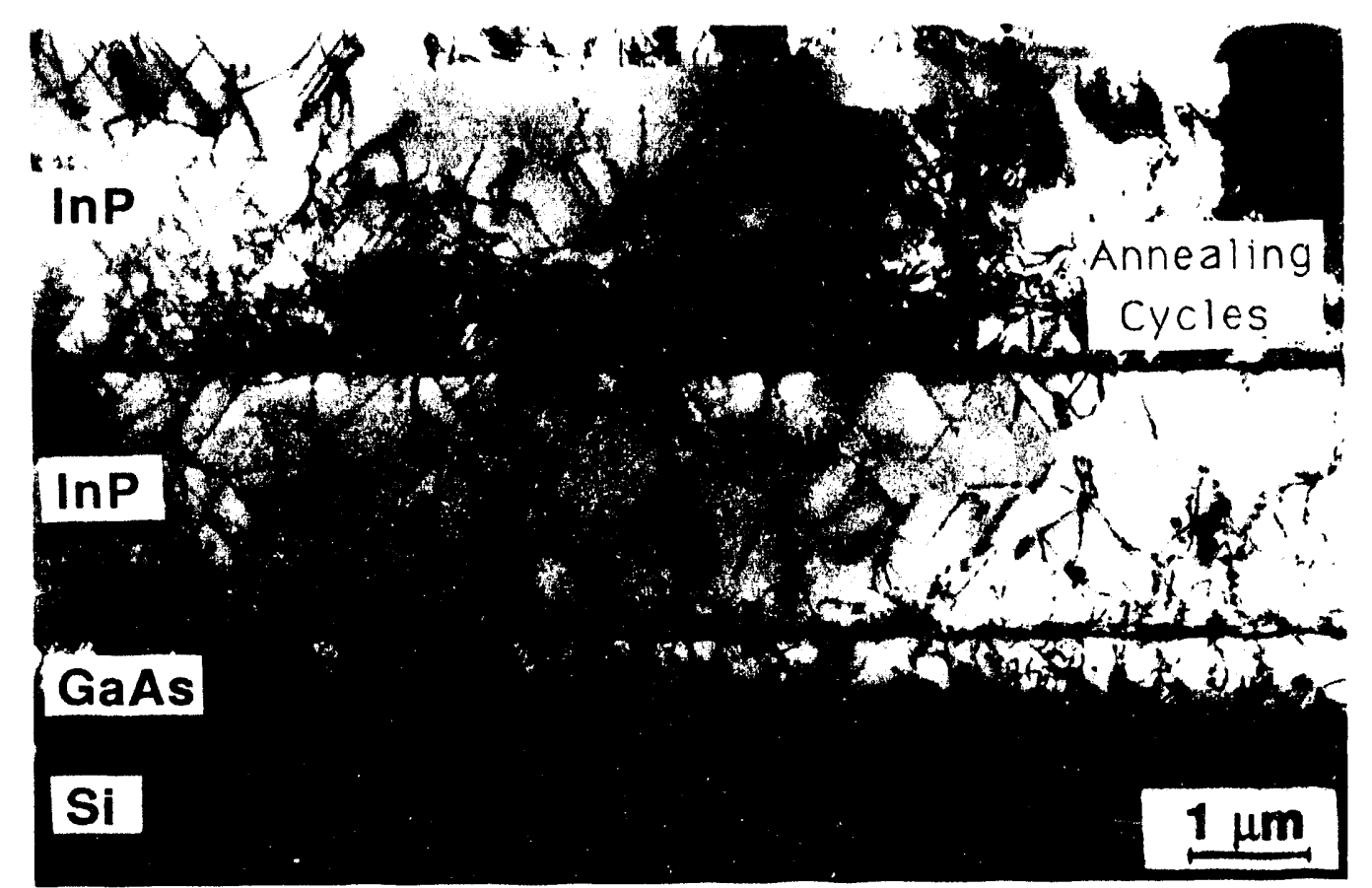

Figure 3.6: TEM cross section showing the deterioration of InP under thermal annealing.

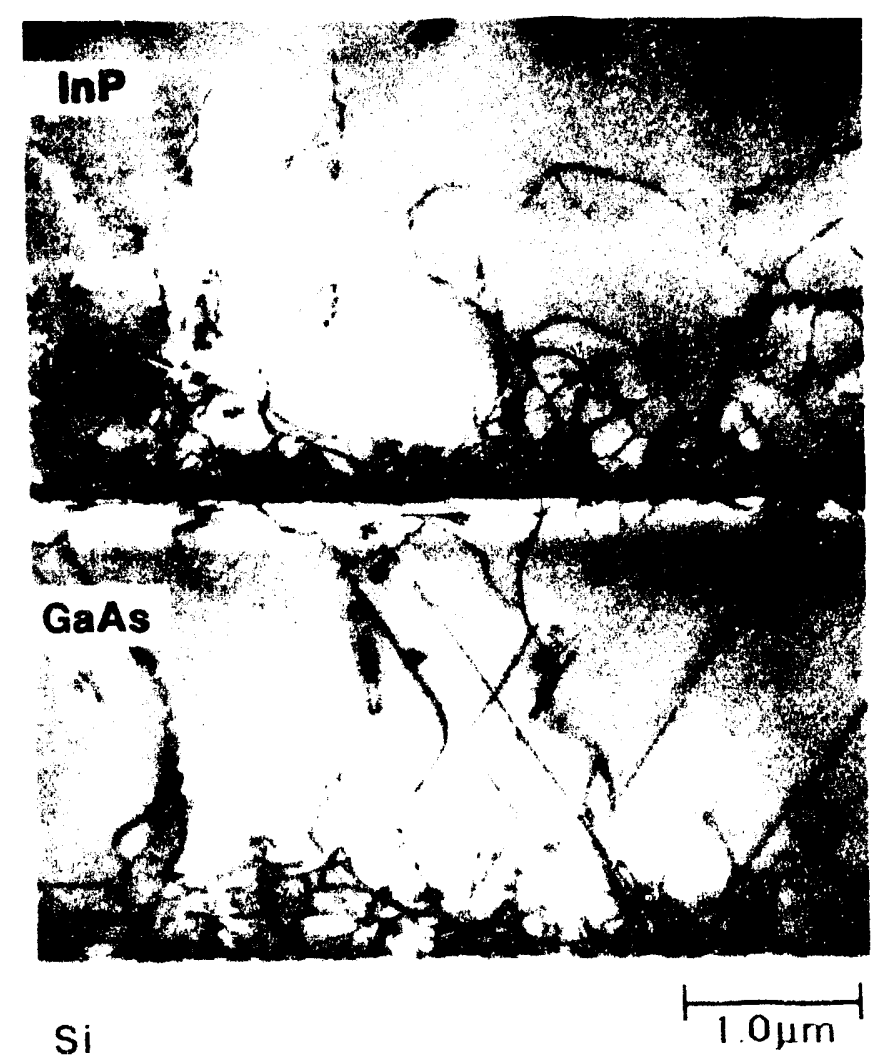

Figure 3.7: TEM cross section of the best annealed InP' layer grown on GaAs/Si. 
relaxation to the bulk lattice parameter. If such a network is gencrated hy the "surface generation mechanism," a threading dislocation density of $>1010 \mathrm{~cm}^{2}$ is expected in the GaAs/InP interface region. Many of these threading defects will interact and annihilate. However. many are expected to propagate to the layer surface, as has been observed above. Therefore, at SERI we have developed GaInAs graded buffer layers to be grown between GaAs and InP in order to relieve this $4 \%$ mismatch over a thicker region ${ }^{1: 3}$. The composition of these layers was varied from that of GaAs to Ga In $_{0,4,5}$ As. which is lattice matched to inP. The graded layer thicknesses used were in the 8 to $20 \mu \mathrm{m}$ range. Figure 3.8 shows a TEM cross section of an InP shallow homojunction cell structure grown on Si using GaAs/8- $\mu \mathrm{m}$ graded GaInAs buffer layers. The graded layer contains an extensive three-dimensional misfit dislocation network, which relieves much of the $4 \%$ lattice mismatch. The dislocations lie chiefly in the (100) growth plane along the two perpendicular $<011>$ directions. They are linked by threading dislocation segments lyir.gin the four inclined $\left\{\begin{array}{lll}1 & 1 & 1\end{array}\right\}$ planes. These threading dislocations are frequently bent over within the graded layer because of the effect of the strain.

The threading dislocation density in the cell region of these structures is 2 $3 \times 10^{7} \mathrm{~cm}^{2}$ as measured from TEM plan-view samples. This is at least an order of magnitude lower than observed in InP grown directly on GaAs. PL lifetime measurements (Fig. 3.9) showed an increase of a factor of 5 in the measured lifetime upon using GaInAs graded buffer layers. There was no detectable difference in lifetime between $\mathrm{Si}$ - and GaAs-based structures. It must be emphasized here that the measured InP layers were umpassivated. Therefore, these lifetime values are possibly limited by surface recombination.

\subsubsection{SEM Studies of Crystalline $\mathrm{CuInSe}_{2}$}

A collaboration with Prof. D. Cahen and his group at the Weizmann Institute of Science, Israel, utilized in-situ SEM experiments in an effort to establish incontrovertible evidence of the "semionic" properties of crystalline CuInSe, (CIS). The term semionic refers to semiconducting materials that have both low, but not negligible, ionic conductivity and high electronic conductivity. Consequently, under specific conditions, voltage biasing in this case, the atoms can be moved or rearranged in the solid and possibly type-convert the electronic material. Some interesting aspects of these materials, especially CIS, are: (1) some ionic mobility occurs at room temperatures (especially $\mathrm{Cu})$ : (2) the mobile species are also native dopants; and (3) the material is semiconducting over a range of stoichiometries. The primary experiments consisted of applying a de bias voltage to a CIS crystal and monitoring the resulting electrical behavior (junction position and strength) both with EBIC and with a current versus voltage (I-V) curve tracer (as the source of the bias) in-situ in an SEM. Although different contact geometries were tried, most 


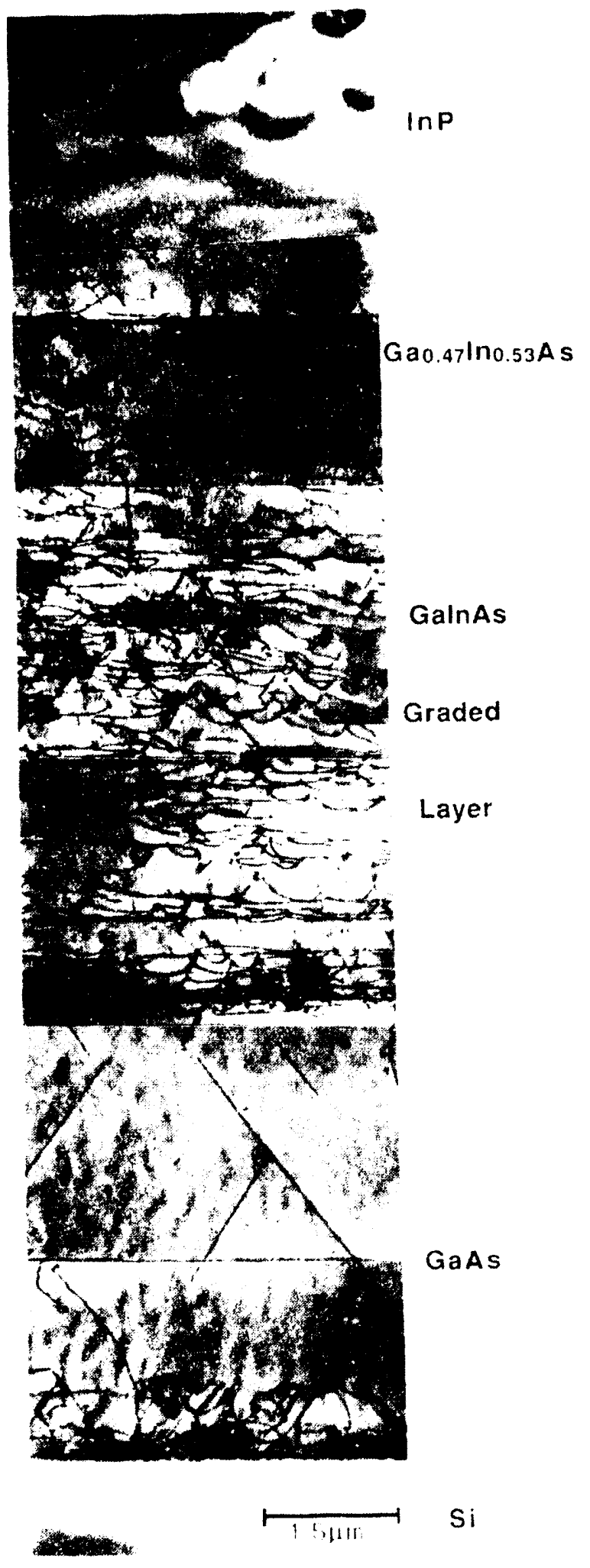

Figure 3.8: TEM cross section of an InP/GalnAs/GaAs/Si structure. 


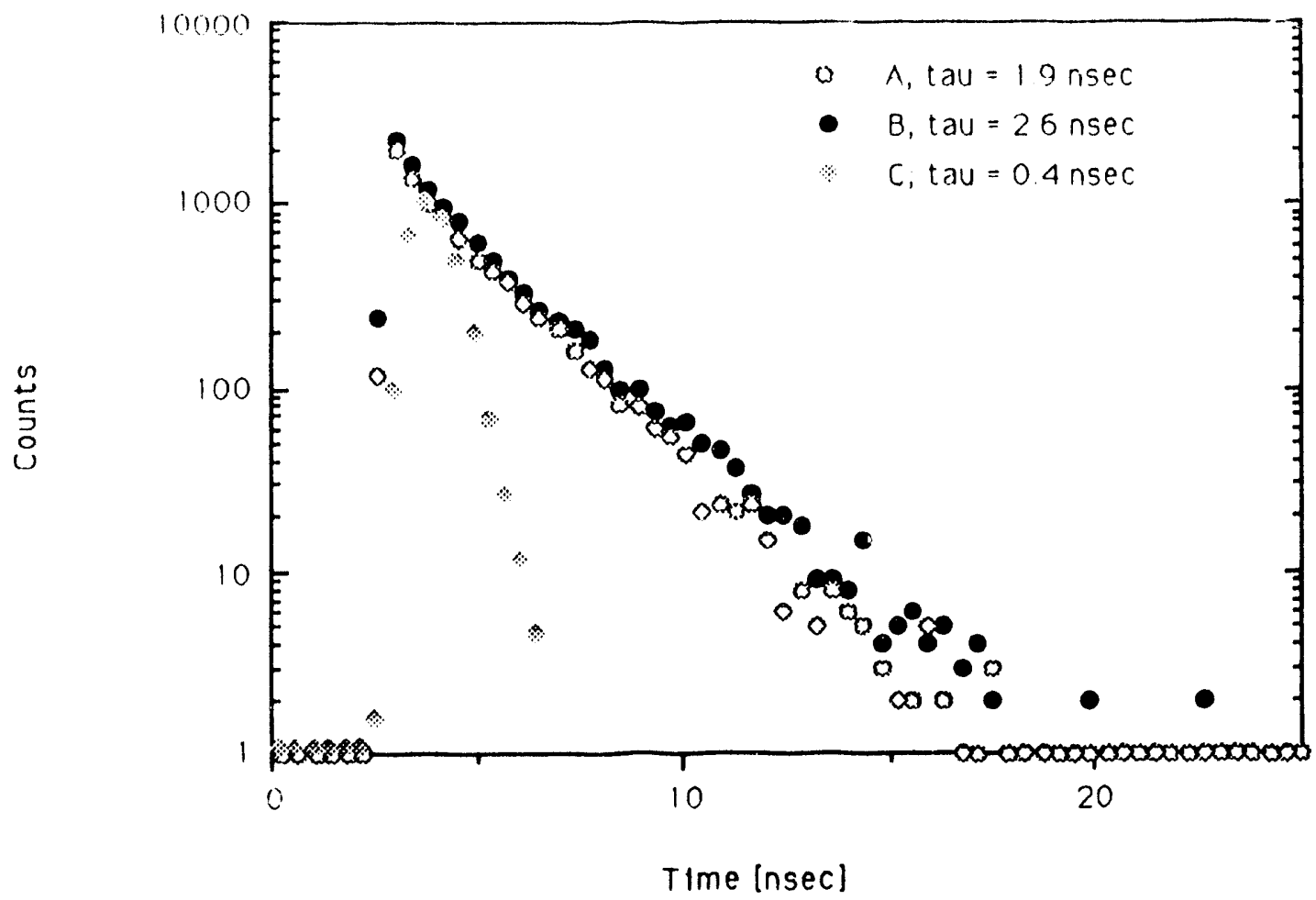

Figure 3.9: Plot of the PL intensity versus time of: (a) an InP layer grown on GaInAs/ GaAs, (b) an InP layer identical to A, grown on GalnAs/GaAs/Si and (c) an InP layer grown on GaAs/Si. Work performed by the Elecro-optical Characterization Group at this branch.

often the bias was applied between a $50-\mu \mathrm{m}$-diameter $i \mathrm{l}$ contact and a larger bark contact. After many trials, we observed under specific conditions that slowly approaching, but not surpassing, something analogous to avalanche conditions resulted in the formation of a $p-n$ junction and/or its further radial movement into the bulk of the crystal. The phenomenon was quasi-reversible. In addition, through a specific sequence of bias direction and amplitudes, the EBIC signature of a $p-n-p$ device was observed in two of the samples. The required bias varied from a few hundred millivolts to 200 $\mathrm{V}$ depending on the sample. The clearest evidence of type conversion and junction formation because of applied bias came through in-situ monitoring of the inaterial/device in cross-sectional EBIC where the strength (measured by electron beam quantum efficiency), location, and geometrical distribution of the junction were observed as a function of bias conditions. There was a persistent problem of nonuniformity within the CIS crystal samples, and, in general, a lack of suitable CIS crystal material for the experiments. This resulted in preferential breakdown along, or with respect to, the material inhomogeneities. Experiments with SERI AgInSe ${ }_{2}$ crystals showed similar results. 
It is postulated that ionic diffusion prosiesses via Cu-vacancies. To test for type conversion and defect type. electroluminescence (EL) and CL studies of the aftected areas are planned. The room temperature ionic mobility bears on thin-film CIS device stability and. even though it is doubted that it will prove to be a problem for solar rells, this issue will be pursued as well. In sum. strong evidence of type conversion because of applied bias to Culnse. was observed and further study is in order.

\subsubsection{SEM Studies of Thin-film Polycrystalline CuInSe}

The device characteristics of a number of SERI thin-film CIS devices were correlated with junction characteristics as determined by junction EBIC (JEBIC) (which includes information on the space charge region with. the diffusion length. and the junction devth). The results showed:

- all of the high short-circuit current (Jsc) devices also had broad JEBIC linescans (essentially, charge collection efficien $y$ profiles as a function of depth into the thin film) indicating that most of the Cis film was collecting (Fig. 3.10a).

- all of the low Jse and the low open-circuit voltage(Voc) devices had a narrower space harge region and junction location $\left(X_{1}\right)$ positioned notably away from the heteroface (Fig. 3. 1Ob).

- that there was no clear corollary between high Voc and the JEBIC protile. and

- the usual variability in the profile on a micron scale and the degree of variability between devices (Fig. 3.11).

The plana: EBiC (a lateral electrical map with the electron beam normal to the plane of the junction) characterication of the same devices demonstrated a considerable variability on both the micro- and the macro-scales (Fig. 3. 121. For example. CdS pinholes, $-5 \mu \mathrm{m}$ in diameter, affected areas -200 $4 \mathrm{~m}$ in diameter, and similarly. a $\sim 200 \mu \mathrm{m}$-wide forder area that paralleled the edge of the CaS contained regions whose fiectron beam quantum vield was eirher much more or much less efficin than the center of the device cungesting lateral ditfision of oxygen from the edge of the $C$ s suring tabmation. Such substantial nommitomities bear further investigation.

\subsubsection{Phosphorus-Doped Epitaxial CdTe Thin Films}

A magre havbare on the development of $p$-type $C$ dTe as a photovoltaic matertal has ben the difficulty trounteres in incorporating sufficiently high trets of dopant atoms. A. Fahrenbruch and co-witers at Stantore

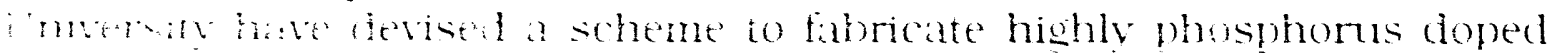

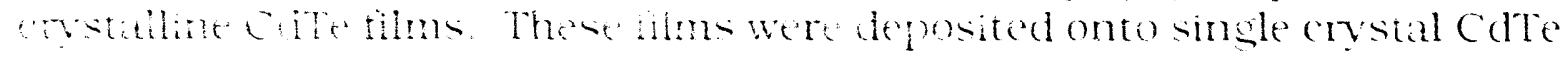

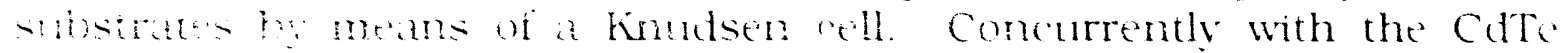



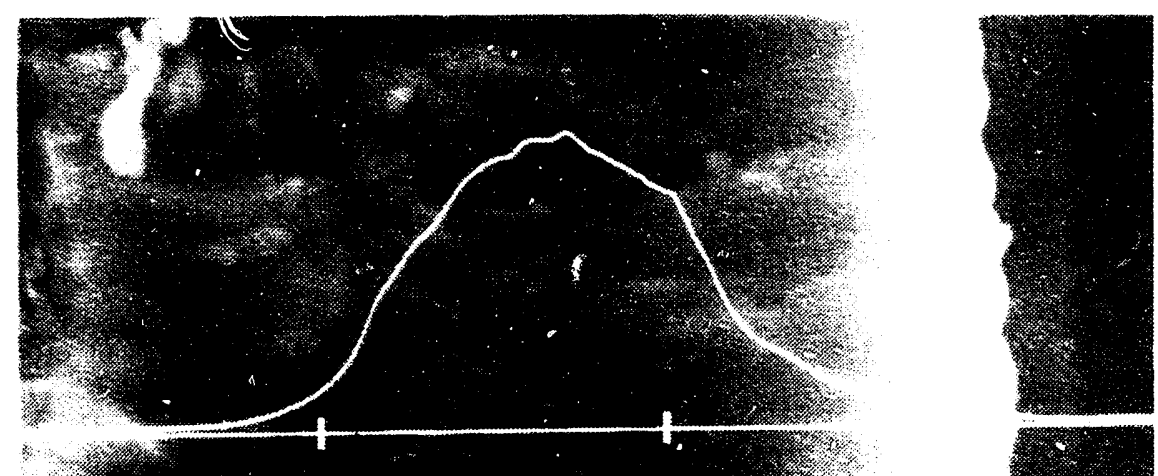

(a)
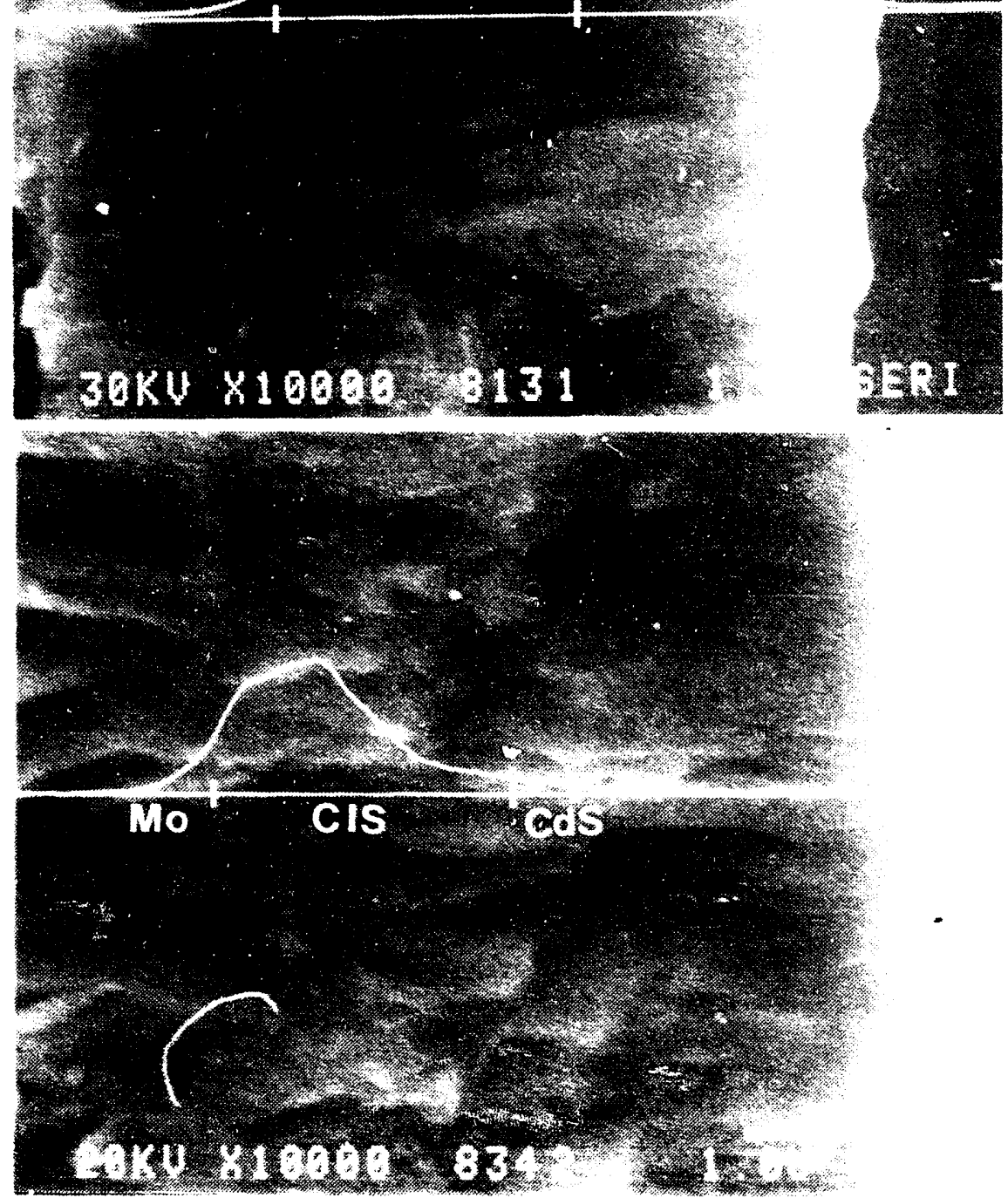

(b)

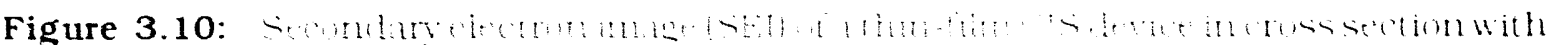

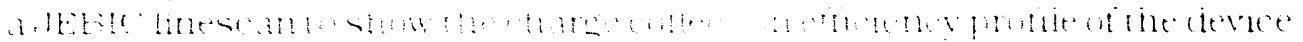

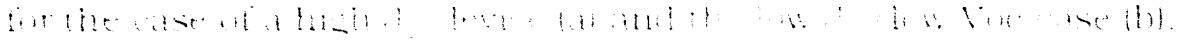




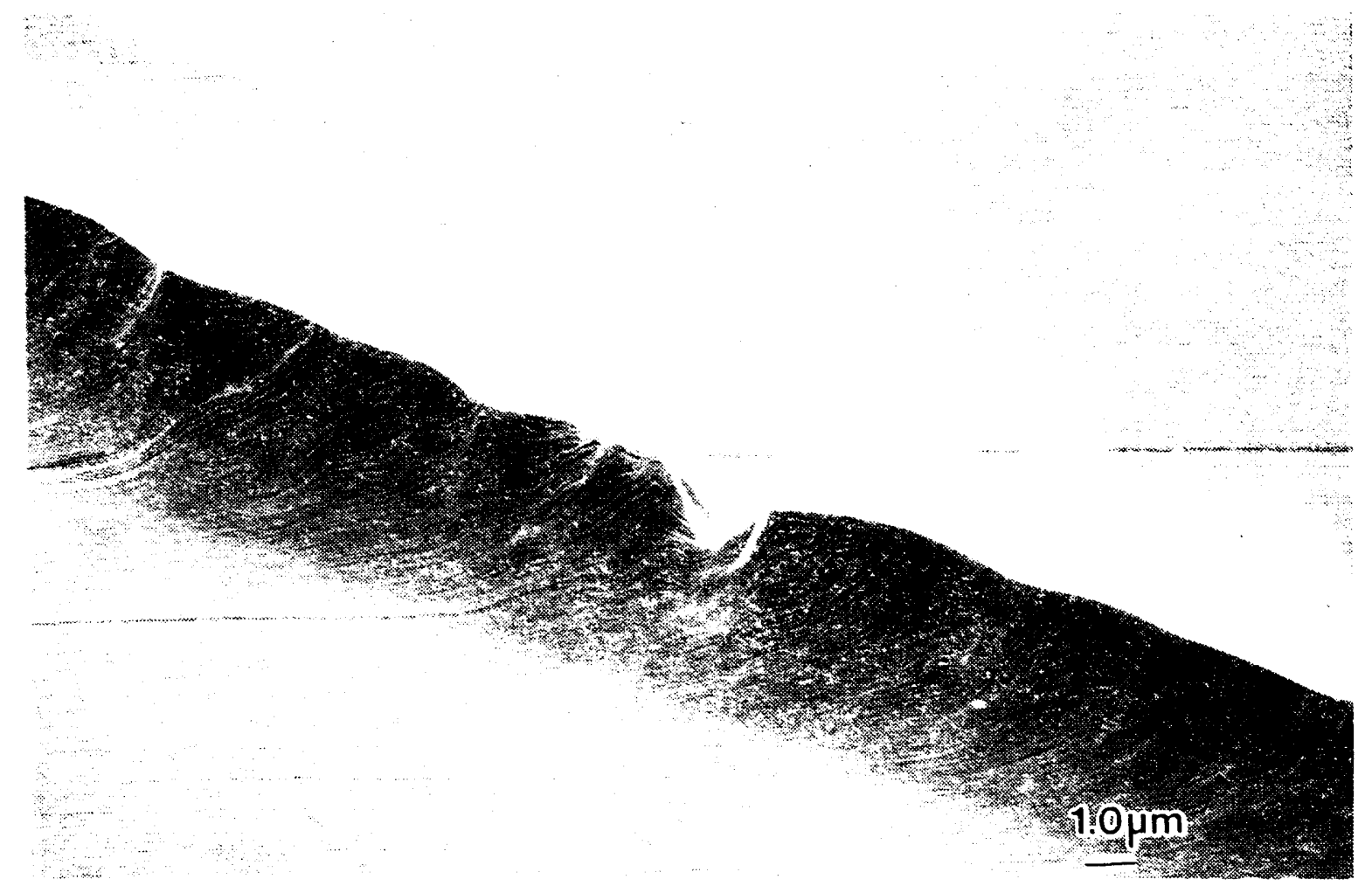

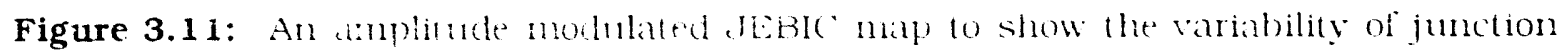

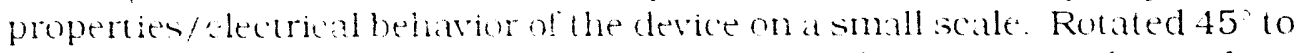
show the collectom profile nore clearly. the praks represent the peak in

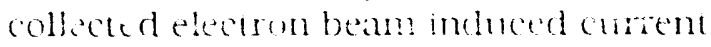

deposition. elemental phosphorus was ionized and incorporated into the epitaxial laver during fiim growth. This growth method yiclded films exhibiting high carrier densities (secondary ion mass spectroscopy [SIMS] measurements indicatte carrier densities on the order of $\left.1-5 \times 10^{17} \mathrm{~cm}^{3}\right)$ but poor photovoltaic performance.

At SERI a number of these ion-assisted doped films were examined using TEM to ascertain whether or not there were any structural features present that would explain the poor photovoltaic pertormance in spite of the high carrier densities. The highly doped plan-view samples showed an extensive defect netrotk that extended upward to the surfate of the epitaxial CdTe laver. The defect densitres measured at the uperes surface of the epitaxial laver were very hiph, on the orite of $10^{\circ}$ ('n - Clearly such a high concentration of strictural defeets wonth act as effecient recombination

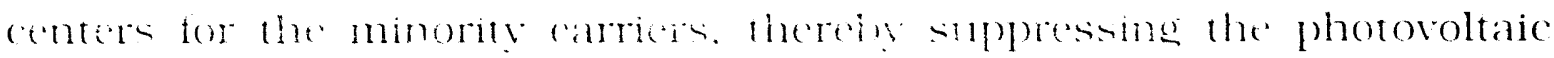

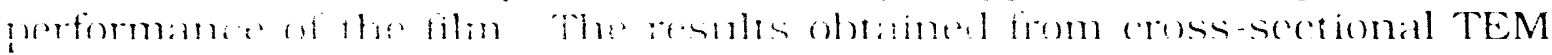

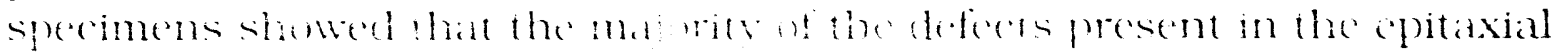




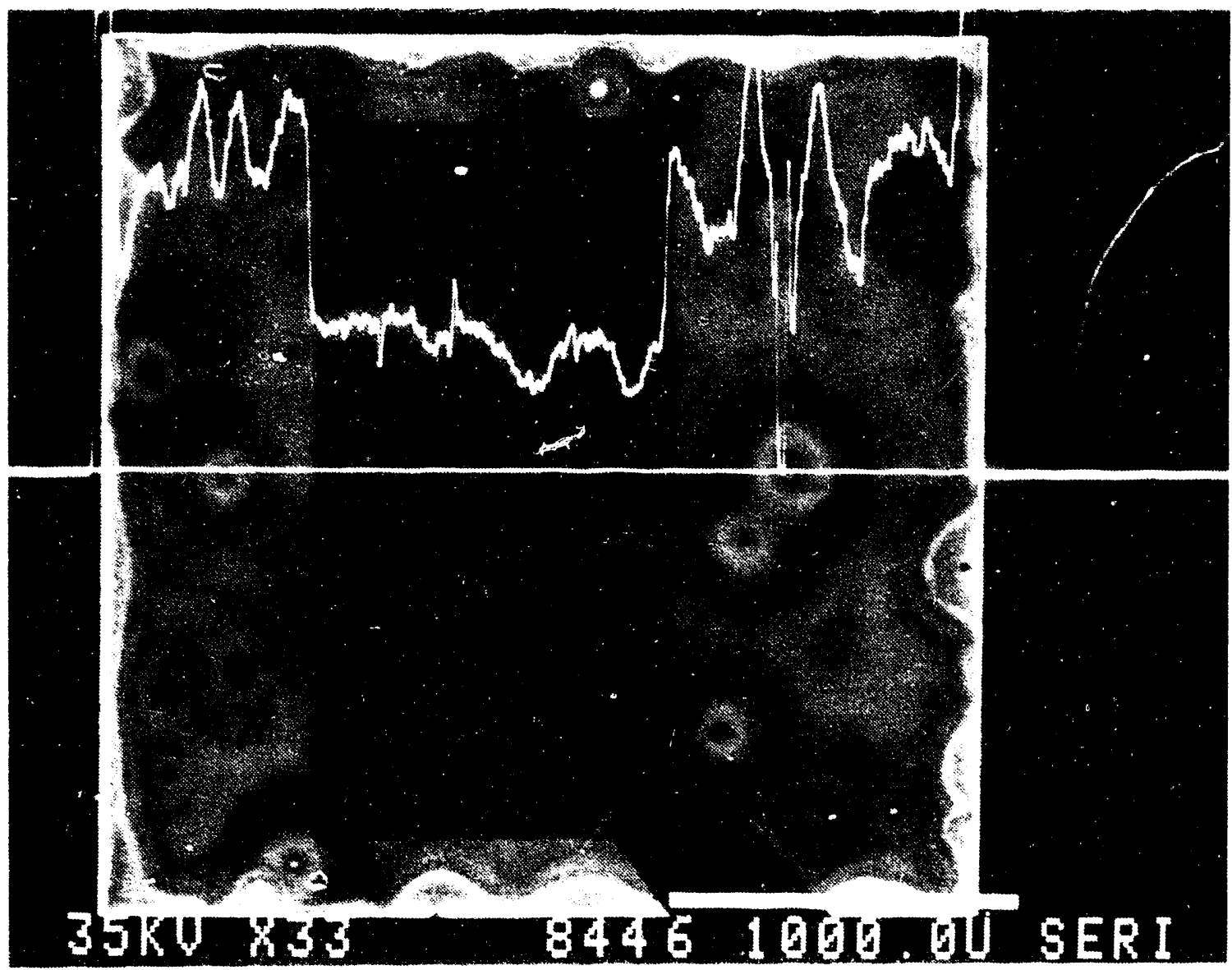

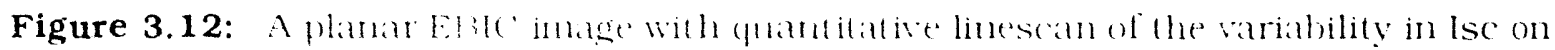

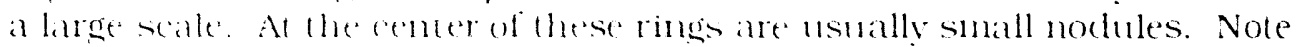

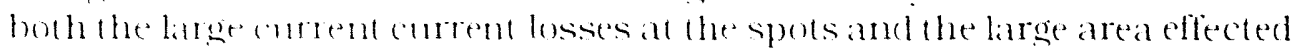
b) 1 hen

layer originate at the substrate film interface (Fig. 3. 13). Even at the highest doping levels, no evidence of phosphoms-rich precipitates or inclusions was observed. Similaty atomic-resolution images of the substrate/film interface showed no indication of an impurity or secondary phase layer. These TEM data suggest that the formation of this defect network is a result of the ionassisted doping rather than the ineorporation of a phosphorus-rich contaminant later at the substrate/film interface.

\subsubsection{Microstructure of Polycrystalline CdTe Thin Films}

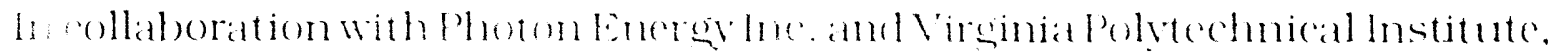

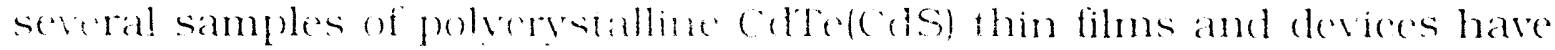
been examined using This. These films were depesited he magnetron

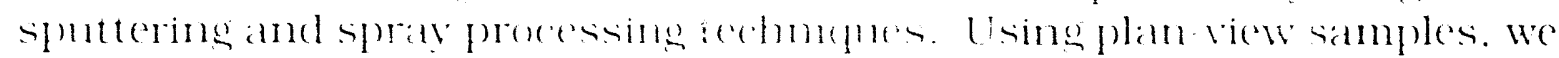

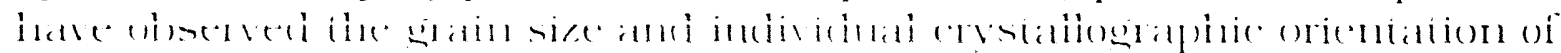




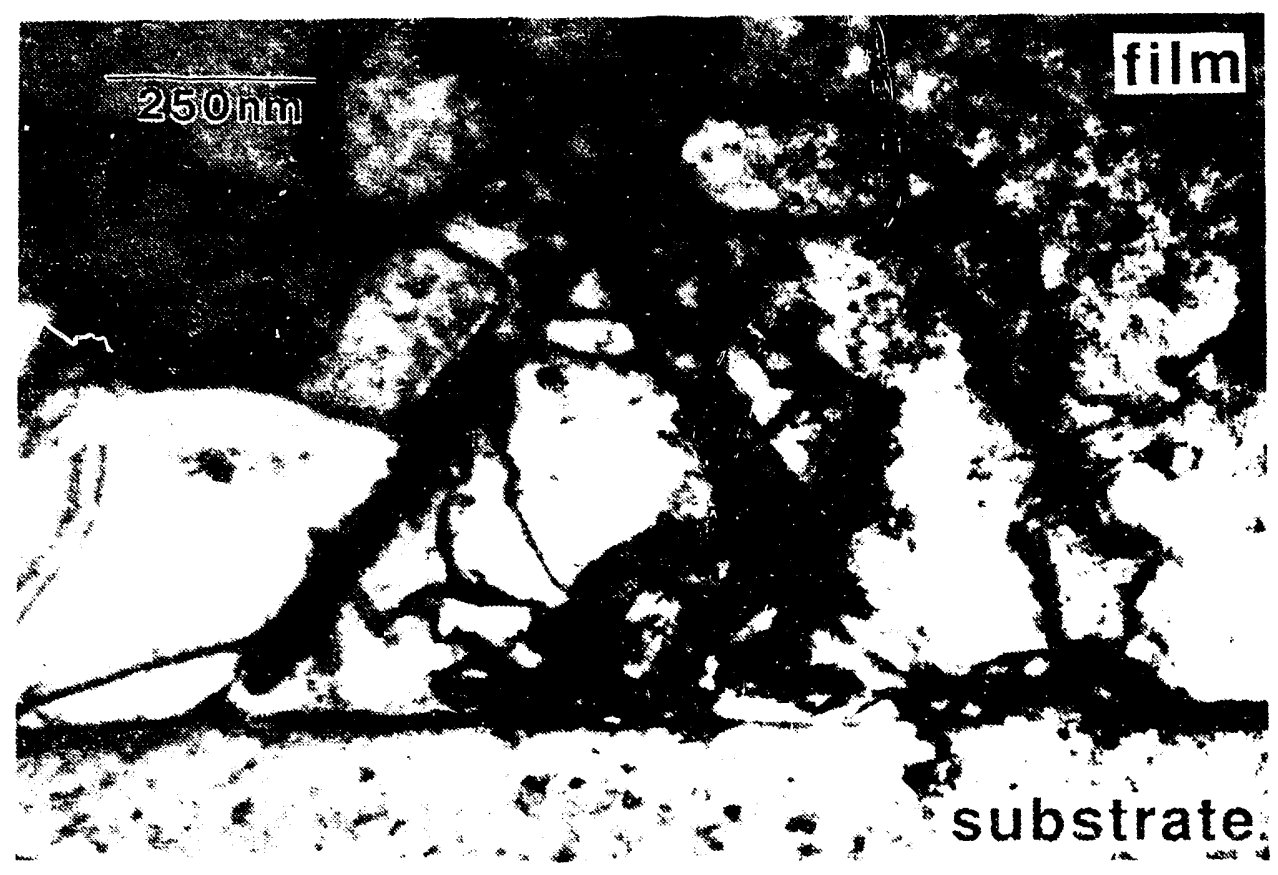

Figure 3.13: Cross-sectional TEM image showing the CdTe substrate/epitaxial CdTe film interface. An extensive defect network originates at the substrate/film interface.

the individual grains. In general the polycrystalline films are composed of a close packed array of single crystal fragments of dimension 0.5 to $2.0 \mu \mathrm{m}$ (Fig. 3.14). Furthermore, the indiridual CdTe crystallites grow with a preferred crystallographic orientation. Most often the [100]. [110] and [1 111$]$ CdTe axes are aligned perpendicular to the substrate surface.

The individual single crystal fragments of the CdTe films grown by magnetron sputtering exhibit a high density of planar defects. Atomic resolution images have shown that these defects are coherent microtwin boundaries (Fig. 3.15). Both the real space electron images and selected area diffraction patterns of single grains (Fig. 3.16) indicate that the twinning occurs perpendicular to the $\{111\}$-type zincblende directions. The individual microtwin lamellac vary in width from 10 to $10^{4}$ atomic layers. Almost all of the CdTe crystallites examined by TEM adopted the cubic zincblende structure. However, certain regions of the magnetron sputtered films exhibited intergrowths of the cubic zincblende phase with the hexagonal wurtzite (hexagonal $\mathrm{ZnS}$ structure) variant of CdTe. The wurtzite variant always occured as the minority phase. The individual hexagonal domains were small. on the order of 10 to $10^{2}$ atomic layers in dimension (Fig. 3.17). This highly twinned growth habit and zincblende/wurtzite intergrowth structure was not observed in similar polycrystalline Cote thin films deposited by spray processing techniques. 


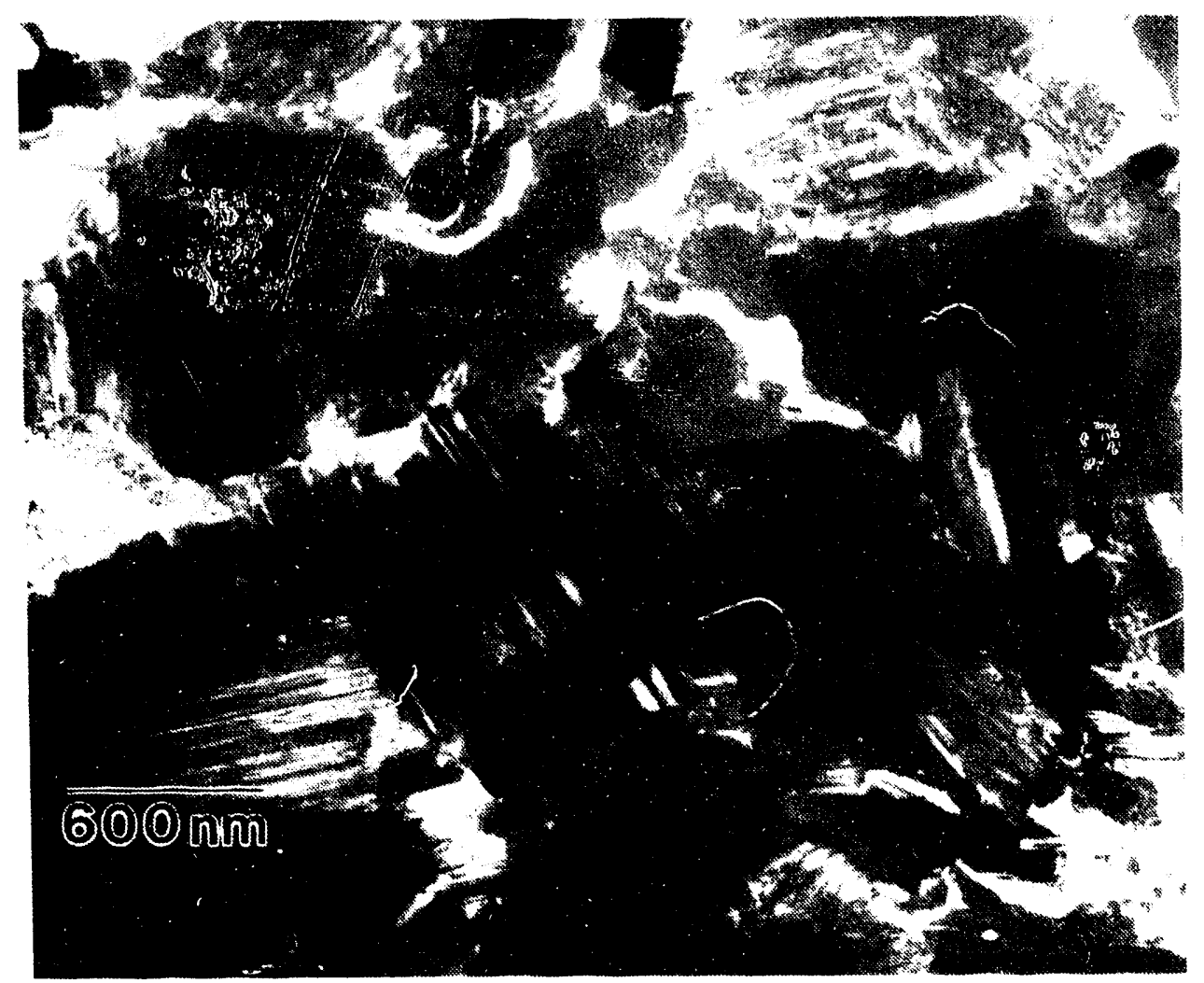

Figure 3.14: Plan-view TEM image of a magnetron sputtered polycrystalline CdTe thin film. A high density of planar defects (coherent microtwin boundaries) are visible in certain of the individual crystallites.

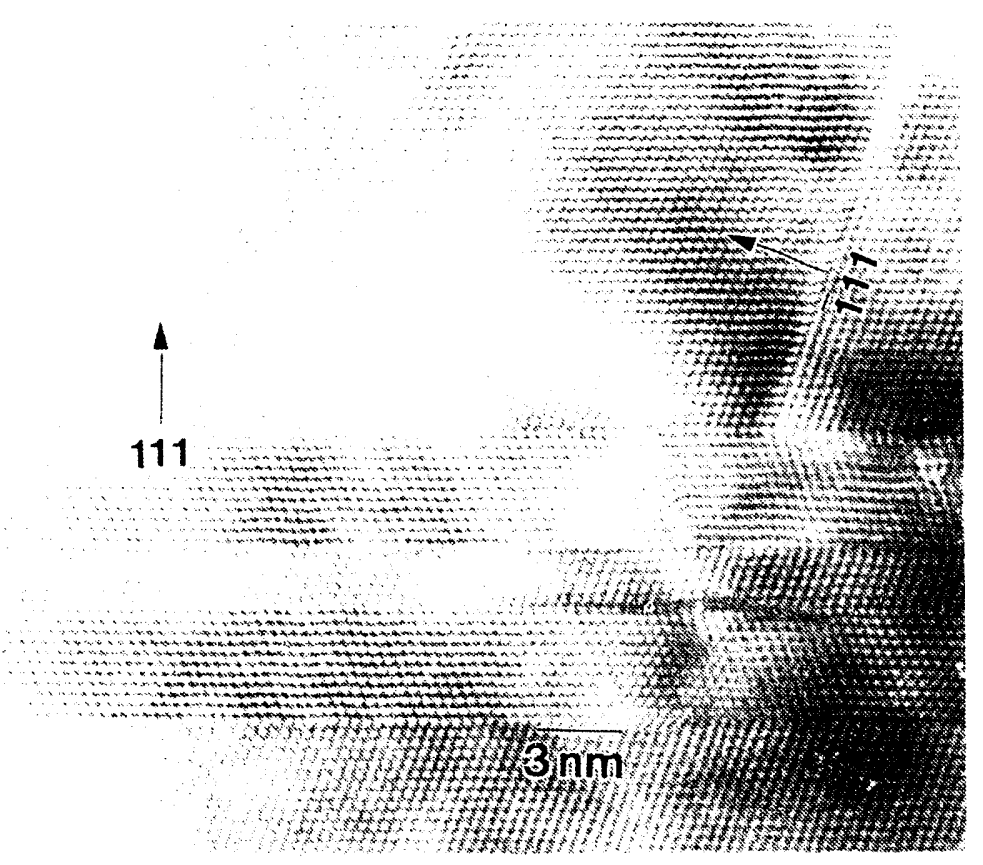

Figure 3.15: Atomic-resohntion image of a single crystal fragment of a magnetron

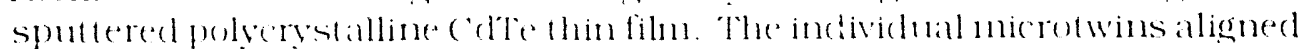

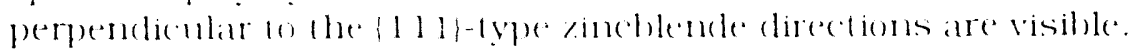




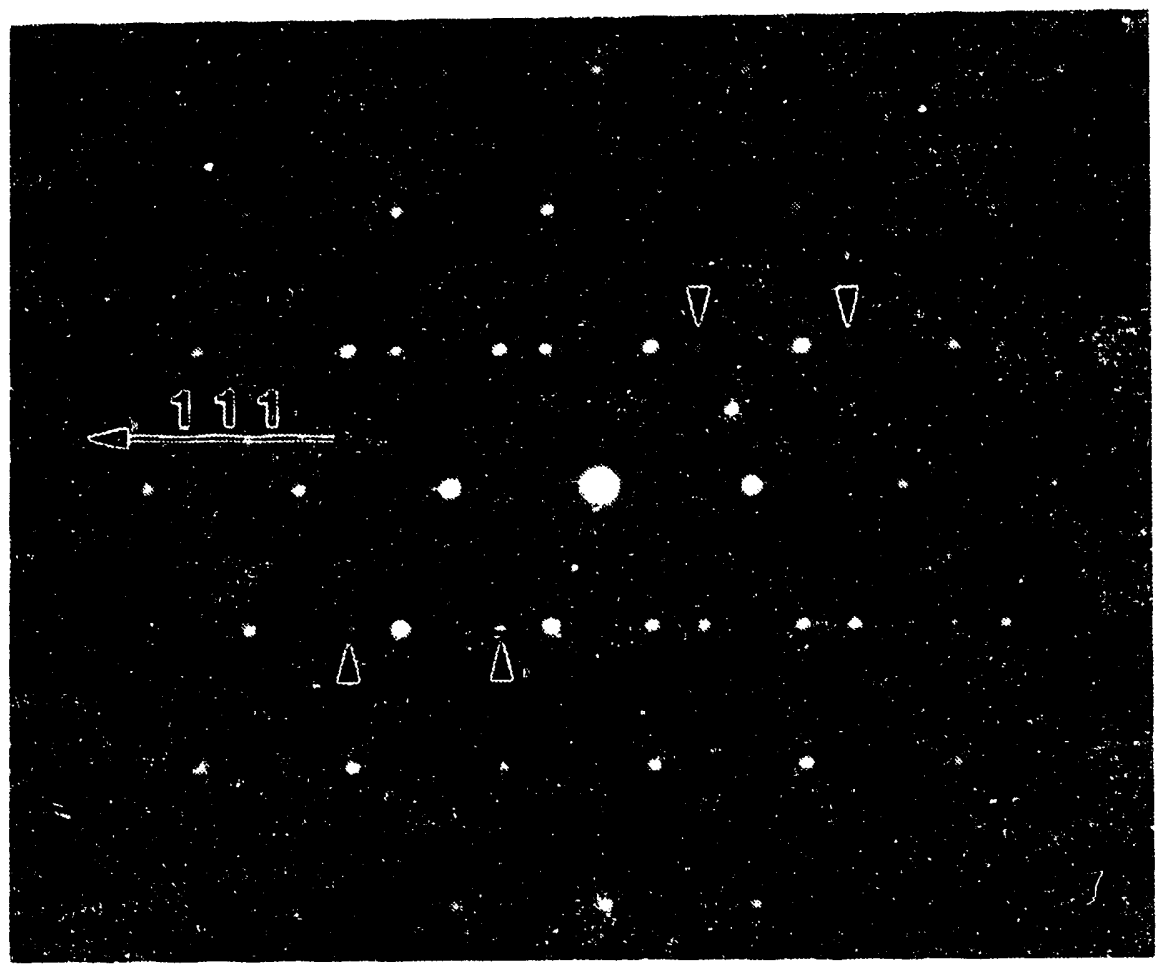

Figure 3.16: Selected area electron diffaction pattern of the single crystal cdTe fragment shown in Figure 3.15. Arrows mark the extra diffraction spots arising from twinning across [1111].

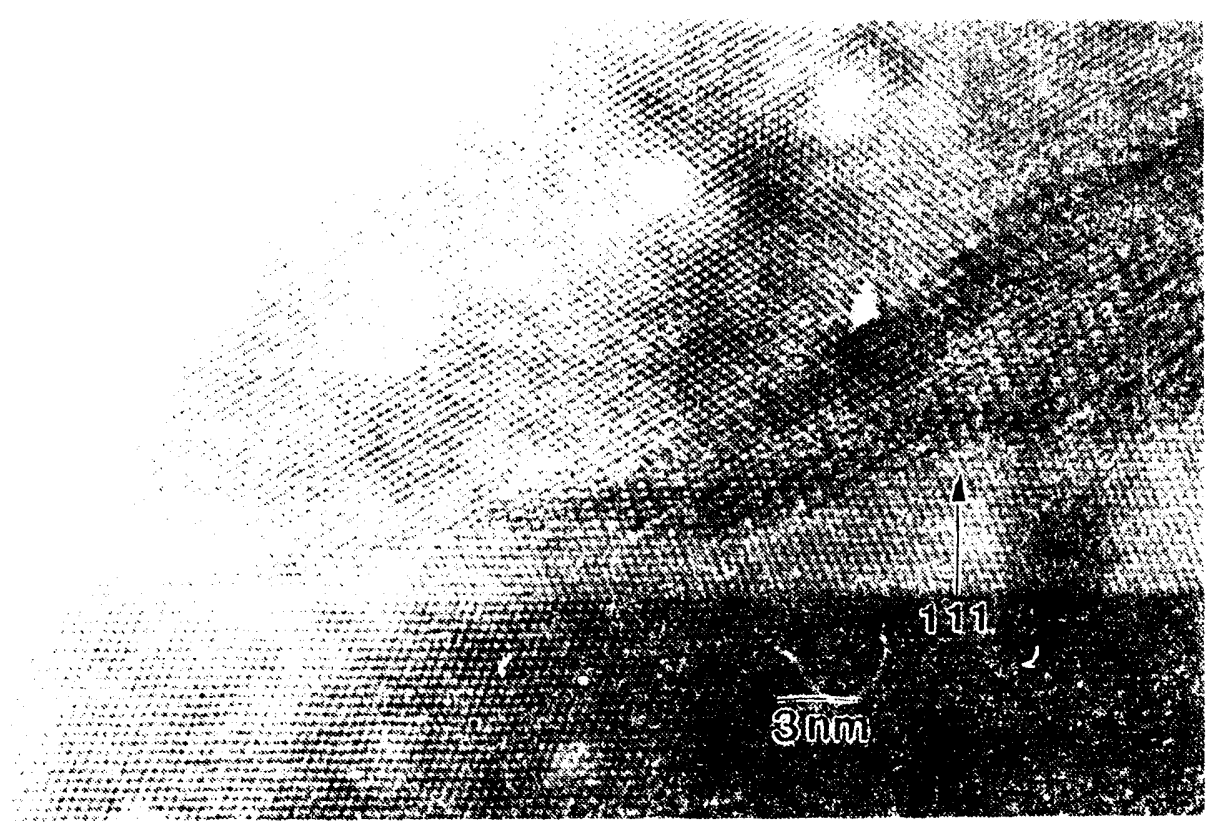

Figure 3.17: Atomic-resolution image of a magnet ron spultered CdTe aystallite showing a $\{11$ \}-type mic rotwin boundany (luwer one-third of the image) and a donain of hexagon d wirtzite (region marked with the 1111 l) arrowhead) intergrown into the cubic sinciblente matrix. 


\subsubsection{TEM Investigation of Hydrogen-Implanted Polycrystalline $\mathrm{Si}$}

Cross-sectional and plan-view TEM examinations were performed to study crystalline defects that occur due to low-energy, high-dose $\left(1020 \mathrm{H}^{+} \mathrm{cm}^{-2}\right)$ implantation. The hydrogen implantation was carried out in a Kaufman ion beam system using a beam energy of 0.5 to $1.5 \mathrm{keV}$ and a beam current of $55 \mathrm{~mA}$ for $15 \mathrm{~min}$. The samples used in this study were high-purity polycrystalline $<112>\mathrm{Si}$ ribbons, implanted at $\sim 300^{\circ} \mathrm{C}$. Cross-sectional examination (Fig. 3.18) revealed a heavily faulted region near the implanted surface. The defect density in this region decreased markedly as a function of depth. Furthermore, no buried damage region was observed. Additionally, a high density of dislocations were observed within some of the grains of the polycrystalline silicon. These are believed to have formed in the as-grown material and many of these dislocations were decorated with hydrogenrelated precipitates. The distribution of the latter is a strong function of depth within the sample. Their density is highest near the implanted surface. tapering off at a depth of approximately $20 \mu \mathrm{m}$.

The defects in the surface region were characterized in greater detail. Figure 3.19 presents a (110) plan-view micrograph and the corresponding electron diffraction pattern of a Si ribbon implanted to a $1020 \mathrm{H}^{+} \mathrm{cm}^{-2}$ at $0.5 \mathrm{keV}$. It clearly shows extended defects that lie on $\{111\}$ planes. The (112) crosssectional micrograph in Fig. 3.20 shows that these planar defects can extend from the surface down to a depth of $1.5 \mu \mathrm{m}$. Tilting the sample revealed the morphology of such defects. showing the extended core that gives rise to lighter contrast. Detailed bright field examinations suggest that these defects are well defined hydrogen plates (i.e., hydrogen-filled vacancy loops) lying on $\left\{\begin{array}{lll}1 & 1 & 1\end{array}\right\}$ planes with a three-dimensional core.

HREM examination (Fig. 3.21) showed that the plates incorporated several broken void segments along the $<111>$ directions. These segments appeared to be connected by stacking faults and microtwins. HREM examination also confirmed the presence of a high density of dislocations in the vicinity of the planar defects. It is concluded that $\mathrm{H}^{+}$implantation to such a high dose results in the precipitation of hydrogen into $\left\{\begin{array}{lll}1 & 1 & 1\end{array}\right\}$ plates. At the relatively high implantation temperature, this is believed to happen after the formation of vacancy loops. Further, at such temperatures the hydrogen pressure may build up within the loops, and hydrogen bubbles may form. The nucleation of such bubbles will exert high stress on the Si lattice and may cause plastic deformation resulting in the formation of the high density of dislocations we observed. 


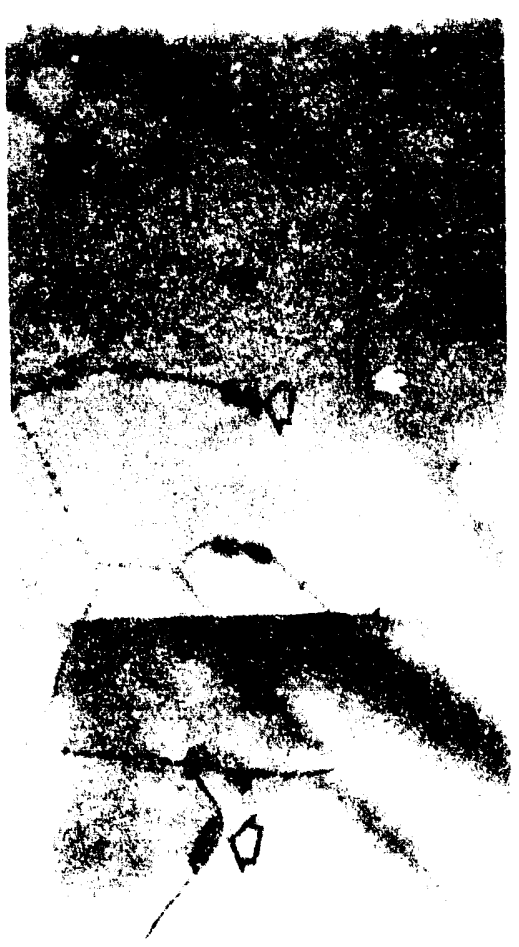

s

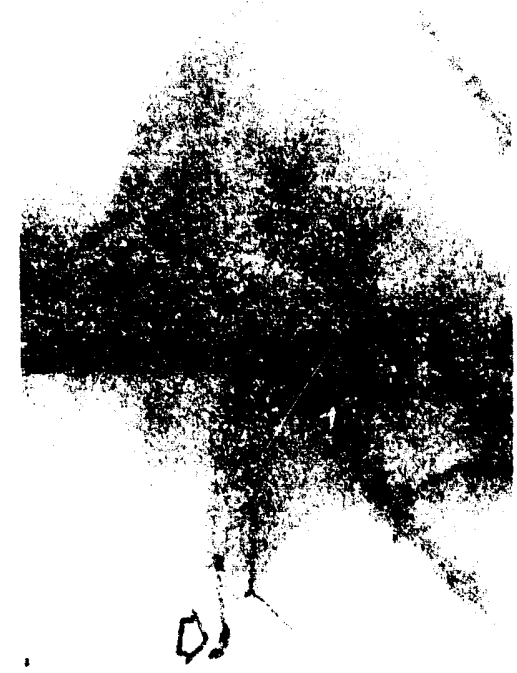

1.0 urn

Figure 3.18: $1112 \mid$ (rosis seron! showin! a hearily dimbared region near the Eimplanted surtace and hetrogen ateoration ol distecations decep in the Si tarroits)

\subsection{ACCOMPLISHMENTS: RELATED RESEARCH AREAS}

\subsubsection{Analysis of High-Temperature Superconducting Films and Devices}

In collaboration with TRW Corporation. Redondo Beach. California. TEM structural investigations of $\mathrm{YBa}_{2,} \mathrm{Cu}_{33} \mathrm{O}_{x}$ (YBCO) thin films and devices are continuing. Researchers at TRW are developing techniques for growing epitaxial YBCO on single crystal $\mathrm{LaAlO}_{3,}(\mathrm{LAO})$ substrates. LAO is one of the few materials possessing a dielectric constant sufficiently low for use in high-frequency applications as well as a favorable lattice match with the $a-b$ plane of YBCO.

Workers at TRW are currently producing thin trilayer structures (LAO substrate/bottom YBCO layer/intermediate LAO layer/top YBCO layer) suitable for use in superconducting fuantum interference devices (SQUIDS). A number of these devices have been investigated using TEM at SERI. Figure 3.22 is a plan-view micrograph showing the norphology of the top layer YBCO surface. A number of grain boundaries (most likely twin boundaries) are visibly perpendicular to the $\{110\}$ type directions. The microtwin grains have a dimension on the order of 10 to $100 \mathrm{~nm}$. The presence of twin boundaries is consistent with the elongation of certain of the higher order reflections observed in the selected area electron diffraction pattern of this region (Figs. 3.23). This diffraction pattern exhibits sharp, well-defined reflections indicative of a highly oriented single crystal laver. The pattern is derived from a relatively large area (on the order of microns) of the top YBCO film. The spacing and symmetry present in the diffraction pattern correspond to the the orthorhombic a and b axes of YBCO. This indicates that the mper YBCO layer is oriented with the orthorhombie: axis perpendicular to the substrate surface. Althongh the YBCO layer is almost entirely a cperpendicular orienterl film, small misoricnted grains are occasionally ohserved. One such region 


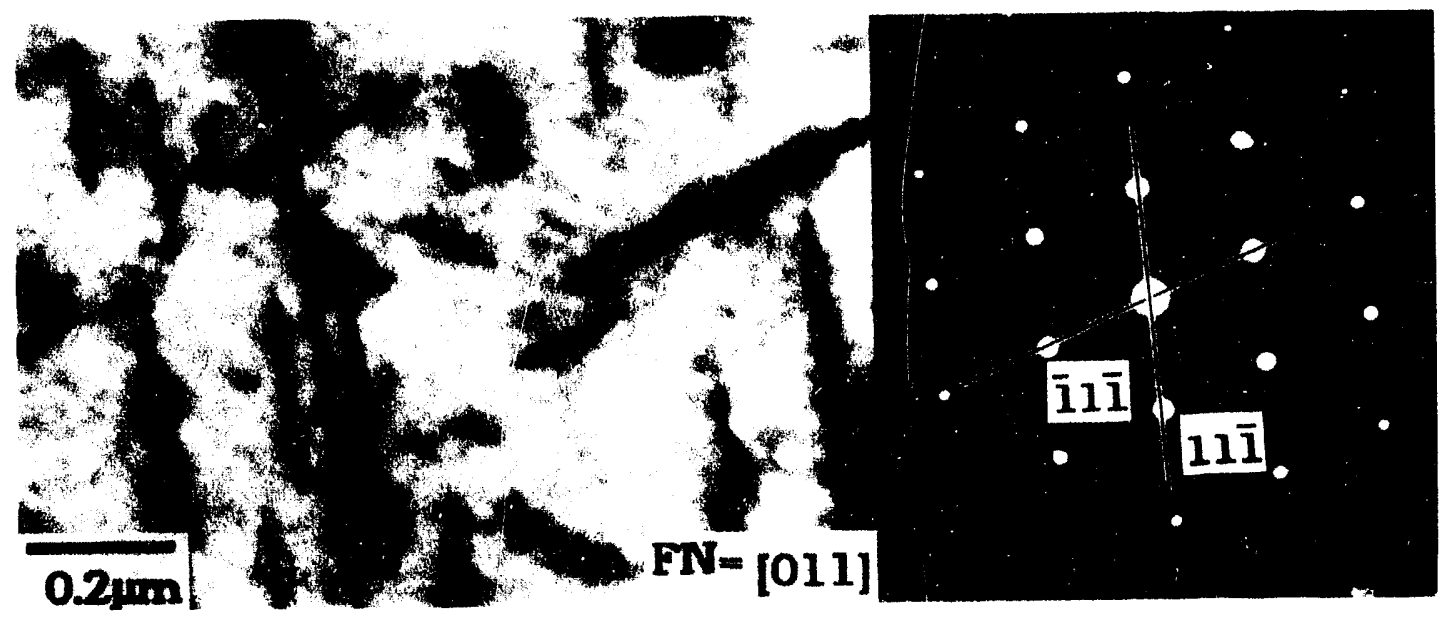

Figure 3.19: $[0111$ plan-view and electron difhaction trace analysis showing extended defects lying on $\{111\}$ planes.

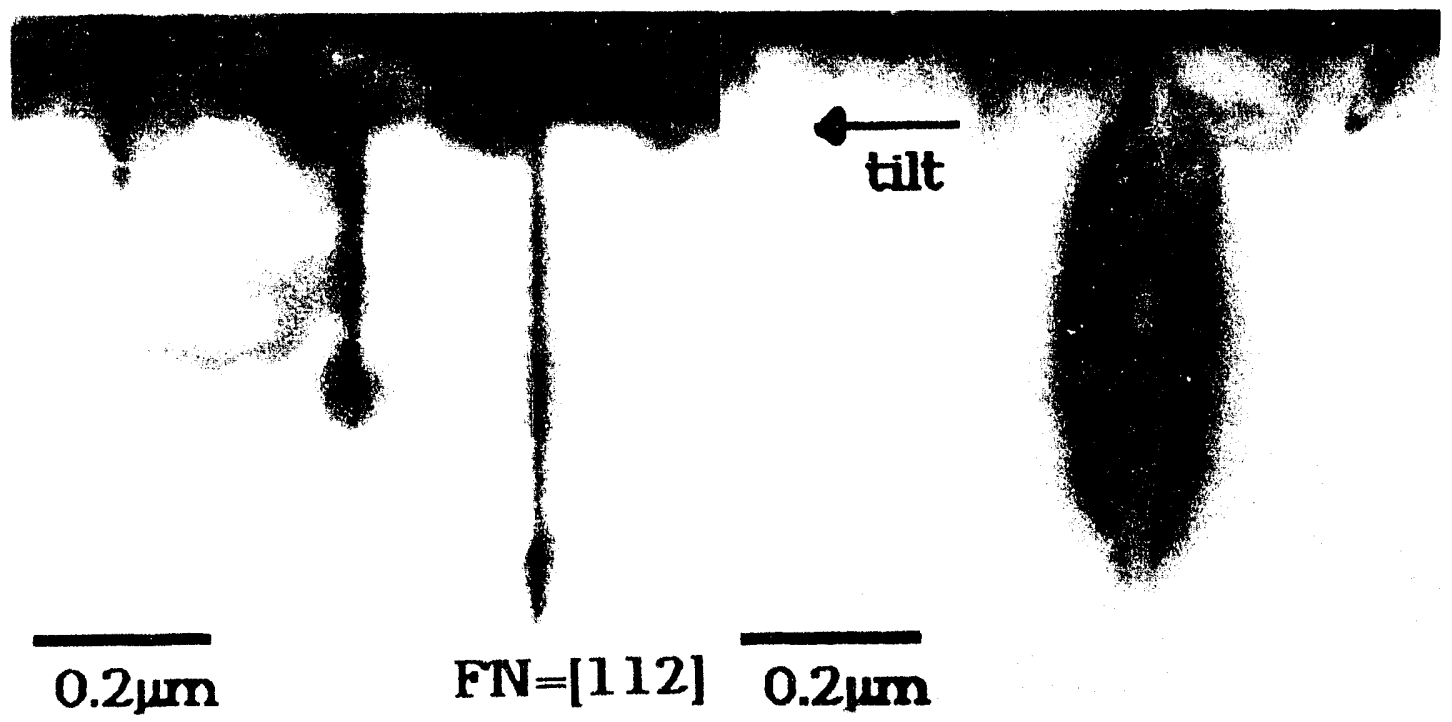

Figure 3.20: [112] cross-section of the surlace region simwing the depth of the extended defects from the implanted sultare

is marked in Fig. 3.24. In this case. the misiligned YBCO grain is oriented with the orthorhombic axis parallel to the suldstrate surface.

Figure 3.25 is a high resolution (ross-sectional TEM image of the top YBCO/ intermediate LAO interface. From the sharpmess and detail of the individual atomic layers present in this image. it can be sect that the (frality of the epitaxy is excellent. The YBCO film grows as a continuous single crystal layer. There is no evidence of any amorphoms region at the substrate surface. Similarly, no indication of the nucleation or precipitation of any secondary phase was observed at the $Y \mathrm{BCO} / \mathrm{L} . \Lambda \mathrm{O}$ interface. The selected area electron diffraction pattem of the YBSCO/LAO interfice region (Fig. 


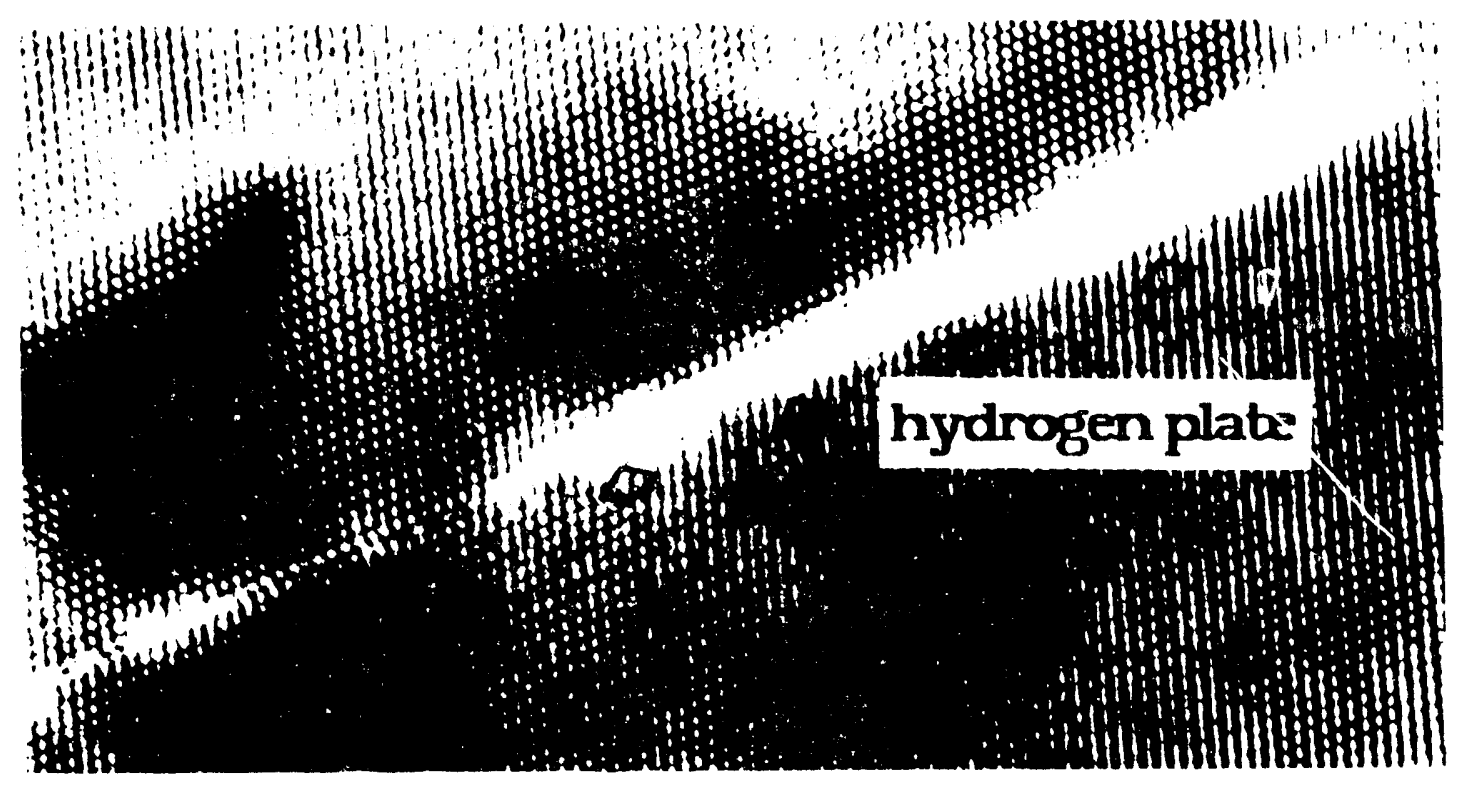

Figure 3.21: 1011] on-axis HREM latlice inage showing it: precipitation of hydrogen into 1111 plate's.

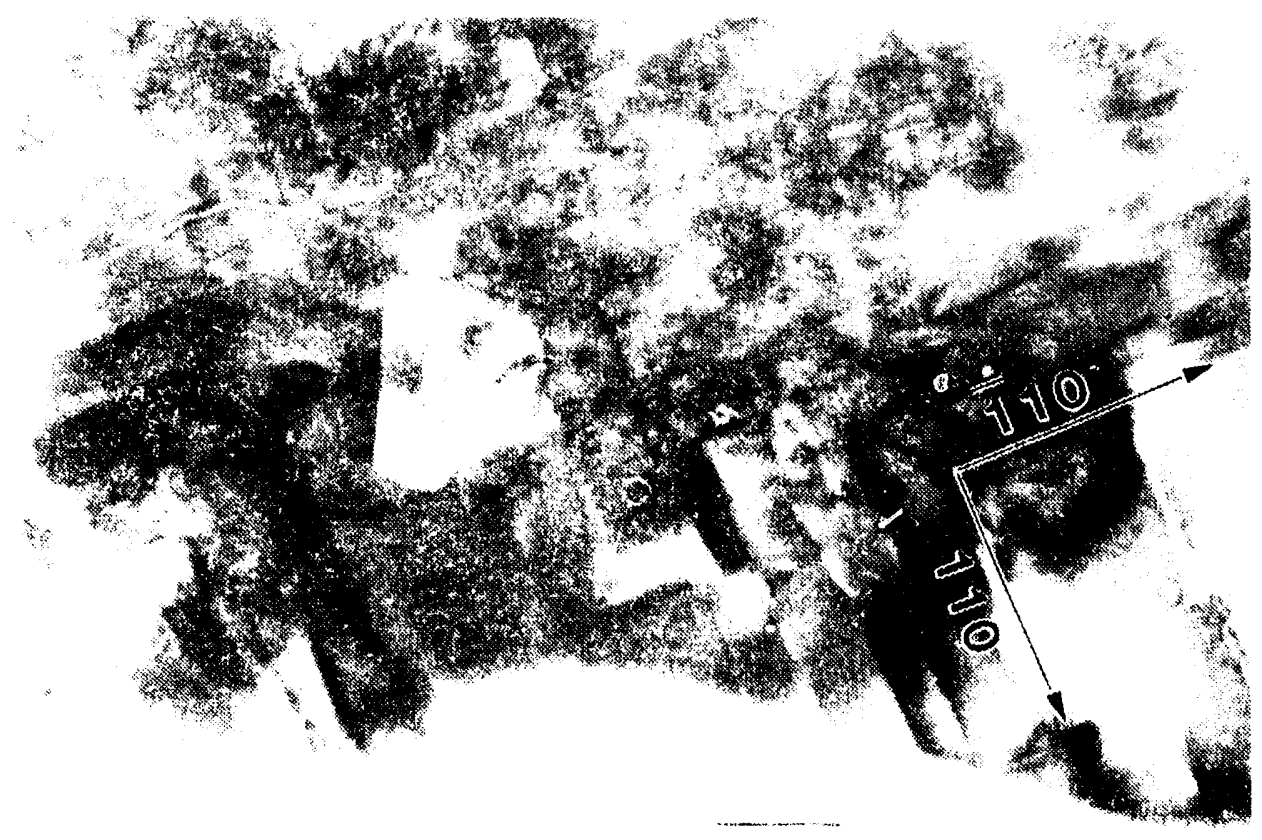

$50 \mathrm{~nm}$

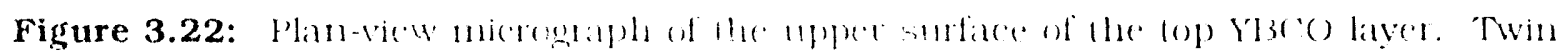

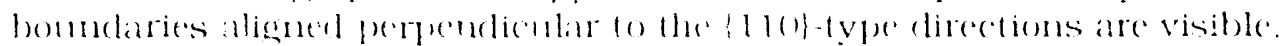




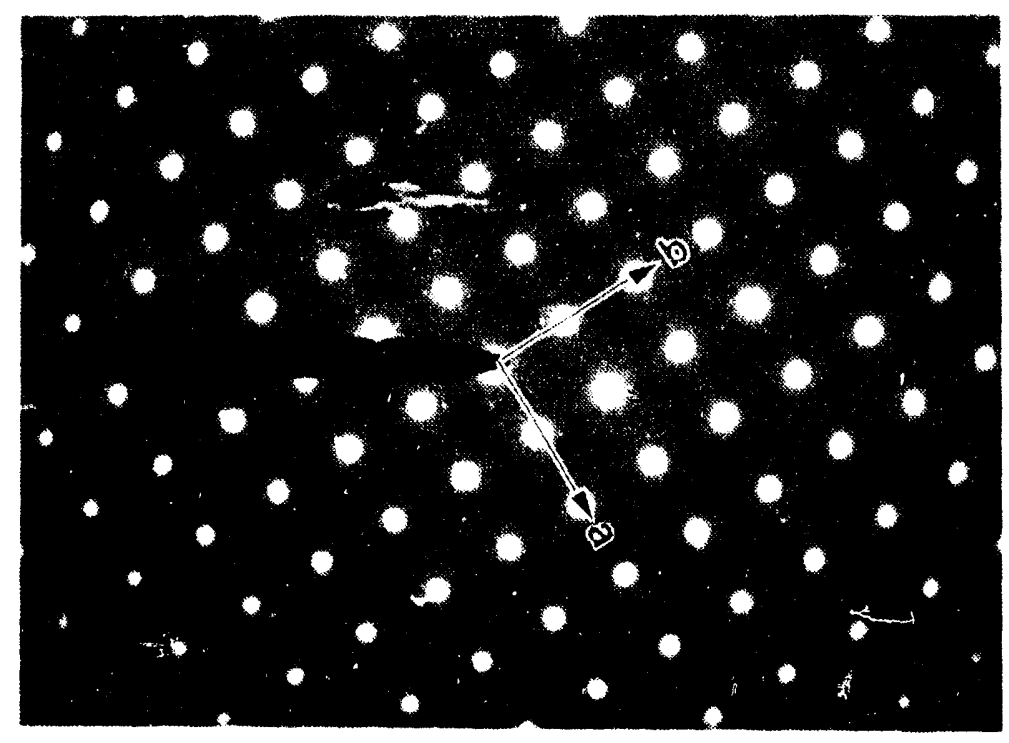

Figure 3.23: Selected area electron diffindion pattem from the uppermost region of the

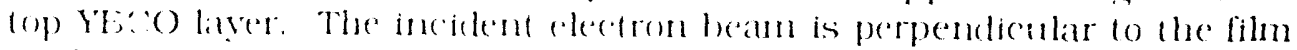
smeface. The orthorhmbir a and haxes lie in the plane of the film surface.

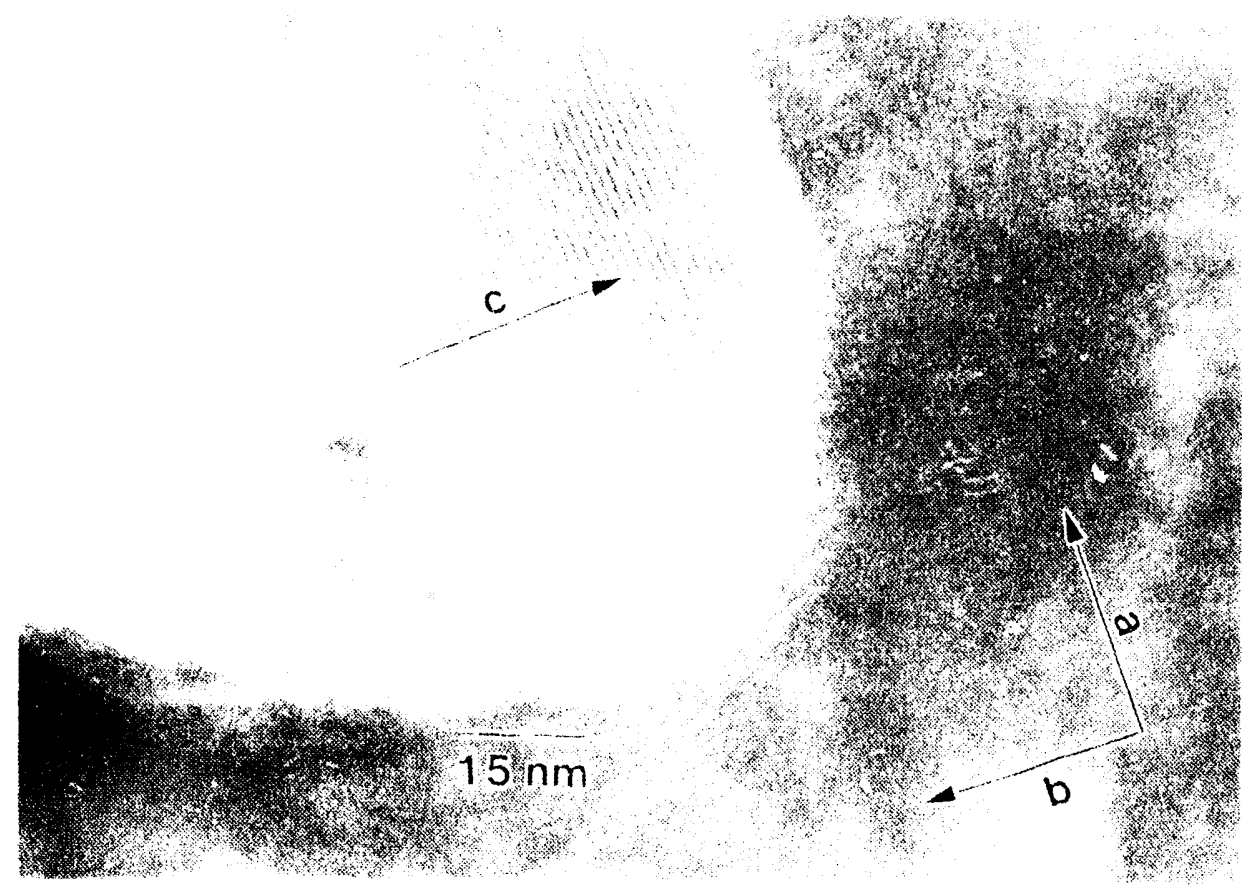

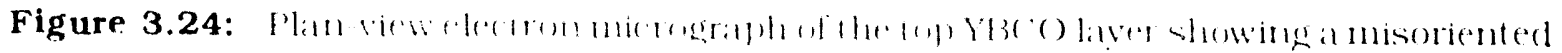

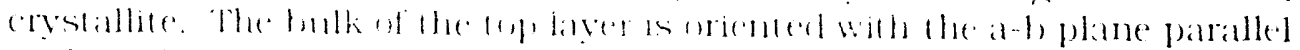

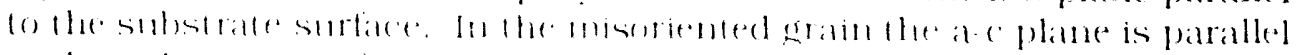

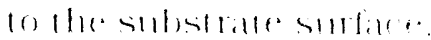




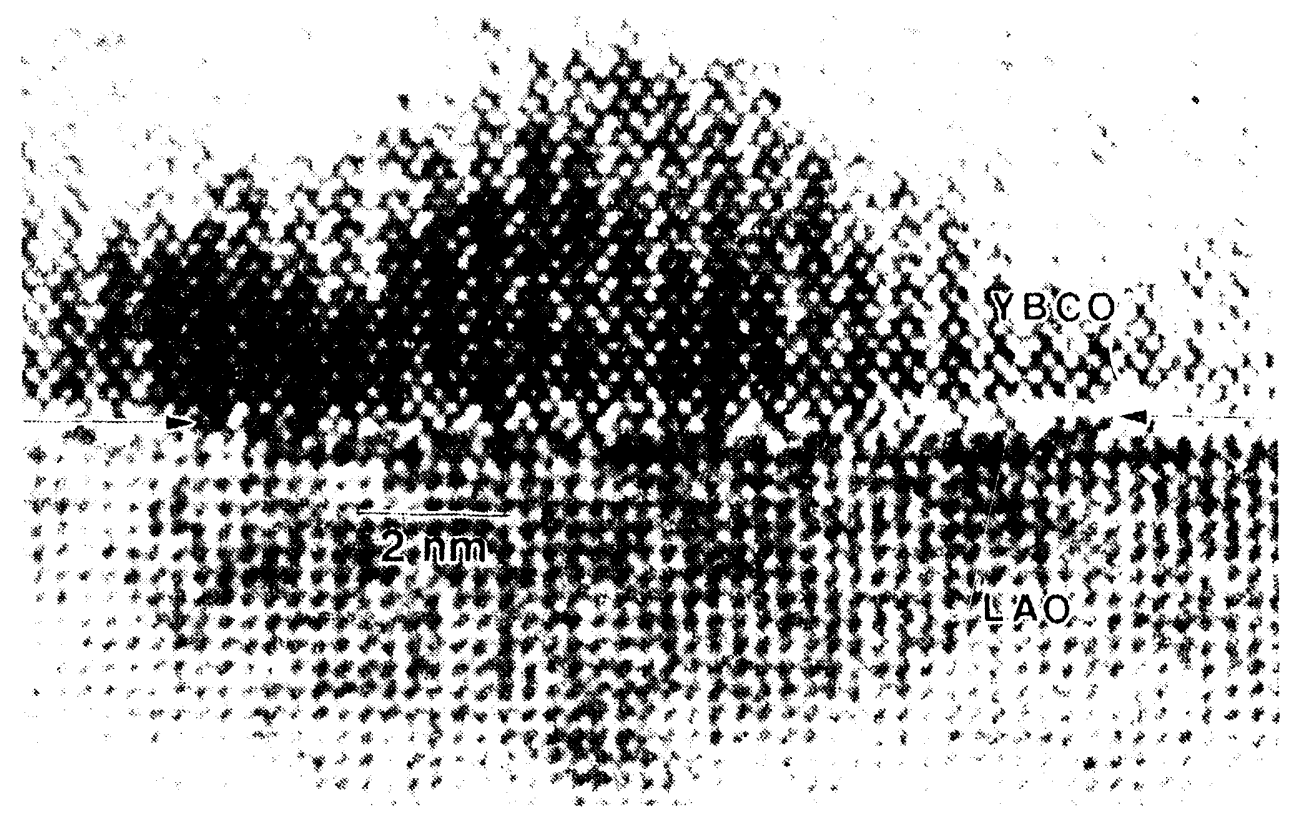

Figure 3.25: High-resolution TEM micrograph showing the epitaxy at the top YBCO/ intermediate LAO interface in a trilayer SQUID device structure.

3.26) confirms the high-quality epitaxy of the YBCO growth. The $\{001\}$-type reflections of the YBCO (closely spaced weak diffraction spots) are seen to align perfectly with the [001] LAO axis. No spurious reflections or diffraction rings are observable.

\subsubsection{The Microstructure of Lead Zirconate Titanate Thin Films}

Ramtron Corporation of Colorado Springs is currently developing Lead Zirconate Titanate (PZT) thin-film structures for use in nonvolatile random access memory devices. A commercial product utilizing this technology is expected to become available within the next 18 months.

SEM and TEM have been used to investigate the film morphology and growth habit as a function of deposition parameters. These films are deposited by vacuum rf-sputtering. Figure 3.27 shows the wide variation in grain size and surface coverage observed as a function of annealing parameters. Electron probe microanalysis has been used to identify the film stoichiometry. This investigation has been crucial in optimizing the design and composition of the sputtering targets used in the deposition process. Reliable control of the $\mathrm{Zr} / \mathrm{Ti}$ ratio in this system is necessary in engineering the electrical switching properties of the ferroelectric films. Microstructural analysis of conventional P'T thin films has been ongoing over the la.t 18 months. At present investigations of new ferroelectric systems are under way. These compounds are variants of the PZT structure containing significant amounts of calcium, niobium, and tin. 


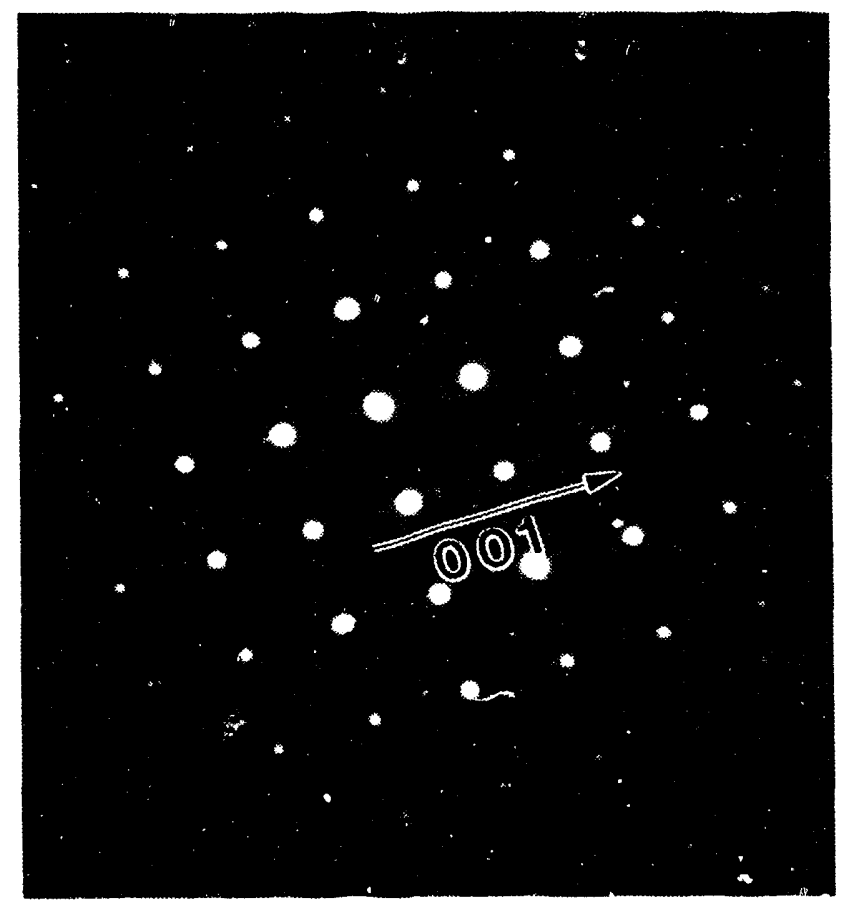

Figure 3.26: Selected area electmon diffantion of he lop haver YBCO/mbermediate LAO laver intertare region. Arrows mark the intertate. 

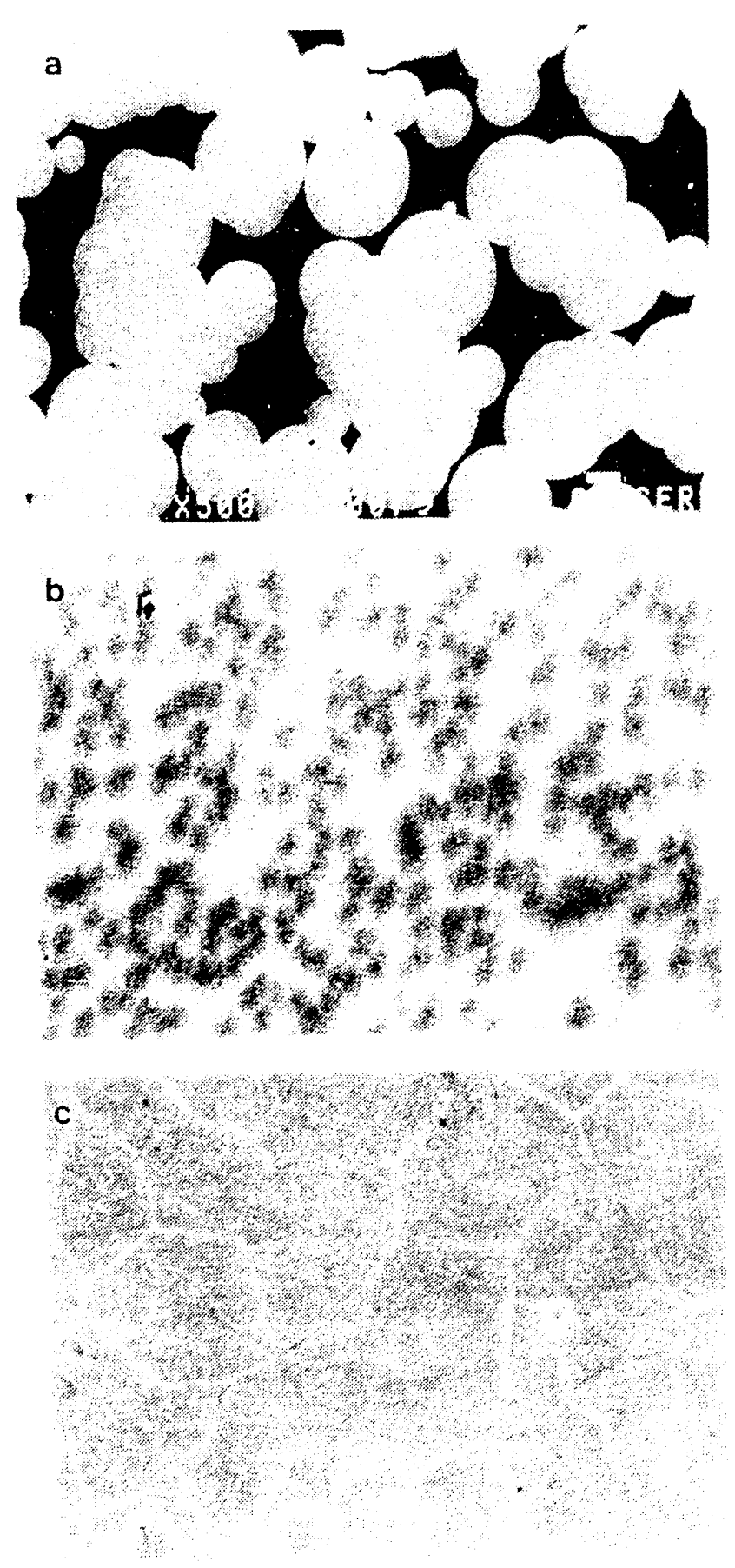

Figure 3.27: SEM images of PZT thin films showing the effect of differing annealing schemes on the film morphology. 
1. Hanoka, J.I., C.H. Seager, D.J. Sharp, and J.K. Panitz, Appl. Phys Lett., Vol. 42, 1983, p. 618.

2. Tsuo, Y.S., and J.B. Milstein, Appl. Phys. Lett., Vol. 45, 1984, p. 971.

3. Miller, J.C., V.T. Quat, and P. Siffert, Solar Cells, Apr. 1988, p. 109.

4. Bell, R.O, and C. E. Dube, 18th IEEE PVSC, 1987, p. 795.

5. Chevallier, J., and M. Aucouturier, Ann. Rev. Mater. Sci., 1988, p. 219.

6. Muller, J.C., Solar Cells, Vol. 17, 1986, p. 201.

7. Sopori, B.L., J. Appl. Phys., Vol. 64, 1988, p. 5264.

8. Leamy, H.J., J. Appl. Phys., Vol. 53, No. 6, 1982, p. R51.

9. Donolato, C., in Polycrystalline Semiconductors, Ed. G. Hartbeke, Spring Series in Solid-State Sciences, Vol. 57, 1985, p 413.

10. ... Pearton, J.S., J.W. Corbett. and T.S. Shi, Appl. Phys., A43, 1987, p. 153.

11. Yamamoto, A., N. Uchida, and M. Yamaguchi, J. Crys. Growth, Vol. 90, 1989, p. 369.

12. Al-Jassim, M.M., T. Nishioka, Y. Itoh, A. Yamamoto, and M. Yamaguchi, Mat. Res. Soc. Symp. Proc., 141, 1988.

13. Wanlass, M.W., P. Sheldon, "Improved Substrate Structures for InP-Based Devices," U.S. patent number 4,963,949, 16 Oct. 1990. 


\subsection{DEVICE DEVELOPMENT}

\subsection{RESEARCH STAFF}

T. J. Coutts, Principal Scientist and Group Leader

M. W. Wanlass, Staff Scientist

T. A. Gessert. Staff Scientist

F. Abou El-Fotouh, Senior Scientist

K. Ramanathan, Senior Scientist

R. G. Dhere, Staff Scientist

X. Li, Post Doctoral Researcher

G. Horner, Graduate Student

J. S. Ward, Graduate Student

\subsection{INTRODUCTION}

The objectives of the Device Development Group are to provide a service oriented activity to advance the fabrication and development of devices fabricated at SERI, or elsewhere within the DOE National PV Program. This includes maintenance of the device fabrication clean-room; provision of a grid design facility; maintenance of equipment within the clean-room (e.g. the mask aligner); provision of an antireflection coating (ARC) design and fabrication service; development of equipment used to advance measurements needed for the above services (e.g. the spectroscopic ellipsometer and other items of equipment discussed below); diagnostics of devices and feed-back to other fabrication groups; development of novel or improved techniques for the fabrication of devices; and the study of materials of current or prospective interest to the program. During the last year, the group has strengthened its involvement in the polycrystalline thin film device area and has initiated collaborations with several of SERI's principal sub-contractors and with inhouse researchers. Although this process was actually begun last year, the pace has accelerated during the current year. Work for others in the high efficiency III-V device area has also increased and this has now led to identifiable benefits to the DOE program.

\subsection{ANALYTICAL CAPABILITIES}

Appendix $\mathrm{D}$ provides a listing of the major instrumentation and test equipment, their features, and specifications.

\subsubsection{Spectrophotometry}

Spectrophotometry is essential for the diagnostics of materials and devices. For thin film devices, it may be used to measure the reflectance/transmittance of window layers thereby aiding the calculation of the internal quantum 
efficiency. This is an important factor in assessing the performance of the junctions in actual cells and in determining the manner of their operation. The Cary spectrophotometer is interfaced to an Apple Macintosh computer which controls the wavelength range over which data are obtained. The reflectance/transmittance data are stored in spread-sheets which may be processed using one of the standard software packages available to the group. It is thus possible to calculate the optical constants of non-absorbing filins on substrates with known optical properties, or of partially absorbing films, such as CuInSe $e_{2}$, on known substrates. This is a valuable facility which is used to assess the quality of films and the consistency of fabrication processes. Modifications to this equipment have recently been made to allow the measurement of the barrier height of metal/semiconductor contacts.

\subsubsection{Ellipsometry}

At the time of writing the last annual report, this equipment was operational over the range $250-850 \mathrm{~nm}$. A liquid nitrogen-cooled germanium detector has now been installed which allows determination of the optical properties of materials over the spectral range $250-1700 \mathrm{~nm}$. Additional modifications will be made to the instrument during the coming year which will increase the speed of data acquisition and analysis. The equipment has been successfully used to analyse the optical constants of single crystal CuInSe ${ }_{2}$ and it has been shown, for the first time, that these are in good agreement with values for stoichiometric thin films. It has also been used to measure the constants of several III-V materials grown in-house. The equipment is available for the benefit of all researchers within the program as a service to assist development of devices.

\subsubsection{Hall Probe}

The Hall probe equipment continues to be used by a variety of groups within and external to SERI for the measurement of the transport properties of their materials. The Device Development Group, however, maintains this equipment and ensures that it remains in operational order. As it stands, the equipment may be operated at temperatures of as low as $10 \mathrm{~K}$ and this is useful for the assessment of charge scattering mechanisms in semiconductors.

\subsubsection{Admittance Spectroscopy}

Development of a novel experiment to be used for the characterization of traps in semiconductors has recently been commenced. Although this is at a relatively early stage, progress has been satisfactory and it is expected that the equipment will be completed within the coming year. 


\subsubsection{Contact Resistance}

The importance of contact resistance in the design of grids for solar cells is commonly underestimated. This is particularly true when the devices are to be operated under concentration. In this situation, the contact resistance becomes much more important. The group has, for many years, had available a system with which it is possible to determine the specific contact resistance of metals to semi-conductors. Techniques are also availabie to assess the quality of metallization and this will be discussed more fully later. Work on contacts to CulnSe 2 has been commenced and it is hoped to clarify and optimize the behavior of these since there is still some uncertainty with regard to their quality. Studies on other materials of relevance are being made and the facility is proving useful in several areas.

\subsection{DEVICE TECHNOLOGY}

The emphasis on technological design has been increased during the past year because of internal collaborations. This has been concerned with top contact grid power loss studies (grid optimization) and antireflection coating (ARC) optimization. The computer aided design facilities at NIST in Boulder are currently used for the fabrication of photomasks for the photolithography of actual grids. In addition to improvements of computer software to facilitate these studies, many grids and ARC's were fabricated. These procedures and facilities were made available throughout the Institute and its sub-contractors, but they were mainly utilized by researchers within Branches 213 and 212.

\subsubsection{Grid Optimization}

A wide variety of grid modeling activities was undertaken and completed this past year. Some of this work was in the form of unique and novel designs for three- and four-terminal devices, which later proved to demonstrate record efficiencies (see Section 4.7). Other grid modeling was performed to provide optimized grid designs and photomasks for researchers in Branch 212 and 213. In addition to these efforts, some fundamental grid studies were completed which will assist the Group's grid modeling studies well into the future. These studies involved not only an in-depth analysis of the effect of non-ideal metal resistance on both one-sun and concentrator solar cells, but also the fabrication of test patterns with which to measure actual metal resistance as a function of deposition technique, line width, etc.

Although the research concerning metal grid line resistance is ongoing, several guidelines have been established for the in-house grid design work. The first is that the plated Au contacts, used for most of the cells produced at SERI. demonstrate about 3-5 times higher resistivity than the ideal or 
pure bulk "textbook" value, thus emphasizing the need to control the plating procedure very carefully. If the plating is not carefully controlled, as might happen if the operat or is not very skilled in plating or if the plating solution is "unbalanced," then the resistivity may be as high as eight times the ideal value. Evaporated metals, on the other hand, seem to be less sensitive to process variations. demonstrating measured resistances of 1.5-1.8 times the ideal values. Thus, if plated contacts are used, and if a metal resistance of at least five times the ideal value is not assumed in the grid modeling, then the contact will not perform as expected. Furthermore, if high-efficiency cell results are sought, evaporated contacts should be used. This may be difficult since the Institute currently has a serious shortage of vacuum systems which are designed for metallization work with the necessary refractory and conduction metals (large E-beam systems).

\subsubsection{Antireflection Coatings (ARC)}

\subsubsection{Development of a Dedicated ARC Deposition System}

The Group has succeeded in producing optical quality thin films using an evaporation system which was designed and built for this purpose by the Group within the past year. Specifically, this system is an NRC Model 3115 (Varian) and both $\mathrm{ZnS}$ and $\mathrm{MgF}_{2}$ have been deposited using thermal resistive evaporation from large (production size) box boats. Initial ellipsometric and spectrophotometric measurements have confirmed that the optical constants are very near those quoted in the literature, and that the spatial uniformity will greatly exceed the current requirements for any photovoltaic (PV) solar cells produced at the Institute ( 3 "x3"). Future spectrometric measurements will analyze these optical constants more thoroughly and these measurements will be necessary for use with optical coating modeling studies being done by the Group.

\subsubsection{Use of $\mathrm{Al}_{2} \mathrm{O}_{3}$ as an Intermediate Refractive Index $\mathrm{ARC}$ Material}

Studies were conducted to determine if electron-beam evaporated $\mathrm{Al}_{2} \mathrm{O}_{3}$ can be used as an optical material in a $\mathrm{ZnS} / \mathrm{Al}_{2} \mathrm{O}_{3} /$ Entech Cover combination. After calibration runs, it was determined that $\mathrm{Al}_{2} \mathrm{O}_{3}$ does form a uniform ARC on $\mathrm{ZnS}$. However, due to the hardness of the $\mathrm{Al}_{2} \mathrm{O}_{3}$ film, it is essential to provide for protection of the contact pads during the deposition; otherwise the fill-factor of the cell will decrease measurably. This ARC stack was successfully employed on a three-terminal, two-junction solar cell which achieved remarkable performance (See Section 4.7). 


\subsubsection{Combination Front- and Back-Side ARC Modeling and Fabrication}

In addition to "normal" $\mathrm{ZnS}$ and $\mathrm{MgF}_{2}$ coatings, several novel ARC structures have been studied. The first of these structures is for use with the top substrate in a four-terminal. mechanically-stacked (i.e., two substrates) solar cell. In this case, the optical coating must not only act as an ARC on the upper surface of the top cell but, in order to maximize the efficiency of the lower cell, must also act as an ARC on the back surface of the top cell. This ensures maximum transmittance of the longer wave, sub-bandgap light used by the lower cell. In this research, modeling was done using the ARC "contour" modeling program assuming a GaAs substrate. Later, two-sided, double layer coatings were actually deposited onto GaAs substrates and cells for use in measurements, and on cells related to Work for Others (see Section 4.7). Although spectrophotometric analysis of these double-sided ARCs has not yet been completed, preliminary results indicate that the model is in agreement with the observed results. Specifically, it has been found that in order to enhance the transmission of light from the back surface of the top substrate, $\mathrm{ZnS}$ and $\mathrm{MgF}_{2}$ can again be used, but the thickness of each layer is roughly double that used on the front surface.

\subsubsection{ARC for Use With Entech Covers}

The second of these novel ARCs are for use with Entech prismatic covers, which have become extensively used at SERI during the past year (see below). In this case it has been found that, since the index of refraction of the cover is $\sim 1.41$, the $\mathrm{MgF}_{2}$ thickness must be reduced to achieve optimum performance. Thus far, it has not been demonstrated that an optimum $\mathrm{MgF}_{2}$ thickness exists for this structure, but modeling studies indicate that it may be possible for the $\mathrm{MgF}_{2}$ to be completely eliminated from the ARC stack. Although this would be acceptable for the InP-based shallow homojunction cells, the ITO/InP cell, always requires a thin layer $(20 \mathrm{~nm})$ to stabilize the electrical properties of the DC magnetron sputtered ITO by protecting them against atmospheric effects. However, in this case too, ARC modeling studies have indicated that the $\mathrm{MgF}_{2}$ should not measurably affect the performance of the cell. It is to be emphasized that practical experience indicates that the predictions of the modelling are not confirmed and that a $\mathrm{MgF}_{2}$ is still required. It is believed that this apparent anomaly is due to the unknown optical properties of the Entech cover.

\subsubsection{Entech Covering Technology Development}

As mentioned above, the routine use of Entech prismatic covers has been initiated at SERI by the Group. This technique was established, and made available to all SERI researchers early in 1990 when two members of the 
Devices Group traveled to Entech, Inc. in Dallas. TX and completed the training required to apply the covers to solar cells. Since then, the technology has been taught to the High Efficiency Group of Branch 212, and successfully used for several different solar cell structures within the Group (see Section 4.7).

\subsubsection{Ellipsometry}

The Group now has a functional wavelength scanning ellipsometer (see Section 4.5.5). However, for daily measurements of thickness and index of refraction for the as-deposited optical materials, single wavelength elliposmetry is still the primary measurement technique. Currently, the group operates and maintains two single wavelength ellipsometers: The first is a Rudolph Research Model 200E and the second is a Gaertner Model L1 16 Automatic Production unit. Although both are theoretically capable of the same degree of precision, the Rudolph Ellipsometer, since it can be aligned and calibrated, within the group, is used for absolute (primary) measurements and only by qualified people within the Group. The Gaertner instrument is used for comparative analysis and for research by individuals outside of the Device Development Group. During the past year, this Gaertner instrument has been used by members of Branches 211,212 and 213.

\subsubsection{Solar Cell Fabrication}

The above mentioned modeling techniques and facilities would not be very useful without the laboratories and procedures necessary to transfer the modeled design to the PV junction/material and thereby create the working solar cell. For this reason, the Device Development Group has established and continues to maintain and improve the Clean Room Photolithographic Laboratory and all associated equipment and procedures necessary for photolithographic processing activities to continue within the Institute.

\subsubsection{Clean Room}

The Clean Room Photolithography Laboratory is located in Laboratory 109 of Building 16. During the past year, use of this laboratory by members of Branch 212 and 213 has increased measurably. Although this is in part due to the ever increasing quality of PV junction material produced by the researchers of Division 210, it is also indicative of the fact that more researchers are viewing quality processing of PV material as being equally important to the end product of producing high efficiency solar cells. Due to this increased use of the clean room, several modifications to it were initiated this past year. The first involves the purchase of a second convection oven, and related timing devices, for use in photoresist soft-and hard-baking procedures. This addition allows at least two separate 
photolithographic process projects to commence in the clean room simultaneously. The second involves the replacement of the original room vacuum pump (used for the chucks on the photoresist spinner and the mask aligner) with a Welch pump requiring less maintenance. Additionally, appropriate switches for this pump were installed inside the clean room such that a user no longer has to leave the room to turn the pump on. This modification should greatly decrease the "down-time" of the room since the Welch pump can run much longer without attention.

\subsubsection{Photolithography Photomask Pattern Generation and Mask Alignment}

In order to perform the photolithography process, it is necessary to have patterns which are appropriate to the specific research project (i.e., solar cell patterns vs. contact resistance measurement patterns), and are consistent with the lithography equipment being used. At SERI, a 1978 Cobilt CA2020 mask aligner is used and this employs 4" square glass photoplates. The patterns are transferred onto the glass plates via a process of "pattern generation", which is performed solely by members of the Device Development Group at the National Institute of Standards and Technology (NIST) in Boulder, Colorado. During the past year, approximately 40 photomasks have been produced by the group for members of Branches 212 and 213.

\subsubsection{Metallization}

The Group has demonstrated the ability to deposit adherent metal grid lines onto transparent conducting oxide (TCO) and semiconductor surfaces which are $5.6 \mu \mathrm{m}$ wide and $4.7 \mu \mathrm{m}$ thick. These dimensions represent significant advancements of the in-house metallization ability (previously best dimensions $\sim 7 \mu \mathrm{m}$ wide and $\sim 1.5 \mu \mathrm{m}$ thick), and make possible an entirely new range of optimized grid structures for use by SERI researchers. Since the metal thickness is substantially greater than the photoresist used to pattern the lines $(2.3 \mu \mathrm{m})$, it is believed that the problem of forming the critical stress induced microcracks at the metal-photoresist conformal cusp has been successfully addressed. Thus, it is expected that the maximum thickness of a grid line which can be formed using this technique has not yet been reached, and aspect ratios of unity may be possible (in the above case, meaning a thickness of $\sim 5.6 \mu \mathrm{m}$ ). This work represents the culmination of several months of effort to understand and improve all aspects of metal pattern definition using additive photolithographic processes. It has also involved adhesion measurement and promotion, as explained below. In addition to these efforts, extensive support measurements were performed by Ric: : Matson (SEM measurements of the photoresist profiles) and John Webb (FTIR analysis of the plasma treated photoresist). 


\subsection{Photolithographic Patterning for Metallization}

The ability to produce photolithographic photomasks with clean, uniform line widths of approximately $4.4 \mu \mathrm{m}$ has been demonstrated by the Group within the past year. This dimension represents a decrease of approximately $50 \%$ over earlier photomasks (previous minimum line width measured approximately $9.0 \mu \mathrm{m}$ ). In addition to a narrower overall dimension, the edge uniformity and definition have been greatly improved. This development, in conjunction with ongoing improvements of the in-house contact-printing photolithography processes, has enabled metal lines with highly uniform widths of 5-7 $\mu \mathrm{m}$ to be routinely fabricated using lift-off processing techniques thereby improving the resistance vs. line width dependence (previous best effort, about $20 \mu \mathrm{m}$ with relatively non-uniform edges). Although these improvements have been beneficial for all grid and test pattern applications, they are essential to future research in concentrator solar cells, for cells with high emitter sheet resistances, and for large area cells. Several photomasks have been produced using these new procedures, including an array of 0.25 $\mathrm{cm}^{2}$ cells incorporating Entech prismatic covering technology for use by the Branch 212 High Efficiency Group.

\section{PiRL and Chlorobenzene Processing}

Considerable effort has gone into the establishment of processes which will allow for the processing of thick $(>2 \mu \mathrm{m})$, narrow $(<10 \mu \mathrm{m})$ grid lines for use in solar cell top grid contacts. Past research has indicated that the only way to produce metallizations with this high aspect ratio is to use vacuum evaporated metals with photolothographic "lift-off" (additive) processing. One final use for this type of metallization would be to replace shadow mask metallization techniques, which are dependent on technologies not available at SERI (i.e., shadow mask manufacture), with in-house photolothographic techniques. Thus far, two different approaches have been attempted to increase the metallization thickness. The first involves the use of a soluble polyimide release layer which underlays the patterned photoresist. Although this technique has thus far only been tested for low temperature processing $\left(<150^{\circ} \mathrm{C}\right)$, very encouraging re-entrant profiles have been established. Additionally, this technique has the desirable feature of permitting high temperature (up to $\sim 400^{\circ} \mathrm{C}$ ) processing, possibly making it useful for in-situ patterning of the active thin-film solar cell materials used by the Thin Film Group of Branch 212.

The second method currently being investigated for use in additive processing involves the use of chlorobenzene to alter the solubility of the top layer of the photoresist, thereby forming the necessary lift-off profile. Although procedures of this sort have already been successfully incorporated at SERI (using toluene instead of chlorobenzene), the present effort is focusing on producing 
more reliable re-entrant profiles with narrower lines, thereby promoting the formation of the microcrack along the patterned photoresist cusp, resulting in the possibility of thicker metal lift-off. Specifically, the use of Shipley $\mathrm{S} 1400$ phtotoresist (with a nominal resist thickness of $2.3 \mu \mathrm{m}$ ) is being used. Results indicate that the basic re-entrant profile produced is far superior to that produced with toluene and that metallizations much thicker than the photoresist thickness can be lifted-off (thus far, $3.7 \mu \mathrm{m}$ has been successfully lifted), attesting to the critical cusp-microcrack formation.

\section{Inclusion of Effect of Relative Humidity in the SERI Clean Room}

The most recent advancements in the metallization related phtotoresist technology were facilitated when it was realized that the extremely low relative humidity of the Clean Room Laboratory (7-12\%) was a significant factor in the poor reproducibility of the photoactive chemical processes (a relative humidity range of $50 \pm 15 \% @ 21^{\circ} \mathrm{C}$ is recommended by the chemical suppliers). To compensate for this laboratory shortcoming, a step involving soaking the samples in deionized water has been added to the process, resulting in greatly improved control over photoresist development time. Also, by raising the water content (and thus the photoactivity) of the photoresist in this way, greater control of the effect of the chlorobenzene treatment (see above) has been acquired, subsequently yielding better reentrant profiles which are necessary for metal lift-off. Although the watersoaking does add an additional step to the normal process, the resultant increased process control has permitted optimization and/or elimination of other steps, resulting in an overall reduction in the processing time. Examples of this include the elimination of a 30 minute soft-bake at $90^{\circ} \mathrm{C}$ in favor of a 15 minute pre-bake at $70^{\circ} \mathrm{C}$ (decreased oven warm-up time), the elimination of a 15 minute pre-exposure toluene soak in favor of a 5 minute post-exposure chlorobenzene soak, and the complete elimination of a 30 minute post bake. These, and other modifications, allow the process to be performed completely in 25-30 minutes (compared to 1.5 - 2 hours for the previously used toluene process).

\subsection{Adhesion of Metallization}

In order to form grid lines with the very high aspect ratios mentioned above (e.g., $5.6 \mu \mathrm{m}$ wide and $3.7 \mu \mathrm{m}$ thick), it is essential that the adhesion be sufficient to overcome the stresses produced by the metal as the processing and measurements commence. For Ag contacts on ITO, it has been found that the use of $\mathrm{Cr}$ or $\mathrm{Ti}$ as an adhesion promoting layer is essential in order to produce line widths of $50-20 \mu \mathrm{m}$. However, it was found that if the line width decreased below $\sim 20 \mu \mathrm{m}$, adhesion loss was again a problem. At about the same time (mid-1990), a project was begun by the Group (funded from an external contract from Spire Corporation) to determine what steps can be 
taken to improve the adhesion of $\mathrm{Cr}$ onto GaInAs. Although this external funding was suspended, because Spire did not receive the funding which had been expected from NRL due to federal budget cuts, some measurements were performed and some results obtained. The following is a summary of the initial results and additional results from the longer-term research on adhesion which is ongoing in the Group.

\section{Effect of Deposition Rate on Stress of Ag Deposits}

After initial meetings with Spire Corporation, it was noted that the contacts produced at Spire were highly stressed. Indeed, the stress was so great that the contact would actually "bend" the InP substrate such that, using a reflection laser measurement system, the chord length could be measured and thus a quantitative value for the stress could be determined. Since most of the metal in the Spire contact stack was $\mathrm{Ag}(5 \mu \mathrm{m}$ of $\mathrm{Ag}$ on top of $80 \mathrm{~nm}$ of $\mathrm{Cr}$ and $\mathrm{Au}$ ), it was believed that the adhesion failure was probably due to the compressive stress built up in the Ag during or after its deposition. Thus, the SERI effort centered on determining if a deposition procedure could be developed to reduce deposition-induced stress. The project was completely successful. By incrcasing the deposition rate of the Ag from $\sim 1-2 \mathrm{~nm} / \mathrm{s}$ (Spire parameter) to $\sim 5 \mathrm{~nm} / \mathrm{s}$, the stress in the Ag was apparently reduced. This reduction was noted by observing the form in which the Ag lifted-off after deposition (highly stressed deposits lift-off in tight curls, unstressed deposits lift off flat). Additionally, this stress reduction was achieved without the added complication of substrate cooling, as was being used at Spire.

\section{Effect of AR Plasma on Cr Adhesion to PV Substrates and TCOs}

It was determined that the adhesion of $\mathrm{Cr}$ to ITO (InP and Si have also shown adhesion improvement) is greatly promoted if the ITO surface is gently cleaned with an Ar plasma before Cr deposition. A Technics Planaretch II system, operating at $30 \mathrm{kHz}$, was used for this purpose. When this procedure was added, it was noted that the adhesion was improved to the point that line widths of $\sim 7 \mu \mathrm{m}$ (and $1.5 \mu \mathrm{m}$ thick) were sufficiently adherent to withstand both an adhesive tape test and scratching with a steel point (to simulate the effect that a measurement probe may have on a solar cell). Since that time, grid lines $\sim 5 \mu \mathrm{m}$ wide and $\sim 4 \mu \mathrm{m}$ thick have been fabricated, and the adhesion promotion provided by the Ar plasma appears to be sufficient for this geometry too.

\section{FTIR Analysis: Effect of Ar Plasma on Photoresist-Coated Substrates}

After determining that the Ar plasma greatiy promotes adhesion of $\mathrm{Cr}$ to semiconductor substrates, an investigation was begun to determine the fundamental mechanism. Since Branch 213 (John Webb) recently acquired 
an FTIR spectrometer equipped with a small spot analysis microscope attachment, a project was initiated to see if FTIR analysis could measure changes in the photoresist-coated substrates as a function of Ar plasma treatment. Initial results of this analysis have suggested a surprising result that. after sequential Ar plasma "cieaning", the substrate surface (that surface which is not coated with photoresist) becomes progressively more covered with a carboxylate species, thereby implying that this species somehow aids $\mathrm{Cr}$ adhesion. The appearance of carboxylate is indeed possible, since the photoresist is composed of similar materials, and the implication that the Ar plasma is, at least to some degree, decomposing the photoresist is certainly plausible. However, the reason why $\mathrm{Cr}$ adhesion is promoted through this process is still uncertain. Using this conclusion as a cautionary guide, the Group has recently completed an in-depth study using the SEM capablities of Branch 213 (Rick Matson) to determine if the Ar plasma damages the re-entrant profile necessary for reliable lift-off. Through this study, it was observed that the Ar plasma does damage the photoresist, thereby supporting the FTIR analysis. Also, the SEM analysis. and subsequent processing experiments indicated that, if the exposure time and plasma power are minimized, (currently $30 \mathrm{sec}$. and 100 Watts, respectively), the damage will not cause problems in metal lift off but is still sufficient to promote $\mathrm{Cr}$ adhesion to the substrate.

\subsection{Specific Contact Resistance $\left(r_{c}\right)$ of Metallization}

\section{Modeling Studies}

Contact resistance research performed during the year included modeling studies which were intended to determine the upper tolerable limit of contact resistance for grid metal on one-sun and concentrator solar cells. The results indicated that for one-sun cells, and line widths greater than $\sim 20 \mu \mathrm{m}$, $\mathrm{r}_{\mathrm{c}}$ must be less than approximately $1 \times 10^{-2} \Omega-\mathrm{cm}^{2}$. However, for narrower line widths $(<\sim 20 \mu \mathrm{m})$, this value of $r$ will cause the contact resistance to be the dominant loss mechanism. A substantial reduction in the loss could be achieved if $r_{r}$ could be reduced to $1 \times 10^{-4} \Omega-\mathrm{cm}^{2}$. Similar calculations for a model concentrator cell (operated at 1000 suns) indicate that unlike the case of the one-sun design, an $\mathrm{r}$ of even $1 \times 10^{-4} \Omega-\mathrm{cm}^{2}$ will still have a significant (although not dominant) effect on the power loss. For this case. $r_{r}$ values approaching those usually required in VLSI would be advantageous. Also. it was shown that even a slight non-uniformity or instability in $r_{r}$ (as might be caused by progressive adhesion loss or interfacial oxide growth) would have a considerable effect not only on the efficiency but also on the maximum concentration ratio achievable before the onset of series resistance effects. 


\section{Values for Contact Resistance Used in Grid Designs}

One of the services which the Group has developed over the years is to provide other researchers at SERI with grid designs and completed photomasks for their solar cell structures. For these designs to be correct, it is essential that the relevant design parameters for PV material (and processes) be known. This is especially true for $r_{c}$. Although in the past, grids have been designed for other SERI researchers by employing "ascribed" values of $r_{r}$ (the end-user often ascribes a very low value for $r_{r}$, which is probably unlikely unless state-of-the-art metallization processes are used), and these have appeared to perform adequately. However, this fortuitous situation probably will not continue if grid cicsigns relying on the more advanced metallization and photolithographic techniques (recently developed by the Group and discussed above) are requested. Since some of these materials are not normally produced within the Group (e.g., GaInP and $\mathrm{CuInSe}_{2}$ ), values for $\mathrm{r}_{r}$ and its dependence on processing parameters are not known. Additionally, although $r_{r}$ measurements can be made by the Group, these cannot be performed until material is made available.

\subsection{Diffusion of Metallization}

A project was begun to study the diffusion of $\mathrm{Cr}$ and $\mathrm{Au}$ metallizations into $\mathrm{Ga}_{0,47} \mathrm{In}_{0,5.5}$ As and $\mathrm{InP}(\mathrm{Cr}$ is used as an adhesion promotion layer, $\mathrm{Au}$ is an electrolytically plated material used by SERI researchers to provide a high conductvity layer). Although the project was initiated by external funding (received from Spire Corporation), it has continued since the development of temperature-stable metallizations is quickly becoming a critical issue for the advanced concentrator work being done at SERI. The two-fold purpose of this study was to investigate the interfacial layers which might form between the metal and the semiconductor (e.g., $\mathrm{CrO}_{\mathrm{x}}$ ) and which are thought to form and promote metal adhesion, and also to determine the extent of diffusion of these metals at elevated temperatures. The diffusion characteristics were investigated both from the standpoint of metals (of a contact stack) into the semiconductor and metals into each other. Thus far in the project, extensive use has been made of the Auger spectroscopy equipment in Branch 213 (Amy Franz). Preliminary results of this analysis indicates that, even during a room temperature deposition, both $\mathrm{Cr}$ and Au diffuse into Ir $\mathrm{P}$ much deeper than was expected.

In order to establish the processing techniques and procedures necessary to produce the results modeled above, it was necessary to establish and/or improve several measurement facilities and procedures essential for PV solar cell processing. 


\subsubsection{Solution Growth of CdS}

Solution grown CdS thin films are now routinely used in the thin film PV industry to fabricate solar cells. Films grown by this method appear to meet the criteria for high efficiency devices: $30-50 \mathrm{~nm}$ in thickness and the ability to provide adequate coverage of the rough CuInSe ${ }_{2}$ films. Thin CdS films have been grown from aqueous solution of $\mathrm{CdCl}_{2}$, thiourea, $\mathrm{NH}_{4} \mathrm{OH}$ and $\mathrm{NH}_{4} \mathrm{Cl}$. Initially, the optical properties of the films grown on glass and quartz

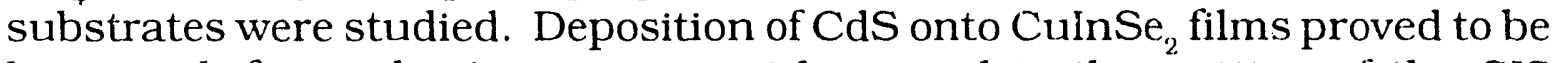
less strightforward. Attention must be payed to the wetting of the CIS surface, the thickness of the deposit and coverage, all of which affect device quality. Work was done to determine the concentrations, temperature, and the duration of the deposition to optimize the CdS properties. Presently, 50to $80-\mathrm{nm}$ films are routinely grown on CIS films. The optical transmission of the films is $80-90 \%$ below the absorption edge. Adequate transmission above the edge is obtained due to the thinness.

\subsubsection{Device Fabrication Procedure}

CdS films were deposited on a few CuInGaSe, ${ }_{2}$ (CIGS) films provided by Boeing High Technology Center. These had been deposited by elemental coevaporation onto Mo coated alumina substrates. After the CdS deposition, the samples were heated in air at $200^{\circ} \mathrm{C}$ for 15 minutes This step is a part of the standard heat treatment procedure required for improving cell performance. After heat treatment, the transparent conducting oxide layer is deposited. A film of dc magnetron sputtered indium tin oxide (ITO) has been used for this purpose. The ITO is deposited in two stages; the first 50 $\mathrm{nm}$ is sputtered in a high partial pressure of oxygen to yield a highly transparent and highly resistivite layer $(10 \Omega-\mathrm{cm})$. The second layer is sputtered at a pre-determined oxygen pressure to yield a conducting film. The high resistivity ITO acts as a blocking layer and minimizes shunting between the conducting ITO and the regions of CIS films not covered by CdS. Although Boeing developed this approach for $\mathrm{ZnO}$, it appears that a serious evaluation of ITO for this purpose has not been made. After sputtering ITO, device fabrication is completed by standard photolithographic procedures. The top contact is provided by electrolpating Au. The devices have a total area of $0.25 \mathrm{~cm}^{2}$ and the grid coverage is $5 \%$.

\subsubsection{Initial results}

The light I-V data of device \#1220, fabricated using the above process, indicate that the CdS thickness is much greater than is optimum. The CdS was grown in two stages; after the first deposition, the sample was removed, washed and the step was repeated using a fresh bath. The CdS was deliberately made thick to minimize shunt paths. The I-V data indicates 
that the process works well. Table 4.1 shows a comparison of the values from device \#1220 and those reported by Boeing on their best device (\#1174H). The open circuit voltage and the fill-factor of \#1220 are close to those of \# 1174, although improvements in $\mathrm{V}_{\mathrm{or}}$ must be made. The current density of \#1220 is much lower, primarily due to the thicker CdS. This limitation is also observed in the external quantum efficiency. Collection is not observed below the absorption edge of CdS and there is considerable room for improvement. Experiments are under way to fabricate devices using thin CdS layers; to evaluate the optical and electrical properties of ITO and to determine the optimum thickness of the various layers by studying the reflectance of the ITO/CdS/CuInGaSe ${ }_{2}$ stack at each stage of processing. Optical measurements on $\mathrm{ZnO}$ thin films show that there is a significant absorption loss, perhaps higher than that found in ITO films. It seems possible that ITO films of the required sheet resistance could be produced at much lower thickness than is the case for $\mathrm{ZnO}$. Investigation of the optical properties of ITO and $\mathrm{ZnO}$ films and the compatibility of each to CIS device technology will be continued during the coming year.

Table 4.1: $\quad$ Parameters of CIS cells with solution grown window layers of CdS.

\begin{tabular}{lll}
\hline \multicolumn{1}{c}{ Parameter } & BAC $1174 \mathrm{H}^{*}$ & BAC $1220^{* *}$ \\
\hline Voc (V) & 0.555 & 0.516 \\
Jsc (mA.cm ${ }^{-2}$ ) & 34.2 & 26.42 \\
Fill-factor (\%) & 65.7 & 61.17 \\
Efficiency (\%) & 12.5 & 8.3 \\
\hline
\end{tabular}

* SERI data, $0.987 \mathrm{~cm}^{2}$ total area, AM1.5 Global, $1000 \mathrm{~mW} . \mathrm{cm}^{-2}$

** SERI data, $0.25 \mathrm{~cm}^{2}$ total area, AM 1.5 Global, $1000 \mathrm{~mW} . \mathrm{cm}^{-2}$

\subsection{EQUIPMENT/ANALYSIS DEVELOPMENT}

\subsubsection{Sputter Deposition Systems}

\subsubsection{ULTEK System Modification}

During the year, this system was configured to enable deposition from two US Gun targets (previously, only a single US gun was available). This modification involved the production of new internal pyrex shielding and the design and manufacture of high-voltage switching equipment so that the power to the two guns could be easily, and safely, switched by the operator. In this configuration, different types of the same TCO material (currently ITO) can be deposited without breaking vacuum, and without undue crosscontamination of the target material. Specifically, one ITO target is sputtered in an $\mathrm{O}_{2}$-rich environment, while the other is sputtered in a $\mathrm{H}_{2}$-rich 
environment. This is a desirable feature for the fabrication of the ITO/InP cells discussed later.

\subsubsection{Vacuum Evaporation Systems}

\subsubsection{NRC 3115 System}

The design and development of this system was completed in mid-1990, and is currently used daily for routine deposition of optical coating materials (see Section 4.4.2). The availability of this system has greatly improved the quality of ARCs used by the Group and increased the frequency which ARCs are deposited on the Group's solar cells. It must also be noted that this machine was developed with minimum cost to the Institute (approximately $\$ 5000$ ) since major parts of it were procured from DOE excess sources.

\subsubsection{Metallization System}

A project has been commenced to design and develop an E-beam vacuum system for use in performing routine metallization of solar cell grids. The grids made with this machine can then take advantage of the Group's recent advances in photolithographic technology by enabling deposition of refractory metals to promote adhesion (initially $\mathrm{Cr}, \mathrm{Ti}$ and $\mathrm{Ni}$ ), diffusion barrier metals (Pd and Pt), and thick films of conduction metals (mostly Ag) for use in stateof-the-art one-sun and concentrator grids. The individual components for this system are presently being procured through a variety of sources including Branch excess, DOE excess, and used equipment vendors. The parts thus far procured are: (1) E-Beam power supply, DOE excess, (cost new $\sim \$ 50 \mathrm{~K}) ;$ (2) Mechanical pump, DOE excess, new cost $\sim \$ 4 \mathrm{~K}$; and (3) multichannel deposition controller, DOE excess, new cost $\sim \$ 10 \mathrm{~K}$. It is hoped that the remaining parts (vacuum system sized for the equivalent of a 24" metal bell jar, and a multipocket E-Beam source hearth) will be procured during the next year.

\subsubsection{Plasma Exposure Systems}

\subsubsection{Technics Planaretch II System}

This system has been used extensively during the past year. The first use of the system is related to the study of plasma-induced type conversion which occurs on the surface of InP. This research has been critical to our understanding of the junction formation process which occurs in the ITO/ InP cell, and especially to the separation of the roles of $\mathrm{H}_{2}, \mathrm{Ar}, \mathrm{O}_{2}$ and plasma damage. The use of this system for this research will be ongoing, and may possibly be extended to other material systems. 
The second use of this system has been for routine Ar plasma cleaning of substrates prior to metallization. Although this is a new application for this system, it has been an extremely important aspect of the recent progress in both the metallization studies and the photolighographic process development project (see Section4.4.3.2). Indeed, much of the progress in the metallization area would not have been possible without this system.

\subsubsection{Technics Model $500 \mathrm{II}$}

Recently, a project has been started in which this system is used to modify the surface of single-crystal CuInSe ${ }_{2}$. After surface modification, the CIS is then analyzed using the scanning ellipsometer facility.

\subsubsection{Spectrophotometry}

\subsubsection{Measurement using the Cary 2300 spectrophotometer}

Work on the data acquisition and control of the Cary 2300 spectrophotometer using a Macintosh Ilx computer was initiated at the end of 1989. The system was operational from the beginning of 1990. Data acquisition can be carried out in two modes: (1) the continuous mode in which the spectrophotometer scans the wavelength range in a conventional manner and the computer collects the data at specified intervals; and (2) the discrete mode in which the computer receives a specified number of data points at each desired wavelength and stores the averaged reading. Operation in the discrete mode results in data with reduced noise but is more time consuming. The data are corrected for zero and $100 \%$ baseline deviations before being stored in a file. In addition, for reflectance measurements using the integrating sphere, the data are normalized using the calibrated NBS standard. Data acquisition and control software have been further modified to allow the automatic activation of the system at a specified time. It is possible to switch on the system early in the morning so that it has warmed-up for 1-2 hours before it is used at the commencement of the working day. This is a great advantage since a warm-up period of this length is required to establish equipment stability.

Routines for data conversion to a format suitable for the HP-1000, used for optical analysis, have been developed. With the new facility, it is possible to calculate real and imaginary parts of the refractive index of transmissive films over a wavelength range of 260-1790 nm. The calculated results for

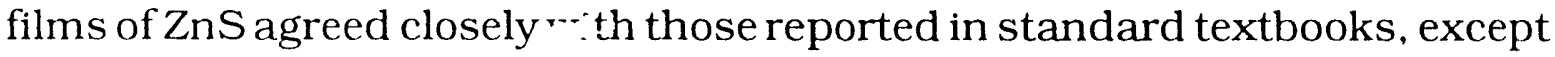
at the long and short wavelength extremes. Sensitivity analysis has been performed to assess the effects of uncertainties in the values of the reflectance $(R)$ and transmittance $(T)$. 
Furthermore, the fabrication and commissioning of equipment for the measurement of optical transmission at high temperatures has now been completed. A custom designed strip heater with provision for sample mounting and a port for transmission of the optical beam has been fabricated and necessary hardware for water cooling of the sample chamber was installed on the spectrophotometer. A simple system of temperature control has been used to assure minimal temperature variation $\left(\sim 5^{\circ} \mathrm{C}\right)$ during the measurement. It is now possible to perform these measurements from room temperature to $325^{\circ} \mathrm{C}$. This facility will be useful to obtain the optical properties of the phases of materials present only at high temperatures.

\subsubsection{Nitrogen purging}

Facilities for nitrogen purging of the spectrophotometer have also been installed and commissioned. Purging is essential to minimize the influence of water vapor absorption bands in the near infra-red region. It is also necessary to prevent oxidation of the samples during high temperature measurements. Using the new facility, optical measurements on vanadium oxide samples have been made. This material is expected to change phase at approximately $260^{\circ} \mathrm{C}$ and it is therefore an ideal vehicle with which to test the equipment.

\subsubsection{Photoemission measurements}

Equipment for the measurement of photoemission is now almost complete. Using the Cary 2300 as a spectrometer, the photocurrent generated by the sample is measured using a lock-in-amplifier. The reference signal is derived from the Cary control electronics. Results obtained show that the measurements could be performed up to $1700 \mathrm{~nm}$ without excessive noise problems. Accurate calibration of the light intensity will be performed using $\mathrm{Si}$ and Ge photodetectors. Some well characterized contact systems, such as GaAs and InP Schottky barriers will be used to establish whether the technique gives reasonable results for the barrier heights. This system will be used to study various metal contacts to CuInSe ${ }_{2}$ and CdTe, and the effects of fabrication and processing parameters on the contact properties.

\subsubsection{Software development}

Software for the calculation of the reflectance (R) and transmittance (T) of single or multi-layer stacks has been developed. For this purpose special Pascal routines for the matrix manipulation of complex numbers were developed. Closed form solutions of $R$ and $T$ for a stack with more than three. layers are very difficult to compute, therefore the matrix method, which uses the interface and the layer matrices for calculation of $\mathrm{R}$ and $\mathrm{T}$ and the light intensity at any point in the stack, was employed. This method can be used 
to process an arbitrary number of layers of known optical constants and thicknesses. This software has been used as the basis for the development of the design of antireflection coatings. For AR coating design, stack optimization incorporates the quantum efficiency of the particular device and the relevant solar spectrum to maximize the short circuit current of the device, instead of only minimizing the optical loss due to reflectance. The output for the AR design routine can be represented as a contour plot to assess the sensitivity of the coating to the thickness of each component layer.

\subsubsection{Spectroscopic Ellipsometry}

\subsubsection{Design Features of the Ellipscmeter}

The improvements of the unique broad band (300-1700 nm) spectroscopic ellipsometer (SE), developed and constructed during the FY 1989, has been completed and the instrument has been used to measure the optical characteristics of various materials. Among these are the polycrystalline thin film materials, CuInSe ${ }_{2}$ and CdTe, (for which single crystal samples have also been investigated), and materials for high efficiency cascade solar cells including InP, GaInAs and GaInAsP. Most of these data are not presently available over such a wide spectral range. The availability of commercial spectroscopic ellipsometers (SE) has been restricted to the UVvisible range from $250-900 \mathrm{~nm}$. The instrument consists of a stepper moto: controlled, rotating polarizer, fixed analyzer setup with an ac detection system developed for accurate measurement of $\psi$ and $\Delta$, the relevant ellipsometric parameters, in the near infrared. The analytical methods include the use of a specially developed computer modeling program which gives $\psi$ and $\Delta$ for a given set of values related to the film thickness (which may be finite or zero) and to the optical properties of the substrate.

The design is based on a stepper-motor-controlled polarizer, fixed analyzer ellipsometer and this configuration exhibits several advantages over rotating analyzer systems (Fig. 4.1). In particular, the position of the monochromator reduces sensitivity to room light, the system is mechanically stable and the computer interface makes efficient use of readily available high-speed A/D $\mathrm{PC}$ boards. It operates in an ac mode using a light chopper to improve the signal-to-noise ratio in the near infra-red (NIR). The light from a 100 watt tungsten halogen lamp is polarized by a rotating prism after first being collimated. After reflection from the sample surface (and the associated changes in amplitude and phase), the polarized beam is directed to the fixed analyzer, and then to the monochromator. The monochromator uses three separate gratings (blazed at $250 \mathrm{~nm}, 600 \mathrm{~nm}$ and $1000 \mathrm{~nm}$ ) to maintain high throughput over the entire wavelength operating range. 


\section{Broadband light source.}

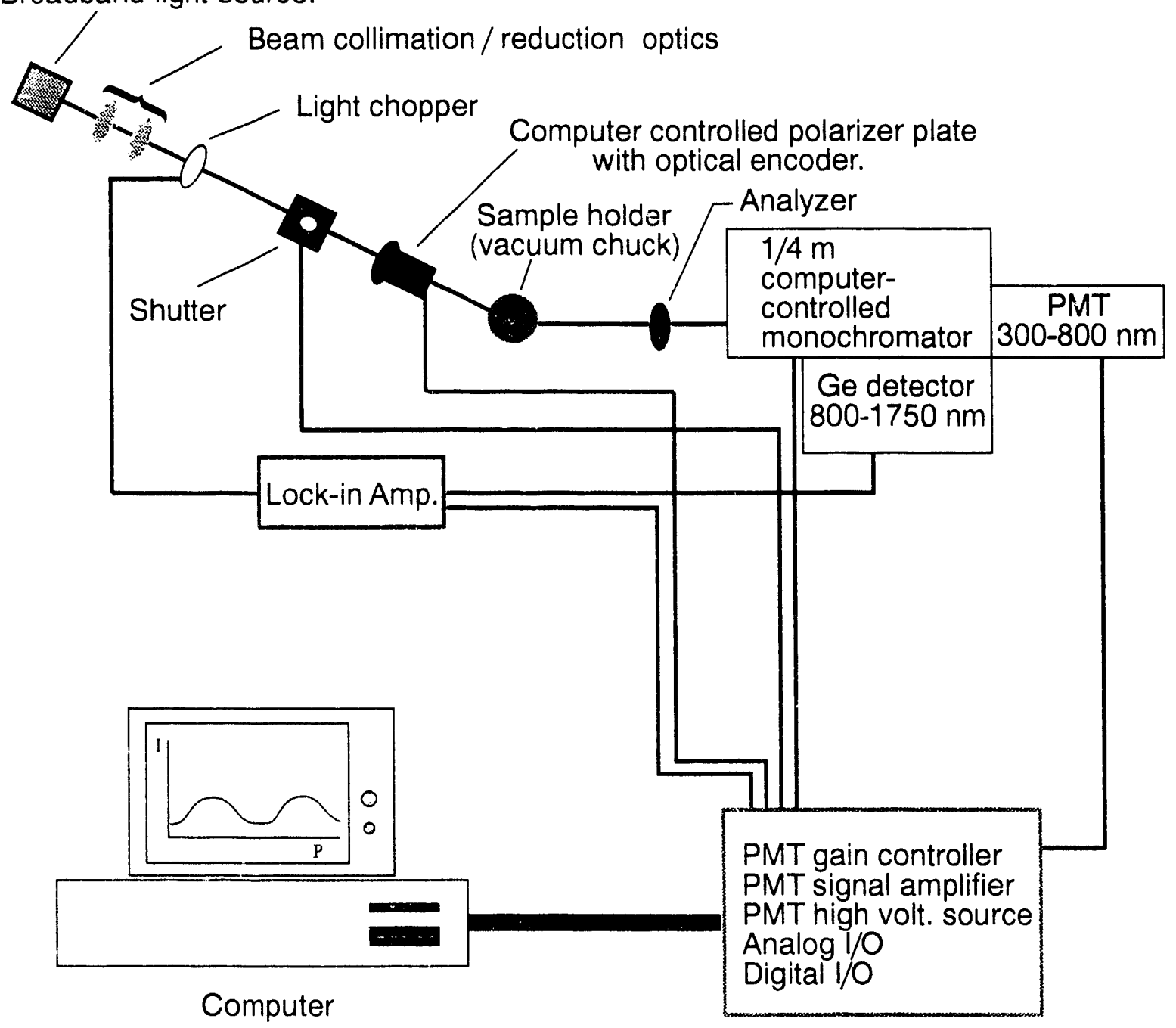

Electronic interface box

Figure 4.1: Schematic diagram of the spectroscopic ellipsometer 
The polarizer assembly consists of a hollow shaft carrying the analyzer prism mounted in a single-race. sealed ball-bearing shaft which, in turn, is mounted in the analyzer/motor housing assembly. The rotating prism assembly is driven with a small, flexible chain by a high resolution stepper motor (12800 steps/revolution). This allows precise positioning of the polarizer plate via an RS232 interface for integrated measurements. An optical encoder provides an 'absolute' position reference (i.e., one that will not drift) for the polarizer positioning system. In its present configuration, the system is able to collect and average 24,000 data points at each wavelength, perform a Fourier analysis on the resulting intensity curves, and present real-time plots in less than 20 seconds. The Fourier coefficients are then used to calculate the standard ellipsometric parameters ( $\psi$ and $\Delta$ ). Commercially available 12-bit analog-to-digital ( $A / D)$ converters are used to interface the detection systems to the computer. The Turbo Pascal programming language is used to achieve $10 \mathrm{kHz}$ sampling rates for highspeed data averaging. The PMT gain is optimized at each wavelength so that the full input range $(0-10 \mathrm{~V})$ of the computer $A / D$ converters is utilized. This minimizes digitization effects of low-voitage signals.

The light source is chopped in the NIR, and the germanium detector signal is routed through a lock-in amplifier before input to the computer. Communications with the lock-in are implemented with a standard IEEE488 interface. Remote control of the sensitivity of the lock-in amplifier allows automatic signal size (again, near loV peak) optimization in the infrared.

Real-time graphics have also been developed to display current system settings, intensity and background curves, and the resultant Fourier fit. Separate graphs are continuously updated to display all of the relevant parameters $(\psi, \Delta, \mathrm{a}, \mathrm{b}, \mathrm{c}, \mathrm{etc}$. ) as a function of wavelength. A larger variance in the Fourier fit, or non-optimal gain settings, trigger an automatic repeat of the measurement at any wavelength.

\subsubsection{Applications of the ellipsometer $[2,3,4,5,6]$}

\section{Optical Constants Of Semiconductors}

In order to undertake adequate modelling, it is necessary to have thorough knowledge of the optical constants of the various materials involved in the solar cells to be studied. Typically, the properties of these materials depend upon the details of their fabrication, and whether they are in thin film form or are single crystals. Whether the test sample is transparent or in bulk form, knowledge of the optical constants of the material is important to an understanding of the fundiamental material behavior and the optimization of device design. The measured optical constants, $\mathrm{n}$ and $\mathrm{k}$, are then used to calculate the absorption coefficient. 
Along with CuInSe ${ }_{2}$ and CdTe, the optical properties of $\mathrm{Ga}_{0.47} \operatorname{In}_{0.53}$ As have been measured in the $350-1650 \mathrm{~nm}$ wavelength range and are shown in Fig. 4.2. The results arc in excellent agreement with previous data published by Aspnes. The optical constants of the quaternary semiconductor compound $\mathrm{Ga}_{0.25} \mathrm{In}_{0.75} \mathrm{As}_{0.54} \mathrm{P}_{0.46}$ are shown in Fig. 4.3. This composition has a bandgap of $0.95 \mathrm{eV}$ and it is lattice matched to InP. This is the first time that these data have been obtained over such a wide spectral range and in the visible, they agree well with the data of Aspnes. The $\mathrm{N}$ and $\mathrm{k}$ values have been used to model the reflectance, and this is found to agree with the measured reflectance to within $2.5 \%$, as illustrated in Fig. 4.4. This work was performed so that some knowledge could be obtained about the materials properties and to assist the development of optimized ARCs. As seen below, this has been particularly valuable for the optimization of performance of the tandem cells which have achieved efficiencies greater than $30 \%$.

\section{Modeling of short circuit current density}

One of the advantages of these measurements is that they can be used for the optimization of AR coatings and in the modelling of the quantity of light

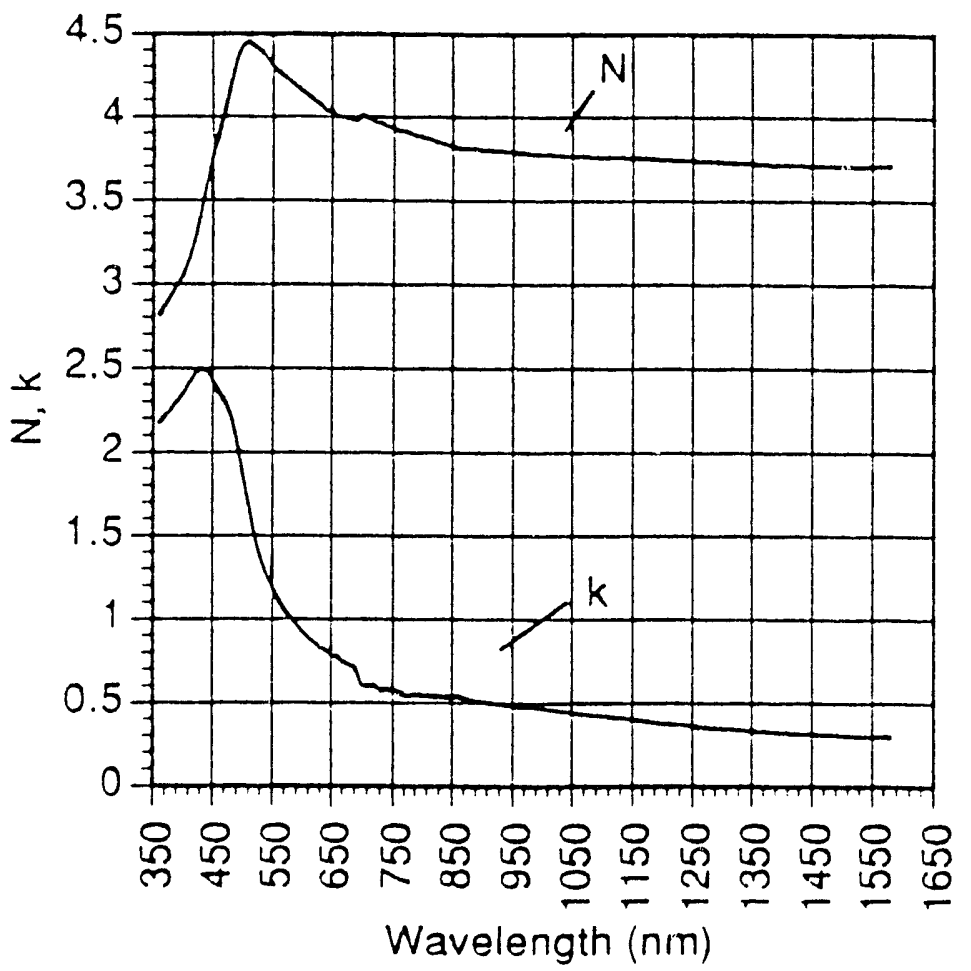

Figure 4.2: The optical properties of $\mathrm{Ga}_{t, 47} \mathrm{In}_{t, 5,3} \mathrm{As}$ lattice matched to InP 


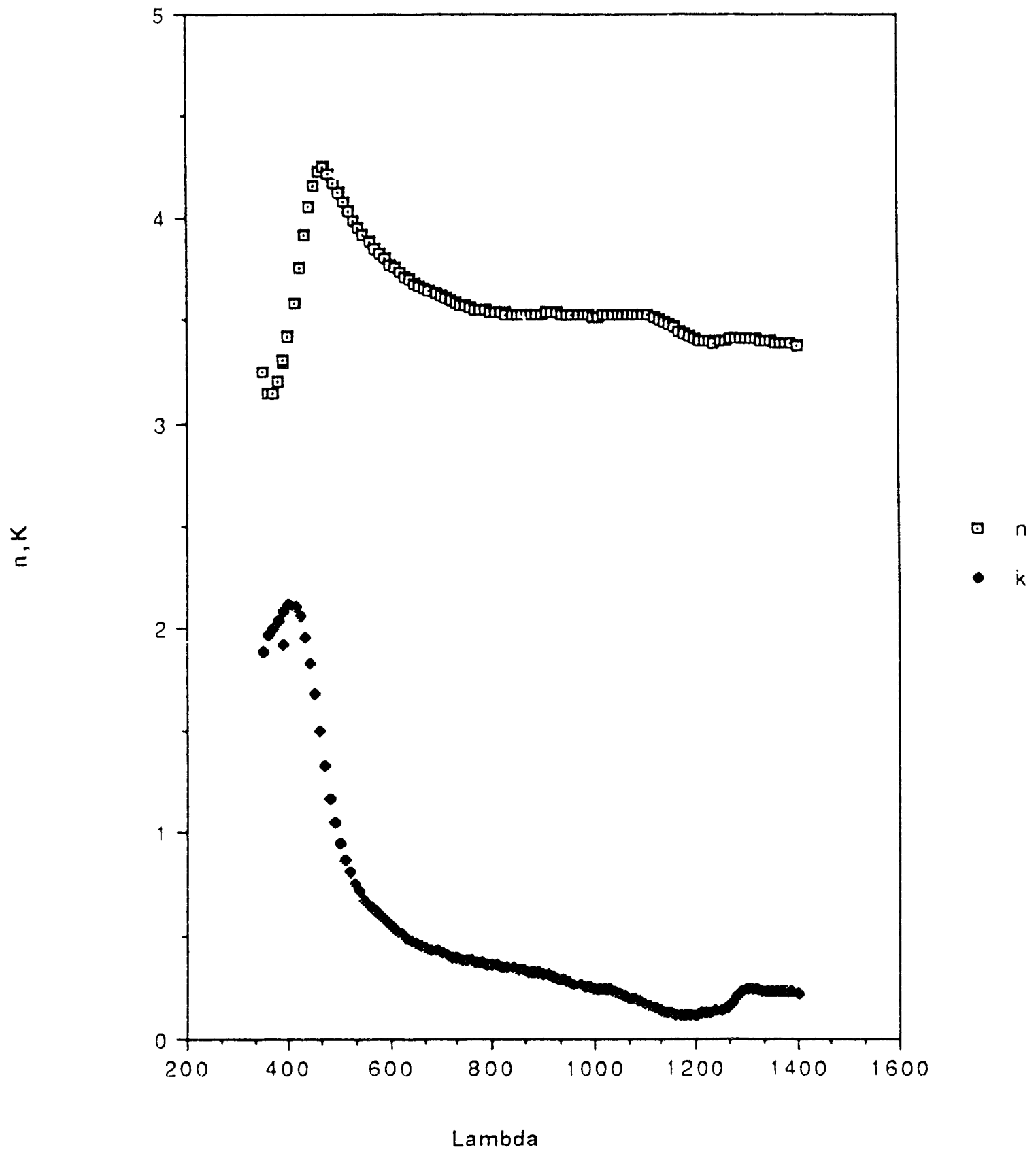

Figure 4.3: The optical constants of the quaternary compound $\mathrm{Ga}_{11.25} \operatorname{In}_{(1) 7,7} \mathrm{As}_{(1,54,4} \mathrm{P}_{1,46 ;}$ 


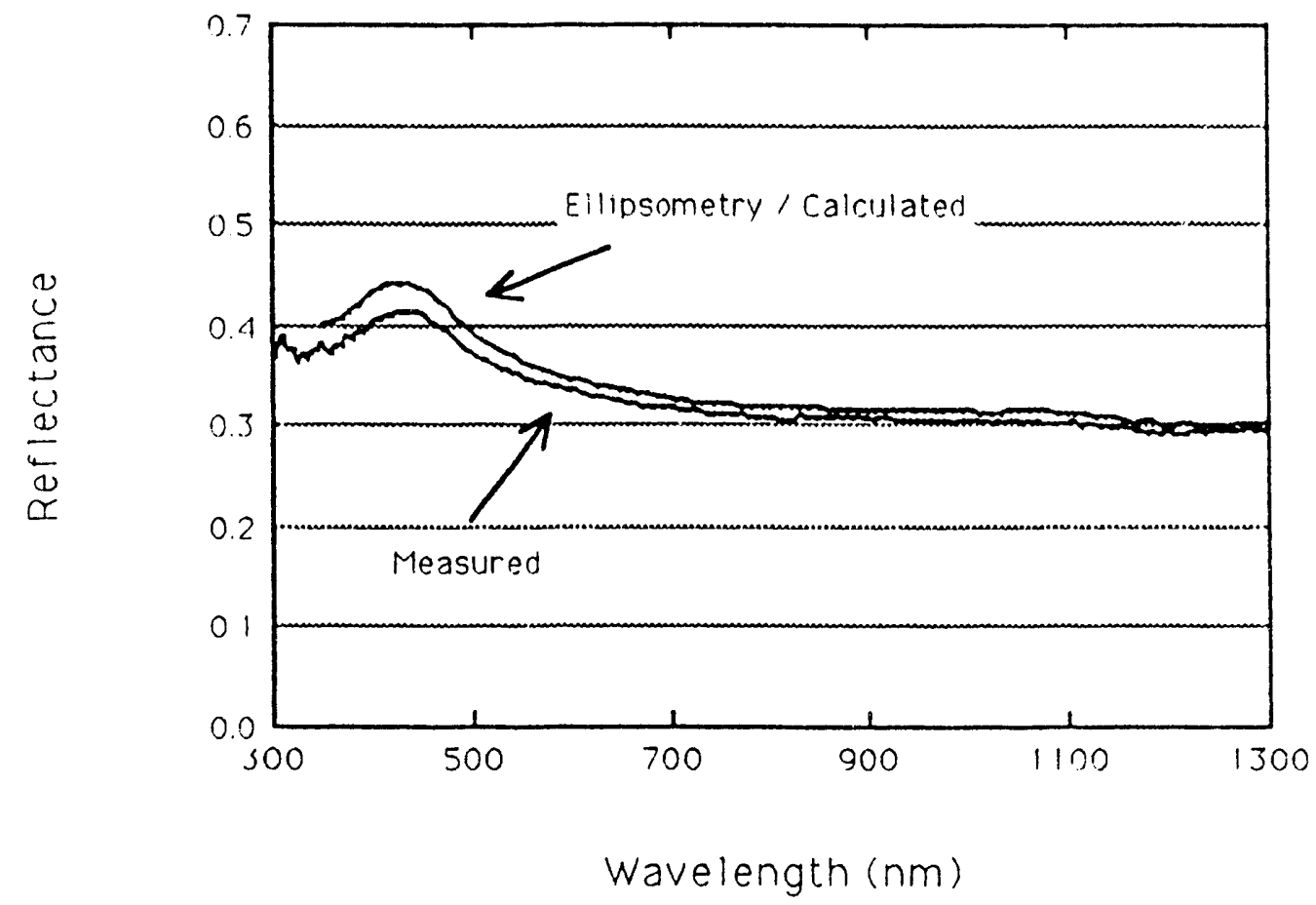

Figure 4.8: The modeled and measured reflectances $\mathrm{Ga}_{11.25} \mathrm{In}_{0.75} \mathrm{As}_{0.54} \mathrm{P}_{0.46}$ agree to within $2.5 \%$.

actually being transmitted into the absorber of solar cells, in order to maximize the output current $J_{s c}$. This depends upon the incident spectrum, external quantum efficiency, reflectance, and the thicknesses and optical constants of all materials in the cell. The current relation is given by:

$J_{s c}=e \int_{0}^{m} \frac{I(\lambda) \times Q E_{e x t}(\lambda)}{(1-R(\lambda))}\left(1-R^{\prime}(\lambda)\right) d \lambda$

where:

$\mathrm{J}_{\mathrm{cr}}=$ short circuit current (Amp $\left.\mathrm{cm}^{-2}\right)$

$\mathrm{I}(\lambda)=$ incident spectrum (photons $\mathrm{nm}^{-1} \mathrm{~cm}^{-2} \mathrm{sec}^{-1}$ )

$\mathrm{l}=$ wavelength $(\mathrm{nm})$

$\mathrm{e}=$ electronic charge (Amp sec)

$\mathrm{QE}(\lambda)=$ external quantum efficiency (electrons/photon)

$R(\lambda)=$ cell reflectance before ARC application (dimensionless)

$R^{\prime}(\lambda)=$ calculated cell reflectance for specific ARC geometry

Two-layer ARCs are often used on solar cells. $\mathrm{J}_{\mathrm{sr}}$ is a function of the thickness of both materials and its' variation must be presented in a contour graph format. In this, the y-axis represents the thickness of the coating with the larger index (i.e., the $\mathrm{ZnS}$ ) and the $\mathrm{x}$-axis indicates the thickness of the lower index material (i.e., the $\mathrm{MgF}_{2}$ ). The data are represented in the form of curves 
of equal $\mathrm{J}_{\text {si. }}$, allowing the maximum current and the thicknesses of the two materials to easily be determined. The $\mathrm{N}$ and $\mathrm{k}$ data for each layer in the structure are used in the calculation of $R^{\prime}(\lambda)$. A $2 \times 2$ complex matrix method has been implemented which calculates reflectance from an n-layer optical stack. given $\mathrm{N}$ and $\mathrm{k}$ of every layer, as a function of the angle of incidence and incident light polarization state. In this work, normal incidence is assumed.

Figure 4.5 shows $J_{\mathrm{sic}}$ contour plots for a GaAs homojunction with a doublelayer $\mathrm{MgF}_{2} / \mathrm{ZnS}$ ARC. A nucleating layer of $\mathrm{MgF}_{2}, 2 \mathrm{~nm}$ in thickness was used and this has been included in the model; this layer is often deposited to enhance growth characteristics of $\mathrm{ZnS}$ on III-V compounds. A similar calculation for an ITO/InP cell reveals that the ITO acts as one of the ARCs, while $\mathrm{MgF}_{2}$ is used as the top ARC. This particular application requires several approximations, since the ITO is an integral (conducting) part of the solar cell. It is assumed that the internal quantum efficiency is independent of ITO thickness (an assumption which will certainly fail for significantly thinner ITO). The results are, however, valid for a broad range of ITO thicknesses centered on $60 \mathrm{~nm}$, since the external quantum efficiency and reflectance data $(R(\lambda))$ were obtained from such a device.
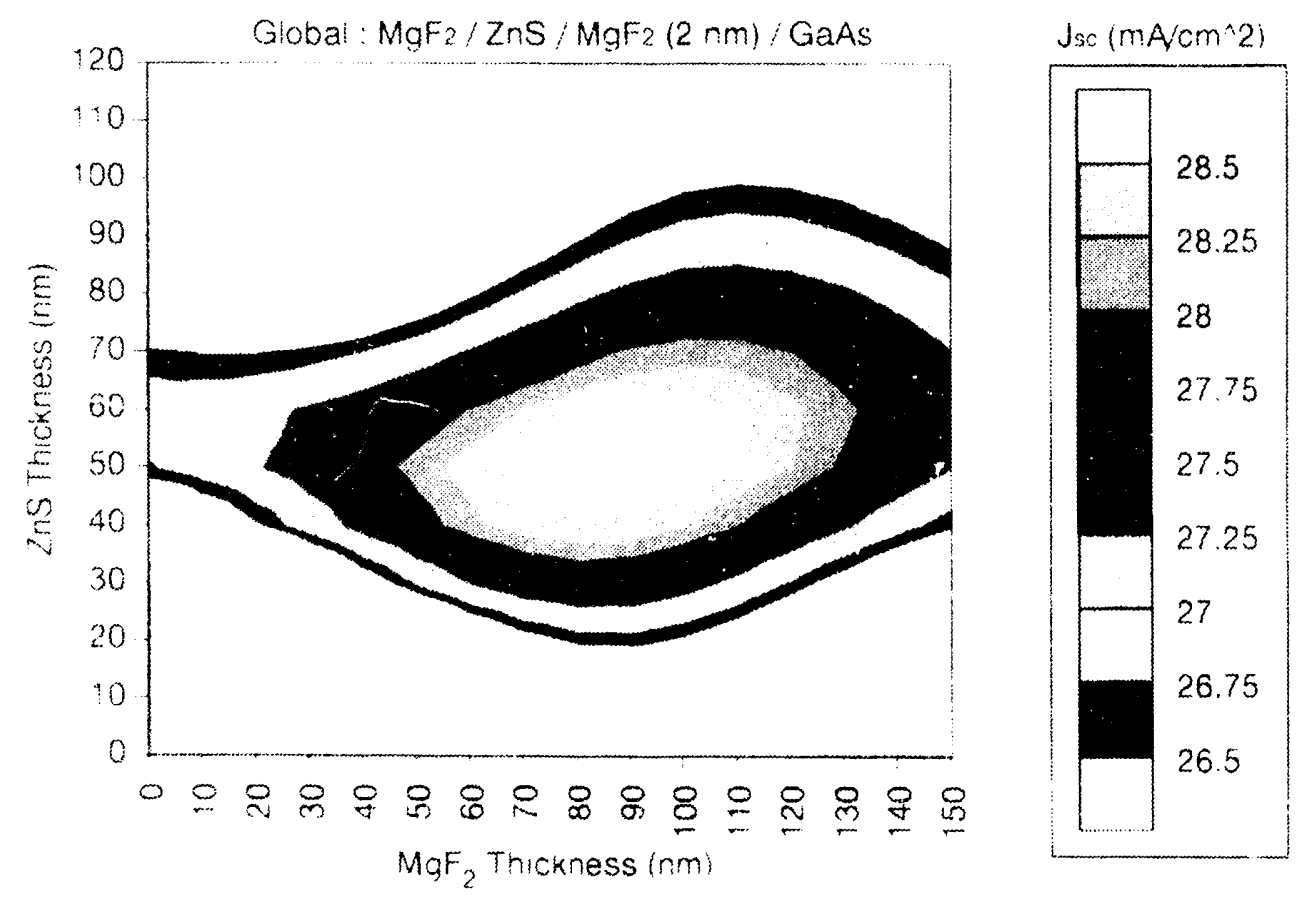

Figure 4.5: $J_{s,}$ contour plots for GaAs homojunction with a double layer $\mathrm{MgF}_{2} / \mathrm{ZnS} \mathrm{ARC}$. 
Recently. Entech prismatic covers have been used to enhance solar cell performance by diverting light from above grid fingers to active areas of the device. The geometric concentration effects have been studied in some detail. but the change in optimal ARC coating thicknesses due to application of such a cover has not been investigated. For concentrator measurements. the incident spectrum is normally incident upon the prismatic cover. The curvature of the cover determines the angle of incidence at the front surface of the cell. The extreme values of angle of incidence may reach $30^{\circ}-60^{\circ}$ although most of the light is much clos $r$ to normal incidence. The materials which are used in Entech plastic covers are quite thick (on the order of 100 $\mu \mathrm{m})$ and non-planar. As important optical property, then, is that these materials do not interfere constructively or destructively. To avoid repetitive integrations over incident angle. the cover has been treated as a noncoherent material with an index of refraction $\mathrm{N}=1.4, \mathrm{k}=0$, under normally incident light.

The effect of a non-coherent cover for the GaAs homojunction is shown in Fig. 4.6. Note that the optimum ARC thicknesses have shifted significantly from their original values. The current will rise substantially due to the elimination of shadow losses (an effect which is not included in these
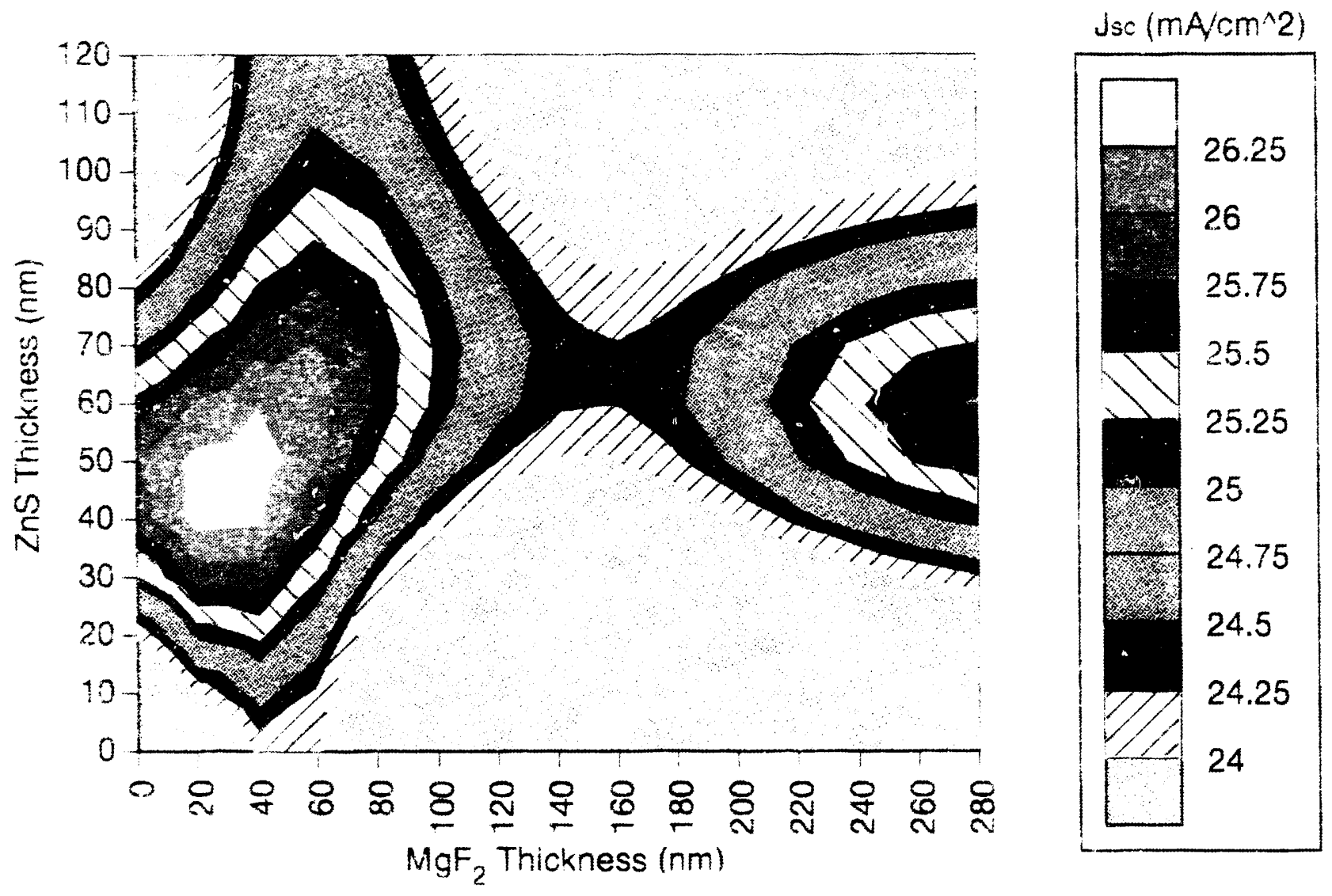

Figure 4.6: The effert of a noncoherent cover on the $J$ comour plots for the GaAs inomingrion 
graphs). but these results indicate that performance may be increased even further with the use of properly designed antireflection coatings.

\subsection{ACCOMPLISHMENTS: PHOTOVOLTAICS}

\subsubsection{Low-bandgap, InP-based solar cells}

\subsubsection{Introduction [97]}

We have continued to perform research on high-efficiency solar cell concepts in support of the DOE program and other externally funded PV programs. Our modeling studies have shown that concentrator tandem cells require infrared-sensitive bottom cell components for high performance. Hence, the objective of this work has been to develop high-performance. low-bandgap solar cells suitable for bottom cell applications in tandem cells designed for terrestrial and space applications. Such cells scavenge power from the nearinfrared portion of solar spectra that is otherwise wasted by conventional photovoltaic materials with higher bandgaps (e.g., GaAs, etc.). The $\mathrm{Ga}_{x} \mathrm{In}_{1-x} \mathrm{As}_{\mathrm{y}} \mathrm{P}_{1-\mathrm{y}}$ alloy system lattice matched to InP substrates has been chosen for this work because the available bandgap can be adjusted over the range which is optimum for such bottom cells. As described below, computer modeling of potential bottom cell performance under a GaAs optical filter has led to the identification of two alloy compositions of particular interest; $\mathrm{Ga}_{0.47} \mathrm{In}_{0.53}$ As $(0.75 \mathrm{eV})$ for space applications and $\mathrm{Ga}_{0.25} \mathrm{In}_{0.75} \mathrm{As}_{0.54} \mathrm{P}_{0.46}(0.95 \mathrm{eV})$ for terrestrial use. Solar cells with state-of theart performance levels have been successfully fabricated in each of these alloys. Additionally, these low band gap cells have been integrated into specially designed high efficiency tandem solar cells. The progress made with $\mathrm{Ga}_{0.25} \mathrm{In}_{0.75} \mathrm{As}_{0.54} \mathrm{P}_{0.46}$ cells is discussed in this section. The work on $\mathrm{Ga}_{0.47} \mathrm{In}_{(0.553}$ As cells is presented in the work-for-others section under NRL.

In recent computer modeling studies of two-junction, concentrator tandem solar cells, we have shown that IR-sensitive bottom cells are required in order to achieve maximum performance levels ${ }^{1}$. As an illustration of this conclusion, the results from a typical modeling calculation are shown in Fig. 4.7. In this figure. the iso-efficiency contours for a two-junction, independently connected tandem cell operated under the direct spectrum at 100 suns, $25^{\circ} \mathrm{C}$ are given as a function of the top and bottom cell bandgaps. Also shown in the diagram are the iso-bandgap lines for single-crystal, direct-bandgap semiconductors presently under consideration for top and bottom cell applications. These include AlGaAs (1.93 eV), GaInP (1.90 eV), GaAs (1.425 $\mathrm{eV})$ and $\operatorname{InP}(1.35 \mathrm{eV})$ for the top cells and GaSb $(0.72 \mathrm{eV})$. GaInAs $(0.75 \mathrm{eV})$, GaInAsP $(0.95 \mathrm{eV})$ and GaAs $(1.425 \mathrm{eV})$ for the bottom cells. The top cell/ bottom cell bandgap coordinates, for typical combinations of these materials, have been identified to illustrate the potential tandem cell efficiencies. 


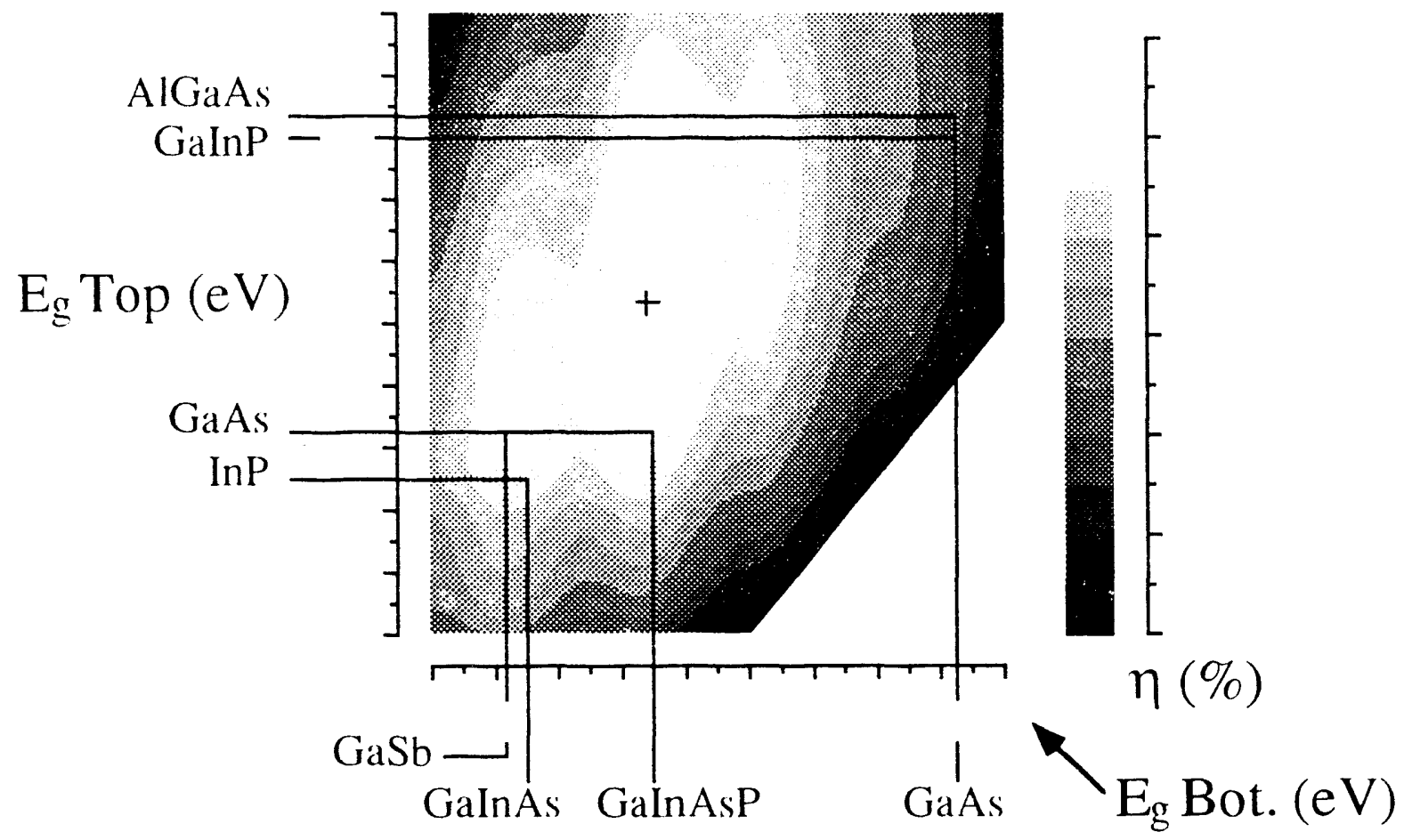

Figure 4.7: Computer modeled iso-efficiency contours as a function of the top and bottom cell bandgaps for an independently connected. two-junction tandem solar cell under the Direct spectrum. 100 suns, $25^{\circ} \mathrm{C}$. Also shown are the iso-bandgap lines for single-crystal, direct-bandgap semiconductors considered useful for tandem cell applications.

In the past, tandem cells, based on GaAs bottom cells, have been the subject of intense research, since lattice matched compositions of high-bandgap alloys such as AlGaAs and GaInP can be readily grown on GaAs substrates. thereby forming monolithic, high/medium-bandgap tandem cell structures ${ }^{2}$. The convenience and elegance of the GaAs-based tandem designs cannot be disputed. however, it is clear from the data shown in Fig. 4.7 that GaAsbased tandems are inappropriate for concentrator applications since the maximum efficiency obtainable with such designs is less than $84 \%$ of the maximum modeled efficiency for two-junction tandems. The relatively low efficiency potential of GaAs-based tandems is easily understood; GaAs is transparent to $53 \%$ of the photovoltaically useful photons (i.e.. photons with energies greater than $0.65 \mathrm{eV}$ ) in the direct spectrum, resulting in a large conversion efficiency loss.

In contrast, from Fig. 4.7 it is evident that concentrator tandems, employing IR-sensitive bottom cells with bandgaps in the $0.7-1.0 \mathrm{eV}$ range, are capable of efficiencies which are $95-100 \%$ of the maximum theoretical efficiency. An additional advantage of tandems using low-bandgap bottom cells. is that a wide range of top cell bandgaps (1.3-2.0 eV) become useful for high-efficiency 
operation, resulting in considerable flexibility in choosing high-efficiency top/bottom cell material combinations. In particular. medium/low-bandgap tandem pairs using simple. technologically mature binary top cell materials, such as GaAs and InP have theoretical efficiencies which are very close to the maximum attainable value, thereby making them extremely attractive candidates for practical applications. In a previous modeling study ${ }^{1}$, it was shown that the above conclusions are largely unaffected at higher operating temperatures $\left(80^{\circ} \mathrm{C}\right)$, since the normalized efficiency temperature coefficients for low-bandgap cells improve substantially at high solar concentration ratios. Therefore, medium/low-bandgap tandem pairs appear to be the preferred choice for concentrator applications.

At the Boeing High Technology Center, recent research into developing GaAs/GaSb mechanically stacked. concentrator tandem cells has resulted in the highest photovoltaic efficiency yet reported ${ }^{3}$. These results corroborate the modeling predictions discussed above. In the work reviewed here, we have extended the medium/low-bandgap, 2-junction tandem concept to two novel, practically relevant materials combinations which have high theoretical efficiencies. These consist of the following: (1) A mechanically stacked, 4terminal GaAs/ $\mathrm{Ga}_{x} \operatorname{In}_{1-x} \mathrm{As}_{y} \mathrm{P}_{1-y}$ tandem utilizing the optimum bandgap of $0.95 \mathrm{eV}$ for the quaternary bottom cell. The composition of the quaternary is approximately Ga $\mathrm{Ga}_{0.25} \operatorname{In}_{0.75} \mathrm{As}_{0.54} \mathrm{P}_{0.46}$. (2) A monolithic, lattice-matched, 3terminal InP/GaxIn 1-xAs tandem. In this case, the bottom cell has a bandgap of $0.75 \mathrm{eV}$ and the composition is $\mathrm{Ga}_{0.47} \mathrm{In}_{0.53} \mathrm{As}$. Both of these designs have theoretical terrestrial efficiencies of $>40 \%$ at concentration ratios of 100 or more. The progress made in developing $\mathrm{Ga}_{0.25} \mathrm{In}_{0.75} \mathrm{As}_{0.54} \mathrm{P}_{0.46}$ single-junction cells and $\mathrm{GaAs} / \mathrm{Ga}_{0.25} \mathrm{In}_{0.75} \mathrm{As}_{0.54} \mathrm{P}_{0.46}$ mechanically stacked tandem cells is reviewed in section 4.6.1.2.

All of the epitaxial device structures described in this report were grown by atmospheric-pressure metalorganic vapor phase epitaxy (APMOVPE) using a home-built system. The system employs a patented reactor vessel design ${ }^{4}$ which yields highly uniform epilayers in all respects, a feature which is particularly important for devices involving ternary and quaternary compounds. Trimethylindium, trimethylgallium, phosphine and arsine were used as the primary reactants and hydrogen sulfide, hydrogen selenide, and diethylzinc were used as doping sources. The carrier gas employed was palladium-purified hydrogen and the growth temperature ranged from $600^{\circ} \mathrm{C}$ to $700^{\circ} \mathrm{C}$. Additional details regarding the epitaxial growth and device processing procedures for the various materials and devices can be found in previous publications ${ }^{5}$. We put a considerable effort into developing the capabilities of the APMOVPE reactor early in the year. In particular, the reactor vessel was scaled up to accomodate a full 2"-diameter wafer and sources were acquired for the growth of lattice-matched $\mathrm{Ga}_{x} \operatorname{In}_{1-x} \mathrm{As}_{y} \mathrm{P}_{1-y}$ layers on InP substrates. Growth techniques were developed for highly 
uniform $\mathrm{Ga}_{x} \operatorname{In}_{1-x} \mathrm{As}_{\mathrm{y}} \mathrm{P}_{1-y}$ layers with excellent optoelectronic properties. $\mathrm{Ga}_{x} \mathrm{In}_{1-x} \mathrm{As}_{\mathrm{y}} \mathrm{P}_{1-y}$ devices with superior characteristics were realized as a result of these efforts.

A system for measuring the efficiency of concentrator solar cells was designed and built in collaboration with the Cell Characterization Group. This system proved to be invaluable in our studies of concentrator tandem cells. The tandem cell efficiency versus solar concentration ratio (C) measurements were performed using the data acquisition system described elsewhere ${ }^{f}$ along with an unfiltered, $1000 \mathrm{~W}$ Xenon-arc light source. Using the short-circuit current $\left(\mathrm{I}_{\mathrm{sc}}\right)$ measured at one-sun intensity under the direct spectrum (ASTM E891-87, $1000 \mathrm{~W} \mathrm{~m}^{-2}$ total irradiance, $25^{\circ} \mathrm{C}$ ), and assuming a linear relationship between the $\mathrm{I}_{\mathrm{sc}}$ measured under concentration and $\mathrm{C}$, the current-voltage characteristics were measured and the efficiency was calculated based on the value of $\mathrm{C}$ determined. The total estimated uncertainty in the efficiency is $\pm 2 \%$ for the medium-bandgap ( $\geq 1 \mathrm{eV}$ ) cells, and $\pm 8 \%$ for the low-bandgap $(\leq 1 \mathrm{eV})$ cells. In order to keep the tandem cell junctions at the standard reference temperature $\left(25^{\circ} \mathrm{C}\right)$ during the currentvoltage measurements, the following procedure was used: (1) Initially, the cell was maintained, at the reference temperature on a thermoelectrically controlled vacuum plate, in the dark, behind a high-speed shutter $(\sim 2 \mathrm{~ms}$ opening time). (2) A high-speed voltmeter was then used to sample (at $\sim 1000$ readings per second) the open-circuit voltage $\left(\mathrm{V}_{\mathrm{or}}\right)$ of the cell as the cell was illuminated using the shutter. The highest measured Voc was taken as the $\mathrm{V}_{\text {or }}$ under concentration at $25^{\circ} \mathrm{C}$. (3) With the cell under continuous illumination, the vacuum plate was then cooled until the $\mathrm{V}_{\text {oc }}$ obtained in step 2 above was reached. The current-voltage data at $25^{\circ} \mathrm{C}$ were then taken. A temperature difference of $\sim 10^{\circ} \mathrm{C}$ between the vacuum plate and the cell junction is typical at a concentration of $\sim 50$ suns.

\subsubsection{Ga $\operatorname{In}_{1-x} A s_{y} P_{1-y} / \operatorname{InP}$ Solar Cells}

A variety of $\mathrm{Ga}_{x} \operatorname{In}_{1-x} \mathrm{As}_{y} \mathrm{P}_{1-y}$ compositions lattice matched to InP have been grown and processed into working cells. However, we have focussed our efforts on concentrator cells in $\mathrm{Ga}_{0.25} \mathrm{In}_{0.75} \mathrm{As}_{0.54} \mathrm{P}_{0.46}$ because it provides the optimum bandgap for bottom cells in terrestrial concentrator tandems. $\mathrm{Ga}_{0.25} \mathrm{In}_{0.75} \mathrm{As}_{0.54} \mathrm{P}_{0.46}$ solar cells have been grown, processed and tested at SERI. Additionally, in a collaborative effort with Varian Associates, cell structures, grown at SERI, have been processed at Varian in a configuration suitable for eventual stacking under high-efficiency GaAs-based cells fabricated at Varian. In this section, the performance of $\mathrm{Ga}_{0.25} \mathrm{In}_{0.75} \mathrm{As}_{0.54} \mathrm{P}_{0.46}$ cells and GaAs $/ \mathrm{Ga}_{0.25} \mathrm{In}_{0.75} \mathrm{As}_{0.54} \mathrm{P}_{0.46}$ mechanically stacked tandems developed at SERI is presented. Additional results obtained at Varian Associates with SERI-grown $\mathrm{Ga}_{0.25} \mathrm{In}_{0.75} \mathrm{As}_{0.54} \mathrm{P}_{0.46}$ cell structures are discussed in the workfor-others section. 
A schematic diagram of the GaAs/ Ga $\mathrm{G}_{0.25} \mathrm{In}_{0.75} \mathrm{As}_{0.54} \mathrm{P}_{0.46}$ mechanically stacked tandem concept is given in Fig. 4.8. The Ga ${ }_{0.25} \mathrm{In}_{0.75} \mathrm{As}_{0.54} \mathrm{P}_{0.46}$ bottom cell is grown lattice matched on an InP substrate and uses InP as a window layer to passivate the emitter surface. An n/p doping configuration has been used to minimize the emitter/window sheet resistance, and emitter grid contact resistance. Positioned on the bottom cell surface is an Entech prismatic cover to eliminate optical losses due to grid obscuration. As shown in the diagram, the quaternary bottom cells have been tested under IR-transparent GaAs filters and also under actual GaAs concentrator cells grown on IRtransparent GaAs substrates. In both cases, the GaAs-based top structure is mirror smooth on the front and back surfaces with appropriate antireflection coatings (ARCs) on each of the surfaces.

Efficiency versus concentration (C) data for a high-efficiency $\mathrm{Ga}_{0.25} \mathrm{In}_{0.75} \mathrm{As}_{0.54} \mathrm{P}_{0.46}$ concentrator cell under a GaAs filter are shown in Fig. 4.9. The efficiency data show the expected increase as $\mathrm{C}$ is increased initially (as compared to the modeled performance data) and then exhibit a broad plateau at about $9.4 \%$ for $\mathrm{C}$ in the 20 -130 range. The fill factor $(\mathrm{FF})$ data show that the cell becomes series-resistance limited at about 30 suns, thus
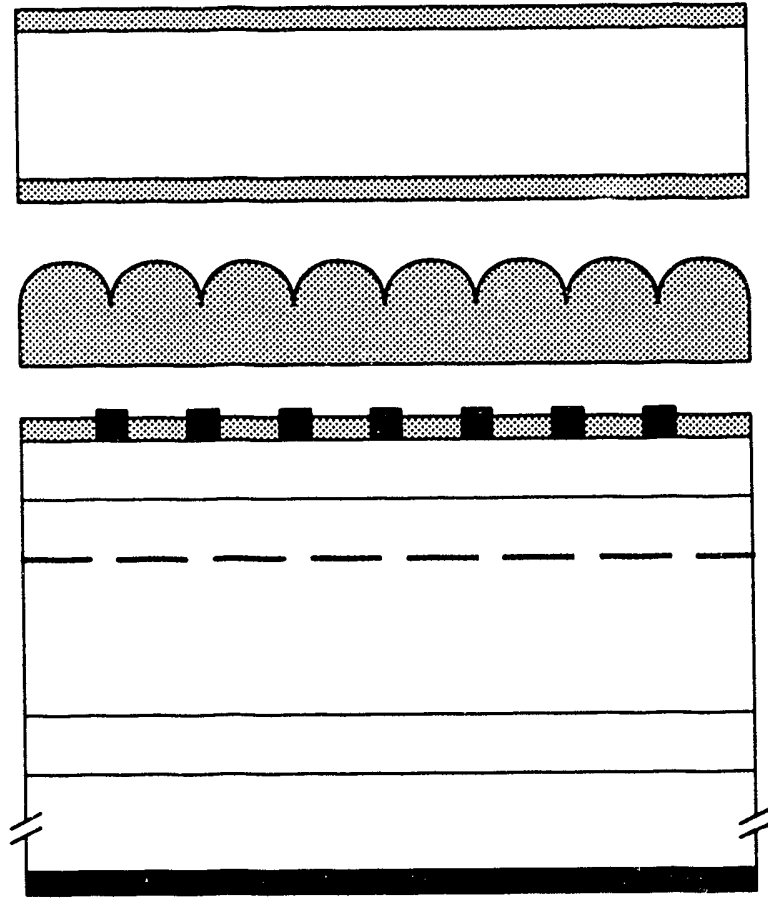

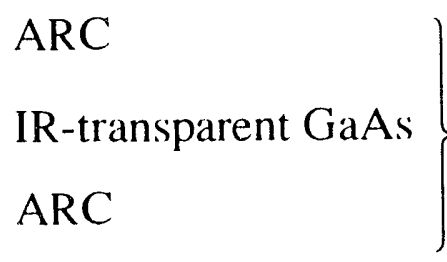

Entech cover

$\mathrm{ARC}$ and grid $0.2-1.5 \mu \mathrm{m} \mathrm{n}^{+}-\mathrm{InP}$ window

$0.2 \mu \mathrm{m} \mathrm{n}{ }^{+}$-GalnA.sP emitter

$4 \mu \mathrm{m}$ p-GaInAsP base

$0.4 \mu \mathrm{m}$ p-InP buffer

$\mathrm{p}^{+}$-InP substrate

Back contact

Figure 4.8: Schematic diagram of the GaAs/Ga $\mathrm{Ga}_{4,2:} \operatorname{In}_{0,7 !} \mathrm{As}_{1,54} \mathrm{P}_{1,46}(0.95 \mathrm{eV})$ mechanically stacked tandem cell concept. Details of the $\mathrm{Ga}_{0,2.5} \mathrm{In}_{0,75} \mathrm{As}_{10.54} \mathrm{P}_{10.45}$ bottom cell construction are shown. The $\mathrm{Ga}_{0,25} \mathrm{In}_{0,75} \mathrm{As}_{0,54} \mathrm{P}_{0.44}$ cells have been tested under a GaAs filter and also in actual tandem cell stacks. 
prohibiting further efficiency gains for higher values of $\mathrm{C}$ and resulting in the broad efficiency plateau. It is clear that GaAs-filtered $\mathrm{Ga}_{0.25} \operatorname{In}_{0.75} \mathrm{As}_{0.54} \mathrm{P}_{0.46}$ cell efficiencies exceeding $10 \%$ at $\mathrm{C} \geq 100$ could be achieved through a reduction in the cell series resistance $\left(R_{s}\right)$. Furthermore, the modeled cell performance data suggest that the efficiency could improve by 1-2 percentage points at low values of $C$ even with the present value of $R_{s}$. An analysis of internal quantum efficiency and absolute external quantum efficiency data for these cells (not given here) shows that the majority of the discrepancy between the modeled and measured efficiency data is due to external optical losses. Therefore, improved optical coupling techniques should lead to higher efficiencies. Nevertheless, the present efficiency boost offered by the $\mathrm{Ga}_{0.25} \mathrm{In}_{0.75} \mathrm{As}_{0.54} \mathrm{P}_{0.46}$ cells is substantial and immediately useful in tandem stacks.

The illuminated current-voltage data for a $\mathrm{Ga}_{0.25} \operatorname{In}_{0.75} \mathrm{As}_{0.54} \mathrm{P}_{0.46}$ concentrator cell at peak efficiency under a GaAs filter is shown in Fig. 4.10. The cell has

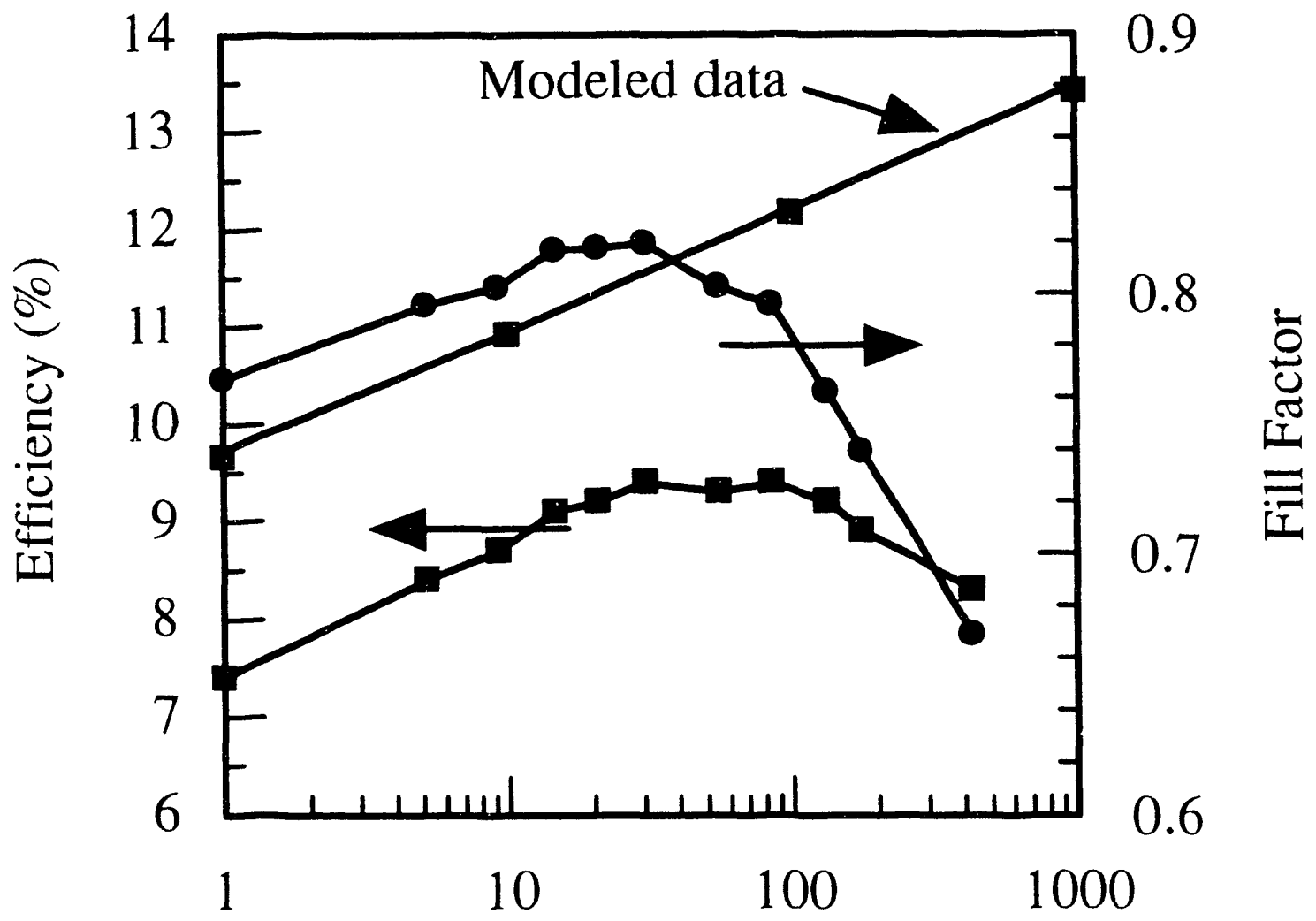

\section{Concentration Ratio}

Figure 4.9: Efficiency versus Concentration Ratio data for a high-efficiency $\mathrm{Ga}_{0.25} \mathrm{In}_{0.75} \mathrm{As}_{0.54} \mathrm{P}_{0.46}$ concentrator cell under an IR-transparent, AR-coated GaAs filter. Also shown are the fill factor data for the same cell along with the modeled efficiency as a function of the concentration ratio. 
an efficiency of $9.4 \%$ at 30.6 suns under the direct spectrum and $25^{\circ} \mathrm{C}$. The high values of $\mathrm{V}_{o c}$ and $\mathrm{FF}(0.658 \mathrm{~V}$ and $82 \%$, respectively) are particularly noteworthy for this low-bandgap cell.

We have been successful in a preliminary attempt to fabricate actual GaAs / $\mathrm{Ga}_{0.25} \mathrm{In}_{0.75} \mathrm{As}_{0.54} \mathrm{P}_{0.46}$ mechanically stacked tandem cells. The cells have been tested under concentration using an aperture to define the illuminated cell area. Efficiency versus $\mathrm{C}$ data for our best stacked tandem are shown in Fig. 4.11. The performance of the $\mathrm{Ga}_{0.25} \mathrm{In}_{0.75} \mathrm{As}_{0.54} \mathrm{P}_{0.46}$ bottom cell in the stack was hampered somewhat by the use of the aperture since only about one third of the total area of the bottom cell was illuminated during the measurement process. Likewise, the quality of the GaAs concentrator top cells, which were used in the stack, are far from state-of-the-art. Despite the obvious deficiencies in the stacked device, the tandem efficiency still exceeded $30 \%$ for $\mathrm{C}$ values ranging from 30 to 100 . A top cell/bottom cell current-voltage data composite for the GaAs $/ \mathrm{Ga}_{0.25} \operatorname{In}_{0.75} \mathrm{As}_{0.54} \mathrm{P}_{0.46}$ tandem at peak efficiency is given in Fig. 4.12. At 39.5 suns, the top cell is $23.1 \%$ efficient and the bottom cell has an efficiency of $7.1 \%$, yielding a tandem efficiency of $30.2 \%$. With improvements in the top cell quality, stacking procedure and optical coupling into the bottom cell, we feel that concentrator tandem efficiencies approaching $40 \%$ may be achieved in the future.

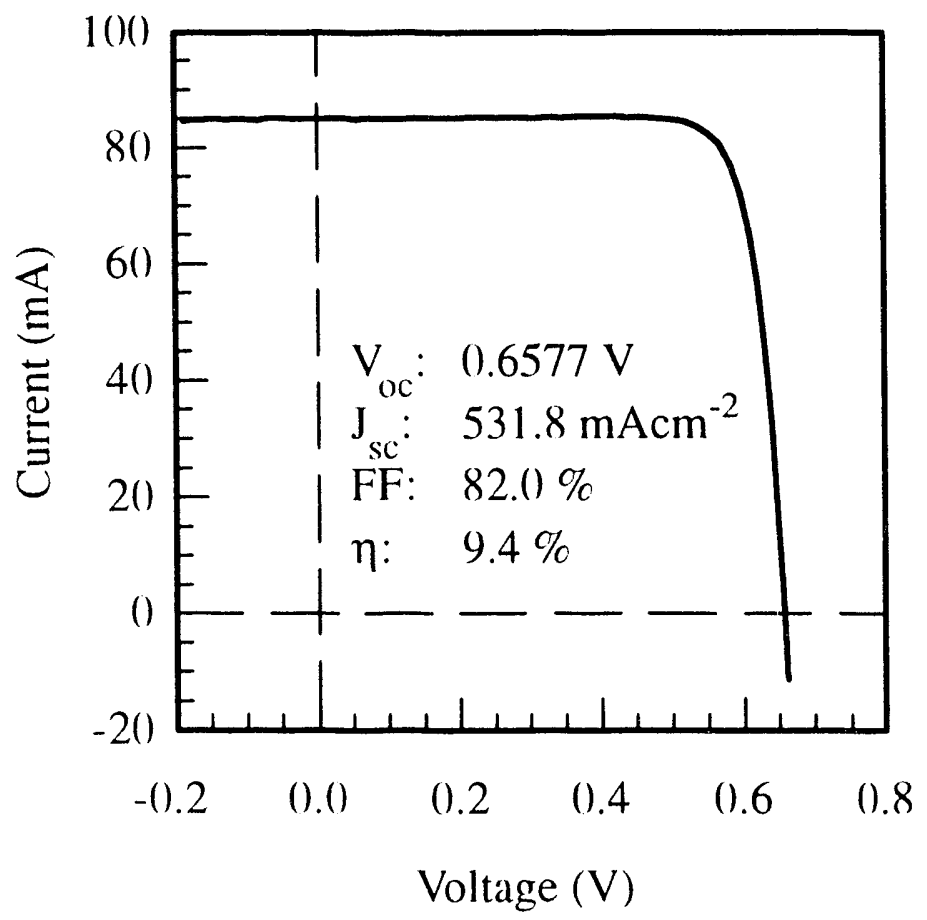

Figure 4.10: Current-Voltage data for a Ga ${ }_{1,25} \mathrm{In}_{0.75} \mathrm{As}_{0.54} \mathrm{P}_{0.46}$ cell at peak efficiency under concentration (30.6 suns, Direct, $25^{\circ} \mathrm{C}$ ). 


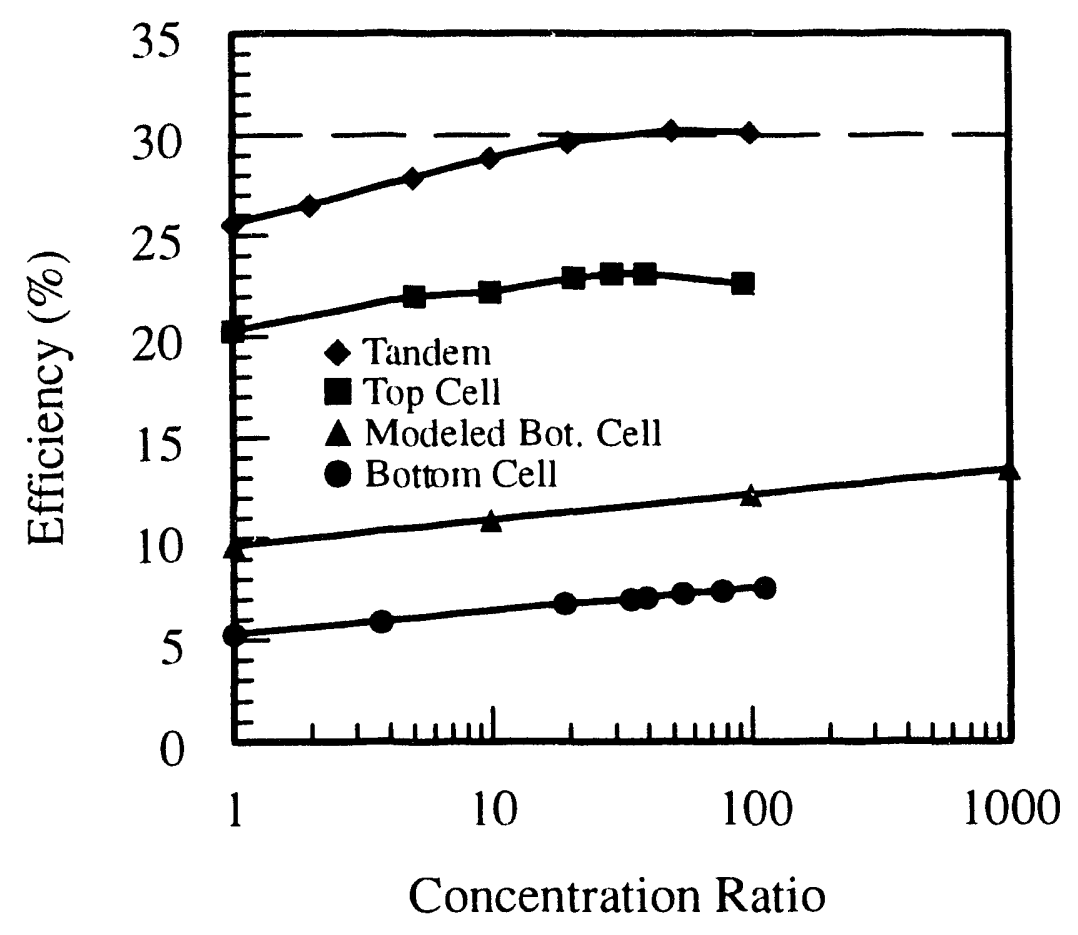

Figure 4.11: Efficiency versus Concentration Ratio data for a mechanically stacked GaAs $/ \mathrm{Ga}_{0.25} \mathrm{In}_{0.75} \mathrm{As}_{0.54} \mathrm{P}_{0.46}$ concentrator tandem cell. The modeled efficiency versus concentration ratio data for the $\mathrm{Ga}_{0.25} \mathrm{In}_{0.75} \mathrm{As}_{0.54} \mathrm{P}_{0.46}$ bottom cell are also included.

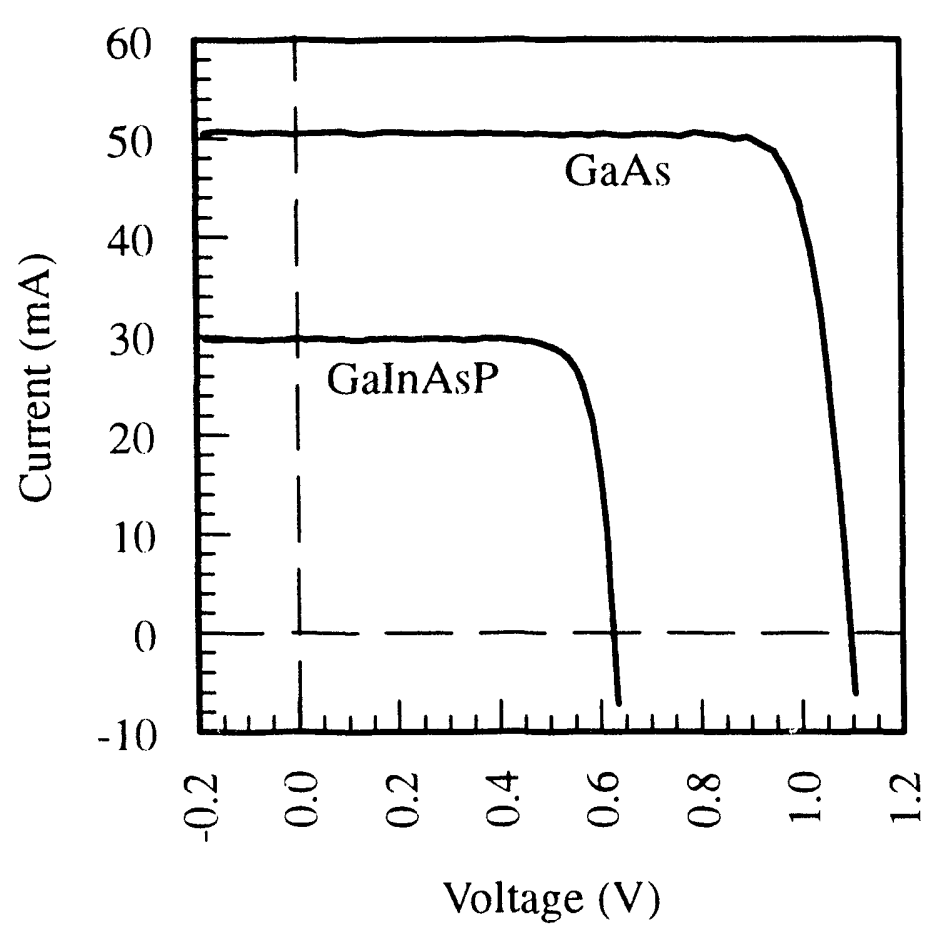

GaAs
$\mathrm{V}_{\mathrm{oc}}: 1.096 \mathrm{~V}$
$\mathrm{~J}_{\mathrm{sc}}: 990.3 \mathrm{mAcm}^{-2}$

FF: $83.5 \%$

$\eta: \quad 23.1 \%$

GaInAsP
$\mathrm{V}_{\mathrm{oc}}: 0.6264 \mathrm{~V}$
$\mathrm{~J}_{\mathrm{sc}}: 556.7 \mathrm{mAcm}^{-2}$

FF: $80.7 \%$

$\eta: \quad 7.1 \%$

Tandem $\eta: 30.2 \%$

Figure 4.12: Composite Current-Voltage data for a GaAs $/ \mathrm{Ga}_{0.25} \mathrm{In}_{0.75} \mathrm{As}_{0.54} \mathrm{P}_{0.46}$ mechanically stacked tandem cell at peak efficiency under concentration (39.5 suns, Direct, $25^{\circ} \mathrm{C}$ ). 


\subsubsection{Summary}

To summarize, $\mathrm{Ga}_{0.25} \mathrm{In}_{0.75} \mathrm{As}_{\mathrm{U.54}} \mathrm{P}_{0.46}$ concentrator cells have efficiencies as high as $9.4 \%$ at concentrations from 20 to 130 suns under a GaAs filter. With a reduction in $R_{s}$, and improved optical coupling, efficiencies exceeding $10 \%$ are anticipated for these cells in the future. Preliminary GaAs/ $\mathrm{Ga}_{0.25} \mathrm{In}_{0.75} \mathrm{As}_{0.54} \mathrm{P}_{0.46}$ mechanically stacked tandems have achieved efficiencies as high as $30.2 \%$ at 39.5 suns. By improving the GaAs top cell quality and the tandem stacking procedure, tandem efficiencies approaching $40 \%$ should be achievable at higher solar concentrations.

\subsubsection{Development of CdTe Thin Film Cells}

\subsubsection{Background}

Work on the preparation of polycrystalline thin films of CdTe using a 2" RF magnetron sputtering with S-Gun system was initiated in Jan. 1990 in conjunction with Dr. A. Onishi (AO) and Dr. A. Riad (AR) of Virginia Polytechnic Institute and State University. The material prepared so far showed very promising properties for PV application

\subsubsection{Objectives}

The main objectives of this study were:

- Preparation of rf magnetron sputtered CdTe polycrystalline thin films with optimum electrical, optical, and structural properties for solar cell aplications.

- Fabrication of CdS/CdTe devices with improved efficiences.

\subsubsection{CdTe Film Deposition}

The CdTe polycrystalline thin films were prepared using one of the two SGun systems, free from electron bombardment on the growing surface, designed and assembled by Dr. Onishi. The heart of the system consists of a 2" rf magnetron sputtering source housed in a tight-fit stainless steel vacuum chamber, which is pumped by a turbomolecular pump. The substrate temperature can be set from room temperature to $550^{\circ} \mathrm{C}$ during deposition. However, higher substrate temperatures resulted in lower film stresses than is observed when the films are deposited using thermal deposition. In order to have good adhesion at high temperature, careful surface preparation and stress-free films are essential. 
Pure Ar or a mixture gas is used for sputtering from the metal or dielectric target. A typical deposition rate of $1-20 \mu \mathrm{m} / \mathrm{hr}$ is obtained with an $\mathrm{rf}$ power of $50-600 \mathrm{~W}$. The substrate is placed off center from the target and positioned close to the target $(2-5 \mathrm{~cm})$. This unusual setting is the key in preventing electron bombardment from the source.

\subsubsection{Film properties}

\section{Electro-optical Properties}

The thickness of the CdTe films, which were deposited on alumina substrates with a $\mathrm{Cu}$ intermediate layer, was varied from $4-9 \mu \mathrm{m}$. The material showed very promising properties (structural and electro-optical) for PV application. A grain size of 2-4 $\mu \mathrm{m}$ ( oxygen doped), and 3-5 $\mu \mathrm{m}$ (Cu-doped) was achieved. Carrier concentrations were in the range of $10^{16}-10^{18} \mathrm{~cm}^{-3}$, with lifetimes of approximately $10^{-10} \mathrm{sec}$. A summary of the electrical properties is shown in Table 4.2 .

The defect configuration of this material is now under investigation using high resolution photoluminescence (PL).

Table 4.2: $\quad$ Electrical properties of sputtered CdTe films

\begin{tabular}{lccccc}
\hline Sample \# & $\begin{array}{c}\text { Grain size } \\
(\mu \mathrm{m})\end{array}$ & $\begin{array}{c}\text { Mobility } \\
\left(\mathrm{cm}^{2} \mathrm{~V}^{-1} \mathrm{~s}^{-1}\right.\end{array}$ & $\begin{array}{c}\text { Carrier } \\
\text { type }\end{array}$ & $\begin{array}{c}\text { Resistivity } \\
(\Omega \mathrm{cm})\end{array}$ & $\begin{array}{c}\text { Carrier } \\
\text { concentration } \\
\left(\mathrm{cm}^{-3}\right)\end{array}$ \\
\hline V6 & 2.8 & 11 & $\mathrm{p}$ & 81 & $7 \times 10^{15}$ \\
V9 & 4.9 & 46 & $\mathrm{p}$ & $1.6 \times 10^{-2}$ & $8 \times 10^{18}$ \\
V12 & 3.0 & 55 & $\mathrm{p}$ & 2.5 & $5 \times 10^{17}$ \\
\hline
\end{tabular}

\section{Structural Properties}

Pian-view samples were characterized by TEM to determine the grain morphology, structure and size. Selected area transmission electron diffraction examination suggested that the smaller grains are copper particles. Small probe X-ray microanalysis will be performed to confirm this. The $6 \mu \mathrm{m}$ thick film has equiaxed grains of approximately $1.0 \mu \mathrm{m}$ in size and with a very high density of intergranular planar defects.

\subsubsection{CuInSe ${ }_{2}$ Single Crystals $[1,2,3]$}

The purpose of the single crystal studies is to establish a foundation for the understanding of the more complex case of polycrystalline thin-film CIS material. In particular, it was intended to identify the origins of the various intrinsic defect states of the CIS semiconductor, including those resulting 
from various surface and heat treatments, and to establish a comparison between thin films and single crystals. It was also intended to clarify the relationship between the type and origin of the dominant defect states, the material chemical composition, and the junction behavior.

As an extension of previous efforts, further discussion is given on the characterization of the defects (their nature and location in gap of the semiconductor) in single crystal (CIS) and polycrystalline material. Defect levels were identified using high-resolution photoluminescence (PL) spectroscopy. deep-level transient-spectroscopy (DLTS), and frequencydependant capacitance-voltage $(\mathrm{C}-\mathrm{V})$ characterization techniques. The nature of the junction was investigated using EBIC. measurements. For the first time, direct evidence for several major defect types $(\mathrm{Cu}$ vacancies, $\mathrm{Cu}$ at In sites, and In at Cu sites), responsible for the majority-carrier type of CIS based upon spectroscopic atomic imaging (provided by L.L. Kaznerski) of the $\mathrm{n}$ - and p-type semiconductor surfaces, has been obtained.

\subsubsection{Photoluminescence of $n$ and $p$-type Surfaces $[2,4,5,6]$}

The PL signals from $n$ - and p-type materials exhibit significantly different signatures, as represented in Fig. 4.13. Many levels have been identified in previous PL studies with proposed acceptor and donor assignments. The major donor level in the CIS is associated with Cu-vacancies; the major acceptor, with Se excess and In at Cu sites.

An energy band diagram has previously been proposed which explains most of the defect chemistry of CIS within a wide range of compositions. This, however, did not account for all of the 12 intrinsic defect states expected in this material. Extension of this effort has resulted in identification of additional levels associated with intrinsic defects which dominate the CIS. The results are summarized in the energy band diagram shown in Fig. 4.14. This diagram was developed utilizing the material composition (as a guide to the likely defect levels), the calculated formation energies of the possible intrinsic defect states, and the published ionization energies. The PL emission from the cleaved surfaces of three p-type CIS single crystal samples with different compositions is shown in Fig. 4.15. The sample with the lowest $\mathrm{Cu}$ content $(\mathrm{Cu} / \mathrm{In}$ ratio $=\mathrm{Dm}=0.837)$ is dominated by a $\mathrm{PL}$ transition due to $\mathrm{In}_{\mathrm{si}}-\mathrm{V}_{(\mathrm{cu}}$ at $0.918 \mathrm{eV}$ (curve a in Fig. 4.15). The second major PL emission at $0.955 \mathrm{eV}$ is attributed to the $\operatorname{In}_{\mathrm{CO}_{11}}-\mathrm{V}_{\mathrm{C}}$ transition followed by the $0.893 \mathrm{eV}$ peak resulting from $\operatorname{In}_{s \mathrm{x}}-\mathrm{V}_{\mathrm{sx}}$. This sample had almost no stoichiometry deviation i.e., there is almost exactly 50 at\% Se.

On the other hand, the sample with an excess of Se and a slightly higher $\mathrm{Cu} /$ In ratio (curve $b$ in Fig. 4.15) $(\mathrm{Dm}=0.896)$, is dominated by the conduction band $\left(\mathrm{CB}-\mathrm{Se}_{(\mathrm{N})}\right)$ recombination responsible for the PL peak at $0.978 \mathrm{eV}$. This 


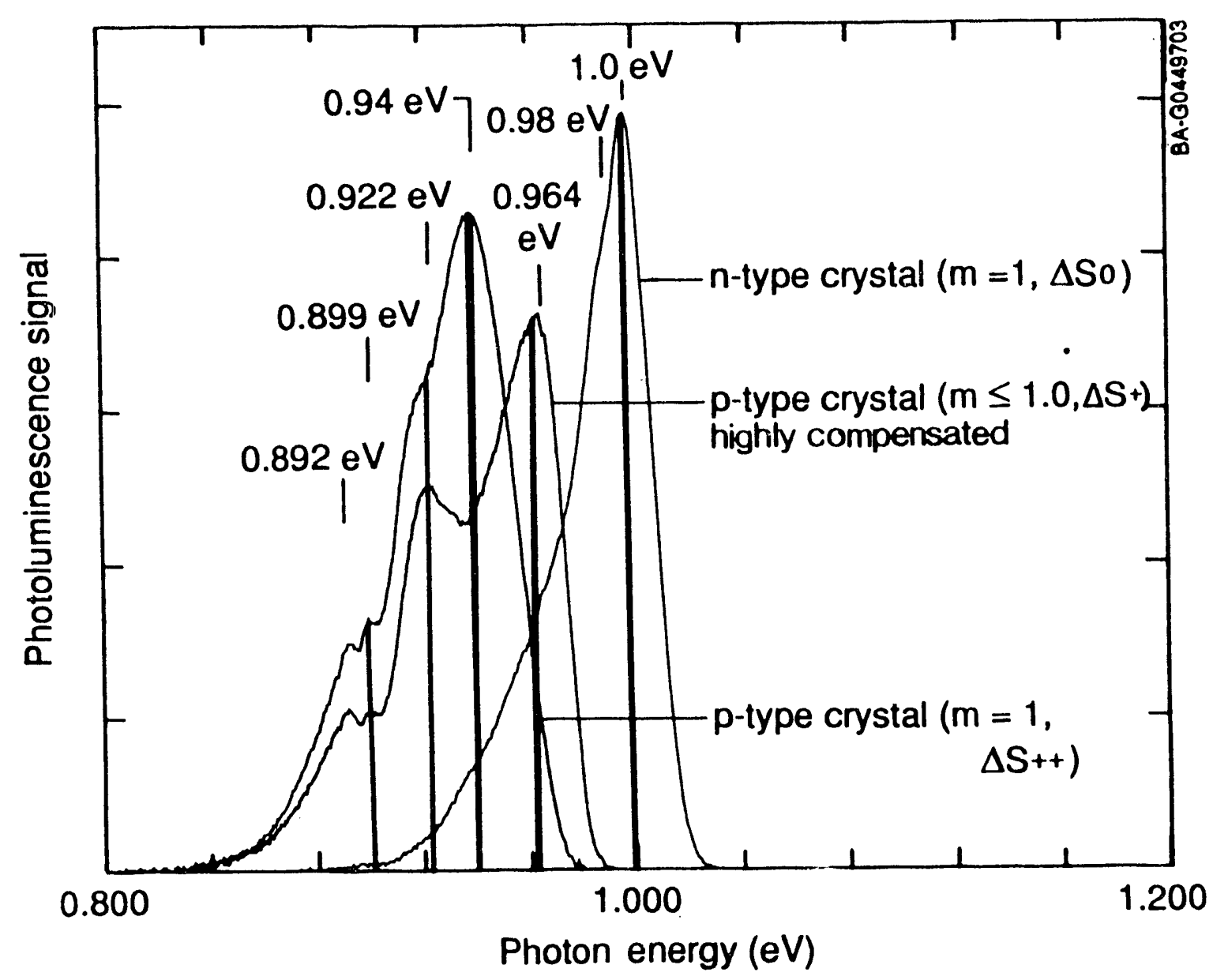

Figure 4.13: Photoluminescence emission from $n$ - and p-type CuInSe ${ }_{2}$ single crystals.

overlaps the PL emission at $0.955 \mathrm{eV}$, which is due to the $\operatorname{In}_{\mathrm{Cu}}-\mathrm{V}_{\mathrm{Cu}}$ recombination, in addition to the signal at $0.926 \mathrm{eV}$, due to $\operatorname{In}_{\mathrm{Cu}_{1}}-\mathrm{Se}_{\mathrm{In}}$ transitions. Curve $c$ of Fig. 4.15 is the spectrum for a sample with an excess of Se (51.09 at.\%). This exhibits the largest $\mathrm{Cu} / \mathrm{In}$ ratio $\left(\mathrm{Dm}=00^{\circ 14}\right)$ and it is dominated by the same emission as sample b, plus a signal at $0.94 \mathrm{eV}$ resulting from the $\mathrm{Se}_{\mathrm{Cu}}-\mathrm{V}_{\mathrm{Cu}}$ transition present at relatively equal concentration.

It is therefore clear that $\mathrm{p}$-type CIS can be grown as $\mathrm{Cu}$ poor material (22.79 at.\%) with near zero molecularity deviation. The p-type crystals with a Seexcess, on the other hand, (curves $b$ and c, Fig. 4.15) can be grown either Cupoor or with a slight $\mathrm{Cu}$-excess, but the defect chemistry and corresponding electrical behavior are considerably different. The $\mathrm{Cu}$-deficient crystals are dominated by $\mathrm{V}_{\mathrm{Cu}}$ defects. These exhibit high carrier concentration $\left(\mathrm{N}_{\mathrm{a}}>5 \times 10^{16} \mathrm{~cm}^{-3}\right)$ and, if combined with $\mathrm{Se}_{\mathrm{Cu}}$ anti-site defects, the carrier mobility is increased $\left(\mu>50 \mathrm{~cm}^{2} \mathrm{~V}^{-1} \mathrm{~s}^{-1}\right)$. The presence of a high density of the $\operatorname{In}_{\mathrm{Cu}}$ defect, on the other hand, reduces $\mu$ substantially. 


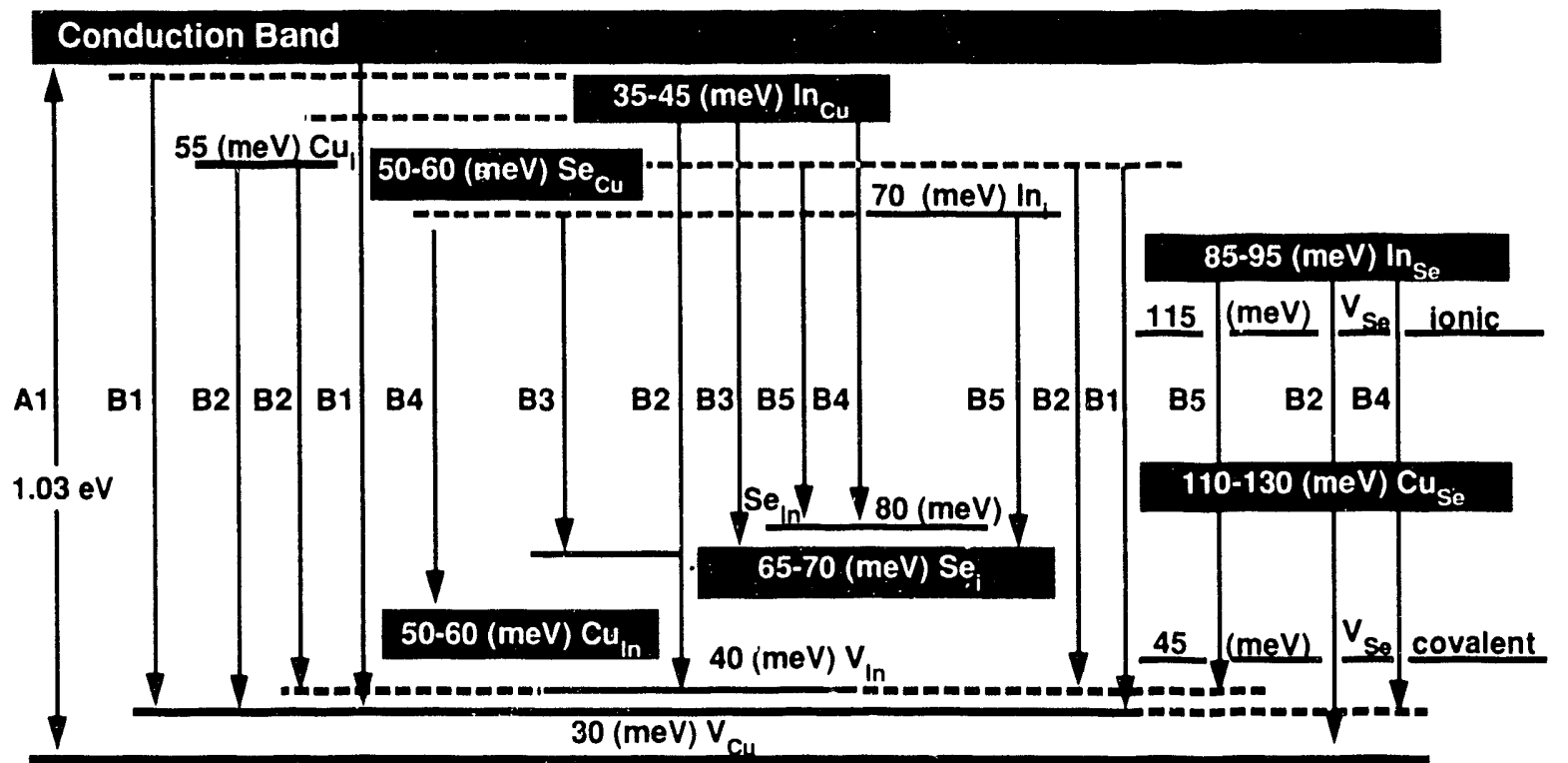

Valence Band

Figure 4.14: Energy band diagram of CulnSe ${ }_{2}$.

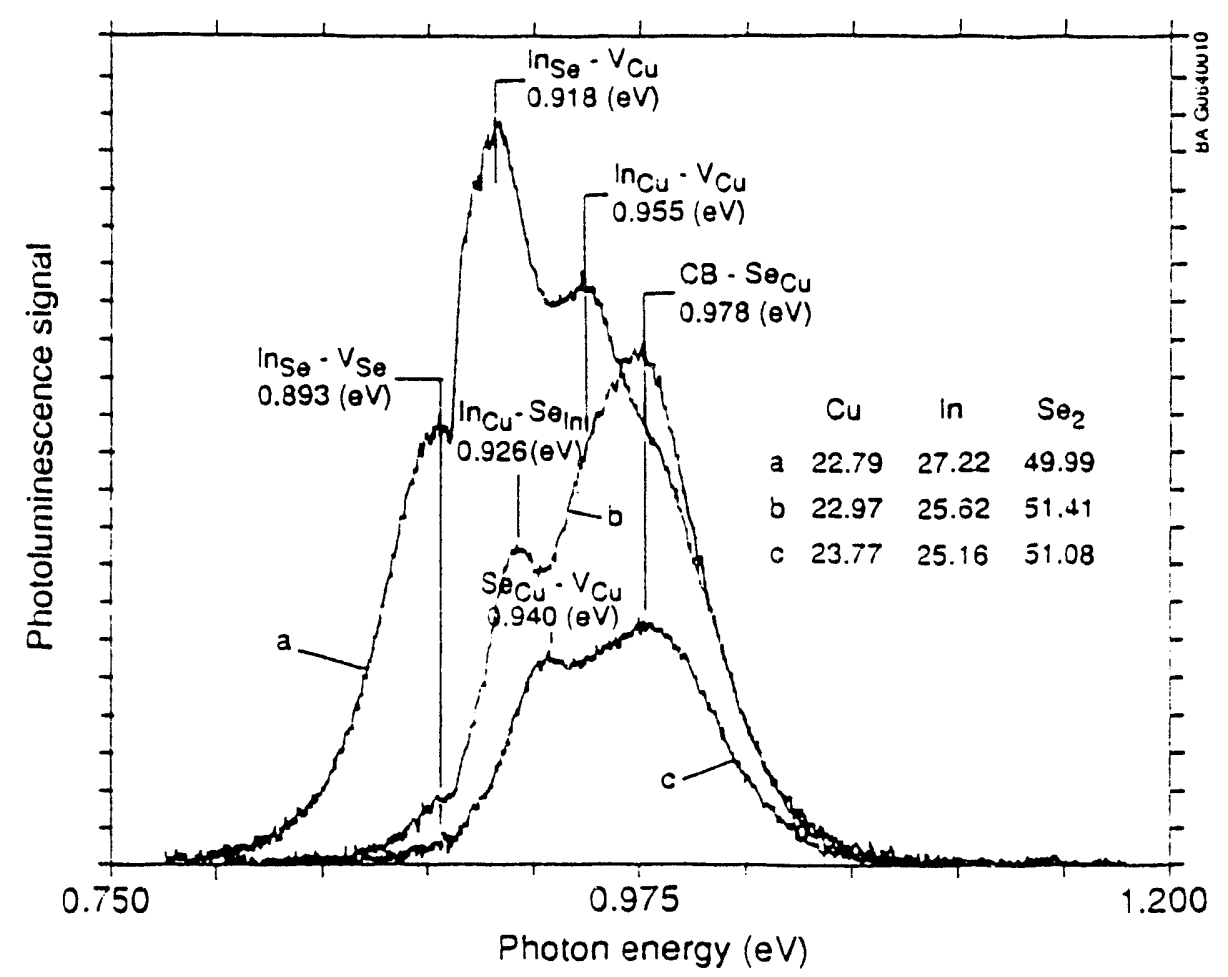

Figure 4.15: PL emission from CIS single crystals of different composition. 


\subsubsection{Spectroscopic atomic imaging}

Spectroscopic sc anning tunneling microscopy (SSTM) provides confirmation of these defects in the n-and p-type crystals. Direct evidence of the $V_{C u}, I_{C u}$, defects responsible for the majority-carrier type in Sample a has been provided by SSTM imaging of the 112 surface (which contains only In and $\mathrm{Cu})$ and the grain boundary of the (112) plane.

Atomic-resolution images of the (112)-metallic CuInSe ${ }_{2}$ cleaved surface of an n-type crystal, grown with approximately a 4 at.\% Cu-deficiency (confirmed by calibrated AES measurements), shows several defect types. The dominant defect is the Cu-vacancy, with singly-deficient and doubly-deficient defects clearly observaiule. These defect leveis dominate the PL spectra of Fig. 4.13, and are considered the major defect responsible for the n-type doping. In addition, evidence for $\mathrm{Cu}$ at In sites is provided in the atomic image. These defects are responsible for bandgap levels that are identified as donors. In comparison, the p-type crystal surface (Fig. 4.15) shows two defects that have been identified as acceptors: In at $\mathrm{Cu}$ sites and In vacancies. This sample had approximately a $2 \%$ In excess. Therefore, these SSTM images confirm the results of the PL data, and provide the first direct correlation between the existence of atomic defects and the resulting doping of a stoichiometry-controlled semiconductor.

\subsubsection{Surface processing effects [13]}

The effect of surface treatments on the CIS single crystal was also found to be dependent upon the initial type and relative concentration of the dominant defect states. Some samples are severely affected by mechanical polishing, while others are affected very slightly. Fig. 4.16 (curves a and c) shows a comparison of the PL emission from sample a of Fig. 4.15 after cleaving and then after mechanical polishing. It is clear from this that polishing created several additional defects in the range 0.954-0.984 eV. Curve a of Fig. 4.16 was taken at an incident power of $7 \mathrm{~mW}$ whereas Curve $\mathrm{b}$ was obtained using a power of $14 \mathrm{~mW}$. Hence, increasing the power also increases the transition energy of the additional states. At lower energy, reduced excitation power causes them to overlap in $\mathrm{In}_{\mathrm{Cu}}-\mathrm{V}_{\mathrm{Cu}}$ emission.

The effect of mechanical polishing on Sample a (which had an In excess) is more pronounced than for Sample b (in content close to 25 at.\%). This is shown in Fig. 4.17. For this, the PL signal at $0.926 \mathrm{eV}$ emitted by the $\mathrm{In}_{\mathrm{Cu}}$ $\mathrm{Se}_{\text {in }}$ transition. dominated after polishing and the $0.952 \mathrm{eV}$ emission due to the $\mathrm{Se}_{\mathrm{Cu}}-\mathrm{V}_{\mathrm{Cu}}$ transition became well defined. The radiative surface recombination state at $0.978 \mathrm{eV}$, which is superimposed on the $\mathrm{CB}-\mathrm{Se}_{\mathrm{Cu}}$ transition. is shifted to $\mathrm{C} .990 \mathrm{eV}$ by increasing the excitation power from 7 
to $14 \mathrm{~mW}$ but it does not dominate the PL emission of this sample as it did for Sample a.

On the other hand, the PL spectrum of Sample c (Fig. 4.18) did not show any additional radiative surface recombination states after polishing. However, the reduction in the integrated intensity of the PL spectrum of the polished surface indicates the formation of non-radiative surface states that possibly quench the radiative recombination transitions. The PL emission of this sample is compared to that from a thin-film sample of similar composition (supplied by Boeing). It is clear that the defect-configuration of the thin-film material is dominated by a strong peak at $0.880 \mathrm{eV}$ (attributed to $\mathrm{In}_{\mathrm{Se}}-\mathrm{Cu}_{\mathrm{ln}}$ ) in $a$ tdition to the main emission from the single crystal sample, including the $0.901 \mathrm{eV}$ signal attributed to $\operatorname{In}_{\mathrm{Se}}-\mathrm{V}_{\mathrm{Cu}}$ recombination. Hall measurements have shown that the carrier mobility of the thin-film material is twice that of the single crystal. The carrier concentration, on the other hand, is higher in the single crystal (Sample c), which is dominated by copper vacancies active in the $\operatorname{In}_{\mathrm{Cu}}-\mathrm{V}_{\mathrm{Cu}}$ transition.

After the deposition of the $(\mathrm{Cd}, \mathrm{Zn}) \mathrm{S}$ window layer on the CIS thin film, the integrated PL emission data were taken and these are shown in Fig. 4.19. It can be seen that there is a substantial increase. This is in contrast to the analogous processing of the single crystal (polished) surface, indicating a

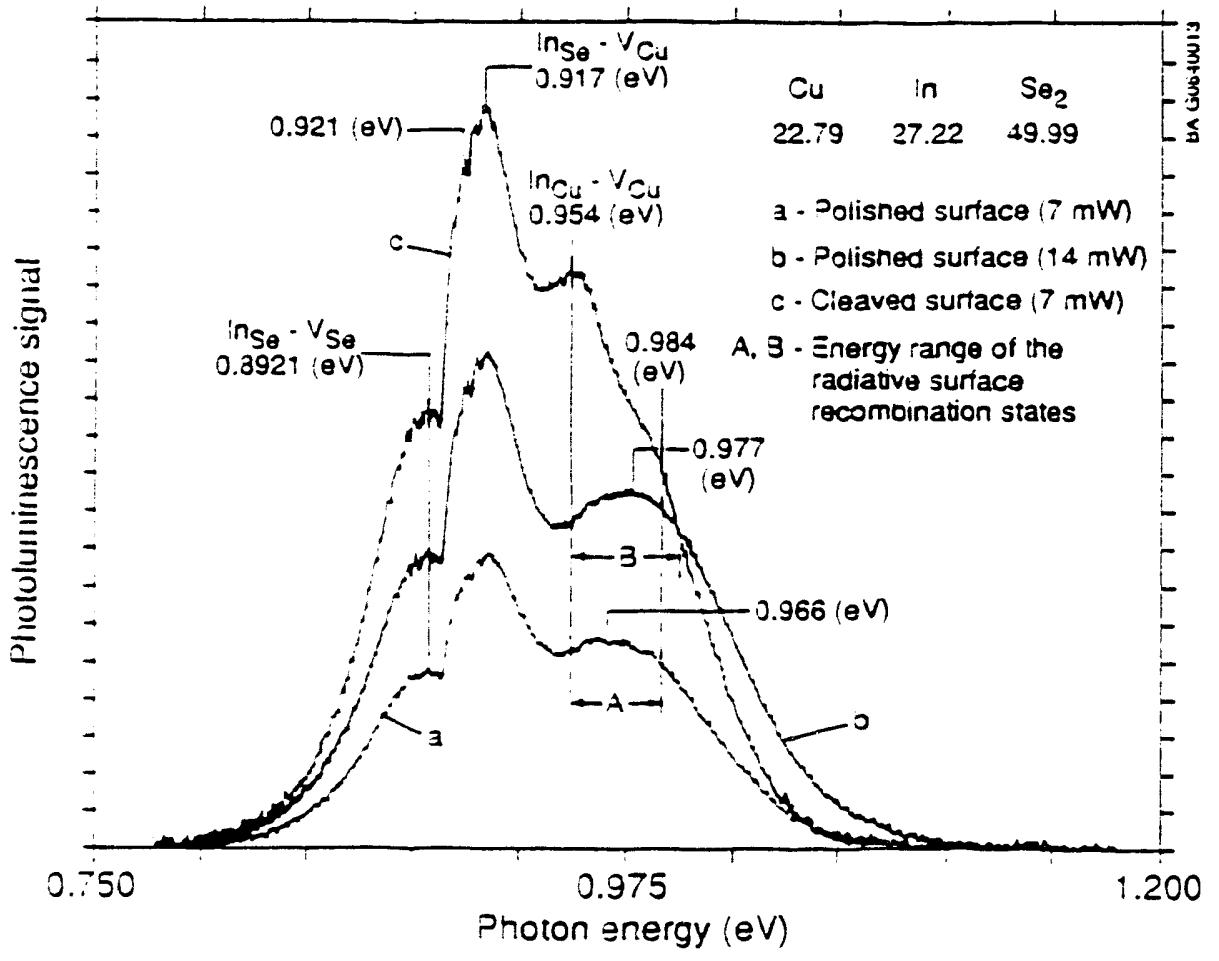

Figure 4.16: Effect of mechanical polishing on In rich CIS single crystal. 


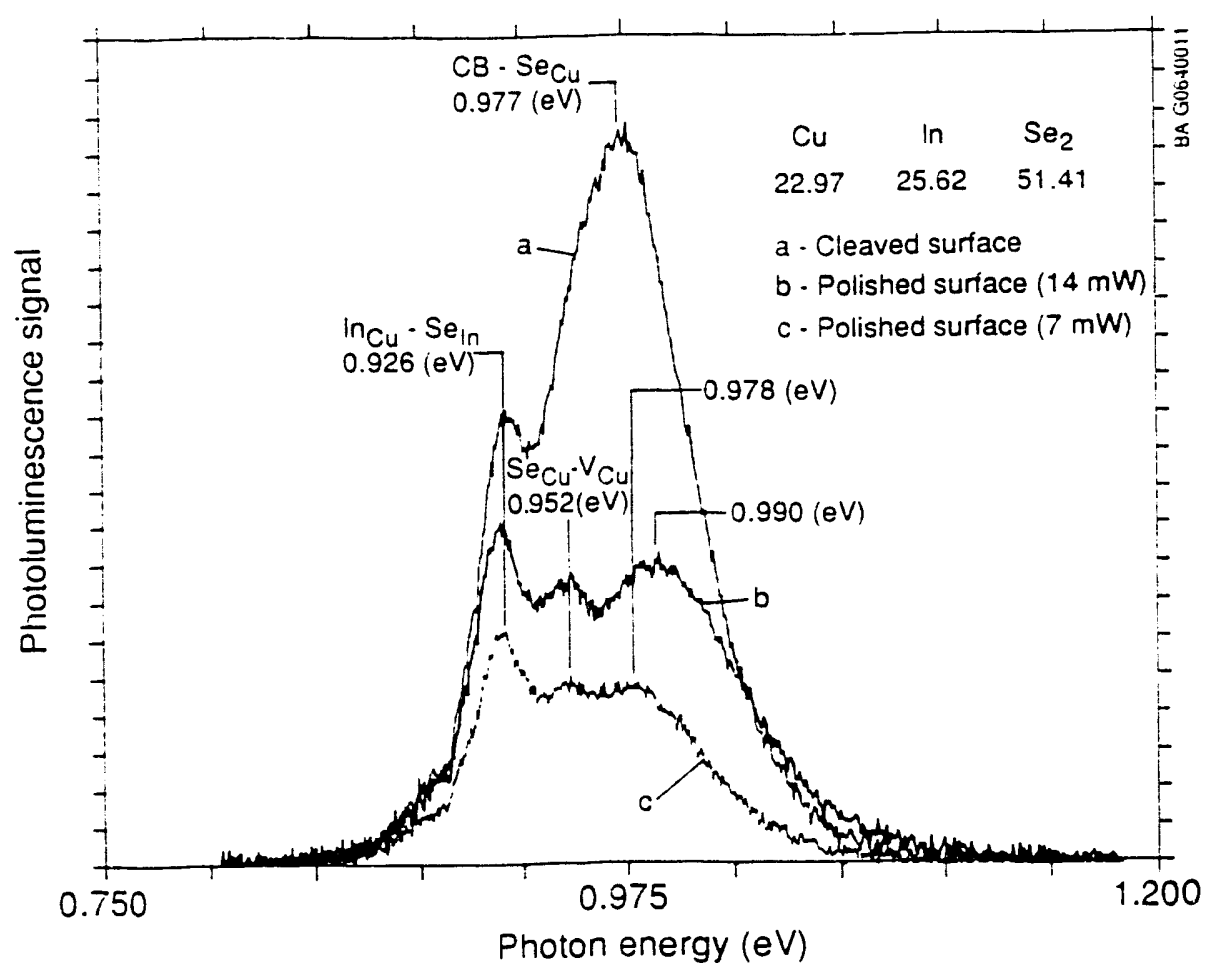

Figure 4.17: Effect of mechanical polishing on SE rich CIS single crystal.

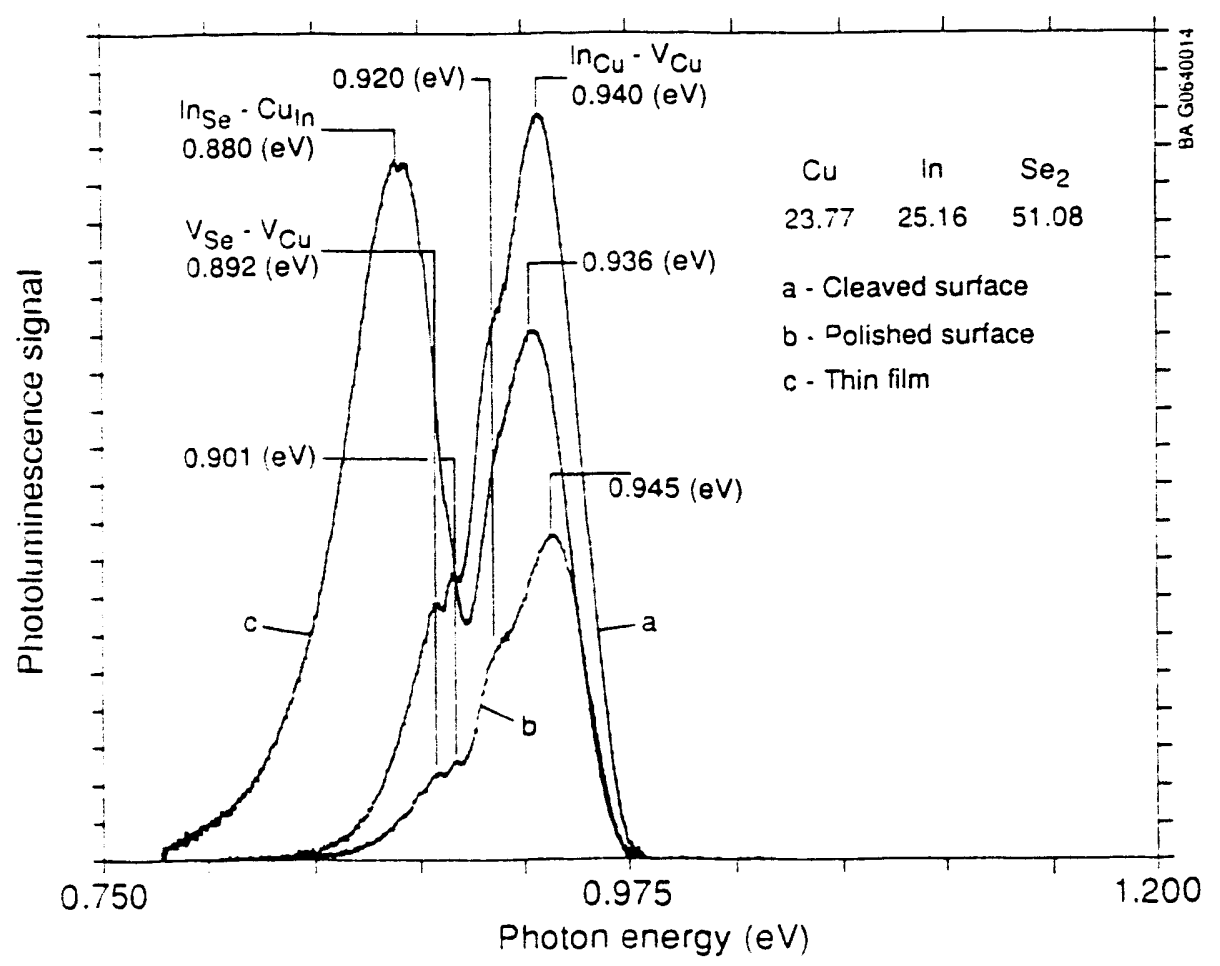

Figure 4.18: PL emission from CIS single crystal (cleaved and polished surfaces) and thin film with the same composition. 


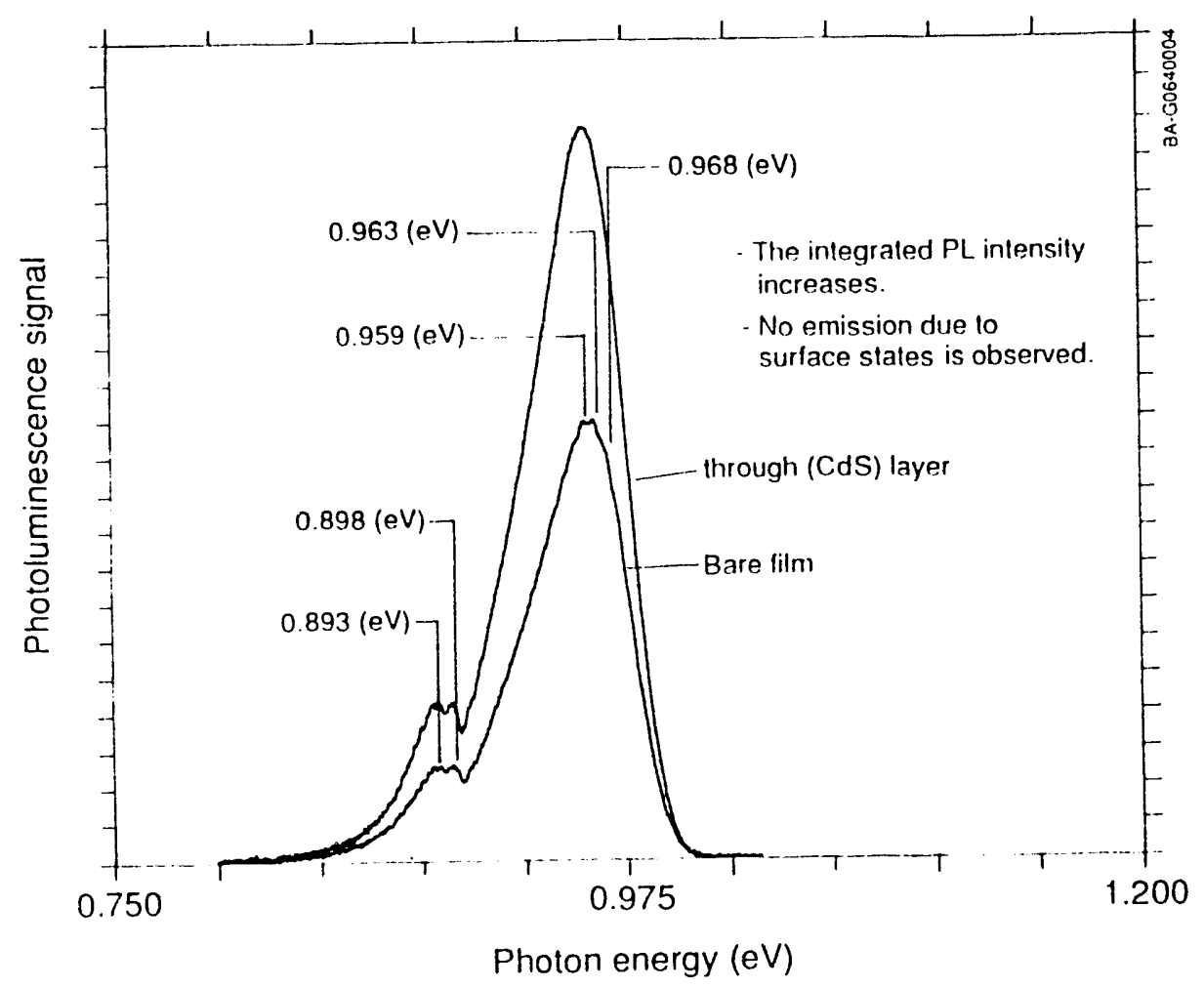

Figure 4.19: PL ernission spectra from CuInSe2 thin films before and after the deposition of the $(\mathrm{Cd}, \mathrm{Zn}) \mathrm{S}$ window layer.

lower surface recombination velocity at the window/thin-film CIS junction. To examine the surface state properties and recombination mechanisms further, metal-CIS junctions were formed.

\subsubsection{Interface Characterization [13]}

\section{Frequency-Dependant C-V}

The frequency-dependent capacitance measurements have also been used to study the interface behavior of both thin film and single crystal junctions. The $\mathrm{C}^{-2}-\mathrm{V}$ plots obtained from the two heterostructure single crystal devices, numbered $\mathrm{C} 2-8$ and $6 \mathrm{~B}$ (their respective conversion efficiencies are 1.2 and $4.1 \%$ ), are shown in Figs. 4.2 .0 and 4.21. It is clear that the characteristics of Device $\mathrm{C} 2-8$ show, not only a decrease in the slope with decreasing frequency, but also a non-linear dependence. On the other hand, device $6 \mathrm{~B}$ had an almost linear characteristic with slight reduction in the slope with frequency. This result confirms the presence of multiple surface states (at higher density in the C2-8 device) with variable distribution. The surface 


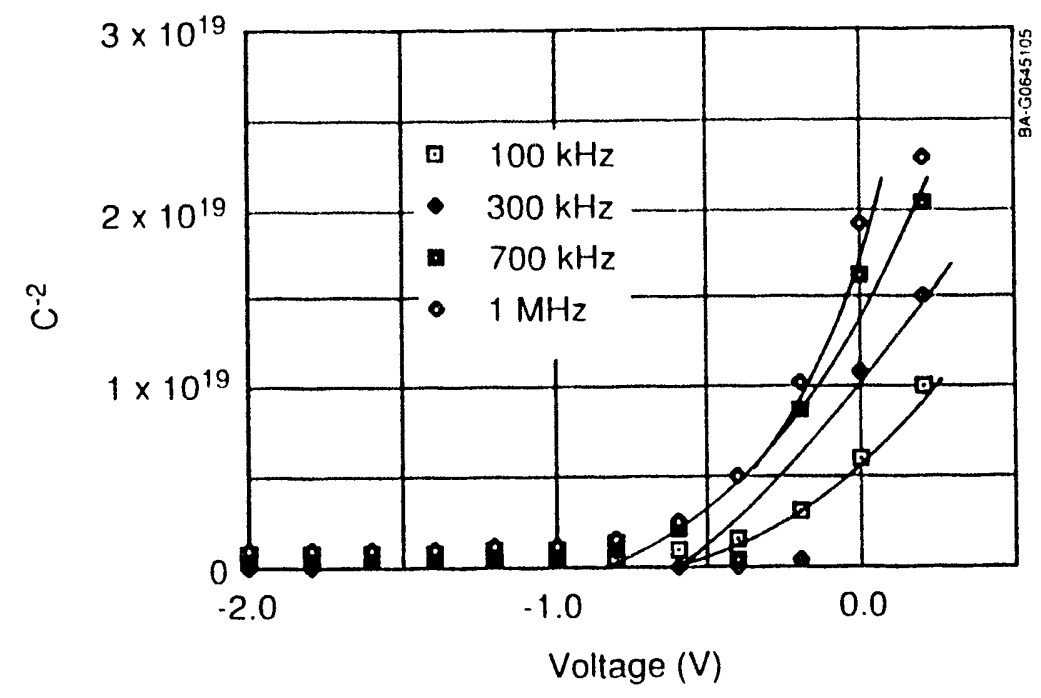

Figure 4.20: Frequency dfependent $C^{-2}-V$ relation of $(C d, Z n) S / C I S$ single crystal device (\#C2-8).

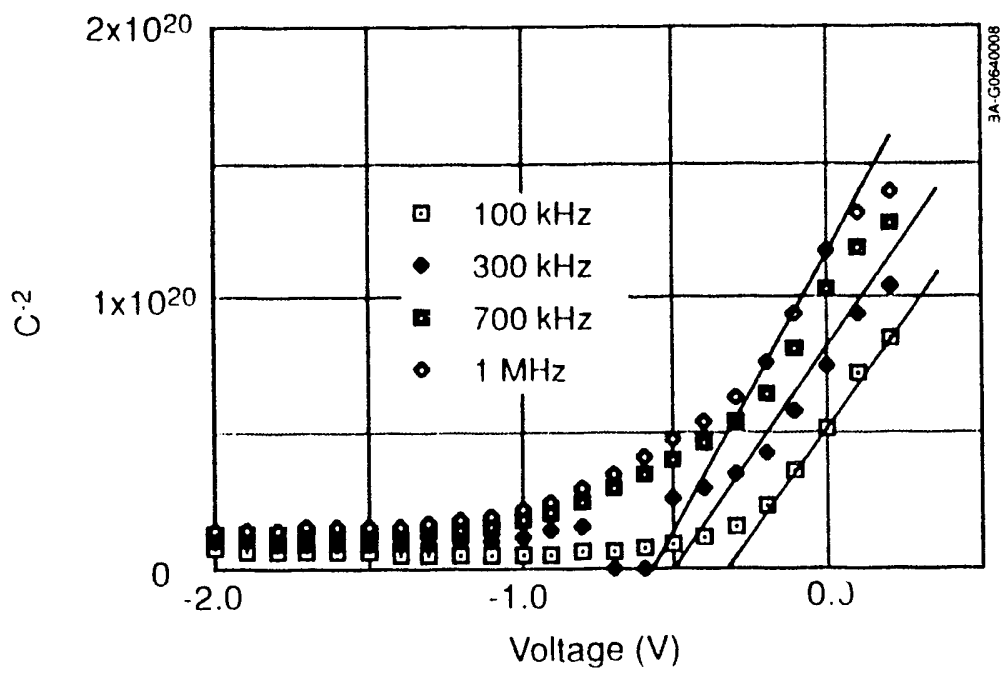

Figure 4.21: Frequency dfependent $C^{2}-V$ relation of $(C d, Z n) S / C I S$ single crystal device (\#6B). 
states include shallow acceptors and deep traps and the density of these decreases with increasing frequency. The $\mathrm{C}^{2}-\mathrm{V}$ characteristics are obtained for devices of the types $\mathrm{Al} / \mathrm{p}$-CIS and $(\mathrm{Cd}, \mathrm{Zn}) \mathrm{S} / \mathrm{p}$-CIS prepared on polycrystalline thin films (supplied by IEC). Although a linear characteristic is obtained from the thin-film Schottky diode, the slope decreases with decreasing frequency. On the other hand, a non-linear characteristic is observed for the heterojunction device (Fig. 4.22) although the capacitance did not vary with the measurement frequency, thus confirming a very low density of deep traps.

The $\mathrm{C}^{-2}$ vs $\mathrm{V}$ characteristics obtained on $\mathrm{Al} /$ single-crystal, p-CIS junctions, presented in Fig. 4.23, show a decrease in the slope with decreasing signal frequency. In comparison, no slope variation is found in the analogous thinfilm case (Fig. 4.24). The presence of an interfacial layer in both the CIS bulk single crystal and thin-film junction is therefore confirmed from comparison of these $\mathrm{C}-\mathrm{V}$ measurements. However, the effect is more pronounced in the bulk single-crystal junctions because of the higher junction recombination.

\section{Deep-level Transient-Spectroscopy (DLTS)}

The DLTS spectra and associated Arrhenius dependencies obtained from the $\mathrm{Al} /$ single-crystal $\mathrm{CIS}$ and the $\mathrm{Al} /$ thin-film CIS Schottky diodes are presented in Figs. 4.25 and 4.26, respectively. These results indicate the presence of only one major hole trap, detected at the same level in the two junctions (at $280 \mathrm{meV}$ and $270 \mathrm{meV}$ in the single crystal and thin-film devices, respectively). However, the trap density was two orders of magnitude smaller in the thin-film diode. These deep levels account for the lower opencircuit voltage for the cells produced on single-crystal (damaged) surfaces compared to the thin-film devices. Figure 4.27 shows the DLTS spectra and Arrhenius plots obtained from single crystal CIS devices of the type (Cd, $\mathrm{Zn}) \mathrm{S} / \mathrm{p}$-CIS and Al/p-CIS respectively. Two major traps were found for the heterostructure device; these occurring $234 \mathrm{meV}$ and $493 \mathrm{meV}$. Only one level at $282 \mathrm{meV}$ was detected for the Schottky diode made from the same crystal. In the case of the thin film heterostructure device, a deep level at $530 \mathrm{meV}$ was detected and this is illustrated in Fig. 4.28. Although the deep trap occurred at approximately the same energy level in the single crystal and thin film heterostructure devices, its density was about 2-3 orders of magnitude larger for the former and this difference accounts for the much lower open circuit voltage, commonly seen for single crystal cells.

It is therefore clear that heating of the CIS during (Cd, $\mathrm{Zn}) \mathrm{S}$ deposition, influences the trap location and density for both thin films and single crystals. However, the original location of the trap levels is dependent on the defect configuration of the starting material and this varies from sample-tosample, even for similar compositions. The PL measurements performed on 


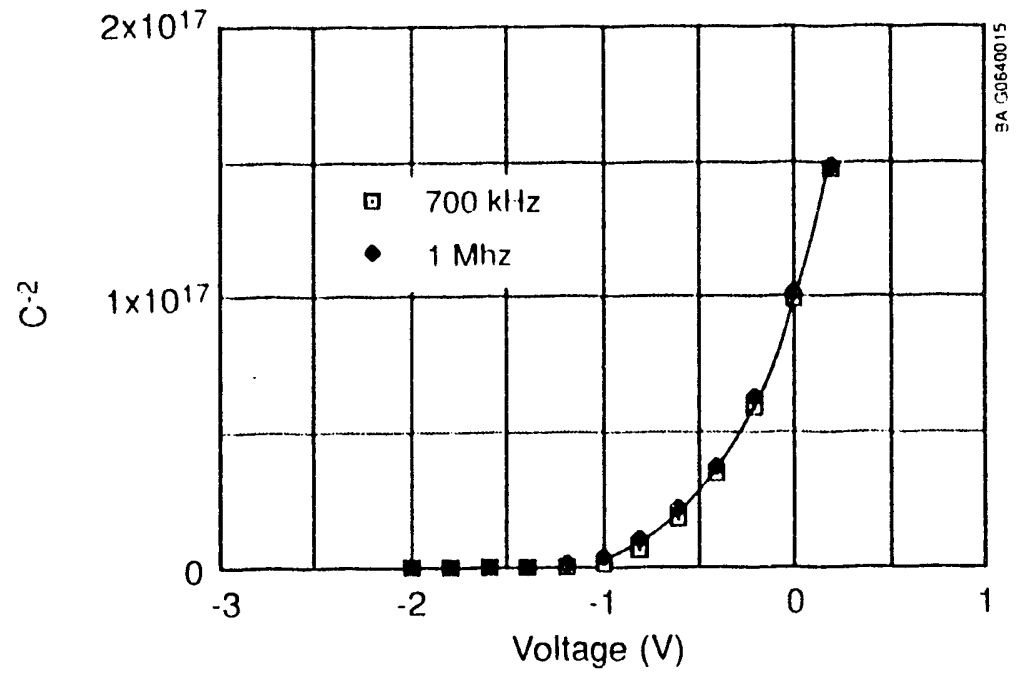

Figure 4.22: Frequency dfependent $C^{-2}-V$ relation of $(C d, Z n) S / C I S$ thin-film device.

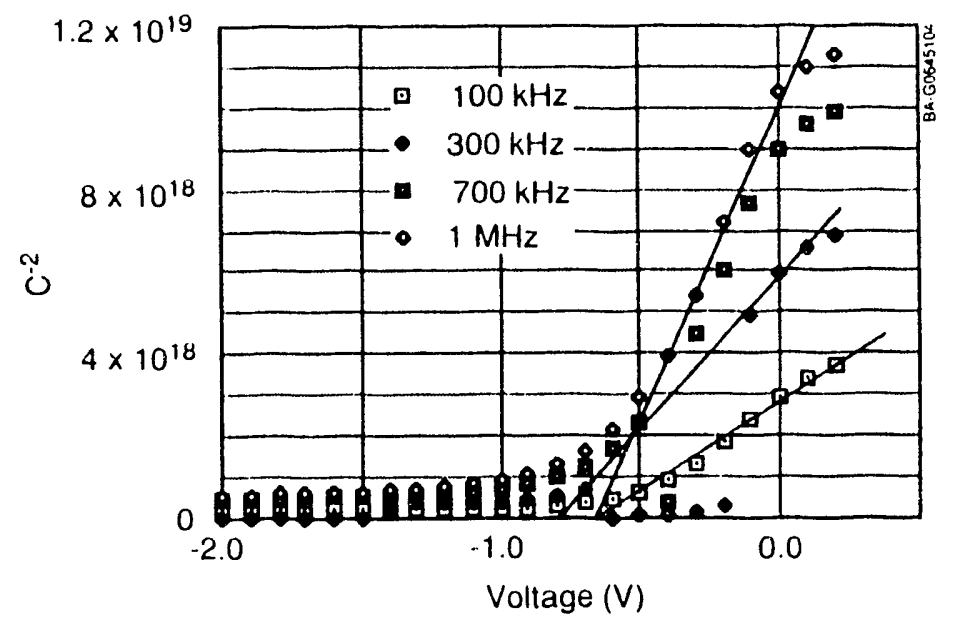

Figure 4.23: $\mathrm{C}^{2}$ versus $\mathrm{V}$ dependence of an $\mathrm{Al} / \mathrm{p}$-type, single crystal CuInSe ${ }_{2}$ Schottky barrier.

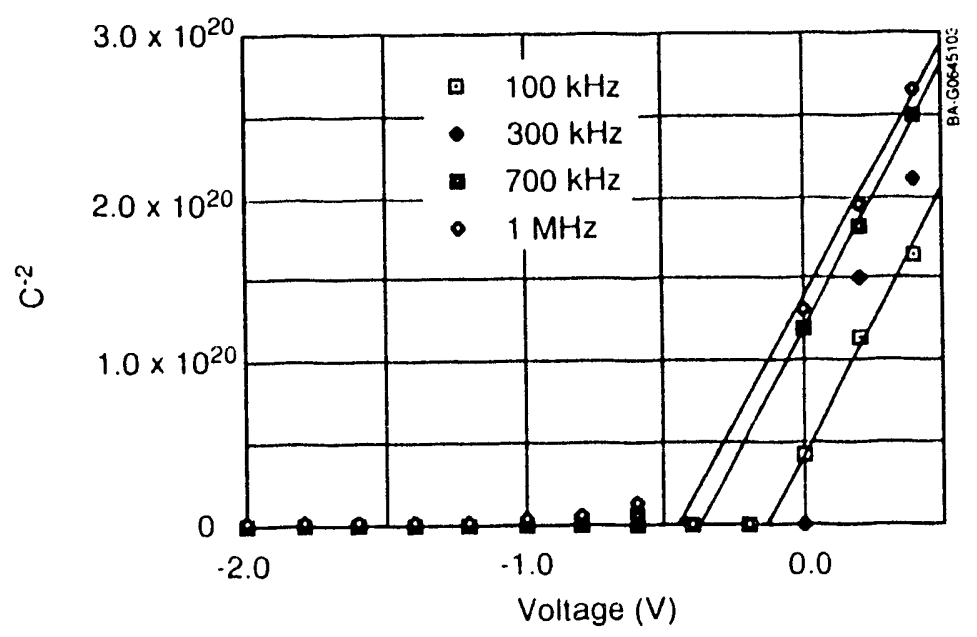

Figure 4.24: $\mathrm{C}^{-2}$ versus $\mathrm{V}$ dependence of an $\mathrm{Al} / \mathrm{p}$-type, single crystal CuInSe $\mathrm{i}_{2}$ Schottky barrier. 


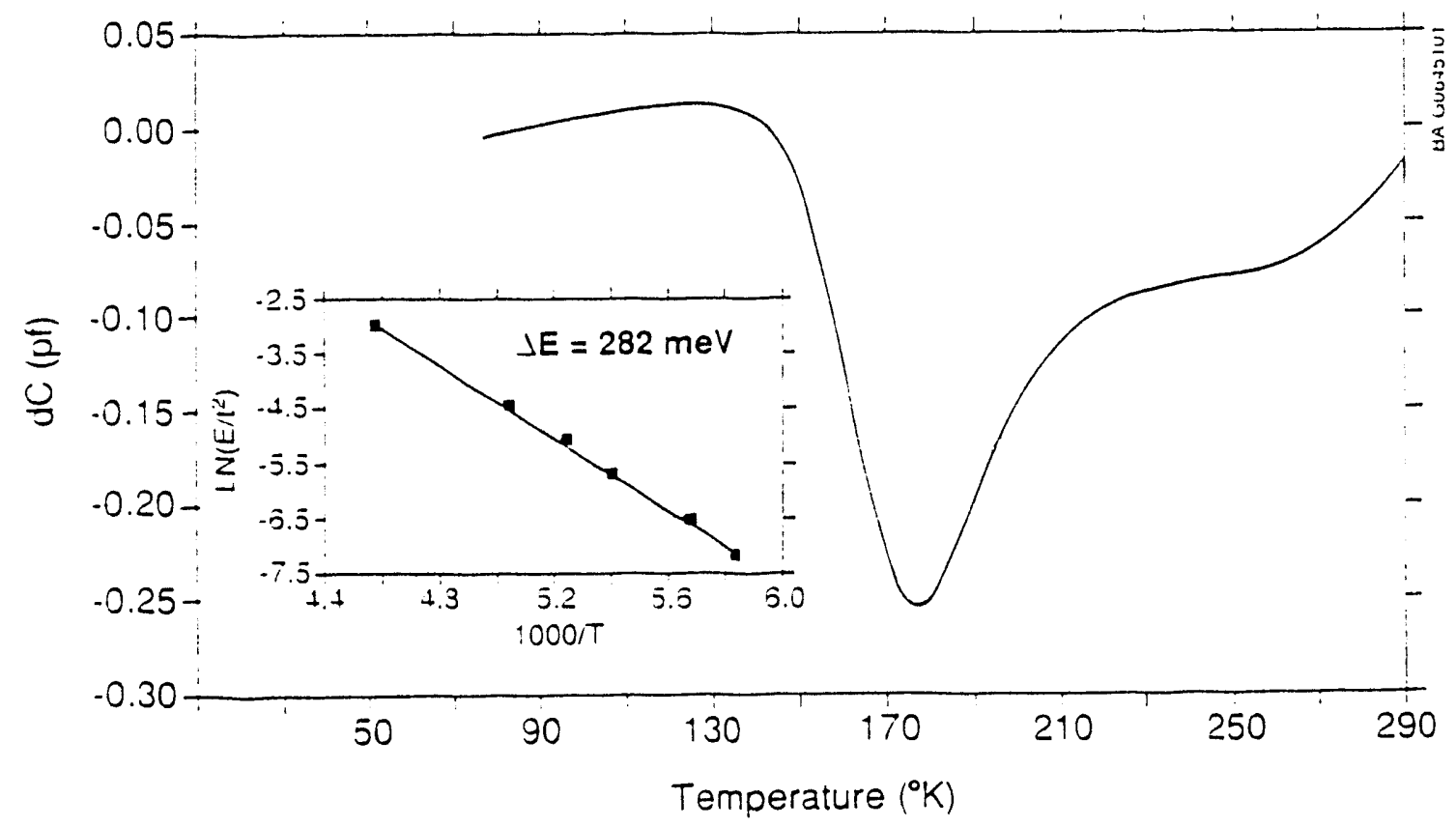

Figure 4.25: DLTS and Arrhenius grapg for an Al/p-type, single cryslal CuInSe ${ }_{2}$ Schottky barrier

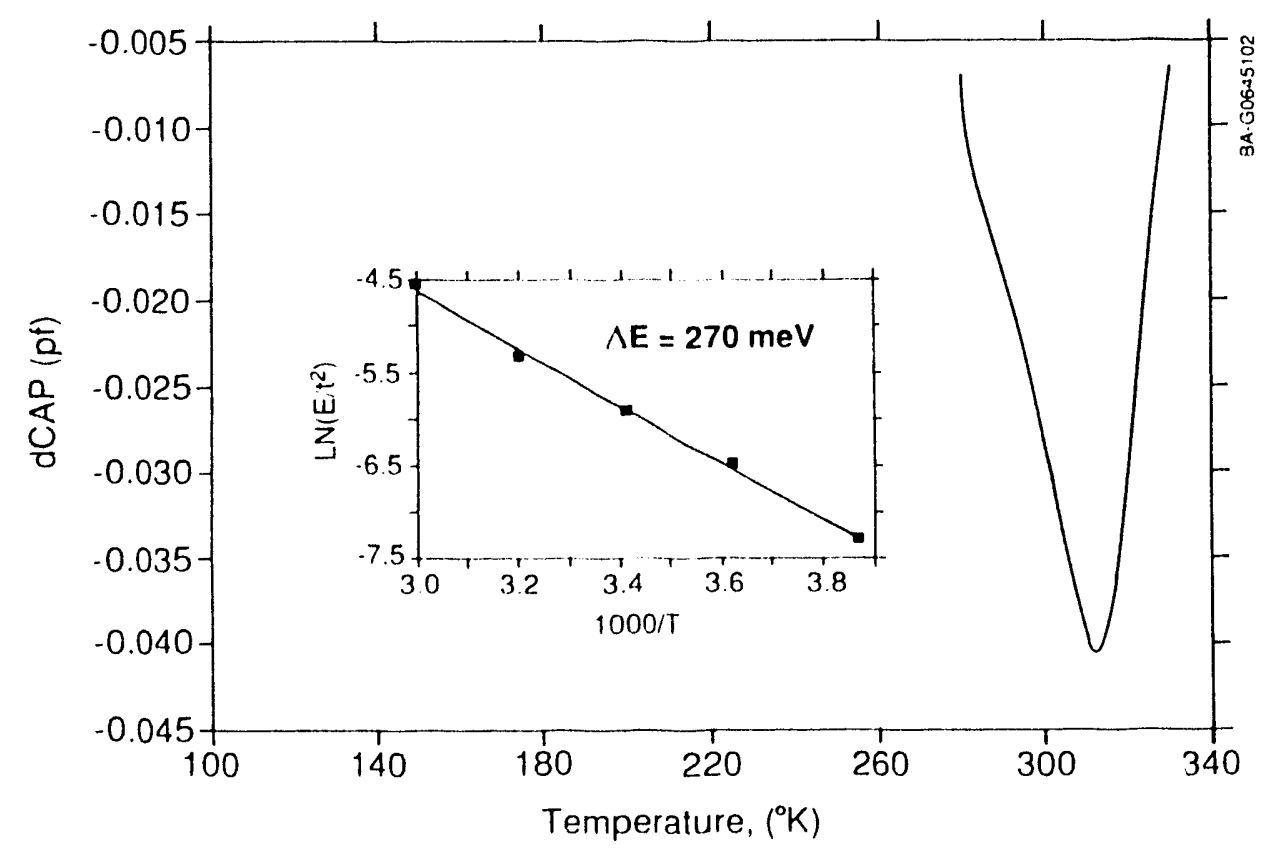

Figure 4.26: DLTS and Arrhenius grapg for an Al/p-type, single crystal CuInSe, Scholtky barrier 


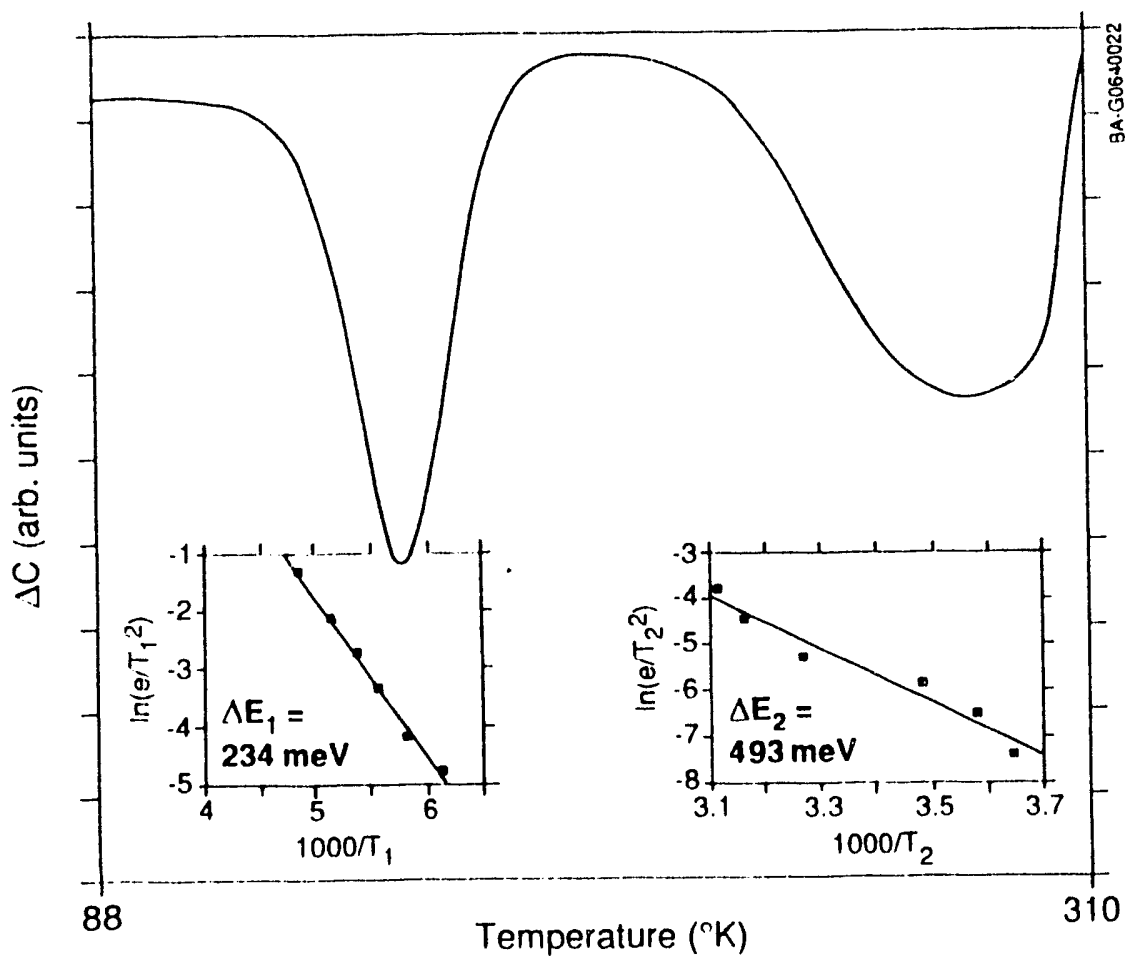

Figure 4.27: DLTS spectrum and Arrhenius plots of (Cd, $\mathrm{Zn}) \mathrm{S} / \mathrm{CIS}$ single crystal device $(23.30,25.47,51.23)$.

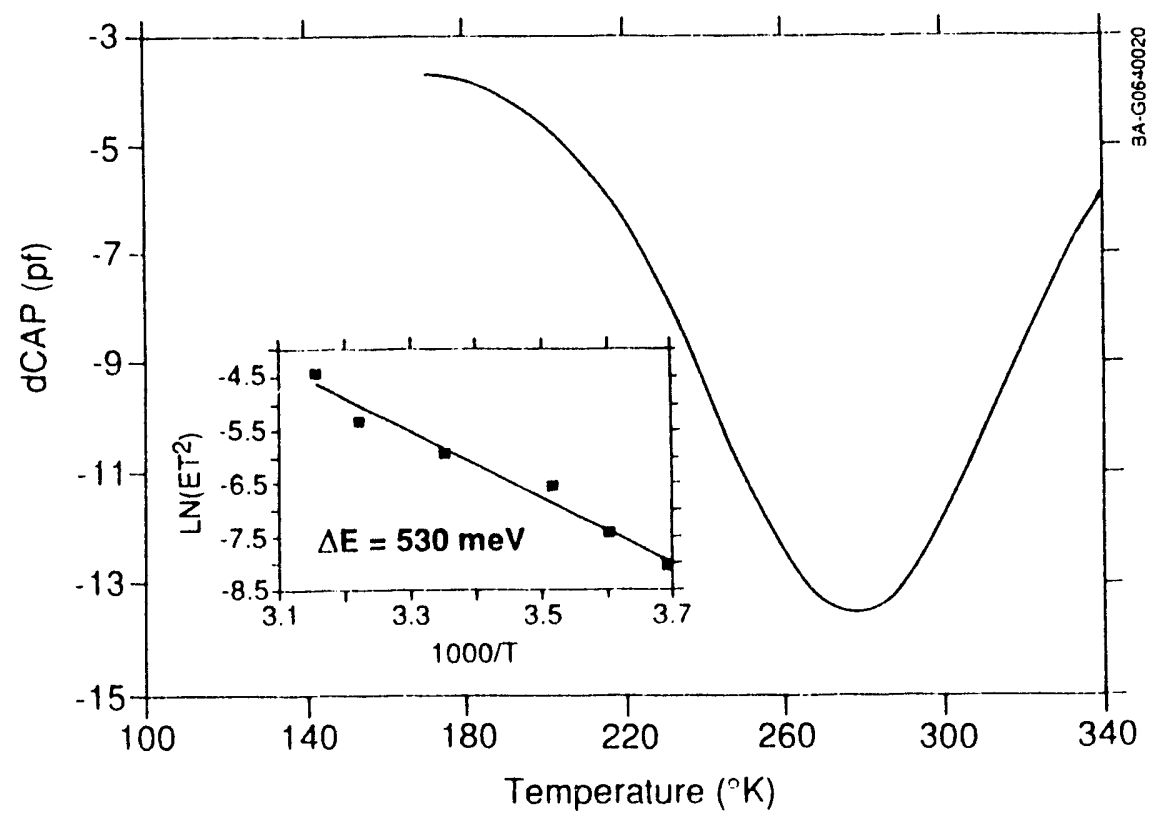

Figure 4.28: DLTS spectrum and Arrhenius plots of $(\mathrm{Cd}, \mathrm{Zn}) \mathrm{S} / \mathrm{CIS}$ thin-film junction (after annealing at $120^{\circ} \mathrm{C}$ for $15 \mathrm{~min}$.). 
various samples of similar composition have shown that these can be dominated by different types and concentrations of intrinsic defects. This could be partially attributed to the preparation conditions which are somewhat uncontrollable because of the ternary nature of the material. In addition, the surface treatment during sample preparation also almost certainly modifies the dominant defect states. Therefore, a systematic study that relates the defect configuration of the material to the growth conditions of both thin films and single crystals is necessary.

\subsubsection{Summary}

The principal observations, as they are summarized below, have been very useful to identify methods to produce improved CIS devices.

- The starting composition of single crystals of CIS provides a guide as to the likely defect levels as evidenced (for example, by the presence of transitions involving copper vacancies in samples which were copper deficient).

- However, energy levels and defect concentrations may be different for samples which have nominally identical compositions; they are evidently more influenced by the detail of fabrication and subsequent thermal or mechanical processing steps.

- Although a Se excess always leads to crystals which are p-type, even with almost exactly 50 at. \% Se, it is possible to obtain p-type conductivity provided the crystals are $\mathrm{Cu}$ poor.

- The effect of mechanical polishing is governed by the initial defect configuration and cannot be predicted in advance.

- It was also found that CIS crystals dominated by $\mathrm{V}_{\mathrm{Cu}_{\mathrm{y}}}$ and $\mathrm{Cu}_{\mathrm{ln}}$ defects are characterized by higher carrier concentration and carrier mobility. This improves the junction characteristic provided that the material is not highly compensated or suffering from a high density of interface states or undesired intrinsic defect states.

- Extrinsic surface states can be produced in the band gap of the single crystal material as a result of surface treatment (mechanical polishing, in particular), heat treatments, and surface oxidation during device fabrication. The origin, energy location, and the density of the surface/interface states created by a specific surface treatment depends not only on the type and amount of surface damage resulting from this treatment, but also on the deviation of the molecularity or stoichiometry of that surface. 
- From correlation between the variation in the defect state density and the effect of heat treatment and surface treatment on the integrated PL intensity, it is concluded that the performance of the bulk crystal devices is dominated by the additional extrinsic surface and deep recombination centers resulting from the formation of the interfacial layer during the device fabrication procedures.

- Single crystal heterostructure cells exhibit poor performance because they have a much greater concentration of traps near the middle of the forbidden gap. It is suggested that these act as efficient recombination centers of excess photogenerated carriers, thus increasing the reverse current and decreasing the open circuit voltage. The high density of this trap is probably associated with the polishing step that is necessary for the fabrication of single crystal cells.

- Simulation of the bulk rate performance at various interface recombination velocities, and interface state densities and distributions is, therefore, necessary for the further elucidation and quantification of these effects.

\subsubsection{Spectrophotometery Measurements}

\subsubsection{Measurements on EVA samples}

Optical measurements were carried out on EVA samples provided by John Pern as part of a study on the degradation mechanisms of the EVA. The data acquisition software was modified to deal with the absorptance measurements. These measurements were made in the normal transmittance mode as well as in the total transmittance mode using the integrating sphere. Several samples were investigated in the study including: virgin samples with no aging history, samples with different periods of exposure; and samples with and without UV stabilizers.

\subsubsection{Measurements on ISET CuInSe ${ }_{2}$ films}

Detailed optical analysis of the component films and the stacks used in the fabrication of CuInSe ${ }_{2}$ cells fabricated at ISET was carried out. Two sets of samples consisting of $\mathrm{CdS}, \mathrm{ZnO}$ and $\mathrm{CdS} / \mathrm{ZnO}$ deposited on glass substrates. Integrated and diffuse reflectance and total transmittance measurements were performed. From the diffuse reflectance data, it was found that CdS films deposited on glass were smooth compared to $\mathrm{ZnO}$ films on glass which exhibited significant surface roughness. CuInSe ${ }_{2}$ films deposited on Mo coated glass substrates exhibited diffuse reflectance values, approximately equal to the total reflectance. implying a very high degree of surface texturing. Texturing effects with the optimal design of the stack have been 
responsible for considerable reduction of reflectance losses in these cells. Absorption in $\mathrm{ZnO}$ was considerably different for the different thicknesses used in these sets.

Similar optical analysis was carried out on CuInSe ${ }_{2}$ cells, jrepared within the Device Development Group, and it was determined that the optical properties were dominated by the CdS layer thickness. For cells with near optimal thickness of CdS, it was possible to reduce reflection losses to approximately $5 \%$ using quarter wavelength coating of ITO. These experiments are still at the preliminary stage and further work is in progress for further optimization of the optical stack and cell performance.

\subsubsection{Measurements on ARCO ZnO}

Optical analysis of a $\mathrm{ZnO}$ film, deposited on a glass substrate, prepared by ARCO Solar was also carried out. The film exhibited slightly less absorption loss for a comparable value of sheet resistance. It is difficult to compare this result, with films from other sources, as an accurate estimate of the thickness of the film is not available.

\subsubsection{Internal Quantum Efficiency of InP Cells}

Specular and integrated reflectance, and transmission measurements were performed on InP substrates and cells. The aim of this was to extract the internal quantum efficiency of the junction from the measured external quantum efficiency and to estimate the transmitted light intensity through the substrate. As mentioned earlier, this light is utilized by the bottom cell in a tandem structure.

\subsection{ACCOMPLISHMENTS: RELATED RESEARCH AREAS}

\subsubsection{NASA Interagency Contract No. C-3000-K}

This ongoing project involves the study and fabrication of ITO/InP solar cells for NASA Lewis Research Center. During the past year, the project has supported approximately $1 / 2 \mathrm{FTE}$ and 1 post-doctoral student. In addition to the development of processes and procedures necessary to meet the contract goals, this external funding has greatly enhanced the the in-house research capability through the development of state-of-the-art technologies which are now available to the Institute.

\subsubsection{Pure Hydrogen Plasma Treatments}

This work made use of non-deposition plasma treatments of InP to help determine how the ITO/InP junction formed. Although, initially, it was 
thought that, if Sn (from the ITO source) was not present in the plasma environment, the junction quality would be poor. Later work using a Sn-free sputter source demonstrated that this was not the case, and relatively goodquality junctions could be formed with an $\operatorname{In}_{2} \mathrm{O}_{33}$ source. After this result, and after realizing that $\mathrm{H}_{2}$ played a significant role in junction fabrication, the non-deposition $\mathrm{H}_{2}$ plasma treatment experiment was conceived. The procedure was to use a plasma etching system (Technics Planaretch II, see above) configured to use pure $\mathrm{H}_{2}$ as the plasma gas. When this was done, device results were remarkably good, culminating in a solar cell with $16.2 \%$ efficiency (Global). This result attests to the fact that: (1) Neither Sn (nor any other species in the ITO target) is required for junction formation to occur; and (2) the ITO/InP solar cell is certainly a buried. shallow-homojunction. Recently, experiments using this same equipment have begun to determine if $\mathrm{Ar}$ can be used to produce a cell with similar performance, thereby indicating that the plasma damage (and not $\mathrm{H}_{2}$ ) is a central component to the type-conversion process.

\subsubsection{TCO Layer Improvement}

Several years ago, it was realized that the addition of controlled amounts of $\mathrm{H}_{2}$ into the ITO sputtering gas greatly improved the performance of the ITO/ InP cell. However, it was also realized that the hydrogen is a strong reducing agent when the plasma interacts with the ITO target, causing the deposited ITO to have poor opto-electrical properties since the deposited material is very oxygen deficient and disordered. To account for this, only the first $5 \mathrm{~nm}$ of ITO rvas deposited in the $\mathrm{H}_{2}$ environment, and the remaining $50 \mathrm{~nm}$ was deposited in an $\mathrm{O}_{2}$-rich environment. However, unless a great deal of presputtering is performed before the $\mathrm{O}_{2}$-rich ITO film was deposited (leading to a considerable decrease in target useful life), the properties of the ITO were still poor. To remedy this situation, a second target has been installed in the system. This enables one target to be sputtered in a $\mathrm{H}_{2}$-rich environment, and the other to be sputtered in $\mathrm{an}_{2}$-rich environment. This procedure has been shown to improve the optical and electrical quality of the ITO layer. thereby leading to improved cell performance.

\subsubsection{Large Area $4 \mathrm{~cm}^{2}$ Cell Development}

Three, $4 \mathrm{~cm}^{2}$ ITO/InP solar cells have been delivered to NASA Lewis Research Center in Cleveland, Ohio. The three cell efficiencies (best to worst) were measured at 15.5\%, 15.1\% and 14.8\% (simulated AMO at SERI). When measured under simulated global conditions, the highest efficiency cell demonstrated $17.8 \%$. These cells have since been measured at NASA (simulation and high-altitude aircraft flight). allowing the SERI measurement to be compared to the official NASA AMO measurements. Additionally, one 
of the three cells will be mounted on the UoSAT-E satellite $1800 \mathrm{~km}, 98^{\circ}$ Inc. orbit) which will be launched mid-1991. This will be the first time that a SERI fabricated solar coll will be placed in earth orbit. These $4 \mathrm{~cm}^{2}$ cells employed newly developed process techniques, resulting in the highest efficiency $4 \mathrm{~cm}^{2}$ III-V cell ever produced at SERI. Because of these new procedures, these radiation hard cells demonstrated a full $1.0 \%$ higher efficiency than similar cells produced using earlier processing practices. Specifically, the new processing involves forming the top contacts using a combination of photolithographic lift-off and plasma processing, resulting very narrow (8 $\mu \mathrm{m})$, thick $(1.2 \mu \mathrm{m})$ and highly adherent grid line metallizations.

\subsubsection{Small Scale Production of $4 \mathrm{~cm}^{2}$ ITO/InP Cells}

A small scale production project has begun in which approximately $50-4 \mathrm{~cm}^{2}$ ITO/InP will be produced. This project is being performed in cooperation with ATT Inc. who is supplying tice Group with the substrate material. At the time of this report, the project has reached the stage of grid design.

Growth studies of $\mathrm{Al}_{x} \mathrm{In}_{1-x}$ As epilayers on InP substrates were continued this year. Eventually, we hope to use these high-band-gap layers as passivating window layers on InP cells to improve the device efficiency. The principle growth parameters investigated were the growth temperature $\left(620-650^{\circ} \mathrm{C}\right)$ and the V-to-III ratio (50-250). Correlations were made between the roomtemperature photoluminescence intensity, surface morphology and the growth parameters. We found that higher growth temperatures and higher V-to-III ratios resulted in smoother films with higher luminescence intensity.

Factors influencing the control of the alloy composition were also investigated. The compositional behavior was found to be very similar to that observed for $\mathrm{Ga}_{x} \mathrm{In}_{1 \cdot x}$ As layers in previous work. Based on these studies, we succeeded in depositing smooth, lattice matched layers of $\mathrm{Al}_{0.48} \ln _{0.52} A$ s on InP substrates. One attempt was made to fabricate an InP homojunction cell with an $\mathrm{Al}_{1.48} \mathrm{In}_{0.5 .22}$ As window layer. The $\mathrm{n}^{+} / \mathrm{p}$ cell utilized a window layer that was $\sim 200$ A thick and an $\mathrm{n}^{+}$emitter layer that was $\sim 900 \AA$ thick. The cell showed reasonable characteristics, however no evidence of surface passivation was observed. We plan to continue this work in the future.

Toward the end of the year, we initiated a study of InP shallow-homojunction concentrator solar cells. This project was designed to serve several research needs for both the NASA and NRL programs. To our knowledge, this is the first time that InP concentrator cells have been fabricated and studied. Our objective in this work is to investigate the cell performance as a function of concentration ratio, temperature and cell design variables such as metallization parameters and junction parameters (i.e., doping levels and layer thicknesses). The goals for this work include: (1) Determine optimum 
designs for InP shallow-homojunction concentrator cells operated under practical conditions: (2) Determine if high-efficiency InP concentrator cells can be fabricated using existing cell processing technologies (i.e., using available Entech prismatic cover materials, metallization schemes, etc.): and (3) Develop improved designs for the InP top cell in the InP/Ga ${ }_{0.47} \operatorname{In}_{0.53} \mathrm{As}$ concentrator tandem cell.

Our preliminary results indicate that we are well on the way to achieving the above-mentioned goals. So far, a small number of cells have been fabricated with different values of emitter thickness and grid line spacing. At $25^{\circ} \mathrm{C}$, the best cells have peak concentrator efficiencies of $24.3 \%$ at 128.7 suns under the direct spectrum, which is equivalent to $21.4 \%$ at 106.5 suns under the AMO spectrum. These are the highest efficiencies reported so far for InP single-junction solar cells. These results are particularly important because they were achieved using off-the-shelf Entech covers and standard epitaxial growth and device processing techniques. Hence, we have shown that the necessary technologies for fabricating high-efficiency InP concentrator cells presently exists. We have also shown that the series resistance for these cells can be reduced significantly by simply reducing the grid line spacing. This effect results in higher efficiencies at higher concentration ratios, an important result for future improvements to the InP/ $\mathrm{Ga}_{0.47}$ In $_{0.53}$ As monolithic tandem cell. We plan on doing more work in this area in the future.

\subsubsection{Spire Corporation}

This contract began in March 1990 and continued until June of 1990. At this time Spire was notified that their contract from NRL (for which SERI was a sub-contractor) may not be renewed and it was requested that work be stopped. It is to be stresset that this was in no way due to a poor technical performance but was concened with funding difficulties. This project primarily involved studying the metallization Spire was using on their epitaxially-grown InP shallow-homojunction space solar cells, which they were manufacturing and delivering to the Naval Research Laboratories. The SERI task was as follows: (1) Determine how to promote the adhesion of a $\mathrm{Cr} / \mathrm{Au} / \mathrm{Ag}$ contact stack to GaInAs: (2) Analyze the diffusion characteristics (at room temperature and elevated temperatures) of the contact stack into the GaInAs and into the InP, and into itself (intermetal diffusion): (3) Determine if another metallization stack would perform better.

\subsubsection{Effect of Ag Stress on the $\mathrm{Cr} / \mathrm{Au} / \mathrm{Ag}$ Contact}

Although this conclusion was arrived at after the contract period ended. the advice was forwarded to Spire. As mentioned above under the Adhesion section. the internal stress of $\mathrm{E}$-beam evaporated $\mathrm{Ag}$ is greatly reduced by increasing the deposition rate from $2-5 \mathrm{~nm} / \mathrm{s}$. 


\subsubsection{Diffusion of $\mathrm{Cr}$ and $\mathrm{Au}$ into InGaAs and InP}

These results were obtained after the contract period. Preliminary Auger analysis indicates that $\mathrm{Cr}$ diffuses relatively deeply into $\mathrm{InP}$. Indeed, even at the deposition temperature (no post-deposition heating), a significant $\mathrm{Cr}$ "tail" is observed to a depth of more than $10 \mathrm{~nm}$ into the InP. The effect is enhanced by post-deposition heating at $300^{\circ} \mathrm{C}$ and $400^{\circ} \mathrm{C}$. The diffusion of $\mathrm{Au}$ into InP is similar to that of $\mathrm{Cr}$. The metallized GaInAs did not arrive at SERI until after the contract ended. and has not yet been analyzed. During this work, it was found that by using Xe instead of Ar as the sputtering gas in the Auger system. sharper profiles were acquired. This observation may prove to be very useful for future work.

\subsubsection{Alternative Metallization Stack}

Since the contract ended abruptly, this question was not addressed. But based on the data analyzed in the post-contract period. it seems that studies should be conducted to replace $\mathrm{Cr}$ with $\mathrm{Ti}$. and the $\mathrm{Au}$ with $\mathrm{Pd}$ or Pt.

\subsubsection{NRL [97]}

A three-year contract with NRL has been secured to develop the InP/ $\mathrm{Ga}_{0.47} \mathrm{In}_{0.5: 5}$ As monolithic tandem solar cell. During the early phases of this program, we have fabricated and studied three-terminal versions of this device to gain an in-depth understanding of the characteristics of the component subcells. We plan to develop a two-terminal version of the tandem in later phases of the project.

The InP/ $\mathrm{Ga}_{0.47} \mathrm{In}_{0,5: 3}$ As monolithic. 3-terminal tandem cell was originally conceived for space applications because it has several advantages. including a radiation-resistant InP top cell. However. it may also be very useful in terrestrial concentrator applications since it has a high theoretical efficiency. The tandem performance under terrestrial conditions is presented here.

An illustration of the $\ln \mathrm{P} / \mathrm{Ga}_{0,47} \operatorname{In}_{0,5: 5}$ As tandem cell construction is shown in Fig. 4.29. The device consists of twelve epitaxial layers which are deposited in a continuous growth sequence. The lattice-matched, monolithic structure consists of three major components. including the $\mathrm{Ga}_{0.47} \operatorname{In}_{0.5: 5}$ As bottom cell, a middle contact region. and the InP top cell. The details and function of each of these components have been outlined previously. The three-terminal cell utilizes a two-level. interdigitated top/middle contact grid system and a contact on the back surface of the InP substrate. The Entech prismatic cover on the cell surface is an integral part of the tandem design. It directs all of the incoming photons away from the top cell gridlines and middle contact trenches, onto the InP top cell surface. 


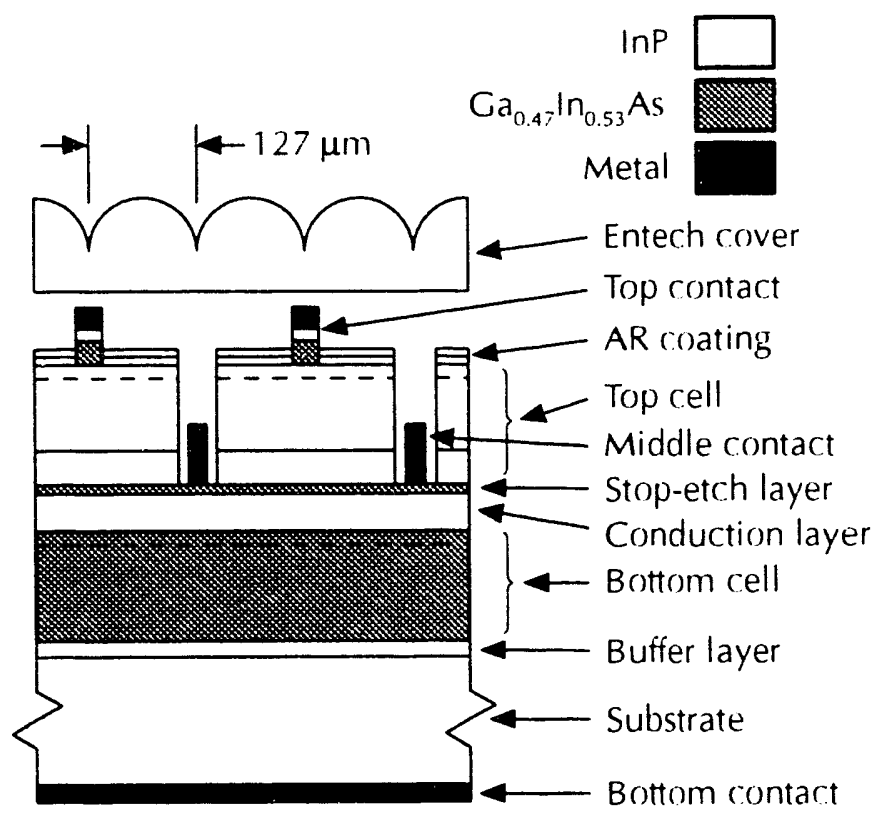

Figure 4.29: Three-dimensional, cross-sectional schematic of the InP/Ga $\operatorname{Ga}_{0.47} \operatorname{In}_{0.5 .3}$ As monolithic, 3-terminal tandem solar cell. Important features include: 1) Two-level, interdigitated top/middle grid contacts, 2) A middle contact which is common to both subcells, and 3) An Entech prismatic cover which eliminates optical losses due to grid obscuration and loss of top cell area.

A limited amount of effort was devoted to developing tandems designed to operate at one-sun intensities. The best cells had efficiencies as high as $26.2 \%$ at $25^{\circ} \mathrm{C}$ under the global spectrum. The equivalent efficiency under the AMO spectrum is $23.9 \%$, which represents a new record for two-junction, monolithic tandem cells. Several improvements are still possible for these cells and the one-sun devices will be pursued more vigorously in the future. We devoted most of our time to concentrator tandems. The results of this work are described below.

In Fig. 4.30, concentrator efficiency data are shown for a high-efficiency InP/ $\mathrm{Ga}_{(0.47} \mathrm{In}_{0.53}$ As tandem cell. The $\mathrm{Ga}_{0.47} \mathrm{In}_{0.53}$ As bottom cell has performance characteristics which are extremely close to the limits predicted by computer modeling. These suggest that further improvements in the $\mathrm{Ga}_{0.47} \operatorname{In}_{0.53} \mathrm{As}$ junction quality appear unlikely. The InP top cell also performs quite well, reaching a broad efficiency peak of $23 \%$ over the 20 to 40 suns concentration range. As $\mathrm{C}$ approaches 100 suns, each of the tandem subcells becomes series-resistance limited, resulting in a broad tandem efficiency maximum approaching $32 \%$ from 10 to 50 suns. The concentrator $\mathrm{J}_{\mathrm{sr}}-\mathrm{V}_{\mathrm{or}}$ data have been used to determine the ideality factors and reverse-saturation current densities for the top and bottom cell junctions. For the InP cell. $\mathrm{n}=1.02$ and $\mathrm{J}_{0}=9.7 \times 10-14 \mathrm{~mA} / \mathrm{cm}^{2}$, and for the $\mathrm{Ga}_{0.47} \mathrm{In}_{0.53}$ As cell. $\mathrm{n}=1.03$ and $\mathrm{J}_{0}=$ $7.0 \times 10-5 \mathrm{~mA} / \mathrm{cm}^{2}$. These values reflect that both junctions are of excellent 


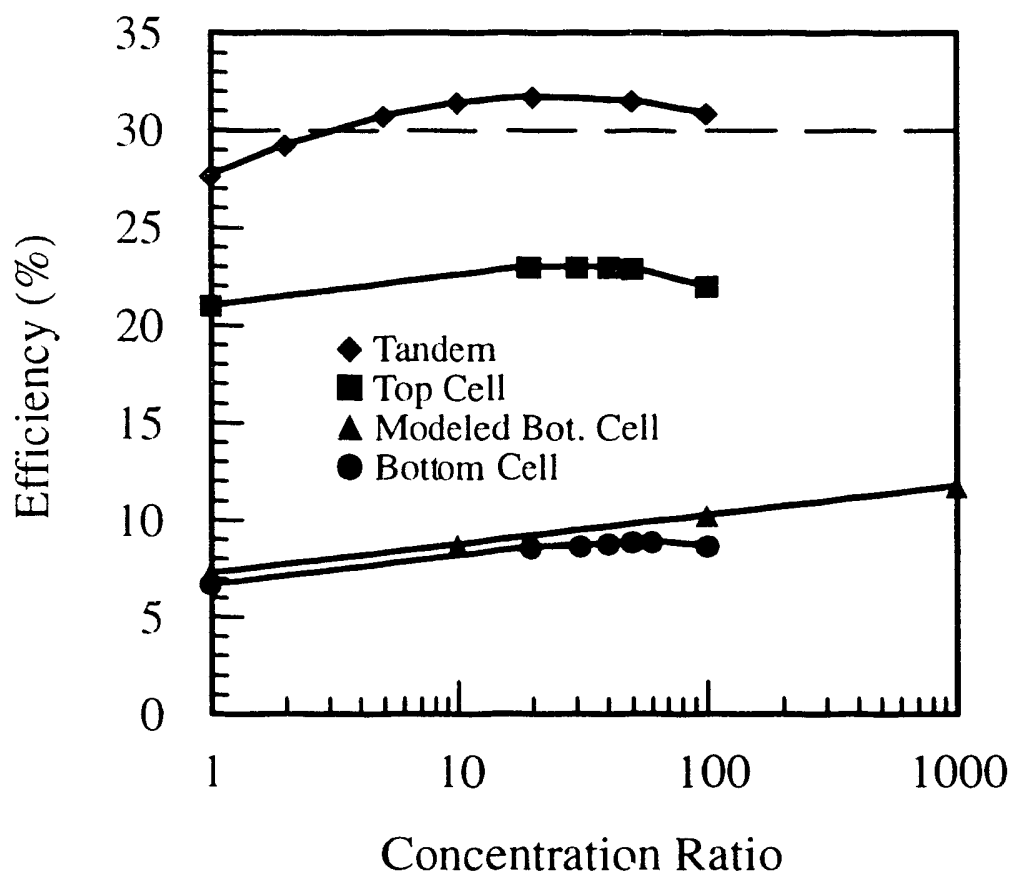

Figure 4.30: Efficiency versus Concentration Ratio data for a high-efficiency InP/ $\mathrm{Ga}_{0.47} \mathrm{In}_{0,53}$ As monolithic tandem cell. The modeled efficiency for the $\mathrm{Ga}_{(1.47} \mathrm{In}_{(1.53}$ As bottom cell are also given.

quality. Further improvements in the tandem cell efficiency are still possible. A reduction of the series resistance in each of the subcells would allow the tandem to operate at a higher efficiency at higher concentration ratios. Passivation of the InP emitter surface would lead to higher top cell efficiencies. Solutions to these efficiency-limiting problems are being pursued and concentrator terrestrial efficiencies $>35 \%$ appear possible for this tandem design.

A composite current-voltage data plot for the $\mathrm{InP} / \mathrm{Ga}_{0.47} \mathrm{In}_{0.53}$ As tandem at peak efficiency is given in Fig. 4.31. At 50 suns concentration, the top and bottom cell efficiencies are $22.9 \%$ and $8.9 \%$, respectively, which sum to give a tandem efficency of $31.8 \%$. The cell was also tested at 100 suns and the corresponding efficiency was $30.7 \%$. Listed below are the noteworthy achievements resulting from this work:

- This is the first solar cell fabricated or measured at SERI to exceed the $30 \%$ efficiency mark.

- This is the first monolithic tandem solar cell to exceed $30 \%$ efficiency. It has the second highest photovoltaic cell efficiency on record, second 


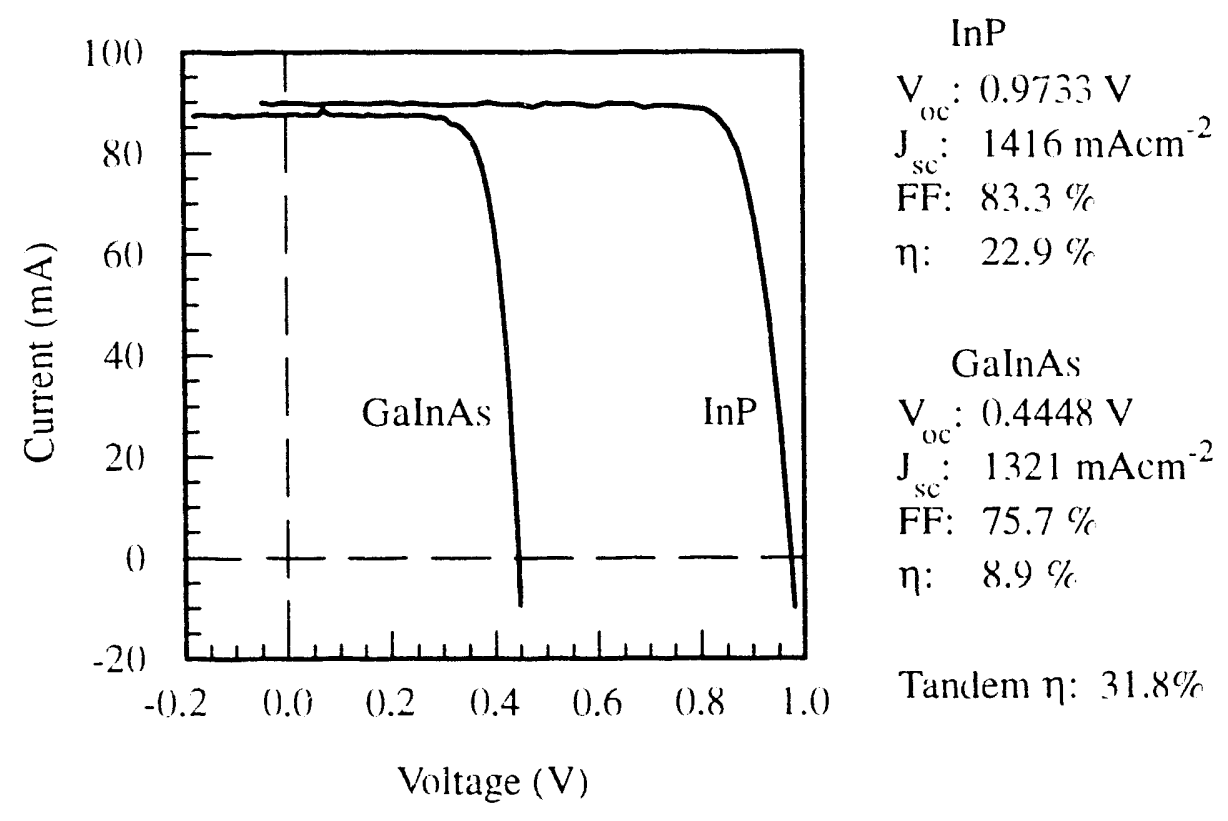

Figure 4.31: Composite Current-Voltage data for an InP/ $\mathrm{Ga}_{0.47} \mathrm{In}_{0.53}$ As tandem cell at peak efficiency under concentration $\left(50.0\right.$ suns, Direct, $\left.25^{\circ} \mathrm{C}\right)$.

only to the results achieved at Boeing for their mechanically stacked GaAs/GaSb tandem cells (measured at approximately three times the concentration ratio of the SERI tandems).

- At the time of measurement, the performances of the component subcells in the tandem represented records for individual InP and $\mathrm{Ga}_{0.47} \mathrm{In}_{0.53}$ As cells. The InP top cell was the first concentrator version to be reported and its peak efficiency of $23.0 \%$ at 40 suns (Direct, $25^{\circ} \mathrm{C}$ ) was the highest yet reported for InP cells. The peak efficiency for the $\mathrm{Ga}_{0.47} \mathrm{In}_{0.57}$ As cell (fully filtered by the InP top cell) was $8.9 \%$ at 60 suns (Direct, $25^{\circ} \mathrm{C}$ ). The high performance levels achieved for the $\mathrm{Ga}_{0.47} \mathrm{In}_{0.5: \mathrm{s}}$ As bottom cell highlight the importance of including an infrared-sensitive bottom cell in any tandem cell design. Due to the relatively low-bandgap combination provided by the $\mathrm{InP} / \mathrm{Ga}_{0.47} \ln _{0.53}$ As tandem cell, it has a distinct advantage in AMO or terrestrial concentrator applications over other GaAs-based tandems which are currently being considered.

- With this cell. we have achieved the 5-year DOE milestone of demonstrating a solar cell with a terrestrial efficiency greater than $30 \%$ at 100 suns concentration. 
The space community is extremely interested in this tandem cell design for future space power applications due to its advantageous features and recently demonstrated high efficiency. In addition, the $\operatorname{InP} / \mathrm{Ga}_{0.47} \operatorname{In}_{0.53} \mathrm{As}$ tandem cell has great potential in ultra-high efficiency terrestrial tandem cell configurations. The results reported above illustrate the utility of InP and its related lattice-matched alloys in the fabrication of high-efficiency tandem solar cells.

In conclusion, monolithic InP/Ga $\operatorname{Ga}_{0.47} \operatorname{In}_{0.53}$ As tandem cells have reached efficiencies of $31.8 \%$ at 50 suns. This is the first report of a monolithic tandem cell with an efficiency greater than $30 \%$. The $\mathrm{Ga}_{0.47} \mathrm{In}_{0.53}$ As bottom cell has near-theoretical performance at low concentration ratios, however, the InP top cell efficiency could be improved substantially with a passivated emitter and an improved ARC. If the top cell series resistance were reduced, tandem efficiencies exceeding $35 \%$ could be realized by operating at high concentration ratios.

\subsubsection{Varian Associates}

A technical services agreement was established with Varian Associates early in the year for the growth of 30 low-band-gap solar cell structures. During the course of this work, $15 \mathrm{Ga}_{0.47} \operatorname{In}_{0.53} \mathrm{As}(0.75 \mathrm{eV})$ and $15 \mathrm{Ga}_{0.25} \operatorname{In}_{0.75} \mathrm{As}_{0.54} \mathrm{P}_{0.46}$ $(0.95 \mathrm{eV})$ device structures were prepared at SERI and delivered to Varian. The structures were processed at Varian in a manner suitable for mechanical stacking under GaAs-based top cells (i.e., single-junction GaAs cells and $\mathrm{Al}_{x} \mathrm{Ga}_{1 \cdot x} \mathrm{As} / \mathrm{GaAs}$ tandem cells). In this way, Varian hoped to form 2- and 3junction tandem cells that would respond to a large portion of the solar spectrum. The low band gap cell structures provided by SERI were the key components in these new tandem cells because they were not available at any other PV laboratory. New record tandem cell efficiencies were anticipated as a result of combining the capabilities at Varian and SERI.

Results for $\mathrm{Ga}_{0.25} \mathrm{In}_{0.75} \mathrm{As}_{0.54} \mathrm{P}_{0.46}$ cells grown at SERI and processed at Varian Associates are shown in Figs. 4.32 and 4.33. The AEQE data show a very high, flat response averaging over $96 \%$ for photon energies less than $1.4 \mathrm{eV}$. These results are consistent with the internal quantum efficiencies calculated for cells fabricated at SERI. Despite obvious optical filtering by the InP window layer for photon energies greater than $1.4 \mathrm{eV}$, the response at the bandgap of GaAs $(1.425 \mathrm{eV})$ is well over $80 \%$. Thus, these cells are nearly perfect for stacking under GaAs-based top cells. The "box-car"-like AEQE also indicates that the InP cladding layers provide excellent minority carrier confinement for the $\mathrm{Ga}_{0.25} \mathrm{In}_{0.75} \mathrm{As}_{0.54} \mathrm{P}_{0.46}$ active layers. The light I-V data shown in Fig. 4.33 illustrate the potential performance of these cells under a GiaAs filter at one sun, AMO $25^{\circ} \mathrm{C}$. For these data, the short-circuit current 


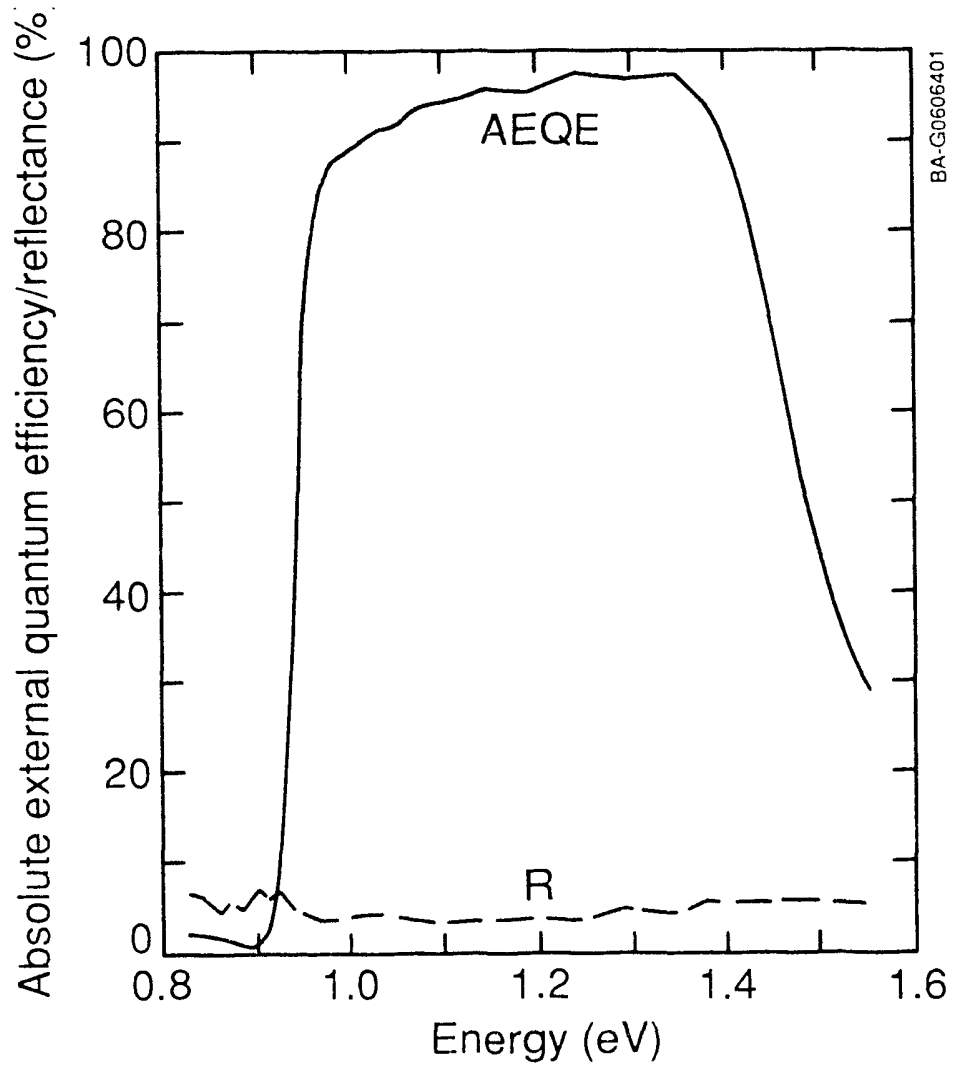

Figure 4.32: Absolute external quantum efficiency (AEQE) and reflectance (R) data for a

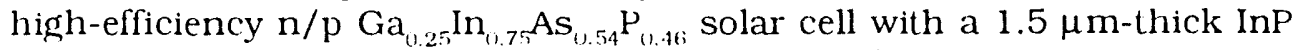
window layer, one-sun grids and an ARC. The cell structure was grown at SERI, and the device processing was performed at Varian Associates.

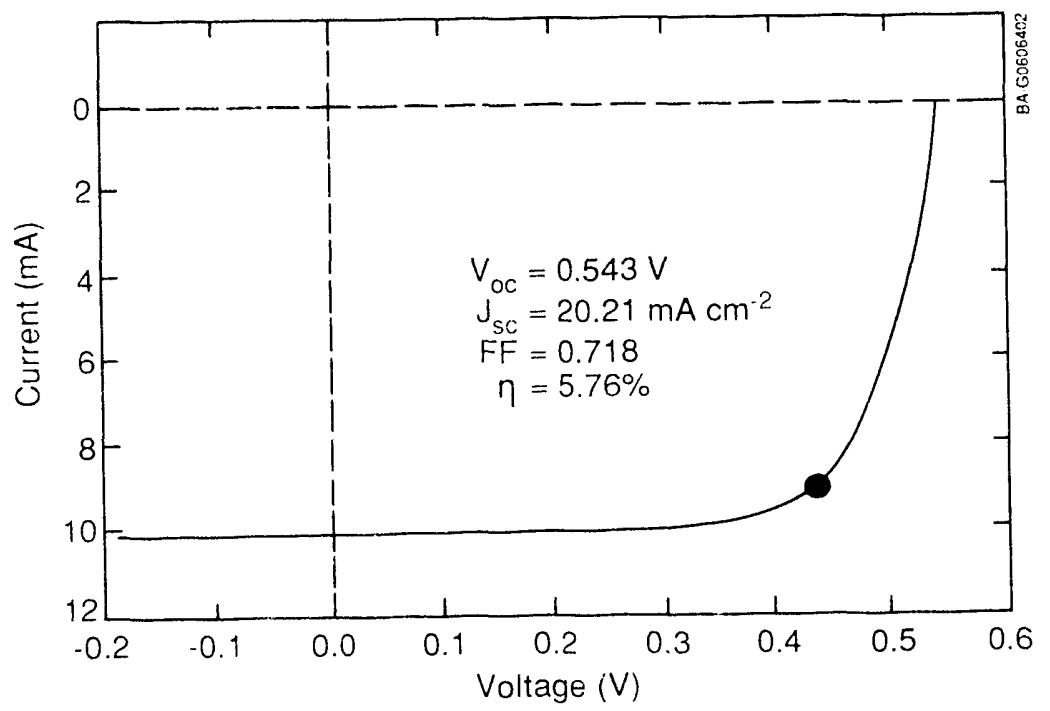

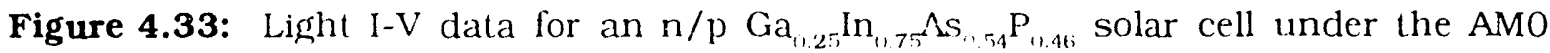
spectrum, one-sun intensity. $25^{\circ} \mathrm{C}$. The cell structure was grown at SERI. processed at Varian Associates, and has a $1.5 \mu \mathrm{m}$-thick InP window and an ARC. The short-circuit current density was set to simulate operation of the cell under a GaAs optical filter. Details are described in the text. 
for the cell was set at a value equal to that obtained through an integration of the AMO spectrum weighted by the AEQE data of Fig. 4.32 truncated at $1.39 \mathrm{eV}$. In this way, a conservative estimate of the performance under GaAs is obtained. The cell gives an efficiency of $5.8 \%$ which would result in a substantial boost to the efficiency of a GaAs-based top cell.

Varian was successful in processing the SERI-grown structures into bottom cells for mechanical stacking. Both $\mathrm{Ga}_{0.47} \mathrm{In}_{0.53} \mathrm{As}$ and $\mathrm{Ga}_{0.25} \operatorname{In}_{0.75} \mathrm{As}_{0.54} \mathrm{P}_{0.46}$ cells were stacked under Varian's $\mathrm{Al}_{0.4} \mathrm{Ga}_{0.6} \mathrm{As} / \mathrm{GaAs} \mathrm{MIC}^{2}$ tandem cells, which were grown on IR-transparent GaAs substrates. The bottom cells were series connected to the tandem top cells. The resulting three-junction tandems were then tested under the one-sun AMO spectrum at $25^{\circ} \mathrm{C}$. To the best of our knowledge, these are the first three-junction, III-V tandem cells ever fabricated. The best $\mathrm{Al}_{0.4} \mathrm{Ga}_{0.6} \mathrm{As} / \mathrm{GaAs} / \mathrm{Ga}_{0.47} \mathrm{In}_{0.53}$ As tandem had an efficiency of $23.8 \%$. The $\mathrm{Ga}_{0.25} \mathrm{In}_{0.75} \mathrm{As}_{0.54} \mathrm{P}_{0.46}$ bottom cells gave even better results: the $\mathrm{Al}_{0.4} \mathrm{Ga}_{0.6 .} \mathrm{As} / \mathrm{GaAs} / \mathrm{Ga}_{0.2 .5} \ln _{0.75} \mathrm{As}_{0.54} \mathrm{P}_{0.46}$ tandems had efficiencies as high as $25.2 \%$, which is a new record for one-sun AMO conditions. Significantly higher efficiencies were expected for this device, however, the fill factor of the tandem top cell was unusually low. A one-sun AMO efficiency of $30 \%$ was the goal for the project. Unfortunately, Varian shut down its GaAs-based solar cell research before the remaining work could be completed.

\subsubsection{DDF Projects}

The group has been exceptionally successful in obtaining funds from the above source during the last two years. Since the criteria for submission to the DDF have been revised, it is now possible for proposals to be developed which may influence the future of the mainstream DOE program. This is known as the Institutional category. Because of this, it has been possible to submit several proposals which previously would not have been appropriate. Because it is now apparent that at least two of these (those concerned with the solution growth of semiconductors) are to be included in the DOE inhouse program for FY 1992, by this measure, the projects can be assessed as successful. The first project concerned with the heteroepitaxialy growth of InP on low cast substrates, was not originally intended to fall into this category although it now seems likely to have an influence on the future program. All three of these projects will be discussed below.

\subsubsection{Heteroepitaxial InP solar cells on GaAs and Si Substrates [98]}

InP solar cells are particularly attractive for space applications due to their resistance to radiation damage and demonstrated high energy conversion efficiency under the AMO spectrum ${ }^{7.8}$. Single-crystal InP wafers, however, have characteristics which make them generally undesirable for solar cell 
fabrication and operation. These include high cost. high fragility, high mass density and low thermal conductivity. Thus, in order to promote the widespread use of InP cells in space, it is critical that techniques are developed for fabricating high-efficiency, thin-film InP cells. Three approaches are currently under investigation for solving this problem. These include; CLEFT" $^{*}$ using a bulk InP wafer, chemical separation ${ }^{10}$ from an InP wafer, and heteroepitaxy onto single-crystal materials with more desirable characteristics. Of the three options, heteroepitaxy may prove to be the preferred choice since, ultimately, large-area thin films of InP may be too difficult to handle and process on a large scale. Furthermore, it is uncertain whether the InP bulk substrates used in the CLEFT and chemical separation processes will actually be reusable. Heteroepitaxial cells have the advantage of being fully compatible with existing cell processing technologies as well as being based on mature single-crystal wafer technologies in materials such as GaAs, Ge and Si.

Due to the large differences in lattice constant and thermal expansion coefficient between InP and the above-mentioned materials, problems generally arise which inhibit the growth of high-quality InP heteroepilayers. For example, the lattice constant mismatch is $3.7 \%$ between InP and GaAs and $7.5 \%$ between InP and Si. Such large mismatches result in high mechanical stresses in the resulting epilayers which, in turn, lead to the generation of a high density of defects. The defects include dislocations, stacking faults and even microcracks. Several techniques have been investigated for reducing the density of defects in the InP layers, thereby reducing their deleterious effects. These have included thermally cycled growth, post-growth annealing and inclusion of an intermediate GaAs layer for the case of InP grown on a Si substrate. Limited success has been realized with these procedures and InP epilayers with dislocation densities of $\sim 3 \times 10^{8}$ $\mathrm{cm}^{-2}$ and minority carrier lifetimes of $\sim 1 \mathrm{~ns}$ or less in undoped material are reported for the best cases when grown on GaAs substrates ${ }^{11}$. Unfortunately, InP layers with these properties are of insufficient quality for the fabrication of high-efficiency solar cells. Using post-growth annealing, the highest efficiency for InP cells grown directly on GaAs substrates is $10.8 \%$ (one-sun, AMO $25^{\circ} \mathrm{C}^{12}$. Even lower efficiencies have been reported for InP cells grown on Si substrates ${ }^{13}$.

In previous work ${ }^{14}$, we reported on the use of a novel structure for the growth of high-quality InP epilayers on substrates such as GaAs, Ge and Si. A full description of the device structure concept is given in reference ${ }^{15}$. The structure utilizes a compositionally graded $\mathrm{Ga}_{x} \mathrm{In}_{1-x}$ As layer disposed between the bulk substrate and the InP device layers. This serves to reduce the dislocation density in the InP device layers substantially when compared to the conventional techniques discussed above. In this work, substrates of GaAs and GaAs/Si were placed side by side in the growth reactor and 
identical structures were deposited on each. The resulting InP epilayers were then characterized using transmission electron microscopy (TEM). electron-beam-induced current (EBIC) and photoluminescence-decay (PLdecay) lifetime techniques to assess the defect density and minority carrier lifetime. $\mathrm{n}^{+} / \mathrm{p}$ shallow homojunctions were grown into the InP layers and solar cells with grids designed for one-sun operation were processed from the structures grown on the GaAs substrates only. Additionally, structures with three different $\mathrm{Ga}_{x} \operatorname{In}_{1-x}$ As graded layer thicknesses $(8,12$ and $20 \mu \mathrm{m}$ ) were grown and characterized: the InP material and solar cell quality was essentially independent of the thickness chosen in this range. With this structure, dislocation densities of $3 \times 10^{7} \mathrm{~cm}^{-2}$ and minority carrier lifetimes of over 3 ns were achieved in the InP layers using either GaAs or GaAs/Si substrates. Furthermore, the InP epilayers were completely free of microcracks in both cases, which is an extremely important result for high-quality solar cell fabrication. InP solar cells with one-sun efficiencies of $13.7 \%$ (AMO, $25^{\circ} \mathrm{C}$ ) and $15.7 \%$ (global, $25^{\circ} \mathrm{C}$ ) were fabricated on GaAs substrates using an $8 \mu \mathrm{m}$-thick $\mathrm{Ga}_{x} \operatorname{In}_{1-x}$ As graded layer. Unfortunately, pinholes in the InP layers grown on the GaAs/Si substrates, resulting from surface contamination prior to growth, precluded the fabrication of cells in this case. However, it seems reasonable to assume that InP cell efficiencies, similar to those achieved using GaAs substrates, should be possible on Si substrates due to the similar dislocation densities, and minority-carrier lifetimes observed in the InP layers grown on either substrate type.

In the remainder of this section, we describe the epitaxial growth, fabrication, and characterization of concentrator heteroepitaxial InP solar cells, grown on GaAs substrates using a compositionally graded intermediate structure, similar to that described above. The cell performance has been determined as a function of the concentration ratio and the operating temperature. We have also investigated the behavior of the cell performance parameter temperature coefficients as a function of the concentration ratio. The details of this work are described in the sections which follow.

A schematic diagram of the heteroeopitaxial (HE) InP solar cell structure grown on a GaAs substrate is given in Fig. 4.34. The structure is initiated with a thin buffer layer of $\mathrm{p}$-GaAs, followed by the $\mathrm{p}-\mathrm{Ga}_{x} \mathrm{In}_{1-x}$ As linearly graded layer (LGL), which has a thickness of $8 \mu \mathrm{m}$, for the results reported here. The LGL is followed by a buffer layer of $\mathrm{Ga}_{0.47} \mathrm{In}_{0.53} \mathrm{As}$ which is lattice matched to InP. The InP solar cell layers are finally deposited at the top of the structure, and these comprise a high-efficiency $n+/ p$ shallow homojunction (SHJ) cell structure (in Fig. 4.34, "BSFL" is an acronym for "back-surface field layer"). A back contact of pure Au is applied to the exposed bottom surface of the GaAs substrate. The top grid contact on the surface of the InP cell emitter is also composed of pure Au. A 2-layer antireflection coating is deposited onto the front surface of the cell structure, 


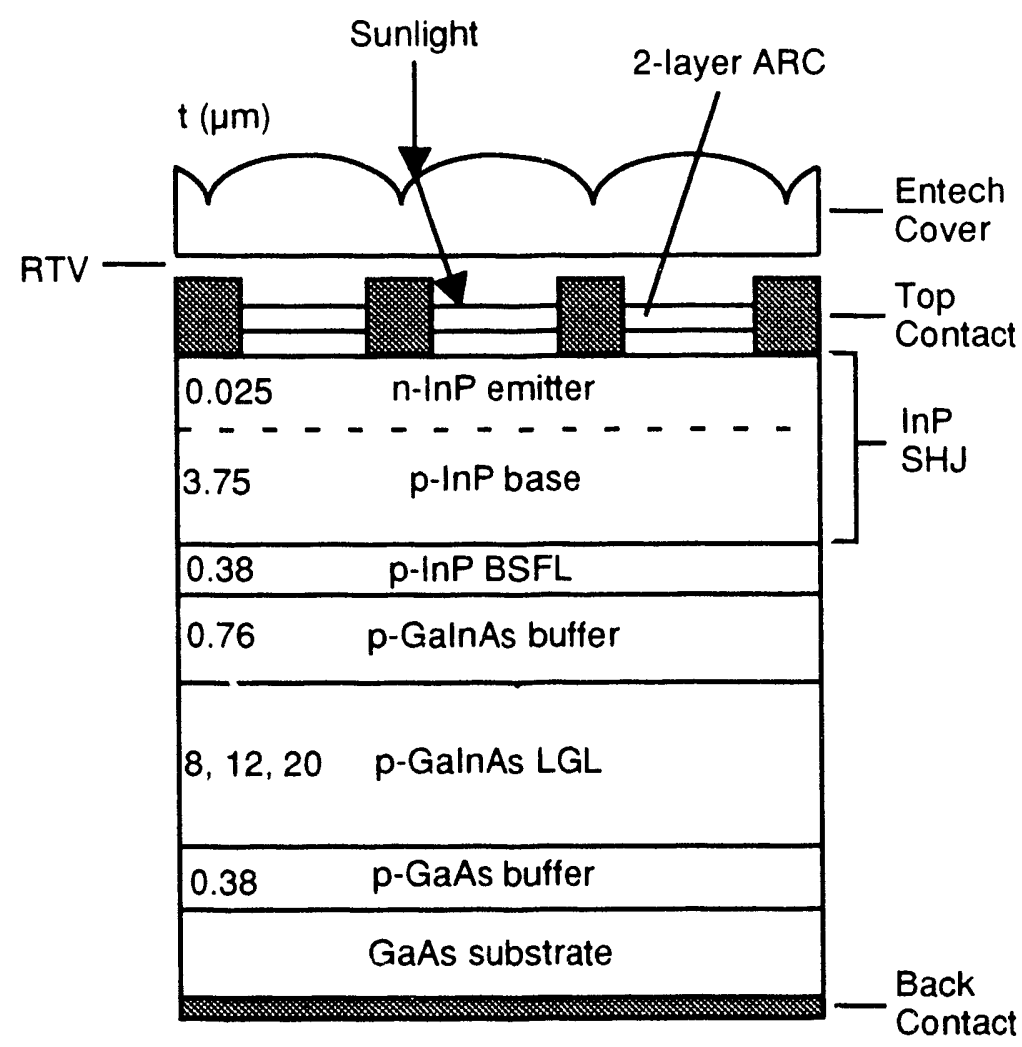

Figure 4.34: Schematic diagram of the HE InP concentrator solar cell structure on a GaAs substrate.

and an Entech prismatic cover is also incorporated into the structure to allow for a high top-contact-metallization coverage ( 20\%). Further details of the device structure are discussed below.

The heteroepitaxial solar cell structures were grown by atmosphericpressure metalorganic vapor-phase epitaxy (APMOVPE) as described previously. $\mathrm{Zn}$-doped p+-GaAs wafers oriented $2^{\circ}$ off the (100) were supplied by Sumitomo Electric, Inc. and used as substrates. These were loaded directly into the growth reactor as received from the vendor (i.e., without any pre-growth cleaning or etching steps). Prior to growth, the GaAs substrates were heated to $700^{\circ} \mathrm{C}$ for 10 minutes with arsine flowing into the reactor vessel. Growth was then carried out at a constant temperature of $650^{\circ} \mathrm{C}$. The structures were grown at a rate of $75-175 \mathrm{~nm} / \mathrm{min}$. in a continuous sequence of steps (i.e., without stop-growth periods at the heterointerfaces). A typical growth run takes about 2.5 hours, including the time required for warm-up and cool-down of the reactor vessel. The entire process is controlled and monitored using a home-built, PC-based control system.

The epitaxial structures were then processed into completed concentrator solar cells using conventional techniques. Ohmic, low-resistance contacts were made to both the back surface of the $\mathrm{p}+-\mathrm{GaAs}$ substrate and the $\mathrm{n+-}$ 
InP emitter surface using electroplated $\mathrm{Au}$ as deposited. The back surface of the GaAs substrate was etched in $1 \%$, by volume, bromine in methanol for 5 minutes at room temperature prior to applying the metallization. The top contact and device mesa geometries were defined by photolithographic techniques using positive photoresist. The top contact grids were specially designed to accomodate an overlying Entech prismatic cover which was originally designed for concentrator GaAs solar cells ${ }^{16}$. A center-to-center grid line spacing of $127 \mu \mathrm{m}$ was used and the individual gridlines have a cross-sectional area of $\sim 125 \mu \mathrm{m}^{2}(\sim 25 \mu \mathrm{m}$ wide by $\sim 5 \mu \mathrm{m}$ high). A busbar is included at both ends of the grid lines in this design to allow for the simultaneous placement of test probes at both ends. This aspect of the grid design results in better performance under concentration. Through the use of the Entech cover, it is possible to cover $\sim 20 \%$ of the cell surface with the grid metallization without incurring any photocurrent losses due to grid obscuration. This allows for ample grid metallization on the cell which results in low electrical power losses within the top contact. As such, the Entech cover has proven to be a very important component in the fabrication of high-efficiency concentrator cells. Electrical isolation of the individual cells was accomplished by etching moats through the $n+/ p$ InPjunction with concentrated $\mathrm{HCl}$. A two-layer antireflection coating of $\mathrm{ZnS}(\sim 55 \mathrm{~nm})$ followed by $\mathrm{MgF}_{2}(\sim 95 \mathrm{~nm})$ was then deposited on the front surface of the device wafer. The concentrator cells were completed by installing the Entech cover. A typical array of completed heteroepitaxial InP concentrator cells is shown in Fig. 4.35. The effect of the Entech cover is also illustrated in this figure. Each individual cell has an area of $0.0746 \mathrm{~cm}^{2}$ which is computed by subtracting the areas of the two busbars from the total device mesa area (this is a standard area definition for concentrator solar cells ${ }^{17}$ ).

The performance of the concentrator cells was characterized by measuring the absolute external quantum efficiency (AEQE), as a function of temperature as well as the illuminated current-voltage characteristics, as a function of the temperature, and the concentration ratio. The latter data sets were used to calculate the dependence of the cell performance parameter temperature coefficients on the concentration ratio. The measurement techniques have been described previously. All of the results reported here are referenced to the AMO spectrum ${ }^{18}$. A discussion of the cell performance is given in the following section.

Initially, the current-voltage characteristics for the cells were measured as a function of temperature under one-sun AMO conditions in order to obtain the necessary information for evaluating the efficiency under concentration (i.e., the one-sun short-circuit current $\left(I_{\mathrm{si}}\right)$ is needed to calculate the concentration ratio for concentrator measurements). To within experimental error, we found $I_{\mathrm{sr}}$ to be independent of temperature. The AEQE data shown in Fig. 4.36 illustrates why $I_{s i}$ is temperature independent. As expected, the 


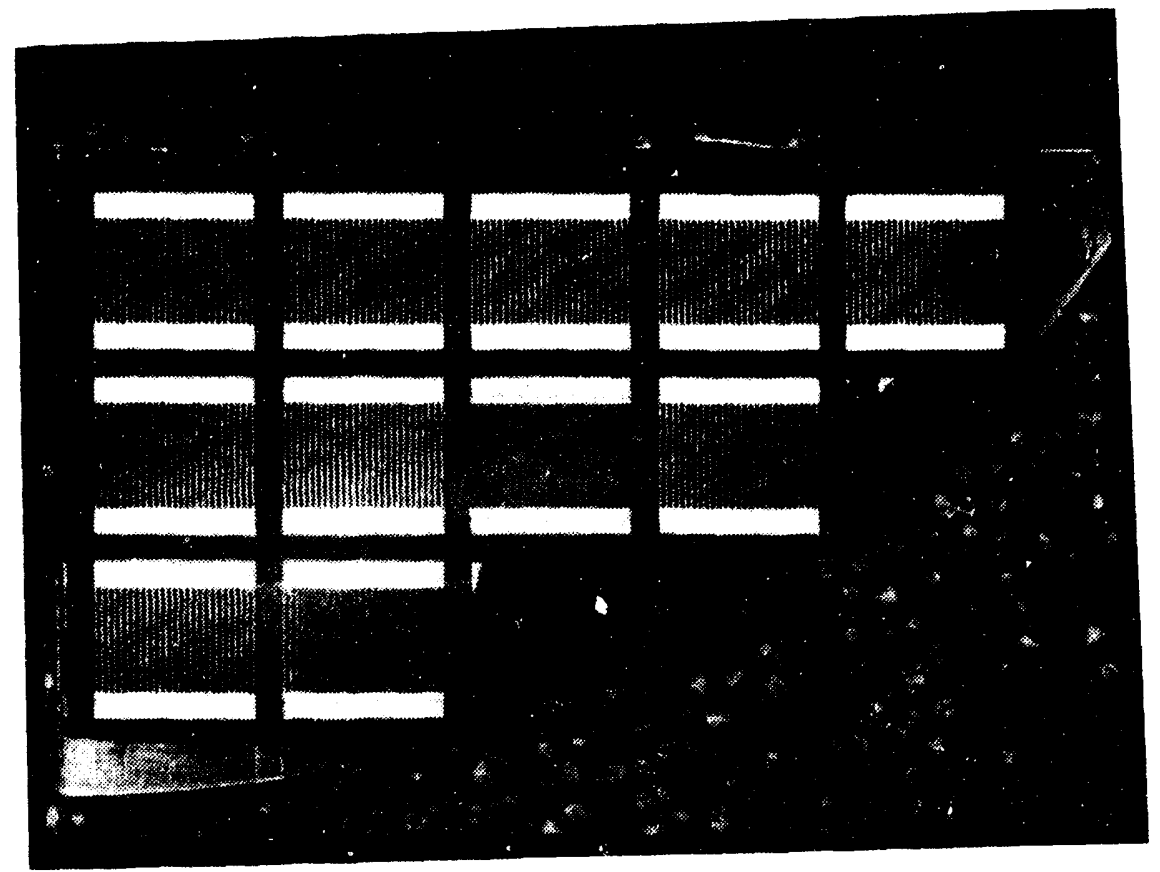

Figure 4.35: Plan-view photograph of a typical array of HE InP concentrator cells. The cell in the center of the micrograph has an Entech cover properly installed.

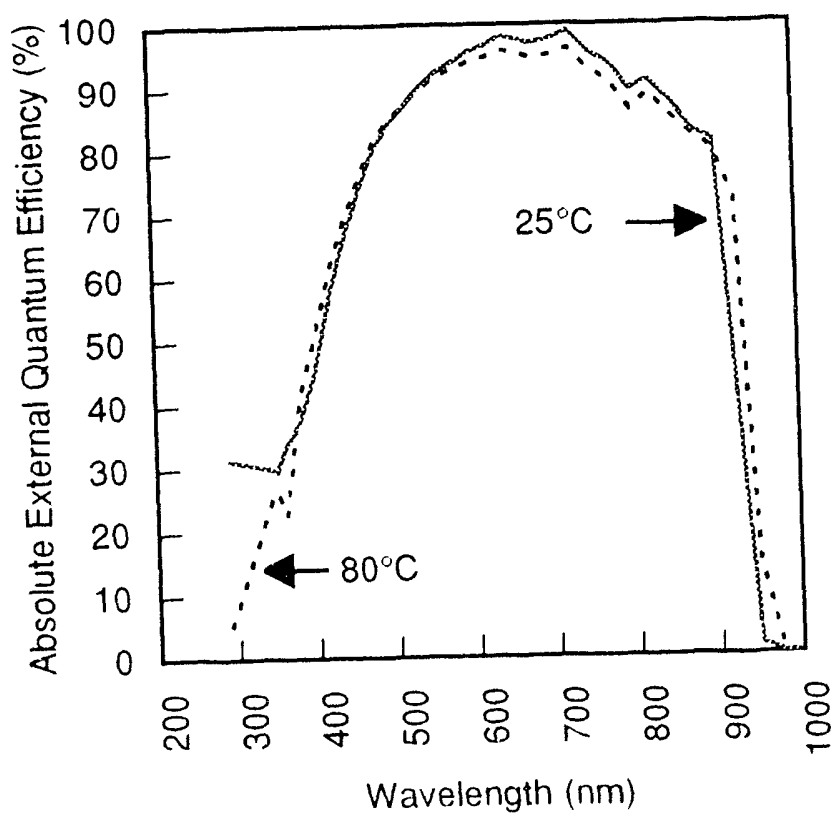

Figure 4.36: AEQE data for an $\mathrm{HE}$ InP concentrator cell at $25^{\circ} \mathrm{C}$ and $80^{\circ} \mathrm{C}$. 
InP band edge shifts to longer wavelengths as the temperature increases and one would normally expect an increase in $I_{s}$ due to this effect. However, a concomitant decrease in the short- and mid-wavelength response is also observed, for these devices, as the temperature increases, which offsets any increase in $I_{s i}$ due to the band gap shift. Thus, $I_{s i}$ remains essentially constant as the temperature is increased. Note that the blue response for these cells is relatively low. This characteristic is typical of shallowhomojunction solar cells, which have a high surface recombination velocity. We have shown in previous work that graded emitter doping profiles can be used to improve the blue response in these cells ${ }^{19}$. However, a technique for effectively passivating the emitter surface needs to be developed in order to realize InP cells with near-theoretical performance characteristics.

The HE InP cell performance was then tested as a function of the temperature. The AMO concentration ratio and the results from these measurements are shown in Figs. $4.37 \&$ 4.38. The AMO efficiency, shown in Fig. 4.37, increases rapidly at low concentration ratios, and then reaches a broad plateau for concentration ratios of $\sim 40$ or more. At $25^{\circ} \mathrm{C}$, the cells have efficiencies of close to $19 \%$ over a broad range of concentration ratios. This value decreases to $\sim 16 \%$ as the temperature is increased to $80^{\circ} \mathrm{C}$. The broad plateau in efficiency can be understood by examining the open-circuit voltage $\left(\mathrm{V}_{\mathrm{or}}\right)$, and fill factor $(\mathrm{FF})$ versus concentration ratio data given in Fig. 4.38. The behavior of $\mathrm{V}_{\text {or }}$ is as expected. In fact, when the $\mathrm{V}_{\mathrm{or}}$ data are plotted against $\ln$ (concentration ratio), a straight line is obtained. However, the FF data indicate that the cells quickly become series-resistance limited as the concentration ratio is increased beyond $\sim 20$ suns. Additionally, this effect appears to be enhanced as the operating temperature is increased. An analysis of the resistance components contributing to the overall series resistance for these cells shows that the emitter sheet resistance is primarily responsible for limiting the concentrator cell performance. A lower emitter sheet resistance or a smaller grid line spacing will be necessary in order to improve this aspect of the cell performance. The broad plateau in efficiency versus concentration ratio is seen to be due to offsetting effects of the $\mathrm{V}_{\text {oc }}$ and $\mathrm{FF}$ as the concentration ratio increases.

Current-voltage data for an $\mathrm{HE}$ InP concentrator cell at peak efficiency are shown in Fig. 4.39. At $25^{\circ} \mathrm{C}$, the efficiency reaches $18.9 \%$ under the AMO spectrum at 71.8 suns. The equivalent efficiency under the direct spectrum is $21.4 \%$ at $~ 88$ suns. The peak AMO efficiency at $80^{\circ} \mathrm{C}$ is $15.7 \%$ at 75.6 suns. These values are very encouraging and demonstrate that HE InP cells have the potential to reach high efficiencies, at high concentration ratios, and high temperatures. Additionally, these results show that the HE cell efficiencies improve dramatically when operated under concentration. 


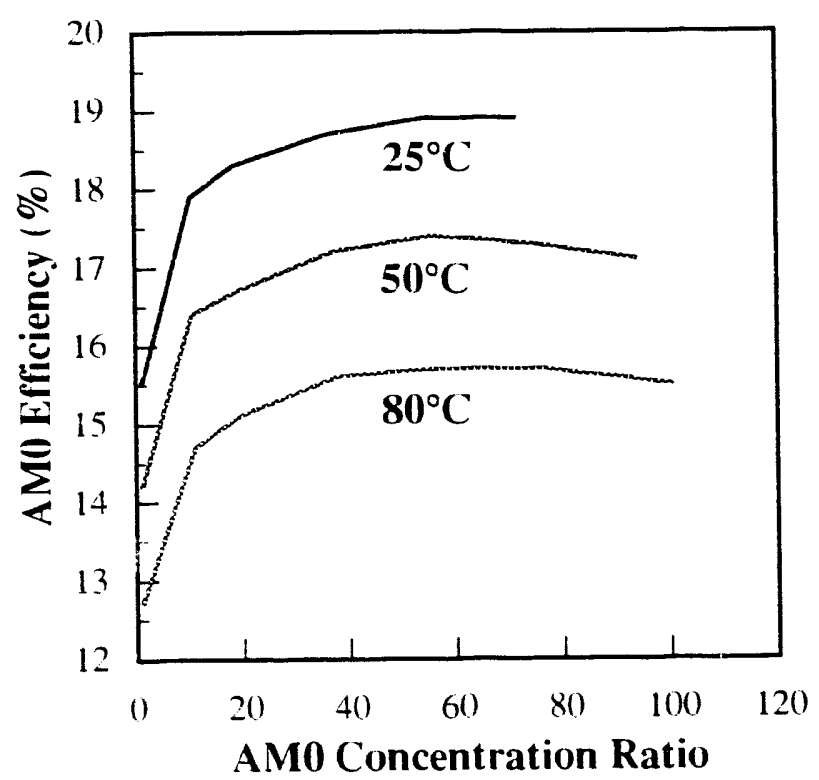

Figure 4.37: AMO conversion efficiency data for an HE InP concentrator cell as a function of the operating temperature and AMO concentration ratio.

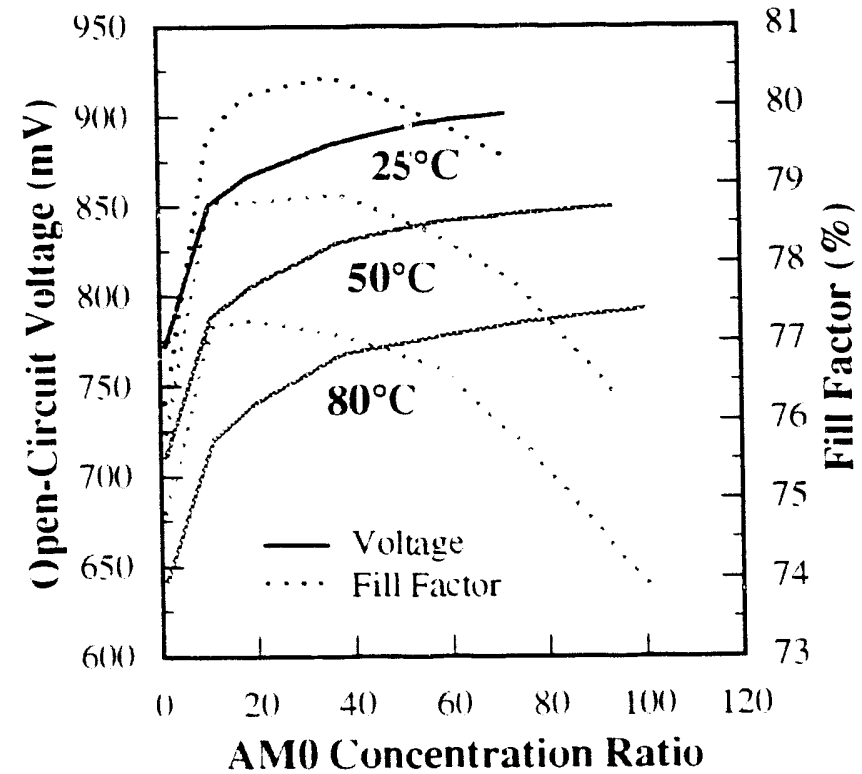

Figure 4.38: Open-circuit voltage and fill factor data for an HE InP concentrator cell as a function of the operating temperature and AMO concentration ratio. 


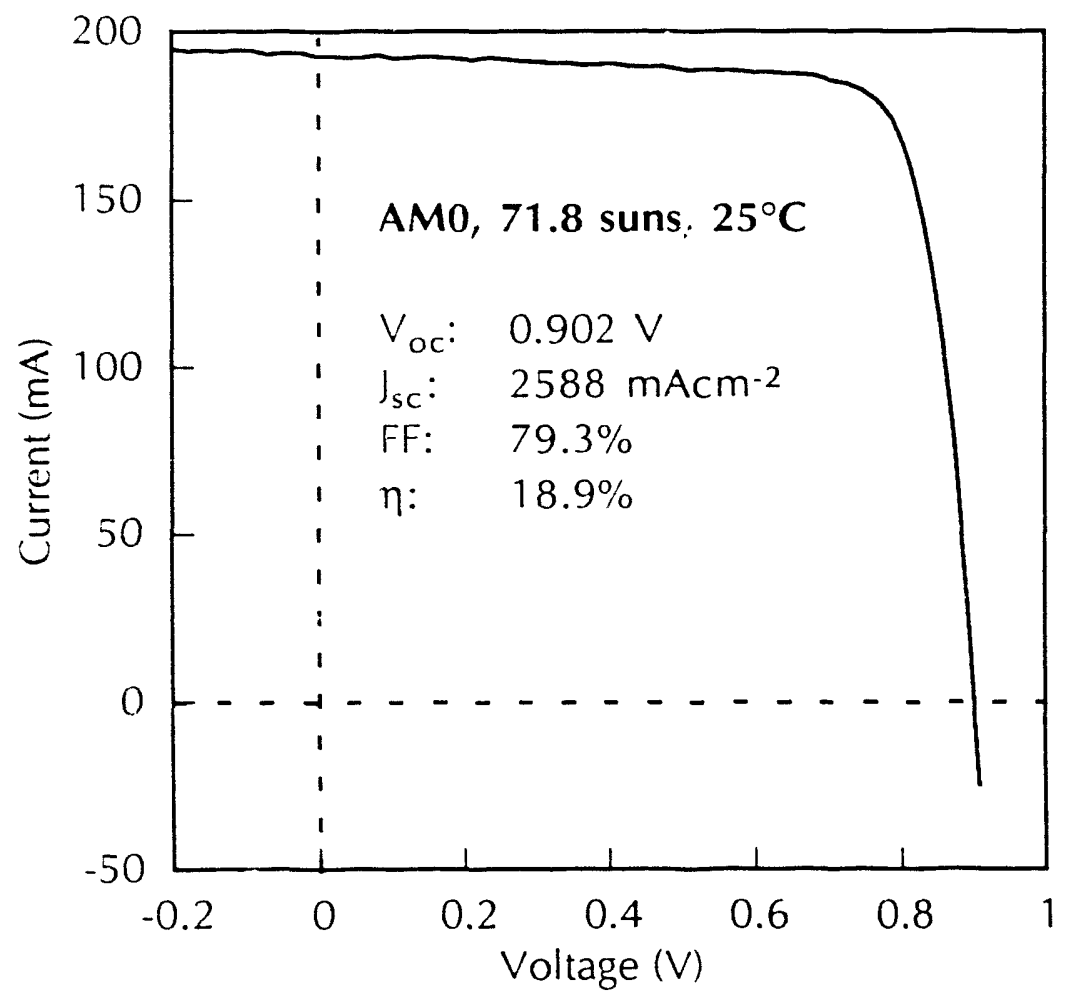

Figure 4.39: Current-voltage data for an $\mathrm{HE}$ InP concentrator cell at peak efficiency under concentrated AMO illumination.

In summary. high-efficiency heteroepitaxial InP solar cells have been fabricated on GaAs substrates using a novel compositionally graded intermediate layered structure. One-sun cells have AMO efficiencies as high as $13.7 \%$ at $25^{\circ} \mathrm{C}$. The concentrator cell performance has been characterized as a function of the temperature and the AMO concentration ratio. Peak concentrator AMO efficiencies of $18.9 \%$ at 71.8 suns, $25^{\circ} \mathrm{C}$ and $15.7 \%$ at 75.6 suns. $80^{\circ} \mathrm{C}$ have been obtained with these cells, which are the highest efficiencies yet reported for InP heteroepitaxial solar cells. The advantages of operating the $\mathrm{HE}$ InP cells under concentration include reduced cell area and higher conversion efficiencies.

The cell performance is presently limited by three main loss factors including: (1) Recombination at the surface of the emitter layer. (2) A high emitter-layer sheet resistance leading to reduced $\mathrm{FF}$ values at high concentration. (3) A high density of threading dislocations in the active cell layers. Improvements in any of these areas will lead to increased cell efficiencies.

Technologically. it would be important, and immediately useful, if the results obtained in this work for InP cells grown on GaAs substrates could be duplicated using Si substrates. Such a result would make HE InP cells a viable contender for space power applications and efforts toward this goal are currently underway. 


\subsection{FUTURE WORK}

We plan to continue making progress in all the above areas assuming that appropriate staff levels are permitted. The objective in the area of equipment development, is to improve the specroscopic ellipsometer so that the spectra can be obtained more rapidly. Interactions with the sub-contracted researchers are already developing and it is anticipated that this will continue. In addition, it is planned to complete the fabrication of the admittance spectrometer within the next year. This equipment could be extremely beneficial in the characterization of junctions and materials.

The group now has an active involvement in the CdTe program and is doing in-house research as well as assisting the sub-contractors. Although this is a relatively small effort at present, it is expected that it will grow during the coming year. The in-house projects will continue to be centered on the growth of films using solution growth and sputter deposition. Both of these are promising and have the potential for mass production. It is hoped that reasonable performance devices will be in production before the submission of the next annual report.

It is also intended to continue the development of device fabrication procedures and to aid researchers in other parts of the program. A service activity has always been available from the group and this will continue to be the case. It is not entirely clear how to achieve this. The areas with which the group can be of assistance include the design and fabrication of grid masks, advice on the use of certain photolithographic techniques, and the design and fabrication of anti-reflection coatings.

There is considerable strength in the III-V materials and devices areas. Considerable progress has been made with the development of an optimum band-gap quaternary material for use under concentrated sunlight. It seems possible that an efficiency approaching $30 \%$ will be achieved within the coming year, provided that there is sufficient time and encouragement to permit this acitivity. Hopefully, this will become an integrated part of the main program.

It is intended to strengthen the Work for Others programs which have been of such great benefit to the development of the group. The reputation of the group with the external sponsors is excellent and this is likely to improve the probability of success in obtaining additional funds. Initial steps towards the technical transfer of the tandem cell technology to industry have been made and it has been made clear by the Naval Research Laboratory that they would regard this as the ultimate success of the project. Although at an early stage of discussion. there is a possibility that additional income will become availahle from a further sponsor of the group's work. 
The group has also received considerable support from the Dirctor's Development Fund and this has benefited us in several ways. The work on the heteroepitaxial growth of InP on low-cost substrates is of great interest to NASA and they would like to include this in their program. Unfortunately. their funds do not permit this at present. However, it is also possible that this work could eventually be included in the main DOE program. The project concerned with the solution growth of CdS has been an outstanding success and work is being done in collaboration with sub-contractors to improve their cells by the incorporation of the solution growth technology. This work is now accepted as part of the group's work for FY 1991, and, to this extent, the outcome is most satisfactory and represents a successful use of DDF funds. The project concerned with the solution growth of CdTe is still at an early stage, although it is probably going to be of interest to the program if either of the deposition techniques being investigated are successful. Again, if this materializes, then the project can be regarded as being successful within the Institutional category of DDF projects.

1. Wanlass, M.W., K.A. Emery, T.A. Gessert, G.S. Horner, C.R. Osterwald and T.J. Coutts, Solar Cells, Vol. 27, (1989) 191-204.

2. Refer to recent proceedings of the IEEE Photovoltaic Specialists Conference for examples of research in this area.

3. Fraas, L.M., J.E.Avery, V.S.Sundaram, V.T.Dinh, J.M.Gee and K.A.Emery. To be published in Proc. 21st IEEE Photovoltaic Specialists Conf., Kissimmee, Fl, 1990, IEE r, New York, 1990.

4. Wanlass, M.W., U.S.Patent No. 4,649,859, March 17, 1987.

5. Wanlass, M.W., G.S.Horner, T.A.Gessert and T.J.Coutts, Proc. 1st Int. Conf. on InP and Related Materials, Norman. OK, 1989. SPIE, 1989, Vol. 1144, 445-458.

6. Emery, K.A., and C.R. Osterwald, "Solar Cell Efficiency Measurements," Solar Cells, vol. 17, 1986, pp. 253-274.

7. Yamaguchi, M., C. Uemure, A. Yamamoto and A. Shibukawa, Jap. J. Appl. Phys., Vol. 23, 302 (1984).

8. Keavney, C.J., V.E. Haven and S.M. Vernon, Conf. Record 21st IEEE Photovoltaic Specialists Conference, 141 (1990).

9. McClelland, R.W., C.O. Bozler and J.C.C. Fan, Appl. Phys. Lett., ol. 37, 560 (1980).

10. Spitzer, M.B., B. Dingle, J. Dingle and R. Morrison, Conf. Record 2 lst IEEE Photovoltaic Specialists Conference, 196 (1990).

11. Vernon. S.M., C.J. Keavney. E.D. Gagnon, N.H. Karam, M.M. AlJassim, N.M. Haegel, V.P. Mazzi and C.R. Wie, Proc. Mat. Res. Soc. Symp., Vol. 198. 163 (1990).

12. Keavney. C.J.. Spire Corp., private communication, Mar. 1991.

13. Keavney, C.J., S.M. Vernon and V.E. Haven. Conf. Record 20th IEEE Photovoltaic Specialists Conference. 654 (1988). 
14. Presented (not published) by M.W. Wanlass at the 2nd Int. Conf. on InP and Rel. Mat., Denver, CO, U.S.A., April 23-25, 1990.

15. Wanlass, M.W., and P. Sheldon, U.S Patent No. 4,963,949, Oct. 16 , 1990.

16. O'Neill, M.J., U.S. Patent No. 4,711,972, Dec., 1987.

17. "Terrestrial Photovoltaic Measurement Procedures", Report ERDA/NASA/102277/16, June, 1977.

18. Wehrli, C., "Extraterrestrial Solar Spectrum," Physical Meteorological Observatory and World Radiation Center, tech. rep. no. 615, Davos-Dorf, Switzerland, July, 1985.

19. Wanlass, M.W., G.S Horner, T.A. Gessert and T.J. Coutts, Proc.1st Int. Conf. on InP and Rel. Mat., Norman, OK, U.S.A., March, 1989, ol. 1144, 445-458, SPIE (1989). 


\subsection{ELECTRO-OPTICAL CHARACTERIZATION}

\subsection{RESEARCH STAFF}

R.K. Ahrenkiel, Group Leader and Principal Scientist

D.J. Dunlavy, Associate Scientist

B.M. Keyes. Staff Scientist

L.L. Roybal, Master Technician

T. Hanak, Research Associate

A. Bakry, Peace Fellow

J. Zhang, Research Associate

D. Levi. Postdoctoral Research Associate

\section{$5.2 \quad$ INTRODUCTION}

This task characterizes both the electrical and optical properties of photovoltaic (PV) semiconducting materials and devices. These investigations provide data to researchers leading to faster development of PV technology. Our facilities specialize in unique minority-carrier characterization measurements. The group also has a facility for the routine electrical characterization of thin-film superconductors.

\subsection{ANALYTICAL CAPABILITIES AND TECHNIgUES}

Appendix D provides a listing of the major instrumentation and test equipment, their features, and specifications.

\subsubsection{The Laser Characterization Facility}

\subsubsection{Time-Resolved Photoluminescence}

The primary function of the photoluminescence (PL) facility is devoted to using time-resolved PL in determining the minority-carrier properties of PV materials and devices. Our facility is used for the measurement of minority carrier lifetime in a variety of PV materials. As such, this facility is unique to the U.S semiconductor and photovoltaic community. The minoritycarrier lifetime is the most important parameter relevant to the performance of PV devices. For special diagnostic structures, this technique produces a direct measurement of bulk lifetime and interface recombination velocity. Our measurements have been used for process development by a number of the in-house workers and subcontractors. The measurement technique used for time-resolved PL is called time-correlated single photon counting. The time-correlated photon counting technique is interfaced to the Macintosh computer and laser printer for improved quality graphics. Device models 
have been developed to relate the raw PL lifetime data to real bulk lifetime and interface recombination velocity of special diagnostic structures.

There are two independent time-resolved PL systems operating in the laboratory. System I uses a mode-locked, frequency doubled Nd:YAG pumped dye laser for excitation and a conventional photomultiplier tube (PMT), which is configured for single photon detection. System II (see Fig. 5.1) uses a mode-locked argon ion pumped dye laser and a high-speed micro-channel plate (MCP) detector. The dye lasers in both systems produce pulses of $\sim 8$ picoseconds (ps) full width half-max (FWHM). The MCP detector has an ultimate system response decay time of $\sim 50 \mathrm{ps}$, compared to $\sim 300 \mathrm{ps}$

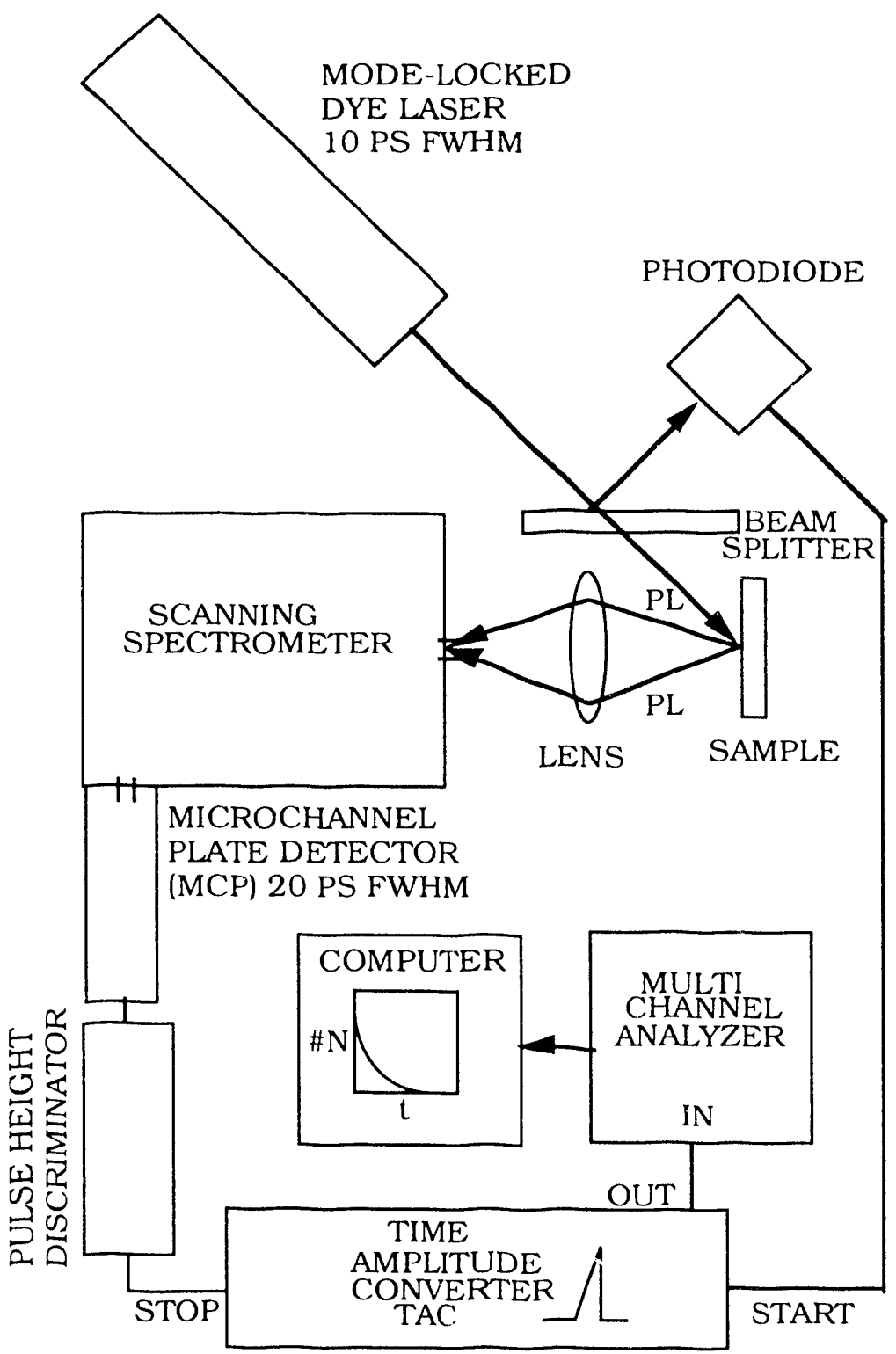

Figure 5.1: Time-resolved PL system. 
for the PMT. The shorter risetime of the MCP provides for tine measurement of lifetimes below $\sim 50 \mathrm{ps}$. The higher speed of system II has allowed the characterization of quantum well structures, III-V material grown on $\mathrm{Si}$, highly doped III-V materials, and polycrystalline materials that exhibit lifetimes in the subnanosecond time domain.

A radio frequency ( $\mathrm{r} f$ ) absorption detector was brought on-line during the past year. This device was built for the determination of lifetime in small band-gap materials that cannot be detected by the PL technique. For Eg < $1.1 \mathrm{eV}$, the PMT and MCP are blind to the band-to-band light emission, and alternative techniques must be sought. The new rf system has given us the capability of diagnosing low-band-gap materials, although it has some severe response limitations to be discussed.

\subsubsection{Measurement Diagnosis}

In addition to data acquisition, an important aspect of the time-resolved PL evaluation of materials is modeling of the data. It is well known that the true bulk lifetime is dependent upon the specific device configurations. The measured PL lifetime in diagnostic structures is less than the real bulk lifetime. A number of device models have been developed for specific structures such as homojunctions ${ }^{1}$, heterojunctions ${ }^{2}$, double isotype heterojunctions ${ }^{3}$, and bulk crystals. These models are available on various computer systems in our laboratory and are applied to the appropriate measured data. The models, when applied to the interpretation of data on real devices, produce the real bulk lifetime. For double heterostructures (DH) structures, the interface or surface recombination velocity, S, is built into the models. The velocity $S$ is usually a significant factor and has been determined by the apppropriate set of of experiments.

The PL lifetime of this structure is related to bulk lifetime and interface recombination velocity by the expression:

$$
\frac{1}{\tau}=\frac{1}{\tau_{N R}}+\frac{1}{\tau_{R}}+\frac{2 S}{d}
$$

Here $\tau$ is the measured PL lifetime, $\tau_{\mathrm{NR}}$ is the bulk nonradiative lifetime, $\tau_{\mathrm{R}}$ the intrinsic or radiative lifetime, $\mathrm{d}$ is the thickress of the active region, and $\mathrm{S}$ is the interface recombination velocity. As the thickness $\mathrm{d}$ becomes larger, the interface recombination contribution drastically decreases. A standard measurement strategy has been to produce two devices of different thickness, $\mathrm{d}$, using the same processing procedure. From these data, values of S and bulk lifetime can be uniquely determined using the above equation. 


\subsubsection{Photoluminescence Spectroscopy}

During the past year we developed a state-of-the-art facility for doing highspeed, high resolution PL spectroscopy of materials at temperatures from 10 $\mathrm{K}$ to about $300 \mathrm{~K}$. These data are analyzed for the determination of a number of important properties. A common use for the measurement technique is for the determination of the band gap in new materials. In addition, photoluminescence spectroscopy is used for identifing optically active impurities in new materials. The great advantage of PL is the ability to perform these measurements nondestructively on finished devices. The measurements can then be correlated with the starting materials to evaluate the effects of processing.

\subsubsection{Diffusion Time-Of-Flight}

A diffusion time-of-flight (TOF) technique, which was developed here for measuring the minority-carrier diffusivity (D), has become more widely requested by collaborating researchers. Our technique is a modification of the Haynes-Shockley technique originally used to measure D in Si and Ge. Owing to the short lifetimes and diffusion lengths of compound semiconductors, our special technique was devised. The technique uses the diffusion time-of-flight of photo-excited carriers across the emitter of a p-n junction to ascertain the minority carrier diffusivity (mobility) and in some cases the lifetime. Transient device models have been developed to fit the experimental data in terms of the minority-carrier parameters.

The excitation consists of a mode-locked Nd:YAG pumped dye laser to produce 8-ps pulses at kilohertz repetition rates. A schematic of the experimental setup is shown in Fig. 5.1. The short laser pulse produces a high concentration of minority carriers near the front surface through a wide-band-gap window layer. The latter provides both surface passivation and a minority carrier mirror. This equipment has been used to determine minority parameters in a variety of III-V semiconductors such as GaAs and AlGaAs. New work on InGaAs will provide information on minority-carrier transport, which has never been previously measured.

In collaboration with workers at Purdue University, the minority-carrier diffusivity in heavily doped $\mathrm{n}$ - and p-type GaAs has been measured. Improvements in the device packaging have allowed us to measure the minority-carrier diffusivity in much shorter emitters than previously possible. The use of shorter emitters results in a measured voltage transient that is less sensitive on the minority-carrier lifetime. This effectively removes one of the variables $(\tau)$ and increases the precision with which the other two (D and S) can be determined. 


\subsubsection{Deep-Level Transient Spectroscopy (DLTS)}

During the past year, there has been an big increase in activity in measuring deep impurity levels in semiconductors. Two DLTS measurement systems have been in constant use in characterizing a number of PV materials. A new SULA Instruments DLTS spectrometer, which was purchased by the Egyptian government under the Peace Fellow program, has been an important addition to our laboratory. Howevever, the instrument was returned to Cairo at the conclusion of the Peace Fellow program in December.

In addition, our home-built DLTS system produces the direct storage of capacitative transients. Our software development allows data fits on multiple level impurity systems and produced some very significant results on the structure of the DX-center in AlGaAs. This computer-automated data accquisition system obtains the entire signal-averaged capacitative transient versus temperature allowing the analysis of complex systems. Using a single temperature scan, data are obtained, which allows a deconvolution of multiple energy level defects such as the DX-center in AlGaAs. Using a nonlinear least squares regression, the analysis provides unambiguous analysis of these complex defect states. Another added feature is the ability to directly measure capture cross sections as a function of temperature. Using the two DLTS measurement systems, a large number of defect studies in PV materials have been undertaken.

\subsubsection{Electrical Characterization of High-Temperature Superconductors}

\subsubsection{Resistance-Temperature Measurements}

We have established a resistance versus temperature (R-T) measurement facility using spring-loaded contacts. The measurement temperature range is $15 \mathrm{~K}$ to $300 \mathrm{~K}$. This equipment is interfaced to and controlled by a MacIntosh computer. The data is stored on disk for later manipulation and graphing. At the present time, we are routinely running a number of transport characterization experiments for the in-house group at SERI directed by Dr. R. Noufi and for several outside research groups.

\subsubsection{Meissner Mutual Inductance}

We have developed a Meissner mutual inductance (MMI) apparatus for measurement of flux penetration through thin-film and bulk samples in an ac magnetic field. The measurement employs phase-sensitive detection, which measures both in-phase and out-of-phase signals. These measurements provide information about film uniformity and critical temperature $T_{.}$. In addition, the mutual inductance measurement provides 
data for performing harmonic analysis of the Meissner eddy current screening effects. These effects are related to the flux penetration depth and the weak link effects.

\subsubsection{Critical Current Apparatus}

We have put into operation a critical current $\left(\mathrm{J}_{f}\right)$ apparatus. This apparatus produces values of $J_{\text {. }}$ as a function of temperature from 4.2 to $300 \mathrm{~K}$ and a function of magnetic field from 0 to $13 \mathrm{KOe}$ with nanovolt sensitivity. A special ultra-low-noise, dc power supply was built and is used in conjunction with a Keithley nanovoltmeter for the required sensitivity for the onset normal resistivity. A Janis varitemp cryostat will be used for sample conling.

\subsection{ACCOMPLISHMENTS: PHOTOVOLTAICS}

\subsubsection{Time-Resolved Photoluminescence}

\subsubsection{AlGaAs/GaAs Guantum Well Structures}

Steady-state and time-resolved PL were used to investigate a number of single quantum wells in collaboration with A. Nozik. The properties of the "hot" luminescence, which arises from the excitation of quantum well states. is significant in research on photoelectrochemical cells. In the current study, we examined the dependence of hot carrier emission on barrier thickness and miniband formation in 20-period quantum well structures. The latter has quantum well structures with $250 \AA \mathrm{GaAs}$ wells and AlGaAs thicknesses of 250,40 , and $17 \AA$. The current work is concerned with determination of hot electron temperatures and the lifetime of the excited states, which wili affect the solar energy conversion efficiency of the devices.

We performed a series of time-resolved experiments on GaAs double heterostructures grown by the Nozik group. We were able to obtain a representation of the carrier density in the GaAs as a function of time and energy. By monitoring the decay at a number of energy levels we can monitor the electron temperature as a function of time and carrier density. The multiple curve technique was used to obtain similar information for the GaAs quantum wells.

\subsubsection{Optimization of Growth Conditions for PV Devices: Spire Corporation}

An extensive series of AlGaAs/n-GaAs/AlGaAs devices were grown by Stan Vernon and co-workers at Spire Corporation. These structures were designed to determine the optimum processing conditions by lifetime evaluation. In collaboration with the Spire Corporation, we investigated the 
effect dislocation density has on minority-carrier lifetime by introducing the latter in a controlled manner.

In one such study, dislocations were intentionally generated by growing a thin mismatch layer of composition GaAsP between the active layer and the substrate. The dislocation density increased with the phosphorolis content as indicated by TEM studies. For this work, Spire provided $18 \mathrm{GaAs} \mathrm{DH}$ devices for lifetime analysis. Nine of these devices were gro:vn on a thin mismatch layer of GaAsP for the controlled introduction of strain-induced dislocations. The active layer was a $\mathrm{n}-\mathrm{GaAs} \mathrm{DH}$ and the minority-carrier lifetime was measured using time-resolved single photon counting. The dislocation densities $\left(\mathrm{N}_{\mathrm{d}}\right)$ varied from $10^{4}$ to $10^{8} \mathrm{~cm}^{-2}$. The $\mathrm{N}_{\mathrm{d}}$ values were measured by M. Al-Jassim et al. by transmission electron microscopy (TEM). Our PL lifetime studies showed that the dislocations had no effect on the minority-carrier lifetime up to $\mathrm{N}_{\mathrm{d}}=5 \times 10^{5} \mathrm{~cm}^{-2}$. The minority-carrier lifetime versus dislocation density were then analyzed and found to obey the currently used model developed by workers at NTT (Japan). For dislocation density $\mathrm{Nd}>5 \times 10^{5} \mathrm{~cm}^{-2}$, the lifetime decreased dramatically. Our work also indicated a laser power dependent lifetime at the lower dislocation densities. The results of this work were presented as an invited talk by S. Vernon (Spire) at the 21 st IEEE PVSC meeting in Orlando, Florida.

Spire also provided p-type, GaAs DH devices with active layer concentrations of $2-10 \times 10^{18} \mathrm{~cm}^{-3}$ for lifetime studies. The confinement layers used were of composition $30 \% \mathrm{Al}, 90 \% \mathrm{Al}$ and $30 \%$ to $90 \%$ asymetrical devices. These structures were grown to model the emitter of the Spire high-efficiency single-junction solar cell. The parameters derived from our measurements included bulk lifetime and the recombination velocities of the $\mathrm{Al}_{x} \mathrm{Ga}_{1-x} \mathrm{As}$ $(x=0.30$ and $x=0.90)$ interfaces. The final results were reported at the PVSC21 and printed in the proceedings.

Time-resolved PL data were also obtained for Spire Corporation on a series of InP DHs. This particular data set evaluated the effect of an InGaAs buffer layer upon the minority-carrier lifetime. The data were analyzed in conjunction with TEM data on the same samples.

\subsubsection{AlGaAs Materials Development: Research Triangle Institute}

A number of ongoing programs use the lifetime measurement to optimize and evaluate the minority-carrier properties of AlGaAs. Extensive work has been done in collaboration with Prof. $M$. Timmons of the Research Triangle Institute to evaluate growth techniques for metal-organic-chemical-vapordeposition-(MOCVD)-grown $\mathrm{Al}_{x} \mathrm{Ga}_{1-x}$ As for a range of compositions $(0.05<x<0.40)$. This has led to an optimization of growth temperatures and starting materials. Time-resolved PL was also used to monitor the difference 
between two trimethylaluminum (TMA) sources used in the Research Triangle Institute MOCVD-growth system. The data clearly showed marked difference in minority-carrier lifetime for films made from different TMA sources. Significant improvement in material quality will be obtained by going to the new vendor for source material. This work demonstrates the usefulnes of the time-resolved PL in rapid optimization of material quality.

\subsubsection{AlGaAs Materials Development: Kopin Corporation}

Many GaAs and $\mathrm{Al}_{x} \mathrm{Ga}_{1-x}$ As double heterostructure samples from Kopin Corporation were measured using time-resolved PL. The Al concentrations of interest were about 0.37 to 0.38 . Historically, the lifetime in $\mathrm{Al}_{x} \mathrm{Ga}_{1-x}$ As has decreased as the aluminum content $x$ has increased. The indication is that recombination centers are introduced with the increased aluminum concentration. In one such study, Kopin Corporation supplied $24 \mathrm{DH}$ devices made by MOCVD for minority-carrier lifetime measurements. The active regions were both GaAs and $\mathrm{Al}_{x} \mathrm{Ga}_{1 . x}$ As. Doping was p-type ranging from $1 \times 10^{16}$ to $8 \times 10^{17} \mathrm{~cm}^{-33}$. The majority of the samples were made as a check on the processing quality-i.e. for quality control. A few $\mathrm{Al}_{x} \mathrm{Ga}_{1-\mathrm{x}} \mathrm{As}(\mathrm{x}=0.38)$ structures were made to assess the effectiveness of some new growth techniques. Some of the Kopin devices produced remarkably long lifetimes as compared with previous samples. The best prior lifetimes were found in Hughes liquid phase epitaxy (LPE) $\mathrm{Al}_{x} \mathrm{Ga}_{1 \cdot x} \mathrm{As}(\mathrm{x}=0.38)$ devices and were about $18 \mathrm{~ns}$. A continuation of the work emphasized the double heterostructure $\mathrm{Al}_{0.8 .8} \mathrm{Ga}_{0.2} \mathrm{As} / \mathrm{Al}_{0.34} \mathrm{Ga}_{0.62 .2} \mathrm{As} / \mathrm{Al}_{0.8} \mathrm{Ga}_{0.2} \mathrm{As}$. Lifetimes as high as 300 ns were observed. These data are being analyzed f $r$ an explanation of the anomously long lifetimes. Theory indicates that the lifetime in AlxGa 1-xAs may dramatically increase near the direct-indirect crossover at $\mathrm{x}=0.35$.

In the course of the $\mathrm{Al}_{x} \mathrm{Ga}_{1-x}$ As work, a strong dependence of the lifetime on the incident light intensity was found. These effects were measured and identified as saturation of Shockley-Read-Hall defects in the bulk of the material. An investigation of power dependence in $\mathrm{Al}_{x} \mathrm{Ga}_{1-x}$ As was pursued as the incident power was varied over many orders of magnitude. Our data indicated that high injection conditions sometimes produce an increase in the Shockley-Read-Hall lifetime to many times the low injection minoritycarrier lifetime. These measurements have strong application to modeling concentrator cells where the incident power level may reach 1000 suns. Our data extended from less than 1 sun to many times that expected in a concentrator cell. The data showed a dependence of the transient PL response upon the incident laser power density. The lifetime varies from 64 ns on the high injection range to 26 ns on the low injection range. These intensity effects are typically found in $\mathrm{Al}_{x} \mathrm{Ga}_{1 \cdot x} \mathrm{As}$ and are the subject of several publications and presentations during the past year. 


\subsubsection{Polycrystalline CdTe: Photon Energy}

During the year we have successfully measured, for the first time. minoritycarrier lifetimes in Cdie polycrystalline films. CdTe films from Photon Energy and Emcore have been investigated using both time-resolved PL and PL spectroscopy. The Photon Energy devices demonstrated the best minority-carrier lifetimes. Photon Enersy's films can be classified as part dense. large grain (up to $5 \mu \mathrm{m}$ ) and part porous, variable grain $(\sim 1$ to $5 \mu \mathrm{m})$. The large grain materials. found in the early stages of df position, exhibit a longer lifetime over the entire time-resolved region than the small grain material found at the surface. Decay data for the large grain material as a function of temperature indicate the presence of two different decay times. The primary decay ( 0.5 to $5 \mathrm{~ns}$ ) appears to be due to radiative recombination. The longer, secondarg decay ( 30 to $100 \mathrm{~ns}$ ) can be attributed to delayed PL from trap released carriers. This two-component decay is also present in the Photon Energy small grain material. The lifetimes for both grain sizes are also dependent on the incident power and tend to increase as the intensity increases. Data from one of the best Photon Energy devices is shown in Fig. 5.2. There was a strong correlation of our time-resolved PL lifetimes with device efficiency.

\subsubsection{PHOTOLUMINESCENCE SPECTROSCOPY}

The PL spectroscopy measurement facility was greatly upgraded during the year by the addition of an optical multichannel analyzer (OMA) detector. This instrument is basically a small computer used to program the operation of diode arrays in the focal plane of a dispersive grating. We have three arrays that cover wavelengths from the near ultraviolet to $1.8 \mu \mathrm{m}$ in the infrared. The advantage of this instrument is that spectral data is collected in parallel over the entire wavelength range. This contrasts with the standarci technique of tuning a grating over the desired wavelength range and collecting single wavelength data at each setting of the monochromator. These data are collected at temperatures of $4.2 \mathrm{~K} .77 \mathrm{~K}$ and $300 \mathrm{~K}$. A recently ordered cryostat will enable data ts ie any temperature between $1.5 \mathrm{~K}$ and $300 \mathrm{~K}$.

1. Recent work in collaboration with Purdue University, using the OMA, produced energy-resolved PL for highly doped MOCVD-grown n-type GaAs. Data was taken on a $5 \times 6$ matrix of samples ranging in thickness from 0.25 to $10 \mu \mathrm{m}$ and in dopant concentration ranging from $1 \times 10^{17}$ to $5 \times 10^{18} \mathrm{~cm}^{33}$. The results are currently being analyzed.

2. The OMA was used to study the PL of $\operatorname{InP}$ and $\ln _{0,47} \mathrm{Gaa}_{05,7}$ As as a

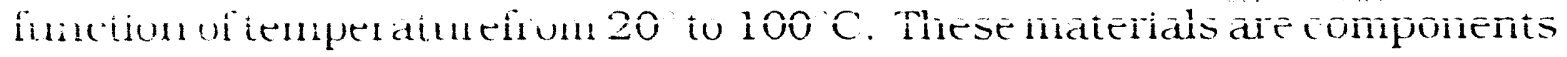
of extremely high efficiency tandem cells currently developed and produced 


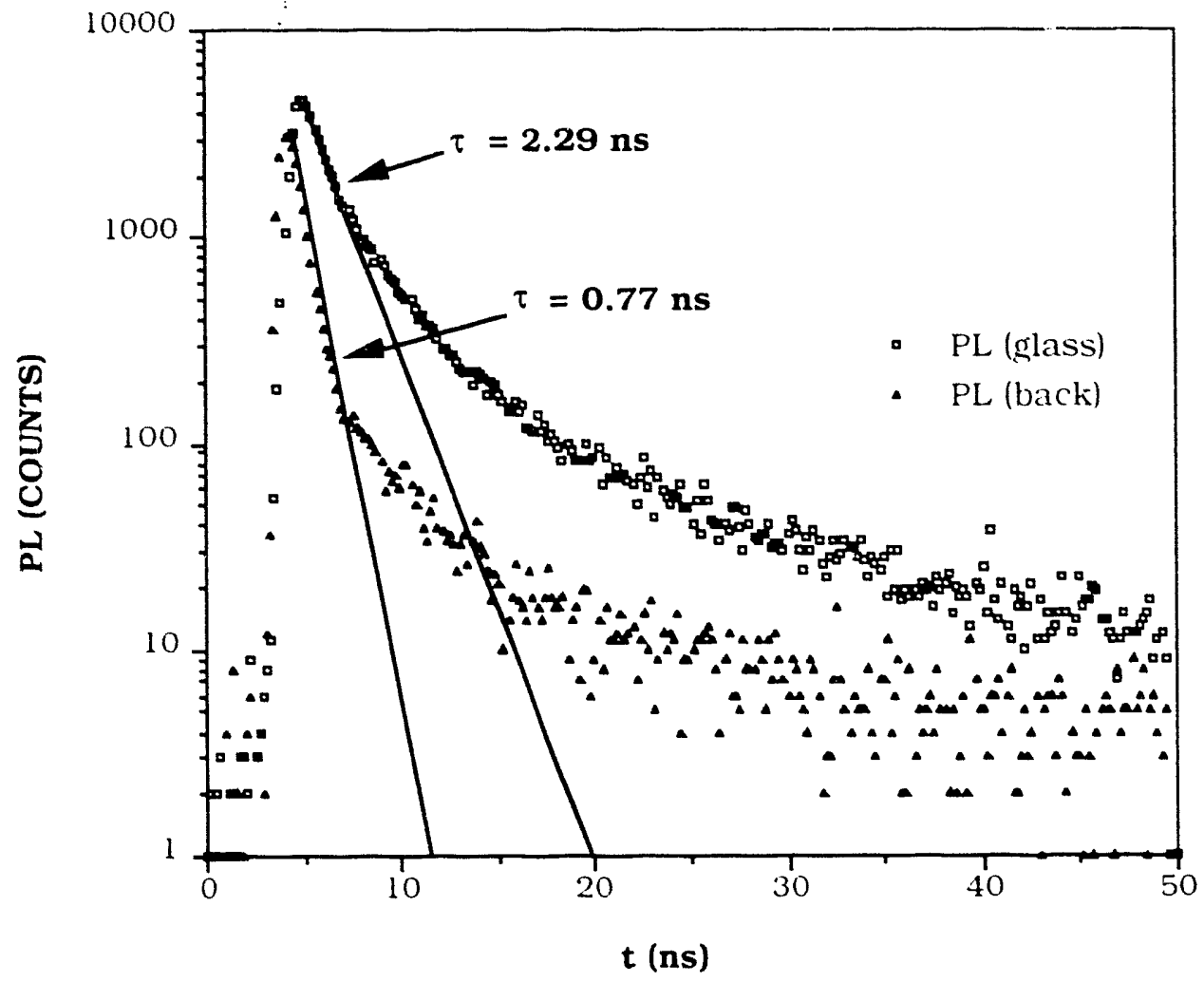

Figure 5.2: Time-resolved PL data of polycrystalline CdTe

at SERI. Determination of the band gap is extremely useful for monitoring the effects that changes in the growth process have on composition.

3. The infrared optical multichannel analyzer (OMA) was used to acquire energy-resolved spectra for CuInSe, and CuIn ${ }_{0.74} \mathrm{Ga}_{0.26} \mathrm{Se}_{2 .}$ (Rommel Noufi. SERI). The band gap is dependent on both composition and growth parameters for these marterials. The PL data should prove to be useful in monitoring and understanding these dependences.

\subsubsection{TIME-ŪT-FLIGHT (TOF)}

Upgrading of the TOF technique has been a high priority task. By working with the trigger and the laser pulse. we have been able to reduce the system response to the point that we can successfully reproduce the ultrafast turnon of a commercial p-i-n photodiode. Employing a variety of sample mounting techniques and bias tee's has also led to major improvements in the response of $\mathrm{p}-\mathrm{i}-\mathrm{n}$ photodiodes made at Purdue. The experimental equipment was then used to investigate heavily doped. p-type GaAs. These TOF measurements were in collaboration with the groul of Prof. $M$. Lundstrom at Purdue University. The goal was to measure the minority- 
carrier diffusivity and lifetime of $\mathrm{p}$-GaAs doped from $1 \times 10^{18}$ to $5 \times 10^{19} \mathrm{~cm}^{-3}$. The results of these collaborations have been the subject of several recent publications.

\subsubsection{DEEP-LEVEL TRANSIENT SPECTROSCOPY (DLTS)}

The use of the DLTS technique has increased considerably during the fiscal year. The increase in acti.ity is partly due to increased demand for measurements by in-house and subcontract workers. In addition, the activity was greatly aided by the Peace Fellowship program, which is run by the Egyptian government. The program has supported a graduate student at SERI and provided a SULA DLTS spectrometer during his stay here. Thus, we kept two SULA spectrometers in operation in order to deal with the larger influx of requested measurements. Some of our highlights follow.

Extensive measurements of impurity levels in p-CuInSe ${ }_{2}$ (CIS) were completed and tabulated. These measurements used the DLTS technique by Assem Bakry under the Peace Fellowship grant. Single crystals were grown by R. Tomlinson of Salford University (England) using the vertical Bridgman technique. The thin films were grown at the Institute of Energy Conversion (IEC) and supplied by R.W. Birkmire. The devices studied included the conventional $\mathrm{Cd}(\mathrm{Zn}) \mathrm{S} / \mathrm{CulnSe}$, heterojunctions and, in addition, Schottky diodes fabricated by A. Bakry. The latter are the first successful Schottky diodes reported on CIS. They were made by evaporating gold on $n$-type material and aluminum on p-type material.

Over ten single crystal devices and four thin film polycrystalline devices were measured. Only majority-carrier defect levels were investigated by DLTS. Minority-carrier levels were not undertaken in this study. The measurements indicated an abundance of majority-carrier impurity levels in the lower half of the band gap of CIS. The concentration levels of many of these defects were as large as $10 \%$ of the background doping level. The data indicate that the electrical properties of CIS are impurity dominated. In single crystal material, defect levels were found at 16.30, and 39, and $485 \mathrm{meV}$ above the valence band (vb). One would not ordinarily expect to see these very shallow levels by DLTS. However, this rare window technique does detect very shallow levels when the capture cross section of the defect is 10 to $20 \mathrm{~cm}^{2}$ or less. The small cross section must be accounted for by a repulsive center such as a double acceptor defect. The thin-film work indicated impurity centers ranging in thermal ionization energy from 136 to $530 \mathrm{meV}$. A number of impurity lf...ls were found that were common to both singlecrystal and thin-film-based devices. Our data dispels the notion that there is no correlation between the defect chemistry of single crystal and thin-film materials. In addition, defect levels were found in heterojunction devices that were not present in Schottky devices madie on the same material. This 
finding supports the earlier suggestions that CdS evaporation produces defects in the underlying CIS.

Extensive DLTS measurements were made on $\mathrm{p}-\mathrm{Al}_{\mathrm{x}} \mathrm{Ga}_{1 . x}$ As for Dr. J. Fan of the Kopin Corp. The quality of $\mathrm{p}-\mathrm{Al}_{x} \mathrm{Ga}_{1-x}$ As has varied during the normal cycling and loading of the MOCVD epitaxial reactors. The defect content of these films were tracked by DLTS in order to correlate processing procedures with material quality. As a result of this work, very high quality $\mathrm{p}-\mathrm{Al}_{x} \mathrm{Ga}_{1-x} \mathrm{As}$ was produced. Also, measurements were made on numerous samples of $\mathrm{Al}_{x} \mathrm{Ga}_{1-x}$ As grown by MOCVD at the Research Triangle Institute. Here the presence of DLTS signals could be correlated with lifetime reductions.

DLTS measurements of epitaxi’ GaInP grown by J. Olson and co-workers were made. The DLTS measurements indicated several deep defect levels, which had not been observed before and might be recombination centers. Past work has shown that GaInP is relatively free of deep levels that can be detected by DLTS.

Several manuscripts have been published on fundamental studies on the $\mathrm{DX}$-center in $\mathrm{Al}_{x} \mathrm{Ga}_{1 \cdot x}$ As. This work describes measurement of the activation energy of the capture barrier for this important metastable defect. The results will help in the selection of the correct microscopic model of this defect.

\subsection{ACCOMPLISHMENTS: RELATED RESEARCH AREAS}

Over 150 measurements of high-temperature superconducting (HTSC) films have been run over the past year. These measurements are primarily in support of the SERI effort to make high-quality electrodeposited YBCO. Measurements include R-T and mutual inductance measurements. We made mutual inductance measurements for groups at Standford University. National Institute of Standards and Technology (NIST) (Boulder), and the Institute of Superconductivity (SUNY, Buffalo). This work has contributed to several publications.

A Directors Development Fund (DDF) has been initiated cluring the past year using high-temperature superconductors as high-speed photodetectors. Initial results have been obtained on YBCO films provided by Emcore Corporation. A readily detectable signal was found at $6328 \AA$ in response to a small HeNe laser. The film thickness, $2000 \mathrm{~A}$. is much too thick to provide a strong response. The film geometry was the standard bridge-shaped critical current structure. Ideally. the film thickness should be $\sim 1 / \alpha$ where $\alpha$ is the absorption coefficient of the incident light. For maximum sensitivity, the device should have a very large aspect ratio (length to width) and be fabricated in a "serpentinc"-type pattem to concentrate the active area. 
A collaboration has been developed with NIST to make such films by laser ablation and photolithographic patterning. These devices will be grown at NIST and tested at SERI. As NIST is very interested in the photodetector technology, this should be a productive collaboration.

\subsection{FUTURE WORK}

- Develop an alternative to rf absorption for the measurement of minority-carrier lifetime in small band-gap semiconductors. A small DDF grant $(\$ 20,000)$ has just been granted for this task.

- Continue to develop the PL spectrometer based on an OMA for the rapid acquisition of spectroscopic data by adding a variable temperature sample stage.

- Begin measurement HTSC high-speed detectors that have been fabricated by NIST.

- Continue the operation of the two time-resolved PL systems for routine lifetime analysis (process development) and for quantum well diagnostics.

- Expand the DLTS facility to include admittance spectroscopy and additional DLTS spectroscopy apparatus.

1. Ahrenkiel, R.K., "The influence of junctions on photoluminescence decay in thin films," J. Appl. Phys. Vol. 62,2937, 1987.

2. Ahrenkiel, R.K., D.J. Dunlavy, and T. Hanak, Solar Cells, Vol. 24, 339, 1988.

3. Ahrenkiel, R.K., and D.J. Dunlavy, "Minority Carrier Lifetime In $\mathrm{Al}_{x} \mathrm{Ga}_{1-x} \mathrm{As}^{\prime}$, J. Vac. Sci. Technol., Vol. A7, 822, 1989. 


\subsection{ADVANCED PHOTOVOLTAIC MODULE PERFORMANCE TESTING AND RELIABILITY RESEARCH}

\section{1 RESEARCH STAFF}

R. DeBlasio, Section Manager

A. Czanderna, Research Fellow

D. Waddington, Senior Engineer

L. Mrig, Senior Engineer

P. Longrigg, Senior Engineer

J. Pern, Senior Scientist

S. Rummel, Associate Engineer

\subsection{INTRODUCTION}

SERI's Advanced Photovoltaic Module Testing and Performance Group has two major roles within the National Photovoltaic Program. These roles involve the performance testing and reliability research of advanced PV submodules and modules, with primary emphasis on photovoltaic (PV) flatplate modules. These efforts are conducted in conjunction with PV research activities originating both within and outside SERI. Specific research activities include testing and investigations of submodule and module performance, stability, energy output, and reliability lifetime characteristics, both under simulated and actual sunlight conditions. The group also coordinates PV thin-film module testing and reliability research for the U.S. Department of Energy (DOE) PV program and develops simulated and outdoor testing methods and diagnostic techniques to understand and determine module performance and potential failure mechanisms. Additionally, the group conducts cooperative testing programs with PV manufacturers and application sectors for comparing test methods and results, and collaborates with consensus stai idards organizations (Institute of Electrical anci Electronics Engineers [IEEE], American Society for Testing and Materials [ASTM], International Electrochemical Commission [IEC]) in developing PV test and measurement standards.

The group conducts its research activities in its five laboratories. These laboratories are: (1) PV Module Outdoor Performance and Characterization Laboratory; (2) PV Module Outdoor Stability and Energy Evaluation Laboratory; (3) Simulation Test and Evaluation Laboratory; (4) Module Encapsulation Research Laboratory; and (5) Accelerated Environmental Test and Evaluation Laboratory. Laboratories 1, 2, and 3 are located at the SERI field evaluation and test area (Fig. 6.1). Laboratory 4 is located in room 174 in building 16 and Laboratory 5 is located in room 156 in the SERI's Field Test Laboratory Building (FTLB). 


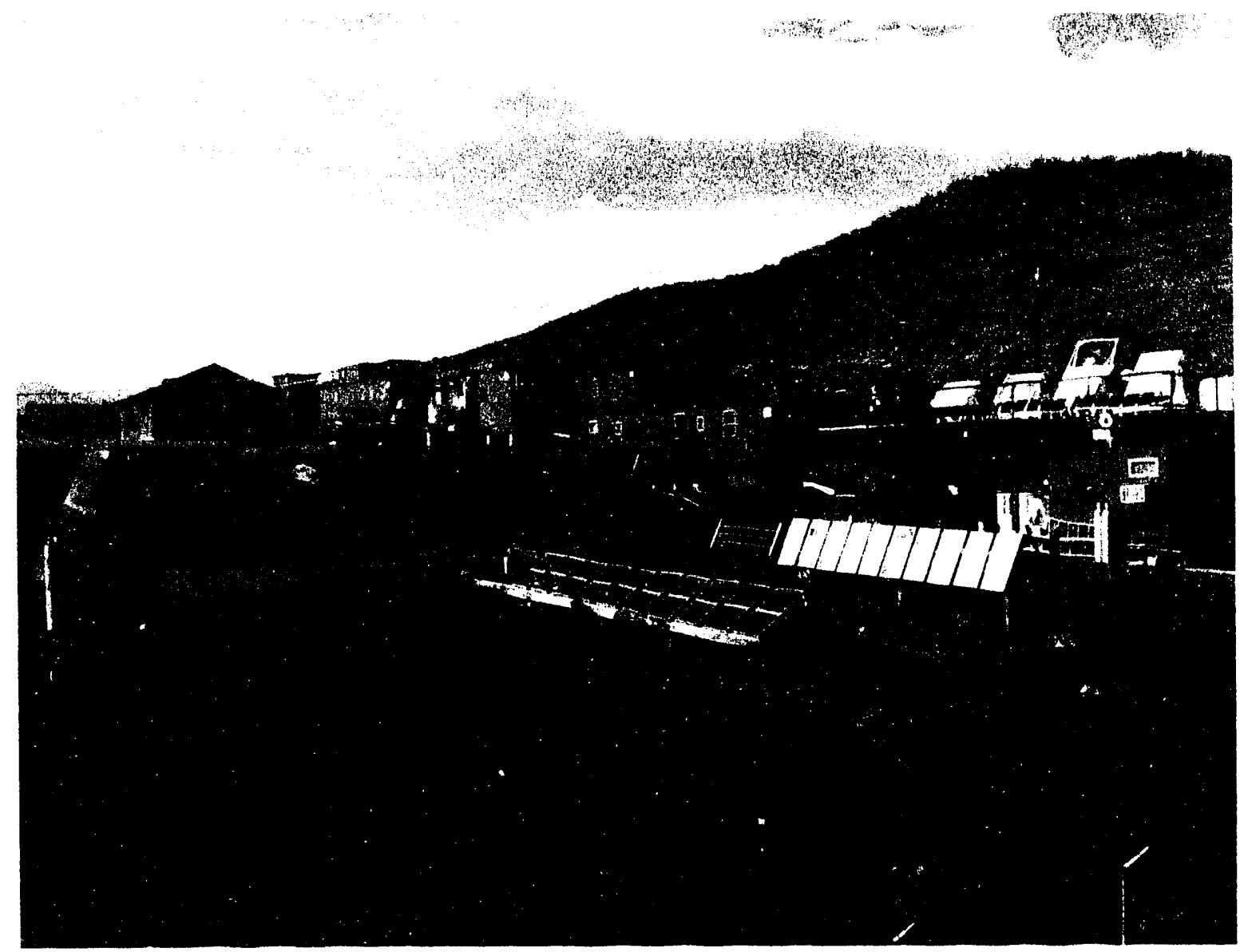

Figure 6.1: SERI PV Module Performance and Test Facility

Module and submodule performance evaluations are closely coordinated with the branch's cell calibration and material/cell diagnostic efforts. In addition, there is interactive support and cooperation with SERI's Solar Radiation Research and Metrology Branch researchers to ensure proper calibrations of solar radiation instruments. These groups provide an all encompassing technical capabilities base in PV module performance testing and reliability research.

\subsection{ANALYTICAL CAPABILITIES AND TECHNIGUES}

Appendix D provides a listing of the operational equipment, their features, and specifications.

\subsubsection{PV Module Outdoor Performance Characterization and Evaluation Laboratory}

The PV Module Outdoor Performance Characterization and Evaluation Laboratory (Fig. 6.2) provides a wide range of outdoor testing capabilities 


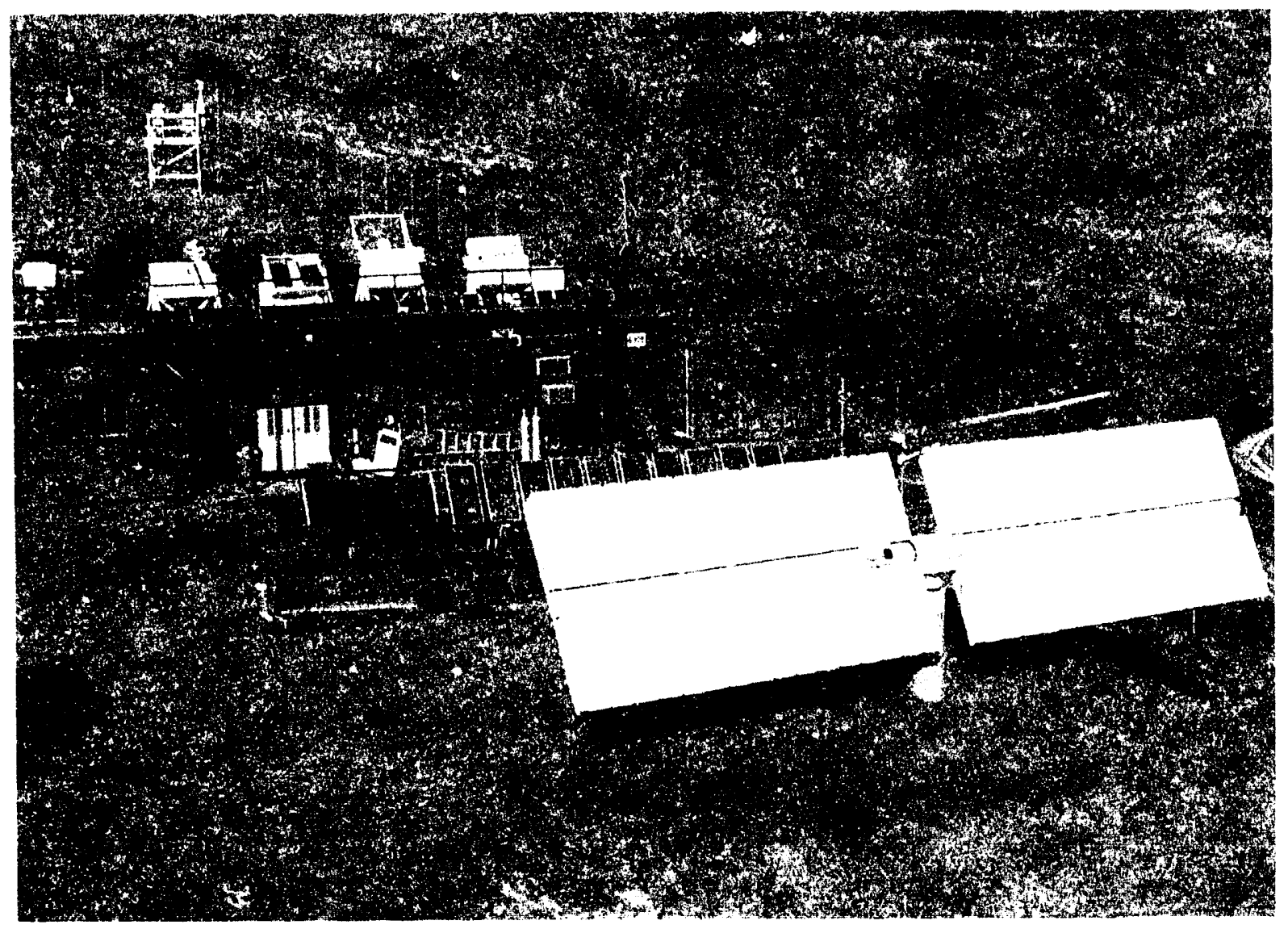

Figure 6.2: $\quad$ PV Module Outdoor Perfornance Characterization and Evaluation Laboratory

used by the research group in conducting test programs on advanced PV devices in support of SERI's subcontracted and internal research. This is accomplished through instrumentation configuration design, fabrication. installation, deployment, and operation of testing systems to conduct routine, exploratory, and experimental test and evaluation programs, as well as the development of outdoor test methods. Laboratory operations and equipment also provide a wide range of flexibility in configuring a variety of testing systems and experiments as a result of the interchangeability of instrumentation within each data-acquisition and control system.

Routinely, tests and measurements are conducted on PV submodules and modules outdoors under natural sunlight to determine their performance for predetermined periods of exposure time and varying operating conditions. Test results on submodule and module performance characteristics and operating parameters typically include current versus voltage (I-V) characteristics. conversion efficiencies, energy output, operating temperatures, solar intensity, and the spectral content of sunlight. Evaluation 
of these results for each test program provides a basis for further diagnostic testing to identify potential module performance degradation mechanisms. Over 900 test programs have been completed since 1983 at this laboratory to verify amorphous silicon thin-film submodule and module performance. Recent results from these tests have confirmed major DOE efficiency milestones, as defined in the DOE Five-Year Research Plan, for submodules and modules having areas up to $1000 \mathrm{~cm}^{2}$. Over $120.000 \mathrm{I}-\mathrm{V}$ performance measurements have also been made on single-junction and multijunction amorphous silicon thin-film modules to determine their stability performance characteristics. Staff members have also conducted outdoor tests on CIS and CdTe modules for long-term stability evaluation.

\subsubsection{PV Module Outdoor Stability and Energy Performance Test and Evaluation Laboratory}

The PV Module Outdoor Stability and Energy Performance Test and Evaluation Laboratory provides new capabilities for field testing modules configured and installed as experimental test arrays deployed outdoors for both fixed and tracking modes of operation. Tests are structured to provide a data base for analysis in determining module and array stability performance and energy output. A testing system, designed and installed by the group, has provisions to evaluate up to 12 fixed arrays of modules configured for array output power levels of $100 \mathrm{~W}$. Typical measurements include $1-\mathrm{V}$ characteristics, de energy output, global radiation, and operating temperatures. Four groups of representative modules deployed in $20-\mathrm{kW}$ arrays for the Photovoltaics for Utility Scale Applications (PVUSA) project under the Emerging Technology 1 (EMT1) program are also being monitored for performance, field failure monitoring, and reliability evaluation. Staff also conduct exploratory examinations on modules and arrays, to evaluate module and array operating characteristics and to determine dc to ac energy conversion under various load conditions.

Results from these studies provide an understanding of module and array performance and the impacts on performance, such as operational modes (fixed versus tracking), environmental effects, module mismatch because of inherent or induced changes in electrical operating characteristics over time, shadowing or hot-spot effects, and module stability performance trends and failure modes. Analysis of these results provides a profile on module and array stability and energy output performance. Additionally, it supports the development of stability, energy, and reliability testing, evaluation, and qualification testing and rating methods. 


\subsubsection{Simulation Test and Evaluation Laboratory}

The Simulation Test and Evaluation Laboratory is utilized to conduct electrical characterizations of submodules and inodules under simulated and standard test conditions. Exploratory tests are also conducted to determine temperature and solar intensity temperature coefficients and stability performance trends for different controlled test conditions. Results from these tests also provide a data base for determining correlations; between module accelerated (simulated) and real-time performance. Typically, before-and-after tests under the solar simulator are conducted at various stages of each study along with such diagnostic tests as infrared (IR) thermography, electrical isolation, and leakage current measurements. Analysis of the results provides information on the need, if any, for further diagnostic testing in the laboratory and provides a data base to quantify degradation mechanisms and failure modes, which are needed to develop realistic life-prediction models for projecting module performance as a function of time and potential life expectancy.

\subsubsection{PV Mudule Encapsulation Research Laboratory}

In 1990 , this laboratory was established in room 174 , building 16 for testing and analysis of EVA (ethylene vinyl acetate) and other polymer encapsulants. The laboratory is now equipped with two Oriel light sources (150 and 1000 W Xe lamps), a Sunlighter Model 150 tester with three GE RS-4 sunlamps, a FEIKER 41-AR Precision Cut-Off machine, an Orion Model 720A pH meter, an oven, and various glassware for wet chemical analysis. More instruments are expected to be acquired in the near future. The current equipment allows testing for the thermal, photochemical, and photothermal stability of the EVA encapsulant alone and dissection and sampling of weathered PV modules.

\subsubsection{Accelerated Environmental Test and Evaluation Laboratory}

This laboratory is used for conducting exploratory reliability tests and evaluation of PV modules under accelerated test conditions. Light-soak testing of PV modules is also conducted in this laboratory to evaluate and study any performance degradation due to continuous or intermittent lightsraking under controlled conditions of environmental temperatures and humidity. This laboratory is also very useful in conducting temperature cycle and humidity freeze tests as required in SERI's "Interim Qualification Tests and Procedures for Terrestrial Photovoltaic Thin-Film Flat-Plate Modules." Results from these tests also provide a data base for determining correlations between module accelerated (simulated) and real-time performance. 


\subsection{ACCOMPLISHMENTS: PHOTOVOLTAICS}

Major efforts by the staff over the past year have included a variety of tasks directed towards module (1) performance tests, (2) qualification test specifications, (3) outdoor stability test and evaluation. (4) reliability research, and (5) technology transfer (Module Reliability Workshop). Emphasis was placed on completing, publishing, and disseminating the thin-film flat-plate module qualification test procedure document developed in conjunction with the DOE PV laboratories and PV industry. This task was assigned to SERI by DOE with the objective of attaining a general consensus on specified tests by including thin-film module manufacturers from the initiation of the project. in the development of tests, and in the review of draft documents.

In addition, a substantial amount of time was expended in support of the PVUSA project. DOE assigned a representative of the group the responsibility to represent DOE and SERI on the project's Technical Review Committee (TRC). This ac ivity required attending numerous planning and review meetings, reviewing proposals for Emerging Technology projects (EMT1 and EMT2) and Utility Scale (US1) projects.

Major efforts were also expended in cooperative research and testing activities that included diagnostic measurement support to PV module manufacturers in evaluating EVA module encapsulant materials, environmental testing of prototype module designs, and performance testing of modules in support of the GM Sunrayce held in June 1990.

Integration and utilization of module reliability research diagnostic measurements capabilities within the branch received a major boost in the past year. This was most notable in the investigation of discoloration phenomenon after prolonged outdoor exposure in the EVA encapsulating material commonly used in crystalline silicon modules.

The above activities and results from these efforts, as well as others, are given in greater detail below.

\subsubsection{Module and Submodule Characterization Testing}

Staff members completed over 140 outdoor and indoor simulated performance characterization tests and solar spectral measurements on PV thin-film submodules and modules delivered from subcontractors. Seventy-nine (79) test reports were gerirated based on these tests. These tests and results contributed to SERI's research efforts in meeting major DOE PV program milestones. 
The staff continued development of a detailed data base on submodule and module performance test results conducted at SERI, which is used by DOE headquarters staff and program planners.

Major efforts by the staff also included continued cooperative characterization and baseline testing with the PV community, which has established SERI as the primary center for standardized baseline module testing. Results of this effort included module and submodule tests and measurement activities with Ametek, Associated Weather Services, Chronar, Florida Solar Energy Center, Glasstech Solar Inc., Mobil Solar Energy Corp., Photon Energy, Siemens Solar Industries, Sovonics (United Solar Systems Corp.), Solarex, Tideland Signal Corp., and Utility Power Group.

Highlights from these activities included tests conducted on a large area $\left(12,300 \mathrm{~cm}^{2}\right)$ a-Si (amorphous silicon) module from Chronar Corp. (now APS) with an aperture area efficiency of 6.23\%: a single-junction a-Si module from Chronar Corp. with an aperture area $\left(873.0 \mathrm{~cm}^{2}\right)$ efficiency of $7.55 \%$; Solarex Corp.'s single-junction a:Si module with an aperture area $\left(933.1 \mathrm{~cm}^{2}\right)$ efficiency of 9.84\%; Solarex Corp.'s triple-junction (a-SiC/a- Si/a-SiGe) submodule with an aperture area $\left(936.4 \mathrm{~cm}^{2}\right)$ efficiency of $8.08 \%$; a CdTe (cadmium telluride) module from Photon Energy with an aperture area $\left(876.2 \mathrm{~cm}^{2}\right)$ efficiency of $5.66 \%$.

The Outdoor PV Test Site module solar simulator (SPIRE 240 SPI-SUN) was replaced with a newer version (240A SPI-SUN). This increases the testing capabilities in the following areas: (1) maximum module voltage from $60 \mathrm{Vdc}$ to $100 \mathrm{Vdc}$; and (2) maximum module current from 15 Adc to 20 Adc. Other improvements in the features of the new simulator are: (1) the controlling and data acquisition system is now an IBM AT, which improves the data handling capabilities; and (2) the xenon lamp life will be increased as the lamp is turned on for about $30 \mathrm{~s}$ during the test and is pulsed at one-eighth the rate of the old lamp.

\subsubsection{Module Stability Performance Testing and Evaluation $[20,21,66,67]$}

Outdoor stability performance tests have been under way at SERI for over five years in order to understand and monitor the progress and improvements in stability performance characteristics of thin-film photovoltaic modules for an environment representative of actual operating conditions. Current results from these tests are illustrated for copper indium diselenide (CIS) and cadmium telluride (CdTe) modules in Figures 6.3 and 6.4, respectively. Detailed results from these and other tests were reported over the past year at the Materials Research Society (MRS ). Intersociety Energy Conversion Engineering Conference (IECEC). Institute of Electrical and Electronic 


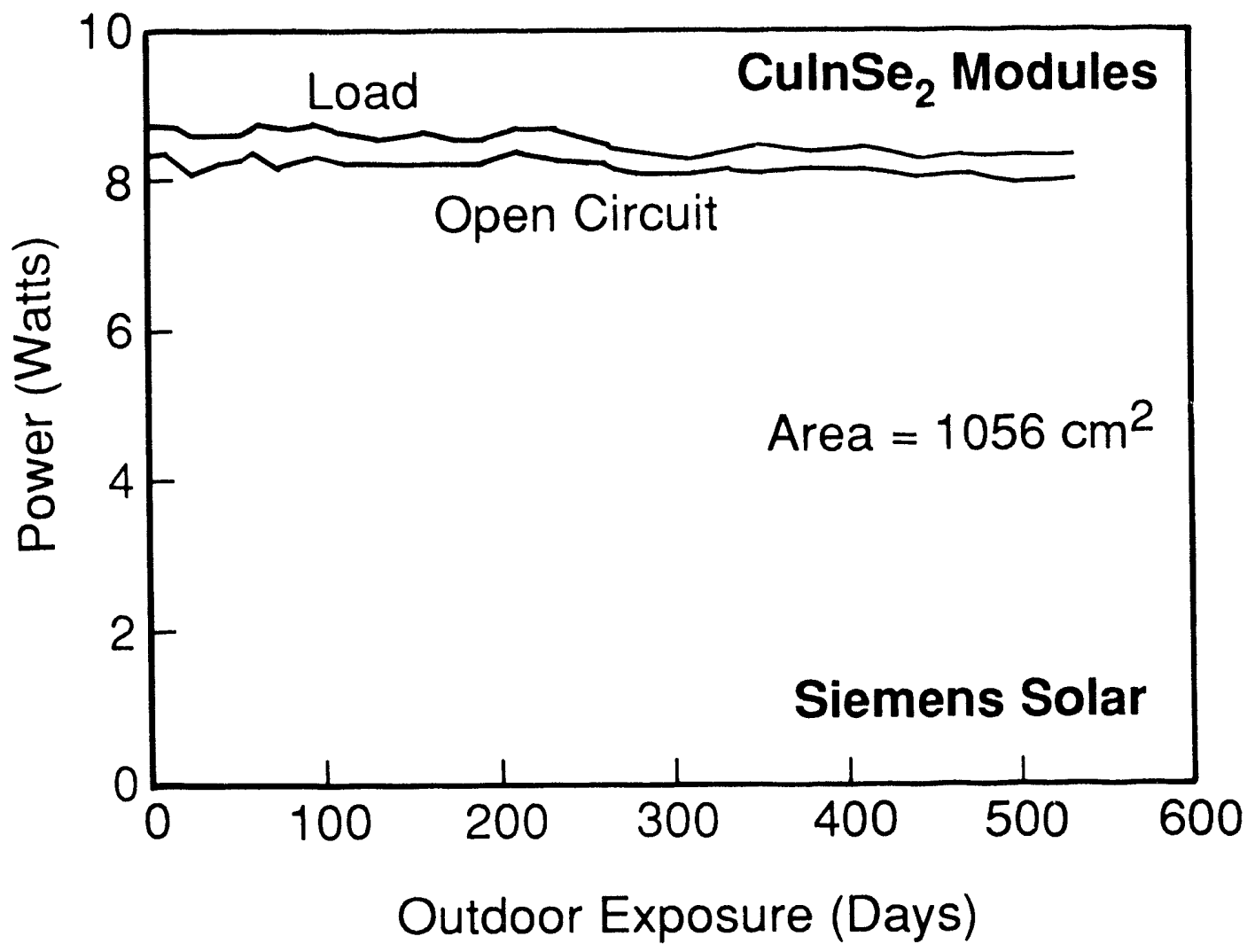

Figure 6.3: CIS module stability performance

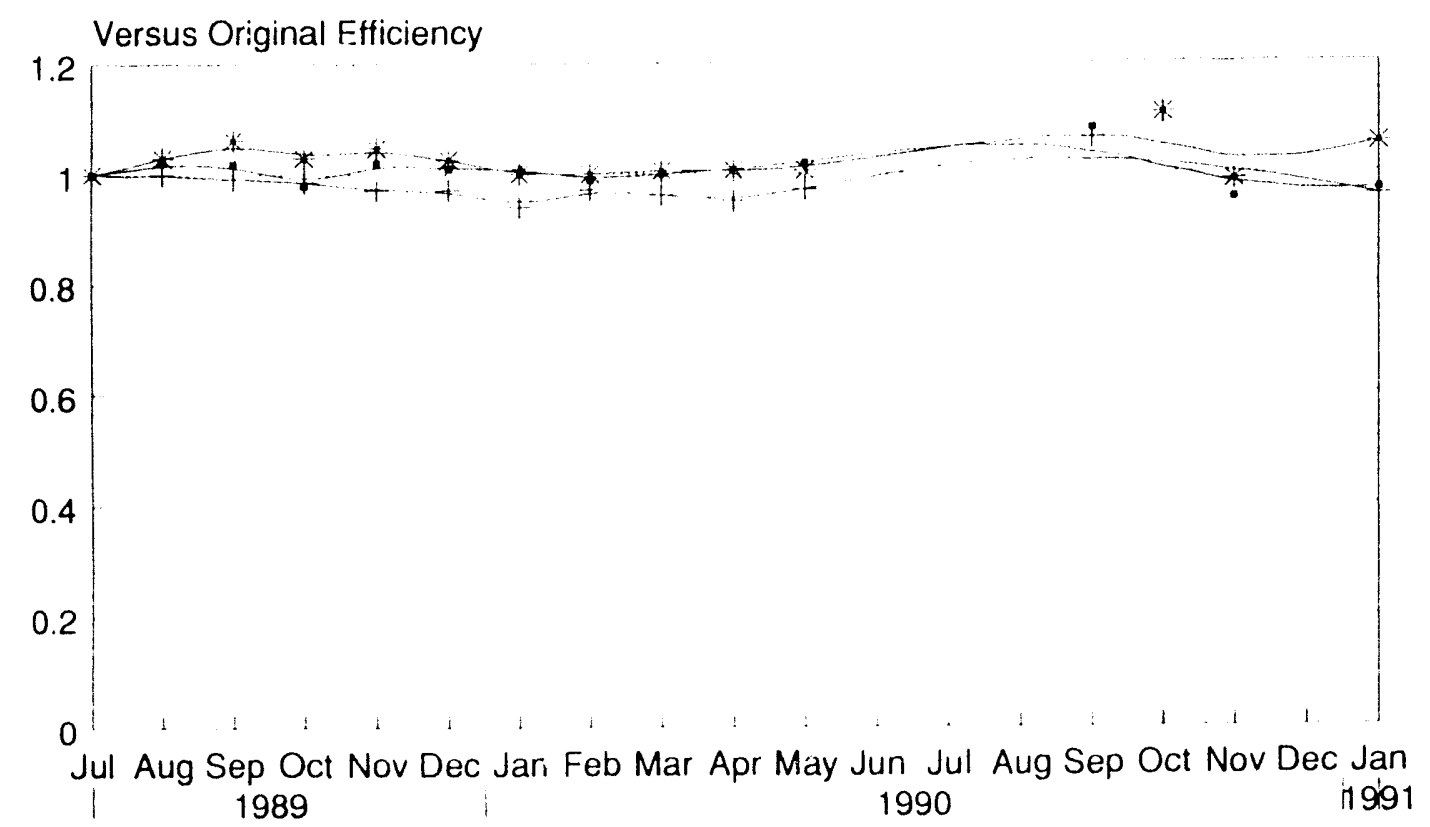

New Simulator Sept. 1990

Figure 6.4: CdTe module stability performance 
Engineers's Photovoltaic Specialists Conference (IEEE PVSC) and other photovoltaic meetings.

\subsubsection{Interim Gualification Test for Terrestrial Photovoltaic Thin- Film Flat-Module Modules [33]}

This document was published in FY 1990 as a SERI Technical Report (SERI/ TR-213-3624). Development of this document was in response to the expressed concerns and general consensus of PV community participants attending the SERI/DOE sponsored PV Thin-Film Module Reliability Testing and Evaluation Workshop, held in Lakewood, Colorado, on 13-14 Aug. 1987. It provides interim qualification tests and procedures to evaluate terrestrial PV thin-film flat-plate modules. The term "interim" is used because thin-film module design and cell material technologies are currently undergoing evolutionary changes in their development, tharefore, the information contained in this document will be updated as the need arises. The effort to manage and coordinate the development of this document was assigned to SERI by DOE in association with the Jet Propulsion Laboratory and the Sandia National Laboratory.

The purpose of the qualification test provided in this document is to evaluate flat-plate thin-film photovoltaic module performance and susceptibility to known failure mechanisms. Emphasis is placed on testing and evaluating module performance characteristics and design features that will affect possible degradation of module performance and physical properties resulting from solar exposure, environmental weathering, mechanical loading, corrosion, and module shadowing.

From the beginning of this effort, emphasis was placed on obtaining PV industry input in preparation and development of the consensus required in completion of this document. Several meetings were held with the PV manufacturers, DOE PV laboratories, and others in the development of this document, and earlier draft versions of this document were reviewed by PV research, development, and application communities in general.

The required qualification test sequence contained in the document is shown in Fig. 6.5. This document was distributed widely to the U.S. PV community, and was also provided to all the attendees of the $1990 \mathrm{PV}$ Module Reliability Workshop held in Lakewood in Oct. 1990.

\subsubsection{Ethylene-Vinyl Acetate (EVA) Encapsulant Degradation}

Ethylene-vinyl acetate (EVA) copolymer used as an encapsulant in photovoltaic modules turns from clear to yellow-brown after five or more years of weathering. EVA films stored in the dark for six years have not degraded. 


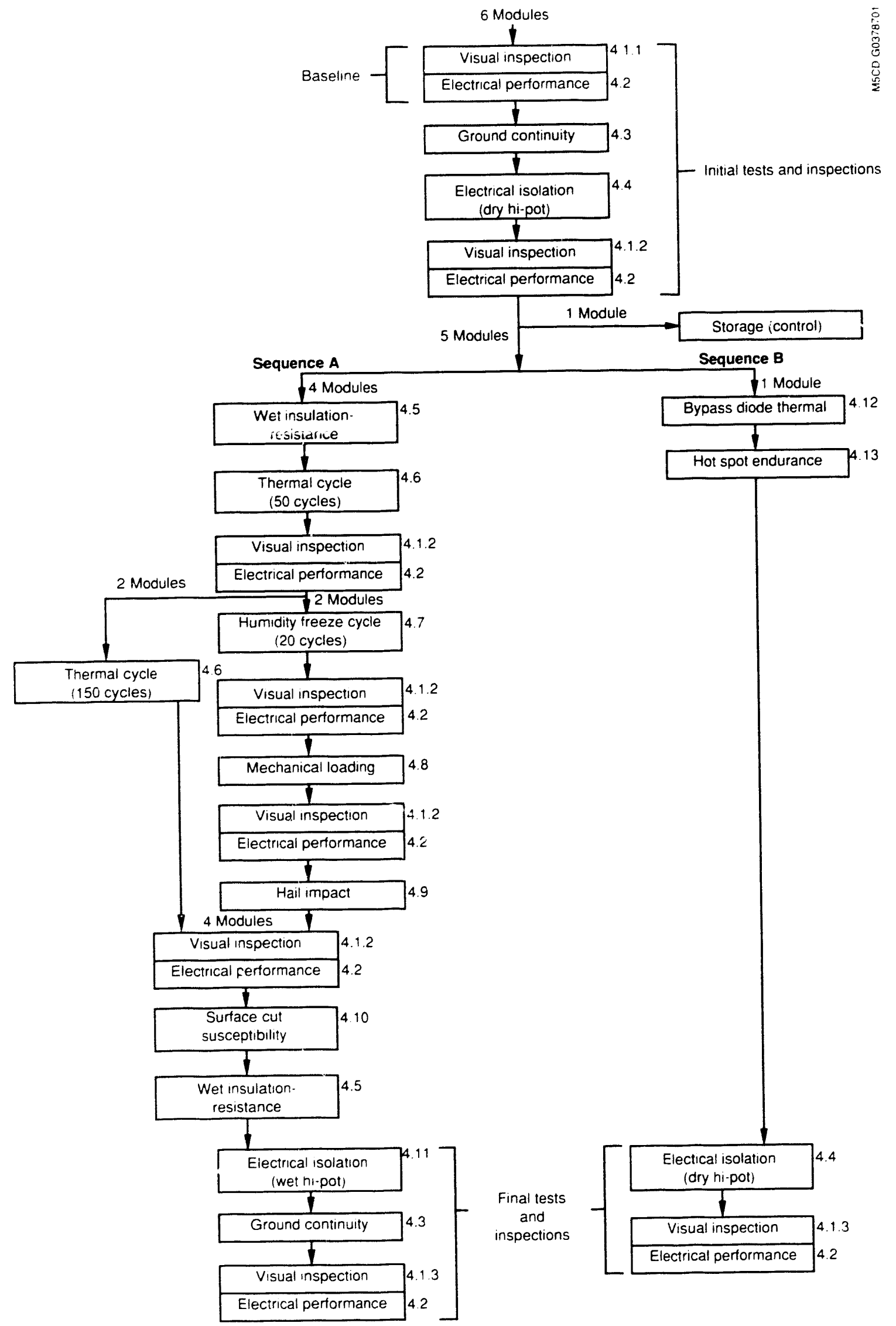

Figure 6.5: $\quad$ Qualification test sequence. 
Various analytical methods were used to analyze and characterize the degraded EVA and to determine the cause of yellowing. In the process of degradation, the gel content increased greatly while the ultraviolet (UV) absorber, Cyasorb UV531, decreased concomitantly. The large increase in the gel content indicates that a cross-linking reaction is dominant in EVA's structural change. Degradation of the polymer near the cover glass surface took place earlier and to a greater extent than that next to the solar cells. The EVA remained clear when it retained more than $70 \%$ of the initial Cyasorb UV531 concentration. Further depletion of Cyasorb during the weathering process resulted in a yellow to brown color in the EVA caused by formation of polyconjugated $(\mathrm{C}=\mathrm{C}) \mathrm{n}$ double bonds of various lengths $(\mathrm{n}>3)$. Acetic acid and other volatile organic components were also produced from the photodecomposition of EVA. The yellowed EVA reduced the photocurrentgenerating capacity of solar cells and the service life of PV modules.

In this work, we studied virgin, partially degraded, and extensively degraded EVA encapsulant films by using a number of analytical methods. Results on the thermal stability, structural effects on EVA by curing processing, the relationship among the extent of degradation, gel content and Cyasorb concentration, and the effects of EVA yellowing on solar cell efficiency will be discussed below.

The polymer encapsulant used in PV modules serves to provide the functions of structural support, electrical isolation, physical isolation/protection, and thermal conduction for the solar cell circuits assembly. Normally the encapsulant materials are made of a visibly transparent polymer to maintain a good optical transmission in a prescribed spectral region. Its long-term stability is important in order to fulfill the above functions over the many years the PV modules are deployed in the field. Ethylene vinyl acetate copolymer (EVA, 33\% vinyl acetate) is almost exclusively used as the polymer for $\mathrm{PV}$ module encapsulation.

\subsubsection{EVA Formulation and Encapsulation}

To provide the desired mechanical strength and long-term stability, the EVA is formulated with a curing agent, a UV absorber. a photo-antioxidant, and a thermo-antioxidant. Tr e trade names of these chemicals and their weight percents added are Lupersol 101 (or TBEC) (1.5\%); Cyasorb UV531 (0.3\%); Tinuvin $770(0.1 \%)$; and Naugard P $(0.2 \%)$. The formulated (stabilized) EVA is extruded into films $\sim 18$-mil thick at $\sim 90^{\circ} \mathrm{C}$ before being laminated at $110^{\circ}$ to $120^{\circ} \mathrm{C}$ onto the stacked assembly of a PV module. Curing is carried out at $140^{\prime}$ to $150^{\circ} \mathrm{C}$ to produce a $65 \%$ to $70 \%$ gel content (degree of crosslinking) on the EVA films. Without curing, the gel content is virtually zero. This typical processing procedure is used for the commercial PV modules 
that have a configuration consisting of a cover glass superstrate. EVA. single crystal Si solar cell circuits. EVA, and backing cover foil.

\subsubsection{EVA Materizis Studied}

The virgin uncured, laminated, and cured EVA freestanding films, and PV modules that were weathered in the field (yielding degraded EVA) were supplied by various PV manufacturers.

Virgin EVA films are formulated with stabilizers. The unstabilized films are made using Elvax 150, which is manufactured by the DuPont Co. To differentiate between the two sides of the EVA film in a module, the surface layer next to the cover glass is referred as the "front side" and the side next to the gridlines and solar cells is the "grid side."

Degraded EVA films were taken from field-exposed PV modules and those stored in the dark for six years, which remained clear. The EVA films exposed outdoors for more than five years developed color ranging from light yellow, to yellow, to yellow-brown, and to brown. When viewed in crosssection, the degraded EVA has a yellow layer on the surface of the front side with the rest of the EVA layer remaining clear to the grid side. The area between neighboring solar cells is referred to as a "vented" region, where the EVA in most cases remained clear.

\subsubsection{Analytical Procedure/Methods}

Extraction with various organic solvents was employed to separate the EVA samples into solution and solid phases. Various analytical instruments were employed to characterize the solution as well as solid phase samples. Methanol and acetone were used to remove soluble components (stabilizers) and low molecular weight EVA particles while keeping the EVA film from swelling. Tetrahydrofuran (THF) was used to dissolve the stabilizers and amorphous EVA from the cross-linked EVA. The solvent-treated films were dried in a vacuum oven at about $40^{\circ} \mathrm{C}$ for at least two days. The gel content was determined from the weight ratio of the vacuum-dried, THF-extracted EVA to the original film. The Cyasorb concentration was determined from the UV absorbance at $326 \mathrm{~nm}$.

\subsubsection{Thermal Stability of EVA}

The thermal stability of freestanding EVA films was evaluated by TGA in dry $\mathrm{N}_{2} . \mathrm{O}_{2}$, and $\mathrm{O}_{2}$ saturated with water moisture. Figure 6.6 shows the nonisothermal thermogravimetric profiles obtained for an unstabilized and a stabilized (cured) EVA at a heating rate of $5^{\circ} \mathrm{C} / \mathrm{min}$. The thermal stability in dry $\mathrm{N}_{2}$ is similar for both films, indicating a similar degradation mechanism 


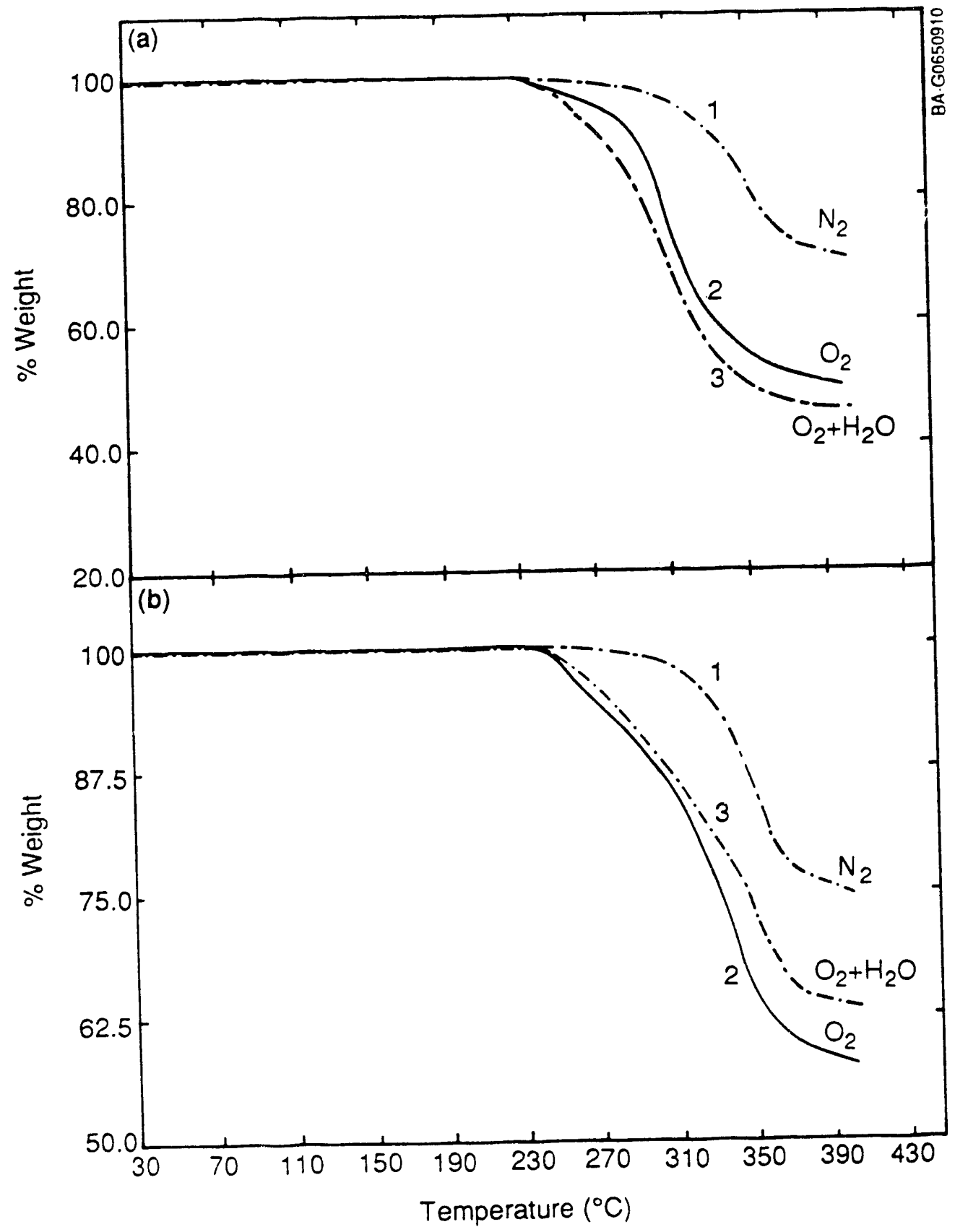

Figure 6.6: Thermogravimetric analysis profiles for (a) unstabilized uncured and (b) stabilized cured EVA films at a heating rate of $5^{\circ} \mathrm{C} / \mathrm{min}$ in the presence of dry $\mathrm{N} 2, \mathrm{O} 2$, and $\mathrm{O} 2$ saturated with water moisture.

of deacetylation. In $\mathrm{O}_{2}$ and moist $\mathrm{O}_{2}$, both samples showed large decreases in the initial decomposition temperature (a drop of $70^{\circ}$ to $80^{\circ} \mathrm{C}$, from $310^{\circ}$ to $320^{\circ} \mathrm{C}$ to $240^{\circ} \mathrm{C}$ ) and large increases in the weight loss, indicating a significantly reduced thermal stability. In addition, a strong smell of acetic acid was detected at relatively low temperatures (ca. $120^{\circ}$ to $140^{\circ} \mathrm{C}$ ). 
Hydrolysis, oxidation, and deacetylation may have proceeded either concurrently or sequentially in wet $\mathrm{O}_{2}$. The reduction of ther nal stability for the unstabilized EVA is greater in wet $\mathrm{O}_{2}$ than in dry $\mathrm{O}_{2}$. The presence of antioxidants in the stabilized EVA gave a higher resistance to thermal oxidation as expected. The cured EVA, because of its cross-linked polymeric chains, was more resistant to hydrolysis in the moisture-saturated $\mathrm{O}_{2}$ than the unstabilized (uncured) EVA.

\subsubsection{EVA Gel Uniformity}

The gel content (degree of cross-linking) on the cured EVA sheets was not uniform. As seen in Fig. 6.7, the distribution of the gel content is not uniform, but Cyasorb concentration is uniform macroscopically. The localized nonuniformity of a cross-linked polymeric structure, as indicated by the nonuniform gel content, was further evidenced by the uneven yellow color developed on the cured EVA strip that was first cleaned with acetone and then stained with trans- $\beta$-carotene in methanol solution. The localized nonuniformity of a cross-linked polymeric structure in EVA is significant because it can result in microscopic fluctuations in the local concentration of UV absorber (Cyasorb) and antioxidants, and, consequently, the stabilization effectiveness.

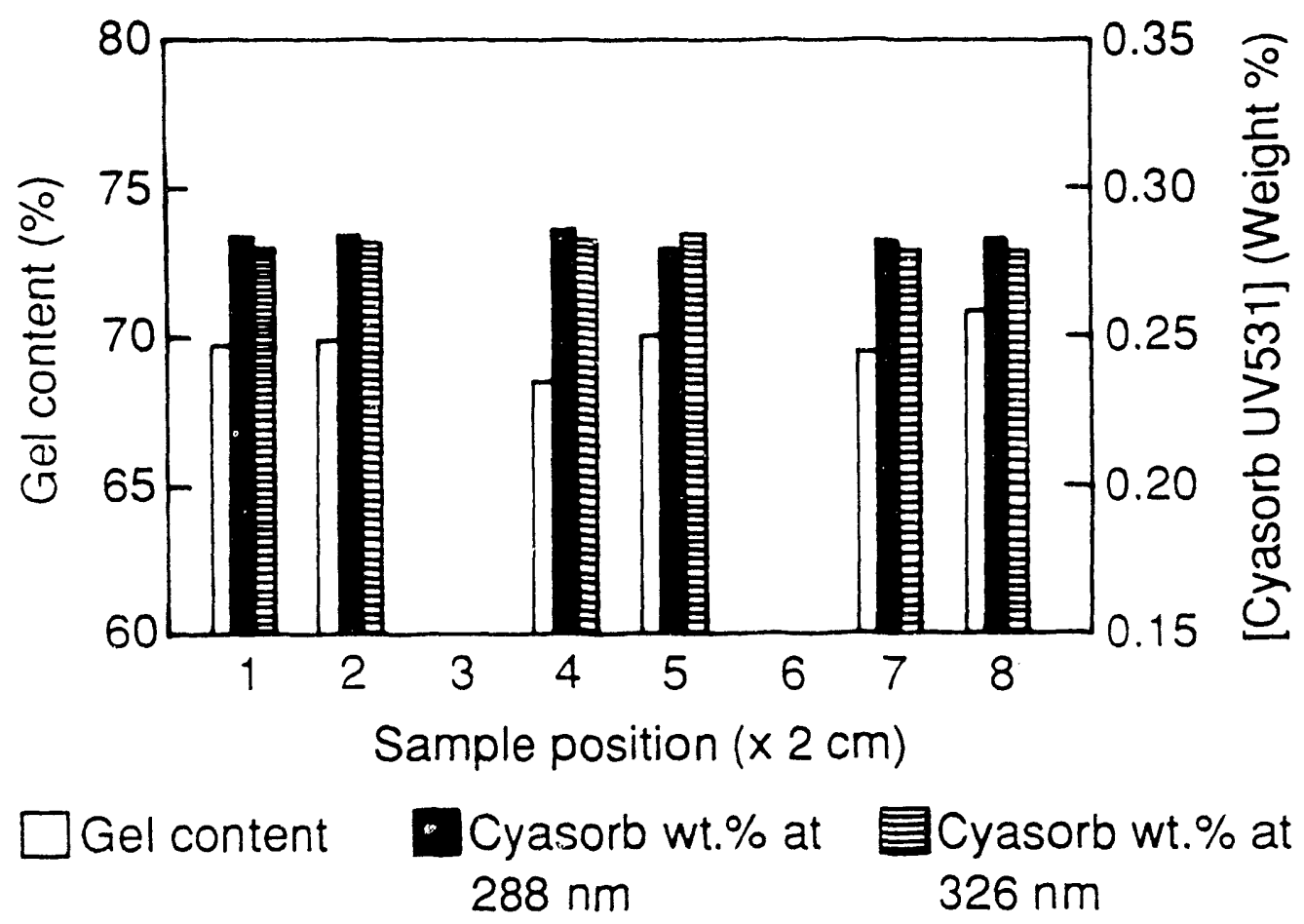

Figure 6.7: Uniformity analysis of gel content and Cyasorb UV531 concentration on a $16 \mathrm{~cm} \times 1 \mathrm{~cm}$ cured EVA strip. Each EVA specimen is of $2 \mathrm{~cm} \times 1 \mathrm{~cm}$ dimension. The sample position corresponds to the location of specimens along the length of the EVA strip. EVA specimens No. 3 and 6 were not analyzed. 


\subsubsection{Quality of Raw EVA Material (Elvax 150)}

Results of UV-visible absorption measurements indicate the Elvax 150 contains absorbing chromophores around $280 \mathrm{~nm}$. After cleaning with methanol or acetone, the absorption peak disappeared. The methanol extract gave an absorption spectrum similar to that of acetophenone or benzophenone. Fluorescence analysis (FA) of the Elvax 150 before and after solvent treatment revealed that the raw EVA already contains UV-excitable chromophores of $\alpha, \beta$-unsaturated carbonyl groups of enone (or -al) type $(-\mathrm{C}=\mathrm{C}-\mathrm{CO}-)$ on the EVA polymer. Hence, the raw EVA material contains a photosensitizer and $\alpha, \beta$-unsaturated carbonyl groups that may serve as potential photosensitizers for the photodegradation of the EVA encapsulant.

\subsubsection{Thermal Processing Effects on EVA}

FA was employed to determine the effects of thermal processing on the EVA structure. The structural change was easily revealed from the changes in the excitation spectra for the thermally processed EVA films. The analysis results, as shown in Fig. 6.8, indicate that the thermal processing in the compounding and extrusion steps $\left(<120^{\circ} \mathrm{C}\right)$ has resulted in some structural change on the EVA. Upon curing $\left(140^{\circ}\right.$ to $\left.150^{\circ} \mathrm{C}\right)$, a new chromophore evolved from the original $\alpha, \beta$-unsaturated carbonyl groups. Accordingly, the structure of the luminescent chromophores in the EVA is modified by the curing step more than by other steps in the thermal processing of EVA formulation and encapsulation. Firstly, near visible light-excitable, new chromophores are produced during the curing step. Secondly, Cyasorb produces an insufficient shielding of the new chromophores. The new chromophores produced from the curing step are excitable by near-visible light (>370 $\mathrm{nm}$ ) as indicated in the FA excitation spectra (Fig. 6.9a).

The UV absorption spectrum of Cyasorb UV531, as shown in Fig. 6.9b, indicates that Cyasorb efficiently "shields" the chromophores of the cured EVA from being excited by the UV light below ca. $360 \mathrm{~nm}$ (Fig. 6.9a). In the spectral region of $\geq 370 \mathrm{~nm}$, the light absorption of the Cyasorb is minimal so that the chromophores on the cured EVA are still excitable by near-visible light, which is evidenced by the increasing intensity from $>350 \mathrm{~nm}$ on curve 2 of Fig. 6.9a. These results indicate that Cyasorb UV531 used in the current EVA formulation cannot shield the new chromophores effectively from absorbing light in the near-visible region.

\subsubsection{Characterization of Degraded EVA}

Two crucial measurements of degradation are derived from UV absorber concentration and gel content analysis. Figure 6.10 shows the results of Cyasorb UV531 concentration (wt\%) and gel content (\%). After THF 

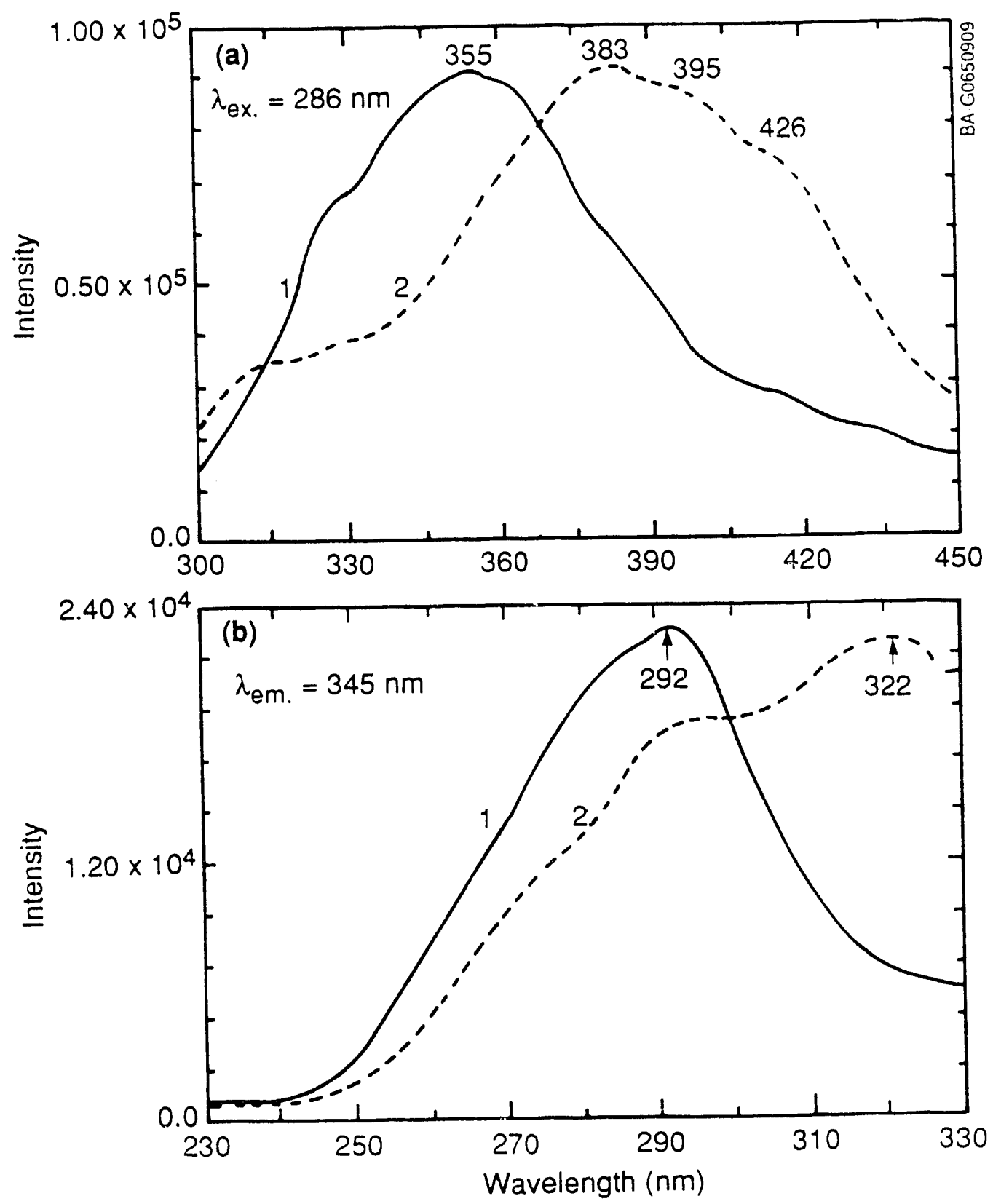

Figure 6.8: Fluorescence analysis of the structural effect of the curing process on EVA. The spectra are for (a) emission and (b) excitation of acetone-or THF-cleaned EVA before (curves 1) and after (curves 2) the curing treatment. The excitation and emission wavelengths are 286 and $345 \mathrm{~nm}$, respectively. 

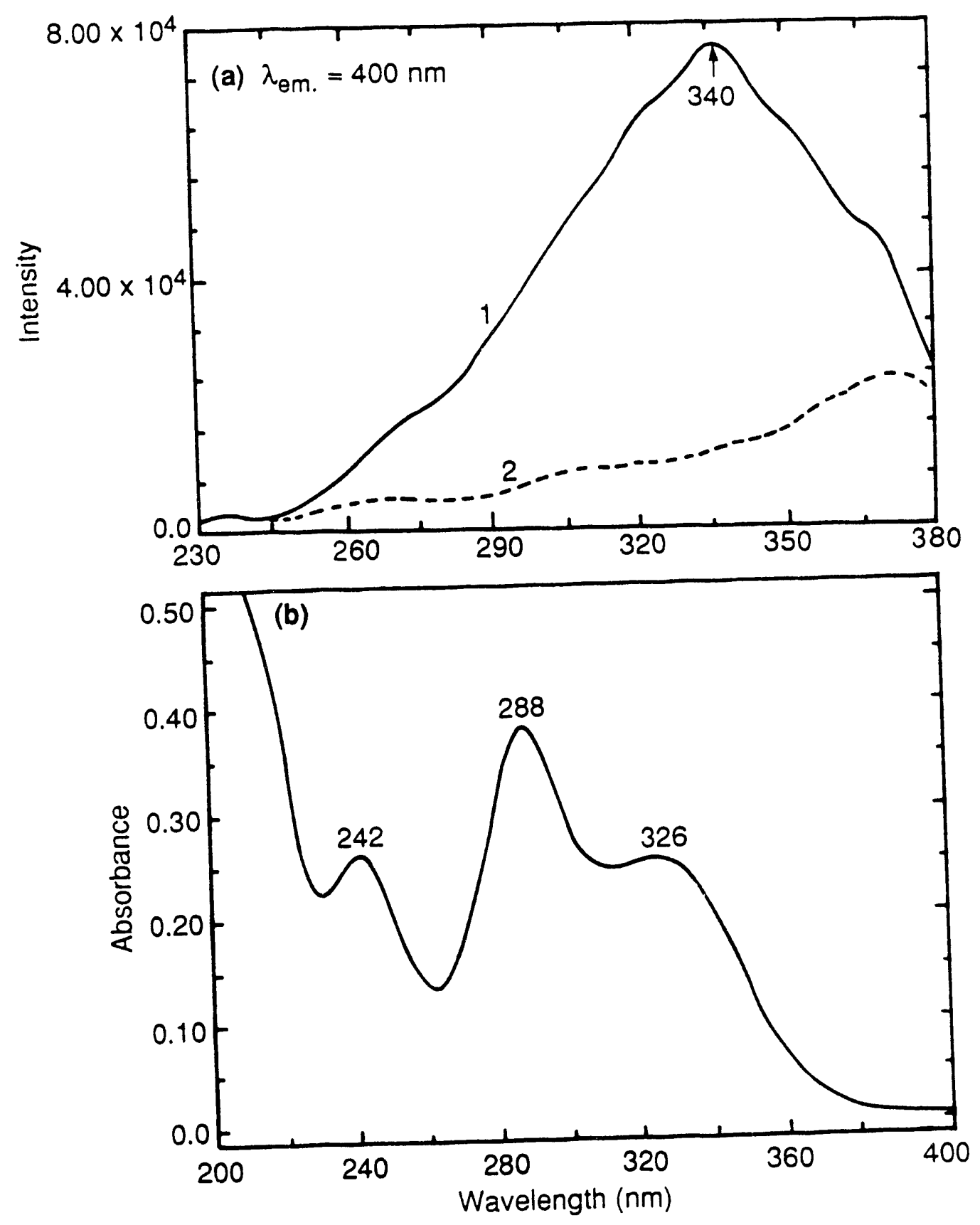

Figure 6.9: Comparison of (a) excitation spectra of virgin cured EVA before and after acetone extraction and (b) absorption of a $2.4 \mu \mathrm{m}$ Cyasorb UV531 in methanol solution. The emission wavelength in (a) is $400 \mathrm{~nm}$. 


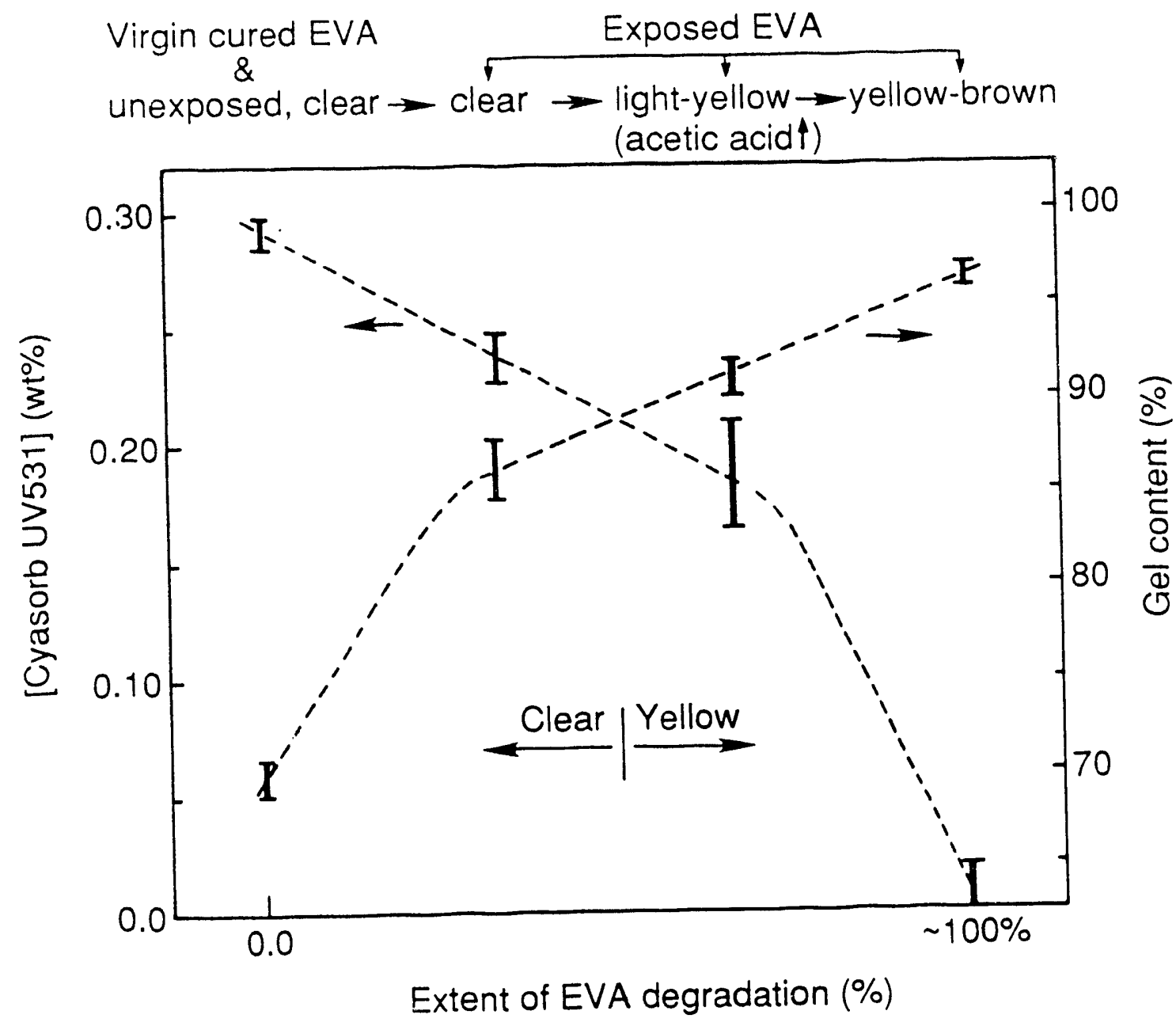

Figure 6.10: Relationships among the extent of EVA degradation, Cyasorb UV531 concentration, gel content, and EVA film color as a summary from the results of analyzing a large number of undegraded and degraded EVA specimens.

extraction, UV absorption measurements were made for the virgin cured, clear EVA stored in the dark for six years, and various degraded EVA films. The results, when compared to the virgin cured films, indicate that the cured EVA films laminated in the PV module and stored in the dark have not degraded. Thus, unweathered modules do not degrade. In addition, no physical loss of Cyasorb UV531 occurred by natural diffusion.

In the process of degradation, the gel content increased from a normal $65 \%$ to $70 \%$ on the virgin cured EVA to $85 \%$ to $88 \%$ on the clear EVA (cell edges), to $90 \%$ to $92 \%$ on the light-yellow EVA, and to $96 \%$ to $97 \%$ on the extensively degraded EVA while the Cyasorb concentration decreased correspondingly. On the extensively degraded EVA, virtually no Cyasorb was observed. The EVA developed a light-yellow, yellow-brown, or brown color, depending on the extent of degradation. In addition to the discoloration, acetic acid and 
volatile organics are present on the degraded EVA. Typically, a large square of yellowed EVA is surrounded by clear EVA ( 0.5 to $1.5 \mathrm{~cm}$ wide) around the edges of the $(10 \times 10 \mathrm{~cm})$ solar cell units.

\subsubsection{Structural Analysis}

The extent of degradation is also determined by FA. Figure 6.11 illustrates the emission spectra obtained with an excitation wavelength of $350 \mathrm{~nm}$ for one extensively degraded, two partially degraded, and one virgin cured EVA. A strong, broad emission peak appeared at a wavelength $>450 \mathrm{~nm}$ for the degraded EVA, in addition to the original one at $\sim 420 \mathrm{~nm}$. As the extent of clegradation increases, the emission intensity of the new peak increases and the peak position shifts from $530 \mathrm{~nm}$ for the light yellow EVA to $560-570 \mathrm{am}$ for the yellow and yellow-brown EVA. The emission in the visible region is due to phosphorescence. Results of FA also confirm that the front side of an EVA degraded much more than the grid side. This is further supported by the results of Fourier transform infrared/attenuated total reflectance (FTIRATR) and x-ray photoelectron spectroscopy (XPS) analyses.

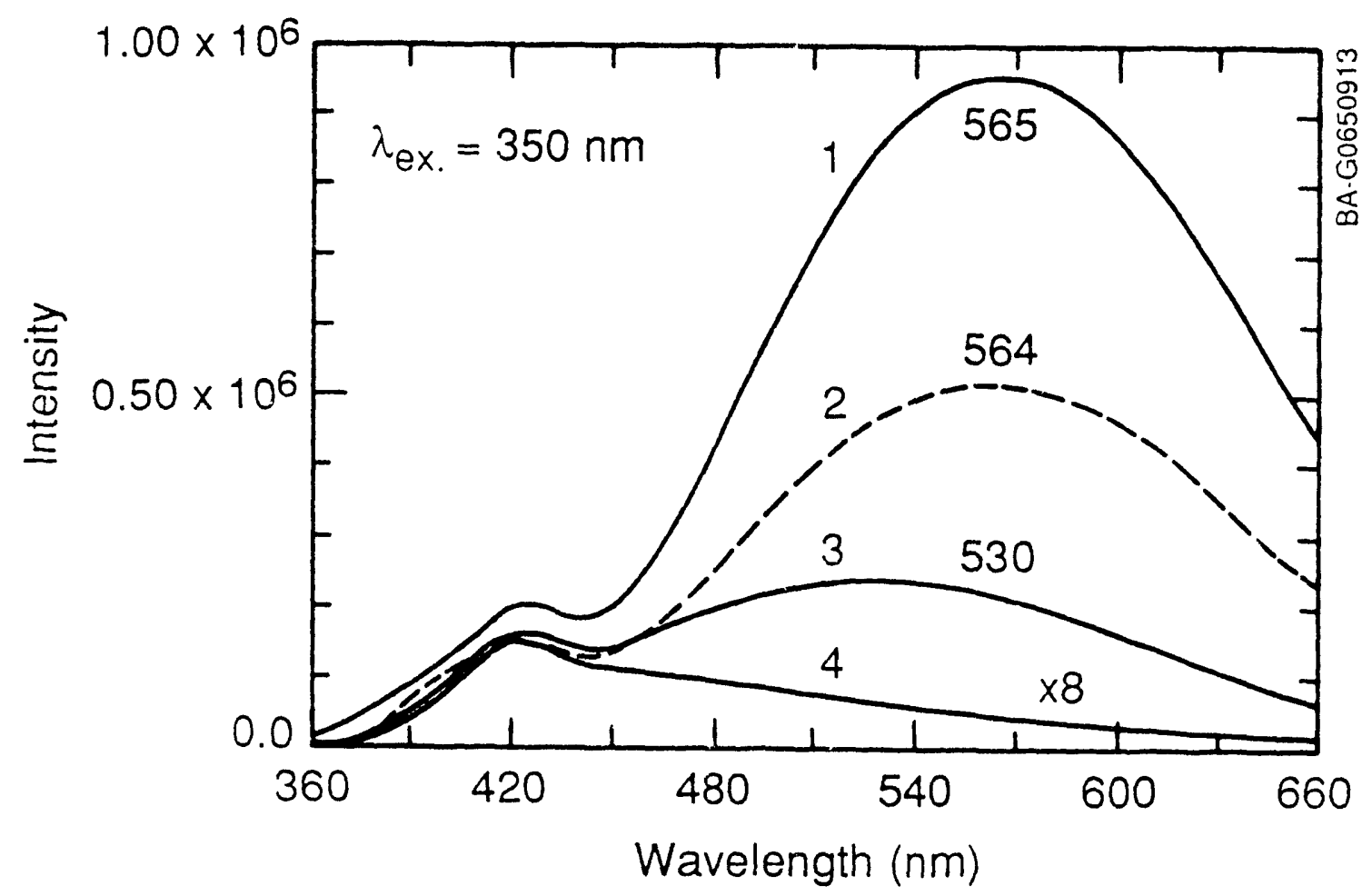

Figure 6.11: Emission spectra for extensively degraded (yellow-brown, curve 1), partially degraded (yellow, curve 2), slightly degraded (light-yelluw, curve 3) and clear EVA films. The excitation wavelength is $350 \mathrm{~nm}$. Curve 4 was multiplied eight times. 
The yellowing of degraded EVA is due to formation of polyconjugated carboncarbon double bonds (polyenes). By comparing the FA results of emission and excitation spectra to those of model compounds, dimethyl 1, 3cyclohexadiene-i, 4-dicarboxylate, and trans- $\beta$-carotene in methanol, the degraded EVA is inferred to consist of mixed polyenes of various lengths with the longest conjugation lengths greater than $n=11$ (i.e., trans- $\beta$-carotene).

\subsubsection{Uniformity of EVA Degradation}

The EVA degradation across each solar cell unit on a weathered PV module was not uniform. Yellowing of the EVA is greater in the central region of each solar cell unit than the cell edges as exemplified in Fig. 6.12a for a partially clegraded EVA film taken from a $4 \times 4$ in. solar cell, where the shaded area indicates a yellow color. Figure $6.12 \mathrm{~b}$ shows that the Cyasorb concentration in the central region was much lower than at the cell edge. Furthermore, there seems to be a boundary on the Cyasorb concentration (ca. $0.21 \mathrm{wl} \%$ ) below which the EVA films become discolored. The FA results shown in Fig. 6.13 further confirm this conclusion.

The Cyasorb may be lost via two possible mechanisms. First, the increase in the gel content can result in a decreased free volume and/or amorphous regions where most of the Cyasorb and other antioxidants reside, causing effective rejection of the stabilizers. The rejected Cyasorb may then diffuse from the central region to the cell edges along the temperature gradient. Second, the Cyasorb may be photodecomposed in the presence of hydroperoxides that are produced by the photooxidation. The hydroperoxides may dissociate into radicals, which can abstract the $\mathrm{H}$ atom from the hydroxyl group on the Cyasorb, resulting in breakdown of the intramolecular hydrogen bonding and therefore the stabilizing capability of Cyasorb.

\subsubsection{Effects of EVA Yellowing on Solar Cell Efficiency}

The extensively degraded and virgin cured EVA films were analyzed for their light transmissions by using a single crystal Si reference solar cell to measure the spectral response (quantum efficiency) and the cell's output current. Results of the spectral response relative to the Si reference cell are shown in Fig. 6.14. The loss of Cyasorb and the light absorbing effect of polyenes on the degraded EVA films are evident. Results are summarized in Table 6.1 for the measured short-circuited currents (Isc) for the single crystal Si reference cell with and without the EVA films. In effect, the solar cell lost $5.7 \%$ to $7.4 \%$ in efficiency when the EVA degraded from clear to yellow. The loss would be larger if a 7059 cover glass plate were placed above the EVA film because the cover glass would filter some of the UV light in the region of 310 to $350 \mathrm{~nm}$. 


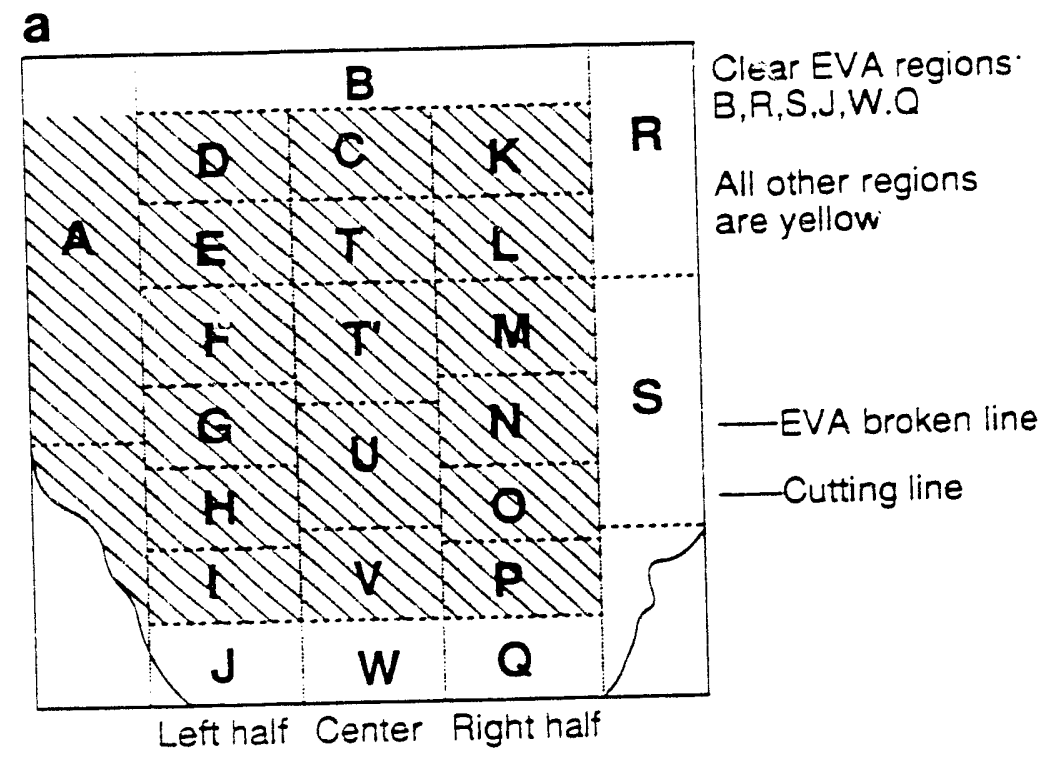

A Partially Degraded EVA Film Mapping

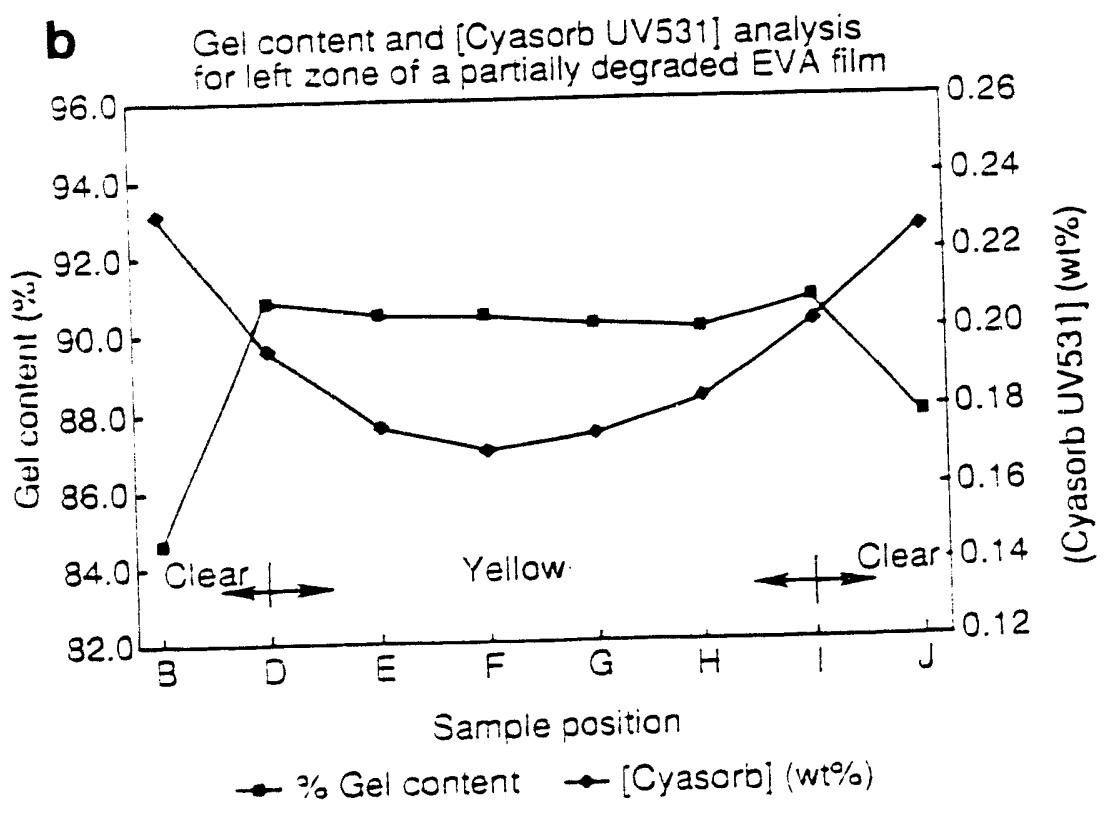

Figure 6.12: (a) Mapping of 24 specimens as labeled from A to W on a partially degraded EVA film that was peeled off from a $4 \times 4$ in. solar cell. The shaded region indicates a yellow color. (b) Results of analysis of the gel content and Cyasorb concentration for the "left half zone" (specimens B through $\mathrm{J}$ ). 


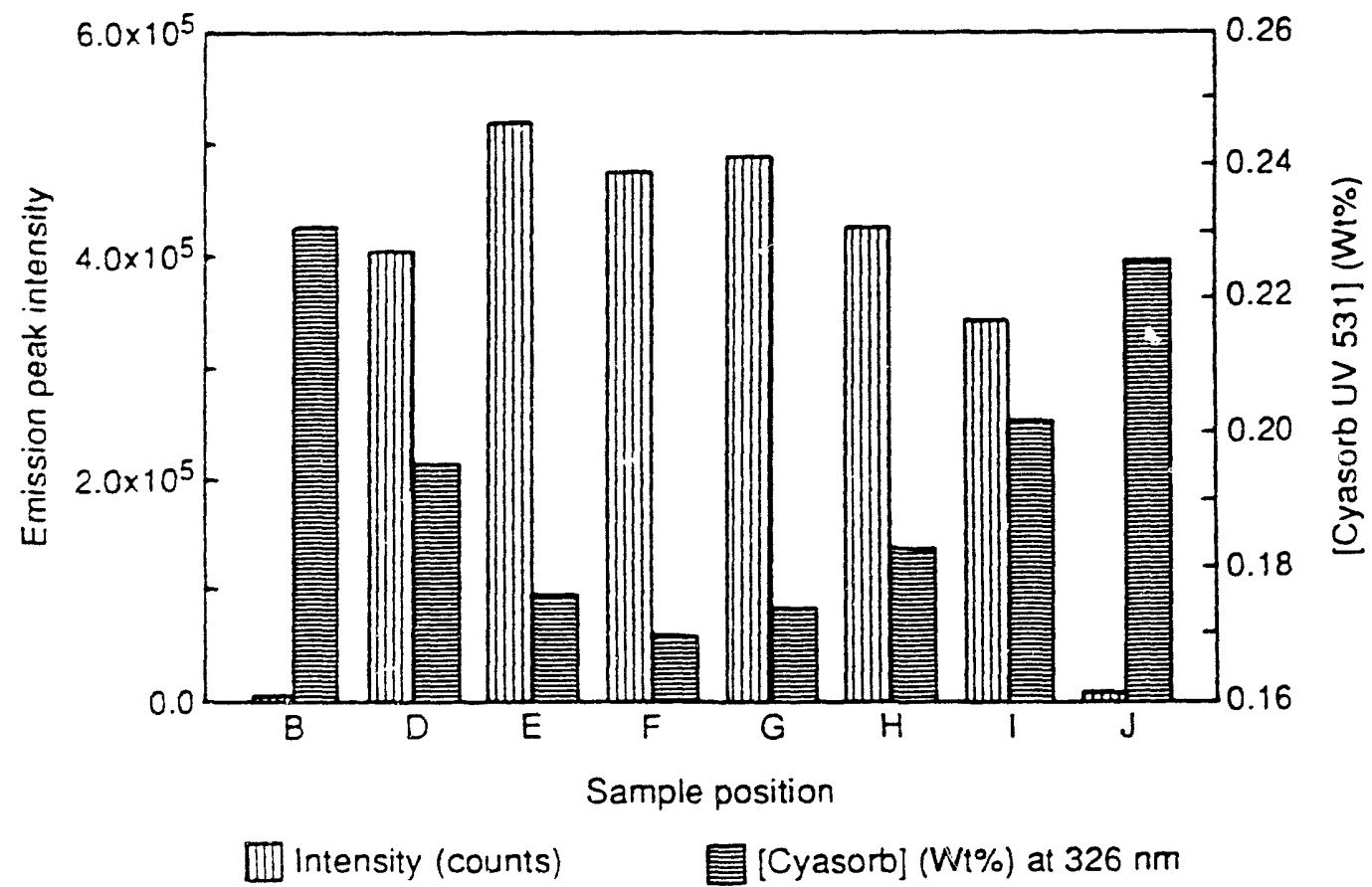

(Excited at $350 \mathrm{~nm}$ )

Figure 6.13: Relationship between the Cyasorb concentration and luminescence intensity obtained for the "left half zone" (specimens B-J) as shown in Fig. 12.

\subsubsection{Conclusions}

The thermal stability of the stabilized, cured EVA was reduced in the presence of $\mathrm{O}_{2}$ and $\mathrm{H}_{2} \mathrm{O}$. Photosensitizer contaminants and UV lightexcitable chromophores are present on the raw EVA material, Elvax 150. The curing step in the thermal processing results in significant structural change in the EVA where new chromophores that are excitable by nearvisible light $(\geq 370 \mathrm{~nm}$ ) are evolved from the initially existing $\alpha, \beta$-unsaturated carbonyl groups. The new chromophores cannot be effectively shielded by the UV absorber Cyasorb UV531.

Weathering-induced degradation of the EVA encapsulant films in PV modules leads to: (1) yellowing of EVA, (2) an increased degree of crosslinking, (3) loss of UV absorber (Cyasorb UV531), and (4) the production of acetic acid and volatile organics. EVA yellowing is directly related to the gel content increase and Cyasorb loss. The extent of degradation is not uniform over the solar cell units. The discoloration results from the formation of 


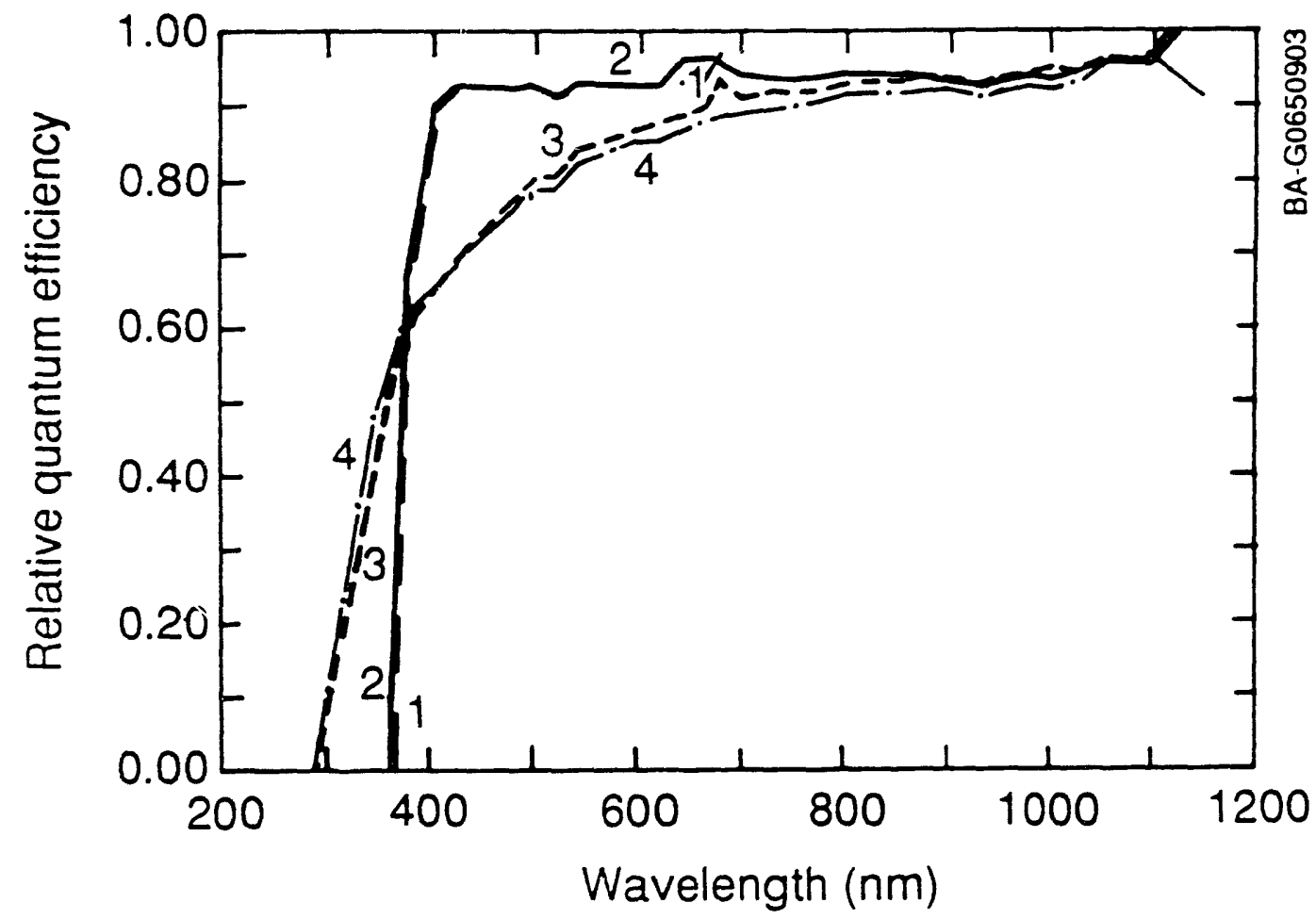

Figure 6.14: Spectral responses measured for a single crystal Si reference solar cell covered with virgin cured (curves 1 and 2) and extensively degraded (curves 3 and 4) EVA films. The virgin EVA films are EVA A9918 (curve 1) and EVA 15295P (curve 2), and the two degraded EVA films differ slightly in their yellow color.

Table 6.1: Short-circuited current $\left(I_{s d}\right)$ of single crystal Si reîerence solar cell ${ }^{a}$ measured with and without virgin and extensively degraded EVA films.

\begin{tabular}{lccc}
\hline EVA Type & I $_{\text {Sc }}(\mathbf{m A})$ & Ratio & Loss $(\%)$ \\
\hline No EVA over cell & 13.733 & 1.000 & -- \\
Virgin EVA A9918 & 12.824 & 0.934 & 0.0 \\
Virgin EVA 15295P & 12.808 & 0.933 & 0.0 \\
Degraded EVA no.1 & 12.087 & 0.880 & 5.68 \\
Degraded EVA no.2 & 11.861 & 0.864 & 7.39 \\
\hline
\end{tabular}

a: An aperture of $0.35 \mathrm{~cm}^{2}$ was used. The EVA film was placed over the aperture. No cover glass was used on top of the cell or EVA, hence allowing the UV-B component of the light to produce current. The Loss (\%) would be larger if the UV-B light were filtered with a 7059 cover glass. 
polyenes of various lengths in the degraded EVA that cause the EVA to become yellow and then brown, depending on the extent of degradation. The polyenes absorb the UV and near-visible light, and luminesce strongly in the visible region, which may partially compensate the loss of solar cell efficiency caused by reduced light transmission.

\subsubsection{Accomplishments}

From our EVA degradation research, we have:

- Demonstrated that the current EVA formulation is not optimal and that new approaches or modifications of current formulation are required;

- Provided several recommendations to the PV manufacturers on the EVAfabrication process to improve the quality of the EVA encapsulant;

- Presented the research results at the PV Module Reliability Workshop (26 Oct. 1990);

- Established the "PV Module Encapsulation Research Laboratory;"

- Identified a non-intrusive analytical method based on fluorescence analysis for diagnosis of the extent of EVA degradation (an invention disclosure was submitted);

- Prepared and submitted an abstract (SERI/TP-213-4087) for the MRS Spring Meeting, 29 Apr. - 3 May, in Anaheim, CA;

- Prepared a draft manuscript titled "Recent Generic Studies of EthyleneVinyl Acetate (EVA) Degradation" that will be submitted for publication in Solar Cells.

\subsubsection{Photovoltaic Module Reliability Workshop [48]}

In an effort to bring together the module reliability effort under way in the PV community and to foster the exchange of knowledge and expertise in the subject, 96 attendees (25 SERI, 2 DOE, 2 Sandia Albuquerque, 1 Jet Propulsion Laboratory (JPL), 5 foreign, and 61 U.S. industry personnel) participated in the 4th SERI/DOE sponsored "PV Module Reliability Workshop" held at the Sheraton Hotel in Lakewood, Colorado on 25-26 Oct. 1990. The three major sessions in the workshop were: (1) Module Performance and Field Experience, (2) Module Research and Testing, and (3) Module Design and Encapsulation Research. Twenty speakers presented state-ofthe-art papers in the entire PV module/system reliability field of crystalline 
silicon, thin-film, and concentrator modules. Qualification testing of the modules, EVA encapsulation research under way at SERI, and module ratings were the major issues for future research discussed at the meeting.

A SERI Conference Proceedings volume (SERI/CP-4079) was published and mailed to all the workshop attendees.

\subsubsection{Standards Development Activities}

Staff members continue to be involved in the development of test methods and standards that can provide a common base for researchers, manufacturers, and users in evaluating and characterizing the performance of PV modules and systems. Senior members of the staff have been very involved with the work of the IEEE PV Standards Board Coordinating Committee (SCC21) and have made important technical contributions, as well as taking leadership roles in the development of national consensus standards. These include current projects on module/array/system performance test, calculation, and rating methods, and module qualification test methods and procedures. These projects reflect the importance of the staff s work at SERI and the unique opportunity for technical transfer of this work to the PV community through the staff interaction and participation in standards activities.

The staff also supports international PV standards efforts through technical interaction and participation at meetings of the U.S. Technical Advisory Group (TAG) on PV systems. SERI (through the DOE PV Program) provided a $\$ 40,000$ subcontract to the Solar Energy Industries Association (SEIA) to continue the management of the US TAG, and to perform duties as the Secretariat to the International Electrotechnical Commission (IEC) Technical Committee 82 (TC-82) on PV systems. The staff monitored the SEIA subcontract and its activities, and participated in the standards making process by attending meetings and providing technical inputs. The importance of this effort is illustrated by recent IEC projects on setting international standards for module qualification tests and module/systems performance tests and evaluation methods.

\subsection{FUTURE WORK}

The group will continue to maintain, improve, and provide testing capabilities and equipment at the Advanced Module Testing and Performance Test Facility. Staff members will also continue to conduct and enhance outdoor, long-term, performance experiments (small arrays of approximately $100 \mathrm{~W}$ ) on amorphous silicon and other thin-film modules (e.g., CIS and CdTe) for performance, stability, reliability, and energy performance evaluation. A major effort will be expended this coming year on developing plans and 
establishing capabilities in module reliability research, and equipping and making operational the reliability laboratories. The group will also undertake the following activities:

- Continue to develop and disseminate measurement procedures and techniques for determining module performance.

- Develop staff and capabilities in PV module reliability research including failure analysis, electrochemical corrosion determination and diagnostic measurement and evaluation techniques.

- Design and fabricate test equipment and apparatus and establish a module wet hi-pot and wet insulation resistance test facility.

- Complete a progress report on testing of EVA and other module encapsulant polymer materials.

- Organize and lead a research forum on module rating issues and test methods.

- Provide technical management and coordination of the DOE PV FlatPlate Module Reliability Research Program, and coordinate DOE laboratory and subcontractor outdoor testing procedures, test objectives, and results.

- Provide technical support through meeting participation and technical input to the IEEE (SCC21) committees and US TAG, and award and monitor the Solar Energy Industries Association (SEIA) subcontract for management of the Secretariat to the IEC TC-82 committee.

- Participate as the SERI/DOE Technical Review Committee member for the PVUSA Project and Niagara Mohawk Project. 


\subsection{CELL PERFORMANCE CHARACTERIZATION}

\subsection{RESEARCH STAFF}

K.A. Emery, Senior Scientist and Group Leader

C.R. Osterwald, Staff Scientist

\subsection{INTRODUCTION}

The performance of photovoltaic devices of all sizes and technologies are evaluated in these laboratories. This group supports the entire photovoltaic community by providing secondary calibrations of photovoltaic cells, efficiency measurements with respect to a given set of standard reporting conditions, efficiency verification of contract deliverables, current versus voltage (I-V) measurements under varying temperature, spectral irradiance, and total irradiance. This group also supports the entire photovoltaic (PV) community by providing information on: PV measurement equipment and systems that are appropriate for the end user. I-V measurement procedures, potential artifacts in the $\mathrm{I}-\mathrm{V}$ results because of equipment or procedures, an analysis of the uncertainty in group performance measurements. This group also performs spectral irradiance modeling and PV performance modeling in support of its ongoing research efforts to reduce the uncertainty in the measured solar spectrum from 300 to $4000 \mathrm{~nm}$, to assist in the optimization of PV devices for a given application, and to explore alternative rating methods.

\subsection{ANALYTICAL CAPABILITIES AND TECHNIGUES}

Appendix D provides a listing of the major instrumentation and test equipment. their features, and specifications.

\subsubsection{Efficiency with Respect to Standard Reporting Conditions}

The efficiency of any photovoltaic device is a function of the temperature, spectral irradiance, and total irradiance. Standards have been developed for terrestrial and extraterrestrial conditions ${ }^{1 \cdot 3}$. The procedures developed by this group allow the performance of any PV device to be measured with respect to any given set of standard reporting conditions. The procedure involves measuring the area using the standard total area definition and then measuring the I-V characteristics ${ }^{4}$. A reference cell, whose spectral response approximately matches that of the test cell, is chosen to measure and set the irradiance of the solar simulator. The requirement that the solar simulator "match" the reference spectrum or that the reference cell "match" the spectral response of the test device has been relaxed by applying a spectral correction factor. 
The intensity of the Spectrolab X25 solar simulator is adjusted using this reference cell until the measured short-circuit current is equal $( \pm 1 \%)$ to its calibrated current. The short-circuit current of the reference cell and intensity monitor (temperature-controlled silicon cell located in the test plane) are then measured at the same time using two HP3456 voltmeters (6 $1 / 2$ digits, 100 line cycle integration time) and averaged over five readings. The test device is then placed in the same location as the reference cell to minimize spatial uniformity error, and the short-circuit current of the test device is then measured at the same time as the intensity monitors. This procedure corrects the measured current for intensity fluctuations and spatial nonuniformity of the light source giving a short-circuit current with a random error of less than $0.03 \%$. The spectral response of the test cell $S_{1}(\lambda)$ is then measured ( 300 to $2000 \mathrm{~nm}$ ) with the bias light intensity adjusted until the measured current is equal to $I_{s c}^{T, c}$. The calibrated short-circuit current of the test device, $I_{\mathrm{sc}}^{\mathrm{T}, \mathrm{R}}$, is then

$I_{s r}^{T, R}=\frac{C V \times E_{r e f} \times I_{s r}^{T, S}}{M \times I_{s i r}^{R, s}}=\frac{I_{s r}^{R, R} \times I_{s r}^{T, S}}{M \times I_{s r}^{R, S}}=\frac{I_{s c}^{T, C}}{M}$

where the spectral mismatch factor is

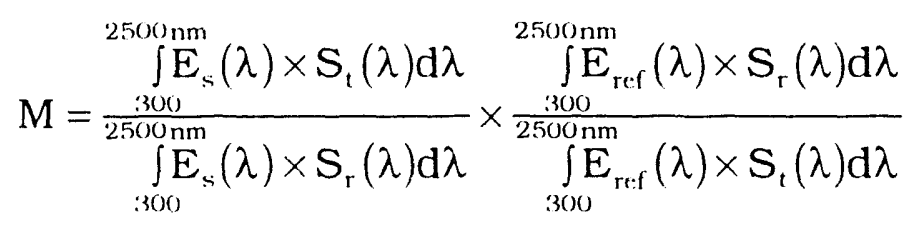

where

$I_{\mathrm{sr}}^{\mathrm{T}, \mathrm{R}} \quad$ is the calibrated short-circuit current of the test cell under the reference spectral and total irradiance

$\mathrm{I}_{\mathrm{sir}}^{\mathrm{K}, \mathrm{K}} \quad$ is the calibrated short-circuit current of the reference cell under the reference spectral irradiance, $\mathrm{E}_{\mathrm{ref}}(\lambda)$, and total irradiance, $\mathrm{E}_{\mathrm{ref} f}$

$I_{s i}^{\mathrm{T}, S} \quad$ is the measured short-circuit current of the test cell under the source or simulator spectrum, $E_{s}(\lambda)$

$\mathrm{I}_{s r}^{R, S} \quad$ is the measured short-circuit current of the reference cell under the source or simulator spectrum. $\mathrm{E}_{\mathrm{s}}(\lambda)$ 
M is the spectral mismatch correction factor

$\mathrm{E}_{\mathrm{ref}}$ is the reference total irradiance, usually $1000 \mathrm{Wm}^{-2}$

$\mathrm{CV}$ is the calibration value of the reference cell

$S_{1}(\lambda) \quad$ is the measured spectral response of the test cell

$S_{r}(\lambda) \quad$ is the measured spectral response of the reference cell

The spectral irradiance of the solar simulator, $E_{\mathrm{s}}(\lambda),(350$ to $2500 \mathrm{~nm})$ is measured periodically using a LI-COR spectroradiometer (300 to $1100 \mathrm{~nm}$ ) and an Optronics Laboratories spectroradiometer (800 to $2500 \mathrm{~nm}$ ).

The I-V characteristics are then measured using four-terminal Kelvin connections to the PV device with a custom data acquisition system designed to give a random error of less than $\pm 0.1 \%$ and a nonrandom error of less than $\pm 1 \%^{4,5}$. Once the short-circuit current of the test device has been obtained, the I-V characteristics are measured and the efficiency is calculated. A fourth order least squares polynomial curve fit to the power versus voltage curve using all points within $15 \%$ of the maximum power point is computed. Setting the derivative of the polynomial equal to zero and solving for its real root closest to the maximum power point allows the maximum power to be more accurately determined than using conventional methods. This is especially important for high fill factor devices. Similar fitting algorithms are used to improve the accuracy of the open-circuit voltage and short-circuit current data points ${ }^{6}$.

When two-terminal multijunction PV structures are evaluated, the spectral correction factor $\mathrm{M}$ must be computed for each junction.

The multiple-source simulator is currently the only technique for determining the I-V characteristics of a two-terminal multijunction solar cell with respect to any given set of reporting conditions. The procedure for measuring a tandem cell under a multisource solar simulator is a simple extension of the single-source method given in Eq. 7-1. The spectral content of the light source(s) is adjusted until the following is true ${ }^{7}$

$I^{R_{1}, R}=\frac{I^{R_{1} . S}}{M_{1}} \cdot \bullet \cdot I^{R_{11} \cdot R}=\frac{I^{R_{n} \cdot S}}{M_{11}}$

The subscript on the superscript $R$ denotes the junction, with 1 representing the top junction and 3 denoting the bottom junction for a triple-junction cell. 
Equation 7-3 assumes that the spatial nonuniformity of the spectral irradiance from each light source is the same. The number of times that the spectral irradiance of the composite light source in the test plane must be measured for the spectral mismatch computations $\left(M_{n}\right)$ can be minimized by having the relative quantum efficiency of the reference cell for each junction closely match the relative quantum efficiency of that junction. For some material systems a single-junction reference cell that has the same quantum efficiency as its two-terminal multijunction counterpart can be fabricated. This is achieved by shorting out all the junctions that are not required except the desired one (top, middle, or bottom) while maintaining the same optical properties. For amorphous silicon based tandems this is not possible so investigators typically purchase optical interference and color glass filters that are placed over a single-crystal silicon reference cell. These reference cells are then calibrated as secondary standards using Eq. 7-1.

\subsubsection{Spectral Response Measurement System}

The spectral response measurement system uses periodic $(440 \mathrm{~Hz})$ monochromatic light directed through 1 of 51 10-nm bandwidth interference filters covering the spectral range from 290 to $2000 \mathrm{~nm}$. The system is capable of providing steady-state light bias levels up to $2 \mathrm{~A}$ and voltage bias levels from $0 \pm 1 \mathrm{mV}$ to $\pm 40 \mathrm{~V}$. The intensity of the bias light is normally adjusted to give the short-circuit current under standard reporting conditions. The custom operational amplifier based data acquisition system allows for a wide range of gains ( 1 to $10^{6}$ ), allowing for a wide range of areas (less than $0.01 \mathrm{~cm}^{2}$ to over $100 \mathrm{~cm}^{2}$ ) with a wavelength dependent error of less than $\pm 5 \%$ and an error of less than $\pm 2 \%$ in the integrated absolute quantum efficiency ${ }^{1.4 .5}$.

\subsubsection{Primary Reference Cell Calibration Facility}

The short-circuit current $I_{\mathrm{sc}}$, total irradiance $\mathrm{E}_{\mathrm{tot}}$, and spectral irradiance $\mathrm{E}_{s}(\mathrm{l})$, are measured at the same time outdoors with the same field of view. The total irradiance is measured with a primary absolute cavity radiometer having a $5.00^{\circ}$ field of view, and the spectral irradiance is measured with a LI-COR LI-1800 spectroradiometer having a $5.00^{\circ}$ field of view. The uncorrected average calibration value $\left\langle\mathrm{CV}_{11}\right\rangle$ is calculated for the $30 \mathrm{I}_{\mathrm{sc}}$ and $\mathrm{E}_{\mathrm{t}, \mathrm{t}}$ readings taken during the $30 \mathrm{~s}$ required to measure $\mathrm{E}_{\mathrm{s}}(\lambda)$. The uncertainty in $\left\langle\mathrm{CV}_{1}\right\rangle$ is reduced by using separate voltmeters that are triggered as a group to record the four short-circuit current for the four cells being calibrated and the total irradiance as measured with the absolute cavity radiometer. The atmospheric constraint for a valid calibration value is that the range (maximum to minimum) over the $30 \mathrm{~s}$ time period in $\mathrm{I}_{\mathrm{sr}}$ be less than $0.25 \%$, in $\mathrm{E}_{\mathrm{tot}}$ be less than $0.07 \%$, and in $\left\langle\mathrm{CV}_{11}\right\rangle$ be less than $0.1 \%$. Once a valid $\left\langle\mathrm{CV}_{11}\right\rangle$ is obtained the short-circuit current is corrected for 
temperature to $25^{\circ} \mathrm{C}$. The temperature of the cell is measured with a thermocouple that is connected to a custom reference plate whose temperature is measured using a precision resistance temperature device (RTD) $(0.01 \%)$ thermometer. The 2.5-cm-thick reference plate is located within $1 \mathrm{~m}$ of the reference cells and is insulated with $5 \mathrm{~cm}$ of Styrofoam. The temperature corrected calibration value is then spectrally corrected giving a single calibration value (CV). The CV is computed at least 20 times for at least three separate days giving a single calibration value using: ${ }^{1,4,5,8,9}$

$C V=\left\langle\frac{I_{\mathrm{sc}}}{E_{\mathrm{tot}}}\right\rangle \frac{\int_{300}^{4000 \mathrm{~nm}} \mathrm{E}_{\mathrm{ref}}(\lambda) \times \mathrm{S}_{\mathrm{r}}(\lambda) \mathrm{d} \lambda \int_{300}^{4000 \mathrm{~nm}} \mathrm{E}_{\mathrm{s}}(\lambda) \mathrm{d} \lambda}{\int_{300} E_{\mathrm{ref}}(\lambda) \mathrm{d} \lambda \int_{300}^{4000 \mathrm{~nm}} \mathrm{E}_{\mathrm{s}}(\lambda) \times \mathrm{S}_{\mathrm{r}}(\lambda) \mathrm{d} \lambda}$

Since the measurement of $\mathrm{E}_{\mathrm{s}}(\lambda)$ does not encompass the limits of the reference spectrum, the measured spectrum is extended using a computer model to encompass the range of the reference spectrum (300 to $4000 \mathrm{~nm}$ ).

\subsection{EQUIPMENT/ANALYSIS DEVELOPMENT}

\subsubsection{Modeling of the Direct-Beam Solar Spectrum from Spectroradiometric Measurements}

The outdoor primary reference cell calibration program requires the directbeam solar spectral irradiance over the 0.3 to $4.0 \mu \mathrm{m}$ wavelength range for each calibration data point. Because the presently available instrumentation, a LI-COR LI- 1800 portable spectroradiometer, can only measure the 0.35 to $1.1 \mu \mathrm{m}$ range, an analysis procedure has been developed to supply the missing spectral range. This procedure computes the direct-beam atmospheric transmittance parameters from the LI-1800 data and, using these parameters, calculates the spectral irradiance ${ }^{10}$.

The spectral analysis procedure uses the molecular absorption models from the LOWTRAN7 atmospheric transmittance code provided by the Air Force Geophysics Laboratory" . LOWTRAN7 breaks the atmospheric transmittance into scattering elements: aerosol, Rayleigh, and molecular absorbers (i.e., $\mathrm{O}_{2}, \mathrm{O}_{3}, \mathrm{CO}_{2}$, and water vapor) (see Fig. 7.1). The spectroradiometer data is first converted to transmittance by ratioing the spectral irradiance to the extraterrestrial solar spectrum. A fit over the 0.752 to $0.774 \mu \mathrm{m}$ range gives the $\mathrm{O}_{2}$ and $\mathrm{CO}_{2}$ absorption functions and the Rayleigh scattering function. Because the direct-beam transmittance is the product of each individual transmittance function, the transmittance data can then be corrected for $\mathrm{O}_{2}$ absorption and Rayleigh scattering. Ozone absorption is calculated from a numerical fit to the 0.400 to $0.566 \mu \mathrm{m}$ region. The ozone fit also gives the 


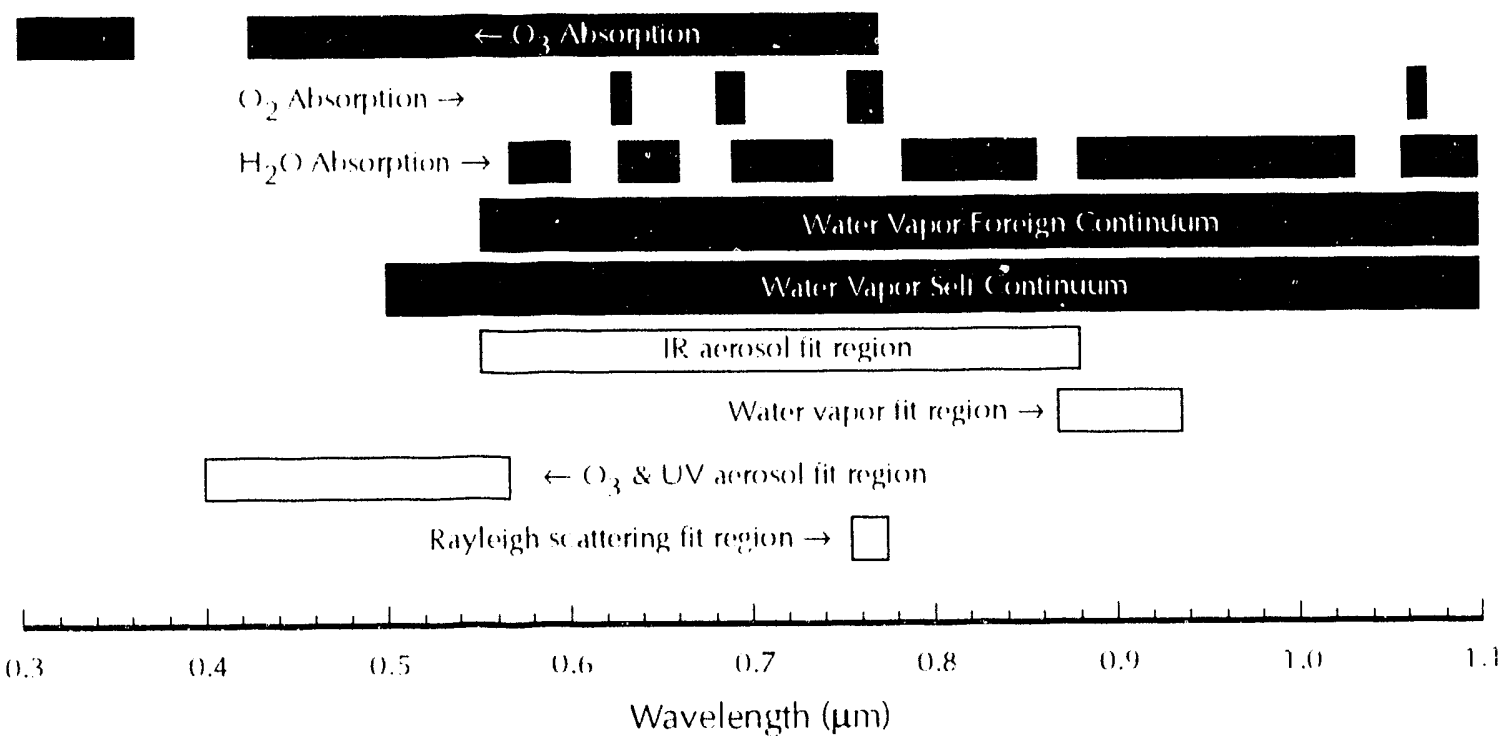

Figure 7.1: Atmospheric transmittance molecular absorption bands and scattering regions as a function of wavelength along with spectral analysis procedure fittings regions.

short-wavelength aerosol scattering function at the same time. After the transmittance data is corrected for ozone absorption, the water vapor absorption and long-wa'velength aerosol function is obtained from a fit of the 0.552 to $0.936 \mu \mathrm{m}$ range. Once the atmospheric transmittance functions are known, the spectral irradiance is produced by multiplying the extraterrestrial solar spectrum by the calculated atmospheric transmittance at each wavelength.

An example of the spectral irradiance analysis is shown in Fig. 7.2, where the heavy line represents the spectroradiometer data, and the light line the calculated spectral irradiance. The agreement between the two is excellent, except beyond $1.0 \mu \mathrm{m}$ where the measured data is too high due to temperature sensitivity of the photodiode detector in the spectroradiometer.

Because the outdoor calibration procedure records the total irradiance as measured with an absolute cavity radiometer, it is possible to the integrate the calculated spectral irradiance and compare this value with the cavity radiometer. Figure 7.3 shows the ratio of the integrated spectral irradiance to the cavity radiometer for an entire calibration data set. The average ratio for this data set is 1.0077 , which is well within the $\pm 2 \%$ error limits of the spectroradiometer. 


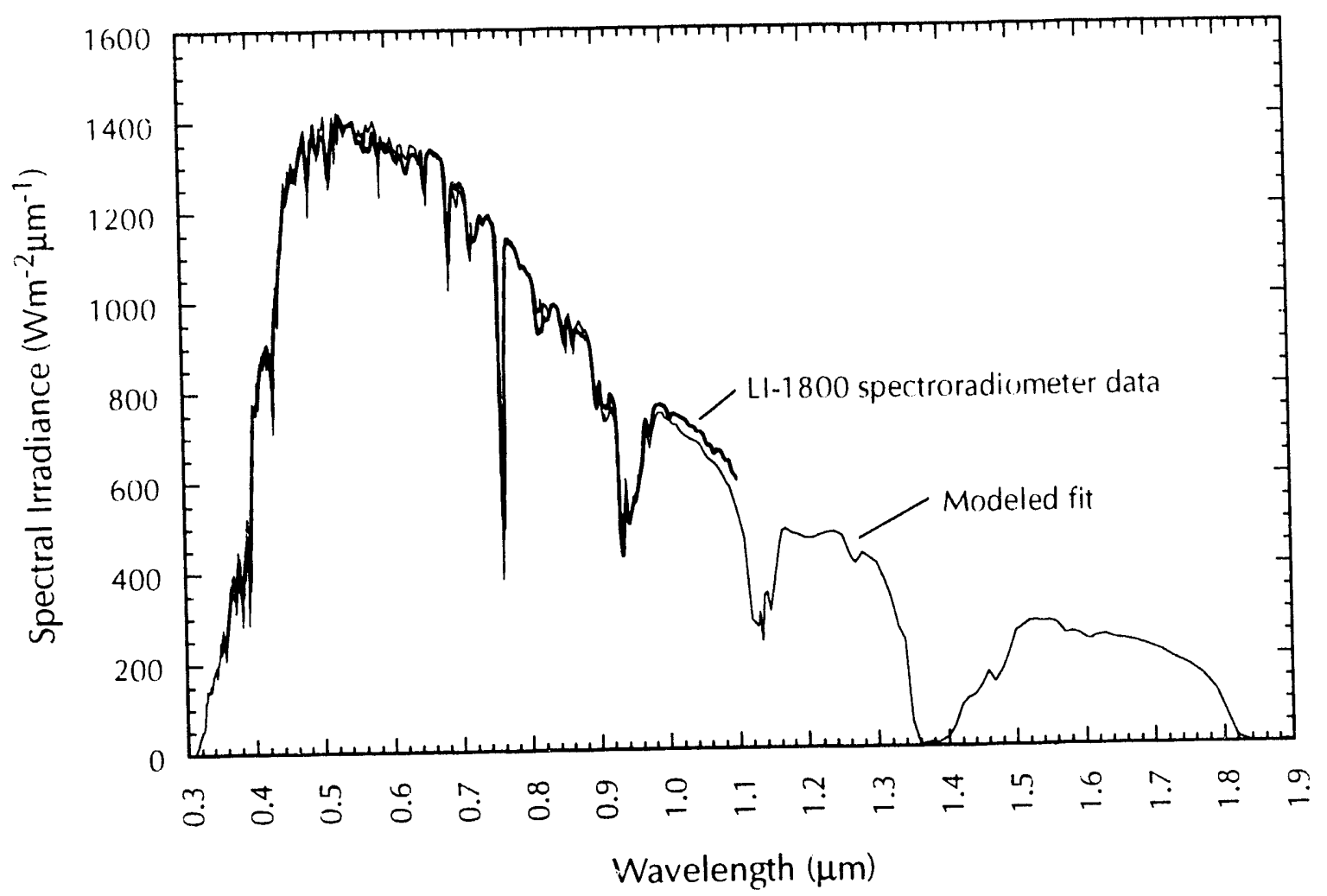

Figure 7.2: Example spectral analysis result showing LI-COR LI- 1800 spectroradiometer data and modeled spectral irradiance as a function of wavelength.

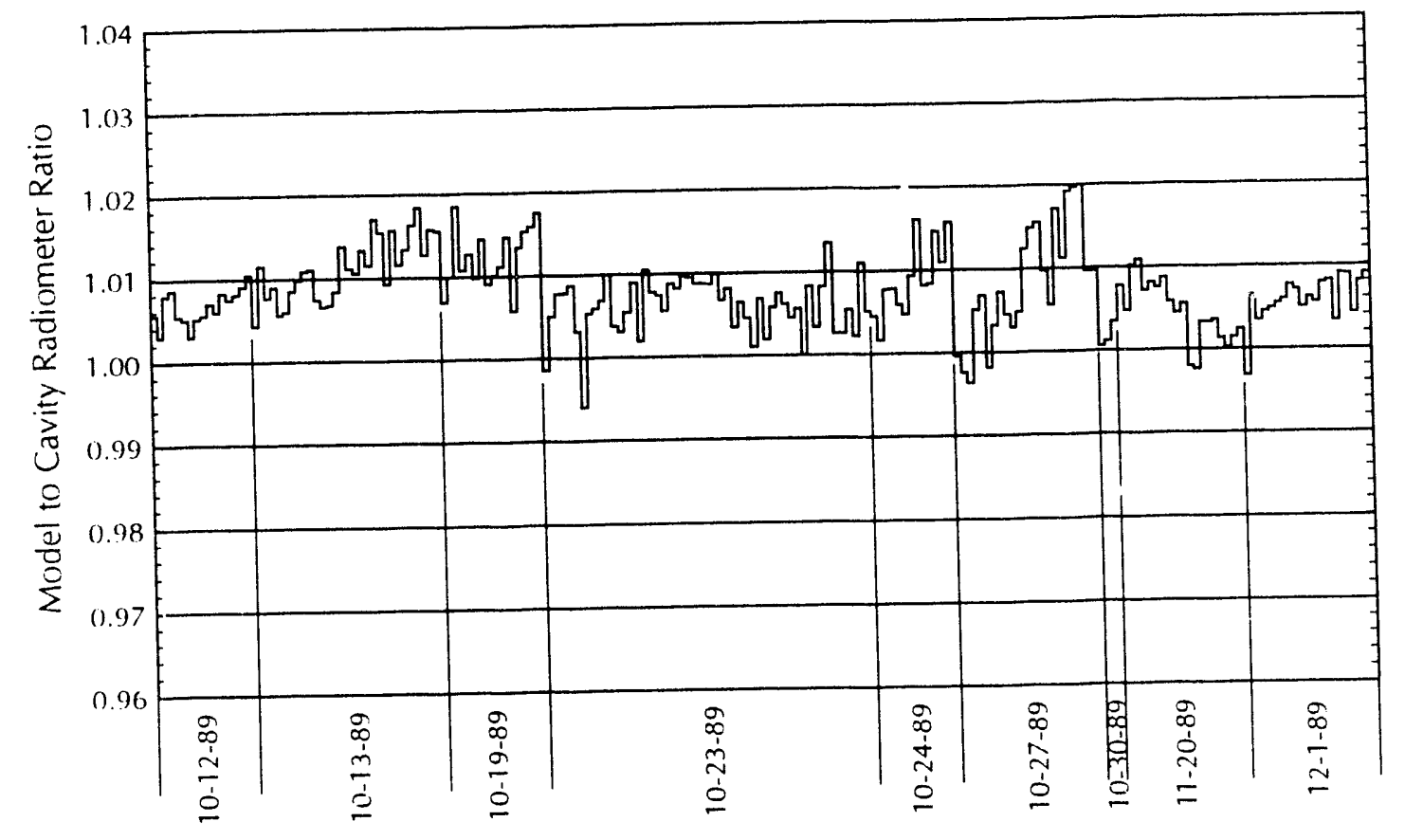

Figure 7.3: Ratio of integrated spectral irradiances produced by spectral analysis to total solar irradiance measured with an absolute cavity radiometer for an entire reference cell calibration data set. 


\subsubsection{1 to 200 Suns Continuous Illumination Concentrator Measurement}

The efficiency versus concentration data are taken with the one sun dataacquisition system ${ }^{1.4 .5}$ and an unfiltered $1000 \mathrm{~W}$ Xe arc light source. Using the $I_{\text {cic }}$ measured at one sun, Eq. 7-1, and assuming linearity, the I-V characteristics are measured. The sample temperature is set to the reference temperature, $25^{\circ} \mathrm{C}$ without illumination. Using a high-speed shutter $(2 \mathrm{~ms})$ and voltmeter, the open-circuit voltage is sampled (1000 readings/s). The highest measured $\mathrm{V}_{\text {oc }}$ is then taken to be $\mathrm{V}_{\text {oc }}$ under concentration. The thermoelectrically controlled vacuum plate is then cooled until this $\mathrm{V}_{\text {or }}$ is reached. A temperature gradient of $10^{\circ} \mathrm{C}$ between the plate and cell is typical. The linearity of $I_{s c}$ with total irradiance is verified by using one of several stainless steel wire meshes mounted in an aluminum frame to attenuate the light a fixed and known amount. A major advantage of this system over conventional flash systems for evaluating concentrator cclls is that the change in voltage with respect to time (bias rate) can be made arbitrarily small. This is important for high lifetime PV structures and high capacitance devices ${ }^{6}$.

\subsubsection{PV Performance Modeling}

A variety of PV models have been developed during FY 1990 to evaluate the performance of PV devices ${ }^{12-14}$. A computer model that accurately predicts the performance as a function of concentration and temperature was developed ${ }^{12.13}$. This model has been instrumental in determining the energy gaps for optimum single-junction, two-terminal or three-terminal multijunction device structures. For the first time this model demonstrated that the optimal energy gaps for tandems shifts appreciable with temperature and concentration. This work has shown why optimum energy gaps for tandem devices were too high and lower band-gap material systems such as GaInAs are better bottom cells. Results from this model are presented in section 4.0 of this report.

The power and energy production of a photovoltaic module or array operating in the terrestrial environment is a function of the total irradiance, spectral irradiance, and cell temperature. The peak power produced under standard reporting conditions $\left(1000 \mathrm{Wm}^{-2}, 25^{\circ} \mathrm{C}\right.$ module temperature, and the International Electrochemical Commission (IEC) or the American Society for Testing and Materials (ASTM) global reference spectrum) is usually greater than the actual measured power in the fif:ld. The purpose of this numerical study is to investigate alternative power and energy rating methods that more accurately predict the cell performance under actual operating conditions. This need is especially important as the variety of commercially available PV devices (a-silicon, CdTe, CulnSe ${ }_{2}$, and other 
single- and multiple -junction structures) increases. There are also a variety of specialized niche markets for PV such as battery charging in a cloudy climate where the rating under standard reporting conditions is inadequate.

A computer model has been developed to simulate solar cell power production using readily available meteorological data: hourly global and diffuse total irradiance, air temperature, wind speed. relative humidity, and time to compute the total and spectral irradiance and the cell temperature. The semi-empirical spectral model consists of a clear-sky simulation code, a normalization procedure, and "cloud cover modifiers" derived from statistical analysis of measured data. This model has been applied to produce hourly spectral irradiance data over a three-year period corresponciing to the climate at Stuttgart F.R.G.

A two-exponential cell model with series and shunt resistances computes the I-V characteristics for single- or multijuncion structures. The model uses a numerical search routine and does not apply simplifying approximations to locate the open-circuit voltage, short-circuit current, and maximum power points. For the case of multipie-junction PV devices the I$\mathrm{V}$ curve for each juriction is computed first and then the two-terminal multijunction I-V curve is reconstructed by summing the voltages at the same current for each junction. The photocurrent is computed using the measured spectral responsivity. The cell temperature in the module is simulated using the air temperature, wind speed, total irradiance and the modules nominal operating cel! temperature (NOCT). The model has been applied to typical production mono-crystalline silicon and state-of-the-art GaAs and thin-film technologies including CdS/CdTe, CdS/CuInSe ${ }_{2}$ a-Si, and $\mathrm{a}-\mathrm{Si} / \mathrm{a}-\mathrm{Si} / \mathrm{a}-\mathrm{Si}: \mathrm{Ge}$ devices. The use of the model to compare energy rating methods for various applications can be seeri from Table 7.1 and able 7.2. From the needs summarized in Table 7.1 the various rating methods that are investigated are summarized in Table 7.2. This study will perform a systematic comparison of these and other rating methods for a variety of PV technologies. 
Table 7.1: Critical operation conditions for the most important terrestrial PV system configurations

PV System Configuration

Critical Period of Operation

\begin{tabular}{|c|c|}
\hline grid-connected, fuel-saving mode & the whole year \\
\hline peak demand supply & $\begin{array}{l}\text { during peak demand (time of day, } \\
\text { temperature) }\end{array}$ \\
\hline remote system for cooling & at high temperatures \\
\hline remote system with storage & $\begin{array}{l}\text { during days or months with low } \\
\text { irradiance }\end{array}$ \\
\hline pump system for agriculture & $\begin{array}{l}\text { during growth time (times when } \\
\text { water needed) }\end{array}$ \\
\hline
\end{tabular}

Table 7.2: Proposals for a multivalue site-specific energy rating scheme

Period

Method

Annual

Monthly max. and min. efficiency

$\sum_{\text {year }} \mathrm{P}_{\max } / \sum_{\text {year }} \mathrm{P}_{\text {insolation }}$

Daily max. and min. efficiency

$\mathrm{P}_{\max } / \mathrm{P}_{\text {Insolation }}$

$\mathrm{P}_{\max } / \mathrm{P}_{\text {insolation }}$

Hourly max. and min. efficiency

$\mathrm{P}_{\max } / \mathrm{P}_{\text {insolation }}$

Month with lowest $P_{\text {insolation }}$

$\sum_{\text {month }} \mathrm{P}_{\max } / \sum_{\text {month }} \mathrm{P}_{\text {insolation }}$

Day with lowest $P_{\text {insolation }}$

$\sum_{\text {day }} \mathrm{P}_{\max } / \sum_{\text {day }} \mathrm{P}_{\text {insolation }}$

Hour with highest temperature

$\mathrm{P}_{\max } / \mathrm{P}_{\text {insolation }}$

Ave. efficiency at $\mathrm{P}_{\text {tnsolation }}=1000$

$\left\langle\mathrm{P}_{\max } / \mathrm{P}_{\text {tnsolation }}\right\rangle$

$\mathrm{Wm}^{-2}$ and at $\mathrm{P}_{\text {insolation }}=100 \mathrm{Wm}^{-2}$

Standard reporting conditions

1000Wm ${ }^{-2}, 25^{\circ} \mathrm{C}$, ASTM E892

global

reference spectrum

Standard reporting conditions at

1000Wm ${ }^{-2}$, NOCT, ASTM E892

NOCT

global reference spectrum 
1. Emery. K.A., and C.R. Osterwald, "Efficiency Measurements and Other Performance-Rating Methods," Current Topics in Photovoltaics Vol. 3. Chap. 4, Academic Press Ltd., London, 1988.

2. Annual Book of ASTM Standards, Vol. 12.02, American Society for Testing and Materials, 1916 Race St., Philadelphia, PA.

3. International Electrotechnical Commission standard IEC 891, IEC 904.

4. Emery, K.A., and C.R. Osterwald, "Solar Cell Efficiency Measurements," Solar Cells, Vol. 17, 1986, pp. 253-274.

5. Emery., K.A., D. Waddington, S. Rummel, D.R. Myers, T.L. Stoffel, and C.R. Osterwald, "SERI Results from the PEP 1987 Summit Round Robin and a Comparison of Photovoltaic Calibration Methods," SERI tech. rep. TR-213-3472, March 1989.

6. Emery, K.A., and C.R. Osterwald, "PV Performance Measurement Algorithms, Procedures and Equipment," Proc. 21st IEEE Photovoltaic Specialists Conf., Orlando, FL, May 21-25, 1990, pp. 1068-1073, IEEE, New York, 1990.

7. Emery., K.A.. "Multi-Junction Amorphous Silicon Solar Cell Calibrations and Testing," Proc. of the 1989 Amorphous Silicon Contractors Review Meeting. 19-20 June 1989, pp. 253-262, SERI tech. rep. CP-211-3514.

8. Metzdorf, J., T. Wittchen, K. Heidler, K. Dhne, R. Shimokawa, F. Nagamine, H. Ossenbrink, L. Fornarini, C. Goodbody, M. Davies, K. Emery, and R. Deblasio, "Objectives and Results of the PEP 87 Round-Robin Calibration of Reference Cells and Modules," Proc. 21st IEEE Photovoltaic Specialists Conf., Orlando, FL, 21-25 May 1990, pp. 952-959, IEEE, New York, 1990.

9. Emery, K.A., and C.R. Osterwald, "Solar Cell Calibration Methods," Proc. SERI 9th Photovoltaics Advanced Research and Development Meeting, 24-26 May 1989, Solar Cells, Vol. 27, pp. 445-453, 1989.

10. Osterwald, C.R., K.A. Emery, D.R. Myers, and C.J. Riordan, "Extending the Spectral Range of Silicon-Based Direct-Beam Solar Spectral Radiometric Measurements,"Proc. 20th IEEE Photovoltaic Specialists Conf., Las Vegas, Nevada, 26-30 Sept. 1990, pp. 12461250, IEEE, New York, 1990

11. Kneizys, F.X. E.P. Shettle, L.W. Abreu, J.H. Chetwynd, G.P. Anderson, W.O. Gallery, J.E.A. Selby, and S.A. Clough, Users Guide to LOWTRAN7, AFGL-TR-88-0177, Air Force Geophysics Laboratory, Hanscom AFB, MA. 1988. 


\subsection{SURFACE INTERACTIONS, MODIFICATION, AND STABILITY GROUP}

\subsection{RESEARCH STAFF}

A.W. Czanderna, Research Fellow and Group Leader

J.R. Pitts, Senior Physicist

F.J. Pern, Senior Scientist

J.T. Stanley, Postdoctoral Research Associate

D. King, Postdoctoral Research Associate

E. Tracy, Staff Scientist

Y. Shinton, Master Technician

C. Fields, Visiting Professional

R.L. Vojdani, Undergraduate Intern

\subsection{INTRODUCTION $[24,26-32,53,83,101-106]$}

The Surface Interactions, Modification, and Stability Group (SIMSG) has been part of the Applied Sciences Branch since Oct. 1989. Some personnel and projects are funded from the Solar Thermal and Solar Buildings programs, in addition to the Photovoltaics Program. Since our funding is secured from several sources outside the Materials Science and Engineering Division, the purpose and/or objectives, background, and accomplishments of these projects will all be addressed in Section 8.7. This is a departure from this reports' format, but will provide continuity for the reader. The purpose of this section is to provide the reader with information about the increased expertise and capabilities that have been added to the Applied Sciences Branch as a result of adding the SliMSG.

The purposes of the SIMSG are: (1) to correlate the composition, bonding, and/or structure and other properties at interfaces with the performance properties of solar energy conversion, opto-, and electronic devices; (2) to understand the mechanisms and processes occurring at interfaces that limit device durability; and (3) to modify the interfaces to improve their properities and/or stability. Specific types of solar-relevant problemsi were highlighted in a previous publication ${ }^{1}$, workshop ${ }^{2}$, and recent review chapter.

Topical areas for study in surface science include radiation induced surface transformations, organized molecular assemblies, polymer/metal (oxide) interfaces, interdiffusion, metallization corrosion, radiation induced surface and near surface reactions, and interface stability in multilayer thin-film devices. The personnel of the SIMSG were involved in FY 1990 tasks as follows: (1) solar induced surface transformations and modifications (SISTM); (2) x-ray photoelectron spectroscopy (XPS) and ion scattering spectroscopy (ISS) of organized molecular assembly (OMA)/copper interfaces; (3) advanced 
desiccant materials research; (4) establishing evaluation criteria and test methods for electrochromic windows; and (5) organizing a surface processing workshop to be held in 1991. Publications or major SERI reports have been completed. In addition, review chapters on ion spectroscopies for surface analysis and on the most widely used methods of surface analysis were completed during the year. In subsections of 8.3, 8.4, 8.5, and 8.7, the ar.alysis, techniques, etc., related to tasks 1 through 5 are ordered sequentially relative to each task, e.g., Section 8.4 .2 will be reserved for techniques related only to task 2 above.

\subsection{ANALYTICAL CAPABILITIES}

The analysis capabilities include IISS, XPS, secondary ion mass spectroscopy (SIMS), Auger electron spectroscopy (AES) , scanning Auger microscopy (SAM), Fourier transform infrared (FTIR) spectrometery, residual gas analysis (RGA), contact angle, ultramicrogravimetry, neutral and ion beams, reaction chambers, solar simulators, excimer dye/laser, a high-intensity (2400 suns) solar furnace, thin-film deposit and control monitors. thin-film deposition, adhesion testing, profilometers, metallographs, X-ray apparatus, and access to capabilities of the surface and optical characterization groups as well as the FTIR Spectroscopic Research Center. Appendix D provides a listing of the major instrumentation and test equipment, their features, and specifications.

\subsection{ANALYTICAL TECHIIGUES $[24,28,83]$}

Descriptions of the surface analytical techniques in the Leybold LHS-10 (XPS, AES, ISS, SIMS) and Physical Electronics $545 \mathrm{C}$ (AES) systems are available, in general ${ }^{3}$, and in considerable detail ${ }^{4}$. The FTIR spectrometery, including using the solar simulator, excimer/dye laser, and reactionchamber techniques, is treated adequately in a recent review.

The use of an RGA, ion beams for analysis and depth profiling, profilometers, and adhesion testing (Sebastian) equipment has also been described.

\subsubsection{Exposing Solid Surfaces to High Flux Densilies of Solar Radiation [101-106]}

Broadband radiation having large flux densities ( $>1 \mathrm{MW} / \mathrm{m}$ ) can easily be delivered to solid surfaces by a solar furnace in a highly controllable way. For example, the radiation can be delivered over large areas or can be shaped to match the demands of a particular process. The incident photon flux and/ or the resulting thermal energy can cause phase changes, atomic migrations, and chemical reactions without greatly perturbing the bulk properties of the materials. Such changes in surface properties are used in a number of 
technologically significant areas such as chemical vapor deposition, surface hardening by phase transformation, and various cladding operations. Prior work has demonstrated the usefulness of using a solar furnace to surface harden sorne types of steels, clad $\mathrm{Ni}-\mathrm{Cr}$ compound alloys to steel from powders, initiate self-propagating, high-temperature synthesis reactions in powders that are predeposited on substrates, and clad $\mathrm{Ni}$-Al compounds from predeposited vacuum coatings. Research in FY 1990 concentrated on extending the work in phase transformation hardening and cladding, and radiative joining and extending the use of a solar beam for studying selfpropagating, high-temperature synthesis reactions and thin-film growth in controlled atmospheres.

\subsubsection{Techniques for Preparing and Studying Organized Molecular Assemblies with Metal Overlayers [27,58]}

In addition to using XPS and FTIR spectrometery, optical multichannel analyzers (OMAs) were prepared by a self- assembly (SA) technique and characterized using contact angle prior to insertion into a vacuum system for XPS characterization before and after depositing copper overlayers.

Static contact angles are measured with a temperature-controlled goniometer. Typically, drops are placed on the surface in the ambient environment and read to obtain sufficient statistical data on the angles for both sides of the drop. Contact angles depend on the change in surface free energy between the wetting liquid and the host surface. There is extensive literature available on contact angles, and their usefulness has been demonstrated for molecular films ${ }^{5}$. Contact angle measurements on OMA films especially provide an assessment of the degree of ordering of the $\mathrm{X}$ groups versus those in the tail (Fig. 8.1), prior to depositing a copper film.

For preparing the OMA, SA techniques are used in preference to preparing Langmuir-Blodgett films. The self-assembled monolayers provide strong chemical bonds between $\mathrm{X}$ and the substrate (Fig. 8.1) and are much more suitable for the subsequent studies. A detailed description of preparing an arrangement shown in Fig. 8.1 will now be given for the SA of 11mercaptoundecanoic acid on gold followed by depositing up to several monolayers of copper. First, silicon wafers (Monsanto, 100) were scored and broken into $10 \times 14 \mathrm{~mm}$ coupons. They were subjected to ultrasonic cleaning for $30 \mathrm{~min}$ in microdetergent solution followed by three rinses in deionized water.

The coupons were then immersed in a hot solution at $80^{\circ} \mathrm{C}$ of $14 \mathrm{H}_{2} \mathrm{O}_{2}(30 \%)$ $\mathrm{H}_{2} \mathrm{SO}_{4}$ ("piranha solution") for $30 \mathrm{~min}$. The silicon wafers were removed, spin rinsed with deionized water followed by absolute ethanol, and dried under ultra-high purity (UHP) nitrogen. The substrates were immediately mounted 


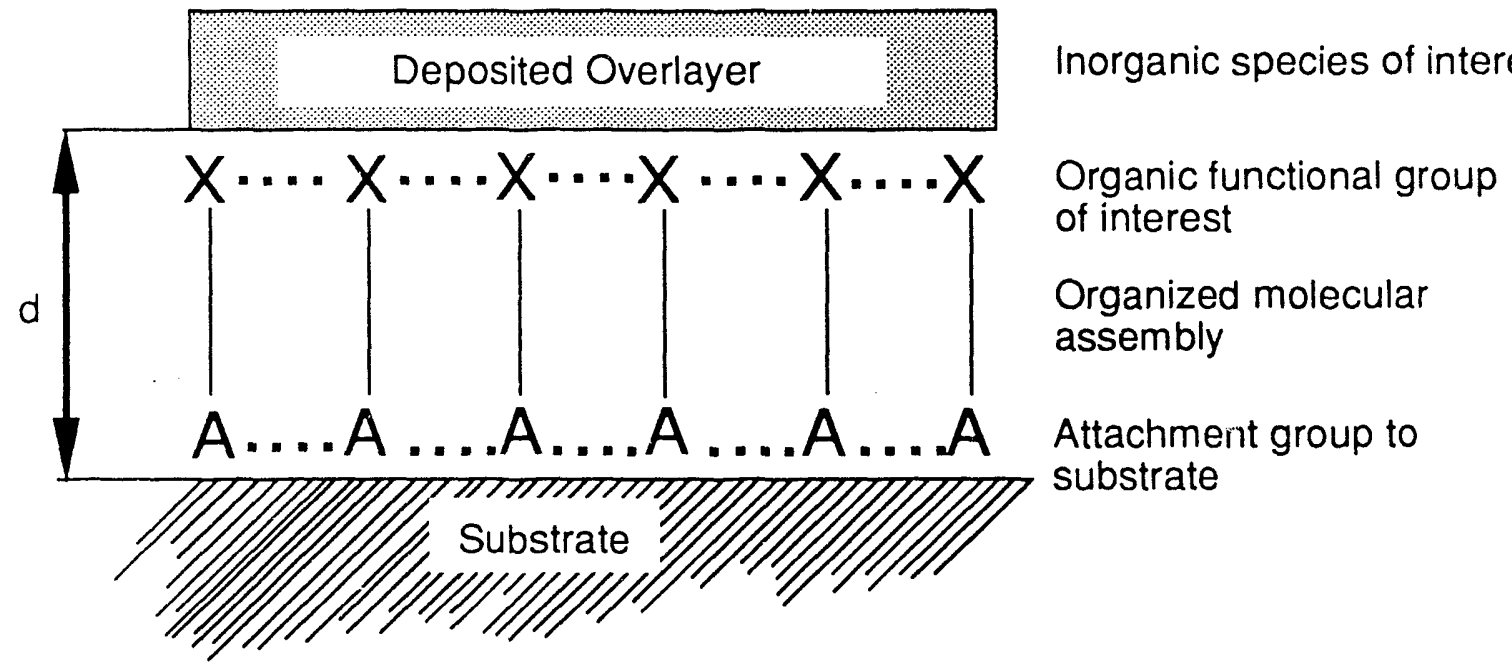

Figure 8.1: Idealized scheme for using an OMA to form interfaces between copper and an organic functional group of interest.

on the sample rod of the LHS-10 system and inserted into the vacuum chamber. The polished side of each substrate was argon ion etched until XPS indicated no carbon or oxygen on the surface of the coupons. The samples were transferred from the analysis chamber directly to the preparation chamber of the LHS- 10 without breaking vacuum. Subsequently, $80 \mathrm{~nm}$ of gold were vacuum evaporated onto each substrate. Evaporation rates were monitored by a quartz crystal oscillator connected to an Inficon IC 6000 deposition controller. The deposition was accomplished without appreciable substrate heating due to the $38 \mathrm{~cm}$ between the substrate and the evaporation crucible. XPS analysis of the substrates indicated clean gold films with no silicon contamination. Survey ISS spot spectra. which were obtained at 3 $\mathrm{nA} / \mathrm{mm}^{2}$ at $1 \mathrm{keV} 3 \mathrm{He}^{+}$, also indicated uncontaminated gold surfaces.

The films were removed from the LHS-10 vacuum system and immediately immersed in $10 \mathrm{ml}$ of a $6.5 \mathrm{mM}$ solution of 11 -mercaptoundecanoic acid in absolute ethanol. All assorted glassware was pre-cleaned in hot piranha solution, rinsed in deionized water followed by absolute ethanol, and dried under UHP nitrogen prior to use. The gold films were allowed to incubate in the acid solution for $48 \mathrm{~h}$ to allow time for the self-assembled monolayers to form. Several OMAs were formed simultaneously and one of the films was checked with a contact angle measurement to assure the criterion for a completed SA process had been met. The samples were removed from the acid solution, spin rinsed with absolute ethanol, dried under flowing UHP nitrogen. and immediately placed in the LHS-10 vacuum system for XPS (or 
ISS) analysis. After XPS analysis, the sample was translated back to the preparation chamber for depositing, in vacuum, the copper overlayer.

For depositing submonolayer overlayers of copper (or any other metal, semiconductor, or oxide), the LHS 10 preparation chamber, quartz crystal oscillator, and Inficon IC 6000 deposition controller are essential. With a vacuum in the $10^{-7}$ torr regime and a shutter covering the sample but not the oscillator, a deposition rate of about $0.1 \mathrm{~nm} / \mathrm{min}$ is established. The shutter is then opened long enough to deposit up to several monolayers of material as desired. The practical lower limit for a controlled deposit is $\sim 0.05 \mathrm{~nm}$ because of the time required to open and close the shutter.

Several other molecules were self-assembled onto gold or aluminum oxide surfaces by procedures similar to those described above. The objective was to form "surfaces" of different X groups prior to depositing copper. In the case of gold, the attachment group forms a thiolate with gold and the $\mathrm{X}$ groups studied were $\mathrm{COOH}$ and $\mathrm{CN}$.

\subsubsection{Water Adsorption/Desorption Using a Guartz Crystal Microbalance $[26,31,32]$}

The experimental apparatus used for measuring the water vapor sorption by a solid is conceptually simple. An arrangement is needed where a solid can be surrounded by pure water vapor (or a partial pressure of water in the ambient atmosphere), and where the number of water molecules that bond to the solid can be measured. A vacuum system can be used to control the gas (vapor) pressure over the solid. There are three basic methods for directly measuring the rate of gas adsorption and the equilibrium amount of vapor adsorbed by a solid: (1) gravimetric, (2) volumetric, and (3) radiotracer. The last is excluded because it would require tritiated water and offers no obvious advantages over gravimetric techniques. Gravimetric techniques have many advantages over volumetric techniques, especially for water vapor sorption studies. Even though water is one of the most difficult vapors to work with when using vacuum systems, the determination of adsorption isotherms and the rate of adsorption and desorption of water can be carried out routinely with gravimetric techniques.

Of the available gravimetric techniques, the beam balance and the quartz crystal microbalance ( $\mathrm{QCM}$ ) are the two best. We chose to use a $\mathrm{QCM}$ for our initial studies because of the commercial availability of QCMs and their ease of use for studying the sorption of water by materials at or near $25^{\circ} \mathrm{C}^{7}$. The experimental system consists of a vacuum system, a residual gas analyzer, a $\mathrm{QCM}$ system with five $\mathrm{QC}$ sensors, and a temperature bath for the $\mathrm{QCM}$. Detailed procedures for determining isotherms and measuring adsorption and desorption kinetics are also given. 
Work on the SISTM task used the solar furnace facilities at Sandia National Laboratories and at SERI. The solar furnace at Sandia was described in a previous report". The High Flux Solar Furnace (HFSF) Facility at SERI employs a unique off-axis, long focal length design, which makes the location and manipulation of experiments in the target zone simple. The heliostats use front surfaced aluminum / glass reflectors (total area $31.8 \mathrm{~m}^{2}$ ). The primary concentrators cover a total area of $11.5 \mathrm{~m}^{2}$, and are also front surfaced aluminum on glass. Both mirror surfaces use UV-enhanced reflective coatings and 'rave a solar weighted reflectivity of 0.91 when clean. Nominal power of the furnace is $10 \mathrm{~kW}$. The beam shape at the focus is a slightly flattened Gaussian, with a $4-\mathrm{cm}$ full width at half maximum (FWHM). Maximum intensities of $250 \mathrm{~W} / \mathrm{cm}^{2}$ after primary concentration and $2100 \mathrm{~W} / \mathrm{cm}^{2}$ after secondary concentration have been measured.

The furnace is equipped with vertically opposed, two-plate attenuators that control the amount of flux or the temperature of the target by trimming the edge beams from the top and bottom of the concentrator array. In addition, there is an air-actuated shutter in front of the target area that fully opens or closes in $0.5 \mathrm{~s}$. Sample positioning is accomplished by a three-axis, computer-controlled, motion table capable of supporting experiments up to $500 \mathrm{~kg}$. Services to the target include chilled water, air, pure gases, electricity, thermocouples, data acquisition, and vacuum. Experiments can be monitored or recorded using a closed-circuit television system. Beam directors, as well as design tools for special applications, are available.

During this year a high vacuum system was assembled and put into service at the HFSF. This system provides atmospheric control over the target zone as well as access to the light. The target chamber is a $10 \mathrm{in.} \mathrm{cylindrical}$ chamber with a high purity $\mathrm{SiO}_{2}$ window to admit the solar beam. A $1501 /$ $s$ turbomolecular pump evacuates the target chamber to the low $10^{-8}$ torr range. The system is equipped with a residual gas analyzer ( 0 to $200 \mathrm{u}$ ) for system diagnostics, or for monitoring reactions in the target zone. Mass flow controllers supply up to four gases in regulated proportions. One of the controllers is designed to accommodate liquid sources. Pressure is controlled by a capacitance manometer coupled with a butterfly valve in the exhaust stream. Currently the system is set up to flow each supply gas in the range of 0 to $200 \mathrm{sccm}$, and to control pressure in the range of $10^{-3}$ to $100 \mathrm{mbar}$. When coupled with the furnace, the system is designed to provide spatial, temporal, and thermal modulation of the growth of thin films.

\subsection{ACCOMPLISHMENTS: PHOTOVOLTAICS}

As stated in the introduction, the projects conducted by the SIMSG in FY 1990 were not generally carried out in support of the Photovoltaic (PV) 
Technology Program. The contributions made by SIMSG personnel to PV projects are included in Sections 6.3.4 and 6.4.5 of this report.

\subsection{ACCOMPLISHMENTS: RELATED RESEARCH AREAS}

\subsubsection{Solar Induced Surface Transformations and Modifications (SISTM)}

The objective of this task is to develop commercially viable markets for solar thermal technologies, especially a solar furnace technology capable of concentrating sunlight by a factor of 1000 or more. The work embodies:

- Performing research to determine which processes represent the best candidates,

- Cost analysis of prospective candidates,

- Research and development of specific processes,

- Demonstration of the technology, and

- Marketing of the concepts to generate interest in the technology.

\subsubsection{Introduction [101]}

Several years ago, when the price of oil dropped below $\$ 20 /$ barrel, interest in generating electricity with alternative sources waned. However, a substantial technology base for concentrating solar energy existed. The natural question to ask was, "Is there anything unique or something that has commercial potential that can use highly concentrated solar radiation?" We embarked upon a study of this question and determined that there is no salient uniqueness to exploit but that there are commercial potentials in areas of technology that require the supply of large amounts of radiant energy. Solar radiation can be directed and concentrated with reflectors that lose only $5 \%$ to $10 \%$ of the radiation at each reflection. Lasers and arc lamps, on the other hand, operate with total conversion efficiencies in the range of $4 \%$ to $9 \%$. Therefore, for processes that require large amounts of radiant energy and where the solar resource is compatible, substantial cost savings might be obtained by using solar furnace technology ${ }^{10,11}$.

We have studied surface modification processes using highly concentrated solar beams in some detail. Most of the work has used solar fluxes in the range from 100 to $250 \mathrm{~W} / \mathrm{cm}^{2}$. Recently, however, there has been a need to raise the available flux using secondary concentrators, and fluxes up to $2000 \mathrm{~W} / \mathrm{cm}^{2}$ have been produced ${ }^{12}$. The potential for obtaining even higher 
fluxes for some applications exists. but we have obtained interesting results at the relatively modest flux levels available for most of our experiments. The topical areas that we have studied include phase transformation hardening. cladding, radiant joining, initiating self-propagating high-temperature synthesis (SHS) reactions, and rapid thermal film growth in controlled atmospheres. Other areas of interest that have been identified are rapid thermal annealing, zone melt recrystallization, rapid thermal processing of ceramic materials, metallization of ceramics, and joining ceramics. Accomplishments in the former areas are described, and the potential of applications in the latter areas is outlined below.

\subsubsection{Phase Transformation Hardening [102]}

Experiments using solar beams to modify the surface properties of metals have been relatively successful. Hardenable steels (A2, 4340, and nitride grade) have been pulsed and scanned to produce hardened zones on the surface. In addition. we have performed some hardening and annealing procedures on some $\mathrm{Cu}$ alloys to demonstrate that near surface regions can be modified without affecting the bulk of the material. These experiments were accomplished on substrates varying in thickness from $1 \mathrm{~mm}$ to $1 \mathrm{~cm}$ and with pulses varying from 1 to $30 \mathrm{~s}$. With fluxes in the range from 100 to $250 \mathrm{~W} / \mathrm{cm}^{2}$. surface regions from 1 to $4 \mathrm{~mm}$ can be fully hardened. However, the heat affected zones (HAZ) are not sharp and extend to the backside in samples with longer exposures. Processing at $250 \mathrm{~W} / \mathrm{cm}^{2}$ is not viable for most surface hardening tasks because of the depth of penetration of the HAZ. It is known that at flux levels four times greater, very nice surface hardening processes can be performed, and applications in selectively hardening plow blades are fully developed ${ }^{13}$.

\subsubsection{Cladding [103]}

Experiments in cladding corrosion and wear resistant materials onto steel substrates have been quite successful. We have been able to clad a variety of powders to 4340, A2, and 1040 steels, as well as to pure iron and nickel substrates. The powders used are commercially available plasma spray powders: NiCr alloy (\#761). 316 stainless steel, WC in a Co matrix, nickel aluminides, and chrome oxide. The principle difficulties in performing the cladding operations is not the flux levels available, but the control of the atmosphere during the cladding operation and the horizontal geometry of the beam line in the available facilities. Powders absorb the radiation very well through multiple reflections and heat rapidly because of relatively poor conduction to the substrate. The geometry of the available furnaces dictated mounting the target surfaces vertically, and this meant that the powders had to be fixed to the surface with a binder. Gravity and the outgassing of the binder caused some difficulties, which had to be overcome by applying the 
appropriate thermal profile. For some samples, substrate oxidation at elevated temperatures prevented the melt from wetting the surface. In these cases, metallurgical bonding did not occur, and it was necessary to go to controlled atmospheres to prevent oxidation and get a good quality clad.

\subsubsection{Radiative Joining [104]}

Certain aluminum/bronze alloys are under development for use in forms to lay up carbon fiber composite structures for aerospace vehicles. There are a variety of reasons that this material development is needed for the composite manufacturing. The most important are that the forms are easily designed by computer aided design (CAD) procedures and cast in near net shape, and the materials may be selected such that they precisely match the coefficient of thermal expansion of the cured composite structure. Further, the expected lifetime of the forms is long compared to the monolithic graphite forms now in use, and they can be assembled from subunits so that the casting procedures are simple and inexpensive. The problem that is solved by radiative joining is that of making the seams gas tight to 100 psig without warping the form. This requirement rules out any conventional means of brazing because the forms have an unusual honeycomb configuration that warps at low temperatures. Thus, a radiative joining technique is the only one that works. Laser welding is effective but expensive compared to the other steps in the process, and it leaves a surface bead that must be postmachined. The material is highly reflective in the infrared, so laser brazing procedures are difficult to carry out. Using absorber coatings for the laser results in so much heat being put into the surface that the near surface melts or evaporates before penetration to braze alloy depth is achieved. A solar beam in the range of $300 \mathrm{~W} / \mathrm{cm}^{2}$ provides the right heating rates and depth of penetration for an effective brazing procedure.

Experiments were carried out on small coupons until the appropriate combination of flux and braze alloy were found. Successful joints wei c nade with these coupons. Initial experiments with a pilot scale test piece using the full honeycomb structure resulted in severe warping of the plates. This was caused by the size of the beam at the Sandia Solar Furnace and the large total thermal delivery onto the target. With a water-cooled aluminum mask in the path of the beam, and the target seam positioned such that .mly a strip 2mm wide was illuminated, a good metallurgical joint was achieved, thereby demonstrating the viability of the process. Optimization of the process remains. This will require a substantial commitment in terms of $R \& D$ effort and equipment. About $2 \mathrm{~kW}$ of total power is needed in a 4-mm diameter beam, with a peak flux of about $500 \mathrm{~W} / \mathrm{cm}^{2}$. Two-axis flexibility in the light optics is also needed (perhaps a fiber optic delivery system!), as well as a three-axis manipulator for the target piece. This would be a nice engineering project with important implications in the future for applications in space. 


\subsubsection{Self-Propagating, High-Temperature Synthesis [SHS] Reactions}

The SHS reactions are characterized by being so exothermic that the heat released upon initiation of the reaction is sufficient to allow propagation of the mixed reactants throughout the bulk until all of the reactants are consumed. The classic example is the thermite reaction. When powders of starting materials or layers of thin films are used, it is sometimes found that the reactions do not propagate uniformly. Further, for some materials the reactions can only be sustained, or the right product can only be obtained. if the reaction mixture is maintained above a defined (high) temperature. Both of these situations can be addressed by supplying additional energy to the reaction mixture prior to and during the reaction. For the production of coatings, delivering radiant energy to the surface of the target is the most convenient and conservative method of supplying the extra energy required to produce the desired phase.

We have studied several SHS reactions designed to deposit high-quality ceramic and intermetallic materials on ordinary metal substrates. The research is in a very early stage, but encouraging results can be reported.

Three types of experiments have been carried nut. Mixtures of pure powders have been glued to $\mathrm{Fe}, \mathrm{Ni}$, Ti, Mo, and 4340 steel substrates. Exposure to a 1 to $6 \mathrm{~s}$ pulse in a solar furnace is sufficient to initiate the reactions, and coatings of various nickel aluminides, $\mathrm{TiB} 2$, and $\mathrm{TiC}$ have been formed. Because these exposures were performed in air, some of the film quality is poor. In some cases, parts of the films either lifted off or did not wet the substrate material. In other cases, so much energy was deposited on the surface of the substrate that substantial melting and surface roughening occurred during the reaction. Other experiments involved exposing multiple layers of vacuum-deposited thin films. In this case, the films were too thin to allow propagation over the whole surface and depended upon the radiative input of energy to drive the reaction. High-quality nickel aluminide films approximately $1-\mathrm{mm}$ thick were formed. The third type of experiment involves reacting preapplied plasma spray coatings with their substrate material to form a new alloy phase on the surface. The specific sample set used plasma sprayed aluminum on $\mathrm{Fe}, \mathrm{Ni}$, and Ti substrates. Exposures were conducted in air. Aluminides with the substrate materials were formed as a result of exposure to the solar beam.

\subsubsection{Thin-Film Growth in Controlled Atmospheres}

Early experiments pointed out the need for inert atmospheres over most target materials to control oxidation. A high-vacuum-compatible target chamber was constructed to allow this. By modifying the chariber to allow 
us to control the flow of reactive gases over the target during exposure to the solar beam, we produced the capability of conducting rapid thermal chemical vapor deposition that results in thin-film growth directly on the target. This system allows programmable control of gas constituents (up to four gases simultaneously), flow rate, pressure, and target temperature. The near-term objective for this research is to explore the growth of several ultrahard coating materials (hard carbon, $\mathrm{TiN}, \mathrm{SiC}$, and $\mathrm{TiB}_{2}$ ) on substrates of steel, $\mathrm{Fe}, \mathrm{Ni}$. and $\mathrm{Si}$. Longer-term objectives include the growth of multilayer electronic materials, especially photovoltaic materials.

Thin films of carbon have been grown on $\mathrm{Ni}$ and $\mathrm{Si}$ substrates using $\mathrm{CH}_{4}$ and $\mathrm{H}_{2}$ as precursors. The carbon growth on $\mathrm{Ni}$ results in a graphitic phase. Experiments with $\mathrm{Si}$ substrates have produced thin films of $\mathrm{SiC}$ and diamondlike carbon films, as well as graphitic carbon, depending upon the growth conditions. The objective of this area of investigation is to define the process parameters required for diamond thin-film growth. Current technology requires using large amounts of energy to grow diamond thin films. This may be an area where the solar-based technique can have a substantial advantage over the more conventional techniques employing microwave plasmas or hot filaments.

\subsubsection{EXPS and ISS of Organized Molecular Assembly/Copper Interfaces $[27,58]$}

The purpose of this internally funded research is to study a new, virtually unexplored subfield of surface science by understanding the chemical and physical interactions at molecularly sharp interfaces between specific organic functional groups (OFGs) and vacuum deposited overlayers formed from inorganic metal, semiconductor, or metal oxide species (IS).

Organized molecular assemblies (OMAs) are being used to form a precisely controlled surface consisting of specifically selected OFGs that are arranged in well-defined spatial distributions with uniform bond orientations. Monolayer-scale thicknesses of IS, such as metals or metal oxides, are vacuum deposited onto an OFG to produce a new interface as shown in Fig. 8.1. The chemical and physical interactions and the dynamics of forming the $\mathrm{OFG} / \mathrm{IS}$ interface are virtually unexplored as a subfield of surface science.

During 1990, the objectives of this task were (1) to attach an 11mercaptoundecanoic acid (MUA) organized molecular assemblies (OMAs) with different functional end groups onto a gold substrate using a SA process, (2) to vacuum deposit $\mathrm{Cu}$ onto the OMA, and (3) to study the $\mathrm{Cu} /$ $\mathrm{COOH}$ interface formed using XPS. 


\subsubsection{Accomplishments $[27,58]$}

The $\mathrm{COOH} / \mathrm{Cu}$ interface was prepared by the $\mathrm{SA}$ of an 11mercaptoundecanoic acid [MUA, $\mathrm{HS}\left(\mathrm{CH}_{2}\right) 10 \mathrm{COOH}$ ] OMA onto a gold thin film supported on a silicon single crystal substrate. The presence of the MUA OMA was established by using contact angle and ISS techniques. Contact angle measurements agreed with values in the literature and ISS established a new criterion for the presence of an OMA. XPS and ISS spectra were recorded before and after forming an OMA of MUA on 20-nm-thick gold thin films on a silicon substrate. The MUA/OMA is attached to gold via the $\mathrm{SH}$ group; the acid group becomes the outer monolayer. At low (1 keV $\left.3 \mathrm{He}^{+}\right)$ current densities, ISS showed no detectable amount of gold on the surface of the OMA. indicating the gold film was completely covered. XPS signals were found on the OMA for carbon, oxygen, sulfur, gold, and a trace of silicon. When using ISS at higher current densities to sputter the OMA, the same elements were detected. Some silicon from the substrate diffuses through the $20 \mathrm{~nm}$ of gold at $22^{\circ} \mathrm{C}$.

For studies of the $\mathrm{Cu} / \mathrm{COOH}$ interface, copper was deposited incrementally onto the $\mathrm{COOH}$ end groups of the MUA in vacuum in SERI's LHS- 10 surface analysis system. After sequential thicknesses of $0.05,0.1,0.2,0.4,0.6$, and $1.0 \mathrm{~nm}$ of $\mathrm{Cu}$ were reached, XPS spectra were recorded and compared with those taken for the MUA alone. Considerable effort was devoted to interpreting the $\mathrm{O}(\mathrm{ls})$ and $\mathrm{Cu}(2 \mathrm{p})$ photoemission peak positions. The most probable interactions between $\mathrm{CU}$ and $\mathrm{COOH}$ include forming the unidentate complex $\mathrm{C}-\mathrm{OHCu}$ or $\mathrm{C}-\mathrm{OCu}$, the bidendate complex $\mathrm{COOH}-\mathrm{Cu}$ or $\mathrm{COOCu}$, and the interaction $\mathrm{C}=\mathrm{O}-\mathrm{Cu}$. The XPS data only support forming the unidentate complex for $\mathrm{Cu}$ up to 0.2 to $0.4 \mathrm{~nm}$ in thickness. At greater $\mathrm{Cu}$ thicknesses, the unidentate interaction is not disturbed and copper remains in the metallic state. The data do not support forming a bidendate copper complex or chemical interaction between copper and the double bonded oxygen atom of the MUA acid group. The maximum charge residing on the copper atom of the complex is plus one with one charged copper atom on the surface per MUA molecule of the OMA. In future work, the $\mathrm{Cu} / \mathrm{CN}$ and $\mathrm{Cu} /$ $\mathrm{OH}$ interactions will be studied in similar detail. Efforts to perform FTIR spectrometery with the old Nicolet 7700 were not productive; the new system 800 will be ideal for metal/OMA interface studies with the purchase of a vacuum chamber with RA capability.

\subsubsection{Benefits to Solar Energy Research}

Many devices for collecting solar energy include using polymers in contact with inorganic oxidized surfaces (e.g., PV modules, membrane mirrors, flatplate collectors). Currently, the interactions at the polymer/metal (oxide) interface are not understood well. This is because it is difficult to prepare, 
keep, and study a polymer/metal (oxide) interface using the modern tools of surface science. This research has provided a positive answer to the most important question - can a metal film, which is deposited onto the functionalized end of organized molecular assemblies, be used to elucidate the interactions and reactions at the polymer/metal oxide interface? While our previous work demonstrated feasibility. the results from this project show detailed interpretations about the new species can be made from XPS and ISS data. Now the elementary and subsequent steps in the chemical degradation of polymeric materials can be studied at the molecular level in controlled experiments. Many technological applications, including optical films. microelectronic devices, adhesion, encapsulation, corrosion protection, and electrode materials can benefit from extensions of this work. These technological needs can now be addressed by seeking scientific understanding at the molecular level of the bonding and reactivity at interfaces between metals, metal oxides, and particular organic functional groups. It is the changes at the molecular level at interfaces that often precede degradative reactions that compromise the performance of solar energy conversion systems as well as other technological devices.

\subsubsection{Advanced Desiccant Materials Research}

The technological objective of this work is to identify a next-generation. lowcost material with which solar energy or heat from another low-cost energy source can be used for regenerating the water vapor sorption activity of the desiccant. The scientific objective of this task is to determine how the desired sorption performance of advanced desiccant materials (ADMs) can be predicted by understanding the role of the materials modifications and their surface phenomena. As in previous years, the funding level precluded addressing any of the scientific objectives.

\subsubsection{Accomplishments $[26,31,32]$}

This summary documents the work accomplished from January through September 1990 as a continuation of a subtask begun in 1985 in the Solar Desiccant Cooling Program. The work was carried out by Y. Shinton and A. W. Czanderna at SERI and by N. Todd Tillman at Eastman Kodak.

This work relates to cooling systems research for improving the performance of desiccant cooling systems (DCSs). These systems process water vapor in an atmosphere to produce cooling. By identifying a next-generation, lowcost, advanced desiccant material. the cost of air conditioning buildings can be lowered by improving the performance of DCSs and eliminating the need to add peak-load electric generating capacity. The purpose of the Advanced Desiccant Materials Project during 1990 was to characterize the sorption performance of ionic salts of polystyrene sulfonic acid (PSSA), and new 
desiccant polymers synthesized. modified, or procured by our subcontractor. Eastman Kodak, for their potential application in solid commercial DCSs (CDCSs). Particular attention was focused on polyAMPSA, its alkali salts. and various copolymer formulations with it. AMPSA is the abbreviation for 2-acrylamido-2-methyl-1-propane sulfonic acid. and is the monomer for polyAMPSA.

Measurements are summarized for the sorption performance of modified polymeric ADM materials with the $\mathrm{BCM}$. The data include the shape of the sorption isotherms, the capacity at 5\% and 60\% R.H., adsorption and desorption kinetics, and if hysteresis is present. Alkali ion salts of PSSA, which were prepared by Kodak, were used for our polymer modification studies. From sorption capacity measurements of the alkali ion salts of PSSA and PolyAMPSA, it was reaffirmed that the isotherm shape depends on the size of the cation present. The best isotherm shapes and capacities are obtained for the acid. lithium salt. and sodium salt, but the optimum preparation scheme has not been deduced.

Twenty-one of the 34 polymer formulations prepared by Kodak have sorption properties comparable to or better than the eight candidate advanced desiccant polymers (ADP) identified in our prior work. The 21 formulations represent four different classes of polymers. two of which are salts or copolymers with AMPSA. One of the four classes is a set of various preparations of salts of PSSA, especially, PSSASS and PSSALS. The final class of polymers includes several cationic polymers (exchange anions) to complement the anionic polymers (exchange cations) such as salts of PSSA and AMPSA. The subcontracted effort with Kodak has been an outstanding success for expanding the number of candidate ADM and elucidating the properties of PSSASS and PSSALS.

Other activities included complementing the final revision of the report entitled "Polymers as Advanced Materials for Desiccant applicants: 1988," SERI/PR-255-3443. by A. W. Czanderna and H. H. Neidlinger and also the final report entitled "Polymers as Advanced Materials for Desiccant Applications: 1989,"SERI/PR-213-3608, by A. W. Czanderna. Information about several of the most promising polymers studied this year was shared with Cargocaire Engineering, Inc. The significant accomplishments this year resulted, in part, from uninterrupted performance of our experimental apparatus and the improvements mare in 1989 for acquiring data with a dedicated IBM-compatible personal computer.

\subsubsection{Benefits to Photovoltaic Research}

The progress on this project provides us with the experimental methodologies for measuring the permeation of gases through polymeric materials. Thus, 
we are positioned to initiate permeation rate studies on candidate polymers for PV and other solar applications when funds are available for studying durability and lifetime predictive issues. These polymers may be as thin as a monolayer of an organized molecular assembly or several millimeters thick. The experimental capabilities are excellent for water vapor permeation studies; water permeation is a known cause for failures in PV modules and silvered mirrors. The Sartorius microbalance, and in some cases, our quartz crystal microbalance, can also be used for numerous other studies involving such topical areas as adsorption, desorption, oxidation, reduction, decomposition, deviations from stoichiometry, absorption, evaporation rates, surface areas, and photodesorption.

\subsubsection{Evaluation Criteria and Test Methods for Electrochromic Windows [29]}

The primary purposes of this section are to summarize the test methods used for evaluating electrochromic (EC) windows, to summarize what is known about degradation of their performance, and to recommend methods and procedures for advancing $\mathrm{EC}$ windows for buildings application. A brief overview is provided for the applications of EC devices in various technologies with a focus on buildings. The parameters used for testing $\mathrm{EC}$ windows are organized into evaluation criteria, performance criteria, and testing methods for laboratory (research), prototype, and durability evaluation. Key technical issues that have emerged are organized into the categories of practical windows, durability testing, and fundamental mechanisms. A brief background summarizes the interest, potential, physical configuration, and operation of an EC window. Evaluation criteria for ranking candidate EC device materials and for complete candidate EC windows are briefly discussed.

The performance criteria for EC windows are organized into two major sections. In the first section, performance parameters are summarized. These are used in research investigations. The parameters are grouped into optical properties, injected charge or ions, open-circuit memory, cycle energy. response time, and substrate effects. A list of questions that need to be resolved for the laboratory performance evaluation is presented. The most important parameters needed to evaluate an EC window are summarized (i.e., optical properties of $T(\lambda), R(\lambda), A(\lambda)$; injected charge/unit volume) and correlated with the optical properties; current versus voltage (I-V) curves, again correlated with the optical properties, and the temperature dependence of the response time.

The seccrid inajor discussion section deals with test methods for evaluating the durability of complete EC windows. Performance losses result from imposed degradation parameters and are summarized under the categories of degradation effects. degradation modes, and degradation mechanisms. 
Degradative parameters include UV, humidity. temperature. air, pollutants, thermal shock, cyclic temperature, and cyclic charge injection and bleaching. Degradation modes can result from thermal shock. prolonged non-use, constant elevated temperatures, and various cyclic changes. Degradation effects include gas generation in some devices, humidity effects, internal water formation, secondary reactions, photoreactions, quenching (fading of color), volume changes, and temperature-dependent effects. Established degradation mechanisms include film dissolution, corrosion, transparent conductor etching, and hydrogen embrittlement.

Performance measurements for real time and accelerated life testing are then discussed. These include measuring the transmittance and reflectance (wavelength dependence) of an EC window in the colored and bleached states, the atomic percent of injected charge, and the time dependence of the change in percent transmittance during coloring and bleaching. The proposed methods and procedures are summarized for real time and accelerated life testing.

As a conclusion, a set of recommendations is made for advancing $\mathrm{EC}$ window technology. especially for durability testing. These recommendations are preliminary but provide a suitable foundation for further refinement.

\subsubsection{Surface Processing Workshop}

\subsubsection{Introduction}

The objectives of the workshop are: (1) to bring together 40 experts from academia, industry, and federally funded laboratories in a workshop setting, (2) to form working groups in $\sim 9$ topical areas of applied surface science with a mixture of expertise in surface characterization and surface modification, and (3) to identify and to prioritize the research needs and opportunities in each topical area of applied surface science.

Surface processing is a subset of applied surface science for (1) preparing tailor-made surfaces for specific end-use applications, (2) characterizing the surfaces, and (3) developing a theoretical framework. Participants will be sent current-status "plenary" summaries of the state-of-the-art in the relevant methods for surface modification and surface characterization prior to the workshop. They will also be sent documents that indicate the crucial importance of surface properties for applications of conservation and renewable energy technologies, energy distribution, energy generation by utilities. buildings, and by other industrial energy users. The unique aspects of these applications areas provide surface scientists with unusual opportunities to carry out focused fundamental research on materials/ systems with targeted end-use applications. 
The present workshop is being organized to respond to the need to identify and prioritize the needs and opportunities in selected areas of applied surface science. It is being organized around the topical areas of corrosion protection, photoelectrochemical systems, solid batteries and fuel cells, polymer/metal(oxide) interfaces, thin-film multilayer solar collectors, accelerated life testing of devices with solid/solid and solid/liquid interfaces, interfacial microchemical characterization needs, conducting polymers, and modification with organized molecular assemblies. The related technologies depend critically on surface properties. For example, the efficiencies, performance, and lifetimes for photovoltaics, high-temperature superconductors, solar thermal concentrators, photoelectrochemical devices, electrochromic windows, and processes/systems used for energy storage and distribution, depend very much on the surface properties of the active materials. Having the ability to tailor-make surfaces for specific applications will have tremendous impacts on these technologies. Concurrently, being able to monitor the changes on these surfaces and characterize the chemical and physical processes occurring on them will allow us to understand the underlying phenomena in microscopic detail and to develop improved materials and surfaces for these applications. It is, therefore, appropriate that DOE/SERI take the leadership role in establishing a generic technologybased, research program in surface processing.

Applications of surface processing include a broad spectrum of energy technologies. Conservation technologies (e.g., buildings, efficient industrial processes, and transportation technologies) will also be candidates for utilizing the benefits of this research program. The National Materials Advisory Board's (NMAB) panel on corrosion estimated a potential savings of several billion dollars annually if surface processing technologies can reduce corrosion problems. Additional billions can be saved if interface reactions that degrade device performance can be slowed or eliminated. The life extension of our aging power generation facilities will also be facilitated as new materials and surfaces are defined through this program.

The potential applications of surface processing are so numerous that it is impossible to list all of them in this relatively short overview. Instead, a few examples that can have significant impacts are listed to provide a sense of the value of a surface processing research program. These examples, which include relevant key research issues, are:

- Solar cell performance: semiconductor surfaces and grain boundaries

- PV module lifetimes: metallization corrosion, encapsulant/metal and metal oxide interface reactions 
- High-temperature superconductors (HTSC): surfaces between the HTSC and the conductor in a thin-film stack is critical to reliability

- Power cables: degradation at the conductor/insulator interface

- Electrochemical energy storage: corrosive degradation at interfaces and catalyst/membrane composite interface properties

- Photoelectrochemical energy conversion: lifetime and anodic photocorrosion

- Solar surface processing: modify/stabilize surfaces using a solar furnace

In addition to the examples mentioned above, a research program in surface processing technologies will greatly impact the microelectronic, semiconductor, corrosion protection, and catalyst industries. Technologies dealing with ultra-thin films, protective and decorative surface coatings, variable conducting masks, and novel bimetallic alloy catalyst systems rely tremendously on the research progress of a surface processing technology. A DOE-supported technology base program will assist U.S. industry substantially in a wide variety of fields and will allow us to maintain and improve our competitive posture in the global marketplace.

\subsubsection{Accomplishments}

The workshop format, plenary sessions, working groups needed, and local arrangements required have been completed. Twenty-one plenary summary authors have agreed to prepare status papers in the areas of basic science support underpinning surface processing, characterization of surfaces, and surface modification methods. The summary papers will be due six weeks before the workshop is actually held. Nine working group chairmen have been identified along with an additional 25 participants. Attendance at the workshop is by invitation only.

\subsection{FUTURE WORK [64]}

Work in FY 1990 for the various tasks are summarized without the use of subsections. For task 1 on SISTM, the plans are to concentrate on the (a) rapid thermal processing of ceramic materials, (b) zone melting recrystallization, (c) metalorganic deposition, (d) rapid thermal processing of electronic materials, and (e) materials science, processing, and construction in space. 
For task 2 on the OMA/copper interface, the work will be extended to include depositing $\mathrm{Cu}$ onto OMAs with the organic functional groups $\mathrm{CN}, \mathrm{CH}_{3}$, and $\mathrm{OH}$ as the $\mathrm{X}$ in Fig. 8.1. The thickness of the vacuum-deposited metal will be increased incrementally from 0.05 to $\sim 2 \mathrm{~nm}$. The OMAs will be attached to $40 \mathrm{~nm}$-thick gold films via SH groups.

For task 3 on advanced desiccant materials, the $\mathrm{QCM}$ may be used to characterize the sorption properties of cationic and anionic polymers. Funding for FY 1991 is still being negotiated between DOE and SERI. Since the $\mathrm{QCM}$ and Sartorious beam microbalance are of considerable value for measurements needed on the Module Reliability Research Task (this report, Section 6), it is planned to use these capabilities on PV materials. The funding level on the desiccant task is only sufficient to provide about $25 \%$ use of the $\mathrm{QCM}$, and no time to complete the assembly and initial testing of the beam balance.

For task 4 on EC windows, the FWP prepared in May 1990, is still being negotiated for funding by DOE/SERI managers. With the dissolution of the Materials Research Branch in 1989, all the expertise on EC windows now resides in the Basic Sciences Branch, except for that of A. Czanderna. The FY 1990 task leader for EC windows will be chosen from one of the experts in the Basic Sciences Branch, and interface issues in ECW will be addressed by the SIMSG as part of an intradivisional team research effort.

For task 5, the objectives of the surface processing workshop will be completed assuming sufficient funds are available to complete the work.

There are interface issues in the Module Reliability Research subtask (Section 6). These issues will be addressed by personnel from both the SIMS and Module Reliability groups.

1. Czanderna, A. W., Solar Energy Materials, Vol. 5, 1981, p. 349.

2. Czanderna. A. W., and R. J. Gottschall, eds., "Basic Research Needs and Opportunities on interfaces in Solar Energy Materials," DOE/OER, CONF. 8006156, Apr. 1981; Special Issue Mat. Sci. Engr. Vol. 53, 1982, pp. 1-168.

3. Czanderna, A. W., Materials Characterization Using Ion, Electron, and Photon Probes, in Industrial Materials Science and Engineering, L. E. Murr, ed., Dekker, NY, 1984, pp. 471-517. (SERI/TR-2552217, July 1984).

4. Pitts, J. R., Silver-Silicon Bonding on Silica Surfaces, SERI/TR255-2410, Apr. 1985, Ph.D. Thesis, University of Denver. 
5. Swalen, J. D., et al., Molecular Monolayers and Films, Panel Report, Division of Materials Sciences, OER/DMS, Langmuir, Vol. 3, No. 9, 1987. p. 932.

6. Czanderna, A. W., and S. P. Wolsky, eds., Microweighing in Vacuum and Controlled Environments, Amsterdam: Elsevier, 1980.

7. Lu, C., and A. W. Czanderna (eds), Applications of Piezoelectric Quartz Crystal Microbalances, Amsterdam: Elsevier, 1984.

8. Czanderna. A. W., and T. M. Thomas, J. Vac. Sci. Technol. A, Vol. 5. No. 9. 1987, p. 2412.

9. Asher. S. E., and P. Dippo, eds., Annual Report: Photovoltaic Measurements and Performance Branch: FY 1989: SERI/TP-2133712, May 1990 pp. 156-158.

10. Kolb, G. J., A Comparison of the Economics of Materials Processing with Solar Furnaces and High-Intensity Arc Lamps, Sandia National Laboratories. Albuquerque, NM, in preparation, 1990.

11. Short. W., Solar Energy Research Institute, Golden, CO, private communication.

12. Lewandowski. A., Solar Energy Research Institute, Golden, CO, private communication.

13. Tan, R. K., N. L. Arrison, D. A. Parfeniuk, and D. M. Camm, Surface Treatment Using Powerful White Light Sources, VORTEK Industries LTD report, 7 Oct. 1988. 


\title{
9.0 FTIR SPECTROSCOPIC RESEARCH AND SERVICE CENTER
}

\subsection{RESEARCH STAFF}

\author{
J.D. Webb, Senior Chemist and Center Manager
}

E.J. Rouse, DOE/AWU Student Fellow

\section{$9.2 \quad$ INTRODUCTION}

The FTIR Spectroscopic Research Center began operations in the Applied Sciences Branch at the beginning of FY 1991. The Center was established to satisfy capital equipment requests from a number of SERI researchers for Fourier transform infrared (FTIR) analytical capabilities. These requests originated in the Materials Science and Engineering Division, the Mechanical and Industrial 'Technology Division, and the Fuels and Chemicals Research and Engineering Division. The diverse sources of these requests gave acquisition of FTIR equipment a high priority. Since sufficient funds were not available to provide each division with its own FTIR equipment, the requestors agreed to share access to a single FTIR system. In order to maximize utilization, SERI's Deputy Director and the research and technology division managers elected to acquire a set of equipment designed to meet the requestor's analytical needs in FTIR spectroscopy for at least the next five years. To ensure access to all interested SERI researchers, a central FTIR research facility was needed. It was also clear that the Center would need a manager to perform analyses, maintain and upgrade the equipment, control access, track costs, and maintain an adequate level of support for the Center, through both existing tasks and development of new sponsors.

Beginning in FY 1988, J. Webb undertook the task, at the request of G. Nuss and with the support of the Deputy Director, of polling SERI researchers in regard to their present and anticipated analytical needs that could be met using FTIR spectroscopy. With input from more than a dozen SERI scientists who expressed interest in using the Center, a set of technical specifications approximately 40 pages in length was developed for the equipment. These formed the core of a Request for Proposal (RFP), which was sent out in January 1990. to FTIR equipment manufacturers who had responded to a solicitation published earlier in Commerce Business Daily. Four manufacturers submitted bids that were determined by the purchasing and subcontract staff to be within the competitive range.

Evaluation of these proposals began at SERI in March 1990. The SERI Technical Evaluation Panel consisted of J. Webb (chair), A. Czanderna, and A. Mascarenhas. The Cost Evaluation Panel was chaired by R. Moore. The 
technical evaluation involved scoring the proposals based on adherence to the technical specifications, results of measurements made by the manufacturers using their proposed equipment on qualification samples provided by SERI researchers. and on telephone interviews with 19 users of the manufacturers' proposed equipment. The cost evaluation involved determining an actual comparison cost for each proposed set of equipment. based on information presented in the different (and often disparate) proposals. Reports of both committees were submitted to the Source Selection official (D. Trujillo), who awarded contracts to Nicolet Analytical Instruments (\$275K) and Hansen Associates (\$25K) in June of 1990. The contracts were supported through FY 1989 general purpose equipment (GPE) funds.

By consensus of operations managers from the three research and technology divisions, and to provide the most appropriate managerial environment for an analytical laboratory, the Center was located within the Applied Sciences Branch. J. Webb transferred into the branch, effective FY 1991, from the Photoconversion Research Branch, to manage the Center. After a search for vibration-free, ground-floor space proved unsuccessful, laboratory 16/387. which already contained one FTIR spectrophotometer. was added to the branch floor space to contain the new equipment.

The Center's equipment began to arrive in July 1990. Significant modifications to the 16/387 floor plan and utilities, again supported by the Deputy Director. were completed in a timely fashion by the SERI Facilities staff, so that equipment installation could proceed on a fast track. Major items of equipment, including the high-resolution FTIR spectrophotometer and the medium-resolution FTIR spectrophotometer with FTIR microscope, were operational by the end of September 1990. This allowed the Center to open as scheduled at the start of FY 1991. Delivery of the FT-Raman accessory to the high-resolution FTIR spectrophotometer, and a vibration-isolated workstation for the far-IR FTIR spectrophotometer, completed the Center's array of analytical equipment in January 1991.

The Center operates as a recharge center at an FY 1991 rate of $\$ 82 / \mathrm{hr}$. Rates for similar services charged by outside laboratories offering FTIR spectroscopy range from $\$ 95$ to $\$ 200 / \mathrm{hr}$. The laboratories at the lower end of this range have equipment inferior in many respects to that available in the Center, and no private, commercial, or university laboratory in the Rocky Mountain region currently has as complete an array of FTIR spectroscopic equipment as SERI's FTIR Center. This versatility was mandated by the diverse range of analytical needs and research interests of SERI scientists, and makes this branch a true "Center of Excellence" in FTIR spectroscopy. The primary components of the recharge cost are staff salaries, 16/387 floor space, and equipment maintenance. Costs for the latter will increase next fiscal year, 
as more of the equipment comes out of warranty. The recharge mode of operation is equitable in the sense that it reflects the real costs of analyses performed or supervised by experienced staff, minus the very significant costs of the equipment itself. The recharge mode also guarantees access to the Center's capabilities on an equal basis to any SERI researcher.

\subsection{ANALYTICAL CAPABILITIES}

Appendix D provides a listing of the major instrumentation and test equipment, their features, and specifications.

The FTIR Center is equipped to obtain the vibrational spectra of many types of solid and liquid samples on a nondestructive basis. Center staff also have access to an FTIR gas analyzer, courtesy of Branch 253. The vibrational spectrum of a sample is useful in both quantitative and qualitative analysis. For example. Center staff have used the FTIR gas analyzer to perform both qualitative on-line analyses of the products of a gas-phase photocatalytic reactor, as well as quantitative analyses of sub-ppm levels of selected reaction products. Other FTIR applications include analysis of dopants, additives, and contaminants, determination of ordering and orientation in polymers and liquid crystals, in-situ observation of reaction kinetics and phase transitions, and time-resolved spectroscopy.

Sample types analyzed at the Center include polymers used as module encapsulants, module indium tin oxide (ITO) surfaces, diamondlike carbon films, amorphous silicon films, catalyst surfaces, polymeric semiconductors, and ordered monomolecular assemblies on surfaces. At the request of researchers throughout SERI, the Center also has incorporated capabilities for vibrational spectroscopic analysis of superconductors, low-level dopants and impurities in silicon and other semiconductors, aqueous ligands, biomass, phase-change thermal storage materials, liquid crystals, desiccants, and microalgae.

\subsection{ANALYTICAL TECHNIQUES}

Common to all the analytical techniques used in the Center is the method of FTIR spectroscopy. Rather than scanning through the infrared frequency spectrum of a sample as is done in traditional dispersive spectroscopy, FTIR spectroscopy involves modulation of the entire blackbody source spectrum by means of an interferometer. After passing through the sample, the modulated source spectrum, or interferogram, is Fourier transformed to yield the frequency spectrum of the source, as modified by sample absorption. By calculating the ratio of this spectrum to a previously collected spectrum of the source without the sample (background spectrum), the transmittance spectrum of the sample is obtained. 
The FTIR spectroscopic method has almost entirely supplanted dispersive infrared spectroscopy over the last decade because of the following advantages'.

\subsubsection{FTIR Advantage: Mechanical Simplicity (One Moving Part)}

There is only one continuously moving component in the FTIR system: the moving mirror in the interferometer. This results in little system wear, high reliability, and low maintenance costs.

\subsubsection{FTIR Advantage: Increased Speed and Sensitivity (Felgett Advantage)}

The interferogram signal is termed "multiplexed" because the detector observes all infrared frequencies within its range simultaneously. As a result. a complete FTIR spectrumi of a sample can be obtained in $1 \mathrm{~s}$, whereas a dispersive spectrophotometer would rtquire at least $10 \mathrm{~min}$. to acquire a spectrum exhibiting similar resolution and signal-to-noise ratio. The signalto-noise ratio of FTIR spectra can be increased by adding more interferogram scans to reveal subtle spectral features impossible to observe using dispersive spectroscopy. Events as short in duration as a few microseconds can be characterized using rapid-scanning and time-resolved FTIR spectroscopy. which yield the entire infrared spectrum of a sample, rather than focusing on a single absorbance band. The Felgett Advantage therefore enables kinetic analyses to be made in real time, as well as enabling faster turnaround of routine samples.

\subsubsection{FTIR Advantage: Greater Optical Throughput (Jaquinot Advantage)}

There are no slits in the interferometer (as in the dispersive unit) to define resolution or to limit the amount of infrared energy reaching the detector. Thus, the optical throughput of an FTIR system is up to 100 times that of a dispersive spectrophotometer operated at similar resolution. This higher throughput translates into higher sensitivity, which in turn makes energylimiting sampling techniques such as reflectance spectroscopy much more feasible on an FTIR spectrophotometer. Outstanding examples of the Jaquinot Advantage at tine FTIR Center include the FTIR microscope accessory and the FT-Raman accessory, both of which are able to produce high-quality spectra from extremely weak signals. With the exception of FTRaman spectroscopy, the Jaquinot Advantage is achieved without significant heating of the sample by the infrared beam, since the interferometer is placed between the source and the sample. 


\subsubsection{FTIR Advantage: Internal Laser Frequency Reference (Connes Advantage)}

FTIR spectrophotometers use an internal helium-neon laser to moniter the position of the moving mirror in the interferometer. Since the wavelength of this laser is accurately known, it also provides an internal frequency calibration standard, which ensures accuracy of any measured data point to within $\pm 0.01 \mathrm{~cm}^{-1}$. This level of accuracy is extremely important when digitally comparing two different spectra to ensure that the differences found are real and not calibration artifacts.

\subsubsection{FTIR Advantage: Freedom from Stray Light and Sample Emission Artifacts}

The freedom from stray light and sample emission artifacts is inherent to interferometric modulation of the source signal and is important for ensuring accurate quantitative analyses.

\subsubsection{Discussion of Analytical Techniques}

Since sample spectra are stored on a computer, the spectra can be manipulated in a number of ways. Standard capabilities include baseline correction. peak integration (useful in quantitative analysis); conversion to reflectance, emittance or absorbance units, optical artifact removal (KubelkaMunk and Kramers-Kronig calculations), absorbance band fitting and deconvolution. signal-to-noise calculations, and digital subtraction of spectra. Spectra can be converted from Nicolet binary format to ASCII format and transmitted via RS. .32 ports to external computers having a Kermit program.

Together with the Connes Advantage, computer storage of the FTIR spectra also enables accurate identification of unknown compounds (qualitative analysis) through comparison of the sample spectra with disk-based spectral libraries. The Center has access to both the Aldrich Condensed-Phase Library (12.000 compounds) and the Aldrich Gas-Phase Library (6000 compounds). enabling rapid and accurate identification of unknown sample constituents. The search algorithms make use of digital subtraction of spectra. operate in several search modes, and require minimal user input.

A number of FTIR analytical techniques are available at the Center to fully utilize the unique advantages of FTIR spectroscopy in the service of research at SERI. In addition to routine transmittance-mode spectroscopy, in which the infrared radiation is transmitted through a sample during analysis, the Center also has available three reflectance-mode spectroscopies: specular 
reflectance (SR) spectroscopy. diffuse reflectance (DR) spectroscopy. and attenuated total reflectance (ATR) spectroscopy.

SR spectroscopy is best suited for analysis of thin films on smooth. IRreflective substrates, and can be sensitive to films or adlayers as thin as a single monolayer. DR spectroscopy is effective in analysis of intractable samples such as powders, fiber masses, or rough surfaces. It too can be sensitive to thin films on surfaces, and is particularly well suited for analysis of catalyst, desiccant. and superconductor surfaces. Both routine DR sample measurements and a DR reaction chamber, in which sample temperature $\left(0^{\circ}\right.$ to $\left.600^{\circ} \mathrm{C}\right)$. ambient atmosphere, and illumination can be controlled. are available at the Center.

ATR spectroscopy involves physically clamping or casting a sample onto an IR-transparent waveguide such as a fiber optic or flat crystal. Sampling is via the infrared "evanescent wave," which extends a few microns beyond the outer surface of the waveguide. The ATR technique is particularly useful in analysis of semi-rigid polymers such as encapsulants for solar cell modules and solar reflectors. The ATR technique is surface-sensitive, and enables "optical depth profiling" of samples to depths of a few microns by varying the incidence angle of the infrared radiation relative to the waveguide. or by changing the waveguide material. Both the ATR and SR techniques can yield orientational information on dichroic samples such as molecular monolayers, liquid crystals. and oriented polymers. The SR. DR. and ATR accessories are mounted on the new high-resolution FTIR spectrophotometer. the Nicolet 800. SR and ATR spectroscopies are also available on the older Nicolet 7199 FTIR spectrophotometer.

\subsubsection{FTIR Microscope}

Two major accessories, the FTIR microscope and the FT-Raman accessory. extend the analytical capabilities of the Center. The FTIR microscope mates a reflective optical microscope of approximately $500 \mathrm{X}$ magnification with a dedicated medium-resolution FTIR spectrophotometer, the Nicolet 710 . The FTIR microscope can image and record complete mid-infrared reflectance or transmittance spectra of samples as small as the infrared wavelengths used $(\sim 10 \mu \mathrm{m})$. In reflectance mode, this nondestructive. spatially resolved surface analysis technique has been useful in characterizing module contamination, and in the transmittance mode. has been important in identifying encapsulant degradation products.

\subsubsection{FT-Raman Accessory}

The FT-Raman accessory enables rapid collection of Raman spectra of solid and liquid samples over a frequency range of 3800 to $150 \mathrm{~cm}^{-1}$. This new 
technique utilizes the Jaquinot Advantage to collect the extremely weak Raman-scattered radiation arising from illumination of the sample with a YAG infrared laser operating at $1.06 \mu \mathrm{m}$. Despite the fourth-order inverse dependence of the intensity of Raman-scattered radiation on the source wavelength, Raman spectra comparable in signal-to-noise to those obtained using visible lasers and dispersive optics can be obtained using the FTRaman accessory, which is attached to the Nicolet 800 FTIR spectrophotometer. The accessory has an advantage over conventional Raman spectrophotometers using visible or ultraviolet sources in that interference by fluorescence is eliminated. However, the accessory is incapable of techniques such as resonance and time-resolved Raman spectroscopy. The accessory enables analysis of water solutions, which are traditionally off limits to vibrational spectroscopy since water is a strong infrared absorber and a weak Raman scatterer. Powders (including catalysts and superconductors) and thin films on surfaces can also be conveniently andyzed using the FT-Raman accessory.

\subsubsection{Far-IR FTIR Spectrophotometer}

With the exception of the FT-Raman accessory, most of the sampling techniques discussed previously are limited to the mid-infrared region $(6000$ to $400 \mathrm{~cm}^{-1}$ ), which includes many absorbances characteristic of covalently bonded organic species. However, many vibrational absorbances characteristic of inorganic, ionically or lattice-bonded species such as semiconductors and superconductors, exist in the far-infrared spectral region (650 to $50 \mathrm{~cm}^{-1}$ ). Many of these absorbances can only be measured at reduced temperatures. To further complicate the analysis, absorbances of some dopants and impurities can only be observed at low temperatures in samples illuminated with band-gap radiation. To address these analytical needs, the Center has available a Nicolet $20 \mathrm{~F}$ far-infrared FTIR spectrophotometer and a Hansen Associates High-Tran cryostatic sample mount. This combination of instrumentation enables the far-infrared spectra of samples up to $25 \mathrm{~mm}$ in diameter and up to $3 \mathrm{~mm}$ thick to be obtained at temperatures as low as $8 \mathrm{~K}$. Quartz windows allow bandgap illumination of samples during analysis to enhance the weak dopant and impurity absorbances. This technique should allow measurement of the latter substances at sub-ppm levels.

\subsection{EQUIPMENT/ANALYSIS DEVELOPMENT}

The entire FTIR Spectroscopic Research Center represents an exciting new capability for the branch and the division in the nondestructive analysis of semiconductor and superconductor materials. The FTIR microscope technique has only been available for about five years. and the FT-Raman technique has been developed within the last three years. Our capability for 
FTIR/ATR/fiber optic characterization of polymer curing reactions represents an even newer technique, which has attracted outside interest and funding. Although we have the newest instrumentation currently available in these three categories, these instruments were all tested prior to purchase with qualification samples provided by SERI researchers, so that most of the pitfalls associated with the purchase of novel equipment were avoided.

\subsection{ACCOMPLISHMENTS: PHOTOVOLTAICS}

Projects completed by the Center in support of SERI PV tasks during the performance period include the following (for clients indicated by parentheses):

- Identification of metal acetates throughout polymer encapsulants of degraded solar cell modules, indicating possible yellowing mechanisms involving the metallic grid (DeBlasio and several module manufacturers). See Fig. 9.1.

- Nondestructive identification of a carboxylate contaminant originating from the photoresist in the apparently clean, $10-\mu \mathrm{m}$-wide grid lines

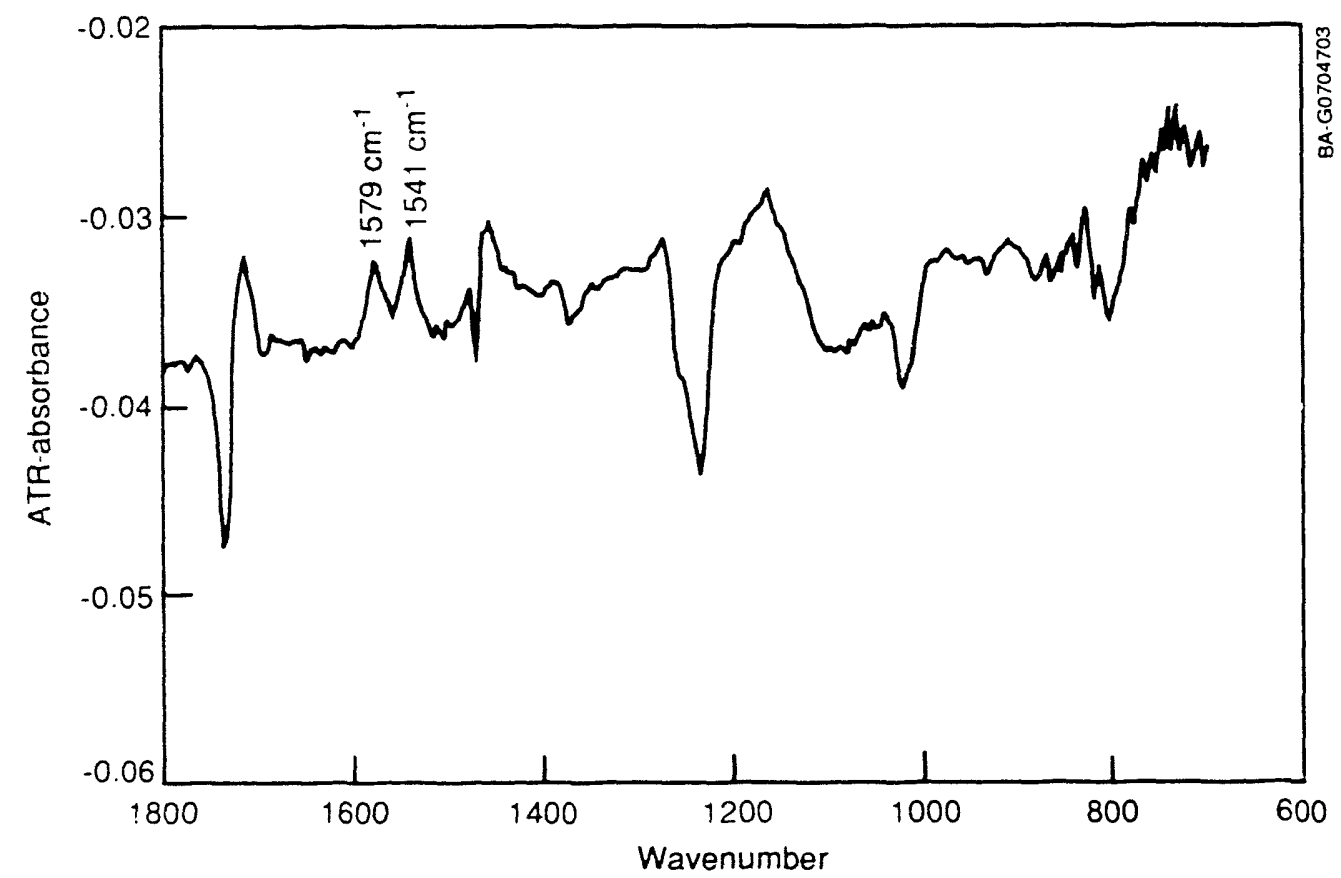

Figure 9.1: Digital subtraction spectrum generated between FTIR-ATR absorbance spectra of outdoor exposed and virgin polymeric module encapsulants. The peaks at 1579 and 1541 wave numbers indicate that metal acetates from decomposition of the module grid metallization have accumulated in the exposed encapsulant. 
etched on an ITO solar cell surface. The contaminant was interfering with adhesion of subsequently deposited grids (Coutts). See Fig. 9.2.

- Identification of hydrocarbon impurities in diamondlike carbon films (Nelson).

- With Jo Rouse, the development of a fast. easy, quantitative analysis method for hydrogen content of anorphous silicon films (Crandall). See Fig. 9.3.

\subsection{ACCOMPLISHMENTS: RELATED RESEARCH AREAS}

- With David King, the Center staff began a work for others (WFO) task in March 1990 for the Science Applications International Corporation (SAIC), as part of the NASA-funded Solid Rocket Motor (SRM) Bondline project. The first-year value of the contract was $\$ 40 \mathrm{~K}$ and was awarded by SAIC in response to a WFO proposal submitted by J. Webb in December 1989. The work involves measurements of the extent of

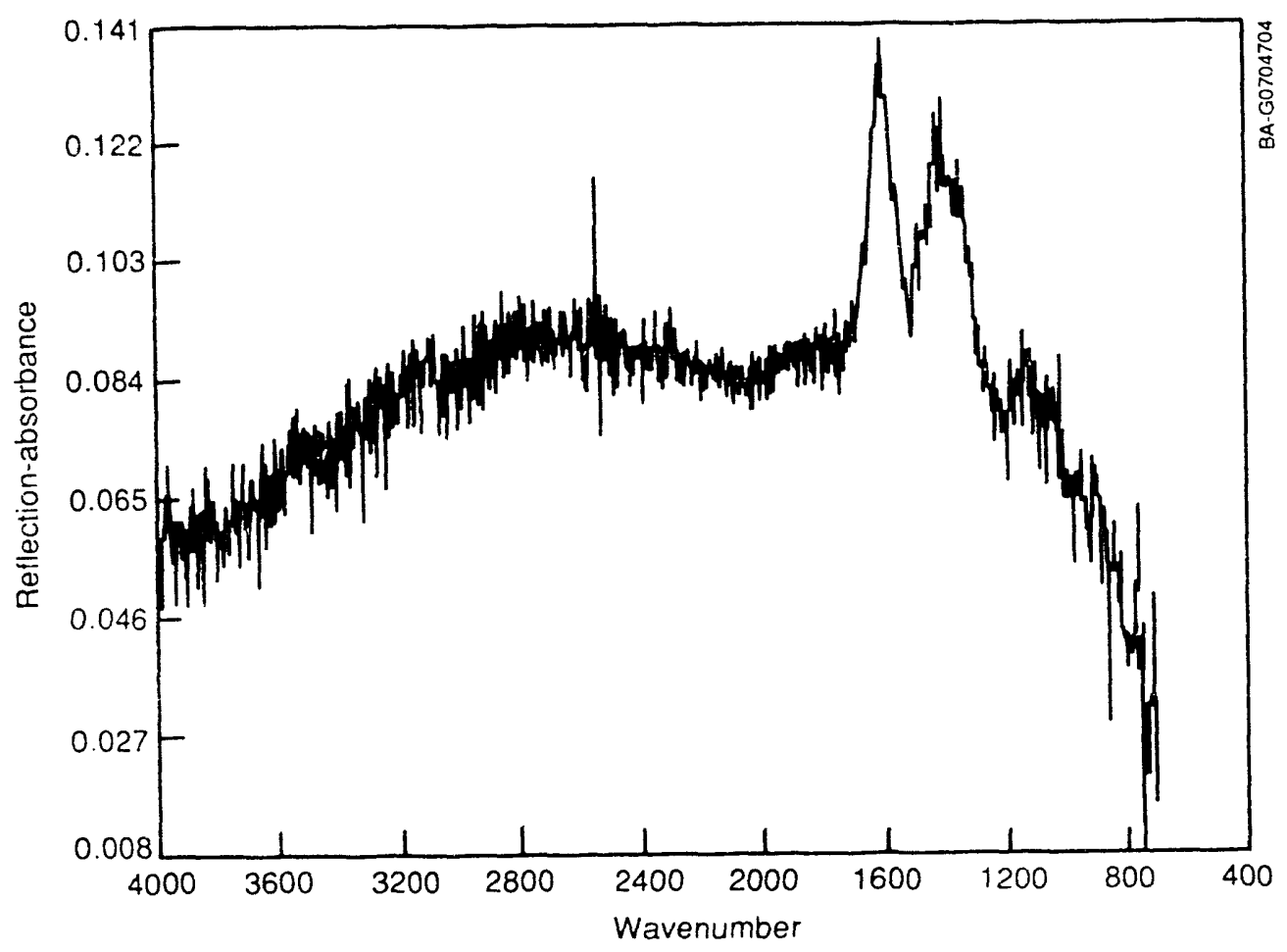

Figure 9.2: FTIR microscope specular reflection-absorbance spectrum showing accumulation of a carboxylate-containing product of photoresist decomposition. This contaminant was detected in the $10-\mu \mathrm{m}$-wide etched grid lines on the ITO surface of a PV cell and is believed to cause delamination of grids applied subsequenty. 


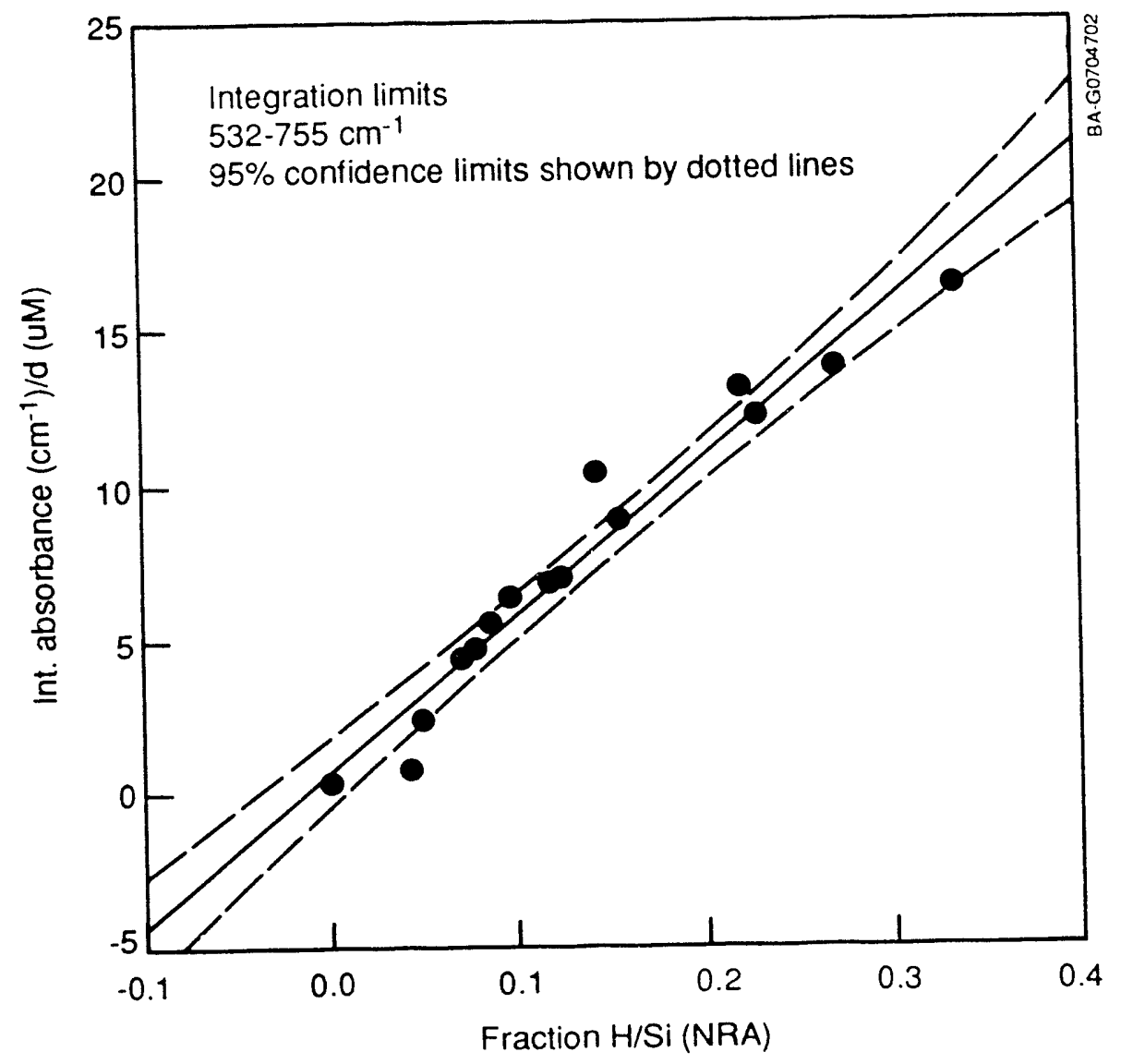

Figure 9.3: FTIR calibration curve for quantitative analysis of hydrogen content in amorphous silicon films.

cure of a polymeric liner compound applied to the interior surface of the case insulation in the space shuttle booster and other SRMs to promote adhesion to the solid propellant. Measurements are conducted using the Center's FTIR equipment (specifically, a novel FTIR fiber optic probe enabling in-situ spectroscopic measurements of extent of liner cure), hardness tests, and a new microwave reflectance technique. which was rated the most promising of a half-dozen alternate techniques proposed by the six other laboratories on the project (Fig. 9.4). SAIC is pleased with our work and has initiated a renewal of our WFO contract, beginning in March 1991, for the same amount.

- Identification of a hydrated hydroxyl species on titanium dioxide surfaces, which correlates with oxidative activity of this semiconductor photocatalyst, as well as identification of reaction product precursors on the photocatalyst surfaces (Blake, Milne).

- Installation and calibration of an FTIR gas analyzer at the new SERI solar furnace, and interfacing the analyzer to a gas-phase photocatalytic reactor for solar-assisted incineration of hazardous waste (Bohn). 
- Characterization of the chain configuration of a polythiophene polymeric semiconductor (Frank).

\subsection{FUTURE WORK}

Future work planned at the Center includes vibrational spectroscopic analysis of superconductors, quantitative analysis of low-level dopants and impurities in silicon and other semiconductors, and analysis of aqueous ligands. biomass, phase-change thermal storage materials, liquid crystals, desiccants, and microalgae. Despite efforts by the Center manager to inform SERI staff regarding the opening of the Center in October 1990, task and project leaders in some of these research areas did not plan FY 1991 funds for Center recharges. Also. some of the research areas mentioned above are either not currently funded or were funded in FY 1991 at a lower level than expected when the Center was planned. The versatility built into the Center, the Center manager's continuing efforts to inform SERI researchers regarding

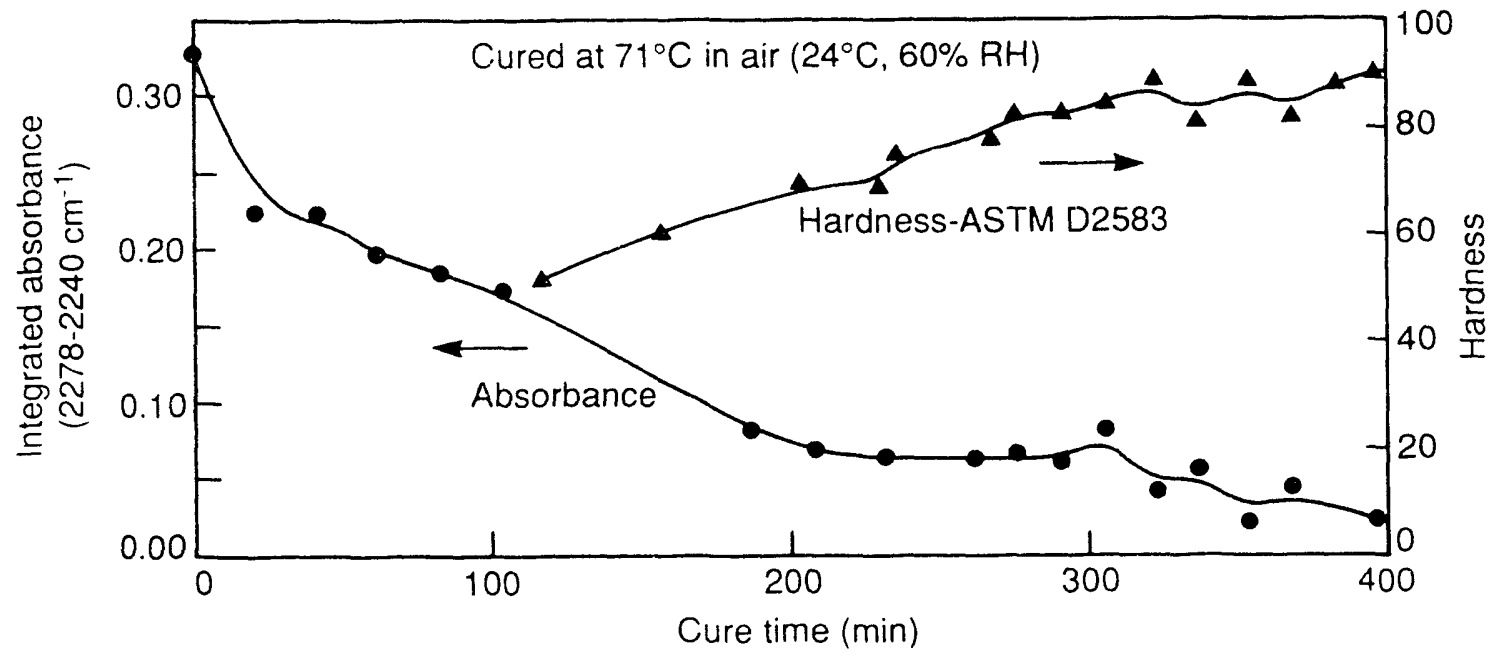

Figure 9.4: In-situ measurement of extent of polymeric SRM liner cure via an embedded FTIR-ATR fiber optic sensor, correlated with simultaneous hardness measurements. Cure of the hydroxyl-terminated polybutadiene (HTPB) resin is indirectly monitored by following the disappearance of an infrared absorbance band associated with the isocyanate curative. 
the Center's capabilities, and the involvement of the Center in new FY 1992 initiatives such as the OER/BES/DMS-funded study. "Spontaneous Ordering in Semiconductor Alloys." should ensure that the Center's operations, and possibly its staffing, will expand next year. The branch manager's efforts to inform the semiconductor industry about the branch's analytical capabilities will also help the Center's operations by bringing in more work, although Center costs and revenues during the first quarter of FY 1991 were close to planned targets.

1. FTIR Theory. Nicolet Corp. publication P/N 269-721903, Madison, WI. July 1986 


\section{APPENDIX A}

\section{STAFF BIOGRAPHIES}

Fourad Abou-Elfotouh (SERI 1986): Senior Scientist. Research and support interests and responsibilities; electro-optical properties (including defect states and their behavior) of semiconductor (thin films and single crystals), fabrication, characterization and modeling of heterojunction devices, solar cell back contacts, development of spectroscopic ellipsometer.

Richard K. Ahrenkiel (SERI 1981): Principal Scientist and Group Leader. Research and support interests and responsibilities; materials characterization by photoluminescence and diffusion measurements, deeplevel-transient-spectroscopy, $\mathrm{C}-\mathrm{V}$ measurements, and superconductor characterization.

Mowafak M. Al-Jassim (SERI 1983): Senior Scientist and Group Leader. $\mathrm{Ph}$.D. Materials Science. Research and support interests and responsibilities; transmission electron microscopy (TEM), scanning electron microscopy (SEM), defect analysis in semiconductors, epitaxial growth by MOCVD and MBE.

Sarah (Sally) Asher (SERI 1985): Senior Scientist, Ph.D. Analytical Chemistry. Research and support interests and responsibilities; surface science; operation and maintenance of SIMS facility.

Assem M. Bakry (SERI 1988): Peace Fellow. Research and support interests and responsibilities; deep-level-transient-spectroscopy (DLTS), defects in semiconductors.

Timothy J. Coutts (SERI 1984): Principal Scientist and Group Leader. Research and support interests and responsibilities; materials and devices fabrication and characterization; development of new analytical equipment; modeling of terrestrial and space solar cells.

Alvin W. Czanderna (SERI 1978): Research Fellow and Group Leader, Ph.D. Research and support interests and responsibilities: surface science, reactions at interfaces, stability and durability of multilayer thin film devices, interactions at polymer/metal (oxide) interfaces, surface analysis, ultramicrogravimetry.

Richard DeBlasio (SERI 1978): Section Manager. Research and support interests and responsibilities; module performance testing, evaluation and reliability research, and the operation of the outdoor module performance test facility and reliability research laboratory. 
Ramesh Dhere (SERI 1985): Staff Scientist. Research and support interests and responsibilities; optical and structural characterization of materials, analysis and modeling of devices.

Patricia C. Dippo (SERI 1980): Administrative Assistant. Research and support interests and responsibilities; preparation of branch correspondence, reports, etc.; day-to-day branch operations.

Donald J. Dunlavy (SERI 1981): Staff Scientist. Research and support interests and responsibilities; minority carrier characterization by photoluminescence and diffusion measurements, design, modification, and maintenance of all test equipment.

Keith A. Emery (SERI 1980): Senior Scientist. Research and support interests and responsibilities; photovoltaic performance characterization by current versus voltage, temperature, spectrum, and intensity. PV calibration, quantum efficiency, PV standards.

Clark L. Fields (SERI 1990) Visiting Professional (Summer), Prof. of Chemistry at the University of Northern Colorado, Greeley. Research and support interests and responsibilities: rapid thermal processing of materials, metalorganic chemistry, boron chemistry, chemical vapor deposition.

Amy B. Swartzlander-Franz (SERI 1980): Associate Scientist. Research and support interests and responsibilities; characterization of solar and electronic devices including superconductors using SAM, AES and XPS.

Steve Gebhard (SERI 1989): Post-doctoral Associate. Ph.D. in Chemistry. Research and support interests and responsibility; characterization of solar and electronic materials using photoemission techniques.

Timothy A. Gessert (SERI 1984): Staff Scientist. Research and support interests and responsibilities; device processing technology, optical and electrical thin film deposition and characterization, metallization science, solar cell grid and antireflection technology modeling and optimization.

John P. Goral (SERI 1986): Staff Scientist. Ph.D. Inorganic Chemistry, Research and support interests and responsibilities; transmission electron microscopy, electron microprobe analysis, structure and properties of photovoltaic materials, defects and interfaces in semiconductors.

Thomas R. Hanak (SERI 1985): Visiting Professional. Research and support interests and responsibilities; deep-level-transient-spectroscopy (DLTS), defects in semiconductors. 
Gregory Horner (SERI 1988): Research Associate. Responsibilities and interests; materials/device processir,; and characterization, software development and computerized instrument control.

Kim M. Jones (SERI 1981): Staff Scientist, A.A. Electron Microscopy/ Materials Science Applications. Research and support interests and responsibilities; transmission electron microscopy, analytical electron microscopy and defect analysis in semiconductors.

Lawrence L. Kazmerski (SERI 1977): Principal Scientist and Branch Manager, Ph.D. Electrical Engineering. Research and support interests and responsibilities; scanning tunneling microscopy and surface and interface analysis research.

Brian M. Keyes (SERI 1988): Associate Engineer. Research and support interests and responsibilities; minority carrier characterization by photoluminescence and diffusion measurements, modeling of the above measurements, computer analysis and interfacing.

David King (SERI 1989): Postdoctoral Research Associate, Ph.D. Chemistry. Research and support interests and responsibilities: surface analysis (especially XPS and ISS), organized molecular assemblies (OMAs), interactions between vacuum deposited monolayers of metals with organic functional groups, FT-IR-RA of OMAs.

Xiaonan Li (SERI 1985): Postdoctoral Associate. Research and support interests and responsibilities; device processing, optical and electrical thin film deposition, and characterization, metallization vacuum technology.

Paul Longrigg (SERI 1979): Senior Electrical Engineer. Research and support interests and responsibilities; module reliability research, testing and failure analysis. Development of techniques to determine and evaluate module failure mechanisms occurring in the field for accelerated stress tests.

Alice Mason (SERI 1984): Master Technician, A.A. Electron Microscopy. Research and support interests and responsibilities; sample preparation for microscopy examination.

Richard Matson (SERI 1979): Staff Scientist, B.S. Physics. Research and support interests and responsibilities; scanning electron microscopy, X-ray microanalysis, defect examination in semiconductors and luminescent properties. 
Edith C. Molenbroek (SERI 1989): Visiting Professional. Research and support interests and responsibilities; hot spot testing of thin-film photovoltaic modules.

Laxmi Mrig (SERI 1979): Senior Electrical Engineer. Research and support interests and responsibilities; analysis and evaluation of module performance test data and developing analytical models for predicting module long term reliability.

Robbin L. Mullins (SERI 1989): Undergraduate Intern, Senior Physics Major. Research and support interest and responsibilities: surface analysis, interfacing instruments and computers.

Art J. Nelson (SERI 1985): Senior Scientist and Group Leader, M.Sc. in Solid State Physics. Research and support interests and responsibility; surface and interface analysis research, surface and grain boundary phenomena in semiconducting and superconducting materials, synchrotron radiation photoemission on crystalline and amorphous materials, growth and characterization of diamond and diamond-like carbon films.

Carl Osterwald (SERI 1982): Staff Scientist. Research and support interests and responsibilities; measurement system design and development, primary photovoltaic reference cell calibration, spectral response, and current versus voltage measurements.

Brian L. Pelton (SERI 1989): Summer Intern, B.S. Metallurgy. Research and support interest and responsibilities: metallurgical modification of cladding layers on steels. [Solar Heat Research Division]

F.J. Pern (SERI 1984): Senior Scientist. Research and support interests and responsibilities; material fabrication and characterization. spectral and electrochemical characterization of thin-film polymers and catalysts, module performance testing and analysis of failure mechanisms.

J. Roland Pitts (SERI 1979) Senior Physicist, Ph.D. Solid State Physics. Research and support interest and responsibilities: surface modification using particle beams, surface and interface science, stimulated desorption, transient thermal processes, optical and physical properties of thin films.

Kannan Ramanathan (SERI 1989): Senior Scientist. Research and support interests and responsibilities; fabrication and characterization of polycrystalline thin films and devices, transparent conducting oxides. Optical and electrical measurements. 
Elizabeth (Jo) Rouse (SERI 1990): AWU Student Fellow. Research and support interests and responsibilities; Fourier Transform Infrared (FTIR) spectroscopy and quantitative analysis.

Lorenzo L. Roybal (SERI 1982): Master Technician. Research and support interests and responsibilities; designs, modifies, machines, maintains, and operates characterization systems for superconductivity. DLTS, C-V/G-V and other test stations.

Steve Rummel (SERI 1980): Associate Engineer. Research and support interests and responsibilities; module performance tests, operates and maintains Spire model solar simulator; module characterization and stability tests, preparation of test reports, maintenance of the module performance data base, and design and development of computer software instrumentation interfaces.

Marta Ruth (SERI 1989): Summer Intern, M.S. Physics. Research and support interests and responsibilities: water permeation of polymeric materials, computer modeling of slow and rapid diffusion processes. [Solar Heat Research Division]

Peter Sheldon (SERI 1979): Senior Scientist, M.Sc. Electrical Engineering. Research and support interests and responsibilities; thin film and semiconductor device research growing a variety of III-V and group IV semiconductors by molecular beam epitaxy (MBE) and electrical characterization of these materials.

Yvonne Shinton (SERI 1984) Master Technician. Research and support interests and responsibilities: metallography, optical microscopy, X-ray diffraction, vacuum microbalance measurements. [Mechanical and Industrial Technology Division].

David Spaulding (SERI 1989): Graduate Intern, M.S. Materials Science. Research and support interests and responsibilities: organized molecular assemblies (OMAs), XPS of copper/OMA interfaces, interactions between copper and specific organic functional groups. [Solar Heat Research Division]

James T. Stanley (SERI 1988) Postdoctoral Research Associate, Ph.D. Materials Science. Research and support interests and responsibilities: transient thermal processing of materials, structural analysis, electron microscopy. evaluation of the physical properties of materials. (Present address: Oregon Graduate Research Center) 
Ed Tracy (SERI 1981) Staff Scientist, B.S. Physics. Research and support interests: thin film deposition via vacumm evaporation, sputtering, and CVD. vacuum technology. laser welding, electrochromics.

Robbin L. (Mullins) Vojdani (SERI 1989) Undergraduate intern, Senior Physics Major. Research and support interest and responsibilities: surface analysis of thin films.

David Waddington (SERI 1978): Senior Electrical Engineer. Research and support interests and responsibilities: module performance characterization and reliability testing and evaluation. Design and development of testing systems, and test methods for characterization of module performance.

Mark W. Wanlass (SERI 1980): Staff Scientist. Research and support interests and responsibilities; metalorganic vapor phase epitaxy, device design, fabrication, characterization and modeling.

Scott Ward (SERI 1989): Research Associate. Research and support interests and responsibilities; device processing and design; generation of photolithographic masks and application of Entech prismatic covers; tandem solar cell development.

John D. Webb (SERI 1978): Senior Chemist and Center manager. Research and support interests and responsibilities; Fourier Transform Infrared (FTIR) spectroscopy. polymer chemistry, and photochemistry; operation, maintenance, and business development for the FTIR Spectroscopic Research and Service Center. 


\section{APPENDIX B}

\section{COOPERATING LABORATORIES}

Ain Shams University, Egypt

Air Force WPL

Airtech Corporation

Alabama Power

ARCO Solar Inc./Siemans

Arizona State University

Armco Research and Technology

ASEC

Astropower, Inc.

AT\&T Bell Laboratories

Ben Gurion University, Israel

Boeing Aerospace Company

PTB. Braumschweig. Germany

Brooklyn Poltechnic Institute

British Petroleum

Brookhaven National Laboratories

Brown University

Cargocaire Engineering. inc.

Charles Evans Associates

Chronar Corporation

Colorado School of Mines

Colorado State University

Coors Ceramic

Cornell University

Eastman Kodak Research Laboratories

Eaton Corporatino

EIC Laboratories

ENEA. Italy

Electrotechnical Laboratory, Japan

Energy Conversino Devices

EXXON Research

Florida Solar Ene-gy Center

Ford Aerospace

Franhofer Institute for Solar Energy, Germany

Fuji Electric. Japan

Georgia Institute of Technology

Grumman Aerospace Corporation

GTE Laboratories

Harvard University 
Howard University

Hughes Research

IBM

Indian Institute of Technology. Delhi

Institute for Non-Ferrous Metals. Beijing. China

Institute of Energy Conversion

Institutto Militar de Engenharia, Brazil

International energy Foundation

Iowa State University

Jet Propulsion Laboratory

Kiefer Electronic Development

King Abduil-Aziz University. Saudi Arabia

Kopin Corporation

Lawrence Berkeley Laboratories

Louisiana State University

Man-Tech Development. Inc.

Martin Marietta

Massachusetts Instittue of Technology

Matsushita. Japan

Midwest Research Institute

MIT Lincoln Laboratory

Mobil Solar

Montana State University

Motorola

NASA Lewis Research Center

National Institute for Standards and Technology

National Instiute for Silicon Technology. Pakistan

National Physical Laboratory. New Delhi, India

National Scientific Balloon Facility (UCAR)

Naval Research Laboratories

Newcastle-Upon-Tyne Polytechnic

Nippon Telegraph \& Telephone. Japan

North Carolina State University

Oak Ridge National Laboratory

Oregon State University

Osaka University. Japan

Pacific Gas and Electric

Pennsylvania State University

Princeton University

PTL (Physical Technical Laboratory). India

Ramtron, Inc.

Rerrsselat: Polytechnic Institute

Research Triangle Institute

Rocky Mountain Laboratories

Sandia National Laboratories 
Santa Barbara Research Institute

Sanyo. Japan

Science Applications International Corporation

Shanghai Institute of Metallurgy. China

University of Sao Paulo, Brazil

University of Texas

University of Utah

University of Washington

University of Wetern Ontario, Canada

University of Wisconsin, Madison

University of Wisconsin. Milwaukee

Utility Power Group

VacTec. Inc.

Varian Associates

Weizmann Institute of Science, Israel

Westinghouse

Wuhan University. China 


\section{APPENDIX C}

\section{FY 1990 APPLIED SCIENCES BRANCH BIBLIOGRAPHY}

1. Abou-Elfotouh. F.A., G.S. Horner, T.J. Coutts, and M.W. Wanlass, "Broad Band Spectroscopic Ellipsometry for the Characterization of Photovoltaic Materials," presented at the 10th PV AR\&D Meeting, Lakewood, Colorado, 23-25 Oct. 1990. To be published in Solar Cells.

2. Abou-Elfotouh, F.A., R.J. Matson, A.M. Bakry, and L.L. Kazmerski, "Study of the Interface Properties of the CuInSe ${ }_{2}$ Single Crystal Heterojunctions and Schottky Barriers," presented at the 8th International Conference on Metallurgical Coatings, San Diego, CA. 16 Apr. 1990.

3. Abou-Elfotouh, F.A., L.L. Kazmerski, A.M. Bakry, and A. Al-Douri. "Correlations of Single-Crystal CuInSe, ${ }_{2}$ Surface Processing with Defect Levels and Cell Performance," 21st IEEE Photovoltaic Specialists Conference, New York: IEEE, 1990, pp. 541-545.

4. Abou-Elfotouh, F.A., L.L. Kazmerski, R.J. Matson, D.J. Dunlavy, and T.J. Coutts, "Studies of the Electrical and Interface Properties of the Metal Contacts to Single Crystal CuInSe ${ }_{2}$," J. Vac. Sci. Technol. A, Vol. 8. No. 4, 1990, p. 3251.

5. Abou-Elfotouh, F., H. Moutinho, A. Bakry, and T.J. Coutts, "Characterization of the defect levels in copper indium diselenide," presented at the 10th PV AR\&D Meeting, Lakewood, Colorado, 23-25 Oct. 1990. To be published in Solar Cells.

6. Abou-Elfotouh, F.A., L.L. Kazmerski, H.R. Moutinho, A.J. Nelson, and A.M. Bakry, "Determination and Observation of Electronic Defect Levels in CuInSe2 Crystals and Thin Films," presented at the AVS 37th National Vacuum Symposium, Toronto, Canada, 8-12 Oct. 1990, J. Vac. Sci. Technol. A, Vol. 9, No. 3, 1991.

7. Ahrenkiel, R.K., D.J. Dunlavy, B. Keyes, S.M. Vernon, S.P. Tobin, T.M. Dixon, "Design of High Efficiency GaAs Solar Cell by Photoluminescence Studies," 2 lst IEEE Photovoltaic Specialists Conference. New York: IEEE, 1990, pp. 432-436.

8. Ahrenkiel, R.K., B.M. Keyes, and D.J. Dunlavy, "Intensity Dependent Minority Carrier Lifetime due to Saturation of Recombination Centers," J. Appl. Phys. . 1990. 
9. Ahrenkiel. R.K.. B.M. Keyes, and D.J. Dunlavy, "Nonlinear Recombination Processes in Photovoltaic Semiconductors." $\underline{\text { Solar }}$ Cells. 1990.

10. Ahrenkiel, R.K., M.M. Al-Jassim, B. Keyes, D. Dunlavy. K.M. Jones, S.M. Vernon and T.M. Dixon, "Minority-Carrier Lifetime of GaAs on Silicon," J. Electrochem. Soc., Vol. 137, 1990, pp. 996-1000.

11. Ahrenkiel, R.K., J.M. Olson, D.J. Dunlavy, B.M. Keyes, and A.E. Kibbler, "Recombination Velocity of the $\mathrm{Ga}_{0.5} \mathrm{In}_{0.5} \mathrm{P} / \mathrm{GaAs}$ Interface," $\mathrm{J}$. Vac. Sci. Technol. A. Vol. 8, 1990, p. 3002.

12. Albin, D.S., G.D. Mooney, J. Carapella, A. Duda, J. Tuttle, R.J. Matson. and R. Noufi, "The Phase Behavior of Evaporated $\mathrm{Cu}$ and In Precursors for Selenization," presented at the 10th PV AR\&D Meeting, Lakewood. Colorado, 23-25 Oct. 1990. To be published in Solar Cells.

13. Albin, D.S., D.S. Mooney, A. Duda, J. Tuttle, R.J. Matson, and R. Noufi, "Enhanced Grain Growth in Polycrystalline CuInSe. Using Rapid Thermal Processing," presented at the 10th PV AR\&D Meeting, Lakewood. Colorado, 23-25 Oct. 1990. To be published in Solar Cells.

14. Al-Jassim, M.M., R.K. Ahrenkiel, M.W. Wanlass, J.M. Olson, and S.M. Vernon. "The Heteroepitaxy and Characterization of InP and GaInP on Si for Solar Cell Applications" presented at the MRS Spring Meeting, San Francisco, CA. 16-20 Apr. 1990.

15. Al-Jassim, M.M.. J.M. Olson, K.M. Jones, and A.E. Kibbler,"The Heteronucleation and Growth Morphology of GaInP and GalnAs Thin Layers and SLS's," Institute of Physics Conf. Series No. 100, 1989, p. 317.

16. Al-Jassim, M.M.. R.K. Ahrenkiel, M.W. Wanlass, J.M. Olson, and S.M. Vernon, "Structural and Luminescent Characterization of InP and GaInP on Si." presented at the 10th PV AR\&D Meeting, Lakewood, Colorado, 23-25 Oct. 1990. To be published in Solar Cells.

17. Asher, S.E., "Secondary Ion Mass Spectrometry Studies of Polycrystalline Thin Film CdTe/CdS Solar Cells," presented at the Microbeam Analysis Society Meeting - XIIth International Congress for Electron Microscopy, Seattle, Washington, 12-17 Aug. 1990.

18. Asher, S.E., "SIMS Investigations of Substrate/Film Interdiffusion in $\mathrm{YBa}_{22} \mathrm{Cu}_{33} \mathrm{O}_{7}$ Films Grown on $\mathrm{SrTiO}_{33}$ and $\mathrm{LaAlO}_{3,}$," presented at the AVS 
37th National Vacuum Symposium. Toronto. Canada. 8-12 Oct. 1990. J. Vac. Sci. Technol. A. Vol. 9. No. 3, 1991.

19. Bhattacharya, R.N., R. Noufi, L.L. Roybal, and R.K. Ahrenkiel, "Y-BaCu-O Superconductor Thin Films via an Electrodeposition Process," J. Electrochem. Soc., 1990.

20. Berry W. . M. Hahn, and L. Mrig, "Post Initial Light Induced Degradation Stability and Performance of Amorphous Silicon Modules," presented at the MRS Spring Meeting. San Francisco, CA, 16-20 Apr. 1990.

21. Berry. W.. M.J. Hahn, and L.C. Mrig, "Comparative Short Term/Long Gield Performance and Stability of Tandem and Single Junction a-Si Modules." 21 st IEEE Photovoltaic Specialists Conference, New York: IEEE. 1990, pp. 1057-1061.

22. Coutts. T.J., M.W. Wanlass, X. Li, T.A. Gessert, and I. Weinberg, "A Review of Recent Progress in InP Solar Cells for Space Application," Proceedings of the 4th International Photovoltaic Science and Engineering Conference, Sydney, Australia, 14-17 Feb. 1989. p. 499.

23. Coutts, T.J., X. Li, T.A. Gessert, and M.W. Wanlass, "A Review of ITO/ InP Solar Cells," Ist International Conference on InP and Related Materials. Bellingham. WA: SPIE, 1989, SPIE Vol. 1144, p. 466.

24. Czanderna, A.W., "Overview of Ion Spectroscopies for Surface Compositional Analysis," Chapter 1 in Ion Spectroscopies for Surface Analysis. edited by A.W. Czanderna and D. Hercules, New York: Plenum, 1990. See SERI/TP-255-3577, Mar. 1990.

25. Czanderna, A.W.. "Overview of Possible Causes of EVA Degradation in PV Modules," PV Module Reliability Workshop, edited by L. Mrig, Lakewood. Colorado, 26 Oct. 1990. SERI/CP-4079

26. Czanderna, A.W., Polvmers as Advanced Materials for Desiccant Applications, SERI/TP-213-3608, Dec. 1990.

27. Czanderna, A.W., D.E. King, and D. Spaulding, "Metal Overlayers on Organic Functional Groups of Self Organized Molecular Assemblies 1. XPS of Interactions of $\mathrm{Cu} / \mathrm{COOH}$ on 11 -Mercaptoundecanoic Acid," (Submitted for Publication), See SERI/TP-213-4170, Jan. 1991.

28. Czanderna. A.W.. "Ion Spectroscopies for Surface Analysis." Electrochemical Society Proc.. Pennington, N.J., Vol. 91. 1991. See SERI/TP-213-3987. Dec. 1990. 
29. Czanderna, A.W., and C. Lampert. Evaluation Criteria and Test Methods for Electrochromic Windows. SERI/TP-255-3537. July 1990.

30. Czanderna, A.W., and H.H. Neidlinger, Thin Film Superconductors for Electric Power Systems. Encapsulation and Stabilization with Conducting Polymers. SERI/PR-255-3572, May 1990.

31. Czanderna, A.W., and H.H. Neidlinger, Polymers as Advanced materials for Desiccant Applications, 1988, SERI/PR-255-3443. Sept. 1990.

Czanderna, A.W., and H.H. Neidlinger, "Polymers as Advanced materials for Desiccant Applications 2. Alkali Ionic Salts of Polystyrene Sulfonic Acid." ASHRAE Transactions, 91, See SERI/TP-2 13-4137. Jan. 1990.

32. Dagan, G., F. Abou-Elfotouh, D.J. Dunlavy, R.J. Matson, and D. Cahen, "Defect Level Identification in CuInSe, from Photoluminescence," Chemistry of Materials, Vol. 2 No. 3, 1990, p. 286.

33. DeBlasio, R.. L. Mrig, and D. Waddington, Interim Qualification Tests and Procedures for Terrestrial Photovoltaic Thin-Film Flat-Plate Modules, SERI/TR-213-3624, Jan. 1990.

34. Emery, K.A., and C.R. Osterwald, "Perforamnce Measurement Algorithms, Procedures and Equipment," 21st IEEE Photovoltaic Specialists Conference, New York: IEEE. 1990, pp. 1068-1073.

35. Fraas. L.M.. J.E. Avery, V.S. Sundaram, V.T. Dinh, T.M. Davenport, J.W. Yerkes, J.M. Gee, and K.A. Emery, "Over 35\% Efficient GaAs/ GaSb Stacked Concentrator Cell Assemblies for Terrestrial Applications." 21st IEEE Photovoltaic Specialists Conference, New York: IEEE, 1990, pp. 190-195.

36. Gessert T.A., X. Li, M.W. Wanlass, and T.J. Coutts, "Progress in the ITO/InP Solar Cell," 2nd International Conference on InP and Related Materials, New York: IEEE, 1990, p. 260, Catalog Number 90CH28597

37. Gessert T.A., and T.J. Coutts, "Requirements of Electrical Contacts to Photovoltaic Solar Cells," Advanced Metallizations in Microelectronics. MRS Spring Meeting, Pittsburgh, PA: MRS, 1990, Vol. 181, p. 301.

38. Gessert, T.A., X. Li, and T.J. Coutts, "Practical Guidelines for Grid Metallization in Photovoltaic Solar Cell Research," presented at the 
10th PV AR\&D Meeting, Lakewood, Colorado, 23-25 Oct. 1990. To be published in Solar Cells.

39. Gessert, T.A., X. Li, M.W. Wanlass, and T.J. Coutts, "Buried Homojunction Solar Cells Formed in p-InP During Sputter Deposition and Hydrogen Plasma Processing," 2 1st IEEE Photovoltaic Specialists Conference, New York: IEEE, 1990, p. 153.

40. Gessert, T.A., X. Li, T.J. Coutts, M.W. Wanlass, and A.B. Franz, "Aspects of Processing Indium Tin Oxide/InP Solar Cells," 1st International Conference on InP and Related Materials, Bellingham, WA: SPIE, 1989, SPIE Vol. 1144, p. 476.

41. Gessert, T.A., X. Li. M.W. Wanlass. A.J. Nelson, and T.J. Coutts. "Investigation of Buried Homojunctions in p-InP Formed During Sputter Deposition of Both Indium Tin Oxide and Indium Oxide," $\underline{\mathrm{J}}$ Vac. Sci. Technol. A, Vol. 8, No. 3, Part 1, 1990, p. 1912.

42. Gessert, T.A., X. Li. M.W. Wanlass, and T.J. Coutts, "Recent Advances in the ITO/InP Solar Cell," Proc. of the 10th Space Photovotlaics and Technology Conference (SPRAT), Cleveland: NASA, 1990.

43. Gessert. T.A.. D.L. Williamson, and A.J. Nozik, "Mossbauer Spectrsocopy Study of the Kinetics of Photoreduction of $\mathrm{Fe}^{3+}$ on CdS Semiconductor Powders," Journal of Physical Chemistry, Vol. 94, No. 5, 1990, p. 1958.

44. Goral, J.P., M.M. Al-Jassim, and K.M. Jones, "TEM of CuInSe, and CdTe Polycrystalline Thin Films." presented at the 10th PV AR\&D Meeting, Lakewood, Colorado, 23-25 Oct. 1990. To be published in Solar Cells.

45. Goral J.P. M. Huffman, and M.M. Al-Jassim. "TEM Investigation of the Ferroelectric Domain Structure in Sputtered PZT Thin Films," MRS Spring Meeting, San Francisco, CA: MRS Symp. Proc., Vol. 200, 1990, p. 225.

46. Goral J.P., M.M. Al-Jassim, D. Albin, J.R. Tuttle, and R. Noufi, “TEM Identification of Secondary Phases in Non-stoichiometric Polycrystalline CuInSe, Thin Films," Proc. of the XIIth International Congress for Electron Microscopy, San Francisco Press Inc., 1990, p. 604

47. Goren, Z.. I. Willner, A.J. Nelson, and A.J. Frank, "Selective Photoreduction of $\mathrm{CO}_{2} / \mathrm{HCO}_{3}$ to Format Aqueous Suspensions and Colloids of $\mathrm{Pd}-\mathrm{TiO}_{2} \mathrm{O}, "$ J. Phys. Chem.. Vol. 94, No. 9, 1990, p. 3784. 
48. Hanak. T.R., R.K. Ahrenkiel. D.J. Dunlavy, A. Bakry. and M.L. Timmons. "A New Method to Analyze Multiexponential Transients for Deep-Level Transient Spectroscopy," J. Appl. Phys.. Vol. 67. 1990, p. 4126.

49. Hanak, T.R., R.K. Ahrenkiel, and M.L. Timmons, "Capture Barrier and the Ionization Entropy of the DX Center in Se-Doped $\mathrm{Al}_{x} \mathrm{Ga}_{1-x} \mathrm{As}$," J. Appl. Phys. Vol. 69, No. 3, 1990, p. 1425.

50. Hanak, T.R., A.M. Bakry, R.K. Ahrenkiel, and M.L. Timmons, "DXCenter in Se-Doped $\mathrm{Al}_{\mathrm{x}} \mathrm{Ga}_{1 \cdot x}$ As." Impurities, Defects, and Diffusion in Semiconductors: Bulk and Layered Structures, MRS Spring Meeting, Pittsburgh, PA: MRS, 1990, Vol. 163, p. 781.

51. Hanak, T.R., A. Bakry. D.J. Dunlavy. F. Abou-Elfotouh, R.K. Ahrenkiel, and M.L. Timmons, "Deep Level Spectroscopy of AlGaAs and CuInSe ${ }_{2}$," Solar Cells, Vol. 27, 1989, p. 347.

52. Jones K.M., M.M. Al-Jassim, J.M. Olson, S.R. Kurtz, A.E. Kibbler, and S.M. Vernon, "The Characterization of $\mathrm{Ga}_{0.5} \mathrm{In}_{0.5} \mathrm{P} / \mathrm{GaAs}$ Solar Cells Grown on Si Substrates," presented at the XIIth International Congress for Electron Microscopy, Seattle, Washington, 12-17 Aug. 1990.

53. Kazmerski L.L., A.J. Nelson, and A.B. Swartzlander, "Depth and Interface Resolution in CdS/CuInSe ${ }_{2}$ Heterostructures," Solar Cells, Vol. 28, No. 3, 1990, p. 273.

54. Kazmerski, L.L., "Thin Film Solar Cells, Evolution in Characterization and Performance," presented at the Special Symposium (Festkoloquium) for Herr Prof. Dr. Ing. Habil Werner H. Bloss in Stuttgart. West Germany, 23-24 Feb. 1990.

55. Kazmerski L.L., "Specific Atom Imaging, Nano-processing and Electrical Nanoanalysis with Scanning Tunneling Microscopy," presented at the 5 th International Conference on Scanning Tunneling Microscopy/ Spectroscopy and The First International Conference on Nanometer Scale Science and Technology, Baltimore. Maryland, 23-27 July 1990.

56. Kazmerski, L.L., H. Moutinho, J. Wissel, and A. Nelson, "Spectroscopic Scanning Tunneling Microscopy of Thin Film Photovoltaic Semiconductors." presented at the 5th International Conference on Solid Films and Surfaces. Providence, Rhode Island, 13-17 Aug. 1990. 
57. Keyes, B.M., D.J. Dunlavy, R.K. Ahrenkiel, S.E. Asher, L.D. Partain, D.D. Liu, and M.S. Kuryla, "Minority-Carrier Diffusion Length of pGaAs Determined by Time-of-Flight," J. Vac. Sci. Technol. A. Vol. 8, 1990, pp. 2004.

58. King, D.E., and A.W. Czanderna, "'He ${ }^{+}$ISS of 11-Mercaptoundecanoic Acid Self Assembled Monolayers onto Gold," Surface Science Letters, Vol. 235, L 329-332, 1990. See SERI/TP-2 13-3699, Apr. 1990.

59. Li, X., T.E. Furtak, T.A. Gessert, M.W. Wanlass, and T.J. Coutts, "A Study of Type Conversion Caused by DC-Magnetron Sputtering of ITO onto InP During Solar Cell Fabrication," Industry-University Advanced Materials Conference, Warrendale, Pennsylvania: The Metallurgical Soc., 1989. p. 34.

60. Lovejoy, M.L., B.M. Keyes, M.E. Klausmeier-Brown, M.R. Melloch, R.K. Ahrenkiel, and M.S. Lundstrom, "Time-of-Flight Measurements of Zero-Field Electron Diffusion in $\mathrm{p}^{+}$-GaAs," Jap. J. Appl. Phys., Voi. 30, No. 2A, 1990, pp. L135-L137.

61. Lundstrom, M.S., M.E. Klausmeier-Brown, M.R. Melloch, R.K. Ahrenkiel, and B.M. Keyes, "Device-Related Material Properties of Heavily Doped Gallium Arsenide," Solid State Electronics, Vol. 33, 1990, pp. 693.

62. Melloch M.R., S.P. Tobin, C. Bajgar, A. Keshavarzi, T.B. Stellwag, G.B. Lush, M.S. Lundstrom, and K. Emery, "High-efficiency $\mathrm{Al}_{0.22} \mathrm{Ga}_{0.78} \mathrm{As}$ Solar Cells Grown by Molecular Beam Epitaxy," Appl. Phys. Lett., Vol. 57, 1990, pp. 52-54.

63. Melloch, M.R., S.P. Tobin, T.B. Stellwag, C. Bajgar, A. Keshavarzi, M.S. Lundstrom, and K.A. Emery, "High-Efficiency GaAs Solar Cells Grown by Molecular-Beam Epitaxy," J. Vac. Sci. Technol. B, Vol. 8 , No. 2, 1990, pp. 379-383.

64. Melloch, M.R., S.P. Tobin, C. Bajgar, T.B. Stellwag, A Keshavarzi, M.S. Lundstrom, and K.A. Emery, "High-Efficiency GaAs and AlGaAs Solar Cells Grown by Molecular Beam Epitaxy," 21st IEEE Photovoltaic Specialists Conference, New York: IEEE, 1990, pp. 163-167.

65. Metzdorf, J., T. Wittchen, K. Heidler, K. Denhe, R. Shimokawa, F. Nagamine, H. Ossenbrink, L. Fornarini, M. Davies, K. Emery, and R. DeBlasio, "Objectives and Results of the PEP'87 Round-Robin Calibration of Reference Solar Cells and Modules," 21st IEEE 
Photovoltaic Specialists Conference. New York: IEEE. 1990, pp. $952-$ 959.

66. Mrig L.. "Stability Performance of Thin-Film PV Modules at SERI," presented at the IECEC Conference, Reno, Nevada, 13 Aug. 1990.

67. Mrig, L.C., and S. Rummel, "Outdoor Stability Performance of CIS and CdTe Modules at SERI." 21 lst IEEE Photovoltaic Specialists Conference, New York: IEEE, 1990. pp. 1038-1042.

68. Nelson, A.J., L.L. Kazmerski, M. Engelhardt, and H. Hochst. "Valence Band Electronic Structure of $\mathrm{Zn}_{1_{3}} \mathrm{P}_{22}$ as a Function of Annealing as Studied by Synchrotron Radiation Photoemission." J. Appl. Phys.. Vol. 67, No. 3, 1990, p. 1393.

69. Nelson, A.J., S. Gebhard, A. Rockett, E. Colavita, M. Engelhardt, and H. Hochst, "Synchrotron Radiation Photoemission Study of CdS/ CuInSe $_{2}$ Heterojunction Formation," Phys. Rev. B. Vol. 42, No. 11. 1990, p. 7518.

70. Nelson, A.J., S. Gebhard, L.L. Kazmerski, E. Colavita, M. Engelhardt, and $\mathrm{H}$. Hochst, "Characterization of the Native Oxide of CuInSe, ${ }_{2}$ Using Synchrotron Radiation Photoemission," Appl. Phys. Lett., Vol. 57, No. 14, 1990, p. 1428.

71. Nelson, A.J., "Soft X-ray Photoemission Investigation of the CdS/ CuInSe ${ }_{2}$ Heterojunction Interface." presented at the 10th PV AR\&D Meeting, Lakewood, Colorado, 23-25 Oct. 1990. To be published in Solar Cells.

72. Nelson, A.J., "Formation and Schottky Barrier Height of Au Contacts to CuInSe ${ }_{2}$, presented at the AVS 37th National Vacuum Symposium, Toronto, Canada, 8-12 Oct. 1990, J. Vac. Sci. Technol. A, Vol. 9, No. 3, 1991 .

73. Nelson. A.J.. "AES Line-Shape Analysis at Grain Boundaries of Oriented Grained $\mathrm{YBa}_{2} \mathrm{Cu}_{33} \mathrm{O}_{7}$," presented at the AVS 37th National Vacuum Symposium, Toronto, Canada, 8-12 Oct. 1990, J. Vac. Sci. Technol. A. Vol. 9, No. 3, 1991.

74. Nelson, A.J., M. Engelhardt, and H, Hochst, "Synchrotron Radiation Photoemission Study of the Electronic Structure of the Filled Tetrahedral Semiconductors LiZnAs and LiZnP." presented at the International Conference on Electron Spectroscopy, J. Electron Spectroscopy and Related Phenomena, Vol. 50, No. 2, 1990. 
75. Nelson, A.J., "Soft X-ray Photoemission Investigation of the CdS/ $\mathrm{CuInSe}_{2}$ Heterojunction Interface," presented at the International Conference on Solid Films and Surfaces. Brown University, Providence. R.I. 13-17 Aug. 1990. To be published in Applied Surface Science.

76. Nelson, A., M. Engelhardt, and H. Hochst, "Radiation Photoemission Study of the Electronic Structure of the Filled Tetrahedral Semiconductors LiZnAs and LiZnP," presented at the 4th International Conference on Electron Spectroscopy, J. Electron Spectroscopy and Related Phenomena, Vol. 51, 1990, p. 623.

77. Nozik, A.J., C.A. Parsons, D.J. Dunlavy, B.M. Keyes, and R.K. Ahrenkiel, "Dependence of Hot Electron Luminescence on Barrier Thickness in GaAs/AlGaAs. Superlattices and Multiple Quantum Wells," Solid State Comm., Vol. 75, 1990, p. 297.

78. Osterwald, C.R., and C. Riordan, "Solar Spectral Irradiance, Analysis and Modeling," presented at the 10th PV AR\&D Meeting, Lakewood, Colorado, 23-25 Oct. 1990.

79. Osterwald, C.R., K.A. Emery, D.R. Myers, and R.E. Hart, "Primary Reference Cell Calibrations at SERI, History and Methods," 21 st IEEE Photovoltaic Specialists Conference, New York: IEEE, 1990, pp. 10621067.

80. Partain, L.D., B-C. Ching, G.F. Virshup, J.C. Schultz, H.F. Macmillan, M.S. Kuryla, K. Bertness, M.E. Klausmeier-Brown, M.W. Wanlass, and T.J. Coutts, "Progress Toward a 30\% Efficient Moriolithic, ThreeJunction, Two-Terminal, Concentrator Solar Cell for Space Applications," 2lst IEEE Photovoltaic Specialists Conference, New York: IEEE, 1990.

81. Partain, L.D., D.D. Liu, M.S. Kuryla, R.K. Ahrenkiel, and S.E. Asher, "Improved Method for Measuring Diffusion Length and Interface Recombination Velocity in GaAs Using Two Emitter Fabrications and Guantum Yield," Solar Cells, Vol. 28, 1990, pp.223-232.

82. Pern, J., "Recent Generic Studies of EVA Degradation," presented at the PV Module Reliability Workshop, Lakewood, Colorado, 26 Oct. 1990.

83. Powell, C.J., D.M. Hercules, and A.W. Czanderna, "Comparisons of SIMS, SNMS, ISS, RBS, AES, and XPS Methods for Surface Compositional Analysis," Chapter 7 in Ion Spectroscopies for Surface 
Analysis, eds. A.W. Czanderna and D.M. Hercules, New York: Plenum Press, 1991. See SERI/TP-213-3865, July 1990.

84. Singh, R., A. Kumar, R.P.S. Thakur, P. Chou, J. Narayan, A.R. Srivatsa, A.J. Nelson, and H.S. Ullal, "Solid Phase Epitaxial Growth of II-A Fluorides on InP by in-situ Rapid Isothermal Processing," Amorphous Silicon Technology - 1989. MRS Conference Proceedings. Pittsburgh, PA: MRS, 1989, Vol. 149.

85. Singh, R., Thakıı R.P.S., Katz A., Nelson A.J., Gebhard S.C., and Swartzlander A.B., "Relationship Between Thermal Stress and Structural Properties of $\mathrm{SrF}_{2}$ Films on InP," Appl. Phys. Lett., Vol. 57. No. 12, 1990, p. 1239.

87. Sopori, B.L., C. Marshall, and K.A. Emery, "Mixing Optial Beams for Solar Simulation - A New Approach," 21st IEEE Photovoltaic Specialists Conference, New York: IEEE, 1990, pp. 1116-1121.

88. Sritharan, S., J. Wilson, J. Pankove, A. Nelson, and R. Matson, "Deposition of Diamond-like Carbon (DLC) by Electron Cyclotron Resonance, Radio Frequency (ECR-RF) Hybrid Plasma Technique," presented at the Electrochemical Conference, Montreal, Canada, 610 May 1990.

89. Swartzlander, A., A.J. Nelson. A.R. Mason, L.L. Kazmerski, N. Saxena, C.M. Fortmann, and T.W.F. Russell, "Auger Line Shape and Electron Energy Loss Spectroscopy Analysis of Amorphous, Microcrystalline and $\beta$-SiC," presented at the 16th DOE Surface Studies Conference, Denver, Colorado.

90. Thornton, J.P., R. DeBlasio, and K. Zweibel, "Photovoltaics - Today's Reality. Tomorrow's Promise," Journal of the Association of Energy Engineers, Vol. 87. No. 3, 1990, pp. 63-70.

91. Timmons, M.L., T.S. Colpits, R. Venkatasubramanian, B.M. Keyes, D.J. Dunlavy, and R.K. Ahrenkiel, "Measurement of AlGaAs/AlGaAs Interface Recombination Velocities Using Time-Resolved Photoluminescence," Appl. Phys. Lett., Vol. 56, 1990, p. 1850.

92. Tobin, S.P., S.M. Vernon, C. Bajgar, S.J. Wojtczuk, M.R. Melloch, A. Keshavarzi, T.B. Stell Wag, S. Venkatensan, M.S. Lundstrom, and K.A. Emery, "Assessment of MOCVD- and MBE-Grown GaAs for HighEfficiency Solar Cell Applications," IEEE Transactions on Electron Devices, Vol. 37. No. 2, 1990. p. 469. 
93. Venkatasubramanian R.. M.L. Timmons. J.B. Posthill. P.M. Keyes, and R.K. Ahrenkiel, "High Quality GaAs on Si Using SeCie/Ge Buffer Layers." J. Crvstal Growth. Vol. 107. 1991. pp.489-493.

94. Vernon. S.M., R.K. Ahrenkiel. M.M. Al-Jassim. T.M. Dixon. K.M. Jones. S.P. Tobin. and N.H Karam. "Improvement of Minority-Carrier Properties of GaAs on Si." III-IV Heterostructures for Electronic/ Photonic Devices. MKS Conference Procee lings. Pittsburgh. PA: MRS. 1989. Vol. 145. p. 349.

95. Vernon. S.M.. C.J. Keavney. E.D. Gagnon. N.H. Karam. M.M. AlJassim. N.M. Haegle, V.P. Mazzi. and C.R. Wie. "Improvement of InPGaAs-Si Quality by Thermal Cycle Growth" presented at the MRS Spring Meeting. San Francisco, CA. 16-20 Apr. 1990.

96. Vernon. S.M.. S.P. Tobin. M.M. Al-Jassim. R.K. Ahrenkiel. K.M. Jones. and B.M. Keyes. "Experimental Study of Solar Cell Performance Versus Dislocatiun Density," 2 lst IEEE Photovoltaic Specialists Conference, New York: IEEE. 1990. pp. 211-216.

97. Wanlass. M.W.. T.A. Gessert, G.S. Horner. and T.J. Coutts. "InP/ $\mathrm{As} \ln _{t, 3,5}$ As Monolithic, Two-Junction. Three-Terminal Tandem Solar Cells." Proc of the 10th Space Photovotlaics and Technology Conference (SPRAT). Cleveland: NASA, 1990.

98. Wanlass. M.W., J.S. Ward. K.A. Emery. T.A. Gessert, C.R. Osterwald, and T.J. Coutts. "Hig'i-Performance Concentrator Tandem Solar Cells Based on Infrared-Sensitive Bottom Cells," presented at the 1Oth PV AR\&D Meeting. Lakewood. Colorado. 23-25 Oct. 1990. To be published in Solar Cells.

99. Wanlass. M.W.. J.S. Ward. T.A. Gesser.. K.A. Emery, G.S. Horner, T.J. Coutts. G.F. Virshup. and M.L Ristow. "Development of HighPerformance GalnAsP Solar Ceils for Tandem Solar Cell Application." 21st IEEE Photovoltaic Specialists Conferenc: New York: IEEE. 1990. pp. $172-178$.

100. Yacobi. B.G. and P. Sheldon. "Analysis of Stress Variations in Epitaxial Films Using Cathodoluminescence Microscopy and Spectroscopy." Adranced Metallizations in Microelectronics. MRS Conference Proceedings. Pittsburgh. PA: MRS. 1990. Vol. 181.

101. Pitts. I. R. I. T. Sranley and C. L. Fields. "Solar Induced Surface Transformation of Materials (SISTM)." Proceedings of the Fourth Incernational Simposium on Solar Thermal Technology. Santa Fe. 
NM. 13-17 Jun. 1988, ed. by B. P. Gupta and W. H. Traugott. Hemisphere Pub. Corp.. New York. 1990. p. 459.

102. Stanley. J. T., J. R. Pitts, and C. L. Fields, "Solar Induced Surface Transformation of Steel Samples," Proceedings of the Fifth Annual Northeast Regional Meeting (TMS): Protective Coatings Processing and Characteriscition. 3-5 May 1989. Stevens Institute of Technology. Hoboken. NJ. ed. by R. M. Yazici, Published by TMS, Warrendale. PA. 1990, p. 43.

103. Pitts, J. R., C. L. Fields, J. T. Stanley. and B. L. Pelton, "Materials Processing Using Highly Concentrated Solar Radiation," Proceedings of the 25th Intersociety Energy Conversion Engineering Conference, Reno. NV. Aug. 12-17. 1990, ed. by P. A. Nelson, W. W. Schertz, and R. H. Till. American Institute of Chemical Engineers, New York. Vol. 6. 1990. p. 262.

104. Pitts, J. R.. T. Wendelin. J. T. Stanley, “Applications of Solar Beams for Materials Science and Processing in Space," ibid., Vol. 1. 1990, p. 553.

105. Stanley, J. T., C. L. Fields, and J. R. Pitts. "Surface Treating with Sunbeams." Advanced Materials \& Processes. Dec. 1990, p. 16.

106. Pitts. J. R., J. T. Stanley. E. Tracy, C. L. Fields. "Surface Modification Technologies Using Concentrated Solar Kadiation." Proceedings of the NAS Workshop on Concentrated Solar Photons, 7-9 Nov. 1990. Golden. CO. ed. by K. Araj. NAS Press, Washington, DC. 1991, in press.

107. Jones. K.M..and M.M. Al-Jassim. "TEM Characterization of III-V Semiconductors Heteroepitaxially Grown on Si Substrates." Proceedings of the 47 th Annual Meeting of EMSA. 1989. p. 688

108. Goral. J.P.,and M.M. Al-Jassim. "TEM Studies of Atomic Ordering in Some Termary Semiconductors with Photnvoltaic Applications," Solar Cells. Vol. 27. 1990. p. 429

109. Olson. J.M., A.E. Blakesiee, and M.M. Al-Jassim. "The Morphology of MOCVD-Growin Semiconductor Multilayers." Strained Laver Super:lattices, ed. by R.M. Biefeld. Switzerland: Trans Tech Publications. 1989. pp. 59-82.

110. Pearton. S.I. K T Short A.T. Macrander. C.R. Abernathv. V.P. Mazzi. N.M. Haegel, M.M. Al-Jassim, S.M. Vernon, and V.E. Haven 
"Characterization of InP/GaAs/Si Structures Grown by Atmospheric Pressure MOCVD." J. Appl. Phys., Vol. 65. 1989. p. 1083.

111. Huffman. M., J.P. Goral. M.M. Al-Jassim, A.R. Mason. and K.M. Jones. "Compositional and Microstructural Characterization of Thin Film Lead Zirconate Titanate Ferroelectrics," Thin Solid Films, Vol. 193/194. 1990. pp. 1017-1022.

112. Goral. J.P.. M. Huffman, and M.M. Al-Jassim. "TEM Investigation of the Ferroelectric Domain Structure in Sputtered Lead Zirconate Titanate Thin Films." MRS Symp.. Vol. 200. 1990. p. 225.

113. M.W. Wanlass. G.S. Horner, T.A. Gessert and T.J. Coutts, "Epitaxial InP and Related III-V Com nounds Applied to Solar Cells," Proc. 1st International Conference on Indium Phosphide and Related Materials for Advanced Electronic and Optical Devices, Norman, OK, March 2022. (1989). SPIE, Vol. 1144. p.445

114. M.W. Wanlass. T.A. Gessert, G.S. Horner, K. A. Emery and T. J. Coutts, "InP/ $\mathrm{Ga}_{0.47} \mathrm{In}_{0.53}$ As Monolithic. Two Junction. Three Terminal Tandem Solar Cells", Proc. 10th. NASA Space Photovoltaic Research and Technology, Cleveland. OH. Nov. 7-9. (1989). to be published. 


\section{APPENDIX D}

\section{MAJOR INSTRUMENTATION AND TEST EQUIPMENT}

Table D-2

\section{Major Instrumentation of the Surface and Interface Analysis Laboratory}

Instrument

XPS/Auger

System(Perkin -

Elmer PHI 55)

Scanning Auger

Microprobe

(Perkin-Elmer PHI

590)

Scanıning Auger

Microprobe

(Perkin-Elmer PHI

600)

Ion Microprobes

(Cameca IMS-3f)

\section{Range/Capacity}

$2 \mathrm{~mm}$ spot size

detection

sensitivity of 0.1

at.\%Li to U, $1 \mathrm{~cm} 2$

sample size.

$0.2 \mu \mathrm{m}$ spot size, SAM detection sensitivity of 0.1 at.\% for $\mathrm{Li}$ to $\mathrm{U}$. SIMS detection of $1 \mathrm{ppm}$ for $\mathrm{H}$ to $\mathrm{U}$, $1 \mathrm{~cm}^{2}$ sample size.

$0.02 \mu \mathrm{m}$ spot size. same detection sensitivities as for PHI 590, $1 \mathrm{~cm} 2$ sample size.

10-200 $\mu \mathrm{m}$ beam spot: Cs, O, or Ar primary ions: all elements and isotopes, $\mathrm{H}-\mathrm{U}$, sensitivities of 1 ppm-1 ppb: $1 \mathrm{~cm}^{2}$ sample size.

\section{Unique Features and Uses}

Performs AES, XPS, UPS, EELS in conjunction with depth profiling quantitative elemental analysis and chemical bonding information, angle resolved photoemission.

Performs SAM and SIMS analysis with depth profiling, quantitative elemental analysis and surface compositional maps, up to 5000X niicrographs of surface features.

Performs SAMS and SIMS analysis with depth profiling quantitative elemental analysis and surface compositional maps, up to 50,000X micrographs of surface features. Hot/cold stage.

Performs high-sensitivity profiling and mass scans with 10-nm depth resolution; mass resolution $(\mathrm{M} / \Delta \mathrm{M})=10,000$ lateral ion imaging resolution of 1 $\mu \mathrm{m}$. 


\section{Table D-3}

\section{Major instrumentation of the Materials Characterization Group \\ Instrument \\ Range/Capacity \\ Unique Features and Uses}

Electron probe X ray microanalyzer (EPMA) Cameca MBX

SEM JEOL JSM $35 \mathrm{C}$

SEM JEOL JSM 840

TEM Philips CM 30
EDS and WDS analysis with an accuracy of $\pm 0.5 \%$

1-49 kV. secondary electron imaging (SEI) 5 nm res. backscattered elect.imaging (BEI) $9 \mathrm{~nm}$ res.

$0.2-40 \mathrm{kV}$, liquid helium cathodoluminescen ce (CL) cold stage. Integrated and spectral CL from $10^{\circ} \mathrm{C}$ to $\mathrm{RT}^{\circ} .1$ from $300 \mathrm{~nm}$ to $1.8 \mu \mathrm{m}$.

$\mathrm{KeV}: 300$. resolution: $2.3 \AA$. Tilt: $\pm 60^{\circ}$
Quantitative compositional analysis of all elements heavier than boron

EDS: compositional analysis $(>\mathrm{Na}$ ). EC:: Crystalline type. orientation and quality. EBIC: Microchararterization of the electrical activity of electronic materials, junction location and diffusion length measurement.

Characterize relative impurity concentrations, defect densities and distributions, bandgap (Eg) and subbandgap (defect) luminescence with high resolution and correlation with topography.

Performs structural. analytical and high resolution examina tion of a wide range of materials 
Table D-4

\section{Major Instrumentation of the Device Development Group}

\section{System}

Atmospheric

Pressure Metal

Organic Vapor

Phase Epitaxy
UV/Vis/NIR Spectrophotometer (Beckman Model 5240)

IR

Spectrophotometer (Perkin Elmer Model 580B)

Research Ellipsometer (Rudolph Model 43603-200E)

Production Eliipsometer (Gaertner Model L1 16)

\section{Range/ Capacity}

Unique Features \& Uses

State-of-the-art home-built system using a patented reactor vessel design. Highly uniform epilayers and abrupt heterojunctions have been demonstrated. All III-V alloys from the Ga In-As-P family have been synthesized doped $n$ - or $p$ type. High performance solar cells and custom diagnostic test structures have also been fabricated in these materials.

$180 \mathrm{~nm}-3200 \mathrm{~nm}$

Performs specular transmittance and absorbance measurements. With integrating sphere attachment also measures total or specular reflection.

$180 \mathrm{~nm}-600 \mathrm{~cm}^{-1}$ Measures transmittance in the IR range.

Thickness meas. of $10 \AA$ to $50000 \AA$. Meas. also $\mathrm{N}$ and $\mathrm{k}$.

Thickness measurements of $10 \AA$ to $50000 \AA$. Also measures $\mathrm{N}$ and $\mathrm{k}$.
Precision measurements of thickness and refractive index of highly reflective substrates or thin films.

Rotating analyzer allows automatic operation for use in trend analysis and uniformity studies. 
Major Instrumentation of the Device Development Group, cont.

System

Hall Measurement Apparatus (Alpha Scientific)

Clean Room (Moore and Hanks)

Mask Aligner (Cobilt Model CA 2020)

Vacuum Deposition system (Perkin Elmer ARC ULTEK)

Vacuum Deposition system (Unifilm Multi-source)
Range/ Capacity

Carrier Concentration and mobility measurements for resistivities $10^{-4}$ to 1 $\Omega$-cm.

Approximately 120

ft. ${ }^{2}$ of Class 100 cleaned environment.
Unique Features \& Uses

Measurement of c.c and mobility for semiconductor thin films and bulk material.

Used to perform photolithography procedure for use in chemical etching, plasma treatment and metallization.

$\mathrm{XY}$ alignment of 0.125 $\mu \mathrm{m}$. Equally fine rotational alignment. Ultra-uniform UV exposure.

Base pressure $\approx 10^{-8}$ torr. Thermal resistive evaporation and 2" DCMS.

alignment and exposure of photolithographically treated materials.

Deposition of metals and oxides for soíar cell back and top contacts and ARC's.

Deposition of metals and oxides onto semiconductors and dielectrics for fundamental materials studies and solar cell fabrication. 
Table D-5

\section{Major Instrumentation of the Electro-Optical Characterization Laboratory}

\author{
Instrument \\ Photoluminescence \\ Spectroscopy \\ System
}

Photoluminescence Lifetime System

Diffusion Time-of Flight System

Superconductivity Test Station

Deep Level

Transient

Spectroscopy

(DLTS)

Capacitance-Voltage Measurement

System

\section{Range/Capacity}

CW \& cavity dumped (40 kHz to $40 \mathrm{Mh} z$ ) excitation sources. detection capability from 400 to $1700 \mathrm{~nm}$. sample temperature range of 4 to $310 \mathrm{~K}$.

Cavity dumped (40 $\mathrm{KH} z$ to $40 \mathrm{Mh} z)$ excitation, detection capability from 700 $1700 \mathrm{~nm} .50 \mathrm{ps}$ time resolution, sample temperature range of 4 to $310 K$.

Cavity dumped (40 $\mathrm{kH} z$ to $40 \mathrm{Mh} z$ ) excitation, detection capability from 700 to $1700 \mathrm{~nm}, 45 \mathrm{ps}$ time resolution.

65-300K. 3.2-22.0 $\mu$ A. 1-10 V rms

DLTS spectra and iransients from 77 to $320^{\circ} \mathrm{K}$

Capacitance as a function of voltage over the frequency range $100 \mathrm{~Hz}$ to 100 $\mathrm{MHz}$.

\section{Unique Features and Uses}

Energy resolved photoluminescence spectroscopy.

Time resolved photoluminescence spectroscopy for minority carrier lifetime and surface recombination measurements.

Minority carrier diffusion, lifetime, and surface recombination measurements.

Tc measurement of superconducting materials

Determination of trap ionization energies. emission rates, and capture cross-sections.

Determination of the effective doping concentration. 
Table D-6

Major Test Equipment of the Advanced PV Module Testing and Performance Laboratories.

\section{Instrument}

Li-cor spectroradiometer model Li-1800

Eppley pyranometer (5) model PSP

Eppley pyroheliometer model NIP

Voltage calibrator

ABACUS PV

module/array

simulator

Spire solar simulator model SPI-SUN 240

\section{Range/Capacity}

$300-1100 \mathrm{~nm}$

UV-IR

UV-IR

$1.0 \mathrm{mV}$ to $10.0 \mathrm{~V}$

Up to $10 \mathrm{~kW}$

\section{Unique Features and} Uses

Spectra measurements. portable

Global radiation measurements

Direct normal radiation measurements

Portable

Outdoor testing of advanced PV modules and arrays under actual operating conditions.

One sun - air mass 1.5 Pulsed xenon light accomodates module sizes up to $2 \mathrm{ft}$ by $4 \mathrm{ft}$. source (avojds excessive heat build-up) light intensity can be adjusted from $70 \mathrm{~mW} / \mathrm{cm}^{2}$ to 160 $\mathrm{mW} / \mathrm{cm}^{2}$.

Temperature range:

-65 to $+177^{\circ} \mathrm{C}$, $\pm 0.25^{\circ} \mathrm{C}$ relative humidity, $20 \%$ to $95 \%$, $\pm 2 \%$, chamber working volume; $31 \mathrm{ft} 3$.

Hughes probeye IR video system, Temperature: $0^{\circ} \mathrm{C}$ to $950^{\circ} \mathrm{C}, \pm 5 \%{ }^{\circ} \mathrm{C}$.
Environmentally of sizes up to 2 by $4 \mathrm{ft}$. conditions PV modules
Thermal mapping of planar and other surfaces 


\section{Major Test Equipment of the Advanced PV Module Testing and \\ Performance Laboratories, cont.}

Polaroid MP-4

Multipurpose

Camera System

Associated

Research Inc., DC

HYPOT Tester

model No. 05220A.
Magnification X26 Versatile

0 to $15 \mathrm{kV} \mathrm{DC}$ at 15 mA max.
DC voltage insulation and current leakage measurements. 


\section{Table D-7}

\author{
Instrument \\ Spectrolab X25 \\ with multi-source \\ attachment \\ Current vs. \\ voltage (I-V) \\ measurement \\ system
}

Major Instrumentation of the Cell Performance Laboratory

Spectral Response Measurement system

PV calibration system

LICOR

Spectroradiometer

\section{Range/Capacity}

$100 \mathrm{Wm}^{-2}$ to 20.000 $\mathrm{Wm}^{-2}$, user controlled spectral and total irradiance

\pm 50 to $\pm 1 \mu \mathrm{V}, \pm 8 \mathrm{~A}$ to \pm 1 pA. $0^{\circ} \mathrm{C}$ to $110^{\circ} \mathrm{C}$. voltage bias rates from steady state to $200 \mathrm{~V} / \mathrm{sec}$
Unique Features and Uses

Solar Simulation under standard or user defined reporting conditions

Efficiency measurements, secondary reference cell calibration. temperature coefficients,diode parameters, I-V measurement artifacts

$300-2,000 \mathrm{~nm}$ expandable to 10,000 nin, light bias to $\sim 5$ suns, voltage bias to $\pm 40 \mathrm{~V}$, current range $1 \mathrm{pA}$ to 4 A, up to $15 \mathrm{~cm}$ diameter monochromatic beam

2 Newtonian trackers, 4 samples at a time biased to I $\mathrm{sc}$, temperature control and monitoring 40 samples at a time if Isc and total irradiance measured separately and under global sunlight

$300-1100 \mathrm{~nm}, 4 \mathrm{~nm}$ resolution, global light (integrating sphere), direct normal light $\left(5.0^{\circ}\right.$ field of view), teflon dome diffuser, fiber optic probe
Quantum efficiency, device parameters
Primary calibration of solar cells under direct or global sunlight; or modules under global light (fixed tilt or normal incidence

required for efficiency measurements and calibrations, simulator spectral characterization 
Major Instrumentation of the Cell Performance Laboratory, cont.

IIP1000 computer,

Macintosh

computers

Large Area Pulsed Solar Simulator
Control of

Measurement

systems, data base

management

$2 \mathrm{~m}$ by $2 \mathrm{~m}$ area at 1000

$\mathrm{Wm}^{2}, \pm 100 \mu \mathrm{A}$ to $\pm 13 \mathrm{~A}$ full scale current, $\pm 1 \mathrm{~V}$

to $\pm 70 \mathrm{~V}$ voltage range
Module

measurements, detect and I-V artifacts related to the pulsed light source 
Table D-8a

Major Instrumentation of the Surface Interactions, Modification, and Stability Group

\section{Instrument}

XPS/ISS/SIMS FAB SIMS/AES

(Leybold LHS-10

System)

Scanning Auger

Microprobe

(Physical

Electronics 545 C)

\section{Range/Capacity}

XPS: $2 * 10 \mathrm{~mm}$ area;ISS: 0.5 to $2 \mathrm{~mm}$ spot size: SIMS: 0.5 to $2 \mathrm{~mm}$ spot size; AES: $1 \mu \mathrm{m}$ spot size. Detection sensitivities of 0.1 to 1 at. $\%$ for XPS, AES, and ISS; of ca. $10-4$ at. $\%$ for SIMS

$3.0 \mu \mathrm{m}$ minimum size for electron beam; detection sensitivity of 0.1 to 1.0 at. $\%$ for $\mathrm{Li}$ to $\mathrm{U}$

\section{Unique Features and Uses}

Depth profiling with a 1 $\mathrm{mm} \leq$ to $1 \mathrm{~cm} \leq$ raster range. Thin film deposition with $\mathrm{QC}$ monitor in a preparation chamber. Translation of sample on heatable (to $800^{\circ} \mathrm{C}$ ) - coolable (to $196^{\circ} \mathrm{C}$ ) rods from preparation or high pressure chambers (to $10 \mathrm{~atm}$ ) into the analysis chamber. Four different surface analysis probes on one instrument. FAB source for FAB-SIMS

AES or SAM analysis while depth profiling. Rapid load lock and multiple sample carousel permits high throughput for routine analyses 
Table D-8b

Major Instrumentation of the Surface Interactions, Modification, and Stability Group

\section{Instrument}

Buartz Crystal

Micro balance

System (Sycon -

SERI)

Beam

Microbalance

(Sartorius 4300)

Residual Gas

Analyzers (Inficon

IQ-200)
Range/Capacity

Range of $106 \mathrm{ng} / \mathrm{cm}^{2}$; detection sensitivity of $1 \mathrm{ng} / \mathrm{cm}^{2}$; practical capacity for hydrophilic polymers is about $1.5 \mathrm{mg}$; for other solids, $100 \mathrm{mg}$; T from 15 to $75^{\circ} \mathrm{C}$; $\mathrm{P}$ from vacuum to 1500 torr

Capacity of 3 to $5 \mathrm{~g}$; detection sensibility of 0.1 to $1 \mu \mathrm{g}$; measuring range of $400 \mathrm{mg}$; T from - 196 to $1000^{\circ} \mathrm{C}$. $\mathrm{P}$ from vacuum to 1500 torr

1-500 a.m.u. with unit mass resolution; less resolution up to 1000 a.m.u.
Unique Features and Uses

Measure adsorption/ desorption of gases on solid overlayers adherent to a gold coated quartz crystal; mass gain or loss during oxidation or reduction; permeation rates of gases through solids up to $100 \mu \mathrm{m}$ thick. Especially good for water vapor sorption studies

Same applications as QCM. Samples (suspended by fibers) can be monitored for outgassing and desorption with an RGA

Monitor gases present or evolved during processing samples in the LHS- 10 or QCM systems 
Table D-8c

Major Instrumentation of the Surface Interactions, Modification, and Stability Group

Instrument

Deposit Thickness

Monitor (Inficon

IC-6000)
Metallograph

(LECO Neophot

21)

Solar Furnace

(SERI)
Range/Capacity

Measure deposition rates $0.05 \mathrm{~nm} / \mathrm{min}$ to over $10 \mathrm{~nm} / \mathrm{s}$ and deposited thicknesses to $10,000 \mathrm{~nm}$ to less than $1 \%$ accuracy

Mag.: $80 \mathrm{X}$ to $2000 \mathrm{X}$ Polarization Quantinet 800 Image Analysis

$10 \mathrm{~kW}$ Maximum Flux $240 \mathrm{~W} / \mathrm{cm} \leq 40 \mathrm{~mm}$ FWHM

\section{Unique Features and Uses}

Monitor vacuum deposited overlayer thicknesses in the LHS 10 preparation chamber for preparing clean metal deposits and subsequent XPS/ISS/SIMS/ AES analysis without exposure to air

Optical microscopy with or without polarized light, Nomarski, prints/ slides and image analysis

Long focal length in pri mary concentrators ( 600 in.). Capable of inserting secondary concentrator to boost flux to the range of 5000 $\mathrm{W} / \mathrm{cm}^{2}$. Fully automated operation and data acquisition 


\title{
Table D-8d
}

\author{
and Stability Group \\ Instrument \\ Profilometers \\ (Tencor alpha-step \\ and Sloan Dektak) \\ Range/Capacity \\ Measurement Range \\ from $100 \mathrm{~nm}$ full \\ scale to $100 \mu \mathrm{m}$ full \\ scale
}

Major Instrumentation of the Surface Interactions, Modification,

Oriel Model 6732

Solar Simulator

Lambda-Physik EMG-50 Excimer Laser and FL-2000 Dye Laser

Polaron Series E6000 Vacuum Coater
Unfiltered spectral output from 0.25 to 2.5 microns at about 17 solar constants (collimated 2"2" output beam)

Monochromatic, coherent output, with wavelengths variable between 197-950 nm (1 $\mathrm{cm}^{2}$ output beam)

$10^{-7}$ torr vacuum capability, sputter or evaporative coating

\section{Unique Features and Uses}

Measures step heights and surface topography with $\pm 1 \mathrm{~nm}$ sensitivity. Auto-level, microscope $\mathrm{X}-\mathrm{Y}$ translation stage for sample positioning 12.5 $\mu \mathrm{m}$ and $5 \mu \mathrm{m}$ diameter tips available

Equipped with filters and dichroic reflectors to limit spectral output to a desired range for photo chemical studies

Complements the solar simulator in photo chemical studies. Excimer is currently set up for $308 \mathrm{~nm}$ output, and dye laser is now tuneable between 260 and $320 \mathrm{~nm}$

Used to deposit thin films (usually of metals) onto smooth substrates. Typical use is to prepare IR-reflective, metal coated substrates for IR external reflection spectroscopy 
Table D-9

Major Instrumentation of the FTIR Spectroscopic Research and Service Center

\section{Instrument}

Nicolet System

800 high -

resolution mid -

infrared FTIR

spectro -

photometer

Nicolet FT-Raman accessory

Nicolet Nic-Plan

FTIR

microscope

Nicolet System

510 medium resolution mid infrared FTIR

spectro-

photometer

Nicolet System 20F medium resolution far infrared FTIR

spectro -

photometer

\section{Range/Capacity}

Frequency range 7800 to $500 \mathrm{~cm}^{-1}$, resolution $0.1 \mathrm{~cm}^{-1}$. sensitivity $10^{-5}$ OD.

\section{Unique Features and Uses}

Collects high-resolu tion, low-noise mid infrared spectra of solid and liquid samples in transmit tance, specular and diffuse reflectance, and attenuated total reflection modes. Also used with FT-Raman accessory.

Frequency range $3800-$ $150 \mathrm{~cm}^{-1}$ Stokes.

Minimum sample size $10 \times 10 \mu \mathrm{m}$, transla tional resolution $1 \mu \mathrm{m}$.

Frequency range 7000 to $400 \mathrm{~cm}^{-1}$ (standard samples). 7800-650 $\mathrm{cm}^{-1}$ (FTIR micro scope), resolution 0.20 $\mathrm{cm}^{-1}$.

Frequency range 650 $20 \mathrm{~cm}^{-1}$, resolution $0.7 \mathrm{~cm}^{-1}$.
Collects Raman spectra of solid and liquid samples in reflective and refractive modes.

Collects mid-in frared spectra of small-area solid samples in reflec tance \& transmittance modes, used with System 510.

Primarily used with FTIR microscope, but has sample compart ment for mid-in frared transmittance analysis.

Collects far-in frared spectra of solid samples in transmit tance and diffuse reflectance modes. 
Major Instrumentation of the FTIR Spectroscopic Research and Service Center, cont.

Instrument

Hansen High-Tran cryostatic sample mount
Nicolet $7199 \mathrm{~b}$

high-resolution mid-in frared FTIR spectrophoto meter

\section{Range/Capacity}

Sample temperature control range 8.0 $450 \mathrm{~K}$.

\section{Unique Features \&} Uses

Provides temperature and illumination control for solid samples during in frared transmittance analysis in vacuo. Used with Systems 20F and 800.

Frequency range 7800 - Collects high resolu $500 \mathrm{~cm}^{-1}$, expandable to $25000-100 \mathrm{~cm}^{-1}$, resolution $0.06 \mathrm{~cm}^{-1}$, sensitivity $10^{-5} \mathrm{OD}$. tion, low-noise mid infrared spectra of solia and liquid samples in transmit tance, specular reflec tance, and attenuated total reflection modes. 


\section{APPENDIX E}

\section{GLOSSARY OF ACRONYMS}

$\begin{array}{ll}\text { ADM } & \text { Advanced Desiccant Material } \\ \text { ADP } & \text { Advanced Desiccant Polymer } \\ \text { AES } & \text { Auger Electron Spectroscopy } \\ \text { AEQE } & \text { Absolute External Quantum Efficiency } \\ \text { AM } & \text { Air Mass } \\ \text { APMOVPE } & \text { Atmospheric Pressure Metal Organic Vapor Phase Epitaxy } \\ \text { AMPSA } & \text { 2-Acrylamide-2-Methyl-1-Propane Sulfonic Acid } \\ \text { ARC } & \text { Antireflection Coating } \\ \text { ASCII } & \text { Decimal computer format } \\ \text { ASTM } & \text { American Society for Testing and Materials } \\ \text { ATR } & \text { Attenuated Total (internal) Reflection } \\ \text { AVC } & \text { Auger Voltage Constant } \\ \text { AWU } & \text { Associated Western Universities } \\ \text { BEI } & \text { Backscattered Electron Imaging } \\ \text { CDCS } & \text { Commercial Desiccant Cooling System } \\ \text { CEM } & \text { Charge Collection Microscopy } \\ \text { CL } & \text { Cathodoluminescence } \\ \text { CV } & \text { Current-Voltage or Capacitance-Voltage } \\ \text { DCS } & \text { Desiccant Cooling System } \\ \text { DDF } & \text { Director's Development Fund } \\ \text { DH } & \text { Double Heterostructure } \\ \text { DLTS } & \text { Deep Level Transient Spectroscopy } \\ \text { DOE } & \text { Department of Energy } \\ \text { DR } & \text { Diffuse Reflectance } \\ \text { EBIC } & \text { Electron Beam Induced Current } \\ \text { EBIV } & \text { Electron Beam Induced Voltage } \\ \text { EC } & \text { Electrochromic } \\ \text { EC } & \text { Electronchanneling } \\ \text { ECR } & \text { Electron Cyclotron Resonance } \\ \text { ECW } & \text { Electrochromic Windows } \\ \text { E } & \text { Bandgap Energy } \\ \text { EDS } & \text { Energy Dispersive Spectroscopy } \\ \text { EELS } & \text { Electron Energy Loss Spectroscopy } \\ \text { EL } & \text { Electroluminescence } \\ \text { EMT1 } & \text { Emerging Technology 1 } \\ \text { EOL/BOL } & \text { End of Life/Beginning of Life } \\ \text { EPMA } & \text { Electron Probe Microanalysis } \\ \text { EVA } & \text { Ethylene Vinyl Acetate } \\ \text { FA } & \text { Fluorescence Analysis } \\ \text { FAB } & \text { Fast Atom Bombardment } \\ & \end{array}$




\begin{tabular}{|c|c|}
\hline $\mathrm{FF}$ & Fill Factor \\
\hline FFT & Fast Fourier Transform \\
\hline FT-IR & Fourier Transform Infrared \\
\hline FWHM & Full Width at Half Maximum \\
\hline GB & Grain Boundary \\
\hline GPE & General Purpose Equipment \\
\hline HAZ & Heat Affected Zones \\
\hline HFSF & High Flux Solar Furnace Facility \\
\hline HREM & High Resolution Electron Microscopy \\
\hline HTSC & High Temperature Superconductor \\
\hline HTPB & Hydroxyl-Terminated Polybutadiene \\
\hline IEC & Institute of Energy Conversion \\
\hline IECEC & Intersociety Energy Conversion Engineering Conference \\
\hline IEEE & Institute of Electrical and Electronic Engineers \\
\hline IR & Infrared \\
\hline IS & Metal Oxide Species \\
\hline ISS & Ion Scattering Spectrometry \\
\hline ITO & Indium Tin Oxide \\
\hline $\mathrm{I}-\mathrm{V}$ & Current Versus Voltage \\
\hline$J_{c}$ & Critical Current \\
\hline$J_{\mathrm{sc}}$ & Short Circuit Current \\
\hline JEBIC & Junction Electron Beam Induced Current \\
\hline JPL & Jet Propulsion Laboratory \\
\hline LPE & Liquid Phase Epitaxy \\
\hline MBE & Molecular Beam Epitaxy \\
\hline $\mathrm{MCP}$ & Microchannel Plate \\
\hline MEE & Migration Enhanced Epitaxy \\
\hline MMI & Meissner Mutual Inductance \\
\hline MOCVD & Metal Organic Chemical Vapor Deposition \\
\hline MQW & Metallization of Multiple Quantum Well \\
\hline MRS & Materials Research Society \\
\hline MUA & 11-Mercaptoundecanoic \\
\hline NIST & National Institute for Standards and Technology \\
\hline NMAB & National Materials Advisory Board \\
\hline NOCT & Nominal Operating Cell Temperature \\
\hline OFG & Organic Functional Groups \\
\hline OMA & Organized Molecular Assembly \\
\hline PEP & Photovoltaic Energy Project \\
\hline photoCVD & Photo Chemical Vapor Deposition \\
\hline PMT & Photomultiplier Tube \\
\hline PSSA & Polystyrene Sulfonic Acid \\
\hline PV & Photovoltaic \\
\hline PVUSA & Photovoltaics for Utility Scale Application \\
\hline PL & Photoluminescence \\
\hline PZT & Lead Zirconate Titanate \\
\hline
\end{tabular}




$\begin{array}{ll}\text { QCM } & \text { Quartz Crystal Microbalance } \\ \text { QC } & \text { Quartz Crystal } \\ \text { QE } & \text { Quantum Efficiency } \\ \text { QMA } & \text { Quadrupole Mass Analysis } \\ \text { R vs T } & \text { Resistance vs Temperature } \\ \text { RF } & \text { Radio Frequency } \\ \text { RFP } & \text { Request for Proposal } \\ \text { RGA } & \text { Residual Gas Analysis } \\ \text { RH } & \text { Relative Humidity } \\ \text { RHEED } & \text { Reflection High Energy Electron Diffraction } \\ \text { RIO } & \text { RHEED Intensity Oscillation } \\ \text { RTD } & \text { Resistance Temperature Device } \\ \text { SA } & \text { Self Assembly } \\ \text { SAIC } & \text { Science Applications International Corporation } \\ \text { SAM } & \text { Scanning Auger Microscopy } \\ \text { SEI } & \text { Secondary Electron Imaging } \\ \text { SEIA } & \text { Solar Energy Industries Association } \\ \text { SEM } & \text { Scanning Electron Microscopy } \\ \text { SHJ } & \text { Shallow Homojunction } \\ \text { SHS } & \text { Self-Propogating, High-Temperature Synthesis } \\ \text { SIMS } & \text { Secondary Ion Mass Spectrometry } \\ \text { SIMSG } & \text { Surface Interactions, Modifications and Stability Group } \\ \text { SISTM } & \text { Solar Induced Surface Transformation of Materials } \\ \text { S/N } & \text { Signal to Noise Ratio } \\ \text { SR } & \text { Specular Reflectance } \\ \text { SRC } & \text { Synchrotron Radiation Center } \\ \text { SRM } & \text { Solid Rocket Motor } \\ \text { SSTM } & \text { Spectroscopic Scanning Tunneling Microscopy } \\ \text { STEM } & \text { Scanning Transmission Electron Microscope } \\ \text { STM } & \text { Scanning Tunneling Microscopy } \\ \text { SWRES } & \text { Southwest Residential } \\ \text { TAC } & \text { Time Amplitude Converter } \\ \text { TAG } & \text { Technical Advisory Group } \\ \text { T. } & \text { Critical Temperature } \\ \text { TCO } & \text { Transparent Conducting Oxide } \\ \text { TEM } & \text { Transmission Electron Microscopy } \\ \text { THF } & \text { Teuahydrofuran } \\ \text { TMA } & \text { Trimethylaluminum } \\ \text { TOF } & \text { Time of Flight } \\ \text { TRC } & \text { Technical Review Committee } \\ \text { UHV } & \text { Ultra High Vacuum } \\ \text { UPS } & \text { Ultraviolet Photoelectron Spectroscopy } \\ \text { US1 } & \text { Utility Scale } \\ & \end{array}$




$\begin{array}{ll}\text { UV } & \text { Ultraviolet } \\ \mathrm{V}_{\text {w. }} & \text { Open Circuit Voltage } \\ \text { VB } & \text { Valence Band } \\ \text { VBM } & \text { Valence Band Maximum } \\ \text { VHV } & \text { Very High Vacuum } \\ \text { WDS } & \text { Wavelength Dispersive Spectroscopy } \\ \text { WFO } & \text { Work for Others } \\ \text { XPS } & \text { X-ray Photoelectron Spectroscopy } \\ \text { XRD } & \text { X-ray Diffraction }\end{array}$




\begin{tabular}{|c|c|c|c|}
\hline $\begin{array}{l}\text { Document Control } \\
\text { Page }\end{array}$ & $\begin{array}{l}\text { 1. SERI Roport No. } \\
\text { SERI/TP-213-4461 }\end{array}$ & $\begin{array}{l}\text { 2. NTIS Accession No. } \\
\text { DE92001201 }\end{array}$ & 3. Recipient's Accession No. \\
\hline \multirow{2}{*}{\multicolumn{3}{|c|}{$\begin{array}{l}\text { 4. Title and Subtitle } \\
\text { FY } 1990 \text { Applied Sciences Branch Annual Report }\end{array}$}} & $\begin{array}{l}\text { 5. Publication Date } \\
\text { November } 1991\end{array}$ \\
\hline & & & 6. \\
\hline \multicolumn{3}{|c|}{$\begin{array}{l}\text { 7. Author(s) } \\
\text { B.M. Keyes and P.C. Dippo, Editors }\end{array}$} & 8. Pertorming Organization Rept. No. \\
\hline \multirow{2}{*}{\multicolumn{3}{|c|}{$\begin{array}{l}\text { 9. Performing Organization Name and Address } \\
\text { National Renewable Energy Laboratory } \\
1617 \text { Cole Boulevard } \\
\text { Golden, Colorado } 80401-3393\end{array}$}} & $\begin{array}{l}\text { 10. ProjectTask/Work Unit No. } \\
\text { PV211301 }\end{array}$ \\
\hline & & & $\begin{array}{l}\text { 11. Contract (C) or Grant (G) No. } \\
\text { (C) } \\
\text { (G) }\end{array}$ \\
\hline \multirow{2}{*}{\multicolumn{3}{|c|}{ 12. Sponsoring Organization Name and Address }} & $\begin{array}{l}\text { 13. Type of Report \& Period Covered } \\
\text { Technical Report }\end{array}$ \\
\hline & & & 14. \\
\hline \multicolumn{4}{|c|}{ 15. Supplementary Notes } \\
\hline \multicolumn{4}{|c|}{$\begin{array}{l}\text { 16. Abstract (Limit: } 200 \text { words) } \\
\text { The Applied Sciences Branch of the National Renewable Energy Laboratory (NREL) provides comprehensive } \\
\text { photovoltaic (PV) materials, devices, characterization, measurement, fabrication, modeling research, and support } \\
\text { for the international PV research community in the context of the U.S. Department of Energy's PV Research } \\
\text { Program goals. This report summarizes the progress of the Branch from } 31 \text { January } 1990 \text { through } 31 \text { January } \\
\text { 1991. The eight technical sections present a succinct overview of the capabilities and accomplishments of each } \\
\text { group in the branch. The branch is comprised of the following groups: Surface and Interface Analysis; Materials } \\
\text { Characterization; Advanced PV Module Performance and Reliability Research; Cell Performance Characterization; } \\
\text { Surface Interactions, Modification, and Stability; and FTIR Spectroscopic Research. The report highlights the } \\
\text { main research projects completed in FY } 1990 \text { including measurements and tests of PV materials, cells, } \\
\text { submodules, and modules. The report contains a comprehensive bibliography of the more than } 110 \text { branch- } \\
\text { originated journal and conference publications which were authored in collaboration with, or in support of, } \\
\text { approximately } 130 \text { university, industrial, government, and in-house research groups. }\end{array}$} \\
\hline \multicolumn{4}{|c|}{$\begin{array}{l}\text { 17. Document Analysis } \\
\text { 8. Descriptors } \\
\text { Photovoltaics ; photovoltaic measurements ; materials testing ; modeling research } \\
\text { b. Identifiers/Open-Ended Terms }\end{array}$} \\
\hline \multicolumn{4}{|l|}{$\begin{array}{l}\text { c. UC Categories } \\
270\end{array}$} \\
\hline \multirow{2}{*}{\multicolumn{2}{|c|}{$\begin{array}{l}\text { 18. Availability Statement } \\
\text { National Technical Information Service } \\
\text { U.S. Department of Commerce } \\
\text { 5285 Port Royal Road } \\
\text { Springfield, VA } 22161\end{array}$}} & & \begin{tabular}{|l|} 
19. No. of Pages \\
299 \\
\end{tabular} \\
\hline & & & $\begin{array}{l}\text { 20. Price } \\
\text { A09 }\end{array}$ \\
\hline
\end{tabular}



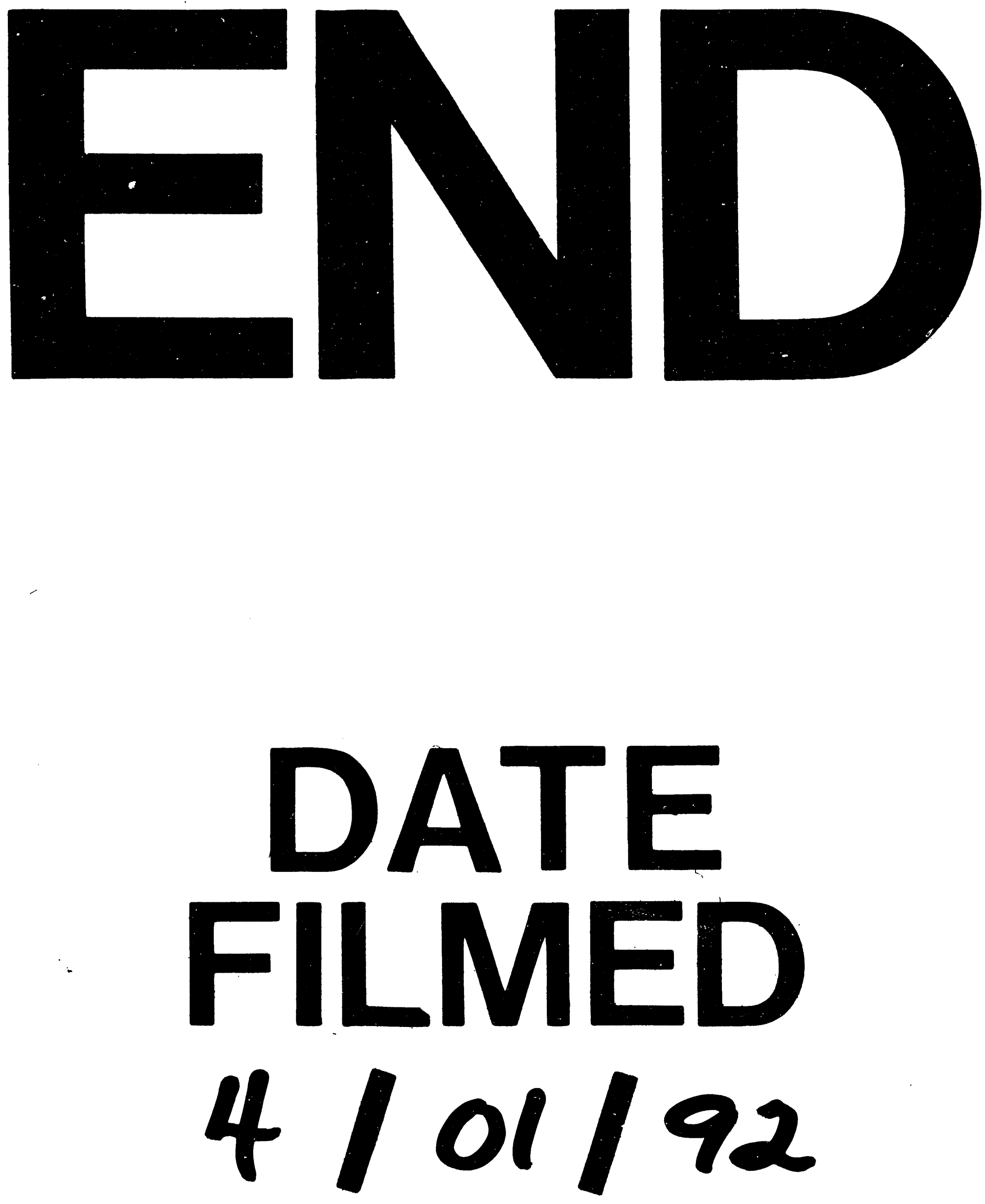